Proceedings of the

\title{
$57^{\text {th }}$ Annual Meeting
}

of the

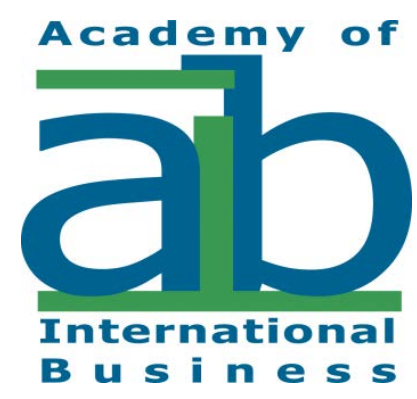

\section{Academy of International Business}

"Global Networks:Organizations and People"

Bengaluru, India

June 27-30, 2015

Editors

Ram Mudambi, Program Chair

Tunga Kiyak, AIB Managing Director

(c) 2015 Academy of International Business 
Proceedings of the $57^{\text {th }}$ Annual Meeting of the

Academy of International Business "Global Networks: Organizations and People" Bengaluru, India June 27-30, 2015

ISSN: 2078-0435

(C) 2015 Academy of International Business

For more information, please contact:

AIB Executive Secretariat

G. Tomas M. Hult, Executive Director, or

Tunga Kiyak, Managing Director Eppley Center 465 N. Shaw Ln Rm 7 Michigan State University East Lansing, MI 48824, USA

Phone: +1 (517) 432-1452 • Fax: +1 (517) 432-1009 E-mail: aib@aib.msu.edu•Web: http://aib.msu.edu/ 


\section{TABLE OF CONTENTS}

Program Acknowledgements......................3

Program Overview................................... 4

Meeting Sponsors.................................... 6

Abstracts ................................................... 7

Saturday Abstracts............................... 9

Sunday Abstracts................................ 10

Monday Abstracts ...............................8

Tuesday Abstracts.............................. 179

Index of Program Contributors............... 251 
(This page is intentionally blank) 


\title{
2015 PROGRAM ACKNOWLEDGEMENTS
}

\author{
PROGRAM CHAIR \\ Ram Mudambi - Temple University
}

PROGRAM CHAIR'S ASSISTANT

T.J. Hannigan - Temple University

TRACK CHAIRS

Kwok Leung - Chinese University of Hong Kong

Erkko Autio - Imperial College Business School

Mary Teagarden - Thunderbird School of Global Management

Simona lammarino - London School of Economics

Susan Mudambi - Temple University

Lucia Piscitello - Politecnico di Milano

L. Felipe Monteiro - INSEAD

Siva Viswanathan - University of Maryland

Akbar Zaheer - University of Minnesota

Gurneeta Singh - University of Minnesota

Ronaldo Parente - Florida International University

Florian Taube - Université libre de Bruxelles

Bjorn Ambos - University of St Gallen

Nandini Rajagopalan - University of Southern California

Nan Jia - University of Southern California

Michael Witt - INSEAD, Singapore

Rene Belderbos - KU Leuven

David Reeb - National University of Singapore

Andreas Schotter - Western University

PRE-CONFERENCE PROGRAM COORDINATOR

Charles Dhanaraj - IMD

DOCTORAL CONSORTIUM CHAIRS

Stewart Miller - University of Texas San Antonio

Shameen Prashantham - Nottingham University Business School China

JUNIOR FACULTY CONSORTIUM CHAIRS

Ruth Aguilera - Northeastern University

Ravee Chittoor - Indian School of Business

RESEARCH CAPACITY WORKSHOP CHAIRS

Jose de la Torre - Florida International University Rishikesh T. Krishnan - Indian Institute of Management Indore Sougata Ray - Indian Institute of Management Calcutta

AIB/JIBS PAPER DEVELOPMENT WORKSHOP

Petra Christmann - Rutgers University, USA

John Cantwell - Rutgers University, USA

Alexandra Vo - Rutgers University, USA

PLACEMENT SERVICES DIRECTOR

di Alhorr aint Louis Univ i , USA

BUCKLEY AND CASSON AIB DISSERTATION AWARD SELECTION COMMITTEE

Mark Casson - University of Reading (Chair)

Anupama Phene - George Washington University

Rebecca Piekkari - Aalto University

Sumit Kundi - Florida International University

TEMPLE/AIB BEST PAPER SELECTION COMMITTEE

Peter Liesch - University of Queensland (Chair)

Elizabeth Rose - University of Otago

Aya Chacar - Florida International University

Ivo Zander - Uppsala University

HAYNES PRIZE SELECTION COMMITTEE

Jane Lu Wenzhen - University of Melbourne (Chair)

Jennifer Oetzel - American University

Olli Kuivalainen - Lappeenranta University of Technology

Klaus Meyer - China Europe International Business School

HOST INSTITUTION

Indian Institute of Management, Bangalore

LOCAL HOST COMMITTEE CHAIR

S. Raghunath - IIM, Bangalore 
2015 PROGRAM OVERVIEW

June 27-30, 2015

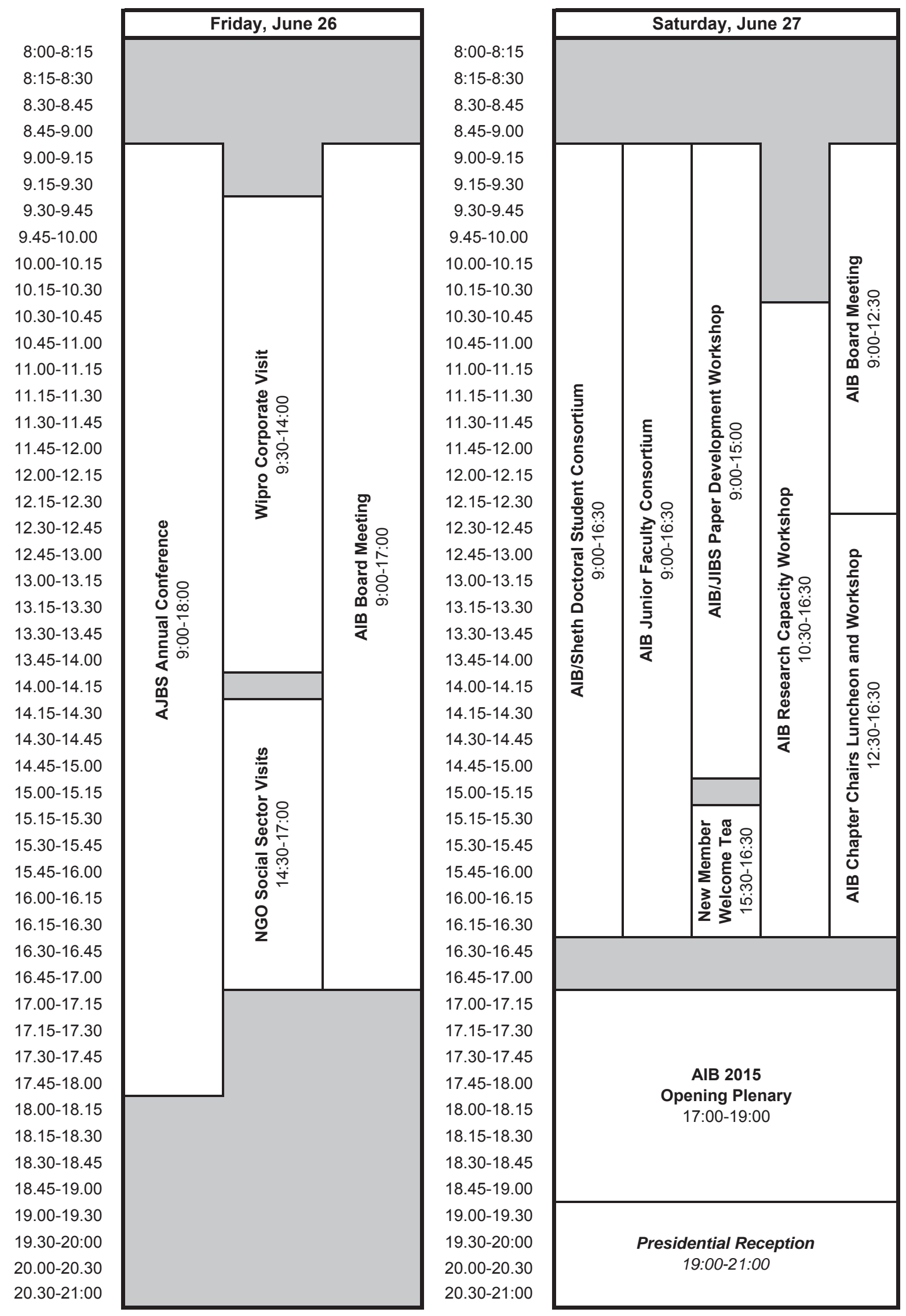




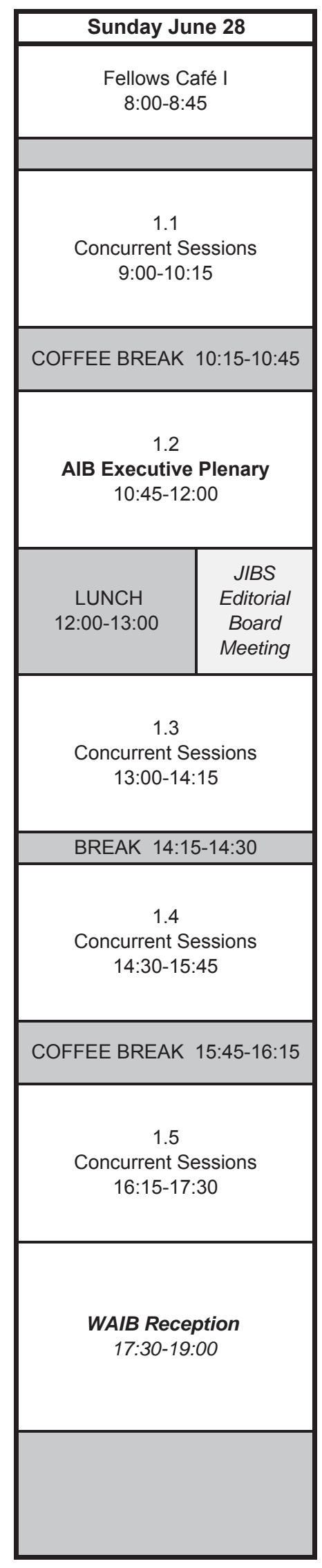

\begin{tabular}{|c|}
\hline Monday, June 29 \\
\hline $\begin{array}{c}\text { Fellows Café II } \\
\text { 8:00-8:45 }\end{array}$ \\
\hline $\begin{array}{c}2.1 \\
\text { Concurrent Sessions } \\
\text { 9:00-10:15 }\end{array}$ \\
\hline
\end{tabular}

$11.45-12.00$

$12.00-12.15$

$12.15-12.30$

12.30-12.45

$12.45-13.00$

13.00-13.15

$13.15-13.30$

13.30-13.45

$13.45-14.00$

14.00-14.15

$14.15-14.30$

14.30-14.45

$14.45-15.00$

15.00-15.15

$15.15-15.30$

15.30-15.45

15.45-16.00

$16.00-16.15$

$16.15-16.30$

16.30-16.45

16.45-17.00

17.00-17.15

$17.15-17.30$

17.30-17.45

$17.45-18.00$

18.00-18.15

18.15-18.30

18.30-18.45

$18.45-19.00$

19.00-19.30

19.30-20:00

20.00-20.30

20.30-21:00
COFFEE BREAK 10:15-10:45

$$
2.2
$$

Eminent Scholar Session

and Concurrent Sessions 10:45-12:00

8:00-8:15

8:15-8:30

8.30-8.45

$8.45-9.00$

$9.00-9.15$

$9.15-9.30$

9.30-9.45

9.45-10.00

10.00-10.15

10.15-10.30

10.30-10.45

10.45-11.00

11.00-11.15

11.15-11.30

11.30-11.45

11.45-12.00

12.00-12.15

12.15-12.30

12.30-12.45

12.45-13.00

13.00-13.15

$13.15-13.30$

13.30-13.45

13.45-14.00

14.00-14.15

14.15-14.30

14.30-14.45

14.45-15.00

15.00-15.15

$15.15-15.30$

15.30-15.45

15.45-16.00

16.00-16.15

16.15-16.30

2.5

Educator of the Year Session and Concurrent Sessions

16:15-17:30

16.30-16.45

$16.45-17.00$

$17.00-17.15$

$17.15-17.30$

17.30-17.45

$17.45-18.00$

18.00-18.15

$18.15-18.30$

18.30-18.45

$18.45-19.00$

19.00-19.30

19.30-20:00

20.00-20.30

20.30-21:00

\begin{tabular}{|c|}
\hline Tuesday, June $\mathbf{3 0}$ \\
\hline $\begin{array}{c}\text { Fellows Café III } \\
8: 00-8: 45\end{array}$ \\
\\
3.1 \\
Concurrent Sessions \\
9:00-10:15 \\
\hline COFFEE BREAK 10:15-10:45 \\
\\
Concurrent Sessions \\
10:45-12:00 \\
\\
\hline LUNCH \\
\hline $12: 00-13: 00$ \\
\hline
\end{tabular}

JIBS Decade Award

and Concurrent Sessions 13:00-14:15

BREAK 14:15-14:30

3.4

Concurrent Sessions 14:30-15:45

COFFEE BREAK 15:45-16:15

3.5

AIB Awards Ceremony and Business Meeting 16:15-17:45

AIB Farewell Reception 17:45-19:00 


\section{AIB 2015 CONFERENCE SPONSORS}

The Academy of International Business thanks the following sponsors for making the 2015 Bengaluru Conference possible through their generous contributions.

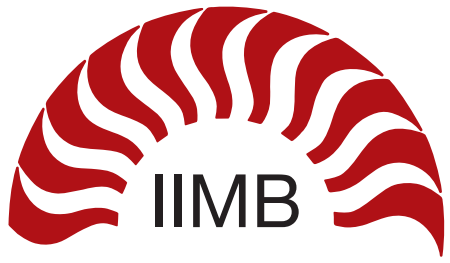

तेजस्वि नावधीतमस्तु $\frac{\text { MICHIGAN STATE }}{\text { U N I V E R S I T Y }}$

Broad College of Business International Business Center

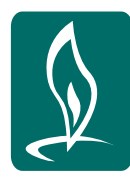

THE SHETH FOUNDATION palgrave macmillan
UNIVERSITY OF LEEDS

UNIVERSITY OF LEEDS

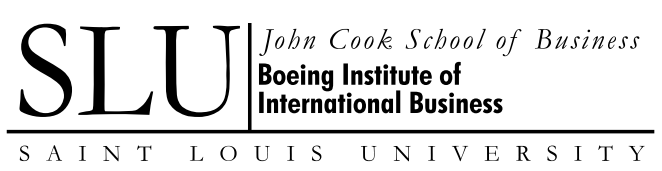

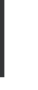

(1)
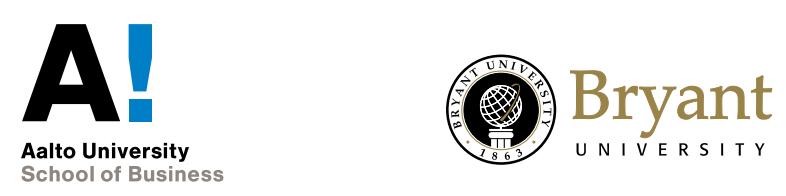

$\underset{\substack{\text { Business school } \\ \text { Henley }}}{ }$

UMELLBusiness 
ABSTRACTS 
(This page is intentionally blank) 


\section{ABSTRACTS FOR SATURDAY, JUNE 27, 2015}

\section{Session: 0.7 - Plenary}

\section{A/ B 2015 Opening Plenary}

Presented On: June 27, 2015 - 17:00-19:00

Welcome Remarks:

Nakiye Avdan Boyacigiller, Sabanci University

Ram Mudambi, Temple University

S. Raghunath, Indian Institute of Management Bangalore

Award Presentation for the 2015 AlB Fellows Educator of the Year Nick Binedell, GIBS Business School

Award Presentation and Keynote by the 2015 AlB Fellows Executive of the Year Kiran Mazumdar-Shaw, Biocon Limited

\section{Al B Fellows Opening Session: Global Networks: Organizations and People}

Presented On: June 27, 2015 - 17:00-19:00

Chair: Ram Mudambi, Temple University

Panelists:

Ram Mudambi, Temple University J ohn Cantwell, Rutgers University Torben Pedersen, Bocconi University

Sri Zaheer, University of Minnesota 


\section{ABSTRACTS FOR SUNDAY, JUNE 28, 2015}

\section{Session: 1.1.1 - Panel \\ Track: 8 - Global Strategy, M\&As and Competitiveness \\ New Competitive Strategies in Emerging Economies: Innovation and Competitive Dynamics of Local Leaders and MNCS}

Presented On: June 28, 2015 - 09:00-10:15

Chair: Sea-J in Chang, National University of Singapore

Panelists:

Sea-J in Chang, National University of Singapore

Rishikesha Krishnan, Indian Institute of Management

Phanish Puranam, INSEAD

Seung Ho Park, China Europe International Business School

This panel symposium provides the most up-to-date discussion of innovation and competitive dynamics between local firms and MNCs in emerging markets. This panel examines how strong local companies evolve in emerging markets, what are their competitive advantages and their weaknesses, and how MNCs can possibly address the competitive challenges of these strong local competitors. These local leaders are not merely copycats, and innovation plays an important role in securing their leadership position. These firms also display a high level of organizational ambidexterity and learning capabilities. Thus, MNCs face great challenges in creating effective organizations to adapt and modify themselves to address these competitive challenges. This panel will enhance our understanding of how both local firms and MNCs manage sustained profitable growth in emerging markets. (For more information, please contact: Sea-J in Chang, National University of Singapore, Singapore: schang@nus.edu.sg)

\section{Session: 1.1.2 - Panel}

Track: Scholars Meet Practice

\section{Business Perspectives on Environmental Sustainability: Challenges and Opportunities}

Presented On: J une 28, 2015 - 09:00-10:15

Chair: G. Tomas M. Hult, Michigan State University

Panelists:

G. Tomas M. Hult, Michigan State University

P. D. J ose, Indian Institute of Management Bangalore

Anil Menon, Cisco Systems

Venkatachalam Anbumozhi, Economic Research Institute for ASEAN and East Asia (ERIA)

Susan Mudambi, Temple University

Damandeep Singh, CDP (Carbon Disclosure Project)

The proposal is to organize two back-to-back special sessions at the forthcoming 2015 AlB Conference program that will serve as a forum for interaction, sharing and exchange of ideas among academics, practitioners, consultants and policy researchers on issues relating to environmental sustainability. The proposed sessions are likely to be of interest to AIB Conference attendees who are currently engaged in research and teaching in the areas of sustainability and corporate social responsibility specifically, as well as the broader community of 
conference attendees. A number of indicators are suggestive of the timeliness and high level of interest in issues relating to environmental sustainability. In recent years, issues relating to environmental sustainability have steadily risen in importance worldwide as principal concerns of consumer groups and individual consumers, for-profit and not-for-profit organizations, governments and non-governmental organizations (NGOs), public interest groups and other stakeholder groups, and researchers in a number of academic disciplines. A cursory examination of numerous articles published in scholarly journals and in the business press on environmental sustainability related issues, and the environmental sustainability reporting initiatives of a growing number of firms worldwide are indicative of its growing importance. Corporate legitimacy in a sustainability oriented environment requires that organizations while striving for a larger market footprint must also concurrently strive for a smaller environmental footprint. By their very nature, issues relating to environmental sustainability are inherently global issues that transcend national borders. Crucial from the standpoint of societal progress toward the achievement of sustainability related goals by countries (e.g. reductions in greenhouse gas emissions, energy intensity per unit of GDP, and water intensity per unit of GDP) is cooperation and collaboration among three key entities - producers of goods and services, consumers of goods and services, and the government. Also of crucial importance is cooperation and collaboration with other entities such as non-governmental organizations, consortia of companies (e.g. World Business Council on Sustainable Development) and consortia of nations (e.g. Inter-Governmental Panel on Climate Change). (For more information, please contact: G. Tomas M. Hult, Michigan State University, USA: hult@broad.msu.edu)

\section{Session: 1.1.3 - Competitive}

\section{Track: 5 - The Geography of I nternational Business and Global Value Chains}

\section{Globalization, Cities and Institutions}

Presented On: June 28, 2015 - 09:00-10:15

Chair: Anthony Goerzen, Queen's University

\section{Language and Globalization}

Palitha Konara, University of Huddersfield

This paper systematically examines the impact of language differences among countries on the globalisation process. Depending on what languages are spoken inside the country and outside the country, the ease of which the country can engage with the rest of the world may vary by country to country. In this paper I introduce the novel concept of language connectedness (LC) of a country that measures the proximity of a country to the rest of the world in terms of languages used in the focal country and languages used in the rest of the world. Then the LC index is constructed utilising the recently released 'Ethnologue global dataset' that contains statistics of country level language speakers for 234 countries and covering 7479 languages. This study demonstrate how LC can determine the level of country's external integration with the rest of the world. Econometric analysis that assess the impact of LC on country level globalization show that LC of a country is the most crucial factor that determines its level of globalisation. Effect of LC was particularly strong for cross border trade, investment and information flows. I thus pose the language barrier as the most crucial barrier to the globalisation process. (For more information, please contact: Palitha Konara, University of Huddersfield, United Kingdom: palitha.konara@hud.ac.uk)

\section{By Accident and by Design: Institutional-MNE Co-Evolution within the Subnational Space}

Sinead Monaghan, Rutgers Business School

Patrick Gunnigle, University of Limerick

J onathan Lavelle, University of Limerick

Contemporary research within international business (IB) has substantially endorsed the co-evolution of institutions in supporting and sustaining multinational (MNE) activity. As research to date has largely ignored 
the subnational dimensions of this process, integrative insights from economic geography (EG) to IB may offer a potential avenue to enlighten our understanding of subnational institutional-MNE co-evolution. Drawing on an interdisciplinary perspective, this paper explores the mutual adaptation of subnational institutions with MNE investment in facilitating subnational institutional co-evolution. Our findings trace considerable changes amongst subnational institutions seeking to both engage with localized MNEs and enhance regional economic development. As such, the evidence suggests active participation and mutual exchange of subnational institutions with inward investment over time. Contributing to the strong body of co-evolutionary research, these findings utilize insights from EG to illuminate the localized activities of institutional-MNE co-evolution. (For more information, please contact: Sinead Monaghan, Rutgers Business School, USA: smonaghan@business.rutgers.edu)

\title{
Geographic Connectivity via Sister Cities: Toward a Theory of Public-Private Value Creation
}

Li Dai, Loyola Marymount University

This paper advances a theory of grassroots institutions, which have the potential to facilitate sustainable value creation across geographic space. In particular, we examine a platform for public-private channel for micro-level linkages that is fundamentally different from the mechanisms for value creation previously identified in management research. Sister cities cultivate and continually reshape the person-to-person ties that bind international communities, and in the process foster long-term relationship residues and trust. The resulting reduction in the institutional distance between countries as well as in the stakeholder distance between private and local interests has implications for firm value creation. We contend that firms can leverage sister cities as a form of grassroots institution to effect favorable value creation conditions not only through increased resource access or network embeddedness but by means of reducing broad-based social divisions. (For more information, please contact: Li Dai, Loyola Marymount University, USA: li.dai@/mu.edu)

\author{
Global Cities and the Establishment of Regional Headquarters \\ Helen Du, KU Leuven \\ Rene Belderbos, KU Leuven \\ Anthony Goerzen, Queen's University
}

The world's major internationally connected metropolitan areas ('global cities') are preferred locations for multinational firms' headquarters. We examine the role of city and firm heterogeneity driving location choices for new regional headquarters (RHQ) establishments. We argue that RHQs are more likely to be established in global cities with a stronger connected position in worldwide inter-city networks related to cross-border operations of producer services firms, and that this relationship is more pronounced in knowledge-intensive industries and for RHQs with an entrepreneurial mandate. A city's geographic centrality within the multinational firm's network of affiliates in the region attracts RHQs, but this effect is mitigated by the strength of cities' international connectivity. Our inferences are drawn from mixed logit analysis of the location choices for 1292 RHQs in 72 global cities, 2003-2012. (For more information, please contact: Helen Du, KU Leuven, Belgium: helendu16@gmail.com) 


\section{Session: 1.1.4 - Competitive}

\section{Track: 2 - Entrepreneurship, Micro-Multinationals, and I nternational New Ventures}

\section{Network Effects on International Entrepreneurship}

Presented On: J une 28, 2015 - 09:00-10:15

Chair: Bruce Allen Hearn, University of Sussex

Does Inter-Firm Collaboration Enhance Export Performance? The Role of Internal Capabilities Youngok Kim, University of New South Wales

Jaspreet N. Bhatia, University of New South Wales

The importance of inter-firm collaboration has attracted much scholarly attention in exporting, but the joint influences of collaborative networks and internal capabilities on export performance remain under-explored. Considering this gap, this study proposes two research questions. First, does collaboration increase export performance? Second, is the relationship between collaborative networks and export performance contingent on a firm's internal capabilities? Using a sample of Australian small and medium-sized exporters, we find that a firm's collaborative intensity is marginally positively related to its export performance. More importantly, the impact of collaborative intensity on export performance is contingent on a firm's internal capabilities. Specifically, we find that the greater the intensity of collaborative networks, the superior the export performance when the firm's technical and managerial capabilities are each greater than smaller, thus demonstrating the complementary effect of networks and internal capabilities on export performance. In contrast, the greater the intensity of a firm's collaborative networks, the poorer the export performance when the firm's marketing capabilities are greater than smaller, which illustrates the substitution effects of networks and internal capabilities on export performance. (For more information, please contact: Youngok Kim, University of New South Wales, Australia: y.kim@unsw.edu.au)

\section{The Role of Network Relationships and Social Capital in Early and Later Foreign Market Entries of High Technology INVS \\ Spiros Batas, University of Northampton - University of Edinburgh \\ Tanja Leppaaho, Jyvaskyla University School of Business and Economics}

Social capital and its dynamic nature can offer plausible explanations to the rapid internationalization of International New Ventures (INVs). This paper tries to shed light on the impact of the three roles of social capital, efficacy role, serendipity role and liability role on international market entries of nine Scottish and Greek high technology I NVs. We observed that the efficacious role of social capital occurred more times in both early and later foreign market entries. The liability role had a very limited effect on internationalization. The serendipity role played an important role in countries with high psychic distance.

(For more information, please contact: Spiros Batas, University of Northampton - University of Edinburgh, United Kingdom: spiros.batas@northampton.ac.uk)

SMES and Networking: A Resource-Based View Perspective Mohammad Mahfuzur Rahman, Shandong University

The aim of this study is to empirically test the resource-based network (RBN) model, and the degree of influence that network resources have on competitive advantages (to both Small to medium sized enterprises (SMEs) and networks), as well as the influence of network resources on SMEs' future network participation. This study builds on the resource-based view (RBV) perspectives that offer an innovative approach to the development and implementation of networking strategies for SMEs. An internet panel administered survey was conducted, involving 298 SMEs from New South Wales (NSW), Australia. We used Structural Equation Modelling 
(SEM) using Partial Least Squares (PLS) path analysis to analyse primary data to investigate the cause-effect relationships between network resources, competitive advantages and network continuance. The findings support the proposed model and demonstrate that network resources and shared vision provide competitive advantages to SMEs, as well as collaborative competitive advantages to the network. The results demonstrate that key network resources-such as sharing the cost of training employees and cost of consultants, along with research and development, export, production, human resources and financial support-are instrumental in gaining competitive advantages over competitors. (For more information, please contact: Mohammad Mahfuzur Rahman, Shandong University, China: mohammad.rahman@sdu.edu.cn)

\title{
Session: 1.1.5 - Competitive \\ Track: 12 - The I nstitutional and Political Environment of I B
}

\section{Politics and Non-Market Strategies}

Presented On: June 28, 2015 - 09:00-10:15

Chair: Phillip Nell, WU Vienna

\author{
Political Ties across Country Borders \\ Kelsey Lynne Syvrud, Florida State University \\ Meghana Ayyagari, George Washington University \\ April Knill, Florida State University
}

In this paper we examine the importance of cross-border political connections using a comprehensive database of contributions to US political campaigns by foreign (non-US) firms from 52 countries during 2000-2013. Foreign firms with political ties to the US have higher profits, greater market share, and are awarded a larger number of government contracts than a matched sample of foreign firms of similar size and in the same industry that do not contribute to US political campaigns. Looking specifically at the Defense industry, where there are greater restrictions on doing business with foreign firms, we find that foreign firms that contribute to US political campaigns are on average awarded 1,867 more contracts and $\$ 1.7$ billion more in total obligated funds than even similar sized US domestic firms in the same industry that do not have campaign contributions. Our results highlight the limited effectiveness of US campaign contribution laws, and provide suggestive evidence of the cost to domestic firms of foreign influence on US politics. (For more information, please contact: Kelsey Lynne Syvrud, Florida State University, USA: KIsO7f@my.fsu.edu)

\author{
The Effects of Diplomacy, Institutions and Political Connections on Firms' Choice of FDI Location \\ J oao Albino Pimentel, HEC Paris \\ Pierre Dussauge, HEC Paris
}

In this paper, we examine when firms choose to mobilize their home-country's inter-governmental ties as a resource for expanding abroad and analyze the factors that make some firms more effective at doing so. We draw on the international investment location and non-market strategy literatures to advance three hypotheses. First, we argue that the existence of inter-governmental ties between a firm's home-country and a focal hostcountry increases the likelihood that the firm will invest in that host-country. Second, we argue that the level of risk of the host-country institutional environment increases a firm's need to rely on its home-country's intergovernmental ties. Third, we argue that a firm holding connections with political authorities in its home-country is more effective in mobilizing the home-country government to protect its international investments, making inter-governmental ties less critical to foreign investment decisions. We test our hypotheses on a sample of international investments made by firms from 14 home countries between 2003 and 2010. Findings provide support to our hypotheses and reveal additional subtleties of the interplay between inter-governmental ties and host-country institutional environment. (For more information, please contact: Joao Albino Pimentel, HEC Paris, France: joao.albino-pimentel@hec.edu) 
The Benefits of Political Capital for Firm Internationalization: Evidence from Russian Firm FDI and Exports

Rajeev Sawant, Baruch College, CUNY

Andrei Panibratov, St. Petersburg University

Lilac Nachum, Baruch College, CUNY

Marina Latukha, St. Petersburg State University

What is the relationship between corporate political ties (CPT) and firm modes of internationalization, exports and FDI? We theorize that CPT archetypes, interpersonal relationships between the firm's owners and political actors (relational political ties or RPT) and organizational linkages through board membership by political actors (knowledge political ties or KPT), differentially determine exports or FDI because these CPT types are conceptually distinct constructs. RPT provides personal access to public authorities while KPT provides knowledge of political markets and leads to a firm specific capability. When the firm uses RPT to obtain political favors, the incentives, interests and preferences of the political actor with whom the relationship exists also determine the use of political favors. Political actors prefer domestic use of political favors because their influence, prestige and power is domestically determined. RPT provides greatest value domestically and the level of political favors is limited. Thus, firms use RPT domestically for exports. KPT is not so constrained and is mobile internationally leading to greater FDI. We test these predictions on a novel data set of Russia MNEs, which have been largely ignored by research to date. Our results broadly support these predictions. (For more information, please contact: Rajeev Sawant, Baruch College, CUNY, USA: rajeev.sawant@baruch.cuny.edu)

\section{Buffered or under Scrutiny? Disentangling the Effect of Size on Subsidiary Political Strategies Patricia Reneé Klopf, WU Vienna \\ Phillip Nell, WU Vienna \\ Jonas Puck, WU Vienna}

This paper integrates institutional and resource based views to investigate political strategies of MNE subsidiaries operating in emerging markets. Our findings show that institutional pressures from public and private stakeholders induce more intense political strategies. While this is consistent with previous findings, we also hypothesize and find that subsidiary size has a dual effect. First, larger subsidiaries do have the capacity and resources to invest into political strategies. Second, however, their size also allows them to react less strongly to external institutional pressures - they are to a stronger extent buffered from the external environment than small firms. Thus, this paper adds to previous research by disentangling the complex effects of size on the use of political strategies - a strand of literature which has been characterized by inconsistent findings in the past. (For more information, please contact: Patricia Reneé Klopf, WU Vienna, Austria: pklopf@wu.ac.at) 


\title{
Session: 1.1.6 - Competitive
}

\section{Track: 3 - International Human Resource Management and Organizational Behavior}

\section{Building and Leveraging Social Capital}

\author{
Presented On: June 28, 2015 - 09:00-10:15
}

Chair: Allan Bird, Northeastern University

La Vie en Rose: Sources and Effects of Positive Emotions in Strategic Alliances Across Cultures - A Boundary Spanning Role Perspective

Linh Khanh Nguyen, HHL Leipzig Graduate School of Management

Vasanthi Srinivasan, Indian Institute of Management Bangalore

Existing studies on the role of emotions in strategic alliances have placed much emphasis on curing the negative emotions that are associated with the formation and operation of an alliance rather on building positive qualities. Drawing on the boundary spanning role research and positive emotions literature, we argue that strategic alliances' operating environment can be sources of boundary spanner positive emotions. Furthermore, we explore various effects of boundary spanner positive emotions at both intrapersonal and interpersonal levels, including strengthening psychological resilience, enhancing cognitive functioning, broadening social cognition, increasing social influence, and facilitating proactive trust building acts. We also examine how such experiences and effects vary across cultures. Our research offers several implications for scholars and business practitioners.

(For more information, please contact: Linh Khanh Nguyen, HHL Leipzig Graduate School of Management, Germany: linh.nguyen@hhl.de)

\section{Managerial Humor as an Affective Event for Gaining Positive Employee Outcomes \\ Nilupama Wijewardena, Monash University \\ Ramanie Samaratunge, Monash University \\ Charmine Hartel, University of Queensland}

Emerging research findings consistently show that mixing positive humor with management brings about positive employee outcomes. Hence, given the ability of managerial humor to expand new grounds in management knowledge, it has become a fruitful domain of research. In light of these developments, this paper examines the outcomes of managerial humor from an affect-based perspective. Using Affective Events Theory and the Broaden and Build Theory of Positive Emotions, we present propositions on the effect of managers' humor on employees' emotions in the short run and on employee Psychological Capital and turnover intentions in the long run. In doing so, we present managerial humor as a possible 'Affective Event' that needs to be managed and intentionally used for achieving desired outcomes at work. Implications for managers and future research directions are also discussed. (For more information, please contact: Nilupama Wijewardena, Monash University, Australia: nilupama.wijewardena@monash.edu)

\section{Linking Positive Social Capital and MNE Performance: The Mediating Role of Job Engagement in a Multi-Cultural} Middle East Context

Thomas J oseph, I ndian I nstitute of Management Udaipur

Raina Chhajer, Indian Institute of Management Udaipur

Using new data from a positive business initiative undertaken in a multinational financial services firm head quartered at Middle East, we demonstrate how employee engagement mediates the relationship between positive social capital and MNE performance. We find that the two of the many forms of social capital, High quality connections (HQCs) and generalized reciprocity are positively related with three dimensions of job engagement namely physical, emotional and cognitive engagement. Using structural equation modeling on a 
multicultural sample of 566 (from eighteen different nationalities), strong support was found for the theory driven hypothesis. Our findings throw light on how innovative approach of strategic human resource management contributes to MNE effectiveness. (For more information, please contact: Thomas Joseph, Indian Institute of Management Udaipur, India: thomas.joseph@iimu.ac.in)

\section{Indian IT Workers on an International Assignment: Role of Organisational Support Dhara Shah, Griffith University}

Although effective organisational support practices during an international assignment constitutes a significant factor in ensuring success of an expatriate assignment, little research has studied this as associated with the onsite-offshore business model that has come to be identified with global outsourcing. The purpose of this paper is to examine the experiences of Indian Information Technology (IT) workers, when they are sent to work on projects in a western country. We did this by using qualitative semi-structured interviews with 54 IT expatriates from Indian IT Multinational Companies (MNCs) who were working in Australia. Results suggest that inadequate support is provided to IT workers while they are overseas and employed at client sites. Such HR shortcomings may be inherent in the onsite-offshore model that is an important feature of I ndian IT business. The implications of the findings are discussed in the paper. (For more information, please contact: Dhara Shah, Griffith University, Australia: d.shah@griffith.edu.au)

\section{Session: 1.1.7 - Panel}

\section{Track: 9 - Emerging Economies and Emerging Economy MNEs}

\section{Multinationals in Big Emerging Markets: Dynamic Challenges, Opportunities and Implications for International Business Theory and Practice}

Presented On: June 28, 2015 - 09:00-10:15

Chair: Sumit Kumar Kundu, Florida International University

Discussant: Luciano Ciravegna, King's College London

Panelists:

Sumit Kumar Kundu, Florida International University

Luciano Ciravegna, King's College London

Ronaldo Parente, Florida International University

Wlamir Xavier, Eastern New Mexico University and UNISUL

J ohn Zhao, Saint Louis University

Helena Barnard, GIBS, University of Pretoria

S. Raghunath, Indian Institute of Management Bangalore

As the organizer of the panel on Multinationals in Big Emerging Markets we seek to understand the challenges faced by MNCs and domestic firms, and invited 4 scholars and experts to discuss both inward and outward internationalization strategies of domestic companies using theoretical lens in International Business. The four nations namely Brazil, China, India, South Africa begun their liberalization and reform process in the time frame [late 70s to early 90s], and domestic companies continuously responded to changes in external environment. The four authors have taken both a macro and micro perspective in analyzing the performance of local and foreign owned companies. The panelist will convey a story that is being played out in Big Emerging Markets and share with the audience idiosyncracies of these markets and decipher some of the commonalities [challenges and opportunities] that exist in the present time. The panelists will also shed light on the relevance of International Business theories to explain the rapid outward internationalization strategies of Brazilian, Indian, Chinese and South African firms, and examine them in relation to the inward internationalization strategies of foreign owned companies operating in BRIC nations. (For more information, please contact: Sumit Kumar Kundu, Florida International University, USA: kundus@fiu.edu) 


\title{
Session: 1.1.8 - Competitive
}

Track: 15 - Teaching I B (Special Track)

\section{Creating Graduate Readiness and Employability}

Presented On: J une 28, 2015 - 09:00-10:15

Chair: Hanoku Bathula, University of Auckland

\author{
2014 U.S. Business Needs for Employees with International Expertise \\ Shirley Daniel, University of Hawaii at Manoa \\ Ben Kedia, Univerity of Memphis \\ Fujiao Xie, University of Hawaii at Manoa \\ Zhou Chen, University of Hawaii at Manoa
}

This study presents the results of a survey of over 800 executives in US companies designed to identify the demand for corporate employees with international competence, including international skills and/or knowledge of foreign languages and world areas. The survey data is analyzed to help U.S. academic institutions evaluate the suitability of their international business degree and executive education programs, and guide modifications in programming, as appropriate. The results clearly call for a continuing need for international business education in the US, with increased emphasis on intercultural communication, foreign language skills and international experience. With the projected growth of international operations, additional international business education programs will need to be developed, particularly programs with a focus on Asia. Additional foreign language experts in Middle Eastern languages are needed for government positions. At the very least, all business graduates need to have an appreciation for cross-cultural differences and a global perspective. Additional outreach from academia to business is desired by business, and training programs, both degree and non-degree, are needed to provide management personnel with the higher levels of global competency needed to address the competitive challenges of the international business environment. (For more information, please contact: Shirley Daniel, University of Hawaii at Manoa, USA: sdaniel@hawaii.edu)

\section{Developing Critical Awareness through Reflection and Mindfulness for Successful International Business Graduates \\ Varina Paisley, University of New South Wales \\ Mark Tayar, Macquarie University}

Critical awareness is the ability to be able to recognise and critically analyse information received within a social context, evaluate competing perspectives and then make appropriate decisions. We propose a model for achieving critical awareness through the development of critical reflection, self-awareness and mindfulness in international business (IB) education. IB education is increasingly complex, given the interconnected and global business world that management graduates will operate in. This means that graduates need to integrate multiple perspectives on global environmental factors and be prepared for cross-cultural interactions with stakeholders, including employees. Through developing critical awareness, IB educators can help students acknowledge the forces that shape their own and others' assumptions, increase self-awareness and use mindfulness in experiences in order to improve their social skills such as cultural intelligence. This is necessary in order to produce successful IB graduates. This article concludes with five sample activities that instructors can adapt and use in their classrooms to develop critical awareness using critical reflection, self-awareness and mindfulness. (For more information, please contact: Varina Paisley, University of New South Wales, Australia: v.paisley@unsw.edu.au) 
The Role of Study Tours in Entrepreneurship Education. The Experience of Universidad EAFIT International Business Students, and Universidad Autónoma de Occidente, in Colombia.

Ricardo Lopez, Universidad Autónoma de Occidente

Maria Paola Podesta, EAFIT

The program "J óvenes con Empresa" left a competency-based model of education, mentorship, and promotion for the companies created, for 38 Colombian universities. Since then, entrepreneurial education has strengthened, and Study Tours arise as pedagogical activities intended to develop and improve the entrepreneurial competencies presented in the aforementioned model. In 2011 two Colombian universities started a joint process of academic visits to Silicon Valley, with results that reflect in both entrepreneurial education and curricular affectation. This paper shows the design and results of these Study Tours regarding the development of four of the thirteen competencies proposed in the model, in the aspects mentioned above, based in an ethnographic methodology. The conclusions tackle the dynamism of the pedagogical strategies, that are continuously feeding back from experiential learning, and its effect on the established education models. (For more information, please contact: Ricardo Lopez, Universidad Autónoma de Occidente, Colombia: ralopez@uao.edu.co)

A Theoretical Approach to a Curriculum for International Business Programs in the Business Schools in Colombia: Study Case at University of Rosario

Andres Mauricio Castro, Universidad del Rosario

Dalsy Yolima Farfan , Universidad del Rosario

Rafael Alejandro Pineros, Universidad del Rosario

Nowadays it has been imperative that the Business Schools' graduates will be prepared for the new world economy linked not only by the trade of goods but also services and Foreign Direct I nvestment from different actors in the business context. Therefore, it is a must that undergraduate students have to be aware of this environment and how they can be affect and take strategic decision for different kind of organizations which want to initiate an internationalization process. The purpose of this paper is to present what are the main bases that an International Business program has to include in their curriculum in order to answer the questions required not only for SMEs but also for MNEs in order to compete in markets abroad since the international business perspective. (For more information, please contact: Andres Mauricio Castro, Universidad del Rosario, colombia: andres.castro@urosario.edu.co)

\section{Session: 1.1.9 - Competitive}

\section{Track: 1 - People and Careers in Cross-Cultural Business}

\section{Cultural Intelligence, Effectiveness in a Foreign Culture, and Global Leadership}

Presented On: J une 28, 2015 - 09:00-10:15

Chair: Andre Anugerah Pekerti, University of Queensland

Relevance of Cultural Intelligence and Communication Effectiveness for Global Leadership Preparedness: Study of Indian Managers

Shoma Mukherji, Management Development Institute

Neera Jain, Management Development Institute

Business environment world over has undergone major transformation in the last fifty years. Global organisations, where operations are not restricted to home or host country, need business leaders with a global mindset and multicultural effectiveness. This paper examines and confirms the relevance on leadership preparedness of global managers of two important constructs - cultural intelligence and communication effectiveness in the context of anxiety and uncertainty management. Adding to extant literature, the study 
empirically confirms the suitability of three assessment instruments. It establishes the importance of cultural adaptability and AUM theory for assessing leadership potential of managers working in multicultural environments with culturally diverse teams. (For more information, please contact: Shoma Mukherii, Management Development Institute, India: shoma126@gmail.com)

\section{Social Networks and Social Support in the Context of International Assignments Tassilo Schuster, University of Erlangen-Nürnberg \\ Benjamin Bader, Leuphana University of Lüneburg}

The social network in which expatriates are embedded is a central factor for a diverse set of desired outcomes, such as psychological well-being, cultural adjustment, job satisfaction, and performance. But at present, the expatriate literature considers social support rather as a theoretical link to explain the link between social network characteristics and performance, or measures it on a global scale in contrast to a meaningful latent construct. By applying confirmatory factor analysis using data from 215 expatriates working for NGOs and 220 expatriates working for privately-held Multinational Companies, this study aims at providing guidance on how social support should be employed in future studies. The study contributes to the expatriate literature by developing a comprehensive conceptual model with regard to social support, recommending a theory-grounded measurement of the construct, and showing that social support should not be employed as unidimensional construct in future studies. Instead, the results suggest to account for three different dimensions that should be measured separately in order to avoid a misdirected, short-falling application of the construct. (For more information, please contact: Tassilo Schuster, University of Erlangen-Nürnberg, Germany: tassilo.schuster@fau.de)

\section{How Do Values Change When Immersed in a Foreign Cultural Environment? Peter Magnusson, University of Alabama Stanford A. Westjohn, University of Toledo Ahmed Al Radadi, Florida International University}

Extant literature on sojourner adjustment has largely focused on cultural artifacts. This conceptual study aims to make the following contributions to the literature. First, we shift the focus from a cultural artifact evaluation of cultural adaptation to a focus on values, particularly individualism-collectivism. Second, we lay out a theoretical explanation to suggest an asymmetrical relationship with respect to value change. We posit that the rate of value change is expected to vary depending on if you transition from a predominantly collectivist culture to an individualist society compared with going from an individualist society to a collectivist society. Third, we identify a series of personality and situational moderating factors that may enhance or attenuate the rate of value change. In the next phase of this study, we plan to test the conceptual framework with Saudi Arabian sojourners participating in Saudi Arabia's large-scale cultural mission. (For more information, please contact: Peter Magnusson, University of Alabama, USA: peter.magnusson@fiu.edu) 


\section{Session: 1.1.10 - I nteractive \\ Track: 5 - The Geography of I nternational Business and Global Value Chains}

\section{Global Production and Knowledge Networks}

Presented On: June 28, 2015 - 09:00-10:15

Chair: Ari Van Assche, HEC Montréal

What Pushes and Limits an OEM Supplier Transforms Under Institutional Change? Roles of Firms' Dynamic Capability and Organizational Inertia

Ming-Chang Huang, Providence University

Hsiang-Lin Cheng, National Chung Cheng University

Regarding the popular business model transformation, i.e., from offshore manufacturing for major clients to developing their owned-brand business, recently adopted by many OEM suppliers as a typical case of radical institutional change, this study elucidates the individual difference among OEM suppliers in the collective institutional change process. Previous studies merely investigate about the network and population characteristics in firms' institutionalization process. Empirical evidence was obtained for 161 Taiwanese OEM suppliers by a mailed survey conducted firsthand. The results reveal a strongly positive correlation between an OEM supplier's perception of institutional coercive and mimetic pressures and its likelihood to radically transform to the owned-brand business operation model, so that this study can assume that such a transformation should be considered as the case of institutional change. In the change process, individual firms' opportunity identification capability of coercive pressures (i.e., conformity to major customers, suppliers and regulatory agencies) can enhance firms' successful implementation of institutional change. Also, explorative learning capability can enhance the better imitation of other successful peers (i.e., mimetic pressure) by the OEM suppliers so that they can change radically. However, the internal organizational inertia, which limits firms to accept another new operation pattern, will weaken the positive impact of OEM suppliers' perception of mimetic pressure on their likelihood to engage in institutional change. (For more information, please contact: MingChang Huang, Providence University, Taiwan: mchuang@pu.edu.tw)

\section{Effect of Outbound Logistics Activities on Delivery Lead Time of Apparel Export Supply Chain Anupama Gupta, National Institute of Fashion Technology \\ Pinaki Dasgupta, International Management Institute Mridula Savitri Mishra, IIFT}

The purpose of this study was to measure the delay lag and cost implications to apparel exporters caused by delay in delivery lead time of apparel export shipments resulting due to issues and challenges involved in outbound logistics activities. The research was conducted in India and 84 apparel export manufacturing units participated in the survey. It was found that both North and South India exporters find customs clearance and road transportation as the most severe outbound logistics activities affecting delivery lead time of apparel exports. For both North and South India, approximately 46- 47 percent of the sea-bound apparel cargo do not face any delay due to issues and challenges of outbound logistics activities. Approximately 40-43 percent of the shipments face delay ranging from one to three days for both North and South India. Around 10-12 percent of the shipments are delayed for four days to seven days and around 1 percent of the sea shipments get delayed for more than seven days due to issues and challenges involved in outbound logistics activities of apparel exports. Apparel exporters belonging to these two clusters were found to have different pattern of spending due to delay caused by logistics issues and challenges. Exporters of North India cluster were found to spend relatively more on transportation whereas exporters of South India were found to relatively spend more on customs clearance. (For more information, please contact: Anupama Gupta, National Institute of Fashion Technology, India: anupamaguptanift@gmail.com) 
Managing Competition and Cooperation: Internationalization Process of HTC Sheng-Tsung Hou, Feng Chia University

Wan-Chien Lien, ChengChi University

The process for a manufacturing firm to shift its business from original equipment/design manufacturing (OEM/ODM) to the built-up of an international brand involves the evolution and interplay between competition and cooperation in the domestic and international contexts. This study draws on the awareness-motivationcapability (AMC) arguments, as proposed by the competitive dynamics perspective, to identify the internationalization process of HTC, an emergent yet flourishing smartphone company. The research reveals a co-evolving process between $\mathrm{HTC}$ and its competitors and strategic partners through managing competition and cooperation. It shows how an OEM firm endeavoured to creating own brand and entering into global market while still remained close relationships with upstream and downstream players in the value chain. The study contributes to the entrepreneurship and co-petition literature by probing into the processes, or "black box", through which a local brand burgeoned in a developing economy yet quickly flourishes in the global markets. A conceptual framework and some propositions are presented for further examinations. (For more information, please contact: Sheng-Tsung Hou, Feng Chia University, Taiwan: samuel.hou@gmail.com)

\author{
How Knowledge Complexity Rises During Paradigmatic Change: The Effects on Knowledge Sourcing and \\ Organization Networks \\ J essica Rae Salmon, Rutgers University \\ John Cantwell, Rutgers University
}

This article analyzes the conditions under which knowledge systems become more complex through recombinatorial efforts. Knowledge grows in part through the combination of existing knowledge streams in novel forms that may entail greater complexity. We suggest that knowledge complexity tends to increase when antecedent knowledge is dispersed across technology fields, geographically, and inter-organizationally. We argue that recombinatorial knowledge structures become more complex in this sense when firms access unfamiliar and formerly unrelated prior knowledge particularly during periods of paradigmatic change. An existing paradigm represents an established path of knowledge accumulation that has become familiar to firms in a given industry or context. When a paradigm shifts the composition of firms in a knowledge-based network becomes more open, is more likely to cross borders, and it tends to become broader and looser in form. (For more information, please contact: Jessica Rae Salmon, Rutgers University, USA:

js1587@scarletmail.rutgers.edu)

\author{
Offshoring-outsourcing, Innovative Capabilities and Technology Leaders: Some Preliminary Remarks \\ Jan Vang, Aalborg University \\ Anders Nielsen, Aalborg University \\ Rasmus Lema, Aalborg University
}

This paper will, within the context of global technological leaders, begin the process of reconciling different perspectives on the reconfiguration of their global value chains. The paper departs from the observation that different streams of research - including operations management research - lead to different explanations concerning the optimal configuration of the company's value chain, for example concerning the scope and scale of outsourcing. Based on a conceptual analysis of the challenges faced by the technological leader the paper concludes by discussing the consequences for their outsourcing and off-shoring patterns. (For more information, please contact: Jan Vang, Aalborg University, Denmark: jv@production.aau.dk) 


\section{Session: 1.1.11 - Interactive \\ Track: 6 - Innovation and Knowledge Management}

\section{Institutions and Innovation}

Presented On: June 28, 2015 - 09:00-10:15

Chair: Deeksha Singh, Rutgers University

Effects of Local Industry on the R\&D-Performance Relationship

Yiyi Su, Tongji University

While R\&D investments are typically conducted in pursuit of firm performance and long-term competitiveness, empirical studies to date have been equivocal in supporting the efficacy of R\&D. Recent research has attributed the equivocality to both industry- and country- factors, paying relatively little attention to the nuanced linkage between the two sets of factors. We contribute to the research by exploring the local industry effects on the R\&D-performance relationship. Our empirical analysis is based on a large panel data of 22,945 company-years from 12 developed and developing countries for the period 2000-2013. Findings indicate that the impact of R\&D on firm performance is influenced by local industry R\&D intensity, capital intensity and dynamism. (For more information, please contact: Yiyi Su, Tongji University, China: suyiyi@tongji.edu.cn)

\section{Local Cluster Growth Through Global Brain Circulation: A Governance Perspective \\ Stephan Manning, University of Massachusetts Boston \\ Cristiano Richter, Federal University of Rio Grande do Sul}

This paper investigates the interplay between geographic cluster growth, in particular in emerging economies, and global connectivity, in particular the role of 'brain circulation' (BC), i.e. talent movement and knowledge exchange with other locations. Using the cases of Bangalore-Silicon Valley, and Sinos Valley (Brazil)-Korea, we examine the role of governance in this process. We find that for larger-scale growing clusters, BC-growth dynamics may unfold as an 'organic process' through self-reinforcing market forces, whereas small-scale embryonic clusters depend on 'strategic coordination' of this process as they lack initial market attractiveness for both individuals and firms. Coordination may include a multi-level governance architecture of ties and talent/knowledge exchange at the firm, university and government level, which leverages local resources across sectors and aligns organizational ties with individual career paths. Our study has important implications for understanding cluster connectivity, the role of governance in cluster growth, and effective catch-up strategies of emerging economies. (For more information, please contact: Stephan Manning, University of Massachusetts Boston, USA: stephan.manning@umb.edu)

\section{Commensalism and Amensalism in University-Business-Government Alliances: The Internal Structure of Innovation Clusters}

Haruo H. Horaguchi, Hosei University

To transcend the traditional dichotomy between competition and cooperation, the theoretical population ecology constructs of Commensalism, Parasitism, and Amensalism were applied to an analysis of the Knowledge Cluster Initiative, a unique social experiment establishing university-business-government alliances for knowledgeintensive innovative clusters in Japan. An analysis of multiple Poisson regressions to check the interdependence of cluster variables revealed that new venture firms served as an input factor rather than output factor. Although the ultimate goal of the Knowledge Cluster I nitiative was to promote new start-ups by the university, the new start-ups increased the number of academic papers, patents, prototypes, and sales of newly developed products. (For more information, please contact: Haruo H. Horaguchi, Hosei University, Japan:

horaguch@hosei.ac.jp) 
The Effects of Industry and Country Variations on the IP Management Strategies of Firms: The Case of India and China

Elizabeth Louise Mason, University of Leeds

This study examines the effect of a nation's institutional system of intellectual property protection and enforcement (ISI) on the IP management (IPM) strategies of foreign firms. A nation's ISI comprises: (i) formal substantive and subordinate laws and (ii) informal rules of behaviour of institutional and economic actors. This study compares how the ISIs of two developing countries, namely, India and China, impact on the IPM strategies of firms from three high-technology industries: biosciences, advanced engineering and softwareelectronics. In depth interviews were conducted with managers of foreign firms that have operations in India or China, or both. This research reveals that a national ISI can have a significant effect on the IPM strategies of firms at both a macro-level (i.e. expansion decisions and entry-mode choice) and at micro-level (i.e. decisions taken in the host country to manage IPRs) and that this effect varies depending on the industry context. We find that the informal rules of behaviour have a greater effect on firm strategy than do formal characteristics of the ISI. We explain why firms adopt different IPM strategies in response to the ISI of each country, and how these vary in their effectiveness by industry and between the two countries.

(For more information, please contact: Elizabeth Louise Mason, University of Leeds, United Kingdom: e.l.mason@lubs.leeds.ac.uk)

\section{With Chopsticks and with Forks: Untangling the Fuzzy Front End of Innovation Projects in Japan and Germany Roman Bartnik, University of Duisburg-Essen}

Project management in the early phase can make or break innovation projects. Are there internationally valid best-practice solutions to this problem? I compare Japanese and German early project phases in 79 firms, arguing that four central differences in their national institutional framework should lead to different ways of organizing the front end phase. Three main findings emerge from the data: First, clear national patterns of organizing front end phases exist in Germany and Japan, in most cases without differences in project success and in direct relation to the respective institutional settings. This coherence indicates that management practices are supported by specific institutions, and that managers will need to replicate or substitute these institutions for transferring practices abroad. Second, I find substantially broader control rights of J apanese project managers. I argue that this high power is linked to high expectations towards J apanese project managers: in an institutional setting which binds all participants much tighter together through company specific human capital, these project managers are expected to achieve compromises across a much broader set of interest groups. Third, some indications of single best practice measures emerge, that appear independent of national institutional settings: extensive use of codetermination and an early determination of areas of expertise are practices employed significantly more often by the more successful companies. (For more information, please contact: Roman Bartnik, University of Duisburg-Essen, Germany: roman.bartnik@unidue.de) 


\section{Session: 1.1.12 - I nteractive}

\section{Track: 12 - The I nstitutional and Political Environment of I B}

\section{Internationalization, Distances, and Entry}

Presented On: J une 28, 2015 - 09:00-10:15

Chair: J ieqiong Ma, Saint Louis University

Sequential Market Entry and Subnational Differences

Maximilian Stallkamp, I vey Business School

Andreas Schotter, I vey Business School

Brian Pinkham, I vey Business School

This research examines the characteristics of multinational enterprises' (MNE) sequential market entries in a country with significant subnational differences. Based on a sample of 4,785 MNE subsidiaries in China spanning the time period from 1990 to 2006, we find that firms' second entries into China tend to differ from their initial entries with respect to location choice, industry, and equity stake. Contrary to what theory would suggest, firms generally choose lower equity stakes for their later subsidiaries, relative to their initial entry. A variation in subnational location choice is associated with a lower equity stake and a longer time lag between entries. We contribute to the literature on sequential market entry by shedding light on the impact of subnational differences on the sequential entry decisions of MNEs. (For more information, please contact: Maximilian Stallkamp, Ivey Business School, Canada: mstallkamp.phd@ivey.ca)

\section{From Country Distance to Revealed Relatedness: Exploring the Impact of Country Differences Aya Chacar, Florida International University Sokol Celo, Suffolk University Stephen Tallman, University of Richmond}

We introduce the concept of "revealed relatedness" as a way to frame and capture more broadly the relatedness between two firms units in two different countries. Revealed relatedness (RR) is the degree to which countries are efficiently combined within the investment portfolios of firms. We argue that similar to country distance, RR can be used as a proxy for the potential joint economies of operations and/or ownership of two average MNEs units in two different countries. We then explore both theoretically and empirically the relationships between country distances groupings and RR arguing that i) country economic differences are an important, ii) stable institutional differences are significant, over and beyond country economic differences, iii) time-varying institutional differences are significant over economic and stable institutional differences, To conduct the empirical portion of the study, we propose a new measure of relatedness between units in two countries and compute it for over 20,000 country pairs. We then explore in a descriptive fashion the drivers of RR using variance decomposition. The empirical results show that indeed country distances explain a significant portion of RR but distances of stable institutions explain the lion's share. (For more information, please contact: Aya Chacar, Florida International University, USA: chacara@fiu.edu)

(De)Institutional Arbitrage: The Effects of Perceived Void Arbitrage Opportunities on Stakeholder Issue Salience J oobin Ordoobody, University of Victoria Alireza Saify, University of Tehran

Prior research assumes that firms manage stakeholder requests in a unified, omnipresent institutional environment and that this environment is out of their control. However, this is not necessarily applicable when firms have access to internationalization mechanisms. By adopting the comparative capitalism approach on institutional theory, this paper extends the logic behind stakeholder issue salience in presence of multiple 
institutional settings. We argue that access to different institutional settings may change the priority of stakeholder issues for the firm. Furthermore, we explain how and when utilization of this access, in order to deal with stakeholders, may become widely spread among firms. (For more information, please contact: Joobin Ordoobody, University of Victoria, Canada: joobin@uvic.ca)

\section{Institutions, Market Entry Strategy and Performance: A Study of Japanese Multinationals}

Daniel Z. Ding, City University of Hong Kong

Naoki Ando, Hosei University

Gloria L. Ge, Griffith University

In this study, we examine the moderating effect of market entry strategy on the relationship between the host country institutions and subsidiary performance. We test our hypotheses using a panel dataset that contains 9160 J apanese overseas investments from 1999-2007 in forty countries. Our results suggest that the better developed formal institutions in the host country are associated with higher performance of foreign subsidiaries, while the cultural distance between the host and home countries is negatively associated with subsidiary performance. The market entry strategy significantly strengthens the performance impact of formal institutions on foreign subsidiaries. We also found that the market entry strategy tends to mitigate the negative performance impact of cultural distance on foreign subsidiaries but not statistically significant. Implications for future research are discussed. (For more information, please contact: Gloria L. Ge, Griffith University, Australia: gloria.ge@griffith.edu.au)

\section{Capital Mobility and Institutional Levels: The Influence of the Corruption on the FDI Flows Ronald de Oliveira Concer, EAESP-FGV}

This study analyzes the impact of corruption levels on foreign direct investment (FDI) flows. We have applied a panel data set in order to determine the duration of FDI flows invested abroad based on the corruption levels of the host countries. We argue that the time of permanence of FDI flows could varies since the existence of corruption levels, once the existence of weak institutional environment would bring more risky situation, and also shaping the kind of FDI projects which means a short or long-term duration. (For more information, please contact: Ronald de Oliveira Concer, EAESP-FGV, Brazil: ronald.concer@gmail.com)

The Legitimization and Reinterpretation of Transnational Business Practices through Traditional Institutions: Consequences of the Importation of American Meritocracy through the Indian Institutes of Management Keshav Krishnamurty, University of Massachusetts Boston

The Indian Institutes of Management (IIMs), established with American assistance, might be seen as the pinnacle of meritocracy. I use the specific case of the two original IIMs to trace: 1 ) historical predispositions toward meritocracy in India, 2) the active exportation of modern, technocratic meritocracy to the IIMs by Harvard and MIT through the establishment of modern business education and practices, 3) the translation of meritocracy into II M practices and a further translation into a specifically Indian set of practices as an attempt at social engineering a new managerial class, and 4) the prospects for reforming the IIM-specific form of meritocracy. (For more information, please contact: Keshav Krishnamurty, University of Massachusetts Boston, USA: keshav.krishnamur001@umb.edu)

\section{Managing BoP as a Business Opportunity. A Longitudinal Field Study Thomas Andre, Ecole Polytechnique Jean-Pierre Ponssard, Ecole Polytechnique \& CNRS}

In the last decade a growing articulation of the business strategy of the firms with some specific global societal challenge in line with its core activities has been observed. This change provides both a need and an 
opportunity for BoP activities to migrate from their preserved status within the CSR department to business operations. We explore the successive steps associated with this change at Schneider Electric through a longitudinal case study. The newly adopted business strategy of the firm clearly facilitates the change in the mindsets all through the company. Still the need for adapting the management systems remains pending. A key result that emerges from our analysis is that BoP activities cannot be directly transferred to operational entities without simultaneously identifying which of the functional department will be in charge of providing the corresponding management systems. (For more information, please contact: Thomas Andre, Ecole Polytechnique, France: thomas.andre@polytechnique.edu)

\section{Session: 1.1.13 - I nteractive \\ Track: 1 - People and Careers in Cross-Cultural Business}

\section{Adjustment and Performance of Expatriates}

Presented On: June 28, 2015 - 09:00-10:15

Chair: Fiona Moore, Royal Holloway, University of London

Repatriation of International Assignees - Where Are We and Where Do We Go From Here? A Systematic Literature Review From 1991 to 2014

Jan Sebastian Knocke, Friedrich-Alexander University of Erlangen-Nuremberg

The issue of repatriation continues to be a challenge for many international firms today. This topic has largely been neglected in literature for a certain time, after it has aroused the interest of academics in the beginning of the 1990's. However, thorough analyses of repatriation issues are scarce in human resources management research. To address this gap, this paper provides a systematic and broad literature review. The intention of this paper is threefold: to analyze repatriation issues published in academic journals, to identify different repatriation research streams in the literature and to suggest fields for future research. The systematic literature review of 93 peer-reviewed empirical articles from 1991 to 2014 reveals an increasing focus on repatriation issues lately. Moreover, the study results suggest that empirical research has mainly emphasized on quantitative studies of career development, organizational support practices and turnover intentions of US samples, while there is a dearth of analysis of other essential ones. (For more information, please contact: Jan Sebastian Knocke, Friedrich-Alexander University of Erlangen-Nuremberg, Germany: jan.knocke@fau.de)

\section{An Examination of Determinants of Expatriate Career Intentions \\ Arpita J oardar, Clark University \\ Guillaume Weisang, Clark University}

This paper draws from Ajzen's theory of planned behavior and examines factors influencing an expatriate's intention regarding future international career. Data was collected about expatriate's perception of his/her taskbased and relationship-based acceptance in the host country, the expatriate's satisfaction with host country, self-efficacy and his/her intention to consider future expatriate assignments. Analysis was conducted using structural equation model based on polychoric correlation matrix of the observable variables. The results of the analysis and their implications for the career of expatriate managers are discussed in this paper. (For more information, please contact: Arpita Joardar, Clark University, USA: ajoardar@clarku.edu) 
In Search of Greener Pastures: Migration Motivations of Talents and Talent Management Practices in Nepal

Kushal Sharma, ESSEC Business School

J ean-Luc Cerdin, ESSEC Business School

We aim to study a previously unexplored area in Talent Management (TM) research - migration motivation of talents from a least developed country. Combining ideas from TM and migration literature, we study how organizations manage talent in an adverse environment. We seek answers to what motivates talents to migrate, whether they are prepared to pursue their careers in an international / multi-cultural environment, and what might their expectations be when they arrive at the host country. Our study has practical implications mainly for Multinational Enterprises that might employ these talents and also for home country organizations that might seek to retain them. (For more information, please contact: Kushal Sharma, ESSEC Business School, France: kushal.sharma@essec.edu)

'Should I stay or Should I go?' An Autoethnographic Account of an International Student's Decision to Transition to a Self-Initiated Expatriate

Namita Rajani, Dalhousie University

Eddy Ng, Dalhousie University

Dimitria Groutsis, University of Sydney

This paper is based on an autoethnographic account of an international student who studied in Canada, and follows her journey from student through to settlement as a self-initiated expatriate. I nitially, personal factors (such as family encouragement), public policy, and employer practices "pushed" the student to repatriate back to her country of origin after her studies. However, personal ambitions (such as travel and career development opportunities) and prospects for a better life "pulled" her back to Canada. Furthermore, personal agency and positive experiences influenced her decision to remain in the host country as a self-initiated expatriate. The present paper adds to existing literature on the "push" and "pull" dynamic of international student retention in the host country. It further enhances our understanding on when and why international students decide to remain in the country following the completion of their studies. (For more information, please contact: Eddy Ng, Dalhousie University, Canada: edng@dal.ca)

\section{Diemo Urbig, University of Wuppertal \\ Philipp Paulus, University of Trier \\ Katrin Muehlfeld, University of Trier \\ Vivien Procher, University of Wuppertal \\ Arjen van Witteloostuijn, Tilburg University}

Social Stays Local - Identifying Typical Profiles of Future Employees of Multinational Enterprises

Multinational Enterprises (MNEs) are regularly criticized for their exploitative firm policies and an adverse impact on the local environments of their host countries. We investigate which types of future young professionals are attracted to work for international firms. Further, we consider whether their typical behavioral and personality profiles might add a complementary perspective to explaining the negative image of MNEs. Specifically, based on Schneider's (1987) attraction-selection-attrition (ASA) cycle, we assume that organizational behavior is based on the collective characteristics of the people that are working for an organization and hypothesize that future young professionals who actively seek to work for MNEs are characterized by a lack of pro-social behavior and by corresponding personality traits. We analyze for a sample consisting of 470 students of a Dutch university whether the degree of pro-social orientation (measured both by means of a self-reported trait and observed behavior) is negatively associated with the intention of working for an international firm. The results largely support our theory. (For more information, please contact: Philipp Paulus, University of Trier, Germany: paulusph@uni-trier.de) 
That Which Does Not Kill Us Makes Us Stronger: Effects of Work and Family Demands on International Business Travelers' Adjustment and Subsequent Career Satisfaction

Mihaela Dimitrova, Oakland University

Margaret Shaffer, University of Wisconsin - Milwaukee

Drawing on an integration of role theory and the challenge and hindrance stressors framework we propose and test a model of the influence of work and family demands on international business travelers' (IBTs) subsequent career satisfaction through work and family role adjustment. We suggest that demands have a differentiated effect on IBTs' work and family adjustment and career satisfaction, with some demands contributing to successful participation in work and family life, while others are seen as road blocks. Through a two-wave study of 209 IBTs, we find general support for our model. As proposed, the work role is found to have a direct influence on IBTs' career satisfaction, while the family role has an indirect influence on career satisfaction through a spillover into the work domain. (For more information, please contact: Mihaela Dimitrova, Oakland University, USA: dimitrova@oakland.edu)

\section{Session: 1.2 - Plenary}

Track: Scholars Meet Practice

\section{Al B Executive Plenary: Global Networks as Forces for Good}

Presented On: J une 28, 2015 - 10:45-12:00

Chair: Ram Mudambi, Temple University

This session features leaders from Unilever, an 86-year-old MNC with deep roots in India, and Narayana Health, a 15-year-old Indian company aspiring to transform healthcare worldwide. It will explore how the two organizations reconcile profitability and social goals, and how they leverage global networks to achieve their purpose.

Moderator:

Ravi Ramamurti, Northeastern University

Featured Speakers:

Harish Manwani, Chairman, Hindustan Unilever Ltd and former COO, Unilever

Ashutosh Raghuvanshi, Deputy Chairman and Group CEO, Narayana Health

\section{Session: 1.3.1 - Panel}

\section{Track: 9 - Emerging Economies and Emerging Economy MNEs}

\section{Leveraging India's Locational Competitive Advantage and the Internationalization of Indian MNEs}

Presented On: June 28, 2015 - 13:00-14:15

Chair: Farok J. Contractor, Rutgers Business School

Discussants: Preet Aulakh, York University and Sougata Ray, Indian Institute of Management Calcutta

Panelists:

Farok J. Contractor, Rutgers Business School

Vikas Kumar, University of Sydney

Charles Dhanaraj, IMD

Shameen Prashantham, Nottingham University Business School China 
Ajai Gaur, Rutgers University

Sougata Ray, Indian Institute of Management Calcutta

Raveendra Chittoor, Indian School of Business

Mehmet Demirbag, University of Strathclyde

Preet Aulakh, York University

As the world's third largest economy in 2014 (World Bank, 2014) India's role in international business as a FDI destination, as well as the internationalization of India-based MNEs is being subject to greater research scrutiny. The first presentation examines economic, demographic and institutional factors that make up India's market, its human capital base, and innovative potential. While the current government has proposed incentives for manufacturing, Contractor, Kumar \& Dhanaraj propose that India may have a greater leverage in the upstream and downstream portions of the value chain. The second presentation by Prashantham focuses on how the internationalization of young Indian firms is aided by home nation network ties, complementing diaspora ties. The third presentation by Gaur examines the propensity of Indian firms to internationalize explained by ownership structure and group affiliation. This, together with CEO characteristics, is also an explanatory variable in the fourth presentation by Chittoor, Aulakh \& Ray focusing on risk-taking behavior in foreign acquisitions as a mode of outward FDI. The fifth presentation by Nair, Demirbag \& Mellahi considers the 'reverse' flow of knowledge from foreign affiliates back to Indian MNE parents as an important strategy motivating outward FDI from India. (For more information, please contact: FarokJ. Contractor, Rutgers Business School, USA: farok@andromeda.rutgers.edu)

\section{Session: 1.3.2 - Panel Track: Scholars Meet Practice}

\section{Challenges of MNC Innovation in India: A View from Practice with a Focus on People Issues}

Presented On: June 28, 2015 - 13:00-14:15

Chair: Abhoy Ojha, Indian Institute of Management Bangalore

\section{Panelists:}

Abhoy Ojha, Indian Institute of Management Bangalore

Rishikesha Krishnan, Indian Institute of Management

Abhijeet Saungikar, 3M I ndia

R. Bhaskaran, Bosch Ltd.

Vinay Dhabolkar, Catalign Innovation Consulting

J aideep Anand, Ohio State University

Multi-national companies (MNCs) have been disaggregating their global value chains and locating various activities in different parts of the world for long. While production capacities and capabilities had significantly shifted to low-income economies, till recently innovation capabilities had largely remained in the high-income economies. However, with changes in demographics, leading to a shrinking work force and also the possibility of flat or declining markets in the high income economies, there is greater attention to countries like India to provide the workforce as well as a growing market for the future. As a consequence, several MNCs have moved significant $R \& D$ facilities to India, sometimes to save costs for R\&D targeted for home markets, and more recently for innovation targeted at India and similar markets. This shift in location and focus of R\&D activities comes with its own set of people related challenges with reference to attracting, recruiting, training, engaging and retaining professionals who are capable of contributing to the innovation efforts. The panellists will share personal insights on these issues acquired while engaging with the process of creating an R\&D setup within their organization, and also promoting an R\&D eco-system in India. (For more information, please contact: Abhoy Ojha, Indian Institute of Management Bangalore, India: aojha@iimb.ernet.in) 


\section{Session: 1.3.3 - Competitive}

\section{Track: 6 - Innovation and Knowledge Management}

\section{Subsidiaries' Role in Knowledge Creation and Transfer}

Presented On: J une 28, 2015 - 13:00-14:15

Chair: Felipe Monteiro, INSEAD

When do Multinational Subsidiaries Develop New Technologies?

Anupama Phene, George Washington University

Stephen Tallman, University of Richmond

This study examines the development of new technologies by multinational subsidiaries. We hypothesize that technological changes in the MNC and host country, in areas specific to subsidiary expertise, have a positive effect, while technological changes in the MNC and host country, at the broad industry level, have a negative influence on subsidiary development of new technologies. We further propose that subsidiary innovative capability acts as a moderator, enabling subsidiaries to respond to technological changes and develop new technologies. We find support for the hypothesized effects of MNC and host country technological changes. Subsidiary innovative capability strengthens the influence of host country technological changes in specific areas of subsidiary expertise and weakens the effects of MNC and host country technological changes at the broad industry level. (For more information, please contact: Anupama Phene, George Washington University, USA: anuphene@gwu.edu)

\section{Transfer between Subsidiaries and Headquarters \\ Sergey Morgulis-Yakushev, Stockholm School of Economics \\ H. Emre Yildiz, Uppsala University \\ Carl F. Fey, Nottingham University Business School China}

Differentiating between Cultural Similarity and Cultural Fit: An Empirical Examination of Dyadic Knowledge

Despite increasing awareness of the positive outcomes of cultural diversity, the extant international business literature has an implicit negative bias against cultural differences. A more balanced assessment of culture's consequences could be achieved by differentiating between the concepts of cultural similarities/differences and cultural fit. We argue that, depending on the intended outcomes of cross-cultural interaction, cultural fit could be achieved by sustaining similarities or maintaining differences. To illustrate our ideas, we look at knowledge transfer within multinational corporations. We develop our theoretical reasoning based on evolutionary economics and test our hypotheses by using a novel methodological technique on a unique dyadic dataset of MNC headquarters and subsidiary pairs. Our findings are in line with our predictions. Implications for theory and future research are discussed. (For more information, please contact: Carl F. Fey, Nottingham University Business School China, China: carl.fey@nottingham.edu.cn)

\section{Subsidiary Ownership of Firm-Specific Advantages, Network Linkages, and Innovation within Multinational Firms} Catherine Magelssen, London Business School

Theories of the firm suggest that the free flow of knowledge is a central advantage to internalizing transactions. However, internal markets for knowledge exist within multinational firms whereby subsidiaries license and contract the firm's key firm-specific advantages (FSAs) to other subsidiaries within the firm. The subsidiaries that own the rights to the FSAs are responsible for developing, managing, and exploiting the FSAs. Using a new, confidential panel dataset on subsidiary-level ownership of FSAs, I examine the effects of subsidiary FSA ownership on innovation. I find evidence that subsidiary FSA ownership is positively associated with innovation, and that transferring ownership rights away from a subsidiary reduces its innovation. The results also suggest 
that tax haven FSA owners adversely affect subsidiary innovation. (For more information, please contact: Catherine Magelssen, London Business School, United Kingdom: cmagelssen@london.edu)

\section{Session: 1.3.4 - Competitive \\ Track: 5 - The Geography of I nternational Business and Global Value Chains}

\section{Global Value Chains, Innovation and Performance}

Presented On: June 28, 2015 - 13:00-14:15

Chair: Marcus M. Larsen, Copenhagen Business School

Creating Shared Value in Global Value Chains: Learning from Impact Sourcing Service Providers in U.S., India and Kenya

Chacko George Kannothra, University of Massachusetts Boston

In this article we seek to understand how positioning in global value chains affects the creation of shared value, i.e. the joint pursuit of both business and social objectives. We look at hybrid organizations- organizations that combine both revenue generation and social welfare as part of their business model. We consider the empirical case of I mpact Sourcing Service Providers (ISSPs) - outsourcing firms that deliver low-cost high-quality services to global clients based on their ability to hire, train and effectively utilize staff from disadvantaged groups of society. Our findings suggest that country-of-origin of ISSPs, in particular distance to global clients and degree of local business sophistication, affect their business option space, their commitment to the social mission and the pressure to grow, all of which, in turn, affect their ability to pursue hybrid models over time. Findings have important implications for our understanding of creating shared value in the context of global outsourcing and global value chains in more general. (For more information, please contact: Chacko George Kannothra, University of Massachusetts Boston, USA: chackog@gmail.com)

How Does the Global Relocation of Business Activities Affect Firm Innovation? An Empirical Analysis of the Impact of Offshoring on Innovation in Danish Firms

Eunkyung Park, Aalborg University

The relationship between offshoring and firm innovativeness has received increasing attention in the literature in the recent years. Some empirical studies investigating the direct offshoring-innovation relationship found positive relationship between offshoring and innovation in various contexts of offshoring such as offshore outsourcing, offshoring of R\&D, and offshoring of services. The current study aims to provide evidence for the effect of offshoring on innovation regardless of the type of activities involved. More importantly, this study attempts to find the impact of offshoring on innovation, not merely a positive association, by controlling for the innovation performance before the firms' engagement in the relocation of the activities. Furthermore, how specific features of offshoring such as the type of activities offshored, the degree of disaggregation, governance mode, and offshoring location are related to the firm innovativeness is also investigated. Empirical analysis on Danish data shows that offshoring firms are more likely to introduce new products/services. Additionally, it is found that the offshore locations and governance mode matter for this relationship. All in all, the results suggest that firms that have diversified the channels and sources of knowledge transfer with a high level of disaggregation of value chain are more likely to innovate. (For more information, please contact: Eunkyung Park, Aalborg University, Denmark: eunkyung@business.aau.dk) 
Global Offshoring Portfolio and Firm Performance

Nidthida Lin, University of Newcastle

Hao Tan, University of Newcastle

Stephen Chen, University of Newcastle

We examine the role of location and function diversity of the firms' offshoring portfolio on the innovation and financial performance benefits the firm obtains from offshoring. The study also investigates the moderating role of the offshoring governance mode on these relationships. Using a sample of US and European firms engaging in offshoring activities, we find that function diversity is a significant determinant of innovation outcome of offshoring firms and offshore outsourcing is a significant moderator of the relationship between function diversity and innovation performance. We also find a significant s-shape relationship between location diversity and innovation performance of offshoring firms. On the other hand, the findings show a significant negative relationship between location diversity and financial performance. Governance mode (outsource vs. captive offshoring) is also found to have a significant moderating effect on the relationship between location diversity and financial outcome of offshoring. Our findings are highly significant for global sourcing and international business research as they shed light on the impact of firms' offshoring portfolios on the outcomes of offshoring activities and firm performance. (For more information, please contact: Nidthida Lin, University of Newcastle, Australia: nidthida.lin@newcastle.edu.au)

The Effect of Cultural Distance on Access to Knowledge and Knowledge Acquisition in International Business Exchange: A Knowledge-based View Mia Hsiao-Wen Ho, Yuzn Ze University

International knowledge acquisition is a context-dependent process. The diversity of the context arising from cultural distance between partner firms can be a source of knowledge transfer due to the potential learning opportunities based on the strategic complementarities. It is however no guarantee that the learning potential can be realized owing to the difficulties and misunderstandings in the process of international knowledge acquisition. In this study, we adopt a knowledge-based view of cultural distance and examine how it influences the firm's access to the transferred knowledge and its acquisition of the knowledge in an international business exchange context. Our findings indicate that a trustworthy partnership can facilitate international knowledge acquisition mainly because it weakens the firm's casual ambiguous perception towards the transferred knowledge and further enhances the firm's learning outcome in a large culture-distant business exchange relationship. (For more information, please contact: Mia Hsiao-Wen Ho, Yuzn Ze University, Taiwan:

mia.hohw@saturn.yzu.edu.tw)

\section{Session: 1.3.5 - Competitive}

\section{Track: 9 - Emerging Economies and Emerging Economy MNEs}

\section{Economic Dynamics in Africa}

Presented On: June 28, 2015 - 13:00-14:15

Chair: Helena Barnard, GIBS, University of Pretoria

Can MNEs Substitute Local Governments in Providing Energy Infrastructures when Host Countries are Failed States? The Case of Sub-Saharan Africa

Matilde D'Amelio, Politecnico di Milano

Paola Garrone, Politecnico di Milano

Lucia Piscitello, Politecnico di Milano

The paper investigates if, in sub-Saharan Africa, MNEs can be substitute for local governments in providing energy infrastructures when host country is a "failed state". Indeed, for MNEs it is important to foster energy 
infrastructures development to run their own business and to gain the legitimacy to operate locally. This is true especially when MNEs come from home countries institutionally (more) developed as they are more likely to possess the needed expertise, and to need to fill a higher gap in legitimacy in host countries institutionally distant. An econometric analysis is performed using a panel dataset, composed by pairs of 15 sub-Saharan host countries and 84 home countries, observed from 2005 to 2011. We find that when host institutions are weak, foreign MNEs can substitute for the traditional options of public production and regulation of private business in the provision of energy infrastructures, nonetheless strong home institutions are necessary. (For more information, please contact: Matilde D'Amelio, Politecnico di Milano, Italy: matilde.damelio@polimi. it)

The Institutional Determinants of Family and Non-Family Involvement in Business Groups - The Case of Africa Bruce Allen Hearn, University of Sussex Lars Oxelheim, Lund University and Research Institute of Industrial Economics Trond Randøy, University of Agder

This study examines the role of retained ownership of business angel (BA), domestic and foreign venture capital (VC), in 202 newly listed firms at initial public offering (IPO) in 22 emerging African economies. We integrate agency-based signalling approaches with institutional arguments and find retained post-IPO ownership is prevalent in non-family group firms while the opposite is true for family business groups. These associations are mediated by institutional quality. We find that elevated government effectiveness and corruption control are associated with family groups retained ownership, which questions the common view of family groups being associated with weaker institutional quality (For more information, please contact: Bruce Allen Hearn, University of Sussex, United Kingdom: b.a.hearn@sussex.ac.uk)

\section{Experimentation in and Internationalization across Mobile Telecommunications Markets of Africa Mohammad J ahanbakht, Carnegie Mellon University \\ Romel Mostafa, Ivey Business School Francisco Veloso, Catolica Lisbon School of Business and Economics}

Research suggests that adaptations of advanced-economy business models to challenging base of the pyramid (BoP) market conditions involve experimentation. We analyze the conditions that facilitate developing country entrepreneurs to learn about business models and the incentive of local and multinational firms to carry out experiments for BoP adaptations. We test our frameworks' implications on the evolution of the mobile telecommunications industry across Africa. Contrary to the economic models that posit one-directional investments from the North to the South, our findings suggest a two-step industrialization process. The spillover of modern-industry knowledge from the North enables a few entrepreneurial firms in the South to actively experiment, and through successful BoP adaptations they gain ownership advantages and internationalize across the South, catalyzing the growth of the industry. (For more information, please contact: Romel Mostafa, Ivey Business School, Canada: rmostafa@ivey.uwo.ca) 


\title{
Session: 1.3.6 - Competitive
}

\section{Track: 10 - Theories of the MNE and of FDI}

\section{Individuals, Skills, Sensemaking, Behavior, and Networks in I B Theories}

Presented On: June 28, 2015 - 13:00-14:15

Chair: Karina R. Jensen, NEOMA Business School

\author{
A Network-based Theory of FDI Clustering and Foreign Subsidiary Performance \\ J ing'an Tang, Sacred Heart University
}

Drawing upon theories of agglomeration economics, institutional ecology and social networks, we develop a network-based theory of FDI Clustering and performance. A FDI clustering is defined as, a foreign peer network (FPN), formed by a group of foreign owned subsidiaries that have the same country of origin and operate same or similar businesses within a common geographic boundary in the host country. We propose that driven by the interplay among institutional, social and economic forces, an FPN develops through four stages in the host country and such development stages partly explain variations in foreign entry performance. (For more information, please contact: Jing'an Tang, Sacred Heart University, USA: tangj3@sacredheart.edu)

\author{
Foreign Direct Investment and Skills-Based Inequality: Some Empirical Evidence \\ Serena Masino, University of Oxford \\ Xiaolan Fu, University of Oxford
}

This study analyses a cross-country panel representing all levels of development to uncover the impact that FDI stocks have on skill-based inequality, measured as wage inequality. It emerges that i) moving from high to low absorptive capacity levels of the hosts increases the reduction effect on inequality. And ii) for every given host group, FDI coming from a more technologically advanced source lowers inequality, while FDI coming from a similarly or less technologically endowed source increase inequality. The study interprets these findings as the interaction between labour demand and scarcity effect conditions created in the host economies by different types of technology transfer embedded in FDI stocks. (For more information, please contact: Serena Masino, University of Oxford, United Kingdom: serena.masino@qeh.ox.ac.uk)

\section{Can International Business Theories be Applied in China? Rui Torres Oliveira, Manchester Business School}

In international business (IB) literature, traditional theories cannot fully accommodate the phenomenon of outward foreign direct investment (OFDI) from developing countries. China, the country that is leading this recent trend, provides a strong focus of study in order to understand whether traditional theories can be applied, and if new developments or a new agenda should emerge. In this perspective paper, we argue that China's OFDI challenges some of the established theories dealing with firm internationalization. On one hand, developing country firms are not expected to act upon the integration process after the acquisition of firms from developed countries; on the other hand, developing country firms will be more resource-augmenting than would be predicted by the traditional resources-based view, the established theory when discussing firms from developed countries. Our findings are based on a case study of the acquisition of a German automotive industry firm by a Chinese company. (For more information, please contact: Rui Torres Oliveira, Manchester Business School, United Kingdom: rui.oliveira@postgrad.manchester.ac.uk) 
Session: 1.3.7 - Panel

Track: 15 - Teaching IB (Special Track)

\section{Engage Them and They'll Understand: Existing Projects and Best Practices of Integrating Experiential Learning in International Business Curriculum}

Presented On: June 28, 2015 - 13:00-14:15

Chair: Vasyl Taras, University of North Carolina at Greensboro

Panelists:

Karen Lynden, Rowan-Cabarrus Community College

Vasyl Taras, University of North Carolina at Greensboro

David M. Berg, Hamline University

Allan Bird, Northeastern University

Virginia Cathro, University of Otago

Susan Forquer Gupta, Monmouth University

James M. Hagen, Hamline University

Huiping Li, Ramapo College of New Jersey

I rina Naoumova, University of Hartford

Gordana Pesakovic, King University

Sonja Sackmann, University Bw Munich

J osé R. de la Torre, Florida International University

Vinh N. Lu, Australian National University

Experiential learning is a "hot" topic in International Business and Cross-Cultural Management Education (IBCCM), but the use of this approach is limited due to a lack of information and understanding on what tools are available and how to incorporate them in the curriculum. The proposed panel will bring together 12 academics who have developed and successfully used various experiential learning projects in IC-CCM education programs. The goals of the panel are to share information about the existing EL projects, share experiences, discuss challenges and best practices of using EL to improve IB-CCM education, provide a networking opportunity for academics interested in the EL approach, and discuss how this panel can contribute to further discussion on the role of EL in IB-CCM education, possibly through special issues on EL in academic journals, targeted conferences and seminars, and better research on the issue. (For more information, please contact: Karen Lynden, Rowan-Cabarrus Community College, USA: karen.lynden@rccc.edu)

\section{Session: 1.3.8 - Competitive}

Track: 1 - People and Careers in Cross-Cultural Business

\section{Cross-Cultural Adjustment of Expatriates and migrants}

Presented On: J une 28, 2015 - 13:00-14:15

Chair: Mary Yoko Brannen, University of Victoria

The Role of Social Network Embeddedness and Social Support for Cross-Cultural Adjustment of Self-I nitiated Academics

Barbara Agha-Alikhani, University of Passau

Based on a mixed-methods design we investigate the question which role embeddedness plays for the social support and adjustment of self-initiated expatriates (SIE). We present the preliminary findings of a case study with 12 self-initiated Academics in a Danish Business School. 
Our findings show that social networks play a crucial role for the emotional well-being of the individuals and that the network embeddedness is perceived as more important than the adjustment to different domains. We complement existing research on social support by the "strategic support" domain and overall contribute to a research stream that is lacking multi-method approaches. Therefor, we add insights into careers and the perceptions of self-initiated academics regarding adjustment and embeddedness processes. (For more information, please contact: Barbara Agha-Alikhani, University of Passau, Germany: barbara.agha-alikhani@unipassau.de)

\section{What Keeps Them Going: Career Anchors and Cross-Cultural Adjustment of Expatriates}

Alexei Koveshnikov, Aalto University

Heidi Marie Wechtler, Macquarie University

In this paper we identify dominant career anchors and examine the relationship between career anchors and cross-cultural adjustment among expatriates in a not-for-profit French organization. Based on a sample of 189 expatriate managers, we find Internationalism, Dedication to a cause, and Lifestyle to be the dominant anchors. Additionally, we show the contingency of dominant career anchors on expatriates' age and current work and life circumstances. These findings challenge some of the key theoretical assumptions of career anchors theory. In regards to how expatriates' career anchors affect their cross-cultural adjustment, we find that different anchors interact differently with different dimensions of cross-cultural adjustment. All in all, our analysis is an initial attempt to explore the complex relationships between career anchors and cross-cultural adjustment and provide an update to the theory of career anchors in the context of expatriation.

(For more information, please contact: Alexei Koveshnikov, Aalto University, Finland: alexei.koveshnikov@aalto.fi)

\section{"Life is Elsewhere": A Longitudinal Diary Study of Female Self-Initiated Expatriates' Cross-Cultural Adjustment Heidi Marie Wechtler, Macquarie University}

This study examines the motives for deciding to work abroad and the trajectory of adjustment of young female self-initiated expatriates. Based on a longitudinal analysis of blogs (online diaries), we identify five themes associated with the expatriate adjustment process: motive, observation, downsides, recovery, and meaning. The study reveals that the trajectory of adjustment of this population seems to follow a U-curve, with culture shocks at around one month and three months in the host country, and a recovery period of up to six months. The analysis also reveals a second type of emotional shock during the first week of arrival, distinct from culture shock. Reflecting the profile of "escapers" found in the expatriation literature, our findings identify a feminist and existentialist perspective related to the specificities of this distinct expatriate population. (For more information, please contact: Heidi Marie Wechtler, Macquarie University, Australia: heidiwechtler@gmail.com)

\section{Researching the Ignored: Lower-Qualified Migrants and Expatriates Lovanirina Ramboarison-Lalao, ESC Troyes Business School J ean-Luc Cerdin, ESSEC Business School Chris Brewster, ISCTE Business School}

This article explores the mobility experience of lower-paid migrants and expatriates through three questions, namely what is the motivation to come to France? What is the motivation to stay there and what is the relationship between this motivation and career success? After clarifying the blurry lines in the definitions between expatriation and migration, we examine the relationship between motivation to migrate and success.

We use qualitative research with 44 lower-paid migrant workers to argue that their career success depends on motivation to settle, in turn explained by motivation to migrate. In line with the theory of motivation to integrate (Cerdin, Abdeljalil, \& Brewster, 2014), we found a relationship between motivation to migrate and 
migration success in terms of career success. However, this theory, derived from the study of highly qualified migrants, needs to be adjusted to be applicable to low-skilled migrants. To that end, contextual factors and locus of control come into play to shed a new light on the migration experience.

(For more information, please contact: Lovanirina Ramboarison-Lalao, ESC Troyes Business School, France: lovanirina.ramboarison@get-mail.fr)

\section{Session: 1.3.9 - Competitive}

\section{Track: 11 - Corporate Governance in IB}

\section{Revisiting the Scope of the Multinational Corporation: Cross-Border M\&A and Divestment}

Presented On: June 28, 2015 - 13:00-14:15

Chair: Ali Taleb, MacEwan University

Value Creation in Cross-Border Acquisitions: Do Outside Directors Make a Difference?

Deepak K Datta, University of Texas at Arlington

Dynah A Basuil, University of Auckland

Our study on the role of independent outside directors in the creation of shareholder value in cross-border acquisitions is based on a sample of 428 cross-border acquisitions undertaken by U.S. firms between 1991 and 2006. Grounded in agency and resource dependence theories, our findings reveal that acquiring firms with outside directors who have longer tenure and hold more external board appointments are able to create greater value for their shareholders in cross-border acquisitions. In addition, our results indicate that cross-border acquisitions by firms with a greater number of outside directors with international experience create greater wealth for their shareholders. However, contrary to what we had expected, we found a negative relationship between the proportion of independent outside directors on the board of acquiring firms and acquisition performance. Our findings also indicate that the positive relationship between the number of external board appointments of outsider directors and value creation in cross-border acquisitions is contingent on the cultural distance between acquiring and acquired firm countries with the observed relationship being stronger in the context of acquisitions made in countries characterized by high cultural distance. (For more information, please contact: Deepak K Datta, University of Texas at Arlington, USA: ddatta@uta.edu)

The Effects of Family Control and Board of Director Characteristics on Cross-Border Acquisition Performance of Emerging Market Firms: Evidence from India 2007-2012

Philsoo Kim, Yonsei University

Young-Ryeol Park, Yonsei University

By employing the resource-based view of the firm and resource dependence theory, this research seeks to both theoretically and methodologically explore the impacts of the ownership control and corporate board factors on cross-border acquisition performance consequences by analyzing a sample of 291 international acquisitions rendered by 184 Indian public and private family firms during the research period of 2007-2012. Our empirical findings indicate that there is a positive relationship between the effect of family ownership and board of directors' acquisition experience on cross-border acquisition performance of Indian firms. This signals that family involvement and strategic expertise of board of directors contribute to family firms' performance gains. In addition, this study also finds that multiple directorships of corporate board members in emerging market family firms hamper cross-border acquisition performance due to their limited expertise in handling acquisition strategy and management burdens to meet diverse firm interests known as 'busy' directorship. (For more information, please contact: Philsoo Kim, Yonsei University, Korea, South: kimseolhyun@yonsei.ac.kr) 
Investor Protection and International Acquisitions: Evidence from US Cross-Border M\&As

Amir Shoham, Temple University

Oded Shenkar, Ohio State University

Agency and shareholder value theory considers investor rights protection a positive factor for firm valuation, and by extension, an inducement for FDI inflows. However, from the perspective of managerial and organization control theory, investor protection in the host country can inhibit the flexibility and independence of MNCs to control their international operations, thereby hindering investment inflows. We examine these opposing views using cross-border M\&A data for US MNCs from 1981 through 2008, and find that legal and regulatory investor protection requiring mandatory dividends and giving anti-director shareholder rights are detrimental to FDI, consistent with management and organizational control theory (For more information, please contact: Amir Shoham, Temple University, USA: amir.shoham@temple.edu)

\section{An Endowment Effect Perspective on Foreign Divestment: The Asymmetric CEO Effect in Family Multinationals} Heechun Kim, Georgia State University

Robert Hoskisson, Rice University

Our study investigates when family CEOs divest less than professional CEOs, and how much influence they exert, by examining the conditions under which their influence varies in family multinational enterprises (FMNEs). With an endowment effect framework, our study analyzes event history data on 859 foreign subsidiaries of 161 Korean FMNEs in the manufacturing sector during bad times of domestic macroeconomic distress between 1998 and 2003. Our results show that family CEOs susceptible to the endowment effect are less likely to divest foreign subsidiaries in which FMNEs have controlling ownership than professional CEOs. But these CEOs have little influence in the divestitures of foreign subsidiaries in which FMNEs have non-controlling ownership. We also find that family CEOs are reluctant to divest foreign subsidiaries that could have been divested when these subsidiaries are located in host countries with high growth opportunities that can compensate for the home country's macroeconomic distress. These findings suggest that the reluctance to divest is likely the result of family CEOs' purposeful strategic choices rather than that of their cognitive blunders. Yet, professional CEOs are found to divest the subsidiaries in the same circumstances. These contrary results suggest that CEOs matter, but in different ways. (For more information, please contact: Heechun Kim, Georgia State University, USA: heechunkim@gsu.edu)

\section{Session: 1.3.10 - I nteractive}

\section{Track: 9 - Emerging Economies and Emerging Economy MNEs}

\section{Knowledge and I nnovation}

Presented On: June 28, 2015 - 13:00-14:15

Chair: Zhi Wang, Manchester Metropolitan University

The Impact of Information Overload on The Entrepreneurs' Behavior in Managing Human Resources: The Mediating Role of Entrepreneurial Self-Efficacy

Manisha Karia, Auckland Institute of Studies

Sanjaya Gaur, Auckland University of Technology

Hanoku Bathula, University of Auckland

Undoubtedly, the entrepreneur is the key to the initiation of the entrepreneurial process and firm performance. Since each entrepreneur varies in their background and abilities, researchers have examined the factors impacting his/her performance. Previous results show that personality characteristics and entrepreneurial selfefficacy have a positive impact on entrepreneurs' performance. In this study, we address two other variables that have not been a focus of attention so far: entrepreneurial information overload and entrepreneurs' HRM 
behavior. We also consider six dimensions of the entrepreneurial self-efficacy separately for their impact. We developed a conceptual framework that proposes a negative impact of entrepreneurial information overload on entrepreneurial self-efficacy and entrepreneurs' HRM behavior. Responses from 402 entrepreneurs of a large emerging economy were subjected to a path-based multiple regression analysis. The results reveal that information overload has a direct impact on entrepreneurial self-efficacy dimensions, except on planning tasks. Although there is no direct effect of information overload on entrepreneurial HRM behavior, there is an indirect effect through the mediating role of entrepreneurial self-efficacy. These results suggest the need for strategies to deal with coping with information overload and also ways of improving entrepreneurs' self-efficacy. This study provides a platform for empirical research for future studies. (For more information, please contact: Manisha Karia, Auckland Institute of Studies, New Zealand: manishak@ais.ac.nz)

\section{Can Knowledge Similarity Be a Substitute for Economic Institution for Foreign Firms in an Emerging Market? Xuelian Piao, Korea University Min J ung Kim, University of Minnesota Jon J ungbien Moon, Korea University}

Institutional theory suggests that firms can improve the performance with better institutional support, and this is particularly the case with foreign firms in emerging markets. In this paper, we attempt to explore the possibility that firms can perform well even in poor institutional environment when they are located where there exist many other firms with similar industrial knowledge or with similar local organizational type. Using panel data on industrial firms and institutional index of subnational regions in China, we empirically find positive relationship between the economic institution and foreign firms' performance, and substitutability of the knowledge similarity and the economic institution in this relationship. (For more information, please contact: Jon Jungbien Moon, Korea University, Korea, South: jonjmoon@korea.ac.kr)

\section{Dreams Come True? The Challenges of Utilizing Knowledge from Advanced Economies in Emerging Market MNES}

J ongmin Lee, University of Reading

Rajneesh Narula, University of Reading

Over the last decade, MNEs from emerging economies (EMNES) have continued to make aggressive moves in cross-border acquisitions particularly in advanced economies. Upon this phenomenon, literature often argues that EMNEs can augment their firm specific advantages by acquiring knowledge-based assets from advanced economies. However this study argues that the physical acquisition of knowledge-based assets and the actual utilization of those assets in EMNEs are entirely different two things, and that EMNEs may suffer from three challenges when they utilize knowledge-based assets obtained from their acquisitions of firms in advanced economies: information asymmetry, lack of absorptive capacity and recombinant capabilities, and organizational inertia. The investigation on these challenges aims to provide some insights to the on-going theoretical debate in the literature as well as to practitioners. (For more information, please contact: Jongmin Lee, University of Reading, United Kingdom: j.m.lee@pgr.reading.ac.uk)

Family Support and Young Entrepreneurs' Start-Up Activities in an Emerging and Developed Economy Context: An Empirical Exploration

Tatiana S. Manolova, Bentley University

Galina Shirokova, Saint Petersburg University

Tatyana V. Tsukanova, Saint Petersburg University

Linda F. Edelman, Bentley University

In this paper we explore the effect of family support on the scope of start-up activities undertaken by young nascent entrepreneurs in the context of developed and emerging economies. Combining the institutional and family embeddedness perspectives and drawing on the nascent entrepreneurship literature, we hypothesize that 
the scope of start-up activities is positively associated with family support in the form of two types of family capital, financial and social. We further argue that the effect of family support on young aspiring entrepreneurs' start-up activities is moderated by the institutional context. To test our hypotheses, we use data from the 2011 Global University Entrepreneurial Spirit Students' Survey (GUESSS) project ( $n=33,925)$. Our findings indicate that the effects of family support on young nascent entrepreneurs' start-up activity are complex and multifaceted. Theoretical and public policy implications are discussed. (For more information, please contact: Tatiana S. Manolova, Bentley University, USA: tmanolova@bentley.edu)

\section{Reluctant Innovation in the Chinese Automobile Industry \\ Peter Thomas in der Heiden, University of Duisburg-Essen \\ Bernd Liesenkötter, University of Muenster}

Green innovation has become a cornerstone of China's economic strategy. Its relevance for addressing the legacy costs of established factor market distortions and ensuring future productivity improvements is uncontroversial. This paper reviews the barriers to innovation in the automobile sector by shedding light on the progress in the development, commercialization and popularization of electric vehicles. Drawing on patent data of Chinese automobile manufacturers and suppliers as well as a series of expert interviews, it suggests the existence of a sailing ship effect - the reinforcement of R\&D activities focused on traditional propulsion systems in reaction to the appearance of electric vehicle technology. After identifying reasons for this resistance and the main obstacles for innovation adoption in the corporate sector, the authors review industrial policy measures to determine whether or not government interference was so far able to remedy prevalent market failures. It is found that because of coordination failures within the government sector, the effectiveness of industrial policy has been greatly impaired. (For more information, please contact: Peter Thomas in der Heiden, University of Duisburg-Essen, Germany: peter.inderheiden@uni-due.de)

\section{Advanced MNEs in the Chinese Pharmaceutical Industry: An Analysis of the Impact on Local Innovation Performance \\ Vittoria Giada Scalera, Politecnico di Milano \\ Alessandra Perri, Ca' Foscari University Venice}

This study analyzes the innovative activity of foreign MNEs operating in the Chinese pharmaceutical industry by exploring (1) their innovative background, (2) their investment choices and the organizational arrangements they use for local knowledge creation and (3) the performance of their local innovative processes.

Our empirical strategy is based on the analysis of the population of pharmaceutical patents issued by the USPTO between 1980 and 2010 and granted to foreign assignees incorporating the scientific work of Chinese inventors. Our results show that the Chinese pharmaceutical industry is populated by a number of extremely competitive MNEs from the advanced economies, which involve in R\&D activities locally and whose presence represents an extraordinary learning opportunity for local firms. Therefore, we conclude that the presence of advanced MNEs in the Chinese pharmaceutical industry is associated with a strong potential for positive externalities that could be highly beneficial for the local innovation system. However, our findings seem to suggest that while the preconditions for the occurrence of FDI-mediated learning processes do, local firms may still be unable to fully benefit from the presence of advanced MNEs, likely because they are not yet provided with sufficient absorptive capacity. (For more information, please contact: Vittoria Giada Scalera, Politecnico di Milano, Italy: vittoriagiada.scalera@polimi. it) 


\section{Dark Triad in Teams - A Study from Iran}

Ricarda B Bouncken, University of Bayreuth

Mehrnaz Karimi, University of Bayreuth

Robin Pesch, University of Bayreuth

Individuals in private and business life suffer from others that expose "dark" personalities such as narcissism, Machiavellianism, and psychopathy. This study is the first to research dark triad at a team level. We conceptualize dysfunctional effects of dark triad on creativity and international knowledge acquisition within teams both of which are crucial for firms in emerging markets. The empirical study of 247 observations from 64 I ranian firms indicates that "team dark triad" decreases team creativity and international knowledge acquisition. Moreover, team autonomy increases the destructive influence of team dark triad on team creativity. (For more information, please contact: Ricarda B Bouncken, University of Bayreuth, Germany: bouncken@uni-bayreuth.de)

\section{Session: 1.3.11 - I nteractive \\ Track: 6 - Innovation and Knowledge Management}

\section{Learning Across Borders}

Presented On: June 28, 2015 - 13:00-14:15

Chair: Nandini Lahiri, Temple University

Perception Gaps between Headquarter and Subsidiary Home Country National Managers on Subsidiary Role: Case of a Japanese Service MNC

J usuke JJ I kegami, Waseda University

Due to the increasing complexity in international management perception gaps between headquarter and subsidiaries have become one of the key issues in IB studies. This paper aims to develop a framework regarding the relationship between headquarter-subsidiary perception gap and subsidiary strategic business unit performance through the case study research of one of the top J apanese service MNC. 134 interviews with expatriates and HCN (Home country national) managers in six core subsidiaries and headquarter were conducted. While most of the existing literatures suggest negative influence of perception gap on subsidiary performance, we found there are some situations where perception gaps influences positively. Even subsidiary overestimation, which is regarded as a cause of negative influence in the existing literatures, has both positive and negative influence on strategic business unit performance. Headquarter overestimation, whose influence was not clearly studied in the past literatures, has both negative and positive influence on performance. (For more information, please contact: Jusuke JJ Ikegami, Waseda University, Japan: jjikegami@waseda.jp)

\section{Cultural Compatibility and the Managerial Knowledge Transfer in Alliance Network Qingfen Fu, Tsinghua University}

Little academic research has been done on the subject of how to deal with the different managerial knowledge from separate alliance partner. Exiting research still have no answer how firm evaluate the importance of different alliance partner, and what kind of managerial knowledge finally been adopted and integrated into the firm's own business. In this exploratory study, we aim to use a multiple case study methodology to examine the focal firm's decision making process of valuation and selection the knowledge from separate alliance partner. Using business group in China auto industry as the research sample, we study in what way they retrieve, synthesize and recombine the managerial knowledge from separate alliance partner. This research gives theoretical implication on the knowledge management and learning theory; and gives practical implication of the alliance management on how to use distinctly different partner knowledge. (For more information, please contact: Qingfen Fu, Tsinghua University, China: fuqf.11@sem.tsinghua.edu.cn) 
The Impacts of Organizational Learning Capacities on Relationship-Specific Innovations: Evidence from the Global Buyer-Supplier Relationship

KyuYeong Choi, Florida State University

Daekwan Kim, Florida State University

Ruey-J er "Bryan" Jean, National Chengchi University

Acquiring knowledge is a critical factor in generating firm innovations. With the emergence of global level sourcing, more firms are stressing the importance of obtaining knowledge from global business partners. While working with global business partners, not only a firm's learning capacity not also its joint learning capability with business partners play an important role in generating relationship-specific innovations. Drawing on the knowledge-based view (KBV) and the social exchange theory, this study investigates the impacts of two organizational learning capacities: absorptive learning, a firm level learning capacity, and joint learning, a relationship level learning capacity, on relationship-specific innovations. In addition, a firm's exploitation/exploration strategy, supplier dependence, and strategic flexibility are further incorporated into the study as moderators. The study model was tested using data collected from 190 electronic firms in Taiwan. The study findings revealed that both absorptive capacity and joint learning capacity positively influence relationship-specific innovations. Furthermore, a firm's exploitation focus enhances the impact of both absorptive learning capacity and joint learning capacity on relationship-specific innovation. In addition, supplier dependence moderates the impact of absorptive learning capacity on relationship-specific innovation positively while that of joint learning capacity negatively. Finally strategic flexibility moderates the impact of absorptive learning capacity on relationship-specific innovation negatively. Several implications of the study findings are offered at the end. (For more information, please contact: Daekwan Kim, Florida State University, USA: dkim@business.fsu.edu)

TMT Foreign Experience and Foreign Subsidiary Ownership Decision: A More Fine-Grained Conceptualization of Experience

Kent Hui, Michigan State University

Yuanyuan Gong, Nanjing University

We draw on the Upper-Echelons theory to predict MNEs' foreign subsidiary ownership decision.

More specifically, we argue that foreign assignment experience of top management team members influences their perceived uncertainty of doing business in a host country, which in turn affects the decision of whether to establish a wholly-owned subsidiary or a joint venture with local partners. Apart from prior studies, we consider three dimensions of experience: quantity, scope, and specificity. We argue that a more fine-grained

conceptualization of experience can enhance our understanding of the learning process of TMT members, and of why they make certain decisions. (For more information, please contact: Kent Hui, Michigan State University, USA: hui@broad.msu.edu)

\section{Building Service Provider Capabilities: The Impact of Clients and Service Types in Service Offshore Outsourcing}

Peter D. Oerberg J ensen, Copenhagen Business School

Kristin Brandl, University of Reading

Manya J aura, Copenhagen Business School

In this paper we study whether and how the interaction between clients and the service providers contributes to the development of capabilities in service provider firms. In situations where such a contribution occurs, we analyze how different types of activities in the production process of the services, such as sequential or reciprocal task activities, influence the development of different types of capabilities. We study five cases of offshore-outsourced knowledge-intensive business services that are distinguished according to their reciprocal or sequential task activities in their production process. We find that clients influence the development of human capital capabilities and management capabilities in reciprocally produced services. While in sequential 
produced senvices clients influence the development of organizational capital capabilities and management capital capabilities. (For more information, please contact: Peter D. Oerberg Jensen, Copenhagen Business School, Denmark: poe.smg@cbs.dk)

\author{
Can Toyota Employee Satisfaction Provide An Edge To Car Dealers in a Changing National Cultural Climate? \\ Ichiro Yamada, St. Andrew's University \\ Osamu Tsukada, Kagawa University
}

This paper investigates the effects of changes in national culture on business practices and ideologies in the automotive industry in J apan. In the face of recent demographic conditions such as population decline and rapid aging, passenger car sales in Japan have been stagnant at 3.5 million per year. Despite these challenging conditions, one dealer in particular, Nets Toyota Nangoku, has doubled its sales/visitors and ranked number one in customer satisfaction among $300 \mathrm{~J}$ apanese Toyota dealers for the past 10 years. In order to learn more about its management and business practices, Nets and 10 other Toyota dealers in Shikoku-prefecture were surveyed and interviewed. Results suggest that Nets' performance may be explained by motivation theory, internal service quality, and organizational culture. Five hypotheses were tested using a questionnaire that provided 1343 responses. The following three hypotheses were statistically verified: (1) employee satisfaction at Nets Toyota Nangoku is higher than at the other Toyota dealers; (2) the motivational force of Nets Toyota Nangoku is also higher; (3) the dimensions of Nets' organizational culture differ from the other Toyota dealers. Based on these results, it can be said that the management's focus on employee satisfaction can provide an edge to other car dealers. (For more information, please contact: Ichiro Yamada, St. Andrew's University, Japan: i-yamada@andrew.ac.jp)

\title{
Session: 1.3.12 - I nteractive \\ Track: 3 - International Human Resource Management and Organizational Behavior
}

\section{Global Mindsets and Expatriate Challenges}

Presented On: June 28, 2015 - 13:00-14:15

Chair: Benjamin Bader, Leuphana University of Lüneburg

\author{
A Study on How Expatriate Styles Influence Subsidiary Effectiveness through Uncertainty Reduction \\ Yong Suhk Pak, Yonsei University \\ Qing Lu Sun, Yonsei University
}

This paper specified expatriate into two styles, commander and coordinator, to examine the whether the different expatriate styles affect differently on host-country nationals' (HCNs) sense of organization uncertainty, and further affect subsidiary effectiveness. Results of a study of 495 HCNs from 19 MNCs operating in China indicate that the commander style positively affects HCNs' uncertainty and the coordinator style is negatively related to HCNs' uncertainty. And uncertainty has mediation effect between two expatriate styles and subsidiary effectiveness. (For more information, please contact: Qing Lu Sun, Yonsei University, Korea, South: miracle_sql@hotmail.com)

\section{The Meaning of Work and Work Meaningfulness among Chinese Employees of a Western MNE Alim Beveridge, Nottingham University Business School China Angela Yazhi Cheng, Nottingham University Business School China}

The meaning of work and work meaningfulness have been studied in various disciplines over the past half century, including sociology, psychology and organizational behavior. Much of this research has focused on employees in a single cultural context, often in the United States and Western Europe. Whether the meaning 
that Chinese employees working in foreign MNEs derive from or make of their work follows similar patterns, and whether the sources of experienced meaningfulness, or perceived significance, resulting from engaging in work are similar or dissimilar has gone unexplored. Research showing that such phenomena are affected by cultural values and norms suggest that important differences ought to exist. We present the results of an inductive study based on over twenty interviews on the meaning of work and work meaningfulness conducted with Chinese employees to show both ways in which their experiences confirm and diverge from existing research findings on these issues. (For more information, please contact: Alim Beveridge, Nottingham University Business School China, China: alim. beveridge@nottingham.edu.cn)

\section{Managerial Attitudes toward Foreignness, Commitment Levels in Foreign Equity and The Level of Multinationality of Spanish Firms}

David Tanganelli, Universitat Internacional de Catalunya

In this study, we investigate how the level of equity invested in foreign ventures and the orientation of the top management teams toward foreign cultures and international markets influence the degree of multinationality of Spanish firms. We postulate and empirically illustrate that a geocentric approach (based on a global mindset that remains perceptive to local differences) is associated with a higher level of internationalization. Conversely, modes of entry that imply a greater commitment relative to the equity investment made in the host country such as the creation of wholly owned subsidiaries are not necessarily related to the highest degree of multinationality. In the current international scenario, the flexibility offered by the establishment of international alliances that imply a lower commitment of equity investment in the foreign country allows for the optimization of the investment made and a significant increase in the level of multinationality. (For more information, please contact: David Tanganelli, Universitat Internacional de Catalunya, Spain: databer@uic.es)

\section{Culturally Creative Minds: Cosmopolitanism as an Antecedent to Creativity in Cross-Cultural Settings \\ Dilek Gulistan Yunlu, Northeastern Illinois University \\ Gretchen Vogelgesang Lester, San J ose State University \\ Rachel Clapp-Smith, Purdue University Calumet}

Research on culture, creativity, and innovation has received significant attention in recent years; however, the connections between these constructs are still ill defined. This study used the creative cognition model to argue that cosmopolitanism, cultural orientations such as power distance and individualism, and a multi-faceted approach towards creativity are positively related within a cross-cultural context. More specifically, we argue that cosmopolitanism is a predilection for generative ideation, and global managers who exhibit high levels of cosmopolitanism are more likely to have high self-expectations of creativity and higher levels of creativity, novelty, and usefulness to their ideas. A survey study along with a creativity exercise showed that cosmopolitanism is significantly and positively related to self-expectations of creativity, novelty, and creativity, but not usefulness. Further, self-expectations for creativity mediated the relationship between cosmopolitanism and creativity and usefulness. The relationship between cosmopolitanism and self-expectation for creativity was moderated by power distance but not individualism. We describe the practical and theoretical implications of these results for future research into culture and creativity. (For more information, please contact: Dilek Gulistan Yunlu, Northeastern Illinois University, USA: d-yunlu@neiu.edu)

\section{Positive Traits and Performance of Internationally Engaged Executives Leonardo Liberman, Universidad de los Andes Lisa Sofia Clefberg, Karolinska Institute}

Existing literature has recognized the importance of positive traits for individuals' satisfaction and their social and personal functioning due to the fundamental role they play in our motivation, learning and wellbeing. The purpose of the present study was to assess the effect that three positive attributes have on work performance among 99 internationally engaged executives. Previous research suggests that the levels of Self-efficacy, 
Optimism and Curiosity influence a wide array of behaviors and performance when coping with challenging situations and in particular those that involve intercultural interactions. We argue that these traits contribute to the performance of executives helping them cope with the daily intercultural interactions that their positions entail. We also contend that identifying, understanding and enhancing such attributes can contribute to a better performance and satisfaction of internationally engaged executives. Significant bivariate correlations were found between work performance and the three traits separately. Moreover, the regression model that we proposed including Self-efficacy, Optimism and Curiosity was statistically significant to predict work performance, the individual attributes with a significant contribution being Self-efficacy and Curiosity. We discuss the practical and theoretical relevance of these finding and also suggest future lines of research. (For more information, please contact: Leonardo Liberman, Universidad de los Andes, Chile: leonardo. liberman@gmail.com)

\section{Returnee Spillover Effect on Corporate Performance: Evidence from Chinese A-share Public Firms $\mathrm{J}$ in $\mathrm{Xu}$, Central University of Finance and Economics Lianxi Zhou, Brock University Guangbao Xie, Bank of China, Anhui Province Branch}

With international returnees coming back in great number, scholars began to pay attention to the influence of returnee spillover effect on corporate preformation at home. This paper, based on a sample of Chinese A-share public firms covering the period from 2001 to 2012, examines whether overseas education background and work experience of intellectual returnees along with other factors would have a positive effect on corporate performance. Through an empirical analysis, the research findings show that intellectual returnees have a significant impact on corporate performance, but with some variations in further analyses of the key variables of interest. Specifically, education background of returnees tends to be negatively related to corporate performance unless the returnees are equipped with certain overseas work experience. Besides, the results also show that indigenous enterprises with better resources as indicated by firm size and age tend to outperform by having returnees on board. This study contributes to the growing body of knowledge on returnee entrepreneurs in emerging economies. (For more information, please contact: Lianxi Zhou, Brock University, Canada: Izhou@brocku.ca)

\section{Session: 1.3.13 - Interactive}

\section{Track: 13 - I nternational Economics, Finance and Accounting}

\section{International and Comparative Finance}

Presented On: June 28, 2015 - 13:00-14:15

Chair: J agadish Dandu, Zayed University

Time Horizon and Corporate Investment: Evidence from Private Firms around the World Serkan Akguc, King Abdulaziz University

J ongmoo J ay Choi, Temple University

We examine the relationship between time horizons and corporate investment, both on the firm and country levels, for private, unlisted firms and publicly traded firms using a unique dataset from 73 countries around the world during the time period of 2003-2012. We show that a longer time horizon (i.e. higher propensity to save and invest) on a cultural and country level also manifests itself as higher investment at the firm level. This is robust to using alternative proxies for the country-level time horizon. Investment behavior of private firms, not public firms, follows a country-level horizon pattern, which is reflective of close monitoring by fewer owners and the absence of stock market pressures in making investment decisions. When we consider time-horizon at the firm-level, we find that firms with a longer time horizon invest more, and this effect is more pronounced for public firms than for private firms, given the former's greater, easier, and cheaper access to capital in the public capital market. We also show that public firms invest more and are more responsive to investment opportunities 
than private firms. (For more information, please contact: Serkan Akguc, King Abdulaziz University, Saudi

Arabia: sakguc@kau.edu.sa)

\author{
Financial Constraints and the Interdependence of Corporate Financial Decisions: A Cross-Country Study \\ Paulo Renato Soares Terra, EAESP-FGV \\ Guilherme Kirch, EA-UFRGS
}

We examine the implications of the models proposed by Almeida and Campello (2007) regarding investment in fixed assets and Acharya, Almeida and Campello (2007) regarding the demand for liquidity in a sample of firms from over 60 countries in the period 1996-2010. Unlike previous studies, we consider the interdependence of financial decisions thus accounting for the direct impact these endogenous variables may have on each other. In addition, we explore the impact of each country's particular legal environment on the pledgeability of assets of the firm as collateral. We specify and estimate a system of simultaneous equations with panel data and firm's fixed effects by three stage least squares (3SLS). Our main findings largely corroborate those of previous studies regarding the interdependence of financial decisions. We also find evidence suggesting that financial constraints have a major impact on the financial decisions of the firm. However, implications regarding the impact of cash flows on investment and cash holdings decisions are only partially confirmed. Our results also suggest that financial constraints manifest themselves in virtually all firms, possibly at different intensities, indicating that such constraints are a matter of degree and not of kind. (For more information, please contact: Paulo Renato Soares Terra, EAESP-FGV, Brazil: paulo.terra@fgv.br)

\title{
The Impact of Crisis on the Determinants of Leverage: European Evidence Victoria Krivogorsky, San Diego state University \\ Gun-Ho J oh, San Diego State University \\ David Deboskey, San Diego State University
}

This paper records the change in the relevance of European institutional and macroeconomic indicators in assessing firms' capital structure before and during the financial crisis. The importance of this study stems from the fact that the risk estimation associated with a firm is more challenging now than at any time in recent history. We go about our examination using cluster analysis and find a significant reduction in the use of debt from pre- to post 2008 period. Among tested variables, after 2008 the level of aggregated direct foreign investment and solvency of national banking systems gain significant impact on the use of bank debt. We also document the evidence that the level of inflation, capacity utilization ratio and design of national tax system impacts the utilization of bank loans. (For more information, please contact: Victoria Krivogorsky, San Diego state University, USA: vkrivogo@mail.sdsu.edu)

\section{Earnings Management and Main Bank Monitoring: Evidence From Japan Hideaki Sakawa, Nagoya City University Naoki Watanabel, Ritsumeikan University}

This paper investigates whether or not main bank systems are effective for mitigating client firms' earnings management behavior in Japan. Although J apanese traditional corporate governance mechanisms are known as relational or bank-dominated systems, its foreign ownership has increased since 1990s. In the transition era of corporate governance, there remains a question that who is the effective gatekeeper to decrease earnings management in J apan. We find that earnings management in firms with main bank relationships tend to be mitigated, compared with firms without them. On the other hand, foreign shareholdings enhance earnings management because of their short-term investment horizons. This implies that main bank systems function as effective gatekeepers under bank-dominated systems and would be expected for substituting roles of the AngloSaxon system. (For more information, please contact: Hideaki Sakawa, Nagoya City University, Japan:

sakawa@econ.nagoya-cu.ac.jp) 


\section{Market Structure and Profitability in Banking Sector: Is China's Case Different? \\ Yanjun Huang, China Foreign Affairs University \\ J iawen Yang, George Washington University}

In this paper, we investigate the structure-performance relationship in China's banking by employing a unique panel data of 168 banks covering 2004-2012 period. Contrary to the previous work in developed countries, our results show that market concentration leads to lower profitability and no significant relationship is observed between market power and profitability, indicating little evidence to support SCP and RMP hypotheses in China's banking. By testing the structure-performance relations across banks with different size, we find that the undermining effect of market share on profitability is significant for the largest banks, independent of concentration; inversely, concentration decreases profitability in the rest of banking sector, rather than market share. In addition, we find that unexpectedly concentration increases bank profitability during financial crisis. By exploring the effects of foreign minority ownership, we confirm that foreign acquisition tends to improve banking profitability under the great banking system reform in China. (For more information, please contact: Yanjun Huang, China Foreign Affairs University, China: huangyanjun@ruc.edu.cn)

\section{Risks Associated with Cross-Listings \\ Hui He Sono, James Madison University \\ Mark Liu, University of Kentucky}

Researchers have documented many benefits for non-U.S. firms to cross-list their shares on U.S. markets, while the costs associated with crosslisting seem relatively small. Many have wondered why more foreign firms do not choose to list their shares in US markets. We show in this paper that there are significant risks associated with cross listing. We find that after crosslistings: (1) stock returns commove more with the international market; (2) the probability of extreme negative stock returns increases; (3) stock returns are more negatively skewed; (4) the above changes are driven by the systematic instead of idiosyncratic part of stock returns. These findings show that crosslisting increases firms' systematic risks. (For more information, please contact: Hui He Sono, James Madison University, USA: sonohh@jmu.edu)

\section{Effect of Regional Trade Agreements - A Study of India-ASEAN Trade with Firm Level Data \\ Amlan Ray, Amrita University \\ Amalendu J yotishi, Amrita University}

India ASEAN Free Trade Agreement (FTA) came into effect from 2010. Free trade is expected to increase production and benefit both for producers and consumers. But, there has been intense debate in the literature on the relative benefits of such FTAs, pluri-lateral agreements that are being negotiated now (e.g. Trans-Pacific Partnership and Trans-Atlantic Trade and Investment Partnership) and the global free trade agreements under WTO, meaning a strict implementation of the MFN clause. Collectively, India-ASEAN trade agreement is expected to cover a market of 1.8 Billion people and slash tariff on 4000 items. There has been trade imbalance between India and ASEAN. In September, 2014, I ndia has also signed a free trade agreement with ASEAN on Services and Investment. This agreement on Services is expected to be complete soon and address the issue of Bilateral Trade I mbalance between India and ASEAN. Our paper tries to find out how the regional trade agreement signed by India is helping it in recovering its industry from decline. This paper attempts to measure import content in Indian exports in different sectors. In this paper, first we have made an attempt to list the sectors which are more often traded between India and ASEAN and then checked the import intensity of those sectors with firm level data for the period of 2003-2013. Our initial finding indicates that India's export-oriented sectors have high import intensity. We make an attempt to check whether India's exports to ASEAN are also high in import intensity and a potential reason for the trade imbalance between these two regions. The findings will have an effect on the existing and future Trade agreements signed by India. (For more information, please contact: Amlan Ray, Amrita University, India: amlanray1@gmail.com) 


\section{Session: 1.4.1 - Panel}

Track: 5 - The Geography of I nternational Business and Global Value Chains

\section{AlB - Regional Science Association International (RSAI) Session}

Presented On: J une 28, 2015 - 14:30-15:45

Chairs: Simona Iammarino, London School of Economics and Lucia Piscitello, Politecnico di Milano

Panelists:

Lucia Piscitello, Politecnico di Milano

Simona I ammarino, London School of Economics

Michael Storper, UCLA

Maryann Feldman, University of North Carolina

The aim of this Showcase Panel is to bring together two of the most distinguished scholars in the field of economic geography and regional sciences representing different theoretical perspectives and disciplinary approaches in the field. Prof Maryann Feldman and Prof Michael Storper will provide their insights and provocative thoughts on the role of geography and space in the current state of the art of conceptualisation and empirical evidence of international division of labour and organisation of global value chain and production networks. As it has been increasingly argued in recent research, geography and space-specific features are becoming increasingly important for multinational enterprises (MNEs) and, in turn, MNEs are becoming progressively more important for local and regional economic development and growth. The pivot on which this vital relationship turns is the creation, diffusion and management of new knowledge. As yet, though, neither a unified or dominant theory of the MNE in space has emerged, nor the different theoretical approaches and methods have taken each other into full consideration to advance our understanding of the geography of MNEs. (For more information, please contact: Lucia Piscitello, Politecnico di Milano, Italy: Iucia.piscitello@polimi. it)

\section{Session: 1.4.2 - Panel Track: Scholars Meet Practice}

\section{Big Data for Innovation, Competition and Productivity}

Presented On: June 28, 2015 - 14:30-15:45

Chair: Pulak Ghosh, Indian Institute of Management Bangalore

Panelists:

Pulak Ghosh, Indian Institute of Management Bangalore

Soumya Kanti Ghosh, Chief Economic Advisor, State Bank of India

Suresh Srinavasan, Co-Founder, Formcept

Ravi Vijayaraghavan, Vice President and Head Analytics, Flipkart.com

Siva Viswanathan, University of Maryland

Big data is no longer a buzzword. It is happening! More and more marketing firms these days, are not only talking about big data, but also trying to develop the talent pool to utilize the effectiveness of big data in informed decision making and increasing the ROI. It is changing the way the marketing firms works, particularly in India, where the data centric decision-making is rather new. Successful organizations are focusing more and more on big data analytics. (For more information, please contact: Pulak Ghosh, Indian Institute of Management Bangalore, India: pulak.ghosh@iimb.ernet.in) 


\section{Session: 1.4.3 - Panel \\ Special Session}

\section{The Institutional and Political Environment of I B}

Presented On: J une 28, 2015 - 14:30-15:45

Chair: Michael Witt, I NSEAD

Discussant: Paul Martin Vaaler, University of Minnesota

Panelists:

Michael Witt, I NSEAD

Ruth Aguilera, Northeastern University

Andreas Schotter, I vey Business School

Markus Taussig, National University of Singapore

Paul Martin Vaaler, University of Minnesota

The importance of institutions and politics for the understanding of IB phenomena has by now become widely accepted. Yet there is considerable room for further elaboration of the relationship between IB and its institutional and political contexts and their co- evolution over time. The objective of this panel is to help along this process by proposing new directions for future research. Our emphasis in selecting scholars was less on name recognition and more on the "freshness" of ideas proposed. Accordingly, our panel consists of a mix of established and rising scholars: Ruth Aguilera, Andreas Schotter, Markus Taussig, and Michael A. Witt. Paul Vaaler has agreed to serve as moderator and discussant. (For more information, please contact: Michael Witt, INSEAD, Singapore: michael.witt@insead.edu)

\section{Session: 1.4.4 - Competitive \\ Track: 3 - International Human Resource Management and Organizational Behavior}

\section{Driving Performance in Global Organizations}

Presented On: June 28, 2015 - 14:30-15:45

Chair: William Newburry, Florida International University

High Performance Work Practices Exist in India: Evidence from Two Organizations Nikos Bozionelos, Audencia Nantes Ecole de Management Vijay Edward Pereira, University of Portsmouth

High-performance-work-practices (HPWP) have been well documented within strongly performing work organizations in developed country economies. Such practices, however, remain under-investigated in emerging economies. This paper aims to work towards filling this void, by empirically evaluating HPWP within two successfully performing Indian public-sector-undertakings (PSUs). Case-study methodology was employed with an "inverse" approach whereby work practices within two successful firms were identified in the first step, and in the second step it was determined whether these fitted into the chosen HPWP framework. It was found that aspects of all HPWP identified in the template framework were present in both organizations, albeit in varying levels and sometimes novel ways. Thus we argue that a combined management strategy of high commitment (HCM), involvement (HIM) and performance (HPWS) allowed the case-study organizations to achieve success and produce profits through their people. The findings also suggest complementation and convergence when it comes to the 'contingency- model' and the alternative 'universalist-model', which are both suitable and successful when it comes to HPWP. The paper contributes to the emerging knowledge of management practices in Indian organizations, which represent a major emerging economy. (For more information, please contact: Nikos Bozionelos, Audencia Nantes Ecole de Management, France: nbozionelos@audencia.com) 


\section{Human Resource Management in India Strategy, Performance and Complementarity Tamer Darwish, University of Gloucestershire Satwinder Singh, Brunel University London Geoffrey Wood, University of Warwick}

This study seeks to explore which types of HR practice are associated with better organisational performance (OP). Whilst the core finding - that specific HR practices lead to better organisational outcomes may not be surprising - we also found an absence of complementarity. Normally, the absence of complementarities would suggest limitations in institutional supports; on the one hand, however, institutional shortfalls are not unique to India and may be encountered in many emerging market settings. In contrast, the great internal diversity of the Indian setting, with strong variations recognised amongst institutions, along with enforcement capabilities, might suggest that these tendencies are particularly pronounced. We also found a strong link between the intrinsic rewards and performance-an unexpected result in a low-income country, where wages are generally low. We suggest that this may reflect the nature of the labour market and the limited (and possibly proportionately shrinking) pool of good jobs, making exit a difficult option for all but the best qualified. Whilst this puts employees in a poor bargaining position in bidding-up pay (making pay rises seem unfeasible), the intrinsic attributes of the job become more important. (For more information, please contact: Satwinder Singh, Brunel University London, United Kingdom: satwinder.singh@brunel.ac.uk)

\section{Equity Portfolio Incentives to CEOs for Downsizing: Differential Impacts on Survivors vs. Victims in Three Countries \\ C Lakshman, Tongji University \\ Linh-Chi Vo, Normandy Business School \\ Rani Ladha, Indian Institute of Management Kozhikode}

Although downsizing research has examined victims' and survivors' reactions and justice perceptions, few studies have examined perceptions of corporate social responsibility (CSR). We examine the impact of CEO compensation for downsizing on CSR perceptions of downsizing decisions made by CEOs in three countries: France, India, and Vietnam. We use a $2 \times 2 \times 2 \times 2$ (performance linked bonuses, internal vs. institutional pressure, loss of human capital-yes/no, and role-victim/survivor) between subjects experimental design to examine factors that influence the CEO's downsizing decision. Results suggest that downsizing resulting in loss of human capital is negatively related to CSR perceptions. Downsizing motivated by deferred compensation and decline in performance linked bonuses are negatively related to survivor commitment, but not to victims' perceptions of fairness. We also find support for convergence across the three countries, with some divergence as a result of power distance. We provide a discussion of the results, limitations, and directions for future research. (For more information, please contact: C Lakshman, Tongji University, China: clakshman2007@gmail.com) 


\section{Session: 1.4.5 - Competitive}

\section{Track: 6 - Innovation and Knowledge Management}

\section{Institutional Environment and Innovation Performance}

Presented On: June 28, 2015 - 14:30-15:45

Chair: Kristin Brandl, University of Reading

International Networks for Developing Exploratory Innovations in Emerging Economies Zaheer Khan, University of Sheffield

This article shows that under weak institutional arrangements and institutional voids in emerging economies, local firms that are part of the global value chains of MNEs develop international network relationships as a balancing mode strategy in order to counter the negative influences of local institutions on exploratory innovations. Using in-depth qualitative analysis, we study 12 motorcycle parts suppliers of Pakistan that are working with some of the leading J apanese and Chinese motorcycle assemblers. The findings suggest that local institutional factors are only favourable for developing exploitative/incremental innovations, and the motorcycle parts suppliers develop international networks with global Tier 1 suppliers, international trade fairs and international institutions as a balancing mode strategy in order to circumvent the negative influence of home institutional factors for developing exploratory/radical innovations. Our study shows the importance of global networks and pipelines as a balancing mode strategy for developing exploratory innovations by emerging economies firms. (For more information, please contact: Zaheer Khan, University of Sheffield, United Kingdom: khan.zaheer@gmail.com)

\section{How Institutional Context Matters for R\&D? An Exploratory Study of Medical Device Development in India Budhaditya Gupta, Harvard University \\ Stefan Thomke, Harvard University}

Today multinationals (MNCS) routinely engage in research and development (R\&D) activities at locations that vary widely in context. While the connection between context and firm performance has been shown, the impact of context on R\&D has not been studied in detail and the mechanisms by which context influences R\&D activities are still somewhat unclear. In this paper, we will explore how context of a location influences experimentation and testing practices fundamental to the R\&D search processes. Based on a detailed field study of medical device development in India, we find that institutional context can dramatically expedite R\&D search through higher fidelity experimentation and testing in the early stages of product development. Other than being one of the early works on how developed-country MNC R\&D centers located in emerging markets adapt the search process given the local institutions, the discussion in this paper points to the innovation impeding institutional voids in the US. We conclude with a discussion on implications for managerial practice and research related to innovation and global R\&D. (For more information, please contact: Budhaditya Gupta, Harvard University, USA: bgupta@hbs.edu)

\section{Public Support for Innovation and the Openness of Firms' Innovation Activities Marcelo F Cano-Kollmann, Temple University \\ Robert D. Hamilton III, Temple University}

This multi-country study explores empirically, for the first time, the relationship between publicly-funded schemes to support innovation and the "openness" of firms' innovation practices. Using survey data from more than 5,000 firms in 29 European countries, we find that support policies for innovation, both monetary and nonmonetary, are related to an increase in the degree of openness of individual firms, both in terms of the number of external partners with whom they collaborate and the number of open innovation activities they perform. But the relationship between support and openness seems to be negatively moderated by the existence of previous 
innovative activity within the firm. Public support has more impact on less innovative firms and less influence when the firm is already innovative, which could potentially indicate the existence of crowding-out effects. Additionally, our novel results suggest that non-monetary support is more strongly linked to openness than financial support. For policy makers facing salient financial constraints, this implies that institutions and government policies could potentially play a more important role than money in fostering open innovation. This paper contributes to the open innovation literature by tapping into the underexplored question of the role of public policies and has relevant implications for academics and policy makers. (For more information, please contact: Marcelo F Cano-Kollmann, Temple University, USA: mck@temple.edu)

\title{
National Trust and the Effects of Firm Size on and Foreign Ownership on Innovation by Firms in Developing Nations
}

Gregory Stephen Hundley, Purdue University

Xiaomei Li, Tianjin University

The nature of trust relationships in a society affects firm-level innovation through impact on the information available to the firm from the external environment and on the firm's motivations to innovate to mitigate the resistance from local customers and work forces. Analysis of a large sample of manufacturing firms in developing nations shows that the effects of foreign ownership are influenced by the national trust environment. The effect of foreign ownership on product innovation is more strongly positive in particularistic societies characterized by high in-group and low in out-group trust where firms may be motivated to engage in local innovation to mitigate for resistance to foreign technologies and product features. The differential effects of out-group and in-group trust on process and product innovation are explored, with product innovation relatively sensitives out-group trust than process innovation while both product and process innovation are equally sensitive in-group trust. (For more information, please contact: Gregory Stephen Hundley, Purdue University, USA: ghundley@purdue.edu)

\section{Session: 1.4.6 - Competitive}

\section{Track: 8 - Global Strategy, M\&As and Competitiveness}

\section{Creating Value from Cross-Border Acquisitions}

Presented On: June 28, 2015 - 14:30-15:45

Chair: Anupama Phene, George Washington University

\author{
International Acquisitions and International Competitiveness \\ Omer Faruk Genc, Abdullah Gul University \\ J ongmoo J ay Choi, Temple University
}

International competitiveness is a major national policy issue, but little work has been done on a firm level. We examine whether a firm's international competitiveness is assisted by international acquisitions using a sample of 3,565 international acquisitions by US firms during 1985-2007. Predictions from management theories such as resource and agency theories contradict each other. After controlling for country factors, we find that international acquisitions enhance the competitiveness of acquirers compared to a control sample of no acquisition, but not necessarily so against domestic acquisitions. The resources and capabilities of acquirers and their complementarity with those of the target firms are important in explaining the success of international acquisitions. The results are robust with regard to different competitiveness benchmarks and endogeneity of acquisition decisions. (For more information, please contact: Omer Faruk Genc, Abdullah Gul University, Turkey: gencomerfaruk@gmail.com) 
How to Make the Most of What You Buy: Value Creation in Cross-Border Acquisitions of Developed Market Firms

(DMFs) by Emerging Market Firms (EMFs)

Yinuo Tang, University of Pittsburgh

Ravi Madhavan, University of Pittsburgh

Cross-border acquisitions are popular tools for MNCs to create value through obtaining strategic assets and exploiting their productivity advantages in foreign markets. As late-comers to global competition and often subject to institutional voids, Emerging Market Firms (EMFs) acquiring Developed Market Firms (DMFs) are usually seeking to upgrade their capability. This study suggests that cross-listing helps EMFs create value in cross-border acquisitions and that acquirers' productivity and institutional experience are critical to enhance the returns. A dataset of 466 Chinese Firms' cross-border acquisitions in the time period of 2000-2010 supported our hypotheses. (For more information, please contact: Yinuo Tang, University of Pittsburgh, USA: yit14@pitt.edu)

\section{Losing the Battle: Unexpectation and Misexpectation in Cross-Border M\&A} Tian Wei, Fudan University

Although the M\&A literature has emphasized the factors and mechanisms involved in pursuing success for acquirers in cross-border M\&A, less is known about why most of acquirers fail to fulfill their expectations. Through an inductive study of five cross-border M\&A cases, we firstly unravel the definition of success for the acquirers and how the acquiring firms achieve this. The data suggest that the success of acquirers should be measured using the approach of the managers' retrospective assessment by focusing on time structure, i.e. value gap and time extension. We then identify the drivers for the failure of acquirers in the acquisition process: triggers in pre-acquisition and value destroyers in post-acquisition. In the course of generating the grounded model, we also uncover the relationships between the drivers and consequences using three propositions to reveal the underlying mechanism for pursuing success. We discuss the implications of the findings for the M\&A theories, the challenges to the traditional research angles and the limitations of this study more generally. (For more information, please contact: Tian Wei, Fudan University, China: weitian1983@gmail.com)

\section{Executive Migration and International Mergers and Acquisitions Yu-Kai (Mike) Wang, Soochow University}

This study examines the impact of executive migration on a firm's acquisition decisions related to particular host countries. Integrating upper echelons theory with learning-by-hiring literature, this study argues that executive migration serves as a conduit for inter-organizational learning. This study proposes that inter-organizational learning associated with migrating executives' international acquisition experience in general and within a particular host country specifically are both positively associated with a firm's decision to undertake acquisitions in the particular host country. It further argues that the influence of the latter type of inter-organizational learning is greater than that of the former. The empirical results support these arguments. In sum, this study contributes to the literature on international acquisitions by uncovering the critical role of migrating executives play in host country acquisition decisions through the inter-organizational learning such migrating executives facilitate. (For more information, please contact: Yu-Kai (Mike) Wang, Soochow University, Taiwan: ywang012@scu.edu.tw) 
Session: 1.4.7 - Panel

Track: 9 - Emerging Economies and Emerging Economy MNEs

\section{Women in Business in India (WAI B Special Session)}

Presented On: June 28, 2015 - 14:30-15:45

Chairs: Gabriele Suder, University of Melbourne and J anet Murray, University of Missouri-St. Louis

Panelists:

J anet Murray, University of Missouri-St. Louis

Gabriele Suder, University of Melbourne

Padmaja Alaganandam, PricewaterhouseCoopers Pvt. Ltd.

Revathy Ashok, I ris Consulting

Alice de J onge, Monash University

Usha Raghunath, Wipro Ltd.

Pamela Kumar, HP Enterprise R\&D

This panel provides professional guidance on the challenges and opportunities faced by women in business. Speakers from the industry and academia who are experts on India will bring their unique perspectives in discussing critical issues faced by women in the business world. They will discuss topics that cover from career progression, succession planning and diversity, succeeding in different career tracks (consulting, technical, and management), achieving equality at the top for women, and accelerating women entrepreneurship. (For more information, please contact: Janet Murray, University of Missouri-St. Louis, USA: murrayjan@umsl.edu)

\section{Session: 1.4.8 - Competitive}

\section{Track: 12 - The Institutional and Political Environment of I B}

\section{New Perspectives on Institutional Distance}

Presented On: June 28, 2015 - 14:30-15:45

Chair: Peter Buckley, University of Leeds

Revisiting Institutional Distance: Towards an Agency-Oriented Understanding J ohann Fortwengel, Free University Berlin

This paper builds on recent calls for theorization of the institutional distance concept by making four interrelated arguments. First, we argue that distance is about the particular kind of relationship between home and host country configurations; second, we introduce the idea that host institutional environments may differ in degree of stability; third, we highlight the role of sub-national variation in terms of varying degrees of institutional thickness; fourth, we argue that institutions can be resources. We develop a model of scope for action of MNCs in relation to these context factors. The paper contributes to existing literature by discussing boundary conditions for agency in light of institutional distance. (For more information, please contact: Johann Fortwengel, Free University Berlin, Germany: johann.fortwengel@fu-berlin.de) 
Testing the Formal Institutional Distance - Subsidiary Performance Link, and the Moderating Effects of Ownership Strategy and Experience: Evidence from 17 Emerging Markets

Vikrant Shirodkar, University of Sussex

Palitha Konara, University of Huddersfield

We examine the link between formal (regulatory) institutional distance and subsidiary performance of foreignowned multinational enterprises (MNEs) operating in 17 emerging markets, based on a large panel dataset consisting of 13975 firms whose performance we traced over the 9-year period: 2004 - 2012. We hypothesise that greater formal institutional distance has a negative impact on subsidiary performance. We also build theoretical arguments regarding the moderating effects of ownership strategy and host country experience on the institutional distance - subsidiary performance relationship. Our empirical findings based in the context of emerging markets support the negative effect of institutional distance on the performance of MNEs' subsidiaries operating in these markets. Confirming our theoretical arguments, our findings also show that the negative effects of institutional distance on subsidiary performance are lesser for subsidiaries with partial ownership (than for subsidiaries with full ownership), and for subsidiaries with greater host country experience. (For more information, please contact: Vikrant Shirodkar, University of Sussex, United Kingdom: v.shirodkar@sussex.ac.uk)

\section{Institutional Logics, Liability of Foreignness, and Poverty Alleviation: The Case of Mining MNEs in East Africa Aloysius Newenham-Kahindi, University of Saskatchewan \\ Charles Edward Stevens, Lehigh University}

Traditional Liability of Foreignness (LOF) research has focused on LOF due to cultural, institutional, or geographic "distance." These distance-based LOF are caused by information asymmetries, which can be reduced by MNE efforts to gain knowledge about the local environment. In contrast, we posit that LOF caused by "friction" are caused by competing institutional logics, and can be resolved through the utilization of intermediaries embedded in both sets of institutional logics-those of the MNE and of the local community. Using case studies of eight foreign mining MNEs investing in sub-Saharan Africa, we find support for this logic. These findings highlight the benefits, as well as the inherent difficulties, of resolving LOF caused by friction due to competing institutional logics. (For more information, please contact: Charles Edward Stevens, Lehigh University, USA: ces213@lehigh.edu)

\section{Partner Selection in International Technological Alliances: The Role of Institutional Distance, Colonial and Economic Ties \\ Sorin Krammer, University of Groningen}

Selection of appropriate partners, especially in an international setting, is a critical ingredient for a successful alliance. I posit that institutional distance influences negatively partner selection in exploitative technological alliances beyond the firms-specific factors documented in the literature. Furthermore, I suggest that the effect of institutional distance on alliance partner selection is moderated by the extent of colonial and economic ties between countries. Based on a longitudinal dataset of firms in the global tire industry, results confirm that MNEs prefer alliance partners from closer cognitive-normative environments, and from similar or stronger regulatory ones. Moreover, my analysis indicates that colonial and economic ties between nations moderate the relationship between institutional distance and partner selection. These findings offer a more comprehensive view of partner selection decisions in an international context, improving our knowledge on the effects of institutions on firm interactions, and their interplay with historical (i.e., colonial) and economic context of countries (For more information, please contact: Sorin Krammer, University of Groningen, Netherlands: sorin.krammer@gmail.com) 


\section{Session: 1.4.9 - Competitive}

\section{Track: 7 - Digital Transformation and Online Markets in I B}

\section{Digital Transformation and Online Markets in I $B$}

Presented On: June 28, 2015 - 14:30-15:45

Chair: Peter W. Liesch, University of Queensland

The Synergy of Entrepreneurial Self-Efficacy and Virtual Self-Efficacy for Entrepreneurs in Emerging Online Markets: An Empirical Investigation

Zeying Wan, Saint Mary's University

Taiyuan Wang, IE Business School

Yinglei Wang, Acadia University

Online markets enable entrepreneurial behaviors on a global basis through standardized payment systems, logistics management, and interface designs. However, such standardized processes and features make it difficult for online businesses to differentiate from each other. How can entrepreneurs succeed in online markets? In this study, we posit that entrepreneurial self-efficacy (ESE) and virtual self-efficacy (VSE) of online business owners play a synergistic role in enabling operational creativity, which in turn is a critical antecedent to online business performance. We found supportive evidence for these hypotheses by analyzing survey data from 267 business owners from Taobao.com, a major peer-to-peer platform in the emerging Chinese online markets. (For more information, please contact: Taiyuan Wang, IE Business School, Spain:

taiyuan.wang@ie.edu)

The Early-Mover Advantage of Exporters at Online B2B Portals

Ziliang Deng, Renmin University of China

Zeyu Wang, Nankai University/Chinese University of Hong Kong

As internet enables enterprises to go international via cross-border business-to-business ecommerce portals, researchers know little about the early-mover advantages at such third-party platforms. As such platforms offer quick, convenient and wide market accesses, early-mover exporters may enjoy advantages over the late movers in learning effects and switching costs. We hypothesize that early-mover advantages may eventually diminish beyond a critical tenure point due to the market education costs, product trial and error costs and asset specificity of the early movers. Moreover we argue that product price and diversity will pose different boundary conditions on the manifestation of early-mover advantages. We collect data on approximately 300,000 businessto-business export transactions conducted by nearly 4,000 firms in 84 months during 2007-2014 at an online portal by web search and mining methods. Employing panel data analysis models, we find strong evidence supporting our hypotheses. The exporters exhibit the highly dynamic nature of the early-mover advantages in cross-border business-to-business ecommerce platforms, and therefore the inclusiveness of such platforms. (For more information, please contact: Ziliang Deng, Renmin University of China, China: dengziliang@rbs.org.cn)

\section{Internationalization of Entrepreneurship and Institutional Reinforcement in a New Global Industry Irina Mihailova, Aalto University Peter W. Liesch, University of Queensland Elizabeth Rose, University of Otago}

In a new industry that does not conform to the standard assumptions of international business - value creation in production and marketing is costly and product adaptation to local markets can be important - Finland-based entrepreneurial firms in the mobile gaming industry have captured global markets at a rapid pace. How and why have these Finnish firms achieved a global market positioning, particularly in the light of Finland's small home market, relative cultural isolation and geographically peripheral location? In the free-to-play games segment, 
where the traditional norms of international business practice do not hold, firms have created a business model in which industry-specific and informal socio-cultural institutional arrangements are reinforced by formal, government-sponsored ones to create the conditions for global leadership. This phenomenon exemplifies the internationalization of entrepreneurship. (For more information, please contact: Irina Mihailova, Aalto University, Finland: irina.mihailova@aalto.fi)

\section{Micro Enterprises in eMarketplaces: Comparing Digital Strategy across Continents Manjula S. Salimath, University of North Texas Vallari Chandna, University of North Texas}

eMarketplaces are disrupting supply chains by directly linking the seller and customer. This has created a seemingly interconnected global business landscape that can be navigated by both buyers and sellers with relative ease and functionality. The number of micro enterprises that are leveraging the convenience of eMarketplaces to set up their online businesses has grown exponentially in the past decade. In addition, eMarketplaces offer opportunities for internationalization that can speed the scalability of micro enterprises. Yet not much is known about these businesses. We explore the type and effect of digital strategies and online visibility on the performance of micro enterprises in eMarketplaces and hypothesize that there will be differences across continents. Based on a random sample of 179 firms representing all six continents (Africa, Asia, Europe, N. America, S. America and Oceania) we tested several direct effects models. The results provide statistical support for hypothesized relationships between digital strategy and online visibility on financial performance of micro enterprises on eMarketplaces. (For more information, please contact: Manjula S. Salimath, University of North Texas, USA: manjula.salimath@unt.edu)

\section{Session: 1.4.10 - I nteractive \\ Track: 6 - Innovation and Knowledge Management}

\section{Learning from Subsidiaries in Emerging Markets}

Presented On: June 28, 2015 - 14:30-15:45

Chair: Pavlina Jasovska, University of South Australia

When are Subsidiaries Motivated to Initiate Innovation under Autonomy?: The Role of Resource Dependence and Power Relation Jason Kyewon Lee, Korea University

Dae-il Nam, Korea University

Drawing from the concept of power-dependence theory, we argue that the effect of foreign subsidiary autonomy on subsidiary innovation is contingent on the power relation of subsidiary and its counterparts. Focusing on the behavioral aspect of subsidiaries, we consider the possibility of subsidiaries deviating from headquarters' expectation to initiate innovation when autonomy is given. To better explain the nature of subsidiary behavior under autonomy, we suggest that resource dependence and power relation within the MNE and the local business network serve a moderating role by influencing subsidiary's motivation towards innovation. (For more information, please contact: Jason Kyewon Lee, Korea University, Korea, South: kyewon.jason.lee@gmail.com) 
Building Innovation Capabilities in Emerging Markets: A Microfoundations Approach to Managing Local Product Development

Shad Morris, Brigham Young University

Daniel Han Ming Chng, China Europe International Business School

Jian Han, CEIBS

James Oldroyd, Ohio State University

This paper examines how foreign firms build innovation capabilities in emerging markets. Adopting a microfoundations approach, we examine how R\&D teams draw upon human, social and organizational capital as first-order resources in building a firm's innovation capability in a foreign market. In particular, we examine what types of experiences, social networks, and organizational controls and coordination mechanisms are necessary to outperform other R\&D teams in the foreign market. Drawing upon over 60 interviews among team members across 15 different foreign multinational firms based in China, we argue that local teams represent a primary source of innovation capabilities for the global firm. In particular, we hypothesize that high performing teams are more likely to have worked for other companies in the local market and be more likely to invest in corporate experience outside of their home market. High performing teams may also be more likely to take more of their valuable research time to talk with local customers and build relationships of trust with corporate colleagues. Finally, high performing teams may be more responsive to increased autonomy rooted in outcomebased incentives and participative management. (For more information, please contact: Shad Morris, Brigham Young University, USA: morris@byu.edu)

\section{Reverse Knowledge Transfer from Emerging Economy Subsidiaries: The Role of Disseminative Capacity Abby Jingzi Zhou, University of Nottingham, Ningbo \\ Carl F. Fey, Nottingham University Business School China}

The purpose of this conceptual paper is to address an important under-researched area in international knowledge transfer, namely the role of disseminative capacity in reverse knowledge transfer from subsidiaries in emerging economies to headquarters in advanced economies. We suggest that the four stages of local subsidiaries' disseminative capacity of identification, articulation, communication and association have positive effects on the four reverse knowledge transfer stages of preparation, initiation, implementation and ramp-up, respectively. Moreover, we go a step further and explore how local subsidiaries can contribute to the development of disseminative capacity via three categories of HRM practices including opportunity enhancing practices, motivation enhancing practices and skills enhancing practices. Our conceptualization has important implications for how local MNC subsidiaries in emerging economies can enhance reverse knowledge transfer to their headquarters. (For more information, please contact: Abby Jingzi Zhou, University of Nottingham, Ningbo, China: jingzi.zhou@nottingham.edu.cn)

\section{Antecedents of Reverse Diffusion of HRM Practices in MNC Subsidiaries: Organizational or Locational Legitimacy? \\ Michal Lemanski, Nottingham University Business School China}

Reverse diffusion of human resource management practices is crucial for competitiveness of multinational companies, and there is a growing interest in this phenomenon. However the understanding of antecedents of reverse diffusion is still incomplete. In particular, empirical evidence of which subsidiaries engage in reverse diffusion is only emerging. We employ the institutional theory framework to investigate location-specific and organization-specific factors affecting propensity of subsidiaries to engage in the process. Using a research design that merges qualitative and quantitative research methods, we put under scrutiny 85 foreign subsidiaries of multinational companies in 5 countries. We identify the sort of a subsidiary in which reverse diffusion is likely to occur, finding that mature and large subsidiaries are best positioned to develop practices that are subsequently diffused to units in other countries, and that in general organization-specific, rather than locationspecific variables are better predictors of reverse diffusion. We discuss implications of such findings for both 
future research and for management practice. (For more information, please contact: Michal Lemanski, Nottingham University Business School China, Poland: michal.lemanski@nottingham.edu.cn)

\section{Experiential Learning, Economic Distance And Subsidiary Performance Of EMNCS: Evidence From Indian Multinationals}

Arindam Mondal, Indian Institute of Management Calcutta

Sougata Ray, Indian Institute of Management Calcutta

Raveendra Chittoor, Indian School of Business

Sarada Devi Gadepalli, Indian Institute of Management Calcutta

This paper explores how direct experiential learning by foreign subsidiaries aid successful international expansion of emerging multinationals (EMNCS). Pursuing a subsidiary level analysis, this study tests all the divergent relationships, explored in the extant literature between subsidiary's own experience and the performance of focal subsidiary in the host economy. Most recent findings in the context of developed country MNCs indicate that the link may exhibit a non-linear form, but there is no consensus on the exact shape of the curve. Precious little is known about this question in the case of EMNCs, most of which are in the state of early internationalization. By integrating organizational (un)learning, international business and strategy literature, this study predicts an new, unified three-stage, horizontal ' $S$ '-shaped relationship between subsidiary experience and focal subsidiary performance for EMNCs. Also we predict that the economic distance between the host and home country will moderate this relationship. These predictions are tested empirically with proprietary, longitudinal data from 695 foreign subsidiaries of 221 Indian EMNCs (those were a part of the BSE 500 index as on November, 2013) for seven years, starting from 2006-07 to 2012-13. We find supporting evidence for most of our hypothesized relationships. (For more information, please contact: Arindam Mondal, Indian Institute of Management Calcutta, India: arindamm11@iimcal.ac.in)

\section{Session: 1.4.11 - I nteractive}

Track: 2 - Entrepreneurship, Micro-Multinationals, and I nternational New Ventures

\section{Capabilities for International Entrepreneurship: Emerging Market and Developed Market Perspectives}

Presented On: June 28, 2015 - 14:30-15:45

Chair: Etayankara Muralidharan, MacEwan University

Cross-National Replication in International Entrepreneurship: Lessons from SKS Microfinance J oshua K Ault, University of Victoria Andrew Spicer, University of South Carolina

We present an exploratory case study of SKS Microfinance's attempt to replicate the global microfinance model in India as a window to build theory about the role of cross-national imitation and innovation in new entrepreneurial ventures. We show that SKS's founder imitated foreign business models when designing his own microfinance organization, but these imitation efforts ultimately failed because of the company's inability to develop and sustain support from local actors. In the end, SKS's ability to replicate the original microfinance model stumbled due to political crisis and state intervention. We build on our case analysis to develop a research agenda that examines the challenges and tradeoffs of managing both global and local legitimacy in the cross-national replication of entrepreneurial business models. (For more information, please contact: Joshua $K$ Ault, University of Victoria, Canada: jault@uvic.ca) 
Born Global Decision - Gradual \& Complex Interaction between Multiple Elements: Evidence from a Software

Product Firm in India

Anish Purkayastha, Indian Institute of Management Ahmedabad

Based on in-depth case analysis of a software product firm in India, this study conceptualize that born global firms are majorly "driven" by multiple standalone drivers like 'leadership characteristics', 'home market push', 'host market pull', 'organizational determinants' and 'strategic decisions'. We have identified an integrated view of interactive nature of various standalone drivers of born global firms. Not all the drivers of born global firms are active throughout the life cycle of the firm. These have varied role in cross section of various stages (innovation, evolution \& formation) of the product and various phases (early, expansion \& maturity) of the firm's journey and play a complex interactive role to fuel and expand into international market in spite of presence of many challenges for born global firms. The research \& practitioners implications \& scope of further extensions are also discussed. (For more information, please contact: Anish Purkayastha, Indian Institute of Management Ahmedabad, India: anishp@iimahd.ernet.in)

\section{How Do Brand Capabilities Facilitate the Internationalization of Firms from Emerging to Developed Economies? Fernando Angulo-Ruiz, MacEwan University \\ Albena Pergelova, MacEwan University \\ William Wei, MacEwan University}

This paper examines the role of branding capabilities in the internationalization of firms from emerging markets. Current literature indicates that firms lacking strong and positive reputation in their domestic emerging markets are more motivated to internationalize into developed economies as opposed to other emerging economies. Using the resource based view of the firm and signaling theory, this paper argues that emerging market firms lacking strong and positive reputation will not be able to internationalize in developed economies or other emerging economies when seeking technology, markets and partnerships with host players. In these cases, these firms need to capitalize on strong branding capabilities. We focus our study on a unique sample of Chinese firms. Our findings indicate that firms from emerging economies that are seeking markets are more motivated to internationalize in other developing economies and branding capabilities provide good signals to enter these markets with full control modes. Our results also reveal that those firms seeking technology are more motivated to internationalize in developed economies through shared control modes; however, contrary to our expectations, branding capabilities provide weak signals to potential partners to realize this motivation. This study suggests emerging markets firms to develop their branding potential in their home markets to then leverage it when internationalizing into a developed or other developing economy. (For more information, please contact: Fernando Angulo-Ruiz, MacEwan University, Canada: fernando.anguloruiz@macewan.ca)

\section{International Small Ventures' Entrepreneurship and Export Market Performance \\ Aytug Sozuer, I stanbul University \\ Gultekin Altuntas, Istanbul University \\ Fatih Semercioz, Istanbul University}

This study investigates small firms' entrepreneurial posture as a determinant of export market performance in an emerging market context. Building on resource-based view, the research proposes that international entrepreneurship, as an organizational culture and a distinct capability, would positively influence performance. Empirical evidence from a cross-sectional survey of 326 international small firms reveals product and customer focus potentially has an explanatory power for foreign market success. The study also speculates on a higher order construct, named "new offer orientation", which is composed of innovation propensity, market orientation, and employee motivation directed to international markets. Moreover, it is inferred that risk assessment and business networking across borders can be complicated for small firms from emerging markets. (For more information, please contact: Aytug Sozuer, Istanbul University, Turkey: aytugsozuer@gmail.com) 
Evolution of a Dynamic Capability in Early International Growth - The Case of a Professional Service Firm

Thorsten Bunz, J ustus Liebig University Giessen

Lucrezia Casulli, University of Strathclyde

Andreas Bausch, J ustus Liebig University Giessen

International new ventures build on strong organisational capabilities to attenuate the challenges in their early international growth and to constantly adapt to changing external conditions. In this study, we explore the evolution of a dynamic capability that performs the aforementioned characteristics in the early internationalisation process of a new venture. Using qualitative evidence from a new professional service firm, we construct a narrative of the changing processes and activities which constitute this dynamic capability and determine its evolution over time. Our findings suggest that the successful evolution of a dynamic capability in early internationalisation depends on its dominant logic, i.e. management's understanding of causal relationships regarding the firm's environment. Ramifications of dominant logic on capability evolution and the nature of dynamic capabilities are discussed. (For more information, please contact: Thorsten Bunz, Justus Liebig University Giessen, Germany: thorsten.bunz@wirtschaft.uni-giessen.de)

\author{
Innovation and Networking Among Entrepreneurs Across Generations of Asian Tigers \\ Jan Vang, Aalborg University \\ Kent W. J ensen, University of Southern Denmark \\ Thomas Schoett, University of Southern Denmark \\ Shamak Rezaei, Roskilde University
}

Much attention has been paid to analyzing the determinants behind the economic development in the different generations of Asian tiger economies. This stream of research has provided valuable insights on the particular generational challenges the tigers face in implementing successful catching up-strategies. The literature has, however, not paid much systematic attention to generational differences in connection to moving from an imitation-based strategy towards an innovation-based strategy. This is especially the case concerning the entrepreneurial companies. Based on the GEM data this paper aims at reducing this research gap by conducting an analysis on the generational differences between the tiger economies entrepreneurs in respect to their innovative performance, their inclination to network and the importance of the quality of the network for the their innovative performance. The paper finds a significant difference of the quality of the networks and its impact on innovation across the tiger generations. (For more information, please contact: Jan Vang, Aalborg University, Denmark: jv@production.aau.dk)

\title{
The Use of Exploited Labor in International Business Subrata Chakrabarty, University of Texas - El Paso
}

Goods and services across industries are increasingly emanating from global supply chains that are dispersed geographically across the world. A key element of managerial thinking has been that firms can use humans, just like other forms of resources, to obtain profits. Profits from the use of resources are higher when firms minimize associated costs. The purpose of this study is to analyze the notion that firms can and do squeeze workers to turn profitable. The key suggestion is that underperforming firms tend to view their in-house employees as a cost burden, tempting them to use exploited external labor in their global supply chains to boost profits. The findings highlight an unfortunate ethically fraught reality - the squeezing of workers for profits. The paper concludes with ethical interrogations based on stakeholder theory. (For more information, please contact: Subrata Chakrabarty, University of Texas - El Paso, USA: chakrabarty@gmail.com) 


\title{
Session: 1.4.12 - I nteractive
}

\section{Track: 12 - The Institutional and Political Environment of I B}

\section{New and Heterodox Themes in IB}

Presented On: J une 28, 2015 - 14:30-15:45

Chair: Mike Peng, University of Texas at Dallas

\author{
An Analysis of Guanxi and the Degree of Internationalization of Chinese Manufacturing Firms \\ Jian Du, Zhejiang Universit \\ Xiaobo Wu, Zhejiang Universit \\ Yongjiang Shi, Cambridge University
}

As a country with a long history, the Confucian culture particularly the concept of guanxi has greatly influenced China's business environment. This paper mainly explores how two types of guanxi ties (guanxi with governments, guanxi with business parterners) affect the degree of internationalization of Chinese manufacturing firms. Based on research using 112 samples, this study indicates that both guanxi with governments and guanxi with business partners exert positive effects on the degree of internationalization of Chinese manufacturing firms. Additionally, through the mediating variables of resource bridging capability and adaptive capability, the positive effect of these two types of guanxi is still significant. In particular, guanxi with business partners moderates the positive effects of guanxi with government and resource bridging capability. Finally, this study finds that market uncertainty weakens the impact of guanxi with governments on resource bridging capability and strengthens the impact of guanxi with business partners on adaptive capability. (For more information, please contact: Jian Du, Zhejiang Universit, China: dujian@zju.edu.cn)

\section{Institutional Changes and Firm Responses in the Global Pharmaceutical Industry \\ Shobha Das, Qatar University \\ Rajshree Chandra, Delhi University}

The patent system forms an integral part of the institutional environment of the pharmaceutical industry. It has supported a business model of MNCs incurring high upfront costs, dealing with uncertainty in commercialization, and earning monopoly rents from patents. However, the patent system and the business model are coming under increasing scrutiny and facing growing criticism. We attempt to understand the existing business model and its problems, the current patent system, and the actual and proposed changes. Using information on the Top 20 firms in the industry, we examine the responses by Big Pharma to the changing external environment. (For more information, please contact: Shobha Das, Qatar University, Qatar: shobha.das@qu.edu.qa)

\section{A New Governance Perspective: Viewing Global Financial Markets as Watersheds W Travis Selmier II, I ndiana University}

This paper conceptualizes financial markets as virtual environments which should be subject to stewardship requirements found in natural environments. As in natural environments, irresponsible self-governance and lax or off-target regulation result in environmental damage and social loss. Watershed governance is proposed as the best-fitting analogy to financial markets governance for seven reasons: 1, scaling the watershed analogy from local to global fits well with financial markets; 2 , each consist of a variety of users who 3, tap hydrologic [capital] resources for many different uses. 4, financial markets, like watersheds, are made up of different types of goods, and so 5, may be better-managed through polycentric institutions with a range of public-private governance arrangements; 6 , governmental, private sector and mixed agents seek to support and manage [sustainable] exploitation in each. 7, environmental degradation comparisons are robust. Upstream-downstream interlinkage and cross-border riparian negotiations illustrate challenging financial market governance issues. (For more information, please contact: W Travis Selmier II, Indiana University, USA: wselmier@indiana.edu) 
To Boldly Go Where No Government and No Corporation Have Gone Before. Internationalization Strategies of Non-Governmental Organizations

Michal Szymanski, University of Victoria

International non-governmental organization are crucial elements in the global fight against some of the socioeconomic challenges, such as hunger, human rights abuses, progressing environmental degradation and lack of easily-accessible health care. Unfortunately, international business and strategic management research on NGOs has been focused on their operations and internal organizational issues, but the process of internationalization has been neglected in the literature. The following paper examines different types of NGOs and investigates their internationalization processes. The paper goes on to propose a mixed method approach to study this phenomenon and suggests potential directions for further research. (For more information, please contact: Michal Szymanski, University of Victoria, Canada: mikeszym@uvic.ca)

\section{Florian Täube, Université libre de Bruxelles Michael Migendt, EBS Business School Florian Schock, EBS Business School Paschen von Flotow, Sustainable Business Institute Friedemann Polzin, Sustainable Business Institute}

Private Equity in Clean Technology: An Exploratory Study of the Finance-Innovation-Policy Nexus

The role of technological innovation in mitigating and adapting to climate change has received growing interest in recent years. Scholars have used innovation system (IS) frameworks to grasp the interdependencies of innovation in such complex settings. However, this literature has somewhat neglected two IS features, namely: (1) the financial framework and (2) negative and unintended feedback mechanisms. Although financing innovation is usually mentioned, it rarely goes beyond venture capital (VC) as part of an entrepreneurial support network. In this paper, we emphasize the importance of these two understudied aspects of IS through an exploratory, qualitative study of private equity and VC in the clean technology (Cleantech, or CT) "industry" in the United States and Germany. Given our focus on clean technologies as well as private equity and VC, our sample includes the United States as the most sophisticated private equity and VC market and Germany as a lead market for environmental technologies. Our study makes one empirical and two theoretical contributions to the literature derived from a comprehensive analysis of policy-finance-innovation interdependencies: empirically, we find that in interdependent systemic relationships such as in the Cleantech sector, different policies neutralize or even overcompensate for one another, leading to a "waste" of economic resources. Theoretically, our model extends the classical IS model to more systematically include financial institutions and considers possible negative feedbacks. These findings lead us to further avenues for research, suggesting the investigation of other relationships for negative feedbacks for similar industry sectors that are asset heavy, such as biotechnology or nanotechnology. (For more information, please contact: Florian Täube, Université libre de Bruxelles, Belgium: ftaube@ulb.ac.be)

\section{Thoughts on the Emerging Institutional Framework for Commercializing Electric Vehicle Technology in China Peter Thomas in der Heiden, University of Duisburg-Essen}

In 2010, the Chinese government initiated a leapfrogging strategy to jump-start technological upgrading in the country's automobile industry and facilitate the transition to electric mobility based largely on indigenous innovation. This move was driven by escalating environmental pressures as well as resource constraints and inspired by the prospect of greatly strengthening the technological capabilities and competitive positions of domestic automakers. The aim was to establish an early lead in the field of electric vehicles featuring high electric power ratios and power grid connectivity. The paper identifies this leapfrogging attempt as a strategic project initiated by the central government and organized in a top down manner. It sheds light on the supporting public policy framework and conducts a preliminary review of the strategy's accomplishments until 
mid-2012. By way of an in-depth examination of guidance policies and incentive structures, the paper highlights the challenge of coordinating promotion plans across the country. (For more information, please contact: Peter Thomas in der Heiden, University of Duisburg-Essen, Germany: peter.inderheiden@uni-due.de)

National Governance, Social Elites on Company Boards, and Board Compensation: A Cross-National Study of Emerging Economy IPO Firms

Bruce Allen Hearn, University of Sussex

Roger Strange, University of Sussex

Jenifer Piesse, King's College London

We contrast behavioural and multiuple agency with institutional perspective in exploring the impact of directors drawn from indigenous social elites on average executive pay. Drawing on a unique hand-collected and comprehensive database of 136 private sector IPO firms from across the African emerging region between J anuary 2000 to J anuary 2014 we find evidence that increasing social elite nonexecutives are associated with higher executive cash-salary where this is inversely mediated by institutional quality. Upon disaggregating our social elites into four constituent elites identified in IPO listings prospectuses we find this result is largely driven by military and governmental social elites. These findings are valuable to policy-makers and managers alike in revealing the importance of domestic indigenous political economy in shaping executive preferences for salary in emerging economies. (For more information, please contact: Bruce Allen Hearn, University of Sussex, United Kingdom: b.a.hearn@sussex.ac.uk)

\title{
Session: 1.4.13 - Interactive
}

\section{Track: 1 - People and Careers in Cross-Cultural Business}

\section{The Role of Culture in International Business}

Presented On: J une 28, 2015 - 14:30-15:45

Chair: Majid Ghorbani, Renmin University of China

\author{
Can Cultural Tightness-Looseness be Measured? \\ Len J. Trevino, Loyola University New Orleans \\ Carolyn P. Egri, Simon Fraser University \\ David A. Ralston, Florida International University \\ Irina Naoumova, University of Hartford \\ Olivier Furrer, University of Fribourg \\ Yongjuan Li, Chinese Academy of Sciences \\ Fidel León Darder, University of Spain \\ María Teresa de la Garza Carranza, Instituto Tecnológico de Celaya
}

This study assesses the internal and external validity of Gelfand et al.'s (2011) recently developed measure of cultural tightness-looseness (CTL). For the six countries and 17 country subsamples under study, confirmatory factor analyses failed to support the unidimensional structure of the 6-item CTL measure. Exploratory factor analyses provided further evidence that the 6 -item CTL measure does not have a strong unidimensional structure across cultures. In addition, inter-rater agreement analyses did not support the use of aggregated scores to construct nation-level scores for the cultural tightness-looseness index. We also found that country rankings of cultural tightness-looseness scores (in total and for subsamples) were substantively different from those reported by Gelfand et al. (2011). We discuss our findings and their implications for cross-cultural research. (For more information, please contact: Len J. Trevino, Loyola University New Orleans, USA: Itrevino@loyno.edu) 
Understanding the Paradox of Cultures: A Yin Yang Perspective

Kunal Kamal Kumar, T. A. Pai Management Institute

Tony Fang, Stockholm University

We aim to highlight a new perspective to understanding the multidimensional construct of 'national culture'. The core of the new perspective lies in the philosophy of Yin Yang that emphasizes inclusiveness and goes beyond dualistic conceptualizations that try charting a country's cultural profile on the basis of pre-determined bipolar dimensions (for ex: Hofstede, 2010). Yin Yang does not challenge the dualistic view; rather, advances it by proposing that the ends of the bipolar dimensions of national culture are not antagonistic in nature. Under the Yin Yang perspective, the bipolar ends, though conflicting in nature, complement one another. Our study deals with two primary research questions: First, is a bipolar dichotomy of national culture(s) worthwhile? And second, how does Yin Yang paradigm help solve inherent cultural paradoxes at national level? Fang's (2012) propositional framework is used as a guide to search for answers to these questions. (For more information, please contact: Kunal Kamal Kumar, T. A. Pai Management Institute, India: kumarkunalkamal@gmail.com)

\section{The Role of Culture in Managers' Conceptualization of Business Networking \\ Lasse Torkkeli, Lappeenranta University of Technology \\ Maria Ivanova-Gongne, Abo Akademi University}

Existing studies in international business tend to neglect to investigate how business culture and the individual cultural background of managers affects their conceptualization of business networks and networking. Studies have found distinct ways of networking to be prevalent in different cultural context, and have noted that effective business networking in many emerging markets tends to mean social networking rather than the organization-specific type favored in network scholarship. The present study investigates this role in an empirical examination of three Finnish and three Russian managers engaged in mutual dyadic business relationships. The results imply that perceptions of the level on which the networking is conducted depend on the cultural background of the managers: Finnish managers conceptualize the practice as an organizational strategy whereas their Russian counterparts bring it down to the level of the individual. We discuss the possible reasons behind these differences, which may arise from the fact that Finnish business networking relies on concepts derived from Westernized business culture, whereas Russian networking is based on the traditional culture. We suggest that the concept of business networking in research may suffer from ethnocentricity, and consider the theoretical and managerial implications.

(For more information, please contact: Lasse Torkkeli, Lappeenranta University of Technology, Finland: lasse.torkkeli@/ut.fi)

\section{Leading and Facilitating Global Innovation within the MNC Karina R. J ensen, NEOMA Business School}

A rapidly evolving and competitive marketplace with culturally diverse customers have created increased demand for MNCs to accelerate global innovation. The capacity to act on consumer insights and reconfigure resources dynamically requires a flexible and responsive network around the world. Leaders are trying to optimize global and local team knowledge in order to improve strategic planning and execution of new products worldwide. How can MNC leaders effectively conceive and execute innovation strategies for international markets? In responding to this research question, a qualitative study has been conducted concerning the role of cross-cultural collaboration for front end innovation, involving interviews with 105 global project leaders at 36 MNCs with headquarters based in Europe, Asia, and North America. Through the development of a model, the study identifies leadership behaviors that influence team success in conceiving and executing innovation strategies for international markets. (For more information, please contact: Karina R. Jensen, NEOMA Business School, France: karina.jensen@neoma-bs.fr) 
The Joint Effects of Societal Culture and Individual Values of Job Attribute Preference: Evidence from Eight Nations

Ji Li, Hong Kong Baptist University

Tao Liu, Southwest University

Yang Yu, Hong Kong Baptist University

J ieying Xu, Hong Kong Baptist University

Arif Mahmood, Hong Kong Baptist University

Wanxing J iang, Hong Kong Baptist University

We conducted a cross-national comparison of how values at two levels (cultural values at the societal level and personal values at the individual level) affect job attribute preferences (hereafter J APS). Comparing samples from East Asian collectivistic cultures and major Western individualistic cultures, we predict that JAPS among men and women can be influenced by both societal values and individual values. Analyzing data from three large scale cross-cultural studies, the World Value Survey (WVS), GLOBE, and the IBM study, revealed several interesting findings: i) For predicting preferences for major job attributes, two societal values (collectivism and masculinity) are useful only when their effects do not conflict with the effect of the most relevant individual value; ii) The effects of individual values are ordered by their relative importance to a given issue; iii) Individual values influence JAPS among men and women in an asymmetric way. (For more information, please contact: Wanxing Jiang, Hong Kong Baptist University, Hong Kong, SAR-PRC: wanxing.jiang@gmail.com)

\section{The Impact of Socio-Cultural Factors on Professional Aspiration in Emerging Markets Grishma Shah, Manhattan College \\ Esi Elliot, Suffolk University}

Research on emerging markets has suggested that socio-cultural factors have a direct influence on marketing , attitudes, and behavior in firms (e.g Sridharan and Viswanathan, 2008). Sridharan and Viswanathan expose the nature of socio-cultural factors to help clarify not only the thought processes and motivations of individuals and consumers but also their philosophies and values within society. Academic research has however yet to explore this notion adequately, particularly from the perspective of psychological career resources. In this study we explore how socio-cultural factors impact career aspirations in emerging market individuals at the micro level. We draw on socio-cultural construction theories (socio-cultural structures, socio-cultural systems and roles) in answering our research question. Specifically, we focus on the socio-cultural factor of collectivism and examine the repertoires of psychological career resources consisting of career preferences, career values, attributes, skills and attitudes linked to experiences of career success. We develop and test hypotheses of family and work collectivism on professional aspirations using more than 1000 valid surveys collected among middle class in Indians. The results suggest from both the family and work domains of collectivism are positively related to career aspirations for the growing middle class population in I ndia. Collectivist relationships are found to overall accrue psychological career resources, which drive career aspirations. Theoretical, managerial and marketing implications are discussed in light of the results. (For more information, please contact: Grishma Shah, Manhattan College, USA: grishma.shah@manhattan.edu)

\section{A Relational Perspective of Nationality Dissimilarity: The Positive Effect on Organizational Identification in International Joint Ventures \\ J iatao Li, Hong Kong University of Science and Technology \\ J ieyu Zhou, Hong Kong University of Science and Technology}

From a relational demography perspective, we examine the effects of nationality dissimilarity in the context of international joint ventures (IJ Vs) and their managers' identification with the IJ V. Different from the majority of prior research which suggests a negative effect of nationality dissimilarity on group dynamics and firm performance, we examine the positive impact of nationality dissimilarity in a special context, the international joint ventures. Specifically, we hypothesize that nationality dissimilarity triggers higher IJV identification. As the nationality dissimilarity increases, managers are more likely to perceive the IJ V as the ideal identification target 
since nationality dissimilarity is a positive trait of IJV. Thus, managers who want to reduce uncertainty and enhance self-esteem would identify more with such IJVs. Further, the positive effect of nationality dissimilarity is stronger for managers of low nationality status, managers who have a close relationship with the leader of the IJ V and managers who identify less strongly with their parent firms. Data from a sample of top managers in 101 Sino-foreign IJ Vs in mainland China largely supported these predictions. (For more information, please contact: Jieyu Zhou, Hong Kong University of Science and Technology, Hong Kong, SAR-PRC: jyzhouaa@ust.hk)

\section{Session: 1.5.1 - Panel Special Session}

\section{China is Predicted to Hit Middle Income Trap by 2030: Can China Build innovation Capacity to Avert Trap?}

Presented On: J une 28, 2015 - 16:15-17:30

Chair: Arie Lewin, Duke University

Panelists:

Arie Lewin, Duke University

Michael Witt, I NSEAD

Douglas B. Fuller, Zhejiang University

Yves Doz, INSEAD

The proposed panel will set up a wide ranging discussion around the World Bank anticipation that China will hit middle income trap by 2030. This represents a daunting challenge for China and of course for the Communist Party. The panel represents key speakers from a conference held at HUST in Hong Kong (December 4-7, 2014) on "The Globalization of Knowledge Creation and Innovation in the Context of Emerging Economies" where the World bank report was the basis for much of the discussions. The panel believes that earlier lessons in economic development from the four Tigers (Singapore, Taiwan, Hong Kong and Korea) are not transferable (cannot be imitated) for China. Founding conditions and history of China, sheer scale, and the importance for maintaining the Communist Party primacy raise serious questions as to their applicability in the case of China. This basic line of thinking is articulated by Dean/Professor J ustin Yifu Lin in his forthcoming opening chapter to a volume (Cambridge University Press 2015). We see the panel as exploring the arguments as to why, why not, and how China might evolve a combination of economic development industrial and socio-political policies which will continue its trajectory of development and avert the World Bank prediction, while continuing CP hegemony. We think that what is unique about this panel is its timeliness, and the opportunity to explore multifaceted elements (micro organization behavior, macro organization and strategy, knowledge creation and innovation, industrial policies, role of founding conditions and history) that we believe will frame wide ranging discussions. Of course the panel presentations have wide implications for all emerging economies. (For more information, please contact: Arie Lewin, Duke University, USA: ayl3@duke.edu) 


\section{Session: 1.5.2 - Panel}

Track: Scholars Meet Practice

\section{Technology \& Science-Based Innovation in Emerging Economies}

Presented On: J une 28, 2015 - 16:15-17:30

Chair: Sai Yayavaram, Indian Institute of Management Bangalore

Panelists:

Sai Yayavaram, Indian Institute of Management Bangalore

Suresh Bhagavatula, Indian Institute of Management Bangalore

Chirantan Chatterjee, I ndian Institute of Management Bangalore

K Kumar, Indian Institute of Management Bangalore

Mariko Sakakibara, University of California, Los Angeles

Mohan Pandey, Bristol-Myers Squibb

S Baskar, Amagi Media Labs Pvt. Ltd.

If US economic growth is over and 'innovation is faltering' as some scholars have argued (Gordon 2012), where will the next wave of global innovation come from? Will it be from emerging economies like India, China and Brazil? How much of that new innovation will be driven by multinationals, domestic firms or by de novo startups? How much of that innovation might be radical vis-a-vis incremental? What might be the institutional impediments to the same? What might be creative ways for innovative enterprises in emerging economies to leapfrog beyond the institutional impediments? Is there enough appetite among investors for risky investments that are required in such innovation in emerging economies? Might there be a moderating role of founding team composition? Using a panel of academic scholars and practitioners this panel proposes to shed light on these questions. (For more information, please contact: Sai Yayavaram, Indian I nstitute of Management Bangalore, India: sai.yayavaram@iimb.ernet.in)

\section{Session: 1.5.3 - Competitive}

Track: 13 - I nternational Economics, Finance and Accounting

\section{Determinants and Effects of Foreign Investment}

Presented On: June 28, 2015 - 16:15-17:30

Chair: Haruo H. Horaguchi, Hosei University

Employment Effects of FDI in Hot Labour Markets: a Cross-Country Analysis

Bettina Becker, Aston Business School

Nigel Driffield, Warwick Business School

Sandra Lancheros, University of Nottingham

James Love, Warwick Business School

We examine the effects of FDI on domestic employment and labour costs, concentrating on high-tech industrial sectors. Using a detailed firm-level dataset covering 6 sectors in 29 countries over the period 2002-10, we find no evidence of crowding out of domestic employment by FDI in 'hot' labour markets, but strong evidence of significant wage effects. These effects are strongest in countries which are typically regarded as having relatively flexible labour markets. In the long run there is some evidence of crowding out in the domestic sector, but this is restricted to transition countries. (For more information, please contact: Bettina Becker, Aston Business School, United Kingdom: b.becker@aston.ac.uk) 
Economic Institutions and the Location Strategies of European Multinationals in their Geographical

Neighbourhood

Simona Iammarino, London School of Economics

Andrea Ascani, London School of Economics

Riccardo Crescenzi, London School of Economics

This paper investigates how the location behaviour of Multinational Enterprises (MNEs) is shaped by the economic institutions of the host countries. The analysis covers a wide set of geographically proximate economies with different degrees of integration with the 'Old' 15 European Union (EU) members: New Member States, Accession and Candidate Countries, as well as European Neighbourhood Policy (ENP) countries and the Russian Federation. The paper aims to shed new light on the heterogeneity of MNE preferences for the host countries' regulatory settings (including labour market and business regulation), legal aspects (i.e. protection of property rights and contract enforcement) and the weight of the government in the economy. By employing data on 6,888 greenfield investment projects, the random-coefficient Mixed Logit analysis here applied shows that, while the quality of the national institutional framework is generally beneficial for the attraction of foreign investment, MNEs preferences over economic institutions are highly heterogeneous across sectors and business functions. (For more information, please contact: Simona Iammarino, London School of Economics, United Kingdom: s.iammarino@lse.ac.uk)

\section{Finance Constraints and Technology Spillovers from Foreign to Domestic Firms Alex Eapen, Australian National University}

Scholars have suggested that positive externalities such as technology spillovers from foreign to domestic firms occur only when the latter have adequate absorptive capacity. However, there has been a rather strong technological focus in their conceptualization of absorptive capacity. A fuller understanding of how domestic firms differ in their capacity to absorb spillovers, we argue, needs greater attention to their finance constraints, and to how these are shaped by the quality of local financial institutions. We present a formal theoretical model showing that inefficient lending confers differential finance constraints on domestic firms that, in turn, impact their realization of positive FDI external effects. Specifically, distortions in lending to domestic firms in the economy pose a critical bottleneck for positive external effects of FDI to materialize. Based on this and a counterfactual simulation, we show that correcting finance misallocation in the economy can unlock significantly more FDI spillover effects. (For more information, please contact: Alex Eapen, Australian National University, Australia: alex.eapen@anu.edu.au)

\section{Limitations of MNC's Operational Flexibility \\ Toshimitsu Ueta, NUS Business School}

Theory of operational flexibility says MNCs can shift manufacturing operations toward countries where cheap input is available upon economic contingencies like currency exchange rate change. While several studies have found related results, the direct evidence of MNCs' operating flexibility upon exchange rate fluctuations has been surprisingly weak and rare. This paper examines this disregarded question: how much do MNCs change the size of manufacturing operations in each subsidiary depending on the currency rate changes? The results show that the flexibility is implemented only with very simple operations located abroad, and call for careful reexaminations of actual flexibility of MNCs. (For more information, please contact: Toshimitsu Ueta, NUS Business School, Singapore: toshimitsu.ueta@u.nus.edu) 


\section{Session: 1.5.4 - Competitive}

\section{Track: 8 - Global Strategy, M\&As and Competitiveness}

\section{Implementation Challenges Across Borders}

Presented On: June 28, 2015 - 16:15-17:30

Chair: Aya Chacar, Florida International University

Organizational Control and Goal Conflicts in Headquarters-Subsidiary Relationships: An Agency Theory Perspective

Adrian Schulte Steinberg, University of St. Gallen

Sven Kunisch, University of St. Gallen

Björn Ambos, University of St. Gallen

In this study, we investigate the interaction of socialization, goal conflicts and behavioral control in headquarters-subsidiary relationships. We take a modern agency perspective and test assumptions of relationship multiplicity, within-subsidiary heterogeneity and multiple principals in an empirical model using 131 observations that cover the full configuration of a European insurance corporation. Our findings suggest that socialization reduces goal conflicts between central and local organizational units, contingent upon task programmability and influence of a second principal. Further, we qualify that goal conflicts mediate the relationships between socialization and behavioral controls only under certain circumstances. The primary contribution of this study is that it advances agency theory to more complex conceptualizations for applications in headquarters-subsidiary relationships. (For more information, please contact: Adrian Schulte Steinberg, University of St. Gallen, Switzerland: adrian.schultesteinberg@unisg.ch)

\section{Spatial Consistency and Temporal Persistence in MNEs' Repetitive Strategic Resource Allocations Majid Eghbali-Zarch, Memorial University of Newfoundland}

Although scholarship regarding organizational capabilities, particularly those manifested as decision rules, has provided meaningful contributions to our understanding of the patterns of strategic decisions, less is known about them in the context of multinational enterprises (MNEs). By focusing on recursive, high-stake strategic resource allocation decisions, we disentangle the time and space dimensions of the deployment of company capabilities. More specifically, we examine the stability patterns in MNEs and their subsidiaries as a result of the deployment of organizational capabilities. We develop two complementary core constructs for our purpose: temporal persistence and spatial consistency. Utilizing two primary dimensions of international strategy, namely expatriate assignment and equity ownership level decisions, respectively representing repetitive and quasirepetitive decisions, we consider the role of degree of repetitiveness in the stability and dynamism of decisions and their influence on firm performance. We find a positive effect on performance for MNEs' spatial consistency across subsidiaries for expatriation (as a repetitive decision), and a negative effect for spatial consistency in equity ownership (as a quasi-repetitive decision). We also observe for temporal persistence in expatriation, a positive effect on performance. (For more information, please contact: Majid Eghbali-Zarch, Memorial University of Newfoundland, Canada: majidez@mun.ca)

Comparison of Corporate Headquarters Influence on Segment Performance Variance between Domestic Firms and MNE Affiliates

Sarada Devi Gadepalli, Indian Institute of Management Calcutta

Arindam Mondal, Indian Institute of Management Calcutta

In this paper, we examine if there are differences in the degree of influence that corporate headquarters wield on the variance of their business segment's performance. We examine the absolute magnitudes, and relative importance of the corporate effects of both the sets of firms vis-à-vis industry, business segment, and business 
group effects in the context of the unique socio-economic, and institutional arrangements of an important emerging economy - India - for the period 2002-2013. We use multilevel analysis to leverage the inherent hierarchical nature of the data and confirm that the corporate headquarters of the multinational firms' affiliates have a greater impact on their segment profitability as compared to the domestic firms. Analysis of the data stratified on the basis of industrial sectors reveals that differences in the knowledge characteristics associated with the industry sectors might influence the relative importance of organizational structural elements. (For more information, please contact: Sarada Devi Gadepalli, Indian Institute of Management Calcutta, India: saradad11@iimcal.ac.in)

\section{Understanding Psychic Distance: A Product of Individual Demographics or Collective Phenomenon? Alexander Leinemann, University of St. Gallen Björn Ambos, University of St. Gallen}

This empirical study, which offers an investigation of the impact of individual-level antecedents on the perceived psychic distance of the individual to foreign countries, adds to the literature in two important ways. First, we empirically investigate different individual-level antecedents. We find that formal education and the command of language reduce the psychic distance perceptions, while we find, in contrast to conventional wisdom, no impact for international experience, work experience and age. Second, we compare the relative importance of individual-level and macro-level antecedents on the formation of individual psychic distance perceptions. Our results suggest that macro-level antecedents are a powerful predictor for individual psychic distance perceptions, while individual-level antecedents only have a comparably limited explanatory power. (For more information, please contact: Alexander Leinemann, University of St. Gallen, Switzerland: alexander.leinemann@unisg.ch)

\section{Session: 1.5.5 - Competitive \\ Track: 5 - The Geography of I nternational Business and Global Value Chains}

\section{Organization of Activities and Industries}

Presented On: June 28, 2015 - 16:15-17:30

Chair: Sinead Monaghan, Rutgers Business School

\section{Export Market Participation: Do Exporters Imitate their Industry Peers? \\ Dirk Michael Boehe, University of Adelaide}

Why do exporters enter particular destination countries? I argue that firms predominantly imitate their industry peers and follow them abroad into their respective destination countries (bandwagon effect). I mitation allows exporters to make less risky bets on foreign markets and access valuable foreign market information at low cost through their industry peers. I further argue that the imitation of industry peers is contingent on the availability of export market knowledge through alternative ways, i.e., their own prior export experience and cross-border information flows. The reason for the substitutive relationship between imitation and alternative information sources is that imitation is the most cost-effective way of exporting for less internationally experienced firms and in the case of destination countries with difficult information access. I test my hypotheses using firm-yeardestination country panel data of Brazilian exporters. This study extends the literature on export market participation by theoretically explaining under what conditions the bandwagon effect leads to export market entry. (For more information, please contact: Dirk Michael Boehe, University of Adelaide, Australia: dirk.boehe@adelaide.edu.au) 
Backward Integration of Foreign Sales Activities into Production: An Operational Flexibility Perspective

Bjoern Schmeisser, WU Vienna

J an Hendrik Fisch, WU Vienna

This study focuses on the decision of expanding foreign sales activities towards production. Complementing traditional theories on foreign direct investment and the internationalization process of the firm, we suggest that multinational enterprises pursue backward integration of foreign subsidiaries also in order to improve arbitrage opportunities in their network of foreign operations. We predict that, rather at the network level than at the local level, fluctuations in exchange rates, differences in corporate income tax regimes, investment incentives granted by host governments, and external knowledge at other foreign locations explain backward integration of foreign sales activities into production. We test the hypotheses using event history analysis on a dataset of 283 manufacturing firms showing 425 cases of backward integration between 1999 and 2012. (For more information, please contact: Bjoern Schmeisser, WU Vienna, Austria: bjoern.schmeisser@wu.ac.at)

\section{Client Co-Production in the Production Process of Offshored Knowledge-Intensive Business Services Kristin Brandl, University of Reading}

Clients co-produce knowledge-intensive business services through transferring and co-creating knowledge. I study the production process of the services and how a geographic relocation (offshoring) impacts client coproduction in this process. Through an empirical analysis of multiple service production processes, I find that client co-production decreases in intensity over time, but despite expectations never stop entirely. Moreover, I find that the interdependent tasks in the production processes of the offshored services, in causation with this continuous client co-production, result in modularization of production tasks, and as a consequence, in standardization of production processes as well as a change of service characteristics. (For more information, please contact: Kristin Brandl, University of Reading, United Kingdom: kristin.brandl@henley.ac.uk)

\section{Session: 1.5.6 - Panel}

Track: 1 - People and Careers in Cross-Cultural Business

\section{Identity Lenses in International Business: Approaches and Opportunities}

Presented On: June 28, 2015 - 16:15-17:30

Chair: Cristina Gibson, University of Western Australia

Panelists:

Cristina Gibson, University of Western Australia

Mary Yoko Brannen, University of Victoria

Lakshmi Ramarajan, Harvard University

Florian Täube, Université libre de Bruxelles

Sarbari Bordia, Australian National University

Andy Chiou, SUNY Farmingdale

The purpose of this panel is to explore the potential and benefits of expanding the use of identity perspectives in International Business (IB). A focus on identity has a long history in management and organizational scholarship, and in the particular field of IB, the interest has naturally gravitated around issues related to cultural identities. But individuals hold other work (e.g., organizational, professional, etc.) and non-work (e.g., gender, race, etc.) identities, which have implications for global workplaces. Working globally confronts individuals and organizations with competing identification references. This multitude of identification options can be both a challenge and a resource, therefore it must be better understood. Moreover, recent global political events reminded us that identities, especially when challenged, are a powerful motivator of behavior. As such, the IB field has the opportunity (and indeed, the obligation) to join in the renewed interest in 
understanding how different identities interact in influencing business and social outcomes. The panelists' input will illustrate the usefulness of identity perspectives in IB, and will provide the platform for a plenary discussion aimed at generating ideas for ways in which the IB field can both benefit from, and contribute to, an identityfocused research agenda. (For more information, please contact: Dan V. Caprar, UNSW Business School, Australia: dan.caprar@unsw.edu.au)

\title{
Session: 1.5.7 - Competitive
}

\section{Track: 9 - Emerging Economies and Emerging Economy MNEs}

\section{Institutional Voids and Natural Environment}

Presented On: June 28, 2015 - 16:15-17:30

Chair: Klaus Meyer, CEIBS

International Voluntary Certifiable Standards in Emerging Economies: Institutional Void Fillers or Non-Tariff Trade Barriers?

Ivan Montiel, Loyola Marymount University

Petra Christmann, Rutgers University

Trevor Znk, Loyola Marymount University

International voluntary standard certifications can fill institutional voids caused by weak government regulations in emerging economies by enabling firms to convey credible product information. However, certifications also create non-tariff trade barriers for non-certified firms. To understand when certifications are void fillers versus trade barriers we hypothesize that three institutional complexity dimensions associated with multiple competing certifications-diversity and dynamism of customer requirements and uncertainty about future standard evolution-decrease firms' certification likelihood. Results using survey data for Mexican exporters' international food safety certifications largely confirm our hypotheses. Thus, when competing certifications exist, they are trade barriers rather than void fillers. (For more information, please contact: Ivan Montiel, Loyola Marymount University, USA: ivanmontiel@gmail.com)

\author{
Institutional Voids and Performance in Emerging Markets - a Configurational Perspective \\ Luciano Ciravegna, King's College London \\ Esteban Brenes, I NCAE \\ Caleb Pichardo, INCAE
}

This study analyzes the antecedents of firm performance in emerging markets. It proposes that variation in institutional voids across emerging markets affects the organizational configurations linked to high performance. It examines a sample of 200 firms based in 12 economies, focusing on the role of family management, vertical integration, collaboration within a network, firm size, and internationalization. This study extends the research agenda on the role of institutions on strategy and performance in emerging market through a multi-causal approach that allows uncovering multiple, non-exclusive mechanisms through which firms succeed in challenges business environments. (For more information, please contact: Luciano Ciravegna, King's College London, United Kingdom: Iuciano.ciravegna@kcl.ac.uk) 
Non-Lienarity of the Environmental Responsiveness Strategy: Is it the Answer?

Yousef Eiadat, University College Dublin

Alejandro M. Fernández Castro, CESUGA

Aidan Kelly, University College Dublin

The impact of strengthening the coercive arm of state regulation on managerial decisions to adopt symmetric environmental responsiveness strategies is well discussed by institutional theorists. The intuitive nature of the relationship is positive and monotonic, i.e., stricter coercive regulatory pressure triggers proactive environmental responsiveness. This paper shows that the overall relationship is curvilinear, i.e., coercive environmental pressure brings significant environmental responsiveness benefits up to a certain optimum level beyond which its ability to trigger proactive environmental responsiveness begins to decelerate, while resistance and symbolic responses accelerate. Finally, results show that a curvilinear regression provides a better empirical model than the standard linear regression and advances the long-standing and contentious debate about the relationship between state regulation and environmental responsiveness. (For more information, please contact: Yousef Eiadat, University College Dublin, Ireland: yousef.husein@ucd.ie)

\section{Political Violence and Greenfield FDI Flowing to the Natural Resources Industries of Developing Countries Enrico Pennings, Erasmus University Rotterdam Martijn Burger, Erasmus University Rotterdam Caroline Witte, Erasmus University Rotterdam Elena Ianchovichina, World Bank}

Previous studies on political violence and natural resources have mainly focussed on how resource abundance can fuel conflict, while largely neglecting the effect political violence has on investment in natural resources. In this paper the effect of political violence on Greenfield Foreign Direct Investment (FDI) in different sector is examined using the predictions from real options theory. We hypothesize that political violence reduces overall FDI inflows, as violence increases uncertainty and operating costs. Yet, this effect is expected to be smaller for secessionist conflicts than for conflict concerning national authority, as secessionist conflicts are more geographically concentrated. In addition, we hypothesize that FDI in the natural resource sector is less sensitive to political violence than FDI in manufacturing and services, since multinational enterprises (MNEs) active in the natural resource sector are more geographically restricted in their location choice and the economic rents associated with natural resource extraction can offset the negative effects of political violence on profits. We find evidence for these hypotheses. In addition, we empirically show that the insensitivity of natural resource FDI to political violence is related to the existence of limited investment options and high resource rents. (For more information, please contact: Enrico Pennings, Erasmus University Rotterdam, Netherlands: pennings@ese.eur.nl) 


\title{
Session: 1.5.8 - Competitive
}

\section{Track: 12 - The Institutional and Political Environment of I B}

\section{The I mpact of I nstitutional Distance}

Presented On: J une 28, 2015 - 16:15-17:30

Chair: Xiaohua Yang, University of San Francisco

Inward FDI and Economic Growth: The Effect of Multiple Measures of Institutional Distance Sarianna Lundan, University of Bremen

Fabienne Fortanier, OECD

Tilo Halaszovich, University of Bremen

The empirical evidence on the growth consequences of FDI for host developing countries has been highly variable. We argue that the link between FDI and growth is influenced by the absolute levels of absorptive capacity and quality of institutions in the host country, as well as by the institutional and cultural distance between the home and host countries. Specifically, we expect that smaller institutional and cultural distances lower transaction costs and hence facilitate spillovers and knowledge transfer. Therefore, we expect to find a negative interaction effect between distance and FDI for economic growth. We test this idea on a comprehensive panel dataset including all major outward investors and more than 140 recipient economies from 1989-2006. We employ newly developed indicators for a variety of formal and informal institutional distance measures. We find different effects for our three investment-weighted distance measures. Cultural distance seems to be of minor importance as compared to institutional and value distance. While our results partly suggest avoiding developing countries with a high institutional distance, a high value distance is not negative per se. In fact, a high value distance is associated with positive growth opportunities. (For more information, please contact: Sarianna Lundan, University of Bremen, Germany: s. Iundan@uni-bremen.de)

\section{Export Expansion Speed, Institutional Distance and Performance Zliang Deng, Renmin University of China Ruey-J er "Bryan" Jean, National Chengchi University Rudolf R. Sinkovics, University of Manchester}

We develop a novel construct of speed of internationalization based on institutional distance, and reexamine the relationship between speed and exporter profitability. We find that when exporters rapidly expand into a destination country with either higher or lower trade freedom, there will be a negative relationship between the expansion speed and firm profitability; but if the exporter is located in a highly liberalized home region, the above negative relationship will be bended from negative to positive. From the positive organizational scholarship perspective, this study identifies why and how institutional distance and internationalization speed may generate positive effects on firm profitability. (For more information, please contact: Ziliang Deng, Renmin University of China, China: dengziliang@rbs.org.cn)

\author{
Institutional Distance, Social Ties, Trustworthiness and Performance: Evidence from China \\ J ieqiong Ma, Saint Louis University \\ J ohn Zhao, Saint Louis University \\ Jie Yang, Saint Louis University
}

Contrast to OECD countries, which have strong third-party enforced exchange systems, emerging markets such as China tend to have weak formal institutions (Eden \& Miller, 2004). According to institutional theory, social ties as informal institution become more important when formal institutions are imperfect and less developed (North, 1990; Mike W Peng, 2003). Using data from 416 Foreign Invested Enterprises (FIEs) from 21 countries in China, this paper finds that formal and informal institutional distance between host and home countries as 
causal factors of social ties and influence political ties with local government and business ties with local business differently in magnitude. Based on social capital theory, this paper further studies corporate voice and entry mode from the perspective of trustworthiness in the relationship between social ties and performance. We find that the effect of political ties and business ties on performance is much stronger in case of low corporate voice and joint venture entry mode. (For more information, please contact: Jieqiong Ma, Saint Louis University, USA: jma17@s/u.edu)

\section{Insidership within Networks: Bridge over Troubled Water of Foreignness? Megan (Min) Zhang, I vey Business School}

This study investigates the controversial question about how cross-national distance influences J apanese MNEs' equity ownership in their overseas subsidiaries. Prior studies adopted aggregated constructs and time-invariant measures of cross-national distance, failing to capture the complexity of the phenomenon. Moreover, although insidership within business networks may moderate the foreignness that MNEs confront in overseas markets, prior studies have not incorporated it into analysis. The present study confirms the explanatory power of multiple dimensions of cross-national distance and MNEs' insidership within networks. It also shows that crossnational distance and insidership within relevant works strengthen or weaken each other's influences on J apanese MNEs' ownership strategy. (For more information, please contact: Megan (Min) Zhang, Ivey Business School, Canada: mzhang.phd@ivey.ca)

\section{Session: 1.5.9 - Competitive \\ Track: 6 - Innovation and Knowledge Management}

\section{Knowledge Management in Alliances}

Presented On: J une 28, 2015 - 16:15-17:30

Chair: Nandini Lahiri, Temple University

Knowledge Acquisition in International Strategic Alliances: The Role of Knowledge Ambiguity Mia Hsiao-Wen Ho, Yuzn Ze University

Although prior research acknowledges that international strategic alliances may help firms to acquire knowledge from foreign partners, it remains unclear why some firms succeed in doing so, but others fail. We extend scholarly understanding regarding the determinants of knowledge acquisition in international alliances by examining the role of knowledge ambiguity, the difficulty of understanding the causal effects of partner's knowledge. Evidence from the analysis of 671 international alliances reveals that knowledge ambiguity negatively affects cross-border knowledge acquisition; however, firms can overcome such negative effect by developing strong inter-partner relationships and by choosing partners that originate from a similar institutional environment. (For more information, please contact: Mia Hsiao-Wen Ho, Yuzn Ze University, Taiwan: mia.hohw@saturn.yzu.edu.tw)

\section{Brokerage Triad Configurations Across Countries: Effects on Innovation Impact and Innovation Radicalness} Exequiel Hernandez, University of Pennsylvania Sarath Balachandran, University of Pennsylvania

Occupying positions that span structural holes across countries in a network of knowledge-based alliances allows firms to access novel resources. However, transferring and integrating knowledge sourced from partners located in different environments is fraught with frictions due to institutional, cultural, and other barriers. Existing research fails to account for the heterogeneity in the ways in which a firm can broker between its partners in different countries. Commonly used ego-network level measures of brokerage overlook national 
boundaries as a source of both novelty and barriers to knowledge integration. We overcome this limitation by taking greater cognizance of heterogeneity at the level of the brokerage triad, where we hold the structure constant and examine the effects of geographic separation across countries. We develop a typology of four unique brokerage triads based on whether the broker and its partners are distributed across the same or different countries. We find that the different types of brokerage triads are associated with significantly different innovation outcomes, both in terms of innovation impact as well as radicalness. Our results suggest that heterogeneity in brokerage configurations within global networks is an important overlooked factor in our understanding of the relationship between global networks and innovation. (For more information, please contact: Sarath Balachandran, University of Pennsylvania, USA: sarath.bc87@gmail.com)

\section{Learning and Knowledge Transfer in China-Africa Alliances: A Social Capital Perspective \\ Abdoulkadre Ado, Laval University \\ Zhan Su, Laval University \\ Roseline Wanjiru, Northumbria University}

This study is conducted on 29 China-Africa alliances from twelve African countries and explores learning management by African partners and the transfer of knowledge from their Chinese counterparts. Based on six social capital knowledge transfer dimensions from three types of network, we analyze dimension applicability and relevance for Sino-African alliances. Using multiple case study method with semi-structured interviews, we found that African partners actively take advantage of multiple facilitating conditions to support learning. Theoretical application of the six social capital dimensions requires adjustment because of interaction dynamics. Strategic alliance network appeared to use or even mix the facilitating conditions from other networks, not necessary at the same level, to gain knowledge. This research provides insights to knowledge-seeking strategic alliances particularly in China-Africa context. Additionally, the paper juxtaposes social capital and organizational theory with empirical perspectives on learning strategies. (For more information, please contact: Abdoulkadre Ado, Laval University, Canada: abdoulkadre.ado.1@ulaval.ca)

\section{Session: 1.5.10 - I nteractive}

\section{Track: 8 - Global Strategy, M\&As and Competitiveness}

\section{Emerging Market Acquisitions: Prospects and Pitfalls}

Presented On: June 28, 2015 - 16:15-17:30

Chair: Omer Faruk Genc, Abdullah Gul University

Strategic Determinants of Equity Participation of International Acquisitions by Emerging Market Firms Young-Ryeol Park, Yonsei University

Philsoo Kim, Yonsei University

Drawing from transaction cost economics (TCE) and organizational learning theory, we theorize and examine the strategic determinants of equity participation of cross-border acquisition made by emerging market firms (EMFs). Analyzing 626 acquisition deals undertaken by 384 Indian firms over 11 years of research period (20002010), our results indicate that the level of R\&D intensity and cross-border acquisition experience held by EMF acquires strongly influence propensity to fully participate in ownership decisions. We discuss our findings and suggest avenues for future studies. (For more information, please contact: Philsoo Kim, Yonsei University, Korea, South: kimseolhyun@yonsei.ac.kr) 
This paper examines how start-up R\&D firms shape organizational boundaries and evolution under the influence of upstream technological uncertainty and internal complementary capabilities. Start-up R\&D firms are often located in the upstream industry value chain. Upstream technological uncertainty and internal downstream and fund-raising capabilities may affect their boundaries. This article integrates insights from property rights, complementary capability and real options to explore firm governance choices, including equity property-rights shared licensing, and equity property-rights shared vertical or horizontal mergers and acquisitions. We argue that equity property rights sharing may be regarded as a source of fund-raising and partnership incentives to obtain complementary capability for reducing uncertainty. Start-up R\&D firms without complementary capabilities may tend towards minority equity property-rights shared licensing. As technical and internal downstream uncertainty is decreased, $R \& D$ firms may shift from vertical integration to complementary horizontal expansion through equity property rights shared mergers (horizontal merger of equals). The evolution of R\&D firm is exemplified by Biogen Idec, Gilead Sciences, and Genentech, the top three biopharmaceutical firms around the world. (For more information, please contact: Ying-Jan Lin, National Taiwan University, Taiwan: d98724007@ntu.edu.tw)

\title{
Acquisitions in the China Retail Industry \\ J ung Ching Lin, National Taiwan University \\ Homin Chen, National Taiwan University \\ D'Arcy Caskey, Feng Chia University
}

How do uncertainties influence firms' preferred size of equity stake in acquisitions (M\&A) of emerging economy retail firms? We use real option theory to empirically analyze this question using a sample of M\&A events in China. Results show that uncertainties resulting from cultural distance and institutional instability lead acquiring firms to purchase smaller equity stakes, but market growth potential results in larger stakes. Furthermore two different moderators of uncertainty, horizontal $M \& A$ and market coverage complementariness, weaken and strengthen the influences of uncertainties on firm's share of acquisition, respectively. Our study contributes to the literature on emerging economies and sheds light on M\&A strategies in the retail industry. (For more information, please contact: Jung Ching Lin, National Taiwan University, Taiwan: dorislin1011@gmail.com)

\author{
How to Select Target Firms in High-Tech Industry: Identifying Value Drivers and Cost Drivers \\ Tian Wei, Fudan University \\ Yuanxu Li, Fudan University
}

This study explores the decision-making process regarding target selection in horizontal acquisitions in the context of the medical technology industry from two aspects: value creation and cost generation. Based on the data gathered from five cases of acquisition undertaken by four medical technology companies, we identified value drivers and cost drivers as the major constructs in the process of target selection. In order to understand the comprehensive nature of this, we further focus on the industry characteristics and firm features as these are closely related to the value drivers and cost drivers, respectively. Technological stability and firm size are therefore found to be two mediators in the relationship between the value drivers and cost drivers. This study answers the call for research to be undertaken on real-life cases of time-critical decision-making problems. An integrated framework and three sets of propositions for these constructs are the major findings of this research. (For more information, please contact: Tian Wei, Fudan University, China: weitian1983@gmail.com) 
Untangling the Relationship between Target Firm Size and Integration Approaches in International Acquisitions: The Role of Organizational Culture

Tian Wei, Fudan University

Youzhen Zhao, Fudan University

The main focus of this study is the contrasting mechanisms through which organizational culture influences the integration approaches in acquiring small and large target firms. The findings of this study contribute to untangle the relationship between target firm size and integration approaches. Two distinct roles are identified through five in-depth case studies: multilevel resistance and power struggling, which can be explained by the organizational power. We further explore the development of integration approaches with each of these two roles. The commonality refers to culture assimilation to achieve organizational identity. The variances include resource rationalization for small target firms, which is purely from a resource-based view and dominance of power winner for large target firms, which is from the organizational power perspective. (For more information, please contact: Tian Wei, Fudan University, China: weitian1983@gmail.com)

\section{Examining Firm and Host Country Influences on Relative Transaction Size in Service Sector Cross-Border Acquisitions: An Empirical Assessment \\ Dynah A Basuil, University of Auckland \\ Deepak K Datta, University of Texas at Arlington}

Based on 290 cross-border acquisitions undertaken between 1991-2006 by firms in the U.S. service industry, our study seeks to identify the factors that impact the size of acquisitions undertaken by such firms in the international context. We used arguments based on the OLI framework to hypothesize how acquiring firm and host country attributes can be expected to influence relative acquisition size (acquisition transaction value as a percentage of acquiring firm asset value). We find that firm characteristics in the form of financial slack and level of differentiation and host country characteristics involving market growth, governance quality, and cultural distance play a significantly role in an acquiring firm's decision related to the relative size of the acquisition to be undertaken. Study implications and contributions are discussed. (For more information, please contact: Dynah A Basuil, University of Auckland, New Zealand: d.basuil@auckland.ac.nz)

\section{Session: 1.5.11 - I nteractive}

Track: 1 - People and Careers in Cross-Cultural Business

\section{Multicultural Experience, Intercultural Competence, and Performance}

Presented On: J une 28, 2015 - 16:15-17:30

Chair: Karina R. Jensen, NEOMA Business School

Biculturals and Third Culture Kids: Apples and Oranges or Two Peas in a Pod? A Critical Review Ebru I pek, Simon Fraser University

Mila Lazarova, Simon Fraser University

This article is a critical review of the non-monocultural literature, focusing on two groups of non-monoculturals: Biculturals and Third Culture Kids (TKCs). These individuals represent a burgeoning demographic and progressively more individuals who have internalized two or more different cultures are shaping the workforce of multinational organizations making these groups of individuals an important subject to examine in business research. Although researchers have argued that non-monoculturals will play an increasingly important role in organizations, there is still very little research about these individuals in business settings and the field is in a nascent stage. For this, the literature from fields such as psychology, sociology and education will be critically reviewed. The main differentiation to monoculturals, namely the internalization of various cultures and the 
outcomes of this internalization, will be examined. The distinction between biculturals and TCKs will be further explored in the paper. The paper will examine in detail the various definitions and the discrepancies between them for the respective group, identify antecedents of non-monoculturals and outline the outcomes of being a non-monocultural. Lastly, the discussion section will combine both fields of bicultural and TCK research, expatiate upon the current challenges in the fields and provide suggestions for future research. (For more information, please contact: Ebru Ipek, Simon Fraser University, Canada: eipek@sfu.ca)

\section{The Impact of Multicultural Experiences and Organizational Diversity Climate on Immigrant Work-Related Outcomes: A Model for Future Research \\ Davina Dias, Monash University \\ Cherrie Zhu, Monash University \\ Ramanie Samaratunge, Monash University}

Cross-cultural encounters are no longer limited to expatriates working overseas because of increased multiculturalism in many countries around the globe. This change in the domestic business environment has stimulated large amount of research in various fields such as international business (IB) and human resource management (HRM) on topics ranging from intercultural competence to organisational diversity climate. However there is lack of cohesive understanding of how individuals with different experiences and cultural capabilities can achieve desired outcomes in such multicultural work environments. To address this gap, this paper proposes a set of hypothesis and a theoretical model based on a literature review. The proposed model highlights multicultural experiences as a predictor of psychological work-related outcomes (job satisfaction, work engagement and job related well-being). It also hypothesises the mediating role individual intercultural competence can play in this relationship and includes organisational diversity climate as a moderating variable. The model addresses the path through which pervious multicultural experiences lead to positive work outcomes and also addresses a boundary condition with regards to intercultural competence. The model therefore advances existing intercultural competence literature and provides practical insights helping organisations to deal with the challenges of doing business in a multicultural context. (For more information, please contact: Davina Dias, Monash University, Australia: davina.dias@monash.edu)

\section{Innovating Synergy from Cultural Diversity: Case of French - Russian MNCS Natalia Guseva, National Research University Higher School of Economics}

Cultural diversity is regarded as the source for innovating synergy, as a reservoir for managerial knowledge, and as an opportunity to gain additional competitive advantages for multinational companies operating in Russia. Cross-cultural differences in multicultural teams is seen with a positive intent as an opportunity to achieve cultural synergy. The proposed approach for innovating cultural synergy is based on a three-stage model. The first stage is to define the most relevant features of the cultural diversity on an example of the French-Russian collaboration. During the second stage, the opportunities of cultural diversity are employed to create new knowledge reservoirs in the management process. The final third stage is to develop and implement new creative managerial decisions and initiatives in order to achieve the synergy effect and to increase multicultural teams' management effectiveness. (For more information, please contact: Natalia Guseva, National Research University Higher School of Economics, Russia: profguseva@gmail.com)

\section{Understanding Cultural Singularities of "Indianness" in an Inter-Cultural Business Setting \\ Francis Laleman, Beyond Borders \\ Ashish Malik, Newcastle Business School}

Analysing data from the Indian Information Technology (IT) industry, this paper advances an understanding of cultural singularities of 'Indianness'. The research context of an intercultural meeting place of IT and business process outsourcing (BPO) firms' overseas subsidiaries, Belgium in this case, allows the authors to identify ten cultural singularities that typify 'Indianness'. This study is a departure from the popular cultural dimension 
frameworks for understanding intercultural business and management as these do not sufficiently describe the contemporary intercultural dynamics that typically take place in workplaces, especially so in offshore and outsourcing environments. A provisional set of parameters for understanding Indian culture, with its relevant impact on business life (customs and manners), business processes, and business deliverables are proposed. (For more information, please contact: Vijay Edward Pereira, University of Portsmouth, United Kingdom: vijay.pereira@port.ac.uk)

\section{A Hierarchy of Cultural Intelligence Antecedents \\ Ilan Alon, Rollins College \\ Michele Boulanger, Rollins College \\ Julie Ann Elston, Oregon State University \\ Eleanna Galanaki, Athens University of Economics \\ Carlos Martinez de I barreta, Universidad Pontificia Comillas \\ J udith Meyers, University of California \\ Marta Muniz-Ferrer, Universidad Pontificia Comillas \\ Andres Velez-Calle, Universidad EAFIT/Rutgers University}

In this article, we aim to further the discussion of the usefulness of the Cultural Intelligence (AKA Cultural Quotient or CQ) concept and of its measurability. We examine the antecedents of CQ and rank them in order of importance, using a recent survey of 1580 respondents from Austria, Colombia, Greece, Spain and the USA. Our research instrument is the Business Cultural Intelligence Test (BClQ), a new, validated measurement tool, involving cognitive, attitudinal and behavioral aspects of CQ. We find the most important antecedent to be the number of countries that business practitioners have lived in for more than six months, followed by their level of education and by the number of languages they speak. Finally, we discuss ways to enhance an individual's CQ that go beyond the traditional and purely cognitive approaches. (For more information, please contact: Andres Velez-Calle, Universidad EAFIT/Rutgers University, Colombia: avelezca@eafit.edu.co)

\section{The Role of Previous Nonwork International Experiences on the Development of Critical Cross-Cultural Competencies for Expatriate Adjustment \\ Ebru I pek, Simon Fraser University \\ Mila Lazarova, Simon Fraser University}

An important matter to be addressed in this paper, is that the literature on Third Culture Kids (TCKs) individuals who have spend a substantial part of their formative years outside their native culture - has stated that TCKs have special cross-cultural competencies and are therefore an ideal source of expatriates; however, save for general claims that these competencies are related to TCKs' experiences in their formative years, research has not dwelled on theoretical explanations for the development of these. Further, recent work in the area of international experiences has suggested that prior nonwork cross-cultural experiences may have a stronger impact on adjustment than work experiences, yet despite such suggestions, the role of nonwork experiences has been rather understudied. These are the issues we explore in this paper. By drawing on social learning theory and the stress-adaptation-growth dynamic framework as our theoretical foundation and consulting the literature on child development, we propose that early cross-cultural experiences create conditions that enable the development of cross-cultural competencies, which in turn facilitate cross-cultural adjustment in adulthood. The paper is a theoretical addition to the current literature by extending the research on TCKs in business and the role of nonwork experiences on cross-cultural adjustment. (For more information, please contact: Ebru Ipek, Simon Fraser University, Canada: eipek@sfu.ca) 
Experiences of International Students in the Australian Higher Education System: An Extreme Groups

Comparison

Andre Anugerah Pekerti, University of Queensland

Miriam Moeller, University of Queensland

Fons van de Vijver, Tilburg University

Tyler G Okimoto, University of Queensland

Using a series of in-depth qualitative interviews of 65 international degree-seeking students studying in Australia, we found that many international students voice great concerns and frustration about the relationship between their expectations and the actual experiences or benefits derived from studying in Australia. A thematic analysis revealed 72 positive and 65 negative themes, covering behavior, services or assistance, general acceptance, cost of living, study/education and lifestyle/leisure. We then selected two small subsamples of students reporting the positive and negative themes, enabling an analysis of their similarities and differences. Results show that all students have expectations that are me and unmet. Results demonstrate that lack of positive intercultural contact led to an overall negative outlook of Australia. In particular, negative experience(s) become magnified, fostering a perception of not fitting-in, and perpetuating negative perceptions of Australia. In contrast, positive contact experience(s) lead to better adjustment through positive perceptions of fit. Positive contact experience(s) with locals fostered a more constructive picture of overall well-being including a prospective future fit sometimes despite past traumatic experiences. Implications for research and higher education practices are discussed. (For more information, please contact: Andre Anugerah Pekerti, University of Queensland, Australia: a.pekerti@business.uq.edu.au)

\section{Session: 1.5.12 - Interactive \\ Track: 10 - Theories of the MNE and of FDI}

\section{Ties, Control, Distance and Diversity: Managing Complexity in MNCs}

Presented On: J une 28, 2015 - 16:15-17:30

Chair: Sylvie K. Chetty, University of Otago

Institutional Ties Ambidexterity and International Exploration and Exploitation

Jie Wu, University of Macau

Livia Markoczy, University of Texas at Dallas

We argue in this paper that emerging market multinational enterprises (EM MNEs) cope with institutional voids by integrating market and nonmarket strategies when they enter into foreign markets. Specifically, we examine the distinct roles of "institutional ties ambidexterity" in EM MNEs' international exploration and exploitation. We further examine the conditions that are likely to amplify or reduce the effect of institutional ties ambidexterity. Longitudinal data for a period of 1999-2013 on a sample of 715 Chinese MNEs provide strong supports for the hypotheses. (For more information, please contact: Livia Markoczy, University of Texas at Dallas, USA: livia.markoczy@utdallas.edu)

\section{Control in the Multinational Enterprise: The Polycentric Case of Global Professional Service Firms Mehdi Boussebaa, University of Bath}

This paper contributes to research on control in multinational enterprises (MNEs) by considering the case of global professional service firms (GPSFs). Drawing on fieldwork in four firms, it argues that GPSFs are seeking greater control over their subsidiaries in order to provide integrated cross-national services to global clients and, in the process, are becoming subject to a center-subsidiary tension similar to that observed in more conventional MNEs. However, and importantly, the paper also argues that the center-subsidiary tension operates differently in GPSFs. This is because central control in this particular context is pursued by not only 
headquarters but also subsidiaries based in core economies where major global clients are headquartered. Such polycentric control leads to the center-subsidiary tension expressing itself along not just the vertical (headquarters-subsidiary) axis but also the horizontal (inter-subsidiary) one and, in particular, along coreperiphery lines. The research and managerial implications of these findings are discussed. (For more information, please contact: Mehdi Boussebaa, University of Bath, United Kingdom: m_boussebaa@hotmail.com)

\section{Where to Locate: The Impact of Local Subsidiary Distance on the Location Choice of Regional Headquarters J unichi Yamanoi, Waseda University Tadashi Hayashi, Kyoto Sangyo University}

A multinational enterprise has increasingly used regional headquarters to manage the operations of its foreign subsidiaries in the distance in response to the recent development of globalization. A regional headquarters is purposed to support global headquarters' activities of monitoring and coordinating local subsidiaries by acting as a local administrator, corporate ambassador, and foreign investor in a focal region. Despite the rapid increase in the number of regional headquarters, it has been little known about the logic behind the location choice of regional headquarters. Which country does a multinational enterprise establish its regional headquarters? From the viewpoint of spatial transaction costs, we suggest that a multinational enterprise is more likely to establish its regional headquarters in a country whose cultural, administrative, and geographic distances with those of local subsidiaries in a region are shorter, because the spatial transaction costs incurred there can be minimized. Using the unique dataset of the Japanese listed manufacturing firms' regional headquarters and local subsidiaries from 1990 to 2009, we found general support for the hypothesized relationships. (For more information, please contact: Junichi Yamanoi, Waseda University, Japan: junyamanoi@yahoo.co.jp)

\section{Institutional Diversity in International Business: Who Shares? \\ Robbert Maseland, University of Groningen}

This paper argues that neither new institutional economics nor the neo/institutional tradition in sociology or organizational studies provides a theoretical basis for the concept of institutional distance, focusing on the negative effects of the amount of institutional diversity. In both perspectives, what matters for firms (co)operating internationally is not the degree of (dis)similarity of institutional structures between contexts, but the presence of commonality in institutional structures governing partners. On basis of this argument, we propose the concept of institutional overlap as more relevant to MNEs, and discuss the implications of an overlap perspective for theories about MNE behavior. (For more information, please contact: Robbert Maseland, University of Groningen, Netherlands: r.K.j.maseland@rug.nl)

\section{The Missing Link between Awareness and Use of Pro-Internationalization Incentives \\ Miguel Matos Torres, University of Aveiro \\ L. Jeremy Clegg, University of Leeds \\ Celeste Amorim Varum, University of Aveiro}

This paper investigates why firms use pro-internalisation incentives towards outward internationalization through focusing on the application process. We pose three research questions: (1) Do firms select public incentives that compensate for a lack of internal resources or capabilities in their possession? (2) Do firms react primarily to internal or external exigencies? And (3) do firms use public support to externalize the increased risk of internationalization, and thereby protect their external activities and investments from loss? We apply a Heckman Selection Model to survey data collected from a sample of Portuguese firms. We find that (1) more use is made of public support the greater are the internal limitations of firms with respect to resources and capabilities, and the more demanding are the conditions in which internationalization takes place; (2) awareness of the availability of support, though positively associated with more demanding conditions of internationalization, is unrelated to any lack of resources and capabilities; (3) the use of public support is 
associated with the opportunity costs of public incentives, and with the increased inherent risk of internationalization. These results indicate important sources of inefficiency within the process of application for policy measures, particularly with respect to the link between awareness and use. (For more information, please contact: Miguel Matos Torres, University of Aveiro, Portugal: miguel.torres@ua.pt)

\title{
Session: 1.5.13 - Interactive \\ Track: 4 - International Marketing, Cross-Cultural Marketing, Social Communities
}

\section{Explorations of Culture and Consumers}

Presented On: June 28, 2015 - 16:15-17:30

Chair: Pinaki Dasgupta, International Management Institute

Effects of Outbreaks of Animosity against the West: What can American B52s do for Local Brand Consumption in China?

Martin Heinberg, University of Duisburg-Essen

Local brands in emerging markets suffer from fierce competition and chronic disadvantages. We investigate if outbreaks of animosity might benefit local brands by raising consumers' willingness to pay and willingness to buy. More precisely, the study introduces the construct "animosity against the West" (AAW), which stretches the animosity construct to an interregional level. On such a level, it is possible to find effects of animosity on local brand consumption, which were not investigated previously. The new construct is validated with a study in China ( $n=244$, SEM). Additionally, we introduce willingness to pay to animosity research. This measurement is built on a real economic exchange and thus better represents cost aspects of substituting one brand for another. A second study in China $(n=676)$ investigates the effect of an outbreak of animosity in an experiment. We find that after an outbreak, AAW enhances the willingness to pay for local products; such an effect is missing in the control setting. This might be due to the cost of substituting Western goods for local ones (e.g. inferior perceived quality and other-signaling value). Moreover, the results suggest that an outbreak of animosity presents a risk to consumers and thus lowers their overall willingness to pay. (For more information, please contact: Martin Heinberg, University of Duisburg-Essen, Germany: martin.heinberg@uni-due.de)

\author{
The Emerging Anti-Consumer: Emerging Correlates of Anti-Consumption Behavior \\ Duraipandian Israel, XLRI-Xavier School of Management \\ Rajesh Iyer, Bradley University \\ James A. Muncy, Valdosta State University \\ Debasis Pradhan, XLRI-Xavier School of Management \\ Abhisek Kuanr, XLRI-Xavier School of Management
}

A specter of mass consumption is engulfing developing societies across the world riding on the waves of liberalization, privatization and globalization. This has prompted individuals from emerging economics to reduce their degrees and patterns of consumption, for a happier and a sustainable future. We call them the Emerging Anti-consumers. The paper explores the evolving anti-consumption behavior in an emerging economy (India). This study investigates the effect of collectivism/individualism on four different categories of anti-consumption. Using a convenience sample of professionals from an Executive MBA program, the study found that anticonsumption attitudes were stronger for those with individualistic values and weaker for those holding collectivistic values. Also there are no studies, which identify relative influence of cultural values on anticonsumption behaviors. This relationship held across for all the four types of anti-consumption that we studied. This study found that the cultural values of collectivism/individualism appear to have a stronger impact on milder forms of anti-consumption than on more radical forms of anti-consumption. For marketers it provides interesting insights into presence of anti-consumerist intentions in developing countries and throws light into its 
cultural correlates for segmentation and targeting. (For more information, please contact: Rajesh /yer, Bradley University, USA: riyer@bradley.edu)

\author{
Can Country of Consumption Information Counterbalance the Negative COO Effect on Product Evaluation \\ Hsiu Ying Huang, Feng Chia University \\ Yi Fang Chiang, Feng Chia University \\ Sheng-Tsung Hou, Feng Chia University
}

A great challenge for emerging-market brand is to overcome the negative quality perceptions associated with their unfavorable country of origins (COOs). This study used an experiment design method to test if country of consumption (COC) information can effectively offset the negative effects of $\mathrm{COO}$ on product evaluation. Moreover, the result demonstrated that the favorable COC information has a greater positive influence when it is combined with unfavorable $\mathrm{COO}$ information than with favorable $\mathrm{COO}$ information. Therefore, the result suggests that COC information serves an effective counterbalance tool for brands without favorable COO images. The result of the study reaffirms the country-of-consumption effect (COC effect) proposed by previous researchers and compliments the conventional "country-labeling" strategy by highlight the importance of demand-side information of a brand. As a result, the COC counterbalance effect demonstrated in this study provides meaningful implications for both academics and practitioners. (For more information, please contact: Hsiu Ying Huang, Feng Chia University, Taiwan: huanghy@fcu.edu.tw)

\title{
National Identity in Marketing: An Integrative Framework \\ Sergio W. Carvalho, Dalhousie University
}

The purpose of this paper is to propose an integrative national identity-based model of consumer behavior through an examination of how citizens as consumers make sense of, interpret and negotiate what their nation and their national identity mean to them not only in routine times but also in times of crises when the national identity has been wounded or threatened by outsiders. Additionally, it attempts to increase the understanding of how consumers position themselves in relation to national symbols or national rhetoric and how these help shape buying behavior. This integrative analysis presents the theories of self-concept, social identity and intergroup relations as roots for consumers' feelings, attitudes and behavioral manifestations linked to national identification. (For more information, please contact: Sergio W. Carvalho, Dalhousie University, Canada: scarvalho@dal.ca)

\section{Exploring CSR practices of MNES in Developing Countries \\ Verena Gruber, WU Vienna \\ Bodo Schlegelmilch, WU Vienna}

Corporate Social Responsibility (CSR) initiatives can provide a mechanism for tapping into the vast consumer markets of developing countries. We investigate how regional headquarters (RHQs) of multinational enterprises (MNEs) in Africa pursue CSR and whether their initiatives are aligned with their own global CSR agendas or tailored to local idiosyncrasies. Following a secondary data analysis of MNEs' CSR and sustainability reports and their homepages, in-depth interviews with their CSR managers in African RHQs are conducted. The paper provides insights into motivations of RHQs to pursue specific CSR initiatives. MNEs need to make considerable adaptations to their global CSR agendas in order to develop initiatives that fit the local setting. We further identify key institutions in developing countries and discuss the potential of collaborations with MNEs in their CSR initiatives. The paper shows how the context of developing countries shapes the translation process of MNEs' global CSR agendas. Companies benefit from the best practice examples provided in this article and learn from the stakeholder collaborations discussed. (For more information, please contact: Verena Gruber, WU Vienna, Austria: verena.gruber@wu.ac.at) 
Perceived Age and Rokeach Value Rankings among Young Adults: A Comparison between India and the U.S. Charles Wood, University of Tulsa

Cole Brown, Indian Professional Research Project

Nicholas Wood, Velocity Consulting

Bruce Alford, Louisiana Tech University

This paper fills a gap in the marketing literature by providing a summary of cognitive ages, ideal ages, and ranking of Rokeach values among young adults in India, and comparing them to a similar sample in the U.S. Intercept survey methods were used to collect data from 244 respondents in Gurgaon, India, and an additional set of Rokeach rankings were collected from U.S. young adults for comparison purposes. The study's findings are combined and compared with prior published work in marketing, revealing interesting cross-cultural differences. Implications for marketing theory and practice - and future research in India - are discussed. (For more information, please contact: Charles Wood, University of Tulsa, USA: charles-wood@utulsa.edu) 


\section{ABSTRACTS FOR MONDAY, J UNE 29, 2015}

\section{Session: 2.1.1 - Panel Special Session}

\section{The MNE-INV Interface: Future Directions}

Presented On: J une 29, 2015 - 09:00-10:15

Chair: Shameen Prashantham, Nottingham University Business School China

Discussants: Peter Buckley, University of Leeds and Becky Reuber, University of Toronto

Panelists:

Shameen Prashantham, Nottingham University Business School China

Erkko Autio, Imperial College

Suresh Bhagavatula, Indian Institute of Management Bangalore

Peter Buckley, University of Leeds

Gary Knight, Willamette University

Rishikesha Krishnan, Indian Institute of Management

K Kumar, Indian Institute of Management Bangalore

Becky Reuber, University of Toronto

Ivo Zander, Uppsala University

This panel seeks to generate ideas about researching the intersection between two exciting international business (IB) phenomena: (1) the accelerated internationalization of born globals and international new ventures (INVs), and (2) the emergence of interfirm networks orchestrated by large multinational enterprises (MNEs). The traditionally siloed approach to studying MNEs and INVs seems no longer tenable if we are to conduct research that is informed by, and relevant to, contemporary business practice, given that present-day MNE interfirm networks frequently include INVs. In addition to surfacing ideas from leading experts in international business and entrepreneurship, the panel includes local academics who will shed light on the I NVMNE interface in Bangalore. (For more information, please contact: Shameen Prashantham, Nottingham University Business School China, China: shameen.prashantham@nottingham.edu.cn) 


\section{Session: 2.1.2 - Panel}

Track: Scholars Meet Practice

\section{Virtual Organizations: Managing Learning and Sharing Knowledge Across Borders}

Presented On: J une 29, 2015 - 09:00-10:15

Chair: Elizabeth Rose, University of Otago

Panelists:

Elizabeth Rose, University of Otago

Abhijit Bhaduri, Wipro Ltd.

Virginia Cathro, University of Otago

Sathya Prasad, Intel

S. Raghunath, I ndian I nstitute of Management Bangalore

Usha Raghunath, Wipro Ltd.

Multinational enterprises (MNEs) are increasingly making use of the rapid developments in information and communication technologies to adjust their approaches to working across borders. The creation of virtual organizations is becoming a standard managerial tool for MNEs, because of the benefits associated with cost, speed, flexibility, and the ability to have the most appropriate people for a given task or project collaborating, regardless of their physical locations. While virtual organizations are no longer unusual in practice, we still have a great deal to learn about how they function, and how this new organizational form can be better employed to support learning and innovation, both within and between MNEs. With a mix of academic and practitioner perspectives, this panel aims to generate a rich discussion that focuses on this emerging phenomenon. The goal is to push forward our understanding of how to facilitate learning and knowledge sharing in the context of virtual organizations. The discussion will be highly interactive, and will emphasize the exchange of ideas among members of the panel and the audience. (For more information, please contact: Elizabeth Rose, University of Otago, New Zealand: elizabeth.rose@otago.ac.nz)

\section{Session: 2.1.3 - Competitive}

\section{Track: 6 - Innovation and Knowledge Management}

\section{Managing Global I nnovation Networks}

Presented On: J une 29, 2015 - 09:00-10:15

Chair: T.J . Hannigan, Temple University

Catch-Up and Connectivity to Global Innovation Systems: A Limited Flying Geese Model?

Ahreum Lee, Temple University

Eunkyung Park, Aalborg University

Connectivity to global innovation systems has been known to be crucial for maintaining competitiveness in the global market. In the context of catching-up, it also provides opportunities for closing the technological gap between advanced and emerging economies. It is therefore expected that increasing connections to global innovation systems accelerates catch-up processes in recently emergent countries. Such connectivity has been demonstrated as a mechanism for catch-up in many national contexts. However, Korea, our empirical context, shows quite different innovation trajectory. Utilizing the patent data from US Patent and Trademark Office (USPTO), we compare the country dispersion of inventors in the electronics and pharmaceutical industry in Korea, Denmark, and J apan to illustrate how globally connected Korea is in terms of new knowledge creation. Our analysis reveals that unlike Denmark, Korea is surprisingly closed to the world when it comes to creation of new knowledge. Its trajectory is similar to that of J apan. Korea's similarity with J apan and its dissimilarity with 
Denmark in terms of connectivity to global innovation systems at the patent level, suggests that the development of innovation trajectory as well as the general industrialization process of Korea conforms to some extent to the "flying geese model" developed by Kojima. (For more information, please contact: Ahreum Lee, Temple University, USA: ahreum.lee@temple.edu)

\section{International Connectivity and Innovation Networks: The Case of Chinese Inventors in the Pharmaceutical Industry}

Vittoria Giada Scalera, Politecnico di Milano

Alessandra Perri, Ca' Foscari University Venice

Ram Mudambi, Temple University

This paper explores the integration of emerging countries into the global system of innovation, as a channel for their technological catch-up. Using data on the innovative activity in the Chinese pharmaceutical industry, we analyze the geographic dispersion of inventor networks linked to China, as a function of the characteristics of the innovative actors that coordinate their inventive work. More specifically, we explore the role of the geographic origin and the institutional type of the innovative actors involved. Based on a dataset of USPTO pharmaceutical patents granted between 1975 and 2010 and reporting at least one Chinese inventor, we find that foreign innovative actors represent a stronger driver of connectivity compared to domestic innovative actors. Our results suggest that - compared to MNEs - universities and research centers spawn more internationally dispersed inventor networks, especially when they are foreign. These findings point to the critical role that foreign universities and research centers may play in the catch-up process of emerging countries. Moreover, the superior ability of foreign single-location firms (compared to their domestic peers) to connect the local context with the global innovation system indicates that the phenomenon of highly innovative international ventures is more likely to happen in the context of developed countries. (For more information, please contact: Vittoria Giada Scalera, Politecnico di Milano, Italy: vittoriagiada.scalera@polimi. it)

\section{Managing Innovation Networks: A Multiplex Analysis of the Global Wind Power Industry}

Snehal Suyash Awate, Indian School of Business

Marcus M. Larsen, Copenhagen Business School

In this article, we treat innovation as a multidimensional construct spanning people, technologies, and geographies. We study how these dimensions interact and impact firms' inventor networks and the ultimate innovation performance. We identify five distinct planes in which inventor networks reside. Specifically, we distinguish between the types of ties that are possible between any two inventor nodes with respect to (i) colocated inventors; (ii) technology cohort; (iii) co-located technology cohort; (iv) distant co-inventors; and (v) colocated co-inventors. We then measure the costs and benefits for the inventors to participate in different planes or being multiplex. Using patent data on the global wind power industry, we find evidence suggesting that being centrally positioned on a multiplex network deteriorates rather than enhances innovation performance. (For more information, please contact: Snehal Suyash Awate, Indian School of Business, India:

snehal_awate@isb.edu)

General Purpose Technologies (GPTs) and the Internationalization of Corporate Innovations - A Cross-Country Analysis

J ohn Cantwell, Rutgers University

Ranfeng Qiu, California State University, San Bernardino

In this study, the concept of General Purpose Technologies (GPTs) is fitted into the context of the changing profile of international corporate innovations and the underlying trajectory of national innovation systems. As GPTs are employed as a "bridge" connecting technologically and geographically separate technologies, the specialization of innovations in GPT fields has important influence on the pattern of geographical distribution of corporate innovations. Based on a USPTO database covering patents granted in the U.S. to the world's largest 
industrial firms between 1969 and 1995, the empirical findings suggest that GPTs, compared with other technologies tend to be created across a wider range of countries. Such geographical dispersion is mainly driven by the fact that increasingly more GPTs invented in different countries are created to support the industry's core areas. Moreover, the specialization of GPTs tends to promote a further internationalization of corporate innovative activities, especially those in the GPT-based industries. Lastly, in those countries in which local industries have largely developed GPTs, the innovative activities tend to taken by firms from a wider range of foreign countries and industries. (For more information, please contact: Ranfeng Qiu, California State University, San Bernardino, USA: rqiu@csusb.edu)

\section{Session: 2.1.4 - Panel \\ Track: 14 - Research Methodology in I B (Special Track)}

\section{Creating and Publishing High-Quality Research on Women and Gender (WAIB Special Session)}

Presented On: J une 29, 2015 - 09:00-10:15

Chairs: Amanda Bullough, University of Delaware and Tugba Kalafatoglu, ESADE Business School

Discussant: J oan P. Mileski, Texas A\&M University at Galveston

Panelists:

Amanda Bullough, University of Delaware

Tugba Kalafatoglu, ESADE Business School

Rosalie Tung, Simon Fraser University

William Newburry, Florida International University

Patricia P. McDougall-Covin, Indiana University-Bloomington

Nakiye Avdan Boyacigiller, Sabanci University

Fiona Moore, Royal Holloway, University of London

J oan P. Mileski, Texas A\&M University at Galveston

With this panel, WAIB (Women of the Academy of International Business) offers a discussion of how scholars can increase their rigor on research topics related to women or gender and improve their chances of getting their papers published in premier journals, like the J ournal of International Studies. While the general topics of women and gender have been fairly well-established from a research perspective (the glass ceiling, work-family balance, discrimination, etc.), there are still many areas of research that remain unpublished in high-quality journals (women in business in adverse environments, businesswomen in politics, institutions, etc.). The panel is made up of experienced editors and reviewers and leading scholars with experience publishing research in these areas, and reviewing and accepting or rejecting articles on these topics. The panelists will openly discuss the issues that they feel contribute to either success or failure with getting papers accepted for publication by premier journals. (For more information, please contact: Amanda Bullough, University of Delaware, USA: bullough@udel.edu) 


\section{Session: 2.1.5 - Competitive}

\section{Track: 11 - Corporate Governance in I B}

\section{Being Innovative and Being Conscientious: What matters?}

Presented On: June 29, 2015 - 09:00-10:15

Chair: Ruth Aguilera, Northeastern University

How Do National Corporate Governance Institutions Affect Slack-Based Innovation Effort in Firms?

Paul Martin Vaaler, University of Minnesota

J oel Malen, Hitosubashi University I nstitute of Innovation Research

Behavioral theory of the firm (BTF) research elaborates on the role slack plays in supporting efforts to innovate in firms, but the BTF fails to account for moderating effects that national corporate governance institutions (NCGIs) may have on this firm-level relationship when studied on a cross-country basis. In response, we develop a cross-level theoretical framework assuming that countries differ in their NCG protections related to two firm stakeholders - shareholders and employees - and that such NCGI protections moderate the firm-level relationship between different types of slack and innovation effort in firms. We test predictions derived from our framework using a sample of 7,083 firms from 29 countries observed from 1991-2005. Two-stage firm- and country-level estimated dependent variable analyses of these slack types and their impact on firm research and development $(R \& D)$ intensity indicate that: 1$)$ stronger NCGI shareholder protections diminish positive financial and human resource (HR) slack effects on R\&D intensity; but 2) stronger NCGl employee protections magnify positive HR slack effects on R\&D intensity. Cross-country research on the relationship between firm slack and innovation effort can and should account for substantial differences in country-level NCGIs that show little tendency toward convergence in the near term. (For more information, please contact: Paul Martin Vaaler, University of Minnesota, USA: vaal0001@umn.edu)

\section{What Determines MNEs' Environmental R\&D? \\ Hyoju Jeong, Korea University \\ J on J ungbien Moon, Korea University \\ J iyoung Shin, Korea University}

We examine determinants of MNEs' environmental R\&D and how environmental regulation moderates them. After analyzing 1,674 firms for 2004-2012, we find that firms with poor reputation in environmental management and long-term oriented compensation tend to have higher levels of environmental R\&D. Moreover, consistent with previous studies that found important regulatory role in environmental R\&D, firms in the biggest greenhouse gas (GHG) emitting industries are likely to engage in environmental R\&D when they participate in emissions trading. However, unlike the previous studies conducted in single country settings, our study suggests that in international setting, the sub-global regulation such as emissions trading may not work as it intended.

(For more information, please contact: Jon J ungbien Moon, Korea University, Korea, South: jonjmoon@korea.ac.kr)

Which "Green" is Better? An Empirical Study of the Impact of Green Activities on Firm Performance Chin-jung Luan, National Dong Hwa University Chengli Tien, National Taiwan Normal University Wei-Lun Chen, National Dong Hwa University

The purpose of this study aims to distinguish an array of green activities and analyze which green activity can generate more performance to companies adopting it. Employing data from the sampled publicly listed firms in Taiwan and regressions to examine the hypotheses, we find that the degree of a firm's R\&D investment fails to 
affect companies on the choice of green activities. The degree of firm internationalization can affect companies on the choice of green activities, and that firms with a higher degree of internationalization will be more likely to employ green certifications. Moreover, a company employing green processes can perform better, followed by ISO 14000, pollution prevention, and lastly, green certifications. (For more information, please contact: Chengli Tien, National Taiwan Normal University, Taiwan: cltien@ntnu.edu.tw)

\section{Are We There Yet? Corporate Social Performance around the World Pre- And Post- The Global Financial Crisis J eremy Galbreath, Curtin Graduate School of Business Mariano Heyden, Newcastle Business School , Australia Trond Randøy, University of Agder}

This paper addresses a paradox: although stakeholder satisfaction is critical for firm success, firm behavior does not always support stakeholder interests. In fact, firms can act in ways that cause harm to stakeholders, with negative implications for wider society. The global financial crisis (GFC) is a good example. This paper posits that intensified institutional pressure post-GFC has impacted the way firms around the world address their societal obligations and responsibilities towards stakeholders, lifting their corporate social performance (CSP). Studying 929 firms across 30 countries, the findings suggest that CSP is improving around the world post-GFC relative to pre-GFC levels. As for specific areas of CSP, contrary to the prediction, the environmental dimension is improving at a greater rate than the social dimension. By country, contrary to the prediction, firms in high risk countries are improving their CSP at a rate greater than firms in medium and low risk countries. The results also demonstrate that banks are improving their CSP at a greater rate than firms in all other industries. The results are discussed along with implications and future research directions. (For more information, please contact: Mariano Heyden, Newcastle Business School, Australia, Australia: mariano.heyden@newcastle.edu.au)

\section{Session: 2.1.6 - Competitive \\ Track: 10 - Theories of the MNE and of FDI}

\section{Managing Distance in the Global Firm}

Presented On: June 29, 2015 - 09:00-10:15

Chair: Douglas Dow, University of Melbourne

The Role of Institutional Distance in FDI Decisions: A Polycentric Perspective

Stewart R. Miller, University of Texas-San Antonio

Kai Xu, Texas A\&M University

Michael A. Hitt, Texas A\&M University

Scholars have sought to integrate institutional theory into the foreign direct investment (FDI) literature. The present study seeks to extend this research by first asserting that FDI decisions are not independent investment decisions, but rather a set of sequential integrated investment decisions. We draw upon work on polycentrism to explain how regulatory distance influences the likelihood of entry decision; cognitive/cultural distance affects the degree of equity investment decision; and normative distance determines the degree of joint venture control ownership. Moreover, we explain how the institutional information spillover from earlier stages in the FDI process benefit decisions made in later stages. (For more information, please contact: Stewart R. Miller, University of Texas-San Antonio, USA: stewart.miller@utsa.edu) 
Institutional Distance as a Source of Arbitrage and Learning: The Case of Ownership Decision in Foreign

Acquisitions

Ajai Gaur, Rutgers University

Shavin Malhotra, Ryerson University

PengCheng Zhu, University of San Diego

In this study, we deviate from the pervasive view that cross-border distance is a source of liability and suggest that institutional distance offers learning and arbitrage benefits for multinational corporations (MNCs). We test this view on equity ownership decisions in cross-border mergers and acquisitions (M\&As). We argue that because institutional distance offers arbitrage benefits to MNCs, foreign acquirers are likely to prefer a shared ownership arrangement to better exploit these benefits as institutional distance increases. However, maintaining shared ownership at very high institutional distances is costly, therefore, the relationship between institutional distance and equity ownership is non-linear. Further, we propose that when firms are more familiar with the external environment (as measured by geographic, cultural and linguistic proximity), they can better derive arbitrage benefits due to institutional differences, and therefore more likely to opt for shared ownership. We test our arguments on a sample of 37,588 cross-border M\&As involving 52 home and 54 host countries over 17 years (1996-2013). (For more information, please contact: Ajai Gaur, Rutgers University, USA:

ajai@business.rutgers.edu)

\section{MNE Dynamics, Distance, and the Role of Space and Place in the Organization of its Locations} Majid Eghbali-Zarch, Memorial University of Newfoundland

Distance is an influential factor in the practice and scholarship of international business and impacts the decisions and activities of Multinational Enterprises (MNEs). As MNEs evolve in the global landscape, the impact of international distance on their activities change. This study aims at investigating this interaction. We argue that for firm-level decision making, distance to the target host country is influenced by the firm and the whole portfolio of its extant footprints. We suggest this as an alternative view to the established measurement of distance (from MNE's home country to the target host country). The extant measure is based on the home host country dyad level as the benchmark for assessment. We use the MNE - host country dyad level to develop a measure with arguably improved predictive validity. Whereas the extant measure has great merit especially in studies such as international trade, our conception results in firm level heterogeneity among firms originating from the same home country. Utilizing the international business and economic geography literatures on location and space, we propose that the spatial evolution of a firm in the global space results in a temporal dynamic in its proximity to a new target host country (For more information, please contact: Majid EghbaliZarch, Memorial University of Newfoundland, Canada: majidez@mun.ca)

\section{Reconceptualizing the Global Factory: An Asymmetrical Network Perspective Liena Kano, University of Calgary Alain Verbeke, University of Calgary}

The global factory has been proposed as a new, highly efficient form of international activity deployment, brought forth by increasing globalization and the macro-level shift of economic activities to emerging economies, and enabled by advances in information and communication technology and managerial practices. It is supposed to facilitate reductions in conventional levels of vertical and horizontal integration that are ownership-based. In contrast, we argue that the global factory as a distinct form of asymmetrical network governance, is associated with benefits and costs, and is therefore not a universal recipe for international competitive success - rather, a number of external and internal conditions must be satisfied for the global factory to emerge and thrive. We develop an integrative perspective on the role of the lead firm head office in the global factory, using an internalization theory perspective, augmented with insights from the business networks literature. We identify the social mechanisms utilized by the lead firm as core actor to facilitate coordination (a bounded rationality economizing challenge), and to reduce the hazards of imperfect effort exerted by network partners (a bounded reliability economizing challenge) in order to achieve the desired level 
of efficiency ascribed to the global factory. (For more information, please contact: Liena Kano, University of Calgary, Canada: liena.kano@haskayne.ucalgary.ca)

\section{Session: 2.1.7 - Competitive}

\section{Track: 13 - I nternational Economics, Finance and Accounting}

\section{Foreign Production, Trade, and Finance}

Presented On: June 29, 2015 - 09:00-10:15

Chair: Susan Feinberg, Temple University

Vertical Product Differentiation and Exchange Rate Pass-Through: Evidence from Trade between China and Japan

Dongmei Hu, Shenzhen University; The George Washington University

J iawen Yang, George Washington University

Zunxin Zheng, Shenzhen University

In this paper, we investigate the impact of vertical product differentiation on exchange rate pass-through (ERPT). We define product vertical differentiation as a spectrum of products that range from high end to low end. Using a comprehensive data set that includes highly disaggregated products traded between China and J apan from J an 2000 to Dec 2008, we proxy product vertical differentiation with unit values of imports and exports and find that J apanese exports to China are more toward the high end while Chinese exports to J apan are more on the low end. Our further empirical tests show that exchange rate pass-through to Chinese Yuan selling prices of J apan imports is incomplete and is strongly affected by the degree of vertical differentiation. In particular, higher pass-through is observed where the vertical differentiation is larger between J apan import and China local-made, which suggests higher end J apan exporters are able to keep their J apanese Yen markup more stable. (For more information, please contact: Dongmei Hu, Shenzhen University; The George Washington University, China: hudm@szu.edu.cn)

Are Emerging Countries Catching Up? The Spatial Geography of Knowledge-Intensive Activities in Global Production of Manufactures

Marcel P. Timmer, University of Groningen

Gaaitzen J . de Vries, University of Groningen

Case-study research suggests a major re-organisation of knowledge-intensive activities across the globe as multi-national enterprises relocate R\&D, design, and marketing. While we know much about the drivers of this process, relatively little is known about the aggregate trends and geographical patterns. This paper provides a novel macro-perspective on the spatial geography of knowledge-intensive activities in global value chains. Following recent insights in the study of international trade, we identify global value chains by means of world input-output tables that describe the flow of goods and services across industries in 40 countries. This is combined with detailed information on the occupation of workers to measure the value added by knowledgeintensive activities. Our results suggest that knowledge intensive activities have become increasingly important in global value chains. During 1995-2003, growth of knowledge-intensive activities in emerging countries averaged only 1 per cent per annum, but it accelerated to 13 per cent in 2003-2011 in particular in Brazil, China and India. However, at the same time knowledge-intensive activities also expanded in high-income countries and a major gap remains. In 2011, value added in knowledge-intensive activities in Europe, Japan and the US is still more than 3 times that in emerging countries. (For more information, please contact: Marcel P. Timmer, University of Groningen, Netherlands: m.p.timmer@rug.nl) 
Debt to Traders! On Borrowing Heterogeneity and the Exporting Activity of Indian Firms

Sumit Majumdar, University of Texas at Dallas

Sushanta Mallick, Queen Mary University of London

Based on a panel of 5,781 Indian firms over the period 1976-77 and 2005-06, this article has examined the impact of different debt types on the levels of exporting undertaken. Classification of debt into relational and transactional categories has been used to divide the types of debt firms borrow. The relational debt types, such as bank borrowings, borrowings from financial institutions and foreign borrowings, positively impact the levels of exporting undertaken. The results establish that for Indian firms, where relational borrowings from these types of borrowers are substantial, such borrowings have influenced their engagement in overseas markets. We also address the issue of whether access to foreign financing aids exporting activity, and present evidence from Indian exporters. We find that companies with such sources of foreign borrowing have higher exporting activity compared to matched companies with only domestic sources of financing. (For more information, please contact: Sumit Majumdar, University of Texas at Dallas, USA: majumdar@utdallas.edu)

\section{Session: 2.1.8 - Competitive}

Track: 4 - International Marketing, Cross-Cultural Marketing, Social Communities

\section{Business and Industrial Marketing Challenges}

Presented On: J une 29, 2015 - 09:00-10:15

Chair: Brenda Sternquist, Michigan State University

\section{A Demand-Strategy Fit Model Based on Industrial Characteristics: Modernization, Consumption Convergence and Glocal Strategy}

Lydia Qianqian Li, Shanghai University

Qiuzhi Xue, Fudan University

Juan Shan, Shanghai University

Glocal strategy wins wide acceptance with high flexibility and comprehensiveness in the debate over global standardization versus local adaptation. But there lacks in-depth study of how to decide the degree of Global strategy, especially in emerging markets with great regional diversity. This study proposed a demand-strategy fit model for global strategy planning. With survey and archival data from China, this study found that as demand characteristic varying from physiological to sociological, consumption convergence represented by consumers' attitude towards global products versus local products, demonstrates different degree.

Modernization is considered as antecedent of consumption convergence. With case studies, it revealed that company's Glocal strategy should be planed according to the consumption convergence, degree of the industry; and the demand-strategy fit influences the company's performance, where the relationship is moderated by market position. (For more information, please contact: Lydia Qianqian Li, Shanghai University, China: qianqian.li@shu.edu.cn)

\section{Impact of Marketing Expertise Spillover on Performance of Domestic Firms and MNES}

Qiang (Steven) Lu, University of Sydney

Chinmay Pattnaik, University of Sydney

Mengze Shi, University of Toronto

This paper studies the spillover effects of marketing expertise on market performance of domestic firms and Multinational Enterprises (MNEs). Specifically, this study examines how adoption of frequency loyalty programs by a domestic firm following an MNE affects the competitive dynamics and the market performance of both firms in a Chinese retail gasoline market. Based on the empirical data from a quasi-field experiment in which the MNE entered the market with a frequency loyalty program and the domestic firm later responded with a similar 
loyalty program, we find that the domestic firm's adoption of a similar loyalty program significantly increased its market share in the regular gasoline market. The domestic firm's loyalty program also increased the market performance of the MNE in the premium gasoline market. This study empirically shows the spillover benefits through demonstration effects to domestic firms as well as MNEs. (For more information, please contact:

Chinmay Pattnaik, University of Sydney, Australia: chinmay.pattnaik@sydney.edu.au)

Export-Specific Investments, Competitive Advantage, and Performance: The Moderating Effect of Domestic Conditions In An Emerging Economy

Vi Dung Ngo, Vietnam National University

Frank J anssen, Université Catholique de Louvain

Leonidas C. Leonidou, University of Cyprus

Paul Christodoulides, Cyprus University of Technology

We develop and test a conceptual model, anchored on resource-based view, institution-based view, and industrial organization, that examines the effect of export specific investments on building a cost-based and product differentiation-based competitive advantage in foreign markets, as well as the impact of these two types of competitive advantage on export performance. Using a sample of 109 Vietnamese exporters, we confirm that human, innovation, marketing, and financial investments help to generate an export competitive advantage (cost-based or product differentiation) that in turn enhances export performance. Most of the links between export specific investments and cost-based or product differentiation competitive advantage were found to be influenced by domestic market conditions, namely institutional quality, infrastructure facilities, level of bureaucracy, and domestic competition. (For more information, please contact: Vi Dung Ngo, Vietnam National University, Vietnam: dungnv@hsb.edu.vn)

\section{Session: 2.1.9 - Competitive \\ Track: 12 - The Institutional and Political Environment of I B}

\section{Institutions and / B}

Presented On: June 29, 2015 - 09:00-10:15

Chair: Marcelo F Cano-Kollmann, Temple University

Status and Dynamics of Host-Country Institutional Quality and The Internationalization Speed of Foreign Firms in Emerging Markets

Moritz Putzhammer, WU Vienna

Jonas Puck, WU Vienna

Thomas Lindner, WU Vienna

The study at hand addresses the call for an expansion of the under-researched area of internationalization speed - i.e. the speed at which firms internationalize further after having already completed a first step beyond their home country's borders. We extend existing firm-level research by adding country-level factors to the analysis. Specifically, we analyze how status and dynamics of host-country institutional quality affect speed. Our findings contribute to the topic of internationalization speed as well as to the institutional quality literature. We find that dynamics of host-country institutional quality have a positive effect on internationalization speed. (For more information, please contact: Moritz Putzhammer, WU Vienna, Austria: moritz.putzhammer@wu.ac.at) 
The Moderating Effect of Industry Concentration on the Relation between Institutions and Firm Performance

David Kallas, Insper Institute of Education and Research

Rodrigo Bandeira-de-Mello, FGV-EAESP

Wlamir Xavier, Eastern New Mexico University and UNI SUL

Carlos Afonso Caldeira, Insper Institute of Education and Research/Getulio Vargas Foudation

We extend the theory by proposing that industry concentration moderates the relation between institutions and firm performance. It is already known that institution matters (Makino, Isobe, \& Chan, 2004) and that promarket reforms positively affect firms' profitability in emerging countries (Cuervo-Cazurra \& Dau, 2009). The explanation is based on transaction costs economics (Coase, 1937; Commons, 1934). However, it is not known whether this effect is the same for all industries. We built a database of 230,222 observations of 10,903 companies in 64 countries in a 23-years interval. Regressions tested the interaction between the HerfindahlHirschman Index ( $\mathrm{HHI})$ and six institutional variables, considering three dependent variables: ROA, ROE and 3year compound annual sales growth rate. We ran fixed effects and hierarchical models. Results confirmed the hypothesis and were significant for the negative interaction between $\mathrm{HHI}$ and four institutional variables: voice and accountability, govern effectiveness, regulatory quality and control of corruption. Industry concentration moderates the effect of institutions on firm performance. The influence is clearer on informal institutions, like democracy protection, consumer rights and control of corruption. We then argue that strategies of expansion within the industry, as market share dominance, mergers and acquisitions and growth may fit better into weak institutional contexts. (For more information, please contact: David Kallas, Insper Institute of Education and Research, Brazil: davidk@insper.edu.br)

\section{Understanding Language in Service FDI vs. Manufacturing FDI \\ Palitha Konara, University of Huddersfield \\ Yingqi Wei, University of Leeds}

This paper systematically examines the differential effects of language on foreign direct investment (FDI) in services and manufacturing sectors. After establishing an analytical framework to compare the role of language in service and manufacturing FDI, we test the central hypothesis using bilateral FDI flows between 21 OECD countries and 75 partner countries during the period 1986-2008, we find strong evidence to support our hypothesis. The findings have clear policy implications. (For more information, please contact: Palitha Konara, University of Huddersfield, United Kingdom: palitha.konara@hud.ac.uk)

\section{Same but Different? Governance Modes for Practice Transfer and their Implications for Host Institutional Dynamics \\ Johann Fortwengel, Free University Berlin}

How do companies engage in the necessary degree of institutional entrepreneurship for the purpose of practice transfer? We argue that Multinational Corporations (MNCs) may try to engage in institutional entrepreneurship on their own, or they may form inter-organizational networks, in order to mold the institutional host environment collaboratively. In a comparative case study, in which we compare two MNCs following these different approaches, we find that while the particular activities involved in the process of institutional entrepreneurship are remarkably similar, their implications for institutional host dynamics in terms of outcome differ in important ways. More specifically, we find that what we term networked institutional entrepreneurship leads to higher degrees of institutional change in the regional host environment. We contribute to existing literature by emphasizing the role of governance mode in institutional change initiatives. (For more information, please contact: Johann Fortwengel, Free University Berlin, Germany: johann.fortwengel@fu-berlin.de) 


\section{Session: 2.1.10 - I nteractive}

\section{Track: 5 - The Geography of I nternational Business and Global Value Chains}

\section{Clustering and Locational Factors}

Presented On: June 29, 2015 - 09:00-10:15

Chair: J essica Rae Salmon, Rutgers University

Internationalisation Processes and Local Resources System. Evidence from Manufacturing Multinational Companies

Mariachiara Barzotto, Ca' Foscari University Venice

Giancarlo Coro', Ca' Foscari University Venice

Mario Volpe, Ca' Foscari University Venice

This article analyses the relationship between the internationalisation processes undertaken by manufacturing companies and the system of local resources, i.e. human capital endowment, supply and users networks, and institutions. First of all, the study aims to explore how the international fragmentation of production has impacted on the generation of the local resources system in the high-income countries. Secondly, our study tries to understand if and to what extent companies engaging in international networks the most have supported the local assets sustainability. The research, conducted through a multiple case study of manufacturing medium-large size companies located Italy, has provided empirical evidence on the interdependence between the local resources system and the internationalisation processes performed by companies operating in the surrounding environment. Findings show the set of companies' possible actions that would promote the local resources sustainability. The article concludes by highlighting some policy issues linked with more awareness among economic actors of the role played by the territorial assets in nurturing the company's performance, and how to encourage the reproduction of the local resources system. (For more information, please contact: Mariachiara Barzotto, Ca' Foscari University Venice, Italy: mariachiara.barzotto@unive.it)

\section{A Beer Index for International Food Security? A Stochastic Actor-Based Modelling of Network Evolutions}

Tiffany S.W. Tsui, Erasmus University Rotterdam

Ronald Sean Wall, Erasmus University Rotterdam

The combination of statistical inferences with network theories is a fast emerging field and offers exciting new opportunities to explore complex systems. This paper adopts a stochastic actor-based modelling framework to analyse the evolution and co-evolution of trade and investment networks at the global scale and to shed light on international policy and business strategies. The two networks are the trading network of the beer industry, which represents a stably evolving network of an economically important sector for the EU countries, and the emerging and dynamic investment network in the food sector with important developmental significance for emerging nations especially in Africa. The longitudinal time series data covers the period from 2003 to 2011. The network dynamics within individual networks as well as between the two networks in response to the endogenous network structures and exogenous environment were modelled. Major findings include 1) exogenous effects such as the impact of economic development, regionalism and urban growth; 2) endogenous effects such as reciprocity, preferential attachment and local clustering; and 3) cross-network dynamics that the trading in beer may 'pave the way' for other investments between nations. (For more information, please contact: Ronald Sean Wall, Erasmus University Rotterdam, Netherlands: wall@ihs.nl) 
The Spatial Expansion Patterns of Domestic and International Acquiring Firms: Evidence from the Indian Chemical Manufacturing Sector

Abhirup Chakrabarti, Queen's School of Business

Amit Baran Chakrabarti, Indian Institute of Management Calcutta

Sougata Ray, Indian Institute of Management Calcutta

The rising incidence of international acquisitions is paradoxical to recent findings that establish the persistent impeding effect of geographic distance on domestic acquisition target selection. We explore this paradox by examining if the spatial patterns of domestic, within country, expansion differ across multinationals and domestic firms. Using a sample of acquisitions of Indian chemical manufacturing firms founded after 1979, we find several intriguing differences in behavior. First, we find that international acquirers are less likely to be constrained by distance compared to domestic acquirers. In addition, we find that international acquirers were more likely to engage in distant acquisitions at an early stage in their acquisition program, less likely to draw benefits from a central geographic location, and less affected by temporal changes in selecting distant targets. These findings that acquisitions by international acquirers are spatially haphazard and less dependent on information resources provide important insights into understanding corporate spatial expansion. (For more information, please contact: Amit Baran Chakrabarti, Indian Institute of Management Calcutta, India: amitbc11@email.iimcal.ac.in)

\section{Foreign Location Choice: Review and Extensions \\ J in Uk Kim, University of Illinois at Urbana Champaign}

International Business (IB) research on foreign location choice has experienced a revival in recent years yet a comprehensive review has been sorely lacking. The purpose of this review is to synthesize the findings of recently published articles on the topic of foreign location choice and offer fruitful directions for future research. Our review consists of three sections: first, we provide a historical overview of this research stream by tracing its origins and analyzing the general trend that has shaped foreign-location choice research. Next, we conduct a comprehensive review of 137 recent articles published in leading IB and business/management journals. We categorize these articles according to common topics and synthesize their main findings of each category in order to bring some cohesion to this fragmented field. Lastly, we identify issues that remain under-researched or require re-thinking some taken-for-granted assumptions. Through this effort, we are able to connect the past, present and future of foreign location choice research and to shed some new light to the IB literature. (For more information, please contact: Jin Uk Kim, University of Illinois at Urbana Champaign, USA: jkim198@illinois.edu)

\section{Does $R \& D$ Internationalization Affect Locational Specialization? Yuanyuan Li, Rutgers Business School}

This paper attempts to examine the relationship between the FDI stock in technology-intensive industry and locational specialization in the corresponding sector. The key question is whether FDI activity stimulates locational specialization or locational diversification? According to Cantwell's competitive international industry approaches and capabilities-based theory, multinational corporations remain the leading role in technological innovation while knowledge spillover is in bi-direction between location and multinational corporations. It is from "local-for-local" to "local-for-global" and gravitational pull that location has the potential to upgrade to specialized center. Additionally, the above mechanism can be moderated by country type, namely competence exploiting type and competence creating type. Therefore, country type functions as the moderator when testing the relationship between FDI and locational specialization. (For more information, please contact: Yuanyuan Li, Rutgers Business School, USA: yuanyuan.li@rutgers. edu) 
Tapping Eastern Direction: Spatial Organisation of the Beer Industry Offshore Centres in Central and Eastern Europe

Pavlina J asovska, University of South Australia

Beer is the most popular alcoholic beverage. The recent industry trend has been characterised by rapid consolidation and global integration of beer markets. Even though beer companies operate under ongoing acquisition deals and rapid change they have been able to separate some of their value chain support services and offshore them into foreign countries. Despite the growing interest in offshoring location choice, the understanding of how these decisions are interlinked to each other and how they relate to overall location strategy of company remains incomplete. This paper analyses offshoring strategies of four biggest beer companies AB I nbev, SAB Miller, Carlsberg and Heineken and what determinants played significant role in choosing their service offshore centres in Central and Eastern Europe. Through secondary data analyses it has been found that offshoring strategy is not only interconnected with beer company major operations, but also offshoring processes are not isolated decisions but strategically create geographical cluster of operations. (For more information, please contact: Pavlina Jasovska, University of South Australia, Australia:

pavlina.jasovska@mymail.unisa.edu.au)

\section{Session: 2.1.11 - I nteractive}

\section{Track: 9 - Emerging Economies and Emerging Economy MNEs}

\section{Competitive Strategies}

Presented On: J une 29, 2015 - 09:00-10:15

Chair: Somnath Lahiri, Illinois State University

How do Manufacturers and Customers Come to Sensitize Servitization in the International B2B Context?

Chih-An Lin, National Taiwan University

Homin Chen, National Taiwan University

Manufacturing senvitization appears internationally and is promoted at a national level in developed, developing as well as emerging countries. Despite the international trend, the external environmental conditions of opportunities and threats are to match internal strengths before manufacturers can expand on servitization. This study uses an overarching resource-advantage theory to discern antecedents to manufacturer's servitization extent and what relational factors moderate the effect of servitization extent on customer's value perception. Results show that the demand and needs for services are not enough for manufacturers to servitize manifestly but only when they are backed up financially and knowledgably with resources and capability specificity. Relational practices and processes vary in their effects to customer perceived value. Insourcing receives higher customer perceived value than outsourcing does. Customer co-creation that is recently popular in strategy and marketing does not significantly contribute to customer perceived value. Moreover, servitization earns higher customer perceived value in developed countries. I mplications and future research direction are also discussed. (For more information, please contact: Chih-An Lin, National Taiwan University, Taiwan: elintc@yahoo.com)

\section{Latecomers' Growth Strategy: The ABCD Framework and Its Application to Samsung Electronics Hwy-Chang Moon, Seoul National University Wenyan Yin, Seoul National University Sohyun Yim, Seoul National University}

The existing studies on competitive advantage have looked into different dimensions of firm competitiveness. By emphasizing that it is important to have a comprehensive view on how firm competitiveness is built through time, this paper introduces a new ABCD (Agility, Benchmarking, Convergence, and Dedication) framework to fill 
the theoretical gap in the literature of growth strategy and competitiveness. The new framework is then applied to explain the success factors of Samsung Electronics Co. (SEC) and examines the success and evolution of its mobile business. The paper concludes that this new framework is more useful than the traditional competitiveness theories to explain the latecomers' growth strategy. (For more information, please contact: Sohyun Yim, Seoul National University, Korea, South: sohyunyim@gmail.com)

\section{Beyond Coopetition: Is There What To Toast In The Institutional Environment? \\ J efferson Marlon Monticelli, Universidade do Vale do Rio dos Sinos \\ Silvio Luís de Vasconcellos, Universidade do Vale do Rio dos Sinos \\ Ivan Lapuente Garrido, Universidade do Vale do Rio dos Sinos \\ Cyntia Calixto, Fundação Getúlio Vargas}

This study aims to highlight how institutions influence the internationalization of firms that adopt coopetitive strategies. Coopetition has been deserving attention on strategic studies, even so just a few studies highlighted the institutional perspective on that. We developed a single case study interviewing managers of wineries and representatives of entities of this industry in Emerging Economy. We proposed a framework approaching institutions-based view of strategy to the coopetiton perspective. Our results demonstrate that firms use coopetition to moderate competition, reducing rivalry, and achieving better performance. In addition, firms cooperate with rivals to compete and create barriers to new entrants. Finally, competitive relations develop over time and according to the influence of the institutions. (For more information, please contact: Jefferson Marlon Monticelli, Universidade do Vale do Rio dos Sinos, Brazil: jeffmarlon@hotmail.com)

\section{The Coevolution of Pro-Market Reforms and Emerging Market Multinationals Alvaro Cuervo-Cazurra, Northeastern University}

I study the relationship between pro-market reforms and the expansion of emerging market multinational companies (EMNCS). Extending institutional economics, I propose a coevolutionary process, whereby promarket reforms in emerging markets induce the transformation of domestic firms into EMNCs and the global expansion of EMNCs, in turn, facilitates the deepening of pro-market reforms in the home country. Specifically, I first explain how pro-market reforms lead to the emergence of EMNCS via international competitiveness, upgrading needs and escape, and then explain how the global expansion of EMNCs leads to a deepening of promarket reforms at home via learning, spillovers and lobbying. I complement these explanations with a discussion of contingencies at the firm (private vs. state, domestic vs. foreign firms), industry (global vs. local industries) and country (developing vs. transition countries) levels. (For more information, please contact: Alvaro Cuervo-Cazurra, Northeastern University, USA: a.cuervocazurra@neu.edu)

\section{From a Township Village Enterprise to a Multinational Corporation: Tracking the Journey of Strategic Changes of} a Non-State-Owned Chinese Firm

Lei Li, Nottingham University Business School China

Wendy Ye, Nottingham University Business School China

Yao Zheng, Nottingham University Business School China

There has been a lack of process-based studies on strategic changes in strategic management and international business in recent years, in particular in the context of emerging markets. This study seeks to understand how a non-state-owned Chinese firm with its core business in the textile and garment industry, has made strategic changes ex ante and ex post its international expansion throughout its lifetime. We have made efforts to track the firm's major strategic changes in terms of the key drivers, the contexts and/or processes and the corresponding consequences. The case evidence reveals the essential roles of social capital and managerial cognition in strategic changes and as a result, makes a tentative contribution to the extension of the existing theoretical framework. (For more information, please contact: Lei Li, Nottingham University Business School China, China: lei.li@nottingham.edu.cn) 
Capability Building of Developing Country MNEs: the Chinese Experience

Huaichuan Rui, Royal Holloway, University of London

In this paper, we take Chinese MNEs (CMNEs) as examples to demonstrate how CMNEs are able to upgrade capability through a dynamic process of transforming CSAs and foreign connection to FSAs. As the three cases show, at the beginning of this process CMNEs were nurtured at their home market in strong CSAs while developing and possessing weak FSAs in technology and management skills. Before internationalising, CMNEs benefited from inward internationalisation which provided opportunities for them to connect and learn from advanced country MNEs. When starting to internationalise, CMNEs, especially large and state owned, benefited from international trade and aided projects, other government supports and various CSAs, during which they were able to improve FSAs. Once operating in the global market, they obtain and upgrade FSAs by learning through practice and forming partnerships. In internationally open bidding projects, they have to learn how to meet the demand of host country, to pass the scrutiny of their supervisors - often advanced country MNEs, and to build a reputation so as to win more contracts. CMNEs were eventually able to compete in the international market with strengthened FSAs. These findings enable us to set up a simplified framework to demonstrate the evolutionary process of the capability building of CMNEs. (For more information, please contact: Huaichuan Rui, Royal Holloway, University of London, United Kingdom: huaichuan.rui@rhul.ac.uk)

\section{Session: 2.1.12 - I nteractive}

\section{Track: 8 - Global Strategy, M\&As and Competitiveness}

\section{Organizing for Success in MNC Settings}

Presented On: June 29, 2015 - 09:00-10:15

Chair: Rida Elias, American University of Beirut

Language and Cross-Border Acquisitions: An Exploratory Study

Rama Krishna Reddy, University of Memphis

Ben Kedia, Univerity of Memphis

The aim of this study is to examine the effects of language on post-acquisition performance of US based crossborder acquisitions. Building on knowledge based view of the firm; our study shows that post-acquisition performance is explained by linguistic distance between home and country of acquisition. In addition, this study explains the moderating role of acquisition experience in linguistic distance - acquisition performance relationship. An analysis of 795 US acquisitions in 33 countries over a period of 4 years demonstrates that linguistic distance has explanatory value, in addition to culture distance. Our analysis also demonstrates that cross-border acquisition experience plays a significant role as a moderator of this linguistic distance - acquisition performance relationship. (For more information, please contact: Rama Krishna Reddy, University of Memphis, USA: rreddy1@memphis.edu)

\section{National Identities and Acquisition Premia in Global M\&A's}

Eugene C. Pyun, Seoul National University

Sung Hun Chung, Seoul National University

Theresa S. Cho, Seoul National University

In this paper, we examined the linkage between the firm's sentiment toward the national identity of its merger partner and the acquisition premium paid. Using a sample of 413 cross-border M\&A's in several countries including the U.S., Canada, Germany, and Korea, we found that the target firms tend to accept lower 
acquisition premia from acquirers with more favorable national identities. Furthermore, acquirers tend to bid higher prices for target firms with unfavorable national identities. In sum, we found a tangible evidence on the effects of subjective favorability of national identities on cross-border acquisition premia. (For more information, please contact: Eugene C. Pyun, Seoul National University, Canada: egpyun@snu.ac.kr)

\title{
Geographical Portfolio Analysis: Opening the Black Box of Risk inside Multinational Corporations Jane Qiu, University of New South Wales
}

Multinational corporations (MNCs) are increasingly concerned with income stream risk, the upturns and downturns in the corporation's financial performance over-time. In the international business literature, however, the relationship between multinationality and income stream risk remains surprisingly undeveloped, both theoretically and empirically. This paper aims to develop a new analytic approach, Geographical Portfolio Analysis (GPA), which advances our understanding on this relationship by opening the black box of risk inside. Cases of IBM, Ford and Disney are presented to illustrate how GPA offers new understandings that often contrast some of our conventional thoughts regarding the multinationality-risk relationship. (For more information, please contact: Jane Qiu, University of New South Wales, Australia: janeq@unsw.edu.au)

\author{
Subsidiary Proactive Initiative Behavior: How the Organizational Context Shapes Subsidiary Managers' Reactions \\ to Headquarters Activities \\ Benoit Decreton, WU Vienna \\ Phillip Nell, WU Vienna \\ Diego Stea, Copenhagen Business School
}

How can the headquarters foster entrepreneurship in their subsidiaries? In this paper, we shed light on this question by using contextual variables to link headquarters involvement in the subsidiary's local context with the proactive initiative behavior of the subsidiary managers. Using a sample of 104 manufacturing subsidiaries in Europe, we investigate how contextual perceptions of subsidiary managers impact their reactions to headquarters involvement. Especially, we find support for our propositions that headquarters involvement is positively (negatively) related to proactive initiative behavior of subsidiary managers if they have high (low) levels of perceived autonomy-support or normative integration. (For more information, please contact: Benoit Decreton, WU Vienna, Austria: benoit.decreton@gmail.com)

\author{
Attention Dissonance in Emerging Market Subsidiaries and Its Effects on Communication in Multinational \\ Corporations \\ Rian Drogendijk, University of Groningen \\ Hammad ul Haq, Uppsala University \\ Desiree Blankenburg Holm, Uppsala University
}

We introduce the notion of attention dissonance, where subsidiaries feel that headquarters' attention is merely expressed at the cognitive level as their actions are not in line with their alleged aspirations. Using comparative case study design, we investigate one European organization with subsidiaries located in emerging as well as advanced markets. In line with the company strategy of shifting focus from advanced to emerging markets, subsidiaries in emerging markets do get recognition in annual reports and internal communication. However, the same subsidiaries feel that this is not reflected in how headquarters act towards them and that knowledge about their activities is lacking. This attention dissonance hinders direct and open communication, which is necessary to realize the economic potential in these markets. (For more information, please contact: Rian Drogendijk, University of Groningen, Netherlands: rian.drogendijk@rug.nl) 
Why do Firms Engage in CSR Internationally?

Kiran Awate, Ohio State University

Anil Makhija, Ohio State University

Mona Makhija, Ohio State University

Despite considerable concern in the management literature over the performance effects of undertaking CSR, we have to date gained little insight on this issue due to conflicting findings. We attempt to address this issue. In this research, we examine which of two opposing theoretical arguments best explains the relationship between CSR and performance. Using a natural experiment context relating to the 2004 Asian Tsunami disaster, we develop two separate models in this regard. Drawing on a strategic approach for the first model, we hypothesize that firms' international exposure, competition and reputation in their industry are positively related to CSR. An agency theory approach is reflected in in the second model which hypotheses that institutional shareholding, tenure of CEO, board independence and managerial ownership are negatively related to CSR choices. Our findings suggest that the agency explanation for CSR outweigh the strategic explanation. Further we examine the effect of CSR activities on the firm performance. Using a matched sample of 471 US companies, we find that firms that did not engage in CSR activities outperformed the firms that engaged in CSR activities on the stock market. These findings have important implications for the debate in management regarding CSR as a source of competitive advantage. (For more information, please contact: Kiran Awate, Ohio State University, USA: awate.3@buckeyemail.osu.edu)

\section{Session: 2.2.1 - Special Session}

\section{Al B Fellows Eminent Scholar Award Session}

Presented On: June 29, 2015 - 10:45-12:00

Chair: Paul W. Beamish, Western University

IT \& IB: Institutional Theory and International Business (Keynote by 2015 AlB Fellows Eminent Scholar Award Recipient)

W. Richard Scott, Stanford University

Commentators:

Yves Doz, INSEAD

Arie Lewin, Duke University

\section{Session: 2.2.2 - Panel}

\section{Track: Scholars Meet Practice}

\section{Managing Digital Transformation in a Global Context}

Presented On: J une 29, 2015 - 10:45-12:00

Chair: Siva Viswanathan, University of Maryland

Discussant: Sandeep Karamongikar, Infosys Technologies

Panelists:

Siva Viswanathan, University of Maryland

Sanjeev Vohra, Accenture Digital

Aditya Menon, Citibank

K. Suryaprakash, Infosys 
Digital technologies are transforming our world - not just the way we communicate, collaborate, and shop, but also the way we experience life. Digital innovations are enabling enterprises to connect deeper with their environment and engage more meaningfully with their consumers, partners and employees. Enterprises can optimize, simplify their processes, operations, increase customer satisfaction and efficiency, and the new world of connected devices is opening completely new possibilities of collaboration and engagement. This panel will discuss the implications of these developments for industries, firms, businesses, consumers, and society in a global context. (For more information, please contact: Siva Viswanathan, University of Maryland, USA:

sviswana@rhsmith.umd.edu)

\section{Session: 2.2.3 - Panel}

Track: 1 - People and Careers in Cross-Cultural Business

\section{The Micro-Foundations of Distance in I B Research: Bringing Social Psychology into the Picture}

Presented On: J une 29, 2015 - 10:45-12:00

Chair: Douglas Dow, University of Melbourne

Panelists:

Douglas Dow, University of Melbourne

Daniel Baack, University of Denver

Björn Ambos, University of St. Gallen

Ilya Cuypers, Singapore Management University

Andre Anugerah Pekerti, University of Queensland

As emphasized by numerous commentators over the past 15 years, the current practices in terms of how the more abstract forms of distance (e.g. psychic distance) are operationalized in international business research is fraught with many problems. In the most famous of these critiques, Shenkar (2001) highlighted eight distinct assumptions or illusions that need to be tested and confirmed. Some progress has been made on this front, but we believe that a shift in emphasis to the 'micro-foundations of distance' is required in order to move the debate forward. Specifically we believe future research needs to explore the factors that shape and bias individual decision-makers' perceptions of distance, rather than focusing on exogenously derived national-level indices. In order to do this, we suggest that researchers should draw more heavily on the social psychology literature in order to develop new theoretical insights. This panel brings together four research agendas that are drawing on social psychology in order to investigate the factors that maybe shaping managers perceptions of distance, plus one research agenda that provides a potential platform for such research. This panel is intended to be a platform to initiate discussion on how we can progress distance research. (For more information, please contact: Douglas Dow, University of Melbourne, Australia: d.dow@mbs.edu) 


\section{Session: 2.2.4 - Panel \\ Special Session}

\section{Middle East North Africa: Facts, Misconceptions and Opportunities for International Business Research}

Presented On: June 29, 2015 - 10:45-12:00

Chair: Melodena Stephens Balakrishnan, University of Wollongong in Dubai

Discussants: Nakiye Avdan Boyacigiller, Sabanci University and Victor Zengyu Huang, Zayed University

Panelists:

Nakiye Avdan Boyacigiller, Sabanci University

Melodena Stephens Balakrishnan, University of Wollongong in Dubai

Victor Zengyu Huang, Zayed University

Adrienne A. I sakovic, Walden University

Christian Tabi Amponsah, Skyline University

Doren Chadee, Deakin University

Ahmed A. Alshumaimeri, King Saud University

Alice de Jonge, Monash University

Ian Michael, Zayed University

The purpose of this PDW is to help scholars interested in the Middle East North Africa Region (MENA) region collaborate on common themes of interests, clear misconceptions or increase awareness of challenges with current theory when applied to the region. MENA is hardly represented in international studies. The future focus is to not only increase output on this region but also refocus studies into those that understand contextual nuances of the region and reinterpret the findings from an emerging market lens. (For more information, please contact: Melodena Stephens Balakrishnan, University of Wollongong in Dubai, United Arab Emirates:

melodenabalakrishnan@uowdubai.ac.ae)

\section{Session: 2.2.5 - Panel}

Track: 9 - Emerging Economies and Emerging Economy MNEs

\section{Al B-CEE Chapter Panel: Challenges for International Business in Central and Eastern Europe}

Presented On: J une 29, 2015 - 10:45-12:00

Chair: Lukasz Puslecki, Poznan University of Economics

Discussant: Piotr Trapczynski, Poznan University of Economics

Panelists:

Lukasz Puslecki, Poznan University of Economics

Marin Alexandrov Marinov, Aalborg University

Piotr Trapczynski, Poznan University of Economics

Svetla Trifonova Marinova, Aalborg University

Andrei Panibratov, St. Petersburg University

Michael Zdziarski, University of Warsaw

Taking into consideration this year's theme of Global Networks: Organizations and People, this panel aims at exploring a better integration of theoretical discussion and empirical evidence on International Business challenges in the Central and Eastern Europe. Three sets of question will be explored: 1) What are the sources of competitive advantage of Central \& Eastern European (CEE) firms? How will this change in the future? In 
which direction are firms developing in this respect? 2) What are the main barriers and challenges for CEE firms operating in foreign markets? 3) What can and should the national governments do to improve the business environment for CEE firms? (For more information, please contact: Lukasz Puslecki, Poznan University of Economics, Poland: lukasz.puslecki@ue.poznan.pl)

\section{Session: 2.2.6 - Competitive Track: 10 - Theories of the MNE and of FDI}

\section{Looking Beyond the Traditional: SMEs and Service Firms in IB}

Presented On: June 29, 2015 - 10:45-12:00

Chair: Kristin Brandl, University of Reading

Family Control, Niche Strategies, and European SME Internationalization

J ean-Francois Hennart, Tilburg University; University of Pavia; Singapore Management University Antonio Majocchi, University of Pavia

Emanuele Forlani, University of Pavia

Most firms around the world are owned and run by families. Yet, surprisingly, there is only limited research on whether this form of governance encourages or discourages internationalization, and empirical evidence to date is inconclusive. While most of the literature has argued that family control discourages internationalization, we suggest that this should only apply to firms that sell mass-market products requiring local production and adaptation, and hence large scale as well as specially trained managers. We argue, on the contrary, that family firms will have greater foreign sales than nonfamily firms when they follow global niche strategies. These strategies focus on high product and service quality and long term relationships with clients, and both are wellsuited to family firm governance. Modelling a firm's foreign sales through a gravity model, we test our hypotheses on a large sample of SMEs from five European Union countries. Our results show that while family control per se reduces international sales for UK, German, Spanish and Italian family-controlled SMEs, it increases them when family-controlled SMEs based in these countries pursue global niche strategies. (For more information, please contact: Jean-Francois Hennart, Tilburg University; University of Pavia; Singapore Management University, Netherlands: j.f.hennart@uvt.nl)

\section{Intra- and Inter-Regional Expansion of Services MNEs: Interdependencies and Contingencies in Internationalization Trajectories \\ Oksana Grebinevych, EMLYON}

In this paper, we propose, substantiate and empirically test a thesis that intra- and inter-regional international expansions are interdependdent. First, by combining insights of semiglobalists on regional aggregation and regionalists on home region orientation, we confirm validity of semiglobal dimension of MNE's internationalization behavior both in home and host regions (controlling for the prior country-level internationalization and firm-specific variables). We, then, characterize the factors that influence intra- and inter-regional internationalization and suggest that firm's propensity to internationalize inter-regionally is a function of quality characteristics of home-based intra-regional expansion of a firm. Using a single-industry data on world population of internationalized grocery retailers, we find support for our models and associated hypotheses. Specifically, we find that depth of home-based intra-regional expansion is negatively associated with firm's propensity to expand inter-regionally. We also find that breadth of home-based intra-regional expansion is positively associated with inter-regional expansion. In addition, our results suggest that MNEs' intra- and inter-regional expansions are not contingent on types of firm's control (family versus non-family firms). (For more information, please contact: Oksana Grebinevych, EMLYON, France: grebinevych@gmail.com) 
Foreign Expansion of Professional Services Firms: A Behavioral Perspective

Eunjung Hyun, Hitotsubashi University

Brian Kim, Yonsei University

Kai-Yu Hsieh, Waseda University

Extending performance feedback theory, this article examines how performance discrepancy from its historical and social aspiration level influences the firm's decision to expand into foreign markets in the context of professional services firms (PSFs). We hypothesize that firms with lower levels of negative performance gap, relative to those with a larger gap, will be more inclined to expand internationally. We argue that this negative association between performance below aspiration and overseas expansion arises because firms nearing their survival point will encounter severe organizational inertia in pursuing market entry into distant regions as a solution to their performance problem. Contrarily, as firms move toward their aspiration level, they will be more inclined to regard foreign expansion as a viable strategic solution and face lesser inertia pushing such initiative. We also consider how recent failures in domestic markets, recent foreign market entry experience, and organizational status affect low-performing firms' likelihood of expanding into foreign markets. We find robust support for our hypotheses using data on U.S. law firms' new market entries, both domestic and overseas, 1985-2012. This study contributes to the international business literature by bringing core insights of the behavioral perspective to the understanding of foreign expansion of PSFs. (For more information, please contact: Eunjung Hyun, Hitotsubashi University, Japan: ej.hyun@r.hit-u.ac.jp)

\section{Session: 2.2.7 - Competitive}

\section{Track: 6 - Innovation and Knowledge Management}

\section{Knowledge Sourcing and Transfer}

Presented On: June 29, 2015 - 10:45-12:00

Chair: Torben Pedersen, Bocconi University

Feasibility, Recipient Ownership and Knowledge Transfer Strategy: A Case of Chinese MNCs in Africa Huaichuan Rui, Royal Holloway, University of London Miao Zhang, Kingston University

The knowledge management of emerging market multinational corporations (EMNCS) has gained little scrutiny in the field of international business research. This paper examines EMNCs' knowledge transfer by using the example of Chinese MNCs (CMNCs) in Africa. We discovered that CMNCs were transferring feasible knowledge to Africa. Such knowledge was practical but not necessarily superior, indicating the importance of recipient initiatives to satisfy local demand. It was affordable but not necessarily sustainable, causing local scrutiny and requiring supervision. It was fresh but might have not been theorized, indicating the necessity of human interaction between the transferor and transferee. "A feasible knowledge transfer through recipient ownership model" is proposed for EMNCs. In this paper, we advance research on MNC's knowledge transfer by considering a situation where the knowledge MNCs possess is less superior but more feasible for the recipients. (For more information, please contact: Huaichuan Rui, Royal Holloway, University of London, United Kingdom: huaichuan.rui@rhul.ac.uk)

\section{Overcoming the Liability of Localness In Innovation}

C. Annique Un, Northeastern University

I analyze differences in the innovativeness of domestic firms and subsidiaries of foreign firms operating in the same country. I argue that domestic firms suffer a liability of localness in innovation, or a competitive disadvantage in product innovation relative to subsidiaries of foreign firms. I propose that this liability of localness is driven by the relatively lower levels of multiculturalism in employees of domestic firms in 
comparison to subsidiaries, which limit the identification, transfer, and integration of a large diversity of knowledge that supports product innovation. However, I also propose that managers of the domestic firms can compensate for this liability of localness in two ways, directly by investing in the training of their employees and indirectly by exporting. Training modifies the mindsets and abilities of employees and enables them to become more cognitively multicultural, which facilitates product innovation. Exporting helps domestic firms learn new ideas available in other countries, which also facilitates product innovation. I test these arguments on a sample of manufacturing firms in Spain and find that, although domestic firms introduce fewer new products than subsidiaries of foreign firms, they introduce more new products than subsidiaries at the same level of investments in language training and of exports. (For more information, please contact: C. Annique Un, Northeastern University, USA: a.un@neu.edu)

\section{The Effects of Privatization on the Characteristics of Innovation Marcelo F Cano-Kollmann, Temple University \\ Yirlier Hyacinthe Some, Universite de Sherbrooke \\ J ean-Claude Cosset, HEC Montréal}

Privatization of state-owned enterprises is equivalent to a paradigm shift that not only implies a change of ownership, but also tends to bring along the adoption of new management practices and changes in the companies' objectives. While the literature has abundantly explored the consequences of relinquishment of government control over different aspects of firm performance, the effects of privatization on innovation have been less studied. Some scholars have found that privatization tends to produce a subsequent reduction in the amount of R\&D investment, but little else is known about specific changes in the patterns of innovation of privatized firms. This is the motivation for this paper. We hypothesize that privatized firms are likely to focus on less technologies as a response to increased pressure for profitability and short-term results. We also argue that, while state-owned firms are likely to source knowledge nationally and conduct more innovation in-house, privatized firms will be more likely to collaborate with other firms and to disperse their innovation activities internationally. Empirical results obtained from a sample of privatized firms from multiple countries are consistent with our arguments. (For more information, please contact: Marcelo F Cano-Kollmann, Temple University, USA: mck@temple.edu)

\section{Session: 2.2.8 - Competitive}

Track: 3 - International Human Resource Management and Organizational Behavior

\section{International Staffing and Retention Challenges}

Presented On: June 29, 2015 - 10:45-12:00

Chair: Anju Mehta, University of Northern I owa

Above and Beyond: Retention of Self-Initiated Expatriates

Dilek Gulistan Yunlu, Northeastern Illinois University

Hong Ren , University of Wisconsin - Milwaukee

Margaret Shaffer, University of Wisconsin - Milwaukee

Katy Fodchuk, Advanced Partnerships

Taking a 'positive psychology' perspective and drawing on Conservation of Resources theory, we apply the concept of "thriving" to explain self-initiated expatriate (SIE) retention. Using longitudinal data from 103 international teachers in the United States, we found that cultural intelligence directly influenced thriving, which in turn led to expatriate actual retention. In addition, cultural intelligence's positive effect on thriving was more pronounced when organizational embeddedness was high. Applying the concept of thriving to explicate SIE experiences, we make several contributions to the global employment literature. (For more information, please contact: Dilek Gulistan Yun/u, Northeastern Illinois University, USA: d-yunlu@neiu.edu) 


\section{International Employee Identity Structure and Context, and Impact on Turnover Intent}

Zhi Wang, Manchester Metropolitan University

Xiaogian Yie, Wuhan University

Stuart Horsburgh, Manchester Metropolitan University

Prior studies suggest that employee identity constructs in social- cultural and organizational environments are structural. The current paper argues for the importance of context in explaining how changes in employment states from expatriation to repatriation have a transactional impact on employee identity, which in turn affects employee turnover intent $(\mathrm{TI})$. Drawing on sample data from 147 recently repatriated employees currently working in multinational enterprises, the results reveal that expatriate role identity influences turnover intent in the repatriation state. Our findings show that changes in identity significantly affect perceived overqualification (POQ), a major factor in determining TI. Our findings also show that contextual mechanisms of identity enact identity reentry from expatriate state to repatriate, moderating the levels of POQ. I mportantly, the paper demonstrates that organizational support (OS) plays a crucial role in mediating the effects of changes in identity on TI. The implication for IHRM is that OS should be seen as an integral part of the organization's competitive strategy, a qualitative shift in emphasis compared with prior studies, where the tendency has been to examine organizational support in the context of an operational support function for international employees. (For more information, please contact: Zhi Wang, Manchester Metropolitan University, United Kingdom: zhi.wang@mmu.ac.uk)

The Impact of MNE Headquarter's Control and Local HR's Knowledge Search on Subsidiary Employee Turnover: Evidence from MNEs Operating in China

Katsuhiko Yoshikawa, London School of Economics and Political Science

Hyun-J ung Lee, London School of Economics and Political Science

This study investigates the relationship between MNE subsidiary employee turnover, local HR's external knowledge search, and the headquarters (HQ)' control over HR activities. We argue that local HR's knowledge search activities play an important role in understanding MNE subsidiary turnover rate. Our sample includes 198 multinational enterprises of US, European and J apanese origin operating in China, the context known to bear high employee turnover. First, we found local HR's knowledge search activities are negatively associated with employee turnover in subsidiaries. Second, consistent with existing literature, we found that HQ's direct control, manifested by the use of expatriates, is positively associated with employee turnover. We also found that local HR's knowledge search activities partially mediated this relationship. On the other hand, we found that MNE's bureaucratic control through HR standardisation was negatively associated with employee turnover via a full mediation by local HR's knowledge search activities. Our study highlights the role of local HR's knowledge search activities in the understanding of MNE subsidiary's employee turnover, which is largely overlooked in the literature. Theoretical and practical implications are discussed. (For more information, please contact: Katsuhiko Yoshikawa, London School of Economics and Political Science, United Kingdom: K.yoshikawa@lse.ac.uk)

\section{Managing Multiple Embeddedness and Global Staffing Policy: A Multilevel Investigation of Korean MNES J ongmin Lee, University of Reading}

This study investigates the MNE's dynamic global staffing policy upon the variation of individual subsidiary maturity and the degree of MNE multinationality. Drawing on the concept of multiple embeddedness and the behavioral theories of the firm, this study aims to provide a comprehensive illustration of the MNE' global staffing decision incorporating managerial concerns of both headquarters and subsidiaries. The results from a multi-level investigation of Korean MNEs showed that the MNE's expatriate staffing policy has a U-curve relationship with the maturity of individual subsidiaries, while it has an inverted U-curve relationship with the degree of geographical dispersion of the MNE activities. The findings of this research demonstrates that MNEs with a high degree of multinationality utilize a strategic selective expatriation policy by decreasing the expatriate staffing level in their subsidiaries on average, while selectively deploying a high number of expatriates to the highly matured subsidiaries. (For more information, please contact: Jongmin Lee, University of Reading, United Kingdom: j.m.lee@pgr.reading.ac.uk) 


\section{Session: 2.2.9 - Competitive \\ Track: 5 - The Geography of I nternational Business and Global Value Chains}

\section{Governance of Global Value Chains}

Presented On: J une 29, 2015 - 10:45-12:00

Chair: Carine Peeters, Vlerick Business School

Identifying Industrial Complexes in Mexico: Implications for Regional Industrial Policy

Amado Villarreal Gonzalez, Tecnolo' gico de Monterrey

Elizabeth Mack, Michigan State University

Miguel Flores, Tecnolo' gico de Monterrey

Given the significant changes in the internal structure of the Mexican economy, and its rise to global economic prominence, the goal of this study is to identify industrial complexes within the country using input-output tables and municipal-level employment data. Results of the analysis highlight five large value chains in the country including electronics components, automotive, chemical, textile and apparel, and agricultural products and processed foods. These value chains are present in distinct portions of Mexico, which suggests directions for regionally based industrial policies based on related variety and smart specialization to move the Mexican economy from a manufacturing to a knowledge and innovation orientation. (For more information, please contact: Elizabeth Mack, Michigan State University, USA: lizmack287@gmail.com)

\section{Ecologically Embedding Global Production Networks: The Cases of Salmon Aquaculture and Palm Oil Christopher R. Whynacht, University of Massachusetts Boston Stephan Manning, University of Massachusetts Boston}

Research into global value chains and global production networks (GPNs) has focused on the interactions and relationships among actors in the production process, but often overlook the origin of the raw materials which form the core inputs of production. Recently there has been efforts to evolve conceptualizations of GPNs, styled as GPN 2.0, particularly to manage risks such as those caused by environmental degradation and climate change. We suggest that many GPNs are ecologically embedded to help provide nuance around the issue of environmental management, risk assessment, and more environmentally responsible governance of global production. Conceptually, this links GPNs with research on sustainable supply chain management, life cycle assessment, and ecosystem services. Our principal contention is that many production networks use biological inputs which depend on enabling environmental conditions and the provision of ecosystem services, both of which serve to anchor the upstream end of the production chain. Two specific cases of production are examined, palm oil and salmon aquaculture, to provide examples of how networks are ecologically embedded. Primary and secondary dimensions are amalgamated into a framework which is then used to discuss implications for the governance of GPNs. Finally, propositions and implications for future research are discussed. (For more information, please contact: Christopher R. Whynacht, University of Massachusetts Boston, USA: christopher.whyna001@umb.edu) 
Organizational Aspirations and Violations of a Social Standard's Minimum Requirements in the Emerging Economies' Clothing Industry

Marc Mueller, University of St. Gallen

J oerg Stefan Hofstetter, University of St. Gallen

Anthony Goerzen, Queen's University

Building on the literature of aspiration-driven behavior, which suggests that performance relative to aspirations (i.e., attainment discrepancy) influences performance corrections, we investigate adaptations of emerging economy supplier social standard compliance attributed to attainment discrepancy between their social performance and organizational aspirations. For this purpose, we rely on social audit reports of the Business Social Compliance Initiative focusing on 66 suppliers from Bangladesh, China, India and Turkey in the clothing industry, which have completed at least four full audits between 2003 and 2012 . Our findings demonstrate that social performance below the aspiration level leads emerging market suppliers to improve social standard compliance whereas social performance above the aspiration level guides emerging economy suppliers to deteriorate social standard compliance. These results suggest that emerging market suppliers are unlikely to live up to a social standard's minimum requirements on a continuous basis. We conducted a further analysis to explore how changes in individual social issues shape adaptations of emerging market supplier social standard compliance for positive and negative attainment discrepancies finding that some social issues are more critical than others. (For more information, please contact: Marc Mueller, University of St. Gallen, Switzerland: marc.mueller@unisg.ch)

\section{Session: 2.2.10 - Interactive}

\section{Track: 11 - Corporate Governance in I B}

\section{Shareholder Protection Around the World: New Challenges, New Solutions?}

Presented On: June 29, 2015 - 10:45-12:00

Chair: Zahid Riaz, Lahore School of Economics

Organizational Distress in an Emerging Market: The Role of Corporate Governance \& Shareholder Expropriation in Chinese Companies

Daniel Han Ming Chng, China Europe International Business School

Qing Ye, China Europe International Business School

We examine the effects of controlling shareholder expropriation and corporate governance on organizational distress in listed companies in China from 2006 to 2012. Our results show that greater controlling shareholder expropriation increases the likelihood of distress. In contrast, governance mechanisms in the form of greater controlling shareholder ownership, executive compensation, and executive turnover decrease the likelihood of distress while managerial monitoring and state-control have little impact. In addition, controlling shareholder ownership and executive compensation mitigate the effect of shareholder expropriation on distress. Our study illustrates the complex nature of corporate governance and organizational distress in a rapidly changing emerging market. (For more information, please contact: Daniel Han Ming Chng, China Europe International Business School, China: dchng@ceibs.edu)

Towards a Global View of Agency Theory: Bringing Principal-Agent and Principal-Principal Perspectives Together Kshitij Awasthi, Indian Institute of Management Bangalore

Agency theory ever since its dawn has been one of the most exciting and highly researched domains. It showed explanatory power in developed economies, however, when applied to alternate institutional context like emerging economies, it needed certain modification because of inherent institutional differences and its impact on corporate governance practices. As opposed to developed economies, most of emerging economies are 
marked by concentrated ownership and weaker legal institutions and external control. This difference led to a separate version of agency theory and emergence of what is called Principal-Principal agency. Together, both these agency models lead to Institution based view of the corporate governance (Pen et al., 2008) and agency theory. However, rather than supplementing each other to extend the agency theory and increase its explanatory power, both traditional and Principal-Principal agency has taken separate directions This has steered the piece-meal development of different flavours of agency theory that attempts to explain behaviour in specific institutional contexts. The present paper, noting the currently prevalent paradigms of institutional differences in agency theory, attempts to bridge these theories in order to enhance generalizability and offer platform for future research. (For more information, please contact: Kshitij Awasthi, Indian Institute of Management Bangalore, India: kshitijawasthi@yahoo.co.in)

\title{
Family-Owned Firms between Agency Conflicts and Stewardship: Corporate Governance Factors Driving Firm Performance \\ Sajal Kabiraj, Dongbei University of Finance \& Economics
}

The performance of family-owned firms has been driven by factors relating to family ownership, family leadership, and external supervision. In this paper, we offer an empirical study investigating the effects of those corporate governance concerns. Surveying 3121 family-owned firms in China, we find that family leadership and external supervision significantly influence the firms' Return on Assets (ROA) and Return on Equity (ROE).

Family ownership significantly influences ROA, but not ROE. Discussing our findings in light of stewardship and agency theories, we especially supplement stewardship theory due to the close alignment between owners and managers in family-owned firms. (For more information, please contact: Sajal Kabiraj, Dongbei University of Finance \& Economics, China: skabiraj@dufe.edu.cn)

\author{
Agency Theory and Corporate Governance in China: A Meta-Analysis \\ Canan Mutlu, University of Texas at Dallas \\ Marc Van Essen, University of South Carolina \\ Mike Peng, University of Texas at Dallas \\ Sabrina Saleh, University of South Carolina
}

Do agency theory-based "good corporate governance" principles apply to China? A straightforward answer to this question is lacking, because evidence is inconclusive across studies. Amassing a pooled database consisting of 81 studies, 272 effect sizes, 200,739 firm and 636,713 firm-year observations between 1991 and 2008, we endeavor to fill this gap by conducting the first meta-analysis on the China literature with two focuses. First, we assess the impact of (i) board independence, (ii) board leadership structure, and (iii) managerial incentives on firm performance, as these elements have been central to both agency theory as well as to Chinese corporate governance reforms. Second, we extend current theorizing by showing support for the temporal hypothesis, which states that over time, with the improvement in the quality of market institutions and development of financial markets, the monitoring mechanisms of the board become more important whereas the incentive mechanisms lose their significance. (For more information, please contact: Canan Mutlu, University of Texas at Dallas, USA: cxm101020@utdallas.edu)

\section{Influence of Board Independence and Type of Concentrated Ownership on CEO Compensation in India Bala N Balasubramanian, Indian Institute of Management Ahmedabad Samir K Barua, Indian Institute of Management Ahmedabad Karthik D, Indian Institute of Management Ahmedabad}

The crisis in the financial and the banking sectors in 2008-2009 brought back into focus the issue of CEO and top management compensation. The unconscionably high compensations, unjustified even remotely by performance, raised concerns about governance of companies. The study, the first of its kind, investigates the efficacy of various measures of board independence for different ownership structures and different types of 
concentrated owners - private domestic, private foreign and government, in controlling CEO compensation in the same economic setting. The sample for the study is the set of companies that were a part of the NSE CNX 100 Index set for the period 2007-2012. Separation of board chair and CEO positions is the single most important governance measure for controlling excessive compensation to CEOs. Gender diversity deflates CEO compensation in case of foreign private companies. Non-executive independent directors are able to control CEO compensation only for private foreign companies when their numbers are large. Compensation committees appear to have no influence on CEO compensation. Institutional block-shareholders positively influence compensation to CEOs of private domestic companies. The results provide actionable feedback on legislation and policies prevailing in the country. (For more information, please contact: Karthik D, Indian Institute of Management Ahmedabad, India: dkarthik@iimahd.ernet.in)

Debt Maturity and Ownership Structure: The Distinctive Effects Of Shareholder Protection and Creditor Rights Paulo Renato Soares Terra, EAESP-FGV

Henrique Castro Martins, UFRGS

Eduardo Schiehll, HEC Montréal

This study examines whether national governance factors distinguishing between shareholder protection and creditor rights interfere on the association between firm's ownership concentration and debt maturity. Using a cross-sectional data set (2013) from 41 countries we found support for our hypotheses. Our results suggest that concentrated shareholder, on average, prefer short-term debt. We found support to the hypothesis of substitution between Board and Creditors monitoring. Finally, we found support to the hypothesis that great disparity between control and cash flow rights leads to creditors expropriation (especially by family members). (For more information, please contact: Paulo Renato Soares Terra, EAESP-FGV, Brazil: paulo.terra@fgv.br)

\section{Session: 2.2.11 - I nteractive \\ Track: 9 - Emerging Economies and Emerging Economy MNEs}

\section{Internationalization Process}

Presented On: June 29, 2015 - 10:45-12:00

Chair: Soma Arora, Institute of Management Technology

The Relationship between International Expansion and Performance in Multilatinas Andres Velez-Calle, Universidad EAFIT/Rutgers University

Fernando Sanchez-Henriquez, Rutgers University

This paper analyzes the internationalization and firm performance relationship (IDP) in Latin American MNEs commonly referred to as Multilatinas. This relationship has been widely studied in various contexts and industries however it has not been tested in Latin America. We found a U-shaped curve that indicates that Multilatinas are entering the second stage of internationalization in which they have overcome the liability of foreignness and therefore their net benefits of expanding internationally are positive. This means that Multilatinas have not reached extensive internationalization and that they will keep expanding at an accelerated pace. Additionally, our results show that Multilatinas benefit from internationalization depth and not from internationalization breadth. (For more information, please contact: Andres Velez-Calle, Universidad EAFIT/Rutgers University, Colombia: avelezca@eafit.edu.co) 
This paper presents an integrated view of the internationalization of emerging-market multinationals by examining the generic internationalizations strategies for a large sample of Chinese manufacturing multinationals. The focus of the investigation is what are the choices and combinations of strategic directions that lead to firm's pursuit of internationalization. We continue our discussion of how the choice of internationalization strategies is shaped by a few contextual factors at the firm level. We finally propose a revised model of generic internationalization strategies as a common scheme for studying the emerging market multinationals. (For more information, please contact: Kun Tan, Minjiang University, China: tankun@nbs.edu.cn)

\section{Emerging Market Multinationals' R\&D Intensity and "Northern" Acquisitions \\ Rajeev Sawant, Baruch College, CUNY \\ Barclay Edward J ames, Universidad San Francisco de Quito School of Business}

Is capability development through acquisitions in strategic factor markets a complement or a substitute to capability development from internal R\&D? We theorize and find a curvilinear relationship between internal R\&D and acquisitions in developed countries in the context of emerging market multinational enterprise's (EMNES). As the level of R\&D intensity increases, EMNE absorptive capacity increases, leading to greater capability enhancing developed country acquisitions, suggesting complementarity. Yet as R\&D intensity increases beyond a threshold, increasing EMNE R\&D leads to lower developed country acquisitions suggesting substitution. Consistent with our theoretical framework, firms with greater proportion of Northern acquisitions and firms with greater R\&D intensity over the threshold both show superior performance since these are alternate means of capability development and act as substitutes. However, we find no evidence that internal R\&D increases the positive effect of acquisitions on performance suggesting that the complementarity of internal R\&D and acquisitions is not reflected in superior performance. (For more information, please contact: Rajeev Sawant, Baruch College, CUNY, USA: rajeev.sawant@baruch.cuny.edu)

\section{Chinese and Indian Acquisitions in Germany: Does Experiential Learning Matter? Simona Gentile-Luedecke, Bremen University Sarianna Lundan, University of Bremen}

Prior studies have established that EMNEs, and especially those from China and India, seem to skip earlier entry modes of internationalization and focus on acquisitions as a primary mode. The issue of experiential learning is thus relevant when talking about EMNEs because, despite having a large scale of domestic operations and local sales, those firms are internationally still relatively inexperienced. What degree of experiential learning do EMNEs have when they acquire developed country firms? To what extent is experiential learning critical for EMNEs in order to successfully acquire firms in developed countries? To answer these questions, this study investigates the particular case of Chinese and Indian acquisitions in the German automotive sector. We find that while Indian firms expand in a sequential manner and utilize internally acquired knowledge gained through previous experience, in the case of Chinese firms the process is more abrupt, and the acquisition is mostly driven by external sources of knowledge. While from the acquirer's point of view the acquisitions aim to fulfill knowledge seeking motivations, for the target firms, the benefits are more akin to market seeking investment. (For more information, please contact: Simona Gentile-Luedecke, Bremen University, Germany: simona.gentile@uni-bremen.de) 
Ram Ranganathan, University of Texas - Austin

Anindya Ghosh, IESE

Harbir Singh, University of Pennsylvania

In this working paper, we examine how the composition of emerging market firms' ownership affects their propensity to undertake external corporate transactions, and, whether it complements or substitutes the effect of a firm's prior experience in undertaking such transactions. In particular, following Khanna and Palepu (2000), we focus on the role of foreign institutional investors - by studying the relationship between such ownership levels and actual strategic decisions, we can isolate and gain a more robust understanding of the effects of external governance on firm actions and performance. Preliminary findings suggest that while foreign institutional ownership and own accumulated experience spur a higher rate of external corporate transactions, the presence of domestic institutional investors dampens it. We also find that relationship between foreign investor ownership and higher corporate transactions is stronger at lower levels of own firm experience with these transactions. (For more information, please contact: Ram Ranganathan, University of Texas - Austin, USA: ram.ranganathan@mccombs.utexas.edu)

\section{Which Countries are Emerging Economies? Evidence from the Heterogeneity of MNEs' Cross-Border Strategies Megan (Min) Zhang, Ivey Business School}

Emerging economies constitute the major growth opportunities for MNEs. However, there is still no conclusion about which countries are emerging economies. By utilizing Citigroup's Global Growth Generator (3G) index and incorporating multiple theoretical lenses, this study investigates the heterogeneity of J apanese MNEs' ownership patterns in developed, 3G and other developing countries, and examines whether this heterogeneity reflects the effectiveness of $3 G$ index. The empirical results suggest that J apanese MNEs increase their ownership levels in 3G countries over time to accommodate to institutional changes; however, they also tend to relax their ownership levels with the increase of subsidiary operating experience. In contrast, Japanese MNEs only increase their ownership levels with the accumulation of subsidiary experience in developed countries. This study shows that $3 \mathrm{G}$ index reflects the heterogeneity of J apanese MNEs' ownership strategies, indicating that it could be an effective tool for MNEs to identify growth-generating countries. (For more information, please contact: Megan (Min) Zhang, Ivey Business School, Canada: mzhang.phd@ivey.ca)

\section{The Influence of Liabilities of Origin on EMNE Cross-border Acquisition Completion Shobhana Madhavan, Amrita School of Business Deepak Gupta, Amrita School of Business}

Extant literature on emerging market multinational enterprises (EMNEs) suggests that they undertake crossborder acquisitions in order to overcome their origin-specific liabilities. We argue that these liabilities of origin themselves influence the success of EMNE acquisition completion. In our conceptual model, we classify EMNE liabilities of origin into two categories, capability liability and credibility liability. We use data from the Thomson SDC Platinum Database to build a logistic regression model to test the influence of these liabilities on the deal completion of cross-border acquisitions made by Indian MNEs during the period 1999-2013. We find a differential impact of the depth and breadth of acquisition experience. The capability liability is mitigated with an increase in the number of successful acquisitions an EMNE makes but not with an increase in the number of countries in which acquisitions have been made. Governance quality of the target firm nation is found to have a positive influence on deal completion. Public sector EMNEs are found to have significantly lower rate of completion. Interestingly, we find that legitimacy building efforts of the Indian IT industry did not seem to have a significant effect on acquisition completion. (For more information, please contact: Shobhana Madhavan, Amrita School of Business, India: shobsdg@gmail.com) 


\title{
Session: 2.2.12 - I nteractive
}

\section{Track: 2 - Entrepreneurship, Micro-Multinationals, and I nternational New Ventures}

\section{Comparative IE Research: Institutions and International Entrepreneurship}

Presented On: June 29, 2015 - 10:45-12:00

Chair: Peter W. Liesch, University of Queensland

\author{
Moderating Effect of Formal Institutions on Individual Resource Commitment to Social Enterprise Entry \\ Sreevas Sahasranamam, Indian Institute of Management Kozhikode \\ Nandakumar Mankavil Kovil Veettil, University of Salford
}

Our study examines the contingent effect of formal institutions namely financial, educational and political systems on the relationship between people's access to resources and the likelihood of them starting a social enterprise. We hypothesize that the individual-level resources would be more influential for starting a social enterprise in those countries where the financial and educational systems are more entrepreneurially oriented. We also hypothesize that the relationship between individual-level resources and the likelihood to start a social enterprise would be stronger in those countries where the political systems are not very well-developed and would be weaker in those countries where the political systems are well-developed. We tested the hypotheses using a multilevel probit regression analysis on a cross-sectional multi-source dataset for the year 2009 and found support for all our hypotheses. The implications of our study are relevant for social entrepreneurs, and policy makers. (For more information, please contact: Sreevas Sahasranamam, Indian Institute of Management Kozhikode, India: mailsreevas@gmail.com)

\section{Internationalisation of Technology-Based Firms from Emerging Markets: Evidence from Brazilian High-Tech New Ventures}

Fernanda Ribeiro Cahen, University Center of FEI

Moacir Miranda Oliveira, University of São Paulo

Felipe Borini, ESPM

What are the factors influencing the accelerated internationalisation of new technology-based firms (NTBFs) from emerging economies? In this paper we address this question by analyzing perceptions of accelerated internationalisation expressed by entrepreneurs of Brazilian NTBFs. This type of firm is typically called born global and they have been reported primarily in high-tech sectors. Existing International Business (IB) theories have been focusing consistently on NTBFs from developed countries and NTBFs from emerging markets remain understudied. A survey was applied to small Brazilian NTBFs $(n=49)$. The results indicate that NTBFs that have an entrepreneur or group of executives with international management skills have more opportunity to have an accelerated internationalisation. In contrast to previous research in the field, the results demonstrate that NTBFs that take more than five years to enter the international market capitalise on their competence for innovation and abilities in international marketing in the internationalisation process. Therefore, this research contributes with new perspectives of studies on NTBFs from emerging markets. (For more information, please contact: Fernanda Ribeiro Cahen, University Center of FEl, Brazil: fribeiro@fei.edu.br)

\section{The Impact of National Culture on Firm Founder's Social Capital Formation Anastasia Veronica Bailey, Ohio State University}

Although national culture is a key contextual variable that is expected to impact relationship seeking behaviors of entrepreneurs, there is a dearth of studies that considers the effects of national culture on the formation of firm founders' social networks. Using data from the U.S. Survey of Small Business Owners and multiple measures of culture, we show how culture influences the knowledge that is applied to business activities by shaping the starting knowledge endowments of the firm, i.e., diversity of founders' social ties. We hypothesize 
that the social network of a firms' founding team is influenced by seven national culture dimensions. We find that cultural norms influence the social networks of founding teams. Our work highlights the differential effects of cultural practices on the knowledge that is brought to bear in the entrepreneurial firm by focusing on culture's impact on the formation of founders' social networks. (For more information, please contact: Anastasia Veronica Bailey, Ohio State University, USA: bailey.833@fisher.osu.edu)

\section{Linking Sub-National Institutions, International Entrepreneurial Capability, and International Performance of Export-Focused SMEs in Emerging Markets \\ Man Zhang, Bowling Green State University \\ Qian Gao, Anhui University \\ Hyuksoo Cho, Chungnam National University}

The differences in Sub-national institutions within complex emerging economies have been increasingly noted. Although the influence of institutional environment on larger MNE's performance of emerging market is well documented, the relationship between sub-national institutions, international entrepreneurial capability (IEC), and international performance (IP) is less researched. In this paper, we seek to address this gap by assessing the effect of relevant sub-national institutions, namely local government support, legal rules, government transparency, and government efficiency on international entrepreneurial capability of export-focused SMEs in emerging markets. We devise and assess a model that links sub-national institutions, IEC and IP. We collect data from export-focused SMEs from two important emerging markets, China and S.Korea. Three rounds of data collection result in 258 completed surveys. We analyze the data and provide discussion and managerial implications on these results. (For more information, please contact: Man Zhang, Bowling Green State University, USA: mzhang@bgsu.edu)

\section{Factor Market Imperfections and Pre-Entry Experience: Employee Spinoffs in the Bangladesh Garment Industry Romel Mostafa, I vey Business School}

Do the advanced economy regularities on employee spinoffs hold in a typical industry of emerging economies where factor market imperfections are pronounced? This paper uses a mixed method approach to study spinoffs in the Bangladesh garment industry. It first employs a qualitative lens to examine the impetus behind their formation. The qualitative findings are then synthesized with literatures on institutions and pre-entry experience to develop testable hypotheses. Evidence from the Bangladesh garment industry indicates that governance and ownership concerns common to emerging economies facilitated spinoff formation, but inefficiencies in capital markets also held back this process. Nonetheless, quantitative analyses find that spinoffs outperformed other entrepreneurial start-ups, indicating that the spinoff process was an effective mechanism for inter-firm knowledge transfer in an economy otherwise characterized by deep institutional deficiencies. Together, the findings broadly suggest that institutions can influence when and what form of organizations come into existence. (For more information, please contact: Romel Mostafa, Ivey Business School, Canada: rmostafa@ivey.uwo.ca)

The Implications of Institutional Forces and Network Competence on Internationalization Outcomes Among SMES

Lasse Torkkeli, Lappeenranta University of Technology

Olli Kuivalainen, Lappeenranta University of Technology

Sami Saarenketo, Lappeenranta University of Technology

Business networking and institutional forces have previously been linked to internationalization of small- and medium-sized enterprises (SMEs). However, the two phenomena have predominantly been examined in separate studies, and a view of SME internationalization accounting for both external institutional forces and networking capabilities of SMEs has been missing. The aim of this study is to respond to this gap, by examining how network competence alongside institutional drivers and barriers impacts the timing and performance of 
internationalization among SMEs. Our empirical sample, gathered in 2008, comprises 119 internationally operating Finnish SMEs across five industry sectors. We apply regression modelling and find that institutional drivers explain earlier start of internationalization among the SMEs, while institutional barriers and network competence do not. In comparison, network competence is a predictor of international performance, but there are no direct effects between the institutional environment and performance. However, cross-relational network competence mediates a relationship between institutional drivers and international performance. The study contributes to research on international entrepreneurship and SME internationalization, by highlighting the drivers of successful international business among SMEs and by clarifying the different impacts of institutional forces and dynamic capabilities during SME internationalization. (For more information, please contact: Lasse Torkkeli, Lappeenranta University of Technology, Finland: lasse.torkkeli@/ut.fi)

\author{
SME Decision-Making on Internationalization: A Critical Review and Research Agenda \\ Linda Hsiu-Yun Hsieh, University of Birmingham \\ Said Elbanna, Qatar University \\ J ohn Child, University of Birmingham
}

The current state of knowledge regarding SME decision-making on internationalization is still embryonic. This paper contributes to the SME internationalization literature by providing a comprehensive and critical review of 65 articles relevant to SME internationalization decision-making. The review led to the development of an integrative framework that incorporates six sets of antecedents (decision, decision-makers, firm, environment, internationalization and network factors), two sets of decision process (decision-making and decision implementation factors), and two sets of outcomes (decision outcomes and organizational outcomes). This paper highlights the underlying themes and contributions of existing studies on SME decision-making on internationalization, identifies gaps in the literature and suggests avenues for future research. (For more information, please contact: Linda Hsiu-Yun Hsieh, University of Birmingham, United Kingdom: h.hsieh@bham.ac.uk)

\title{
The Internationalisation of Location-Bound Service SMEs Niina Nummela, University of Turku Helena Turunen, HAMK University of Applied Sciences
}

This study explores how location-bound service SMEs - such as tourism companies - internationalise. It extends the existing classifications of service firms and challenges previously applied theories in a novel context. We suggest that key dimensions in the internationalisation of these firms are the available resource base, the company network and its embeddedness, and their entrepreneurial capabilities; and propose an internationalisation framework model supported by three illustrative case examples from Finland. The framework differentiates between passive developers, emerging developers and international entrepreneurs. The study makes several contributions. First, it provides a refined view on the internationalisation of service firms. Second, it highlights the significance of entrepreneurs in the process. Third, the findings indicate that both the significance of location-specific resources and entrepreneurial capabilities may differ in the course of internationalisation. We also suggest managerial implications for both the industry and policymakers supporting the internationalisation of location-bound service firms. (For more information, please contact: Niina Nummela, University of Turku, Finland: niina.nummela@utu.fi) 


\section{Session: 2.3.1 - Special Session}

\section{Peter J. Buckley and Mark Casson Al B Dissertation Award Presentations}

Presented On: J une 29, 2015 - 13:00-14:15

Chair: Anupama Phene, George Washington University

Presentations by the 2015 Peter J. Buckley and Mark Casson Al B Dissertation Award Finalists. The finalists are listed alphabetically. The Peter J. Buckley and Mark Casson Al B Dissertation Award is being sponsored by Henley Business School, University of Reading and the Centre for International Business, University of Leeds (CIBUL).

Why do Regional Headquarters Live and Die? (Ph.D. Awarded by Aalto University) Perttu Kähäri, Aalto University

Regional headquarters can be defined as an intermediate organizational unit with a mandated role, geographic scope and location. Regional headquarters represents a dynamic phenomenon within multinational companies. Guided by the research question - why do regional headquarters live and die -the purpose of this thesis is to understand and explain why RHQ exist and why they sometimes cease to exist. The thesis builds on four essays examining the dynamism of regional headquarters with various methods and applying different perspectives. The first essay presents regional headquarters in their dynamic context of corporate headquarters, subsidiaries and local environment. The second essay explains the processes that regional headquarters apply to add parenting value and presents evolutionary patterns that the value follows. The third essay investigates the effects of trust and distance on the retention and loss of the regional headquarters mandate. The final essay explores an entire population of regional headquarters in relation with its three environments. The empirical studies use a longitudinal approach in line with the dynamic research question. This thesis argues that regional headquarters are inherently dynamic, because they are embedded in three environments, terminable and sensitive to change. Their role, location, and geographic scope are interdependent, and change in one attribute will affect other attributes. Regional headquarters exist because they add value to their parents and subsidiaries at a lower agency cost than other alternatives. However, regional headquarters will die if they stop adding value, if they lose their parent's trust, or if an abrupt change in the corporate or external environment affects their position within the organizational structure of the multinational company. (For more information, please contact: Perttu Kähäri, Aalto University, Finland: perttu.kahari@aalto.fi)

\section{Should Birds of a Feather Flock Together? Trade-Off of Agglomeration by Nationality in Foreign Markets (Ph.D. Awarded by University of Michigan) \\ Heeyon Kim, National University of Singapore}

This study examines how national identity affects firm behavior and performance as firms expand internationally. Prior studies show that firms often follow the location decision of other firms from the same home country when moving abroad, which can lead to agglomeration by nationality in foreign markets. While foreign location choices are well understood, we know less about the consequences of agglomeration by nationality, an important question because shared nationality, while facilitating foreign market entry, may also have unintended negative consequences. This study fills this gap by studying the positive and negative consequences of agglomeration by nationality and the behavioral mechanisms that account for the performance consequences. I, first, argue that agglomeration by nationality can lead to a trade-off for firms by enhancing the performance of exploitative innovation but hindering explorative innovation. Second, focusing on the negative consequences on explorative innovation, I explore behavioral mechanisms such as forming homophilic relationships and imitating strategic decisions that mediate the effect of agglomeration by nationality on firm's explorative innovation performance in foreign markets. Finally, I explore firm-level characteristics that make some firms less (or more) likely to be negatively affected by agglomeration by nationality. Using a sample of 
non-U.S. pharmaceutical firms that conduct R\&D in the U.S. from 1980 to 2006, I find empirical support for my theoretical arguments. (For more information, please contact: Heeyon Kim, National University of Singapore, Singapore: heeyon@nus.edu.sg)

\author{
Property Rights Theory and the Ownership of Firm-Specific Advantages: The Implications of Contracting and \\ Licensing within the Multinational Firm (Ph.D. Awarded by Rutgers University) \\ Catherine Magelssen, London Business School
}

Firm-specific advantages (FSAs) play a critical role in the theory of the multinational firm. Firms establish foreign operations when their FSAs are not suitable to outsource or license in the market. However, the same assets deemed unsuitable for external contracting and licensing are extensively contracted and licensed within the multinational firm. The parent and/or subsidiaries that are the economic owners of the assets contract other entities within the firm to perform activities such as $R \& D$, manufacturing, and distribution and pay them a guaranteed return for their activities. Internal ownership of FSAs has implications on the risks borne, incentives, resource allocation, and power distribution within the firm. Using a unique, confidential dataset on the internal transactions of multinational firms, including intra-firm product flows, economic ownership of FSAs, financials, and detailed specifications of the activities of subsidiaries within the firm, I examine the determinants of the structure of economic ownership of FSAs as well as the impact of FSA ownership on innovation within the multinational firm. (For more information, please contact: Catherine Magelssen, London Business School, United Kingdom: cmagelssen@london.edu)

\title{
Global Subunit Specialization: An Organizational Perspective (Ph.D. Awarded by McGill University) Carlos Adrian Rodriguez, INCAE
}

The last 20 years have seen fundamental changes in the organization of firms' value chain. A large body of knowledge has been developed to inform the geographical ("offshoring") and organizational ("outsourcing") allocation of firm activities; however, much less research has explored the value chain disaggregation as an organizational process, in particular the design choices involved as the new subunits form and expand. My thesis seeks to contribute in this area, by analyzing organizational design issues during the formation and evolution stages, in fully owned and outsourced business units. The three empirical papers on this thesis are situated as follows: First paper examines the formation of a fine-sliced business unit, which result from the processes of geographical and organizational disaggregation in the firm's value chain activities. It focuses on the impact that the initial geographical and functional scope of an offshoring arrangement poses over its performance. This paper combines the resource-based view and complexity theory in large sample hypothesis testing to identify four impacts of fine-slicing on the subunit's performance: the number of geographical locations, the number of related functions, the structural complexity, and the ownership mode effects. By its focus on performance at the project level rather than measuring performance at the firm level, Paper I contributes with a fine-grained understanding of performance effects involving the allocation of related and unrelated activities. Second paper moves forward to analyze the evolution within the fine-slices. Specifically, this paper explores how previous performance affects the patterns of future offshore internationalization. This paper draws on the Internationalization Process Model with Performance Feedback Theory to explain the geographical and functional expansion of fully owned and outsourced offshoring in large sample hypothesis testing. The results of Paper II show that while the attainment of financial aspirations affects geographical expansion, it is the fulfillment of operational aspirations that explains functional diversification within a country. Moreover, I find that while ownership structure moderates the relation between financial aspirations and geographical expansion, the relation between operational aspirations and functional expansion is similar under both, fully owned and outsourced organizing modes. Third paper explores how changes in the organizational architecture affect capability development. By comparing two successful offshoring firms that followed highly divergent strategies, this qualitative analysis compares and contrasts the architectural mechanisms used in fully owned and outsourced offshoring arrangements on their different stages of development. Despite their divergence in strategies, I find striking coincidences in the architectural tools used in their initial stages, which became more divergent as their capabilities solidified. Beyond that, I find that organizational changes are, in turn, affected as 
offshoring capabilities develop. Overall, the studies in this dissertation contribute to the analysis of organizational choices as firms realize the fine-slicing of their value chains. Moreover, it contributes to a better understanding of the design choices that help enhance a subunit's performance, identifies the drivers of functional and geographical scope changes in offshoring operations, and helps identify the organizational design mechanisms used to foster the development of offshoring capabilities. (For more information, please contact: Carlos Adrian Rodriguez, INCAE, Nicaragua: carlos.rodriguez@incae.edu)

\section{Essays on the Relationship Between Host Market Corruption and Multinational Enterprise Strategy (Ph.D. Awarded by Western University) \\ Michael A. Sartor, Queen's School of Business}

This dissertation is guided by three research questions. First, how does host market corruption impact the equity-based market entry strategies implemented by multinational enterprises (MNEs) with respect to their foreign subsidiary investments? Second, does host market corruption increase the likelihood of market exit? Third, can MNEs implement strategies which reduce the likelihood of market exit under conditions of more pervasive host market corruption? In the first essay, I synthesize insights from institutional theory and integrative social contracts theory to disaggregate the concept of government corruption into two dimensions (grand and petty). My theory pertaining to informal institutional pluralism suggests that discrete institutions (such as government corruption) within a host market can be conceptualized as pluralistic phenomena constituted by distinct dimensions which exert a disparate impact on the foreign entry strategy of MNEs. The results support aspects of this theory. In the second essay, I build on the concept of informal institutional pluralism, categorizing corruption into two dimensions (public and private) to study its impact on the structure of equity-based foreign subsidiary investments. My theory proposes that the primary mechanism that drives the distinct approaches to foreign entry is the firm's anticipated reliance on different sources of bargaining power to reduce information asymmetries that it expects to encounter in the host market. In the third essay, I study the relationship between host market corruption pervasiveness, the subsidiary localization strategies implemented by MNEs and the likelihood of host market exit. In this context, the strategic insights proffered by resource dependence theory (RDT) and institutional theory (IT) are characterized by distinct spatial orientations. While RDT predicts that subsidiaries will implement proximal (or, host market-oriented) localization strategies, IT suggests that distal (or, home market-oriented) localization strategies are better-suited to reducing the likelihood of exit from increasingly corrupt host market environments. I find that a proximally-oriented partnering strategy heightens the likelihood of market exit under conditions of more pervasive host market public corruption, but not more pervasive private corruption. Conversely, a distally-oriented expatriate strategy increases the likelihood of market exit under conditions of both more pervasive public corruption and private corruption. Taken as a whole, this dissertation introduces new theory, constructs and insights into the relationship between host market corruption and the equity-based foreign entry strategies of MNEs. (For more information, please contact: Michael A. Sartor, Queen's School of Business, Canada:

michael.sartor@queensu.ca) 


\section{Session: 2.3.2 - Panel \\ Special Session}

\section{Organizing for Global I nnovation}

Presented On: J une 29, 2015 - 13:00-14:15

Chair: Felipe Monteiro, INSEAD

Panelists:

Felipe Monteiro, INSEAD

Kazuhiro Asakawa, Keio University

Yves Doz, INSEAD

Jaeyong Song, Seoul National University

Phanish Puranam, INSEAD

If any firm has the possibility of innovating, MNCs have the potential advantage of doing so on a global basis. The idea that MNCs create value from the internalization of knowledge assets can be traced back to the pioneering work of Hymer (1960), Caves (1971) and Buckley and Casson (1976), among others. The conventional view was that the MNC created knowledge in the home country, which it then diffused worldwide in the form of new products, processes and practices. In the last two decades an increasing interest in knowledge as a strategic resource has shed new light on how the MNC creates value from knowledge and how MNCs can be organized to source knowledge and innovate on a global basis. The change in the focus of research away from the dyadic headquarters-subsidiary relationship in MNCs has led to a new conceptualization, understanding and appreciation of subsidiaries. As foreign subsidiaries increasingly play a pivotal role in tapping external knowledge from different parts of the world, the importance of understanding how MNCs organize for global innovation is paramount. The purpose of this panel is to be broad, allowing for agenda-setting discussions which provide an overview of extant research on how MNCs organize for innovation and present a research agenda on this multifaceted topic. (For more information, please contact: Felipe Monteiro, INSEAD, France: felipe.monteiro@insead.edu)

\section{Session: 2.3.3 - Panel}

\section{Track: 9 - Emerging Economies and Emerging Economy MNEs}

\section{UNCTAD-WAI B Panel on MNCs, Gender Equality and Development}

Presented On: June 29, 2015 - 13:00-14:15

Chairs: Hafiz Mirza, UNCTAD and Gabriele Suder, University of Melbourne

Discussant: J anet Murray, University of Missouri-St. Louis

Panelists:

Axèle Giroud, UNCTAD

Gabriele Suder, University of Melbourne

Priyanka Sudarshan, WIPRO Limited

Premila Nazareth, UNCTAD

Sarianna Lundan, University of Bremen

Janet Murray, University of Missouri-St. Louis

Gender is a core element of the current Millennium Development Goals and will remain core to the forthcoming Sustainable Development Goals (SDGs). Although multinational enterprises are expected to be key actors to help achieve the SDGs, through their investment and operations, little attention has been given so far to their impact on gender equality and women empowerment, especially in developing countries. A recent report 
published by UNCTAD suggests that MNEs can help reduce gender-based inequalities and promote women's empowerment. However, more research is vital to show how this can occur. This panel aims to raise awareness about the necessity for further research to better understand how MNEs can raise women's economic empowerment in developing countries. The work conducted by UNCTAD and the Division of I nvestment and Enterprise is important and path-breaking in this respect. This UNCTAD-WAI B Panel will launch a much-needed debate on this forward-looking research agenda, to better understand how potential benefits of MNCs for women can be achieved maximized. Existing research conducted in the fields of International Business and Development will be presented, together with activities and programs currently developed and implemented by UNCTAD. (For more information, please contact: Axèle Giroud, UNCTAD, Switzerland: axele.giroud@unctad.org)

\section{Session: 2.3.4 - Competitive}

Track: 4 - International Marketing, Cross-Cultural Marketing, Social Communities

\section{Brands and Branding in International Markets}

Presented On: J une 29, 2015 - 13:00-14:15

Chair: Brian R. Chabowski, University of Tulsa

International Spinoff Effects of League Sponsorship

Merel Walraven, Fontys University of Applied Sciences

Tammo H.A. Bijmolt, University of Groningen

Ruud H. Koning, University of Groningen

Despite the increasingly global scale of sports sponsorship, research on international sponsorship effects in multiple countries is scarce. This study adds a cross-national dimension to existing research and allows investigation of the role of country participation in sponsorship's success. Data on sponsor equity of the Heineken sponsorship of UEFA Champions League were collected in nine different countries all over the world with differing degrees of participation in the competition. Our findings suggest that sponsor equity can be created in multiple countries through international broadcasting irrespective of country participation in the league. This implies that sponsorship may indeed be a suitable instrument in a global branding strategy. Yet we find important differences in the degree of sponsor equity between countries, implying that sponsorship may be more effective in one country than in another. (For more information, please contact: Merel Walraven, Fontys University of Applied Sciences, Netherlands: m. walraven@fontys.nl)

\section{Do Brands have Nationalities? Investigation of Consumers' Categorization of Foreign Brand Origins Abraham Koshy, Indian Institute of Management Ahmedabad Harsh Dadhich, Indian Institute of Management Ahmedabad}

Research finds that consumers have limited brand origin recognition accuracy (Balabanis \& Diamantopoulos, 2008; Samiee, Shimp \& Sharma, 2005). However, the country-of-origin and brand origin literature is replete with evidence that origins influence purchase decisions and brand evaluations. This paper offers a new theoretical framework to resolve this paradox. It builds on the category learning theory and argues that consumers will form similarity-based clusters of countries to better assimilate origin related cues. These clusters will be used for inferences and predictions regarding the country-of-origin of a brand. In two studies, this papers tests the hypothesis that consumers will form these clusters and the perceptions towards them.

(For more information, please contact: Harsh Dadhich, Indian Institute of Management Ahmedabad, India: harshd@iimahd.ernet.in) 
Marketing Mix Drivers of Brand Equity: Differences between Foreign and Domestic FMCG Brands in China Martin Heinberg, University of Duisburg-Essen

Markus Taube, University of Duisburg-Essen

The literature has established that a product's country of origin influences brand equity and the dimensions of brand equity (brand quality, brand loyalty, brand awareness). However, there is a dearth of research concerning the moderating effects of foreign and domestic brand ownership on the marketing mix drivers of brand equity.

The study investigates the role of marketing mix drivers to shape brand equity for FMCG products in China and explores possible differences in the strength of these drivers because of a foreign or domestic brand ownership. The study includes 817 consumers from three cities in China and a representative set of 25 real FMCG brands (12 domestic, 13 foreign). We analyze the data with variance based structural equation modeling (PLS-SEM approach). Results indicate that price is an equally strong driver for foreign and domestic brands. Moreover, foreign brands enjoy an advantage in distribution strategy shaping brand equity, whereas advertising is superior for domestic brands to build brand equity. The results support the theoretical notion that firm-specific advantages for foreign firms and location-based competitive advantages for domestic firms transfer to the efficiency of the marketing practices of these firms to create brand equity. (For more information, please contact: Martin Heinberg, University of Duisburg-Essen, Germany: martin.heinberg@uni-due.de)

\section{Session: 2.3.5 - Panel \\ Special Session}

\section{Alan Rugman's Contributions to I B}

Presented On: J une 29, 2015 - 13:00-14:15

Chair: Paul W. Beamish, Western University

\section{Panelists:}

Paul W. Beamish, Western University

Paul Martin Vaaler, University of Minnesota

Ruth Aguilera, Northeastern University

Jonas Puck, WU Vienna

Timothy Devinney, University of Leeds

(For more information, please contact: Paul W. Beamish, Western University, Canada: pbeamish@ivey.uwo.ca) 


\section{Session: 2.3.6 - Panel}

\section{Track: 8 - Global Strategy, M\&As and Competitiveness}

\section{The Role of Firm-Specific Assets and Organizational Capabilities in the Context of Multinationality-Performance Relationship: Critical Insights}

Presented On: June 29, 2015 - 13:00-14:15

Chair: Sumit Kumar Kundu, Florida International University

Panelists:

Ahmet Kirca, Michigan State University

Sumit Kumar Kundu, Florida International University

Farok J. Contractor, Rutgers Business School

J ean-Francois Hennart, Tilburg University; University of Pavia; Singapore Management University

Lei Li, Nottingham University Business School China

The purpose of this panel is to focus on the theoretical foundations of the M-P research and discuss the role of firm-specific assets and organizational capabilities, as well as the theoretical and methodological challenges and opportunities in the context of M-P relationship. After four decades of extensive research on M-P, it appears to be the right time to take stock and reflect on what we know so far, the current state of research and to provide a roadmap for future research, practice, and education. Specifically, in this panel will (1) review four decades of $M-P$ research with a focus on the role and nature of firm-specific assets and organizational capabilities, (2) provide insights into current and future challenges and opportunities in this stream of research, and (3) provide cutting edge thoughts on the M-P topic for future research. The session format for this panel will be in the form of paper presentations followed by questions from the audience and answers to these questions. In mapping out the 90 minutes that would be available for the session, we envision allocating 15 min $\neg$ utes to each talk, and then 30 minutes for the audience to engage in a Q\&A session with the panel. (For more information, please contact: Ahmet Kirca, Michigan State University, USA: kirca@broad.msu.edu)

\section{Session: 2.3.7 - Competitive}

\section{Track: 9 - Emerging Economies and Emerging Economy MNEs}

\section{Institutional Theory and Emerging Markets}

Presented On: J une 29, 2015 - 13:00-14:15

Chair: Wlamir Xavier, Eastern New Mexico University and UNISUL

Curvilinear Effect of Managerial Learning on New Product Innovativeness in an Emerging Economy: The Moderating Role of Multilevel Institutional Support

Xiaoyun Chen, University of Macau

Lin Yuan, University of Macau

Combining organizational learning theory and institutional theory, this study examines how managerial learning affects the new product innovativeness of high-tech firms in an emerging market and assesses whether such an effect is conditional on institutional support. Based on a survey of 174 high-tech firms in China, the study finds that managerial learning helps firms increase the innovativeness of their new product but that such an effect declines at high levels of managerial learning. Furthermore, institutional support at the government level enhances the effect of managerial learning on new product innovativeness; while organization-level institutional support reduces the effect of managerial learning on new product innovativeness. This study sheds further light on the driving forces of new product innovativeness for firms in an underdeveloped institutional environment. (For more information, please contact: Xiaoyun Chen, University of Macau, Macau: xychen@umac.mo) 
Does the Quality of Institutions and the Regulatory Bodies Influence Deal Duration in M\&As?

Yingdan Cai, University of Groningen

Hans van Ees, University of Groningen

Kees van Veen, University of Groningen

Sathyajit Gubbi, University of Groningen

The speed with which a merger and acquisition (M\&A) deal is completed or the number of days taken from the announcement of the deal until its completion is an important barometer of the success and efficiency of a deal. In this paper, we apply the institutional-based view to propose that in the context of acquisitions by emerging market firms, the deep embeddedness in the institutional environment is likely to influence deal duration. Our theoretical model speculates on the effect of the quality of home and host country institutional environment in cross-border acquisition deal duration. We further propose that the number of regulatory agencies involved has a bearing on deal duration. The hypotheses are tested using a unique sample of both domestic and international acquisitions with non-zero duration dates by Brazilian firms between 2000 and 2014. The findings supported our conjectures. (For more information, please contact: Yingdan Cai, University of Groningen, Netherlands: y.cai@rug.nl)

\section{The Strength Of Institutional Theory: EMS Adoption Yousef Eiadat, University College Dublin \\ Alejandro M. Fernández Castro, CESUGA \\ Aidan Kelly, University College Dublin}

The role of institutional factors in affecting EMS adoption is well discussed in the organizational literature. What is less understood is the perceived strength or the relative contribution of each institutional factor to determine the strongest institutional incentive for EMS adoption. Using data from the chemical sector in J ordan, this research seeks to contribute to filling this void. More specifically, it examines and empirically tests whether firms that are motivated mainly by coercive factors adopt an EMS to the same extent as firms that are mainly driven by mimetic or normative factors. Our results show that institutional mimicry influences EMS adoption decision to a far greater extent than normative and coercive forces. Studying the relative contribution of the institutional forces would enrich and sharpen our understanding of the institutional theory to a much greater degree. (For more information, please contact: Yousef Eiadat, University College Dublin, Ireland: yousef.husein@ucd.ie)

\section{Investment Liberalisation, Technology Take-Off and Export Markets Entry: Does Foreign Ownership Structure Matter? \\ Yundan Gong, Aston University \\ Sourafel Girma, Nottingham University \\ Holger Görg, Kiel Institute for the World Economy; University of Kiel; University of Aarhus \\ Sandra Lancheros, University of Nottingham}

In the run up to its accession to the WTO in 2001, China has undergone a far-reaching investment liberalisation. As part of this investment liberalisation process, existing restrictions on foreign ownership structure and mandatory export and technology transfer requirements imposed on foreign firms have been lifted in a number of industries. We exploit these facts to identify the causal effects of foreign acquisitions on export markets entry and technology take-off and to evaluate whether the level of foreign ownership plays a role in stimulating these changes. Using doubly robust propensity score reweighted bivariate probit regressions to control for the selection bias associated with firm level foreign acquisition incidences, we uncover strong but heterogeneous positive effects on export activity for all types of foreign ownership structure. We also find that minority foreign owned acquisition targets experience higher likelihood of $R \& D$, providing evidence that joint ventures between foreign owners and Chinese firms can contribute positively to China's "science and technology take-off". (For more information, please contact: Yundan Gong, Aston University, United Kingdom: y.gong1@aston.ac.uk) 


\section{Session: 2.3.8 - Competitive}

\section{Track: 2 - Entrepreneurship, Micro-Multinationals, and I nternational New Ventures}

\section{Institutions and Entrepreneurship in Different Country Contexts}

Presented On: June 29, 2015 - 13:00-14:15

Chair: Manjula S. Salimath, University of North Texas

Effects of Societal-level Performance Orientation, Self-expression, and Social Desirability on Entrepreneurs EarlyStage Internationalization

Etayankara Muralidharan, MacEwan University

Saurav Pathak, Michigan Technological University

André Laplume, Michigan Technological University

This study examines the influence of three normative institutions, performance orientation, self-expression and social desirability, on the propensity of entrepreneurs to internationalize their ventures at the inception stage (i.e., born global). We employed multi-level modeling techniques using 21,418 individual-level responses obtained from the Global Entrepreneurship Monitor (GEM) survey for 39 countries from 2001 to 2008, and supplementing with country-level data obtained from the World Values Survey (WVS) and the Global Leadership and Organizational Behavior Effectiveness (GLOBE) study. The results demonstrate that while performance orientation and self-expression increase the likelihood of early-stage internationalization, entrepreneurs in societies that view entrepreneurship as less socially desirable are more likely to be born-global entrepreneurs. In short, the study promotes new theory and empirical findings on the relationship between institutions and entrepreneurial agency, which are relevant for public policy and yield insights for practitioners. (For more information, please contact: Etayankara Muralidharan, MacEwan University, Canada:

muralidharane@macewan.ca)

\section{Effect of Institutional Voids on Entrepreneurial Opportunity \\ Susan L Young, Seton Hall University \\ Christopher Welter, Xavier University \\ Michael Conger, Miami University}

Discovery-type and creation-type opportunities may co-exist in the same geographical space, but are there institutional environments where the formation and exploitation of one type of opportunity may be more effective than another? This paper empirically examines how national differences in institutional efficiency, determined by aspects of a nation's regulatory environment, influence the nature of opportunities developed within these environments. We find that efficient institutions related to property rights and labor choices support the formation of discovery opportunities, while the presence of institutional voids regarding business formation and financing are more likely to force entrepreneurs to create opportunities. (For more information, please contact: Susan L Young, Seton Hall University, USA: susan.young@shu.edu)

\section{The Impact of Private Equity on Executive Salary and Compensation Packages in Emerging Economy IPO Firms} Bruce Allen Hearn, University of Sussex

Building on behavioural and multiple agency as well as institutional perspectives this paper explores the level of cash salary of executives and the likelihood of performance-contingent bonuses and benefits (perks) being included in their compensation packages at IPO. Drawing on a unique hand-collected and comprehensive database of 136 private sector IPO firms from across the African emerging region between January 2000 to January 2014 we find evidence that retained cash flow ownership post-IPO of Business Angel (BA) investors is associated with lower salary. The opposite is true of foreign-originated VC. Furthermore VC retained ownership and high institutional quality are associated with increased likelihood of the inclusion of benefits (perks) and 
bonuses within executive compensation packages. These results are valuable to entrepreneurs and policymakers in terms of the institutional and governance arrangements necessary for the optimal design of executive compensation arrangements in emerging economies (For more information, please contact: Bruce Allen Hearn, University of Sussex, United Kingdom: b.a.hearn@sussex.ac.uk)

\section{SMEs' Exports: Exploring the Soft Side of Capital \\ Stephanie Elisabeth Mansion, J ustus Liebig University Giessen \\ Andreas Bausch, J ustus Liebig University Giessen}

SMEs' capacity to export is perceived to be a major societal asset, fostering employment, value creation and innovativeness. Appreciating antecedents and outcomes of SMEs' export involvement is hence of paramount analytical and practical interest. In this vein, a growing literature explores the pertinence of human and relational capital for explaining differences in SMEs' export behavior. By meta-analytically integrating 151 studies, this analysis revealed positive and statistically significant effects of human capital endowment and relational capital. Concurrently, multivariate meta-regression uncovered that the influence of this "soft side of capital" is context dependent. (For more information, please contact: Stephanie Elisabeth Mansion, Justus Liebig University Giessen, Germany: stephanie.mansion@wirtschaft.uni-giessen.de)

\section{Session: 2.3.9 - Competitive \\ Track: 1 - People and Careers in Cross-Cultural Business}

\section{The Role of Culture in Intercultural Interaction and Work Behavior}

Presented On: J une 29, 2015 - 13:00-14:15

Chair: Douglas Dow, University of Melbourne

What Difference Does It Make? Ignoring Complexity in National Culture: The Case of Taiwan Fiona Moore, Royal Holloway, University of London

This paper shows how national culture itself, rather than being an undifferentiated category, is extremely varied, even among members of the majority culture of the same nation, explores how this can make a difference to both theory and practice, and suggests means for reevaluating earlier works on cross-cultural management and cultural distance in light of this situation. Through an ethnographic case study of Taiwanese professionals in London, conducted through interviews with 23 individuals, participant-observation at Taiwanese community events, and netnographic work on Chinese and Taiwanese social media, I will argue that not only can a single group with, ostensibly, a single national culture, interpret what it means to be a member of that culture in strikingly different ways, but that these differences have a definite effect on this group's ability to do business across borders. (For more information, please contact: Fiona Moore, Royal Holloway, University of London, United Kingdom: fiona.moore@rhul.ac.uk)

"I J ust Don't Feel Comfortable Speaking English": The Language Barrier through the Lens of Foreign Language Anxiety

Nathalie Aichhorn, WU Vienna

Despite the proliferation of language-sensitive IB research, we are lacking a complete understanding of the challenges of language standardization. We conducted an exploratory study at two Austrian MNCs based upon 22 in-depth interviews with top managers, non-managerial staff, and operatives. We find that the introduction of English can induce strong feelings of anxiety among non-native speakers of English. This can lead to communication avoidance, communication withdrawal, and code-switching. We illustrate how these (re-)actions can instigate a vicious cycle, which can reinforce negative emotions. Our study contributes to emerging research on the role of emotional factors as drivers of language barriers. (For more information, please contact: Nathalie Aichhorn, WU Vienna, Austria: nathalie.aichhorn@wu.ac.at) 
Cultural Values and Managers' Hiring Decision in Twenty-Four Countries: Does Societal Culture Matter?

Ji Li, Hong Kong Baptist University

Tao Liu, Southwest University

Yang Yu, Hong Kong Baptist University

J ieying Xu, Hong Kong Baptist University

Arif Mahmood, Hong Kong Baptist University

Wanxing J iang, Hong Kong Baptist University

Based on the theory of universal human values as developed by Schwartz $(1992,1994)$, we conducted a crossnational comparison of two levels of values (i.e., the cultural values at the societal level and personal values at the individual level) in terms of their effects on managers' decision of employment discrimination (hereafter DED). We argued that, when studying the relationship between cultural values and DED, one should consider not only those commonly-studied societal values, such as individualism/collectivism (hereafter I/C), but also personal values at the individual level. Analyzing data from over ten-thousand managers across 24 countries, we show that the effects of individual value are more robust than those of societal/institutions -level values in terms of their influence on DEDs. For instance, different from the findings from such a good journal as JIBS, we found that societal value of I/C has no significant relationship with either gender or immigrant discrimination. Moreover, the effects of both societal and individual values seem to be ordered by their relative importance to relevant discrimination, which supports the argument of universal values by Schwartz (1992, 1994). (For more information, please contact: Arif Mahmood, Hong Kong Baptist University, Hong Kong, SAR-PRC:

14485206@life.hkbu.edu.hk)

\section{Session: 2.3.10 - I nteractive}

\section{Track: 8 - Global Strategy, M\&As and Competitiveness}

\section{Making Global Partnerships Work}

Presented On: J une 29, 2015 - 13:00-14:15

Chair: Anoop Madhok, York University

Differentiated Impacts of Perceived J ustice on Inter-Organizational Contracting: The Role of Contract Complexity and Trust

Cheng-Min Chuang, National Taiwan University \& Overseas Chinese University

Chih-Fang Chiu, National Taiwan University

This research investigates how perceived justice of transaction partners affects the contracting issues including the extent of contract complexity, contract learning, and contracting capabilities, in inter-organizational exchanges. Based on an empirical study of 97 buyer-supplier contracts, we find that both the distributive and interactional justice facilitate contract negotiation with a greater extent of exemplified contract complexity, which in turn influences the contract learning and contracting capabilities. In addition, trust will moderate the association between contract complexity and contracting capability. (For more information, please contact: Chih-Fang Chiu, National Taiwan University, Taiwan: juju0802@gmail.com)

\section{Cultural Determinants of Alliance Management Capability - An Analysis of Japanese TNCs in India}

Sumati Varma, Sri Aurobindo College (Eve), Delhi University

Kalpana Narain, Director, Full Spectrum Consulting

Richa Awasthy, International Management Institute

Rishika Nayyar, PGDAV College, Delhi University

The present study examines the role of national and organizational culture in alliance management in the context of three prominent J oint Ventures between India and J apan which reached diverse alliance outcomes. It 
uses the case study method as a tool for an initial rich exploratory analysis (Yin 2013) of alliance management capabilities that may later be tested on a larger dataset. The study has finds that national and organizational culture are both important factors of alliance management capability. It highlights the specific role of trust, consensus in decision making, communication and relationship building as key constituents of alliance management capability. This paper thus contributes to an important strand of literature on alliance management in the context of two important Asian players from the developed and emerging markets. Its focus on cultural factors as determinants of alliance management helps to establish a managerial blueprint leading to positive alliance outcomes for such ventures in future and establish a roadmap for increased interaction between India and J apan. (For more information, please contact: Sumati Varma, Sri Aurobindo College (Eve), Delhi University, India: varmasumati@yahoo.co.in)

\section{Dynamic Alliance Formation and Maintenance: A Structural Stability Perspective Kuen-Shiou Yang, Asia University}

Different from prior strategic alliance literatures adopting Mainstream Economics, Transaction Cost Paradigm, Resource-Based View, Organizational Learning, Institutional Theory, Strategic Position Approach, Network Perspective, and Logistical Relationship, this research adopts a structural stability perspective to study alliance formation and maintenance. The analytic model applied to study structural stability is Heider's "P-O-X Model" which is an important model of Social Network Analysis (SNA). The analytic model introduces different type of structural balanced and imbalanced structures which present different degree and content of structural stability. Through our result, we find that structural stability does influence the possibility of alliance formation and maintenance. More specific, (1) the possibility of firms to form a new alliance with the other to achieve a potential structural balance situation is higher than a structural imbalance one when there is no any relationship between them; (2) the possibility of firms to maintain an existed alliance with the other in a balanced triad is higher than in an imbalanced one; (3) the highest structural stability structure exhibits highest possibility of alliance formation and maintenance than other structures. Our research may contribute to strategic alliance and social network analysis research in both theory and methodology parts. Research limitations and future directions are also discussed and provided in this research. (For more information, please contact: Kuen-Shiou Yang, Asia University, Taiwan: kyang@asia.edu.tw)

\section{Enjoy Swimming with Sharks: The Alliance Strategy of Japanese Small-And Medium-Sized Trading Companies Tianyou Hu, National University of Singapore Andrew Delios, National University of Singapore}

This study examines the outcomes of alliances between small and large firms from the perspectives of small firms. We propose that small firms have to sacrifice part of their independence in alliance strategy to exchange for the financial and network resource of their large partners. Using a data of J apanese small-and medium-sized trading companies, we find that allying with Sogo Shosha (general trading companies) enable those smaller firms to expand fast to foreign markets and form alliances with industrial firms that are affiliated in large business groups. But such ties with Sogo Shosha would constraint small firms in partner selection and modify their trajectories of international expansion. (For more information, please contact: Tianyou Hu, National University of Singapore, Singapore: ht@u.nus.edu)

\section{Do Similar or Complementary Competitors form More Alliances? Competitive Dynamics among Semiconductor Leaders \\ Sonya H. Wen, Tamkang University}

Do similar or complementary competitors form more alliances? We proposed multidimensional distance determinants to explain why competitors form alliances, by applying the literature of competitive dynamics and resource-based view, in the context of leaders competing in the same technology-intensive industry. Two competing hypotheses of resource similarity versus resource complementarity to increase the propensity of 
alliance formation among competitors were developed and tested by a sample of 600 firm-dyads of 25 semiconductor leaders in two decade-periods during 1990 and 2009. Our empirical results supported the hypothesis that similar competitors with less learning, experience, functional, cultural, and financial distance, form more alliances than complementary competitors with longer distance. Extending the framework of competitive dynamics from competing firms to cooperative alliances, this study suggests resource similarity increases not only competitive tension but also cooperative incentives, especially among industry leaders. (For more information, please contact: Sonya H. Wen, Tamkang University, Taiwan: sonya.wen@gmail.com)

\section{Pursuing the Stretch Goals of Low Opportunism and High Adaptation in Interorganizational Relationships Pei-Li Yu, National Cheng Kung University}

Achieving high interfirm adaptation and low ex-post opportunism enable superior interorganizational relationships performance, however, simultaneously pursuing both of these objectives presents a considerable challenge. To address this issue, we link transaction cost economics to organizational design theory and propose an integrated model for specifying the link: coordination mechanisms and interorganizational relationships performance. We extend research on the relationships performance by providing a framework for integrating the role of coordination mechanisms in reconciling conflicting interests in opportunism and adaptation between firms. We posit that the direct effect is mitigated by a variable specific to the interfirm context, namely cultural differences. The findings indicate that coordination mechanisms that involve routine and hierarchy can lead to better interfirm relationships performance and the positive interaction effects between routine of coordination mechanism and a lower cultural difference can reduce opportunism. (For more information, please contact: Pei-Li Yu, National Cheng Kung University, Taiwan: r4895113@mail.ncku.edu.tw)

\section{Learning to Contract in Repeated Inter-Organizational Exchanges: What and How \\ Cheng-Min Chuang, National Taiwan University \& Overseas Chinese University \\ Chih-Chuan Yeh, National Taiwan University}

Contract is regarded as knowledge repositories in governing inter-organizational exchanges, and transaction partners can learn from prior interactions to have the contract to be specified in greater detail. This paper examines the conditions under which this learning effect is most likely to be significant. Using a survey of on the 70 suppliers and foreign agents with a Taiwanese leading company in tool industry, it is shown that both contract specificity and contract complexity increase with partner's prior interactions. A U-shaped relationship between prior interaction and contract detail is also identified. This learning to contract effect is most prominent in exchange commitment section (for coordination purpose) than enforcement and safeguarding section (for control purpose). In addition to the typical task-specific learning in contract, this research also explore the partner-specific learning from prior interactions, and confirm the indispensable role of relational factors played in contract learning, suggesting a complementary nature between contractual governance and relational governance in inter-firm exchanges. (For more information, please contact: Cheng-Min Chuang, National Taiwan University \& Overseas Chinese University, Taiwan: cmchuang@ntu.edu.tw)

\section{Globalization and the Appointment of Internationally Oriented CEOs Yannick Thams, Suffolk University \\ Aya Chacar, Florida International University Margarethe Wiersema, University of California, Irvine}

This study argues that globalization places demands on firms' managerial capabilities, influencing the board's appointment of an internationally oriented CEO. A CEO's international orientation is defined as the extent and diversity of an individual's exposure to various country environment(s). We argue that a CEO's international orientation is an important managerial capability allowing for an international perspective and mindset, provides a more expansive cultural knowledge base, and greater cultural sensitivity and professional ties. We propose and find that greater levels of country globalization, firm international diversification and the undertaking of 
major foreign acquisitions are more likely to lead to the appointment an internationally oriented CEO in a sample of global US and European firms. (For more information, please contact: Yannick Thams, Suffolk University, USA: ythams@suffolk.edu)

\section{Session: 2.3.11 - I nteractive}

\section{Track: 6 - Innovation and Knowledge Management}

\section{Knowledge Sharing and Transfer within Multinational Companies}

Presented On: J une 29, 2015 - 13:00-14:15

Chair: T.J . Hannigan, Temple University

How does the Promotion of Knowledge Sharing Work within and across MNC's Business Units?

Angels Dasi, University of Valencia

Frank Elter, Telenor Research

Paul N. Gooderham, Norwegian School of Economics

Jarle Hildrum, Telenor Research

Torben Pedersen, Bocconi University

The ability of an organization to make available knowledge from one unit to another contributes to organizational performance (Szulanski, 1996) and has been conceptualized as critical for the existence of multinational corporations (Kogut \& Zander, 1993). However, organizational complexity inherent to MNCs establishes internal boundaries that difficult knowledge sharing. In the literature, we identify three broad categories of factors that are viewed as enabling MNCs to overcome internal organizational barriers for knowledge sharing - individual motivation, governance mechanisms and organizational values. In this paper we argue that these factors work differently within, as opposed to across, MNC's units. We propose four hypotheses that are tested on a unique data-set based on questionnaire response from over 15,000 employees in the multinational telecom company, Telenor. Our basic contention that factors that promote knowledge sharing work differently in the intra- and inter units contexts is supported but with various caveats. Explicit and formal factors such as HR-tools, openness-oriented values and extrinsic motivation are more relevant for promoting knowledge sharing across MNC's units, while factors associated with empowerment and socialization such as job design, innovative values and intrinsic motivation are more bound to the promotion of knowledge sharing within the unit. (For more information, please contact: Angels Dasi, University of Valencia, Spain: angels.dasi@uv.es)

\section{How Healthy Intra-Firm Competition Shapes Knowledge Sharing Dynamics Barbara Brenner, Danube University Krems \\ Shalini Rogbeer, European Business School}

While intra-firm competition is omnipresent among MNC subsidiaries, without an active orchestrating role of the parent, the intensity of competition largely determines knowledge sharing behavior which may trap subsidiaries in exceedingly unhealthy sharing patterns. Consequently, the MNC may benefit by ensuring a certain level of 'healthy competition' that allows for an optimal moderate level of knowledge flows. Our study aims to shed light on how knowledge sharing dynamics within the MNC can be actively shaped by headquarters intervention under conditions of healthy competition. Our case study evidence shows that by establishing clear corporate-wide rules, time lines, and responsibilities, the headquarters can frame competition in a way that allows for constant innovation and renewal while at the same time keeping knowledge flow volumes at an optimal level. (For more information, please contact: Barbara Brenner, Danube University Krems, Austria: barbara.brenner@donauuni.ac.at) 


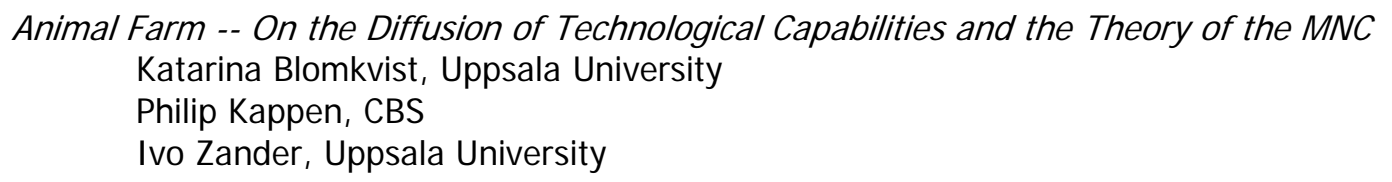

This paper is an empirical investigation of patterns in the emergence and diffusion of technological capabilities in the multinational corporation. It draws upon a database containing the complete patenting history of 24 Swedish multinationals over the 1890-2008 period, allowing for the identification of when and where in the multinational network new technological capabilities first emerged, and when and to where they subsequently diffused into other units of the multinational network. Observed differences between diffusion patterns for technological capabilities emerging at headquarters and in foreign subsidiaries are discussed in the light of extant theories on the nature of the MNC. We conclude that a theory of the MNC should recognize the shift towards more equal conditions and positions of various units of the multinational group, but that within this overall development some units remain more equal than others. (For more information, please contact: Katarina Blomkvist, Uppsala University, Sweden: katarina.blomkvist@fek.uu.se)

\title{
Intangibles Trade and MNCS Francisco A Moris, George Washington University
}

This paper studies the role of MNCs operations in country-level flows of disembodied technology, or intangibles trade, in the form of transactions in R\&D services captured in balance of payment statistics. The main conceptual premise, that MNC subsidiaries have a substantial effect on these flows, was supported by empirical findings using panel econometrics. The paper introduces the concept of 'reverse knowledge transfer' from IB research to the study of trade in intangibles, and integrates micro and macro theoretical perspectives on MNC $R \& D$ strategies, knowledge economics, and two-way trade and FDI to understand these flows. Consistent with these considerations, the empirical model treats panel countries as both trade partners and FDI host/home countries, and considers simultaneously two types of MNC subsidiaries, domestic owned and foreign owned, with the U.S. as reference country. The study suggests potential benefits from an integrated conceptual approach for enhancing monitoring of these aggregate flows for science and technology (S\&T) policy research purposes. (For more information, please contact: Francisco A Moris, George Washington University, USA: fmoris@nsf.gov)

\author{
Parents' Knowledge and Patterns of Affiliate's Knowledge Sourcing in Host Country \\ Se Ho Cho, Rutgers Business School \\ Hyun Gon Kim, Rutgers Business School
}

Why and when do foreign R\&D affiliates source knowledge more actively from their parent firms? Our study investigates factors that influence the extent to which foreign R\&D affiliates source knowledge from their parent firms. We develop theoretical hypotheses based on the patterns of foreign affiliates' knowledge sourcing and technology capability related factors for multi-levels: firm, industry, and country level. We test our theoretical arguments using their $M \& A$ and patent citation data from Fortune Global 500 firms. Our findings suggest that parent firms' technological capability and industry intensity positively affect the foreign affiliates' knowledge sourcing from their parent firms. The degree of ownership for foreign affiliates is meaningful moderator for the relationship between parent firms' technological capability and the patterns of foreign affiliates' knowledge sourcing. However, how home-host country difference on technological capability affects the pattern of foreign affiliates' knowledge sourcing is unclear. (For more information, please contact: Se Ho Cho, Rutgers Business School, USA: shcho1981@gmail.com) 
Aligning International Transfer Assignments with Service Offshoring Strategies

Carine Peeters, Vlerick Business School

Florence Duvivier, Universite libre de Bruxelles

This paper develops a conceptual framework that links the extent of international transfer assignments to the characteristics of service offshoring strategies in terms of drivers, task complexity, governance mode, and host country location. We argue that offshoring strategies are associated with different needs for control, coordination and transfer of tacit knowledge. Those needs are in turn best served by using a different combination of international assignments. The model suggests that opting for extensive international transfers when the offshoring strategy does not require doing so, exposes firms to unnecessary extra costs. On the contrary, limiting the use of international transfers below the level required to guarantee cross-border control, coordination and knowledge transfer increases the risk of not being able to integrate the offshored services. Therefore, the adequate use of various forms of international assignments (such as expatriation, inpatriation and virtual assignments) constitutes an important capability for the offshoring organization to be able to integrate globally dispersed value chain activities whilst at the same time containing costs. (For more information, please contact: Carine Peeters, Verick Business School, Belgium: carine.peeters@vlerick.com)

\section{Session: 2.3.12 - Interactive \\ Track: 9 - Emerging Economies and Emerging Economy MNEs}

\section{Economic Growth / Performance}

Presented On: J une 29, 2015 - 13:00-14:15

Chair: K. S. Momaya, Indian Institute of Technology Bombay

Impact of Tourism on the Environment and Economic Growth: Evidence from a Panel of Sub-Saharan Africa Tantatape Brahmasrene, Purdue University North Central

J ung Wan Lee, Boston University

This paper integrates tourism, economic growth and environmental issues in a multivariate format. Unlike recent research on this topic, a panel data of selected sample nations of sub-Saharan Africa is adopted by using cointegration and panel regression models. The current research discovers both long-run equilibrium and shortrun dynamics between economic growth, tourism, energy use and carbon emissions in sub-Saharan Africa. Furthermore, tourism and energy use show a highly significant direct impact on economic growth. In addition, tourism, energy use and economic growth yield a highly significant positive effect on carbon emissions. Dissecting the region into oil producers and non-oil producers further suggests that the economic growth of sub-Saharan Africa has been accomplished by strong growth in tourism and energy use. However, there is highly significant evidence that in oil producing countries, $\mathrm{CO} 2$ emissions are directly affected by energy use and economic growth and not by tourism. For non-oil producing countries, tourism and energy use but not economic growth incur a highly significant positive impact on carbon emissions. (For more information, please contact: Tantatape Brahmasrene, Purdue University North Central, USA: tapeb@pnc.edu)

Twenty Years after TRIPS: A Transitory and Post-TRIPS Impact of Technology Resources on Export Performance of Indian Pharmaceutical Industry

Satyanarayana Rentala, Pondicherry University

Sreevas Sahasranamam, Indian Institute of Management Kozhikode

The Indian pharmaceutical industry has undergone many dynamic changes during the past two decades even since India became a signatory to the TRI Ps agreement. In this paper, we investigate the effect of technology resources on the export performance of Indian pharmaceutical industry during two phases of intellectual property reforms. We find that internal $R \& D$ expenses, import of capital goods, and royalty payments impact 
the export performance of Indian pharmaceutical firms differently in the transitory-TRIPs (1995-2004) and postTRIPs (2005-2014) periods using panel dataset of 615 firms. However, the effect of import of raw materials is same across the two periods. The results indicate that different technological capabilities have a varied impact on the export performance of Indian pharmaceutical industry in the transitory-TRI Ps and post-TRI Ps periods. (For more information, please contact: Satyanarayana Rentala, Pondicherry University, India: rentsatya@gmail.com)

\section{Explaining Foreign Affiliate Performance of Emerging Multinationals: The Effects of Firm Resources and Institutional Distance}

Piotr Trapczynski, Poznan University of Economics

Marian Gorynia, Poznan University of Economics

This study investigates the performance effects of firm resources and institutional distance in the context of FDI undertaken by firms from Poland. The main findings show that among the intangible resources, managerial capabilities drive the success of infant multinationals. Conversely, the role of technological capabilities does not turn out to be significant. Moreover, while FDI experience appears beneficial to affiliate performance, hostcountry experience is non-significant with a negative sign, signalling a potentially detrimental influence of excessive reliance on earlier experience. Moreover, the study suggests that the direction of formal institutional distance between should be taken into account in future studies. (For more information, please contact: Piotr Trapczynski, Poznan University of Economics, Poland: piotr.trapczynski@ue.poznan.pl)

\section{Creating Shared Value through Impact Sourcing: A Process View Chacko George Kannothra, University of Massachusetts Boston}

This paper examines the potential and challenges of 'Creating Shared Value' (CSV) - the joint generation of social and economic value - in the increasingly important context of 'impact sourcing' - hiring and training of staff from disadvantaged communities for outsourcing jobs. We develop a 'process view' of CSV than acknowledges the critical role of hybrid organizations in implementing CSV models in practice. We show that CSV implementation relies on the continuous management of tensions, collaborative strategies with community organizations, and contingent adjustments over time. We inform practice and research on CSV and social responsibility in global outsourcing and beyond. (For more information, please contact: Chacko George Kannothra, University of Massachusetts Boston, USA: chackog@gmail.com)

\section{"Emerging" Through Foreign INVESTMENT: Investment Development Path Estimation of "MINT" Economies Emine Beyza Satoglu, Rutgers University}

This article aims to analyze the relationship between the foreign investment, both inward and outward, and the development levels of the emerging market economies: Mexico, Indonesia, Nigeria and Turkey. (MI NT) Dunning's IDP (Investment Development Path) theory has been used as the basis for empirical analysis covering the recent globalization era: 1990-2013. The fixed effects longitudinal data analysis for the four countries demonstrated that MINT economies are at the 2nd stage of IDP. At the same time, increasing level of inward FDI flows into these countries prove the potential of these economies to be represented as "second generation of fast growing developing countries" after the BRICS. Thus, this study aims to improve our understanding on the emerging economies by focusing on a new group (MINT) and it demonstrates the interaction of inward/outward FDI and the GDP growth in that group of countries.

(For more information, please contact: Emine Beyza Satoglu, Rutgers University, USA: eminebeyza@gmail.com) 
The Landscape of Export Promotion Institutions in the South-South Trade

Sivakumar Venkataramany, Ashland University

Balbir Bhasin, University of Arkansas at Fort Smith

Global financial crisis, the slowdown in the industrialized economies, and the unemployed surplus liquidity in the vaults of multinational corporations and financial services firms have caused a high level of uncertainty in the markets. As a result, the South-South trade and investment relations have gained increasing importance as primary drivers of growth in global trade and destinations of future investment flows. The scope of this paper is to measure the growth of trade in the countries in which export promotion is pursued by an export-import bank or a development financial institution. This study proceeds to analyze the trade-related outcomes such as income, employment, poverty, environment, health, and gender. (For more information, please contact: Sivakumar Venkataramany, Ashland University, USA: svenkata@ashland.edu)

\section{Sustainable Waste Management in a Frontier Country: The Case of Cote d'I voire} Ulf Henning Richter, Nottingham University Business School China

Solid waste management is considered one of the most crucial health and environmental problems faced by African municipal governments (Achankeng 2003, Okot-Okumu 2012). Uncollected solid waste in urban areas creates a favorable environment for insect and vermin to spread air and waterborne diseases such as dengue fever and diarrhea. If waste is collected but not disposed of properly, leachate contaminates the surrounding soil and adjacent water, posing further health risks to the local population (Courtois 2012). The District of Abidjan, Cote d'I voire, is currently generating around 1.5 million metric tons of municipal waste per year. In the case of continued development, in 25 years the amount of waste will be tripled to four times larger than the current amount, and that for the next 20 years it is expected to be generated an aggregated quantity of waste of approximately 75-100 million tones. We pro-pose the construction of an Innovative Recycling Center in Abidjan, Côte d'I voire, for (1) the production and marketing of compost and recyclable materials from suitable waste, (2) the stabilization of residual waste for proper landfilling, (3) the support of know-how transfer, (4) the dissemination of knowledge, and (5) training and education. (For more information, please contact: Ulf Henning Richter, Nottingham University Business School China, China: ulf.richter@nottingham.edu.cn)

\section{Session: 2.3.13 - I nteractive}

\section{Track: 2 - Entrepreneurship, Micro-Multinationals, and I nternational New Ventures}

\section{Emerging Market Perspectives to International Entrepreneurship}

Presented On: June 29, 2015 - 13:00-14:15

Chair: K. Indu Rao, University of Twente

Internationalization of New Technology-Based Firms: Analysis of Managerial Perceptions of Barriers Fernanda Ribeiro Cahen, University Center of FEl

Somnath Lahiri, Illinois State University

Felipe Borini, ESPM

What barriers constrain internationalization of new technology-based firms (NTBFs) in emerging markets? In this paper we address this question by analyzing perceptions of barriers to internationalization expressed by top executives of Brazilian NTBFs. The NBTFs belonged to high technology, medium-high technology or technologyintensive service sectors. Our analysis of data collected through questionnaire survey revealed three important barriers: external institutional barrier and internal organizational capability barrier and human resource barrier. These barriers are consistent with the institutional theory, resource-based view of the firm and human capital theory. In addition, we found that Brazilian NTBFs can be partitioned into three clusters with varying extents of perceptions of the above barriers. We conclude by discussing various contributions and limitations of the study 
and providing directions of future research. (For more information, please contact: Somnath Lahiri, Illinois State University, USA: slahiri@ilstu.edu)

\author{
Strategic Paths of Born Globals: A Study of Indian IT firms \\ Karthik D, Indian Institute of Management Ahmedabad \\ Rajesh Upadhyayula, Indian Institute of Management Kozhikode \\ Rakesh Basant, Indian Institute of Management Ahmedabad
}

Extant literature argues that post entry internationalization strategies and performance implications of born globals is an under researched area. In this paper, we examine the internationalization strategies of born global firms and performance implications thereof through strategic group analysis of hand collected data from Indian IT firms. We find that there are four distinct strategic paths chosen by born global firms. While some paths are performance neutral, others have performance differences. Our analysis shows that the nature of services and ways of mobilization / deployment of resources plays a salient role in the positioning of born globals along the dimensions of geographic scope and extent of foreign operations. We find that firms that move interchangeably between these two dimensions have similar performance outcomes. (For more information, please contact: Karthik D, Indian Institute of Management Ahmedabad, India: dkarthik@iimahd.ernet.in)

\title{
Determinants of Internationalization by New Ventures in India \\ Abrar Ali Mohammad Usman Saiyed, Indian Institute of Management \\ Rakesh Basant, Indian Institute of Management Ahmedabad \\ Karthik D, Indian Institute of Management Ahmedabad
}

This study contributes to research gaps identified by researchers (Fernhaber, Mcdougall, \& Oviatt, 2007; Jones, Coviello, \& Tang, 2011; Keupp \& Gassmann, 2009a; Kiss, Danis, \& Cavusgil, 2012). They emphasized to study INV phenomenon which integrates various fields, theories. They also showed importance of longitudinal study which includes various organizational, industry and environmental factors in the context of emerging markets. This study tries to understand effects of different organizational, industry factors in the context post liberalization era of India. The study has given an integrative framework which combines International New Ventures (INV), International Business (IB) and Industrial Economics theories to give understanding of internationalization process in INVs in India. It is a longitudinal study which has used secondary data on India firms. A Panel data multiple regressions was used to analyze two dataset; one with 413 firms (complete case) and 1033 firms (non complete case). The results show that firm's size and technology capabilities are critical for INV's International Intensity in I ndia while Industry factors like Local Industry I nternationalization help firms to explore internationalization due to isomorphism. New ventures in highly competitive industries were less prone to try international business. New ventures in less competitive industry, high knowledge intensive industry, high growth industry and highly internationalized industry are more prone to try international business quickly post their incorporation. New Ventures in transition economies with less restriction and in permissive policy regime period get encouragement to explore international business. (For more information, please contact: Abrar Ali Mohammad Usman Saiyed, Indian Institute of Management, India: abrarali@iimahd.ernet.in)

Trials of International Entrepreneurship: Overcoming Liabilities of Smallness, Newness and Origin in Emerging Market SMEs

Maria-Alejandra Gonzalez-Perez, Universidad EAFIT

Eva Cristina Manotas, Universidad Nacional

Intensification of trade and commercial diplomatic relations amongst countries, as well as trends of harmonization in domestic and international production and consumption dynamics, have led entrepreneurs to participate in foreign markets. Aiming to identify how size, age, and exports to the destination country influence the intensification of firm internationalization, the recurrence rate of exports to a particular country was calculated, taking into account for each company the variables of age and size. These data were obtained from 
both official data from the Colombian Ministry of Trade, and from a face-to-face survey designed to determine patterns of internationalization in a sample of 50 SMEs. With the collected data, a descriptive and recurrence analysis was applied to 1165 exports events for two Central American countries during the period 2000 to 2012. This study allowed to estimate the recurrence rate and to model the behavior of the exports to Central America. This study found the sample divided in two groups:(i) a group of young companies with high export intensity, and which internationalized at an early stage; and (ii) a group of incumbents, more older, with a moderate export intensity. Findings of this study contribute to the existing literature on international entrepreneurship.

(For more information, please contact: Maria-Alejandra Gonzalez-Perez, Universidad EAFIT, Colombia: mgonza40@eafit.edu.co)

\section{Slack Resources and the Emergence of Early Internationalizing Firms} Hadi Fariborzi, University of Calgary

This study explores the role of slack, especially in human resources of the firms on their capability to internationalize early in their life cycle. I analyzed data from the panel study of 4928 firms in Kauffman Firm Survey and contribute to international business and entrepreneurship studies by arguing that slack in human resources positively moderates the relationship between innovative entrepreneurial capabilities of the firm and its internationalization. I will discuss results using theories from international entrepreneurship, internalization theory and also Penrose's theory of growth. (For more information, please contact: Hadi Fariborzi, University of Calgary, Canada: hadi.fariborzi@ucalgary.ca)

\section{Session: 2.4.1 - Panel \\ Track: 5 - The Geography of I nternational Business and Global Value Chains}

\section{The Geography of International Business and Global Value Chains}

Presented On: June 29, 2015 - 14:30-15:45

Chair: Mark Lorenzen, Copenhagen Business School

Panelists:

T.J. Hannigan, Temple University

Mark Lorenzen, Copenhagen Business School

Anders Oerding Olsen, Copenhagen Business School

Michael Storper, UCLA

Snehal Suyash Awate, Indian School of Business

In 2013, we began to articulate a research agenda at the intersection of International Business, Economic Geography and Innovation (iBEGI N). We focused on the importance of connections between cities, clusters, organizations and people, as the basis for local innovation. The convergence of these literatures is a function of their underexplored complementarities: organizations, locations, and the space that connects them represent complex global innovation systems. The internal dynamics of cities and clusters bring about changes to firm behavior, and the activities of firms bring about changes within locations, and the evolution of both impacts the connections that tie the system together. These connections, which may be based on organizations or autonomous individuals, are crucial conduits through which knowledge moves out of clusters and around the world. This panel will seek to expand on the iBEGIN framework, and will put forth four unique approaches. The first presentation will address co-inventor networks of connections spanning locations and firm to generate globally sourced innovation. The second presenter will explore the role of inventor diasporas in driving knowledge connections. Third, we will examine how policy programs facilitate connections, impacting local innovation performance. The last two presentations address the institutional and structural preconditions for connections to arise and for particular configurations of connectivity. (For more information, please contact: T.). Hannigan, Temple University, USA: tj.hannigan@temple.edu) 


\title{
Session: 2.4.2 - Competitive \\ Track: 10 - Theories of the MNE and of FDI \\ Managing Liabilities of Foreignness and Origins
}

\author{
Presented On: J une 29, 2015 - 14:30-15:45 \\ Chair: J aeyong Song, Seoul National University \\ Imitative Behaviour and the Influence of Foreign MNEs on Local Firms' Environmental Innovation \\ Yoo J ung Ha, University of York
}

This paper investigates the extent to which inward foreign direct investment (FDI) by multinational enterprise (MNE) influences environmental innovation of indigenous firms in the host country. Indigenous firms adopt imitative behaviours to conform to institutional pressures valuing environmental issues and use foreign MNEs as benchmarking organisations. Using firm-level data from the Korean Innovation Survey of 2010, this paper confirms the positive greening effect of FDI by MNEs. Our three findings in particular are: 1) the greening effect of FDI is positive and an inverted U-shaped if the MNE adopts globally integrated strategy to environmental innovation, 2) the greening effect is positive and linear if the MNE adopts internationally dispersed strategy to environmental innovation, and 3) the greening effect is more marked among the indigenous firm whose current environmental performance ranking is higher than its peers. Findings will make contributions by zooming in the role of MNEs as a mimetic isomorphic force that influences local environmental innovation and broader institutional changes in the host country. (For more information, please contact: Yoo Jung Ha, University of York, United Kingdom: yoojung.ha@york.ac.uk)

\section{Liability of Regional Foreignness and Geographic Scope: An Empirical Assessment \\ J in Uk Kim, University of I llinois at Urbana Champaign \\ Ki Bum Noh, Korea Economic Research Institute (KERI)}

Liability of regional foreignness (LORF), which refers to the idea that the cost of doing business abroad is substantially lower within than outside the home-region, is a central concept underlying the theory of the regional multinational enterprise (MNE). However, the validity of LORF remains contested because the concept and measurement of LORF have remained underdeveloped. The purpose of the paper is to consolidate the link between the concept and measure of LORF and empirically assess its impact on MNE activities with special attention to firm-specific advantage. Using a sample of 158 Global Fortune 500 firms, we find that LORF constrains the geographic scope of MNEs yet those with stronger non-location bound FSAs tend to be less limited by LORF. We draw implications and significance of our findings for regionalization research as well as international business research in general. (For more information, please contact: J in Uk Kim, University of Illinois at Urbana Champaign, USA: jkim198@illinois. edu)

\section{Overcoming Locational Disadvantage: Identity Work and Category Manoeuvring by Emerging Economy Multinationals \\ Anirvan Pant, Indian Institute of Management Calcutta \\ J Ramachandran, Indian Institute of Management Bangalore}

Although a substantial literature has developed around how emerging economy MNEs (EE MNES) develop and deploy locational advantage, we have scarcely any understanding of how they overcome locational disadvantage. Specifically, we argue that the extant literature on EE MNEs is advantage-centric and locationagnostic. Examining the question of locational (dis)advantage in IB from the lens of the categorization literature in organization theory, we ask: How do EE MNEs overcome their liabilities of origin, specifically, the negative 
evaluations implicit in their categorization as firms from emerging economies? Drawing upon a comparative case analysis of two MNEs from the information technology industry in India, we develop a process model that highlights linkages between identity work conducted by EE MNEs and their ability to manoeuvre around the negative attributes of their locational category. (For more information, please contact: Anirvan Pant, Indian Institute of Management Calcutta, India: anirvan@iimcal.ac.in)

\section{Session: 2.4.3 - Competitive}

\section{Track: 9 - Emerging Economies and Emerging Economy MNEs}

\section{Networks, Technology and Entry}

Presented On: J une 29, 2015 - 14:30-15:45

Chair: Vittoria Giada Scalera, Politecnico di Milano

Institutional Complexity and Organizational Response via Technological Boundary Spanning

Jie Wu, University of Macau

Seung Ho Park, China Europe International Business School

Shuaihe Zhuo, University of Macau

Drawing on the literature on institutional complexity and the literature on technological boundary spanning, we examine how institutional complexity arising from international expansion affects organizational response via technological boundary spanning. We suspect that a high degree of institutional complexity arising from emerging market (EM) multinational enterprises' (MNEs) expansion to multiple foreign markets has a positive effect on organizational response via technological boundary spanning. This positive effect is positively moderated by top management team (TMT)'s heterogeneity and intense competition in technological domain. We further suspect that the interactive effect of institutional complexity and TMT heterogeneity is stronger for EM MNEs encounter low levels technological competition than those encounter high levels of technological competition. We test our hypotheses using a sample of 760 Chinese firms expanding to 99 overseas markets in the period 2001-2010. The results provide strong supports for our hypotheses. The theoretical implications, the limitations and future directions are provided. (For more information, please contact: Jie Wu, University of Macau, Macau: jiewu@umac.mo)

\section{Entry Mode, Networks, and Reforms \\ Luis Alfonso Dau, Northeastern University}

This empirical paper studies how MNEs may learn through their choice of mode of entry and international subsidiary network configuration as means to increase their responsiveness to pro-market reforms in their home market. The paper proposes that modes of entry and network configurations that facilitate knowledge acquisition provide firms from developing countries with marked institutional voids an advantage when responding to such institutional changes. The analyses use data for the largest Latin American companies from 1989-2008. The findings provide evidence for a positive moderating effect of joint ventures, acquisitions, subsidiary network control, and subsidiary network centralization on the relationship between reforms and profitability. (For more information, please contact: Luis Alfonso Dau, Northeastern University, USA: I.dau@neu.edu) 
Technology Catch up in Emerging Economies: Emerging versus Mature Technologies

Shantala Samant, Virginia Tech University

Donald Hatfield, Virginia Tech University

Pooja Thakur Wernz, Virginia Tech University

This paper examines how emerging economies (EE) overcome their latecomer disadvantages and catch up technologically with developed economies (DE). Specifically, we examine the nature of technologies that EE firms innovate in (namely emerging or mature technologies) and investigate if the role of country governments and independent firm activity in EEs impacts the outcome of technological type. Specifically, we theorize that centralized governmental processes will tend to imitate mature technologies which have been accepted in developed countries, while decentralized business investments will be more likely to focus on less certain, emerging (and less imitative) technologies. Data from 57 countries on patents filed with USPTO from 2000 to 2008 is used to test our hypotheses. We find that government R\&D efforts are more likely to focus on mature technologies, whereas R\&D efforts by independent firms are more likely to focus on emerging technologies. We also find that firms are more likely to innovate in mature technologies, and that EE firms may have higher propensity to innovate in mature technologies than DE firms. (For more information, please contact: Shantala Samant, Virginia Tech University, USA: ssamant7@vt.edu)

\author{
The Role of Network Embeddedness and Organizational Learning in Subsidiary Development \\ Ming Pu, Harbin University of Science and Technology \\ Pek-Hooi Soh, Simon Fraser University \\ Kexin Bi, Harbin University of Science and Technology
}

Subsidiary development can benefit from the knowledge flows arising from the networks embedded within MNCs or in external host environments. To extend the network model of subsidiary development, this study examines whether or not internal and external embeddedness lead to different levels of subsidiary development because of a subsidiary's learning process. We verified the model using survey data from 119 foreign-owned subsidiaries operating in China. The results show significant and positive relationships between internal embeddedness and subsidiary competence, and between external embeddedness and subsidiary scope. Moreover, organizational learning fully mediates only the relationship between internal embeddedenss and subsidiary competence. This study implies that a subsidiary is capable of enhancing its levels of scope and competence by creating and maintaining relationships flexibly and through a learning process. (For more information, please contact: Ming Pu, Harbin University of Science and Technology, China: puming2004@163.com) 


\section{Session: 2.4.4 - Competitive}

\section{Track: 11 - Corporate Governance in I B}

\section{Being Accountable, Being Transparent, and Creating Value}

Presented On: June 29, 2015 - 14:30-15:45

Chair: Paul Martin Vaaler, University of Minnesota

\section{A Comparative Analysis of Disclosure Practices between Foreign Multinational Corporations and Australian Domestic Firms}

Zahid Riaz, Lahore School of Economics

Sangeeta Ray, University of Sydney

Pradeep Kanta Ray, University of New South Wales

Firms face disadvantages when they operate in foreign markets. Multinational corporations (MNCs) address disadvantages of foreignness by imitating local firms. This research examines whether MNC-subsidiaries are embedded into the local institutional context and the extent to which these subsidiaries respond to local laws and standards of disclosure of director and executive remuneration in host country. This comparative analysis examined the hypothesis that globalized firms were less likely to respond to increased disclosure requirements needed to address a higher level of information asymmetry. The analysis of disclosure level of MNC-subsidiaries vis-à-vis domestic firms suggests that, other things being equal, MNCs are less responsive to increased disclosure requirements than their local counterparts in Australia unless they have substantial interactions with domestic product-markets. These results demonstrate that MNCs are willing to incur the additional cost of increasing disclosure if the benefits of increasing disclosure level justify it: that is, a clear presence of demand for information from product-markets. Another interesting finding relates to the negative association between lower regulatory distance and disclosure level of director and executive remuneration.

\section{Corporate Responsibility as Institutional Mirrors or Substitutes? A Study of Four European Union Countries Marjo Siltaoja, University of J yväskylä Carolyn P. Egri, Simon Fraser University \\ Olivier Furrer, University of Fribourg Mika Haapanen, University of Jyväskylä Knud Sinding, University of Southern Denmark Ruth Alas, Estonian Business School}

In this study, we examine the boundary conditions of mirror and substitute hypotheses regarding the influence of institutional pressures on the adoption of CR practices across countries. Specifically, we propose that the institutional mirror hypothesis relates to regulative, coercive pressure exerted by formal institutions on firms to adopt CR practices, whereas the institutional substitute hypothesis relates more to normative pressures exerted by stakeholders in a given context. We test hypotheses with a sample of 573 firms in the EU countries of Denmark, Estonia, Finland, and Latvia. Our findings indicate that strong coercive and normative pressures in a single-country context promotes the development of CR practices. In contrast, the presence of only strong regulative or only strong normative pressures is likely to lead to a more limited adoption of CR. 
rom Responsibility to Opportunity: Reorienting Samsung's Social Responsibility through Creating Shared Value

Hwy-Chang Moon, Seoul National University

Yeon Lee, Seoul National University

J immyn Parc, Sciences Po Paris

Along corporate social responsibility (CSR), there has been a transition to embody strategy and competitiveness on top of ethical and philanthropic side to it. A practical framework - creating shared value (CSV) was presented, shedding heightened interest and recognition as the most compelling CSR initiative for sustainable business. Complementing the implications rendered by CSV, corporate social opportunity (CSO) framework was introduced as the more accurate end-value. The main objective of this paper is to apply and assess the strategic approaches of firms in helping social problems by using the four stages and four elements for CSO. For a case study, social contribution activities of Samsung Electronics Co., Ltd. were reviewed and analyzed. The paper concludes that social role played by Samsung has agreeably followed the four stages of CSO. Especially when going to foreign markets, Samsung has engaged in many activities that mainly enhance corporate image among others. This shows that Samsung still needs to take on more synergistic approach by realigning the value chain activities, focus on core areas, and cooperate with other cluster in order to enhance corporate competitiveness while helping the society. (For more information, please contact: Jimmyn Parc, Sciences Po Paris, France: jimmynparc@gmail.com)

\section{Corporate Social Engagement at the Base of the Pyramid: Microfinance and Entrepreneurship Jasjit Singh, INSEAD \\ Pushan Dutt, INSEAD}

Companies increasingly support rural microenterprise as a way of achieving impact at the base of the pyramid (BOP). We examine one such effort in the context of a for-profit microfinance company in Sri Lanka. The company carefully monitors the purpose for which its microloans are provided, allowing us to track whether a loan funds income-generating activities in general and microenterprise-related activities in particular. Microloans provided for income-generating purposes are found to indeed be associated with better household-level outcomes. However, these benefits are at least as large - if not larger - when funding traditional means of rural livelihood than when funding microenterprises. Unlike studies of conventional entrepreneurship, we find no evidence of microenterprises in the BOP setting offering a more attractive upside either. Within microenterprise loans, the benefits are larger for existing microenterprises than new ones, suggesting lack of complementary assets and skills as a potential bottleneck for these. While caution is necessary in drawing strong causal inference from archival data, our cumulative findings suggest a need to temper the disproportionate emphasis often placed on microenterprise development as being the most preferable way of supporting BOP livelihoods. (For more information, please contact: Jasjit Singh, INSEAD, Singapore: jasjit.singh@insead.edu) 


\section{Session: 2.4.5 - Panel}

Track: 12 - The I nstitutional and Political Environment of I B

\section{Economic I nequality and International Business}

Presented On: J une 29, 2015 - 14:30-15:45

Chair: Thomas Andre, Ecole Polytechnique

Panelists:

Ivan Montiel, Loyola Marymount University

Thomas Andre, Ecole Polytechnique

Raquel Antolin-Lopez, University of Almeria

Hari Bapuji, University of Manitoba

Ahreum Lee, Temple University

Nathaniel Curtis Lupton, Fordham University

Etayankara Muralidharan, MacEwan University

Despite widespread concern about rising levels of economic inequality around the world, both in developed and developing countries, the engagement of business scholarship with this topic has been minimal. This is particularly surprising given the active engagement of IB scholars to understand the institutional context of business and the positive role they played in studying the BOP markets. Our intent in this AlB panel is to bring together scholars interested in the intersection between economic inequality and international business.

Drawing on successful previous workshops, panels and symposiums conducted at AOM and IABS, we propose an interactive panel. Specifically, the session will involve (i) an introduction the topic by two scholars actively researching the relationship between business and economic inequality, (ii) structured roundtable discussion on three issues relevant to international business, which can potentially address economic inequality, i.e., social entrepreneurship in global supply chains, partnerships between MNEs and developmental agencies, and innovations in/from emerging markets, and (iii) integration of the roundtable discussions to propose a research agenda for the future. (For more information, please contact: Ivan Montiel, Loyola Marymount University, USA: ivanmontiel@gmail.com)

\section{Session: 2.4.6 - Competitive}

\section{Track: 13 - International Economics, Finance and Accounting}

\section{Multinationals and Financial Markets}

Presented On: June 29, 2015 - 14:30-15:45

Chair: Sumit Majumdar, University of Texas at Dallas

\section{A Comparative Analysis of Stock Market Development: The Impact of Formal and Informal Institutions Robert Lindorfer, WU Vienna \\ Anne d'Arcy, WU Vienna \\ Igor Filatotchev, WU Vienna}

In light of theory and evidence that the functioning of stock markets affect firm's financing decisions, and economic growth, we aim to link institutional quality to capital markets. In that respect, we analyzed 3,244 newspaper articles to run a comparative empirical analysis on the relation between the mindset of a population towards capital markets, as a proxy for informal institutions, the Heritage Foundation's Index of Economic Freedom, as a proxy for formal institutions, and stock market development in Austrian and Poland. Our results show positive and significant direct effects of formal and informal institutions on stock market development, 
where both differ for Austria and Poland. Furthermore, we theoretically explain and empirically examine how both institutional dimensions interact with each other, by showing that the societal mindset of a population towards capital markets positively influences the relation between formal institutions and stock market development. (For more information, please contact: Robert Lindorfer, WU Vienna, Austria: robert.lindorfer@wu.ac.at)

\section{Financial Constraints and Firm Export in Latin American Countries Jagadish Dandu, Zayed University \\ Maria Barulina, University of Texas at El Paso \\ Barkat Ullah, Rhode Island College \\ Zuobao Wei, University of Texas at El Paso}

In this paper, we empirically estimate the effect of financial constraints on firms' export behavior in terms of probability to export and export intensity. Using World Bank Enterprise Survey (WBES) data for 22,255 firms from 31 Latin American countries for 2006-2010, we find that older, larger firms with a share of foreign ownership, and those having a line of credit and an overdraft facility are more likely to export than smaller, younger, domestically-owned firms that are financially constrained. We also find that younger, larger firms with a share of foreign ownership, and those having no line of credit or overdraft facility export more of their products than their older competitors that have access to a line of credit or an overdraft facility. Our paper provides the first concrete evidence in a cross-section, cross-country setting that financially constrained firms are less likely to export. (For more information, please contact: Jagadish Dandu, Zayed University, United Arab Emirates: jagadish.dandu@zu.ac.ae)

\section{International Diversification of Public Defined Benefit Pension Plans: Influence of Plan and State Characteristics} Raj Aggarwal, University of Akron

John W. Goodell, University of Akron

Prior literature on the determinants of the international diversification of defined benefit (DB) pension plan assets seems non-existent. This paper is an attempt to fill this gap with panel analysis of data for the period 2001-2011. As expected, we find that higher ratios of international equity to domestic equity in these pension plans are positively related to plan characteristics of higher leverage (less use of equity versus debt), lower inflation expectations, lower future return assumptions, higher underfunding, and higher fund size and age. However, more interestingly we document that international diversification of public DB plans is strongly negatively related to the state cultural measures of collectivism, power distance, and uncertainty avoidance and that these cultural differences are economically important. This study makes important contributions not only to our understanding of the determinants of the international diversification of DB pension funds but also to the role of cultural differences across US states in influencing financial decisions. The novel and important evidence presented here should be of much interest to policy makers, capital market participants, managers, and beneficiaries of pension plans. (For more information, please contact: Raj Aggarwal, University of Akron, USA: aggarwa@uakron.edu) 


\section{Session: 2.4.7 - Competitive}

\section{Track: 3 - International Human Resource Management and Organizational Behavior}

\section{Crossing Cultures}

Presented On: June 29, 2015 - 14:30-15:45

Chair: Abraham Stefanidis, St. John's University

Cultural Propensities and Ethically Questionable Negotiation Tactics: A Four Country Analysis

Abraham Stefanidis, St. J ohn's University

Moshe Banai, City University of New York

Ursula Schinzel, United Business Institutes

Ahmet Erkus, Bahcesehir University

The study empirically tests the question of whether employees' tendency to endorse the use of questionable negotiation tactics is a related to their national or of their individual cultural values. More specifically, it compares the attitudes toward the use of ethically questionable negotiation tactics of 293 Turkish negotiators who reside in Turkey with those of 181 Turkish negotiators who reside in Germany, and the attitudes of 327 Greek negotiators who reside in Greece with those of 186 Greek negotiators residing in Cyprus. The attitudes were measured by survey questionnaires translated from English to Turkish and Greek. Turkish negotiators in Turkey scored significantly higher on vertical and horizontal individualism, on vertical collectivism and on their tendency to use 'pretending' and 'deceiving' negotiation tactics, than Turkish negotiators in Germany. Greek negotiators in Greece scored significantly higher on horizontal individualism and on vertical collectivism, and on their tendency to use 'pretending' and 'deceiving' negotiation tactics, than Greek negotiators in Cyprus. Turkish negotiators in Germany and Turkey together scored significantly higher on their tendency to use 'pretending', 'deceiving' and 'lying' negotiation tactics than Greek negotiators in Greece and Cyprus together. We conclude that both national and individual cultural values may influence business negotiation tactics. Caution should be taken when relating behavior in organizations to national culture alone. (For more information, please contact: Abraham Stefanidis, St. John's University, USA: stefania@stjohns. edu)

\section{The Moderating Effects of Transformational and Transactional Leadership Styles on Commitment and Satisfaction across Countries Youngsam Cho, Korea University Mannsoo Shin, Korea University}

In this study, the effects of two leadership styles on organizational commitment (OC) and job satisfaction (JS) are compared across countries. Based on culturally endorsed implicit leadership theory (CLT), we developed and empirically tested a two-by-two matrix model to identify differences in leadership effectiveness depending on the cultural context. Bass (1985) argued that transformational leadership (TFL) is built on a base of transactional leadership (TSL); therefore, a positive moderating effect may exist between these two leadership types. However, House (1996) challenged that argument by insisting that TSL has a negative moderating effect on employee outcome. In this study, we suggest that the moderating effect of TSL may differ depending on the culture. The results of our analysis of four groups classified according to the perceptions of subordinate workers (315 Korean and 181 U.S. employees) of TFL and TSL styles showed highest values for both OC and JS in the high-TFL and high-TSL groups in the Korean sample, while values for the high-TFL and low-TSL groups were highest for U.S. employees. To elucidate these findings further, we investigated the moderating effects of various subdimensions of TFL and TSL. The results indicated that TFL and TSL had positive moderating effects on OC and JS in Korea. However, no such moderating effect was evident in the U.S. sample. These results were consistent with the results of the two-by-two matrix. The findings of this study provide important insight into how managers can lead Korean and U.S. employees more successfully. (For more information, please contact: Youngsam Cho, Korea University, Korea, South: zegal82@korea.ac.kr) 
Exploring Modes of Leadership Adjustment in a Cross-Cultural Context

Chin-J u Tsai, Royal Holloway, University of London

Chris Carr, University of Edinburgh

Sasiya Supprakit, Deloittte UK

Kun Qiao, Dalian University of Technology

The adjustment of leadership approach according to conditions in the host country has been argued to be essential for leadership effectiveness in a cross-cultural context. However, virtually no empirical research has studied the adjustment of cross-cultural leadership. This paper presents a study that explores modes of crosscultural leadership adjustment and investigates forces influencing modes of adjustment. Data were collected from senior expatriate managers working in Thailand. Nicholson's (1984) theory of work role transitions was used as the theoretical foundation to explore work role requirements (consisting of role discretion and novelty of job demands) as potential predictors of modes of cross-cultural leadership adjustment. The results show that a majority of expatriate executives made adjustment to their leadership approaches and tried to change Thai employees, showing the exploration mode of adjustment, and that role requirements, characteristics of Thai employees, and local hierarchy system, as well as leaders' perceptions, all influenced expatriate leaders' modes of adjustment. Based on our findings, a theoretical framework and a number of research propositions were developed. The implications of the findings are discussed. (For more information, please contact: Chin-Ju Tsai, Royal Holloway, University of London, United Kingdom: chin-ju.tsai@rhul.ac.uk)

\section{Exploring Cross-Cultural Skills for Expatriate Managers from Chinese Multinationals: Congruence and Contextualization \\ Dan Wang, Monash University \\ Di Fan, Deakin University \\ Susan Freeman, University of Adelaide \\ Cherrie Zhu, Monash University}

This study explores what cross-cultural skills are essential for Chinese expatriate managers working in varied host countries, and how home and host contextual factors play a role in constraining the effective application of cross-cultural skills. Given the surge of Chinese outward investment, it is timely to study the competence of expatriate managers accompanying Chinese multinationals' overseas expansion. We conducted in-depth interviews with Chinese expatriate managers (25), their foreign colleagues (15) and other relevant informants such as expatriate supervisors and cross-cultural consultants (10). The findings demonstrate that Chinese expatriates working in different countries require different skills to be effective, and whether certain skills could positively or negatively affect expatriate performance depends upon the nature of home and host contextual incongruence. This study extends social learning theory by incorporating contextual factors to explain the applicability of cross-cultural skills, and the findings have significance for expatriate management from emerging markets. (For more information, please contact: Dan Wang, Monash University, Australia:

dan.wang@monash.edu) 


\section{Session: 2.4.8 - Competitive}

Track: 4 - International Marketing, Cross-Cultural Marketing, Social Communities

\section{Theory Development in International Marketing}

Presented On: June 29, 2015 - 14:30-15:45

Chair: Brian R. Chabowski, University of Tulsa

The Interdisciplinarity of Cross-National Research

Brian R. Chabowski, University of Tulsa

Saeed Samiee, University of Tulsa

G. Tomas M. Hult, Michigan State University

Comparative cross-national research is among the oldest approaches of scientific inquiry in the social sciences. We investigate the foundations of published works in cross-national research (CNR) in international business (IB), sociology, and psychology with an eye on leveraging their rich traditions for future IB research. Our approach aims to contribute and address repeated calls for taking an interdisciplinary approach in IB. We examined published CNR in 106 scholarly journals and identified 817 articles with 51,738 citations in IB, 477 works with 30,017 citations in sociology, and 835 articles with 54,519 citations in psychology. We use metric multidimensional scaling to examine the co-citation data for each field and identify knowledge and approaches commonplace in sociology and psychology to propose future research paths in IB. In particular multilevel analysis, which is scarcely used in IB, offers the potential of unfolding new dimensions within the field. Based on the emergent results from MDS solutions, we recommend multiple future paths for CNR, particularly with respect to relationship marketing, market entry, and related domains. (For more information, please contact: Brian R. Chabowski, University of Tulsa, USA: brian-chabowski@utulsa.edu)

What's the Use of Globalness? - Analyzing Leading MNCs' Globalness in India, Japan, and the United States Bernhard Swoboda, Trier University

J ohannes Hirschmann, Trier University

While multinationals are increasingly forced to adapt offered value in foreign markets little is known whether they can use their advantage of being global and how perceptions of global corporations drives consumer behavior. To expand on this issue, in this study consumer data from India, Japan, and the U.S. is analyzed regarding the evaluation of leading foreign and domestic multinationals. Results show that perceived brand globalness only enhances consumer loyalty toward the corporation by affecting consumers' functional and psychological value. These value creation routes change according to firms' origin but also differ between countries. Further insights follow from segmenting consumers concerning their level of ethnocentrism. (For more information, please contact: Bernhard Swoboda, Trier University, Germany: b.swoboda@uni-trier.de)

\section{Leapfrogging vs. Switching to New Technologies in Developed \& Emerging Markets Deepa Chandrasekaran, University of Texas at San Antonio \\ Gerard J . Tellis, University of Southern California \\ Gareth M. James, University of Southern California}

The authors propose the Leapfrogging Adoption (LeAd) model. The LeAd model extends prior multi-generation diffusion models by providing for a separate rate of disengagement allowing consumers to hold both the old and new technology at the same time and by decomposing new technology adoption into switching \& leapfrogging. Analysis on 10 pairs of successive technologies in 100+ countries indicates that leapfrogging forms a substantial proportion of adoption, especially in emerging markets. Contagion is significant-- Higher new technology 
penetration in prior countries positively affects leapfrogging and switching. Precommitment is significant--Prior penetration of the old technology in the same country positively affects switching but negatively affects leapfrogging. The authors discuss implications. (For more information, please contact: Deepa Chandrasekaran, University of Texas at San Antonio, USA: deepa.chandrasekaran@utsa.edu)

\section{Session: 2.4.9 - Competitive}

\section{Track: 8 - Global Strategy, M\&As and Competitiveness}

\section{When do Cross-Cultural Mergers and Acquisitions Succeed?}

Presented On: June 29, 2015 - 14:30-15:45

Chair: Aseem Kaul, University of Minnesota

Operationalizing Cultural Differences with Country Level Scores: The Moderating Role of Contextual Factors Manish Popli, Indian Institute of Management Indore

Vikas Kumar, University of Sydney

Cultural distance is one of the most widely used distance construct in international business. However, its application as having linear and homogenous impact on organizational actions and performance has long been questioned. In this paper, we attempt to redress this weakness by examining the moderating effects of firmlevel capabilities and industry context, on the impact of cultural differences on firm-level outcomes. Drawing on the organizational learning theory with cultural friction perspective, we hypothesize that the 'cultural experience reserve' of a focal firm is a potential firm-specific capability, mitigating the negative impact of cultural differences on cross-border deal abandonment. Second, we hypothesize that the firm's industry context impacts the uncertainties associated with cultural differences. Our findings based on a sample of 197 Indian services sector firms support our theoretical predictions. (For more information, please contact: Manish Popli, Indian Institute of Management Indore, India: manishp@iimidr.ac.in)

\section{Culture's Consequences for Acquisition Performance: An Analysis of Statistical Artifacts, Moderators and the} Context of Emerging Markets

Daniel Rottig, Florida Gulf Coast University

This paper provides a quantitative synthesis and integration of the existing empirical body of literature on culture and acquisition performance based on a meta-analytical approach that synthesizes 189 effect sizes from 24 independent samples with a total sample size of $\mathrm{N}=5,496$ acquisitions. In so doing, this study provides mean true score correlations for the direct relationships between both organizational and national cultural differences and acquisition performance, examines methodological and contextual variables that moderate these direct relationships, and provides an exploratory study of national culture's impact on acquisition performance in the context of emerging markets. Results of this study reveal the impact of the type of acquisition performance measure used, as well as the type of respondents tapped into when measuring cultural differences, on the findings of empirical studies examining the effect of culture on acquisition performance. Results further reveal the importance of the nationality of the acquirer, as well as economic development of the target company (i.e. developed vs. emerging markets) on the relationship between national cultural differences and acquisition performance. (For more information, please contact: Daniel Rottig, Florida Gulf Coast University, USA: drottig@fgcu.edu) 


\section{The Effect of Cross-Border M\&A Activity and M\&A Cultural Diversity on CEO Pay}

Padma Rao Sahib , University of Groningen

Desislava Dikova, Vienna University of Economics and Business

Hans van Ees, University of Groningen

This paper links two streams of literature, specifically, one concerning executive compensation that is primarily rooted in finance and the other that is related to cross-border M\&A activity and the role of national culture. We demonstrate that, while the usual 'suspects' such as firm size and firm growth are important determinants of CEO pay, cross-border M\&A activity and the cultural diversity of an M\&A portfolio of multinational firms are important and hitherto ignored determinants of CEO compensation. We further disaggregate CEO remuneration into different compensation components (e.g., fixed and variable pay), suggesting that cross-border $M \& A$ activity and the cultural diversity of the M\&A portfolio have pronounced effects on the long-term and variable component of CEO pay while fixed pay components are influenced much less. Using a panel dataset on CEO pay from a sample of stock listed firms in the Netherlands, we ascertain support for our hypotheses. We find that although cross-border M\&A activity and cultural diversity of the M\&A portfolio have a positive effect on CEO pay, the effects of M\&A cultural diversity on CEO pay differ across pay components. (For more information, please contact: Padma Rao Sahib, University of Groningen, Netherlands: p.rao.sahib@rug.nl)

\section{Cross-National Distances and Acquired Subsidiary's Post-Acquisition Identity Change in Emerging-Market Firms' Cross-border M\&As \\ Rushiun Liou, Texas A\&M University -Central Texas \\ Rekha Nicholson, University of the West of England}

To address the unique challenge facing emerging-market multinationals (EMFs) in managing their foreign subsidiaries, we propose and evaluate the foreign subsidiary identity transitions that are driven by the competing demands of parent stakeholder and local stakeholder. Lacking international experience, EMFs often grant foreign subsidiaries substantial autonomy to be flexible to respond to diverse stakeholder demands, shaped by the potentially large national differences between the host market and the home emerging economies. Utilizing a sample of worldwide mergers and acquisitions conducted by EMFs originated from BRICS, we establish that various cross-national distances, including economic distance, and formal and informal institutional distance, do not consistently encourage the acquired subsidiaries to take on the parent's identity, which is reflected by the acquired subsidiary's corporate name change after the acquisition event. While economic distance and formal institutional distance increases the likelihood of an acquired subsidiary's corporate name change, informal institutional distance decreases the likelihood of corporate name change. (For more information, please contact: Rushiun Liou, Texas A\&M University -Central Texas, USA: r.liou@tamuct.edu) 


\section{Session: 2.4.10 - Interactive}

\section{Track: 3 - International Human Resource Management and Organizational Behavior}

\section{Leading the International Enterprise}

Presented On: June 29, 2015 - 14:30-15:45

Chair: Anna Katharina Hildisch, University of Goettingen

Success of Single Founders in Entrepreneurial Firms from the Emerging Economy of China: The Perspective of Tertius I ungens Orientation

Liqun Wei, Hong Kong Baptist University

$X \mathrm{Z}$ Zou, London Business School

J unbang Lan, Hong Kong Baptist University

We propose that single founders' tertius iungens orientation is critical to the new venture success in China. Specifically, we establish a moderated mediation model: single-founders' need to belong is a critical antecedent of tertius iungens, whereas their followers' need to belong moderates the effect of founder's tertius iungens. We tested these hypotheses based on a two-wave and multi-sourced data from 101 Chinese entrepreneurial firms. Findings revealed that single founders' tertius iungens orientation is positively related to their firms' performance. Moreover, among firms with followers who are high on need to belong, there is an indirect effect of single founders' need to belong on firm performance through single founders' tertius iungens orientation.

(For more information, please contact: Liqun Wei, Hong Kong Baptist University, China: weiliqun@hkbu.edu.hk)

\section{Leadership Development Practices as Drivers of Attitudinal Outcomes Violetta Khoreva, Hanken School of Economics Dmitri Melkumov, Hanken School of Economics}

In this article, we examine the relationships among leadership development practices and behavioural outcomes such as affective commitment, acceptance of increasing performance demands and commitment to building competencies. Affective commitment is expected to mediate the relationship between leadership development practices and acceptance of increasing performance demands as well as between leadership development practices and commitment to building competencies. Our analysis of 330 high-potential employees in eight Nordic multinational corporations revealed that leadership development practices were positively related to acceptance of increasing performance demands, commitment to building competencies and affective commitment. We also found that affective commitment fully mediated both the relationship between leadership development practices and acceptance of increasing performance demands, and between leadership development practices and commitment to building competencies. The research and practice implications of the findings are discussed. (For more information, please contact: Violetta Khoreva, Hanken School of Economics, Finland: violetta.khoreva@hanken.fi)

Role of Personal, Social, and Contextual Variables in Employee Turnover under Continuous Change: An Investigation of Indian Outsourcing Industry Anju Mehta, University of Northern I owa

In today's highly dynamic business environment, adapting to change is a critical dynamic capability for a firm's survival and success. Therefore, managing change effectively is becoming increasingly important for managers. Organizational change has generally been associated with negative employee reactions and behaviors. The knowledge that change can be difficult and disruptive necessitates an understanding of the critical factors influencing employee outcomes under change. In this study, we examined the role of personal, social, and contextual variables in employee work outcomes under conditions of continuous change. One of the industries that faces constant change is the outsourcing industry. We collected data from 350 employees working in six outsourcing companies in India. Results indicated that personal variables were significant in predicting both 
primary and secondary outcomes. However, social and contextual variables had limited predictability for primary outcomes. Implications for research and practice, study limitations, and directions for future research are discussed. (For more information, please contact: Anju Mehta, University of Northern Iowa, USA: anju.mehta@uni.edu)

\title{
Caught Between Two Worlds: Subsidiary Managers and Executive Attention Dwarkaprasad Chakravarty, I vey Business School
}

Executive attention is known to be a scarce resource. MNE subsidiary managers responsible for revenue generation often need to attract attention from their corporate headquarters executives as well as from local market customer and partner executives. We examine how such executive attention may be shaped by both intrinsic characteristics as well as the agency of subsidiary managers. We also investigate the nature and efficacy of attention shaping subsidiary manager practices. We draw upon theories of attention, ecology, institutions, and contingency to frame conceptual arguments and propositions. Our conceptual framework also considers how competition, cross national distance, subsidiary performance, and executive characteristics contribute to shaping the relationship between subsidiary manager characteristics, attention shaping practices and executive attention. (For more information, please contact: Dwarkaprasad Chakravarty, Ivey Business School, Canada: dchakravarty.phd@ivey.ca)

\author{
Employee's Perceptions at Post M\&A Integration: Changing Perceptions of Interpersonal and Procedural Justice \\ on Organizational Identification \\ Ralf Bebenroth, Kobe University \\ Nir Kshetri, University of North Carolina at Greensboro \\ Tomoki Sekiguchi, Osaka University
}

This study sheds light on how employees perceive organizational change after a cross-border acquisition. Specifically, we tested how interpersonal and procedural justice influence organizational identification in target organizations. We extend previous findings from the literature, that organizational justice positively influences organizational identification, precisely that procedural justice matters more at an earlier stage of postacquisition integration. In contrast, Interpersonal justice is more salient in its later stage. We also find evidence that there is a mutual reinforcement of justice criteria at the integration period. I mplications and future research directions are discussed. (For more information, please contact: Ralf Bebenroth, Kobe University, Japan: rbeben@rieb.kobe-u.ac.jp)

\section{Leadership in Tight and Loose Cultures: A Conceptual Exploration Rakesh Mittal, New York Institute of Technology}

Impact of culture on leadership processes is of interest to both scholars and practitioners, particularly in the context of an increasingly globalizing world. One of the important views of culture is the notion of cultural tightness-looseness that denotes the pervasiveness of social norms in a society and how strictly they are enforced. Concept of tight and loose cultures links external societal constraints with individuals' psychological processes. Therefore, it is particularly salient to a discussion of effective leader behavior, which is enacted at individual level in a particular cultural context. But cross-cultural leadership research so far has not focused on this manifestation of culture. This paper is a conceptual examination of the linkage between cultural tightnesslooseness and various leadership behaviors. Several propositions are offered, to posit how tightness or looseness of a society might impact the acceptability of different leadership dimensions that have been established in the leadership literature. For example, it is posited that Charismatic/value-based leadership will be viewed as contributing to outstanding leadership more in loose cultures than in tight cultures. On the other hand, Autonomous leadership would be viewed as contributing to outstanding leadership more in tight cultures than in loose cultures. Theoretical and practical implications are discussed. (For more information, please contact: Rakesh Mittal, New York Institute of Technology, USA: rmittal@nyit.edu) 


\title{
Session: 2.4.11 - Interactive \\ Track: 9 - Emerging Economies and Emerging Economy MNEs
}

\section{Cultural Diversity}

Presented On: J une 29, 2015 - 14:30-15:45

Chair: Vijay Edward Pereira, University of Portsmouth

\author{
A Dialectical Process View of Cultural Diversity and Geographic Diversity for Emerging Market MNES \\ Yadong Luo, University of Miami \\ J uan Bu, University of Miami \\ Vladislav Maksimov, University of Miami
}

We investigate input states, outcome states, and necessary conditions in the process by which diversity improves performance in emerging market multinational enterprises (EM MNEs). We focus on geographic diversity and cultural diversity and acknowledge their dialectical nature in bringing about both opportunities and adversity. We argue that (1) EM MNE's goal diversity triggers greater geographic or cultural diversity, (2) geographic, but not cultural, diversity has a direct positive performance effect, and (3) generating greater positive net gains from cultural or geographic diversity requires enabling mechanisms such as strategic resilience and integration capability. Our analysis of 199 EM MNEs generally supports these arguments and also suggests that geographic diversity is more amenable to the above forces than cultural diversity. We draw important implications for the relationship between diversity and MNE performance, as well as for managers of EM MNEs. (For more information, please contact: Juan Bu, University of Miami, USA: j.bu@umiami.edu)

\section{Substitutes or Compliments: Relationship-Based Strategy and Innovation Strategy of Private Firms in China Xiaoyu Zhou, ShanghaiTech University \\ Daomi Lin, Sun Yat-sen University \\ Song Wang, Zhejiang University}

Based on the institution-based view, this paper examines the interrelationship between relationship-based strategy and innovation strategy, and the circumstances under which relationship-based strategy and innovation strategy are more likely to be substitutive or complementary in the context of institutional transition. Using data based on a series of nationwide surveys conducted on privately owned firms in China, we find that firms that are engaged in relationship-based strategy are more likely to adopt innovation strategy. This complementary relationship is strengthened by the market supportive institution and mature infrastructure, but weakened by firm state-dominated legacy constraints. We also find that firm slack resources have significant impacts on firm institution-based strategy. (For more information, please contact: Xiaoyu Zhou, ShanghaiTech University, China: zhouxy@shanghaitech.edu.cn)

International Diversity and Performance in Knowledge Intensive Indian Firms: Moderating Role of 'Speed' and 'Cultural Distance'

Naveen K. Jain, University of Akron

Debmalya Mukherjee, University of Akron

Lin Yuan, University of Macau

Multinationality and performance (M-P) relationship has generated mixed empirical results, with conclusions ranging from linear (positive or negative) to non-linear (U- or S-shaped). Thus, scholars have called for more finely-grained research by exploring the boundary conditions of this relationship. This is especially true for emerging market firms (EMF) that may follow a different internationalization path than developed-country firms. We explore 'internationalization speed' and 'added cultural distance' as moderators influencing the process of 
aggressive internationalization of EMFs. Our findings based on 650 international location choices made by 32 large software companies from India, suggest that speed of internationalization positively moderates the M-P relationship in the case of knowledge-intensive services EMFs, and that the positive relationship is stronger for firms in the later stage of internationalization. We also find support of the "performance-enhancing" argument of cultural distance as a moderator and suggest that MNEs are often able to enter culturally distant markets because of the numerous organizational advantages their foreign operations can provide. (For more information, please contact: Debmalya Mukherjee, University of Akron, USA: dmukher@uakron.edu)

\section{Explaining Differences in Developing Countries' Compliance to Trips: Effect of Local vs. Foreign Firms and Supra-National Institutions \\ Kristin Brandl, University of Reading \\ Izzet Darendeli, Temple University \\ Robert D. Hamilton III, Temple University}

We study the different approaches of developing countries to fully comply with TRIPS and consequently secure intellectual property standards in the countries domestic innovation systems. We question the impact of local firms, foreign firms and supra-national institutions on this compliance. Though a study of ratification periods of 60 developing countries (from 1995 to full compliance) we are able to conclude that the pressure of foreign firms in developing countries results a faster TRIPS compliance, especially when supra-national institutions impact the country; domestic firms pressure for a slow TRIPS compliance independent from the impact of supra-national institutions. (For more information, please contact: Kristin Brandl, University of Reading, United Kingdom: kristin.brandl@henley.ac.uk)

\section{A Multi-level Conceptualization and Empirical Examination of Reputation Risk in Latin America William Newburry, Florida International University}

Reputation Risk refers to the risk of a devalued reputation arising from negative perceptions on the part of a multitude of firm stakeholders, such as customers, potential employees, shareholders, investors or regulators. However, while reputation risk is being increasingly recognized as an important risk that firms face, our understanding of the factors that contribute to this risk are limited. Drawing on the social-constructionist view of reputation, within this paper, we develop theory based on factors impacting information availability regarding companies at multiple signal levels and test some of the most important sources of reputation risk stemming from firm-, industry- and country-level factors in the context of Latin America. (For more information, please contact: William Newburry, Florida International University, USA: newburry@fiu.edu)

\section{Cultural Heterogeneity in the Emerging Economies and Its Effects on Foreign MNES: Conceptualizing an} Aggregate Culture Model to Understand the Cultural Differences Between Tier 1 and Tier 2 Cities Komal Kiran Kalra, University of Victoria

J ust like the global economy is segregated into developed, emerging (developing) and underdeveloped economies, the various regions within the emerging economies can also be categorized as developed, developing and underdeveloped. Emerging economies like India, China and Brazil are culturally heterogeneous and there are some regions which are more economically developed than the others. Multinational Enterprises have been exploring new regions within the emerging economies and it is important to understand the cultural differences between these regions. By introducing the concept of Tier 1 and Tier 2 cities, I would explore the cultural differences between the developed and developing cities in India (a perfect example of a culturally heterogeneous country). Using case studies and ethnography, I would analyze how such variations affect a Multinational's organizational culture, and also try to understand how aggregate cultural models could be conceptualized to accommodate subnational variance. (For more information, please contact: Komal Kiran Kalra, University of Victoria, Canada: komal@uvic.ca) 


\title{
Session: 2.4.12 - Interactive
}

\section{Track: 10 - Theories of the MNE and of FDI}

\section{Motives, Choices and Consequences in Foreign Direct I nvestments}

\author{
Presented On: June 29, 2015 - 14:30-15:45 \\ Chair: Alvaro Cuervo-Cazurra, Northeastern University \\ Institutional Arbitrage as Motive for Outward FDI \\ J ohn Manuel Luiz, University of Cape Town \\ Helena Barnard, GIBS, University of Pretoria
}

With a few exceptions, notably Witt and Lewin (2007), the assumption in international business research has tended to be that firms experience the institutional context of the home country as positive or at least neutral. Therefore the well-known motives for internationalization are pull factors in the host country: The availability of natural resources, markets, greater efficiencies or created assets. Those motives do not take into account how the home country conditions may shape an interpretation of the attractiveness of a foreign market. Yet outward FDI (OFDI) can be motivated not only by the attractiveness of a given host location, but also by conditions in the home country. We provide empirical evidence of OFDI as a form of institutional arbitrage. In particular, we examine institutional arbitrage that occurs when OFDI to locations that are perceived to be stable increases when the home country suffers instability. Our findings suggest an important alternative explanation for OFDI from less to more developed countries - home country instability rather than firm capabilities. We discuss the usefulness of institutional arbitrage as motive for FDI, and how the notion of institutional arbitrage relates to the differentiated MNE network, to locational attractiveness, and to firm strategy. (For more information, please contact: John Manuel Luiz, University of Cape Town, South Africa: john.luiz@gsb.uct.ac.za)

\section{The Chicken or the Egg? Migrants and Foreign Direct Investment: an Exploratory Study Pallavi Shukla, Rutgers University \\ J ohn Cantwell, Rutgers University}

The purpose of this study is to examine the extent to which migrants in a host country exert a gravitational pull on investments of multinational firms of their country of origin (COO). More importantly, we examine whether the patterns and the relationship between migration and investment of multinational firms from high-income countries is different from those of low-income countries in a host country. To this end, we use panel data to examine (i) the effect of prior migrant stock on the inward FDI stock of foreign firms and (ii) the effect of prior FDI stock on the migrant stock in the United States. We find that presence of migrants from both high- and low-income countries is a predictor of future inward FDI (into the host country) by firms from migrants' countries of origin and that this effect is stronger for high-income countries. Second, we find that prior FDI from countries (into the host country) is a significant predictor of future people flows specifically from low-income countries, suggesting migration of labor through organizational pipelines for low-income countries as opposed to high-income countries. (For more information, please contact: Pallavi Shukla, Rutgers University, USA: pallavi.shukla@rutgers.edu)

Entry-Mode and Growth Perspectives in the Offshoring of Business Services: Learning or Inertia? Filippo Albertoni, Politecnico di Milano

Stefano Elia, Politecnico di Milano

Lucia Piscitello, Politecnico di Milano

Most of the research on entry-mode has been focussed on transaction costs, strategic resources, and institutional and cultural distances. Several studies have recognized the role of previous experiences in 
international business processes and the interdependence of entry-mode choices; however, the existing literature does not indicate wether companies grow when they iterate their choices, and it has traditionally attributed the replication of previous choices to the need of reducing uncertainty and transaction costs. We argue that this strategic behaviour might be linked with companies locked-in in the blind self-imitation and replication of routines that is incapable to lead to any business expansion. Conversely, some companies are able to learn from their previous experiences and foster their own growth. Using data from the Offshoring Research Network, this paper tests when companies iterating their entry choices grow. We find that the ritualistic imitation of previous choices does not increase the probability of growth of the present offshoring initiative; conversely, the adoption of a strategy fostering learning and developing knowledge over time leads to expansion. (For more information, please contact: Filippo Albertoni, Politecnico di Milano, Italy: filippo.albertoni@polimi. it)

\section{Where do MNEs Locate their Foreign Direct Investments? An Assessment of the Empirical Literature Bo Bernhard Nielsen, University of Sydney \\ Christian Asmussen, Copenhagen Business School}

Most studies of FDI are aggregated at country or regional level and shows, historically, whereto (i.e. which country or region) FDI flowed with little focus on the underlying determinants rendered important by MNES making these investments. Such ex post studies mask the relative importance of factors at macro, micro and firm levels that play a role in FDI location choice. Based on a systematic review of the extant literature, we integrate insights from urban economics and economic geography with international business literature to provide a better understanding of the multi-faceted drivers of location choice for FDI. We review underlying theories and empirical evidence of agglomeration, institutions, global cities and firm-specific advantages to expose a complex interplay between characteristics at firm and (micro and macro) locational levels that may determine why certain firms locate particular types of international activities in specific micro-locations. By synthesizing the theoretical rationales and empirical evidence for MNE location choice, we offer potential new directions for FDI location research. (For more information, please contact: Bo Bernhard Nielsen, University of Sydney, Australia: b.nielsen@econ.usyd.edu.au)

\section{Success of Fast Foreign Direct Investment Expansion Se Ho Cho, Rutgers Business School}

Conventionally, international business researchers have paid attention to incremental internationalization. They assumed that as firms learned more knowledge and information of foreign markets, such firms could reduce uncertainty and the risk of failure. However, dynamic environment and high competition requires firms to move quickly against competitors. In particular, late movers should engage in fast foreign direct investment (FDI) expansion to catch up to advanced technologies and competitive advantage of the first or earlier movers. Born global firms quickly internationalize due to their high technologies. In this sense, the importance of fast FDI expansion becomes a firm's essential strategy. Despite the growing importance of fast FDI expansion, there is a lack of literature on this topic. Therefore, this study attempts to find circumstances and conditions for firm success in fast FDI expansion. This article studies not only the importance of fast FDI expansion on firm performance, but also under what conditions this type of FDI is necessary, such as less time of learning in foreign markets. Multi-level variables, such as, team, firm strategy, and macroeconomic levels are discussed as moderators for the relationship between speed of FDI expansion and firm performance. Theoretical and practical implications are discussed in the conclusion. (For more information, please contact: Se Ho Cho, Rutgers Business School, USA: shcho1981@gmail.com) 
Country-Of-Origin, Government-Based and Consumer-Based Advantage and Disadvantage of Foreignness, and Foreign Investment

Alvaro Cuervo-Cazurra, Northeastern University

C. Annique Un, Northeastern University

We analyze how a firm's country of origin affects its investments abroad. Integrating the resource-based view and the country-of-origin literature to view the country of origin as a resource, we explain the advantage (disadvantage) of foreignness, i.e., the relative benefit (liability) a subsidiary of a foreign firm enjoys over domestic firms when its country of origin is liked (disliked) by individuals in the host country. Separating government-based from consumer-based advantage and disadvantage, we propose that whereas the government-based advantage induces the firm to invest more to benefit from incentives, the consumer-based advantage induces the firm to invest less to maintain foreignness; whereas the government-based disadvantage induces the firm to invest less to reduce risks, the consumer-based disadvantage induces the firm to invest more to localize. (For more information, please contact: Alvaro Cuenvo-Cazurra, Northeastern University, USA: a.cuervocazurra@neu.edu)

\section{Session: 2.4.13 - I nteractive}

Track: 4 - International Marketing, Cross-Cultural Marketing, Social Communities

\section{Digital Marketing in the Global Marketplace}

Presented On: June 29, 2015 - 14:30-15:45

Chair: Susan Mudambi, Temple University

Consumer Engagement with Firms through Social Media: A Comparative Study

Constanza Bianchi, Universidad Adolfo Ibanez

Andres Cuneo, ESADE Business School

Lynda Andrews, Queensland University of Technology

Social media has reached global proportions, yet little is known about consumer's engagement with firms through these technological platforms in different countries. This research addresses this limitation and examines Chilean and Spanish consumers' intentions to engage in social media platforms drawing on the theory of reasoned action as a theoretical model. Specifically, this study develops and tests a model of consumer attitude and engagement with firm Facebook pages. The findings provide an understanding of the main drivers of social media engagement for consumers in two different countries. (For more information, please contact: Constanza Bianchi, Universidad Adolfo Ibanez, Chile: constanza. bianchi@uai.cl)

\section{Can Social Media Marketing Improve Customer Relationship Capabilities and Firm Performance? Dynamic Capability Perspective \\ Zhan Wang, Saint Louis University \\ Hyun Gon Kim, Rutgers Business School}

Social media are becoming ubiquitous and need to be managed by organizations to meet their strategic goals. Businesses are finding it necessary to modify their approach toward Customer Relationship Management (CRM) and develop new marketing capabilities that facilitate customer satisfaction. The purpose of this study is to explore and examine how social media marketing usage impacts firm performance through improving marketing adoption strategies and increasing marketing assets and capabilities. This study will contribute to existing literatures by connecting firms' social media marketing with business performance by arguing that social media usage can improve firms' customer satisfaction and social CRM capabilities based on Resource-Based View (RBV) and dynamic capabilities theory. (For more information, please contact: Zhan Wang, Saint Louis University, USA: zhanwang@s/u.edu) 
Turning Complainers into Fans: Customer Complaint Handling in Multi-Channel Marketing

Alexander Rossmann, Reutlingen University

Kumar Rakesh Ranjan , Indian Institute of Management Tiruchirapalli

Praveen Sugathan, Indian Institute of Management Bangalore

The emergence of social media as a prominent alternative and useful complement to the traditional channels of service delivery motivates this research to contribute to the understanding of firm's multi-channel distribution strategy in two ways: a) conceptualize complaint handling quality across traditional and social media channels, and b) test the impact of channel type on key marketing outcomes like customer loyalty, positive word-ofmouth, and cross-purchase intentions. The theoretical framework comprising these relationships is tested against data collected from two different channels (hotline and social media) of a German telecommunication service provider to offer insights into tractable channel migration metrics. (For more information, please contact: Praveen Sugathan, Indian Institute of Management Bangalore, India: praveen.s@iimb.ernet.in)

\section{The Influence of Green Word-of-Mouth (WOM) on Green Purchase Intentions: The Mediation Effects of Consumers' Conformity Behaviors Sheng-Hsiung Chang, Tamkang University}

The paper aims to explore not only the influence of green word-of-mouth (WOM) on green purchase intentions, but also the mediation roles of green consumers' informational interpersonal influences and normative interpersonal influences. The research setting of this study is conducted on Taiwanese consumers who had the purchase experience of green products. This research employs an empirical study by means of the structural equation modeling. The empirical results suggest that green WOM is positively related to consumers' green purchase intentions, and equally important, consumers' informational conformity behavior and normative conformity behavior play as the role of mediators. Thus, this study suggests that companies must strengthen their green WOM reputations to enhance consumers' purchase intentions. In addition, this study also expects to contribute to the literature that consumers' conformity behaviors are important factors in the green word-ofmouth and green purchase intentions relationship. (For more information, please contact: Sheng-Hsiung Chang, Tamkang University, Taiwan: shchang@mail.tku.edu.tw)

\section{Can the Internet Help SMEs in Reducing Export Barriers? \\ Gianpaolo Baronchelli, University of Bergamo \\ Sara Castelnuovo, Clifford Chance Pte Ltd \\ Valeria Caviezel, University of Bergamo \\ Antonio Majocchi, University of Pavia}

Exporting is one of the oldest and most common setup of economic activity used by firms to start doing business in a foreign market. Notwithstanding companies still face various impediments during their export activities that could reduce export performance: e.g. lack of information, custom duty, transport and documentation, payments (Bennet, 1997,Leonidou, 2000, 2010). Several researchers, (Hamill and Gregory, 1997; Sinkovics et al., 2013) argued that an effective use of the Internet could provide a low-cost 'gateway' to international markets. Our research on 137 firms from I talian organic sector shows that the Internet is a strategic mean for companies operating in foreign markets. At same time, through the use of clustering technique - with VOS viewer software - we find that firms' international experience - in terms of number of markets where the companies are exporting - could improve the impact of the use of the Internet in companies' internationalization strategy. (For more information, please contact: Gianpaolo Baronchelli, University of Bergamo, Italy: gianpaolo.baronchelli@unibg.it) 
A Study of Television Advertising in Brazil

Marin Alexandrov Marinov, Aalborg University

Svetla Trifonova Marinova, Aalborg University

Dan Alex Petrovici, University of Kent

John B. Ford, Old Dominion University

More research is needed to shed light on how and why ad avoidance happens so that advertisers can enhance the chances of their media being processed and having a significant effect upon viewer brand recall and preference. The purpose of this study to examine the perceptions of viewers in an important emerging economy, Brazil, with the hope of better understanding perceived advertising intrusiveness and advertising avoidance. Based on reactance theory a model investigating perceived intrusiveness and ad avoidance is developed. Eleven hypotheses are developed and tested based on research conducted in two of the largest Brazilian cities. Research findings are discussed, managerial implications drawn, study limitations addressed and avenues for future research indicated. (For more information, please contact: Marin Alexandrov Marinov, Aalborg University, Denmark: marinov@id.aau.dk)

\section{Session: 2.5.1 - Special Session}

\section{Challenges Confronting Emerging Market I B Educators (A/B Fellows Educator of the Year Session)}

Presented On: June 29, 2015 - 16:15-17:30

Chair: Sushil Vachani, IIM Bangalore

Challenges Confronting Emerging Market IB Educators (AIB Fellows Educator of the Year Session)

Nick Binedell, GI BS Business School

Nakiye Avdan Boyacigiller, Sabanci University

Sushil Vachani, IIM Bangalore

Vidya Yeravdekar, Symbiosis International University

Bernard Yeung, National University of Singapore

\section{Session: 2.5.2 - Competitive}

\section{Track: 5 - The Geography of I nternational Business and Global Value Chains}

\section{Networks and Economic Geography}

Presented On: J une 29, 2015 - 16:15-17:30

Chair: Snehal Suyash Awate, Indian School of Business

\section{Economic Geography and Networks: Role of Local and Non-Local Ties in Cluster Evolution \\ Florian Täube, Université libre de Bruxelles \\ Petra Sonderegger, independent}

The organization of geographic clusters plays an increasingly significant role due to the presence of network ties that exist within the location and beyond. This has proven to be particularly true for knowledge-intensive industries, where the organization of resources - people and technology - has been a primary driver for firm and regional performance. With the help of a longitudinal case study of the IT cluster in Bangalore (India), we investigate the effect of local and non-local network ties on its evolution. We argue that local and non-local networks play a clear role in cluster evolution. We propose a U-shaped relationship between cluster evolution phases and the distance among the network tie members. Our study also outlines the role that embedding, expansion, and extension of ties plays in transitioning cluster from one phase to the other. The consideration of 
non-local ties is rather nascent in the cluster literature and promises to enhance the understanding of how clusters develop at both levels - policy as well as firm (For more information, please contact: Florian Täube, Université libre de Bruxelles, Belgium: ftaube@ulb.ac.be)

\section{The Geographical Dispersion of Inventor Networks in Peripheral Economies Marcelo F Cano-Kollmann, Temple University \\ Ana Teresa Tavares-Lehmann, University of Porto}

In this paper we explore the dispersion of inventor networks in peripheral economies, a topic that has received relatively little attention in the literature. As global value chains fragment into geographically dispersed activities, opportunities arise for peripheral economies to participate in these processes of innovation. However, different types of knowledge creation have distinct network properties. More codifiable innovative activities can be carried out through collaboration by internationally dispersed teams. On the other hand, activities that are more dependent on tacit knowledge are more likely to require the co-location of knowledge workers. This implies that, for peripheral economies, innovation that relies mostly on tacit knowledge will provide the local innovation system with limited connectivity benefits. We hypothesize that, while this is generally true, "leading" innovative multinational enterprises may possess more sophisticated capabilities for transnational collaboration than less innovative firms. Therefore, innovation in activities involving tacit knowledge may show different network characteristics depending on who performs them: leaders or "laggards". Our results, based on two European peripheral economies, are consistent with our hypotheses. (For more information, please contact: Marcelo FCano-Kollmann, Temple University, USA: mck@temple.edu)

\section{The Changing Geography of Value Creation: Evidence from Mobile Telecommunications \\ Marcus M. Larsen, Copenhagen Business School \\ Timo Seppälä, Research Institute of the Finnish Economy \\ Jyrki Ali-Yrkkö, Research Institute of the Finnish Economy}

We explore through an innovative trade-in task case study of how Nokia-one of the historically most important mobile phone manufacturers in the world-disaggregated and geographically distributed the production of three mobile phones. We find that the value creation across the three studied models shows a rapid shift in tangibles and manufacturing as well as intangibles and design knowledge away from advanced economies to being captured in the emerging economies in which manufacturing occurs. Further, our evidence shows that this transfer has proceeded on a step-by-step basis - beginning with manufacturing knowledge and ending with the transfer of system knowledge - that enables the products to be developed and manufactured for the global market. We argue that these processes pose new requirements and challenges for contemporary MNCs. (For more information, please contact: Marcus M. Larsen, Copenhagen Business School, Denmark: mml.smg@cbs.dk)

\section{Trans-Local Connectedness and Local Cluster Dynamics \\ Ekaterina Turkina, HEC Montréal \\ Ari Van Assche, HEC Montréal}

Recent work points out that both local and trans-local linkages are important for an industrial cluster's economic prospects, yet data limitations have made it difficult to verify this claim. Taking this observation as a starting point, we have developed an original dataset that maps the network of local and trans-local firm linkages in three knowledge-intensive industries (aerospace, IT/Telecom and Biotech/Biopharma) across North American and European clusters over the period 2002-2014. The dataset further distinguishes between three types of firm ties: intra-firm investment linkages; inter-firm buyer-supplier linkages and inter-firm partnership linkages. Our dataset provides a rich array of new stylized facts that shed light on the link between a cluster's trans-local connectedness and its local dynamics. We also investigate how a cluster's position in the global cluster network affects its innovative performance. (For more information, please contact: Ari Van Assche, HEC Montréal, Canada: ari.van-assche@hec.ca) 


\section{Session: 2.5.3 - Competitive \\ Track: 9 - Emerging Economies and Emerging Economy MNEs}

\section{Internationalization and Performance}

Presented On: June 29, 2015 - 16:15-17:30

Chair: Amit Karna, Indian Institute of Management Ahmedabad

Internationalization Scope, Context, Time, and Performance - Extending the Research Agenda to Multilatinas Luciano Ciravegna, King's College London

Mauricio Melgarejo, INCAE

Luis Lopez, INCAE

This paper tests the effects of internationalization on the performance of multilatinas. The paper integrates the insights of studies of regional internationalization with those of the institutional perspective, examining the effects of time, political risk, and regional strategy on the performance of 454 firms based in four Latin American economies between 1995 and 2012. The findings show that the performance of internationalized firms improves over time, and that firms expanding regionally perform better. (For more information, please contact: Luciano Ciravegna, King's College London, United Kingdom: Iuciano.ciravegna@kcl.ac.uk)

The Role of Dynamic Capabilities in Enhancing the Performance Effects of Internationalization of Emerging Market Firms: A Study of Indian Firms

Anish Purkayastha, Indian Institute of Management Ahmedabad

Amit Karna, Indian Institute of Management Ahmedabad

Sunil Sharma, Indian Institute of Management Ahmedabad

Dhiman Bhadra, Indian Institute of Management Ahmedabad

Based on the marginal benefits and marginal costs arising out of internationalization, its overall effect on performance is widely accepted to have a horizontal S-curve shape: the relationship is negative at low degree of internationalization, then positive and finally again negative at high degree of internationalization. The relationship has been tested across different empirical settings including on firms from emerging markets, where the strength of the relationship differed, primarily due to the lack of empirical evidence of firms with higher degree of internationalization. The weaker home-country institutional environment of emerging market firms makes it more difficult to overcome the initial negative performance attributed to internationalization. In this paper, we argue that with the help of dynamic capabilities such as sequential ambidexterity and strategic flexibility, the emerging market firm is able to improve the positive effects of internationalization. We test this hypothesis using data on internationalization of 846 Indian firms between 1995 and 2014. Our results support the horizontal S-curve relationship including the high internationalization with a negative performance effect. We also find support for a moderating role of dynamic capabilities (combined effect of sequential ambidexterity and strategic flexibility) as enhancers of performance effects of internationalization. (For more information, please contact: Amit Karna, Indian Institute of Management Ahmedabad, India: karna@iimahd.ernet.in)

\section{Relationship between Internationalization and Innovation in Emerging Economy Firms Pooja Thakur Wernz, Virginia Tech University \\ Shantala Samant, Virginia Tech University}

Emerging economy multinationals (EMNEs) are increasingly internationalizing their operations as well as moving from imitation towards innovation. We study the impact of internationalization on innovation in the context of EMNEs. Specifically, we propose a curvilinear relationship between outward foreign direct investment and patenting activity of these emerging economy firms. Data on Indian biopharmaceutical firms from 1997 to 2013 
is used to empirically test our hypotheses. Our sample consists of all biopharmaceutical firms that have previously established at least one foreign subsidiary and patented with either the Indian Patent Office or the United States Patent and Trademark Office (USPTO). Controlling for firm specific characteristics such as firm size and $R \& D$ expenditure, we find that innovation decreases in the initial stages of internationalization. However with increased internationalization, the impact of international expansion on innovation becomes positive. In the third and final stage of internationalization, the costs of international expansion outweigh the benefits and the impact on innovation becomes negative. We contribute to the literature on EMNEs which has primarily focused on the internationalization of these firms. As these firms begin to innovate, it is important to study the impact of internationalization on their ability to innovate. (For more information, please contact: Pooja Thakur Wernz, Virginia Tech University, USA: pthakur@vt.edu)

\section{Rapid Internationalization of Service Firms from an Emerging Market through Contextual and Strategic Lenses Naveen K. Jain, University of Akron Nitin Pangarkar, National University of Singapore Lin Yuan, University of Macau Vikas Kumar, University of Sydney}

In this study, we examine the drivers of internationalization speed of I ndian software firms which have been known to exhibit high speed of internationalization even to culturally distant countries. Drawing from the LLL perspective proposed for EMNEs and supplementing it with the arguments from the absorptive capacity and organizational inertia perspectives, we argue for inverted $U$ shaped relationships between geographic scope and added cultural distance on the one hand, and speed of internationalization, on the other hand. We also argue for an interactive effect between added cultural distance and the regularity in adding cultural distance. Our analyses of internationalization patterns of 32 leading Indian software firms over the period 2000 to 2009 support all our key hypotheses. Our study provides a nuanced understanding of the internationalization by software firms and suggests that country and industry contexts are important in understanding internationalization. (For more information, please contact: Naveen K. Jain, University of Akron, USA: njain@uakron.edu)

\section{Session: 2.5.4 - Competitive}

\section{Track: 10 - Theories of the MNE and of FDI}

\section{Decision-Making When Going Internationally}

Presented On: June 29, 2015 - 16:15-17:30

Chair: Sumit Kumar Kundu, Florida International University

\section{Experiential Learning and Internationalization Paths of a Firm's Sourcing Activities} Carlos Adrian Rodriguez, INCAE

This article analyzes internationalization processes in a firm's sourcing activities. I expand Internationalization Process Model by using Performance Feedback Theory in order to explain subsequent sourcing of a firm's information-intensive activities, under in-house and outsourced entry modes. I suggest that, while the fulfillment of profitability aspirations affects geographical expansion across countries, it is the achievement of operational performance aspirations that explains the sourcing of additional activities within a country where the firm already operates. This study tests these relations in a dataset of offshoring projects undertaken by 230 firms in the period between 1995 and 2012. Overall, I find strong evidence of experiential learning effects on a firm's sourcing trajectory. Moreover, I conclude that, while the entry mode (i.e. in-house, outsourced) modifies the interpretation of profitability aspirations for geographical expansion, the achievement of operational performance aspirations positively influences functional expansion within a country. (For more information, please contact: Carlos Adrian Rodriguez, INCAE, Nicaragua: carlos. rodriguez@incae.edu) 


\author{
Risk Dynamics And Firms' Decision-Making Ininternational Entry Mode \\ Yanbin J iang, Zhejiang University \\ Kun Fu, Imperial College \\ Shaheen Akter, TMCD, ODID, University of Oxford \\ Jing Li, Zhejiang University \\ Xiaobo Wu, Zhejiang Universit
}

This paper explores the impact of risk dynamics on firms' decision about choices of foreign entry mode. It explains how we can better understand this decision by explicitly incorporating the dynamics of political risk in countries. Further, we employ a behavioral theory of firms to investigate how experience and macro environment moderate the relationship between political risk trend and entry mode decision-making. We draw evidence from 775 foreign investment cases made by China firms in overseas markets. Research findings revealed that firms chose entry modes based on changes in rather than static level of political risk in a host country. Specifically, firms are more likely to choose entry modes that render better control, e.g., WOSs (wholly owned subsidiaries) when there is a reduction in political risk in a host country. Otherwise, they prefer entry modes that bring complementary resources, e.g., JVs (joint ventures). (For more information, please contact: Yanbin Jiang, Zhejiang University, China: yanbinjiang@126.com)

\title{
SME'S International Market Entry: How do They Make Decision? \\ Gianpaolo Baronchelli, University of Bergamo \\ Olli Kuivalainen, Lappeenranta University of Technology \\ Ali Ahi, Lappeenranta University of Technology \\ Mariella Piantoni, University of Bergamo
}

Making the right decision on international market entry mode is of utmost importance to an internationalizing firm. However, there is a lack of analysis concerning the actual decision making process, specifically with regard to internationalization of Small and Medium-sized Enterprises (SMEs). Accordingly, based on four decisionmaking process (DMP) models, namely, reactivity, incrementalism, bounded rationality, and real options, we study the DMP of SMEs on international market entry mode and the subsequent change in strategy. In addition, we explore the factors affecting the use of a certain entry mode decision-making approach and seek to find out how the decision-making process adopted by the firms affect their international market development strategy. To do so, we conduct a qualitative study of six SMEs from Finland and I taly, taking into consideration their DMP and internationalization strategies. Our findings indicate that SMEs that adopt a more rational process in their decision-making are more likely to succeed in foreign market. (For more information, please contact: Gianpaolo Baronchelli, University of Bergamo, Italy: gianpaolo.baronchelli@unibg.it)

\section{The Determinants of Strategic Asset Seeking Foreign Expansion of Emerging Economy Firms \\ Faisal Ahsan, I ndian I nstitute of Management Lucknow \\ Ashutosh Sinha, Indian Institute of Management Lucknow}

It has now been well developed in international business literature that emerging economy firms' foreign expansion is motivated to seek strategic assets also. However, there is limited research on the factors that shape up the strategic asset seeking motive of foreign expansion. In this study, using a multi-theoretic approach we study firm level, industry level and institutional level factors that influence the strategic asset seeking behavior of emerging economy firms. Using a sample of 415 I ndian firms, comprising both manufacturing and service firms, we find that a firm's technological capability, ownership structure; its exposure to foreign competition and pro-market institutional reforms influence the motive for foreign expansion.

(For more information, please contact: Faisal Ahsan, Indian Institute of Management Lucknow, India: fpm12005@iiml.ac.in) 


\title{
Session: 2.5.6 - Competitive
}

\section{Track: 12 - The I nstitutional and Political Environment of I B}

\section{The Role of the State and Corruption}

\author{
Presented On: June 29, 2015 - 16:15-17:30
}

Chair: Michael A. Sartor, Queen's School of Business

Do State-Owners Facilitate or Constrain Corporate Strategies of Internationalization? The Role of Institutions Bo Bernhard Nielsen, University of Sydney

Saul Estrin, London School of Economics

Klaus Meyer, CEIBS

Sabina Nielsen, Copenhagen Business School

National institutions shape the ability of civil society and minority shareholders to monitor and influence insider decision-makers in state owned enterprises (SOEs), and thereby SOEs' strategies of internationalization. We argue that if such controls are weak, state owners choose self-aggrandizement over profit maximization and these tend to be predominantly domestic.Yet, with more effective controls, SOEs choices will be more similar to private owned firms with respect to internationalization. We conceptualize the institutions enabling control over firms by developing Williamson's hierarchy of institutions from informal via formal to governance. Based on a matched sample of 153 majority state owned and 153 majority privately owned firms from 40 different countries, we confirm our hypotheses. Our findings suggest that if home country institutions enable effective control, the internationalization strategies of SOEs and POEs converge. (For more information, please contact: Bo Bernhard Nielsen, University of Sydney, Australia: b.nielsen@econ.usyd.edu.au)

\section{SOE Internationalization and Resource Dependence: The Case of Vale Suzana Rodrigues, Erasmus University \& FUMEC University Marleen Dieleman, National University of Singapore}

We advance the IB literature in the area of SOE internationalization by investigating it from the perspective of resource dependence. We show that SOE dependence on home governments drives strategy, and affects its internationalization. Although internationalization can be a form of "escape" from home government constraints, our study suggests it is a double-edged sword because internationalization also alters the power balance between SOE and home government and provides rationales for the government to increase its control. Utilizing a longitudinal case of Vale from Brazil we analyze how shifts in government - SOE dependences affect the latter's internationalization trajectory over time, and generate new theoretical insights on dynamics of SOE internationalization. (For more information, please contact: Suzana Rodrigues, Erasmus University \& FUMEC University, Brazil: srodrigues@rsm.nl)

The Law, Corruption and Reinvestment Decisions: The Transitional Periphery in Comparative Context Mehmet Demirbag, University of Strathclyde Martina McGuinness, University of Sheffield Geoffrey Wood, University of Warwick

A range of studies has found that corruption has a significant impact upon FDI decisions, however to date there has been scant investigation into longer term investments made by firms and relative proclivity to reinvest. Further, there is particularly little work on reinvestment choices on the transitional periphery of post-state socialist countries and how this might differ from the more stable transitional economies of central and Eastern Europe. Utilising 2005 World Bank Enterprise Survey data, this study explores the relationship between corruption and MNEs' strategic decision to reinvest profits. From an institutionalist starting point, we find 
variation in the impact of different dimensions of corruption upon reinvestment; pervasive corruption impacts negatively upon reinvestment, but its effects are more pronounced in the transitional periphery. Perceived robust legal institutions have a positive correlation with reinvestment, but again, the negative effects are most pronounced on the transitional peripheral state socialist states. We ascribe this disparity to greater institutional fluidity, and explore why this context has particularly adverse effects. Finally, we find that firm level attributes of increased size and age are important factors in positive reinvestment decisions, appearing to mitigate the worst consequences of this fluidity. (For more information, please contact: Mehmet Demirbag, University of Strathclyde, United Kingdom: mehmet.demirbag@strath.ac.uk)

\section{Investing in Bribes: Role of International Anti-Corruption Regulations \\ Srividya J andhyala, ESSEC Business School \\ Fernando Oliveira, ESSEC Business School}

International institutions that transcend national borders are increasingly governing multinational firms' global transactions. We focus on international anti-corruption regulations to analyze if pressures from such institutions will lower the bribing probability of multinational and domestic firms in host countries. In contrast to prior literature examining this issue, we build a formal model that explicitly accounts for the competitive interactions among multinational and domestic firms. These interactions shape the available rents and returns from bribing. Our model indicates that the presence of multinational firms decreases competition for rents among both types of firms. As punishment costs for multinational firms increase, the bribing probability of domestic firms increases. Further, as the proportion of multinational firms increases, both domestic and multinational firms have a higher probability of bribing. Implications for institutional design and the role of conjectures about firm behavior in lowering corruption levels are discussed. (For more information, please contact: Srividya Jandhyala, ESSEC Business School, Singapore: srividya.jandhyala@essec.edu)

\section{Session: 2.5.7 - Competitive \\ Track: 2 - Entrepreneurship, Micro-Multinationals, and International New Ventures}

\section{Capabilities Perspective to International Entrepreneurship}

Presented On: J une 29, 2015 - 16:15-17:30

Chair: Gary Knight, Willamette University

Microfoundations to the Capabilities Development and Deployment Activities in Firm Foundation and Early Internationalization

Jay Weerawardena, UQ Business School

Gillian Sullivan Mort, La Trobe University

Peter W. Liesch, University of Queensland

In the context of the foundation of new firms, we unravel how knowledge development and deployment activities are created and applied to facilitate early entry into overseas markets. Adopting a capabilities view and the microfoundations approach our focus is the interplay between dynamic and operational capabilities and the role individual founders play in these interactions. We find that complementary knowledge routines built by the founders provide the organization for building the new firm, and its early internationalization is driven by the capabilities introduced into the firm and how these capabilities are deployed for innovation to set in place the preconditions for early internationalization. (For more information, please contact: Peter W. Liesch, University of Queensland, Australia: p.liesch@business.uq.edu.au) 
Competence: Not Attributes but Understanding-Based and Task Accomplishment

Peter Lamb, La Trobe Business School

Peter W. Liesch, University of Queensland

J orgen Sandberg, UQ Business School

Drawing upon the internationalisation context, we present a new approach at defining competence in international business. In owner-managed small firms, we find that competence for internationalisation is defined by understandings of firm internationalisation and their interplay with the work done, not as firm-based or individual qualities that the entity-based explanations report. We uncover four qualitatively different forms of internationalisation competence: competence as envisioning, calculating, differentiating and narrating. We illustrate how each competence is organised into distinctive structures upon which the owner-managers rely in internationalising their firm. In contrast to the orthodoxy which treats competence as defined by two separate entities, the knowledge and skills possessed by the owner-manager on the one hand and the work to be done on the other, we conceptualise and demonstrate competence as relational and something one does. (For more information, please contact: Peter Lamb, La Trobe Business School, Australia: p.lamb@latrobe.edu.au)

\section{Entrepreneurial Initiative-taking and Improvising for Business Model Innovation: Subsidiary-Enabled Dynamic Capability for Cross-Divide Entry \\ Peter Ping Li, Copenhagen Business School \\ Yangfeng Cao, Copenhagen Business School \\ Jens Gammelgaard, Copenhagen Business School \\ Tony Fang, Stockholm University}

Business model innovation (BMI) plays a critical role in building up competitive advantages when medium-sized multinational enterprise (MMME) from advanced economies enter emerging economies given the sharp distinctions in the operating contexts between advanced and emerging economies as the two sides of global divide. Prior research has highlighted the salient role of foreign subsidiaries in the global network of multinational enterprise (MNE). However, little is known about how MNEs from advanced economies specifically facilitate BMI at the subsidiary level when operating in emerging economies, especially in the case of MMNEs. Adopting the method of comparative and longitudinal case study, we have tracked the specific processes of BMI among six Danish MMNEs' subsidiaries in China. We have proposed a theoretical framework with initiativetaking and improvising as two primary enablers for BMI via dynamic capability as their core mediator. This occurs at the subsidiary level in the special context of cross-divide entry from advanced economies to emerging economies, especially for the latter's mid-end market. This study contributes to the emerging research stream of international strategic entrepreneurship. (For more information, please contact: Peter Ping Li, Copenhagen Business School, Denmark: ppl.int@cbs.dk)

\section{Session: 2.5.10 - I nteractive \\ Track: 4 - International Marketing, Cross-Cultural Marketing, Social Communities}

\section{Marketing Challenges in Multinational Environments}

Presented On: June 29, 2015 - 16:15-17:30

Chair: Rakesh Mohan J oshi, Indian Institute of Foreign Trade

New $2{ }^{\circ} \mathrm{C}$ Era: Multinational Corporations' Proactive Strategies for Climate Change Actions

Su-Chuan Yen, National Chung Hsing University

Yu-Ching Chiao, National Chung Hsing University

Will multinational corporations (MNCs) have more proactive strategic management while dealing with climate change actions? Some empirical studies focused on organizational level resource-based view (RBV) indicate that 
the resources impact MNCs' financial performance by the MNCs' strategic decision making. Transaction cost economics (TCE) approach explicitly regards efficiency as the fundamental element in determining the nature of organization and the organizations' own substitutes for structuring efficient transactions when market fail. Building upon the above theories, this article attempts to fill the gap of studies on the relationships among RBV, natural resource-based view (NRBV) and proactive strategic management (i.e., investment on new facilities, resource innovation alliance and organizational restructuring). While confronting climate change actions, and MNCs with asset specificity (a characteristic of TCE) differ in their strategic management for climate change actions. (For more information, please contact: Su-Chuan Yen, National Chung Hsing University, Taiwan: dora@yourcarbon.com.tw)

\section{Nonlinear Relationships between $R \& D$, Firm Size and Corporate Social Responsibility Yu-Shu Peng, National Dong Hwa University Altan-Uya Dashdeleg, National Dong Hwa University}

Although there are a number of studies exploring the antecedents of corporate social responsibility, few of them pay attention to the nonlinear relationship between them. To fill the theoretical gap, this study particularly examines the nonlinear impacts of two firm-level factors (R\&D and firm size) on corporate social responsibility (CSR). We collect research data from 240 manufacturing firms in US and J apan during the period from 2003 to 2011. 2,160 firm-year observations were used to test the proposed hypotheses. The empirical findings confirm the inverted U-shaped relationship between firm size and CSR, and S-curve relationship between R\&D and CSR. The observed difference across US and Japan subgroups indicate that the institutional difference also plays an important role on the relationship. (For more information, please contact: Yu-Shu Peng, National Dong Hwa University, Taiwan: yspeng@mail.ndhu.edu.tw)

\section{A Comparative Study of Power of Large State-Owned and Private-Owned Sellers (Manufacturers) over Small Buyers (Distributors) under an Oligopolistic Industry Setting - The Case of the Steel Industry in China \\ Wei Liu, Manchester Business School \\ Claudio De Mattos, Manchester Business School \\ Nitin Sanghavi, Manchester Business School}

This paper investigates the differences between state-owned enterprises (SOEs) and private-owned enterprises (POEs) regarding the use of coercive and non-coercive power on smaller buyers (distributors) as well as the impact of countervailing power of distributors. We propose a model that has the generation of conflict and/or satisfaction as predicted outcomes. The model also incorporates the capability for conflict resolution from the smaller firms (distributors) that are driven by Chinese business culture (e.g., guanxi), trust and communication. Appropriate hypotheses are developed. (For more information, please contact: Wei Liu, Manchester Business School, United Kingdom: wei.liu-2@postgrad.mbs.ac.uk)

Female Business Travelers' Unsatisfied Wish List? An Empirical Study of the International Hotel Industry Katharina Maria Hofer, Johannes Kepler University Linz

Elfi Furtmueller, Innsbruck University

Nicola Graf, J ohannes Kepler University Linz

This study investigates female business travelers' unsatisfied needs in the international hotel industry. Building upon the gender-based and services marketing literature, we employ a qualitative research design for in-depth exploration of the topic. To date the concept of service-dominant logic has been largely neglected in research on the international hotel industry. We conducted 30 in-depth interviews among experienced female travelers, employing a semi-structured interview format. The interviews were transcribed, and the analysis included operations of categorization and integration. It is noteworthy that most issues stated by the interviewees refer to the physical environment of the hotel, including security factors. In clear contrast, the service personnel who worked at the hotels and their routine services were mentioned with less emphasis. Supplementary services and 
recreational offerings turned out to be a central issue, the former offering considerable potential for differentiation from competitors. Although some studies have previously investigated the topic of female business travelers' needs, this study makes several novel contributions, highlighting specific areas to create and upgrade service value for businesswomen. To the best knowledge of the authors, it is the first study to examine businesswomen who travel internationally and their experiences in upscale hotels. (For more information, please contact: Katharina Maria Hofer, Johannes Kepler University Linz, Austria: katharina.hofer@jku.at)

\section{Export Pricing Strategy in Higher Education Services: A Conceptual Framework and Research Propositions Terry Wu, University of Ontario Institute of Technology Vik Naidoo, University of Sydney}

Although export pricing is an important consideration for an international marketer, it is still an underresearched area in the international marketing literature. It has been attributed in the scholarly literature that this area has received little attention due to a lack of a formal export pricing theory and to the reluctance of organizations to discuss their export pricing strategies with researchers. This study attempts to bridge this gap in the literature. With a focus on the higher education services industry, this paper uses a grounded-theory approach to develop a conceptual framework of export pricing strategy building on interviews with 24 marketing managers from 6 different countries. This study makes several contributions to the export pricing literature. First, this study contributes to an area which has received little attention to date in spite of an overwhelming recognition of the importance of export pricing as a key determinant of export performance. Second, this study uses a multi-country approach, while most of the research in export pricing has been conducted from a singlecountry perspective. Last but not least, we add to a largely unexplored area of international marketing with respect to higher education. (For more information, please contact: Terry Wu, University of Ontario Institute of Technology, Canada: terry.wu@uoit.ca)

\section{Masstige Index for Measuring 'Brand Value' in Foreign Markets Justin Paul, University of Puerto Rico}

We develop an instrument titled Masstige Mean Index (MMI) as a measure to examine the effectiveness of "mass prestige" (masstige) strategy in foreign markets and building brand for premium products. We collected data from 600 people living in US, France and India. We posit that higher the Masstige Mean Index (MMI) value of a brand, higher the likelihood that potential customers recall that as a 'top of mind' brand. Lower MMS implies that the firm has to go long way in their efforts to build the brand. We argue that a firm can measure brand value using MMI., and compare the index value in foreign countries to derive insights on its popularity. (For more information, please contact: Justin Paul, University of Puerto Rico, USA: justin.paul@upr.edu) 


\section{Session: 2.5.11 - Interactive}

\section{Track: 3 - International Human Resource Management and Organizational Behavior}

\section{Crossing the Cultural Divide}

Presented On: June 29, 2015 - 16:15-17:30

Chair: Fabian J intae Froese, University of Goettingen

The Role of Organisational Values in Managing Employee Retention in Local and Foreign BPO Firms

Alfred Presbitero, Deakin University

Hernan Roxas, Deakin University

Doren Chadee, Deakin University

This paper examines one of the issues confronting the BPO industry: employee retention. Traditional view suggests that employee retention can be achieved through superior HRM practices. However, this view has often overlooked the role of context in which HRM practices are implemented. Drawing from a sample of local and foreign BPO employees in the Philippines, this study looks into how organisational values impact employee retention. Results show that organisational values partially mediate the relationship between HRM practices and employee's intention to stay in the organisation. The theoretical as well as the practical implications for international HRM are discussed. (For more information, please contact: Alfred Presbitero, Deakin University, Australia: alfred.presbitero@deakin.edu.au)

\section{Organizing Language Use: Practices of Plurilingualism \\ Stephanie Jo Kent, UMass Amherst \\ Jeffrey Kappen, Drake University}

Given the rising interest in issues surrounding language use and policies in contemporary organizations, this paper aims to enrich theorizing on language in global organizations by presenting homolingualism and plurilingualism as key tensions that exist within organizations in which multiple languages are used. This theoretical framing has the advantage of highlighting relational nature of communication when multiple languages are in use. These language choices and discursive strategies, embedded within organizational policies and interpersonal relations, lead to the creation of linguascapes. Drawing on examples from fieldwork at the European parliament and MNC sites, we then discuss the implications of plurilingualism for contemporary global organizations in the areas of human capital, knowledge transfer and innovation. (For more information, please contact: Jeffrey Kappen, Drake University, USA: jeffrey.kappen@drake.edu)

\section{Disruption and Synergy: Cultural Friction in Leadership Beliefs and Foreign Invested Enterprise Survival Pamela Tremain Koch, Grand Valley State University Bradley J ames Koch, Grand Valley State University Tanya Menon, Ohio State University Oded Shenkar, Ohio State University}

Building on the dynamic cultural friction approach, we argue that cultural differences may be either synergistic or disruptive. Differences in more culturally central leadership dimensions will contribute to disruption while differences in less central dimensions have synergistic potential via complementarities, effective third culture development, and creative diversity. Analyzing data from 274 foreign invested firms in China, we found support for these hypotheses. Given that Participative and Team-Oriented leadership dimensions are deeply embedded in China's traditional Confucian culture, differences in these dimensions increased firm death while differences in the less culturally central Charismatic, Autonomous and Self-Protective dimensions increased firm survival. (For more information, please contact: Bradley James Koch, Grand Valley State University, USA: kochb@gvsu.edu) 
How We Do Things Around Here: the Relationship Between Societal and Ideal Organizational Culture

Catherine Kwantes, University of Windsor

Cheryl Boglarsky, Human Synergistics International

Societal culture shapes what is viewed as an ideal setting for better employee performance, or ideal organizational culture. This project was undertaken in two phases. Study I drew on an employed student sample and examined cultural values (Hofstede, 1980) and beliefs (Leung et al, 2002) as predictors of respondents' ideal organizational culture (Cooke \& Lafferty, 1987). The results suggest that both values and beliefs predict preferences for ideal organizational cultures. . Study II examined descriptions of ideal organizational culture from employees at various types of organizations in 15 countries. Respondents' descriptions clustered into two distinct cultures, with Anglo cultures the majority in one cluster (Cluster 1: US, UK, Canada, Australia, Germans, Poland, Sweden, Hong Kong) and Latin cultures in the other (Cluster 2: Spain, Colombia, Argentina, Mexico, Hungary, China, France). No significant differences emerged on preferences for Constructive organizational cultures, but Cluster 2 endorsed both Passive/Defensive and Aggressive/Defensive aspects of organizational culture strongly than the other. These differences corresponded with higher levels of power distance and uncertainty avoidance in Cluster 2 and higher levels of Individualism in Cluster 1. Implications are discussed. (For more information, please contact: Catherine Kwantes, University of Windsor, Canada: catherine.kwantes@uwindsor.ca)

\author{
An Examination of Relationships between Management Practices and Performance: Evidence from India and \\ Malaysia \\ Takashi Sakikawa, Niigata University \\ Kaushik Chaudhuri, Shiv Nadar University \\ Azam Arif, Universiti Utara, Malaysia
}

Based on evidence from India- and Malaysia-based manufacturing firms, this study explores the impact of management practices on performance by taking into consideration two nations' different national culturesalthough they both are from the Southern Asia cultural group. Our statistical results revealed the efficacy of universalistic, configurational, and contextual perspectives in strategic human resource management (SHRM) research. By responding to the call for SHRM research in the global context, this study contributes to the development of the burgeoning field of international SHRM. (For more information, please contact: Takashi Sakikawa, Niigata University, Japan: saki@mot.niigata-u.ac.jp)

\author{
Multilevel Moderating Effects of Career Orientation, Supportive Climate on Psychological Empowerment and \\ Affective Commitment in China \\ Yi Yang, Yonsei University \\ Yong Suhk Pak, Yonsei University
}

This paper has built a multilevel moderating model to understand the implementation of empowerment and its impacts on affective commitment in China. Theoretically, we have developed the organizational supportive climate into two types: vertical and horizontal supportive climate; and investigated the moderating effects of individual's career orientation, organizational supportive climate and firm nature. Based on 879 samples nested in 35 firms, we used the hierarchical linear model (HLM) to test our multilevel moderating model rigorously. The results show that vertical supportive climate will enhance the relationship between empowerment and affective commitment; while horizontal supportive climate will eliminate the relationship. Moreover, comparing with Chinese-invested enterprises (ClES) and foreign-invested enterprises (FIES), we find that vertical supportive climate has stronger positive impacts in $\mathrm{ClEs}$, while horizontal supportive climate has stronger negative impacts in ClEs than FIEs. (For more information, please contact: Yi Yang, Yonsei University, Korea, South:

yangyi0403@gmail.com) 
Personality and Expatriate Job Performance: The Moderating Role of Expatriate Cross-Cultural Adjustment

Odette Christie, University of Dallas

Sri Beldona, University of Dallas

This paper examines the relations between personality traits and expatriate job performance and the moderating role of expatriate cross-cultural adjustment in that relationship. This paper proposes that the Big Five personality: openness to experience, conscientiousness, extraversion, agreeableness and neuroticism have significant and positive impact on expatriate job performance and three dimensions of cross-cultural adjustment: general adjustment, interaction adjustment and work adjustment moderates the relationship. The implications of this paper encourage multinational companies to systematically develop programs and interventions to support expatriates cross-cultural adjustment on international assignments. We suggest possible ways that MNCs can employ to mitigate the propensity of international assignment failure among its managers. (For more information, please contact: Sri Beldona, University of Dallas, USA: sbeldona@udallas.edu)

\section{Non-Traditional Managers in International Assignments \\ Cynthia Saldanha Halliday, Florida International University \\ Kowoon Kim, Florida International University \\ Yue Zhao, Florida International University \\ Mary Ann Von Glinow, Florida International University}

Although there is an increasing demand for qualified candidates for international positions to fulfill the needs of multinational enterprises (MNEs), as well as constant changes in the demographics of the workplace, very little has been written about non-traditional international managers. In this paper, we focus on non-traditional managers, particularly those with different family compositions, sexual orientation and from different religious groups, and analyze their likelihood of success in international assignments based on their degree of diversity, the length of the international assignment and the cultural distance between the host and home countries. Based on our study, we develop eight propositions to guide international human resource managers in the selection of non-traditional managers and encourage scholars to develop future empirical research to test them. (For more information, please contact: Cynthia Saldanha Halliday, Florida International University, USA: chall061@fiu.edu)

\section{Session: 2.5.12 - Interactive \\ Track: 9 - Emerging Economies and Emerging Economy MNEs}

\section{The Role of Governments and Institutions}

Presented On: June 29, 2015 - 16:15-17:30

Chair: Alice de J onge, Monash University

Learning from Formally Constructed Relationships with Single Networks-Nodes during Internationalization to Institutional Distant Markets

June Borge Doornich, University of Nordland

This paper investigates organizational learning from relationships during internationalization. The purpose is to explore processes of constructing relationships when actors internationalize to institutional distant markets. Through case analysis of Western multinational companies offering high technology and complex solutions in emerging markets, the paper brings novel insight to organizational learning from relationship when companies already represented at global level establish subsidiaries in institutional distant markets, from an interpretive approach. The findings demonstrate that dyadic bilateral relationships are purposely constructed between an intrapreneur and a single network-node in the foreign market. There is an important interplay between the relationship at an organizational level, as relationships are constructed in formalized agreements, and at 
individual level, as it becomes even more important to ensure trust based relationships in the dyad. Institutional distance is found to be the main uncertainty during internationalization, where psychical distance related to dissonance in business culture and ambiguity in red tape constrain internationalization and organizational learning. It is argued that dyadic relationships is formally constructed, as foreign actors are not admitted in larger business networks. Nonetheless, it becomes equal important to develop trust and loyalty in the dyad, as networks in emerging markets are group-oriented where traditions friendships becomes important. (For more information, please contact: J une Borge Doornich, University of Nordland, Norway:

june.borge.doornich@uin.no)

\section{Examining How Firm-Level and Country-Level Factors Affect Lean Capability Development of Emerging Market Firms?}

Crystal X J iang, Bryant University

Omar Malik, Higher Education

Madan Annavarjula, Bryant University

Drawing on the dynamic capabilities view (DCV) and institutional theory, we examine how firm-level and country-level institutional factors matter to EMFs' lean capability development. Based on a sample of 1,702 firms from Brazil, China, and India, we find that EMFs' internationalization enhances the positive relationship of firms' human capital development and lean capability development. At the institutional level, our results suggest that regulatory institutions reinforce the positive effect of process management on EMFs' lean capability development; however, normative institutions (power distance) negatively influence the effect of firms' human capital management on lean capability development. Our cross-country analysis suggests that comparing to Indian and Brazilian counterparts, China's regulatory and normative environments (a higher level of power distance) hamper the positive effect of firm-level resources on their firms' dynamic capability development. (For more information, please contact: Crystal X Jiang, Bryant University, USA: cjiang1@bryant.edu)

\section{How Do Government Ownership and Political Connections Affect the Financial Market Performance of Chinese Acquirers?}

Wenjun Tu, University of Nottingham, Ningbo

Xiaolan Zheng, University of Nottingham, Ningbo

Lei Li, Nottingham University Business School China

This paper integrates the resource dependence theory (RDT) and agency theory (AT) to examine the impacts of government ownership and political connections on value creation of Chinese cross-border merger and acquisition (CBM\&A) from 1999 to 2013. We find that Chinese CBM\&As create value for Chinese acquirers in general. Government ownership has a significant influence on performance, the magnitude of which varies from one period to another while political connections have significantly positive impact on value creation all the time. We look into the principal-principal and principal-agent problems to explain the significant negative influence of government ownership on the short-term stock performance after $M \& A$ announcement before 2008. Moreover, we reveal that the politically-connected firms can easily obtain extra resources and reduce environment uncertainty. Furthermore, we find that politically connected-CEOs do not influence market performance of acquirers while politically connected-board, especially political connected-outsider directors, has significantly positive influence on value creation of CBM\&As. (For more information, please contact: Wenjun Tu, University of Nottingham, Ningbo, China: zx06477@nottingham.edu.cn)

Leadership Mechanisms for Strategic Conformity to Ambidexterous Decision-Making under Family Ownership: Evidence from Emerging Economies Hong-J en C. Chiu, National Taiwan University

Do top executives differ in ambidexterity of making strategic decisions by the degree of conformity to stewardship by family ownership? If yes, could leadership mechanisms be designed effectively in various control 
configurations and institutional contingencies? These inquiries motivated this research. Firms with greater controlling-family's involvements have greater strategic conformity, and their CEOs tend to be more accountable. In addition, CEOs under family members' stewardship create value from core businesses. Instead, CEOs of non-family firms focus on maximizing firm value. Moreover, both small firm size and concentrated ownership moderate positively the relationship between strategic conformity and firm performance under family ownership. Finally, GDP per capita and English legal origins mediate positively the relation between leadership mechanisms and firm performance. (For more information, please contact: Hong-Jen C. Chiu, National Taiwan University, Taiwan: hongjen@ntu.edu.tw)

\section{Market Entry Strategies and Performance of Chinese Firms in Germany: The Moderating Effect of Government Support}

Dirk Holtbruegge, Friedrich-Alexander University of Erlangen-Nuremberg

Sue Claire Berning, Friedrich-Alexander University of Erlangen-Nuremberg

This study uses data at the firm level in examining the relationship between market entry strategies of Chinese MNEs and their performance in Germany. Based on the learning theory of internationalization we develop six hypotheses on the impact of different market entry modes on performance, and on the moderating effect of government support on this relationship. The hypotheses are tested against a dataset of 556 Chinese MNEs that have invested in Germany. The findings show a significant effect of location choice, activity mode, establishment mode and the duration of market presence on performance. Furthermore, strong interaction effects of government support on these relationships are revealed. Our study contributes to the market entry literature in general and provides new insights to the analysis of market entry strategies of emerging market firms in particular. (For more information, please contact: Sue Claire Berning, Friedrich-Alexander University of Erlangen-Nuremberg, Germany: sue.claire.berning@fau.de)

\section{Public-Private Partnerships: From Social Dilemmas to Value Co-creation in Emerging Market Shih-Chieh Fang, National Cheng Kung University Hsin-Hui Chou, National Cheng Kung University Chih-Fang Chiu, National Taiwan University Chih-Hao Tsai, National Sun Yat-sen University}

There has been a surge in research of public-private partnerships (PPPs). However, the understanding of how to develop, manage and assess PPPs in order to co-create value for governmental and business organizations and the society remains constrained. We contend that the characteristics of publicness, heterogeneity and collective actions in PPPs will inevitably result in situations that self-interests of individual organizations are placed above the public welfare; such phenomena are seen as "social dilemmas". From a perspective of valuebased strategy, we further argue that, social dilemmas between public and private organizations can accelerate to build a platform that provides information accessibility and transparency, with which mutual dialogue and negotiation is facilitated; we term it as a "value co-creation platform". Drawing on a case study of Pier 2 Art Center's cultural innovation, we identified and analyzed three types of social dilemmas occurring in the crosssector partnerships between Bureau of Cultural Affairs Kaohsiung City Government and operators of Pier 2 Art Center: cooperating dilemma, sharing dilemma and evaluating dilemma. We found these dilemmas evoked tensions to create a value co-creation platform, successfully generating economic and social value. The findings permit us to develop theoretical and managerial implications. (For more information, please contact: Chih-Hao Tsai, National Sun Yat-sen University, Taiwan: billy.tsai88@gmail.com) 
Corporate Expansion during Pro-Market Reforms: The Role of Business Group Affiliation and Unrelated

Diversification

Deeksha Singh, Rutgers University

Chinmay Pattnaik, University of Sydney

Elif Ketencioglu, Optus

This study examines how emerging economy firms pursue growth in the domestic market during pro-market reforms. Grounding on the literature on institutional voids and business groups, we propose that group affiliation and pro-market reforms are positively related to the scale of new investments, while diversification into unrelated industries is negatively related to the scale of new investments. Further, we propose that the effect of business group affiliation is contingent on pro-market reforms and whether new investments lead to unrelated diversification or not. Given that pro-market reforms provide greater opportunities to focus on existing lines of businesses, we expect pro-market reforms to negatively moderate the positive effect of business group affiliation on the scale of new investments. Likewise, given that unrelated diversification loses its appeal as a result of institutional transition, we expect a negative interaction between group affiliation and unrelated diversification in affecting the scale of new investments for corporate expansion. Our empirical findings based on a sample of 3,479 projects for corporate expansion in India during 1995 to 2007 provide robust empirical support for the hypothesized relationships. (For more information, please contact: Deeksha Singh, Rutgers University, USA: deeksha.singh@rutgers.edu)

\section{Session: 2.5.13 - Interactive Special Session}

\section{International Business Research in India}

Presented On: June 29, 2015 - 16:15-17:30

Chair: Elizabeth Rose, University of Otago

An Investigation on Effects of Intangible Assets on Degree of Internationalization Bikramiit Ray Chaudhuri, Indian Institute of Management Calcutta Santanu Bhadra, Indian Institute of Management Calcutta

To further knowledge about the effects of intangible assets on degree of internationalization, this paper examines the importance of three intangible assets - namely, corporate reputation, absorptive capacity, and stakeholder influence capacity, and their synergistic interactive effects on organizational degree of internationalization. The results show that all the intangible assets and interactive effects between them have significant effects on degree of internationalization except the interactive effect between stakeholder influence capacity and absorptive capacity. (For more information, please contact: Bikramjit Ray Chaudhuri, Indian Institute of Management Calcutta, India: bikramjitr12@email.iimcal.ac.in)

Foreign Ownership, Royalty Payments and Expropriation of Minority Shareholders: Evidence from India Aditya Mohan Jadhav, T. A. Pai Management Institute Durga Prasad, T. A. Pai Management Institute Srikanth P., T. A. Pai Management Institute

This paper examines the expropriation of minority shareholders invested in listed subsidiaries of foreign firms by excess royalty payments by the subsidiaries to their parent multinational companies (MNC). Using the data of 4673 Indian firms over a period of 12 years, we examine the impact of royalty payment on firm performance viz., asset turnover ratio, net profit margin and return on capital employed. We document that royalty payments made by foreign subsidiary firms is higher than the royalty payments by Indian firms. We also report that 
royalty payments have a negative impact on firm performance. We report a negative impact of royalty payment on firm performance is differs for foreign subsidiary firms in comparison to domestic firms. The results show that royalty payments impact firms' earnings negatively. The reduced net earnings to the parent MNC are offset by higher royalty payments. But the minority shareholders are left with reduced earnings indicating expropriation through royalty payments. (For more information, please contact: Aditya Mohan Jadhav, T. A. Pai Management Institute, India: adityajadhav@tapmi.edu.in)

\section{Is there Any Relationship between Foreign Direct Investment, Carbon Emission, Economic Growth, Trade and Energy Consumption: A Study Based on Japan}

Ruhee Singh, Indian Institute of Foreign Trade

Sweta Srivastava Malla, Indian Institute of Foreign Trade

After the 2011 nuclear disaster Japan did not sign up to the Kyoto Protocol in order to suspend implementation of carbon emissions reduction. J apan being the fifth largest carbon emitting country has motivated to do this study in order to explore the long run relationship between FDI, trade, Carbon emissions, economic growth and energy usage. This study empirically examines causality between carbon emissions, FDI, trade, economic growth in terms of Gross Domestic Product (GDP) and energy usage using a time-series data of J apan from the World Bank for the period 1977-2012. The study explains that there is a unidirectional causality between FDI inflow and Carbon emissions, FDI inflows and energy usage, trade and energy usage, and FDI inflows and trade. (For more information, please contact: Ruhee Singh, Indian Institute of Foreign Trade, India:

ruheesingh18@gmail.com)

\section{Tax Havens: Conduits for Corporate Tax Malfeasance \\ Akanksha J alan, Indian Institute of Management Bangalore \\ R. Vaidyanathan, Indian Institute of Management Bangalore}

This paper is an effort to demystify tax havens- what they mean, what they offer and why they could be harmful. Owing to the havoc these havens have wreaked in the recent past, they have become the subject of much public policy and debate. Earlier known only for facilitating the stashing of illegal wealth of the super-rich across the globe, their use by corporations has recently come to light. Starbucks, Apple, Walmart - no big name has been spared. This paper offers a detailed analysis of how tax havens facilitate corporate tax planning and how the latter pervades corporate decision-making right from their start. Tax havens with all the secrecy and flexibility they offer contribute significantly to such corporate behavior by making it easier for corporations to go around the tax rules of their home countries. This behavior is specifically more pronounced for multinational corporations, given their global presence and complex business operations. The term 'malfeasance' has been used to indicate the 'abuse' that results from the tax arbitrage multinational corporations resort to. While hightax economies find their tax bases shrinking, tax haven economies flourish by inviting tax-evaded funds from corporations in the former. Finally, the paper also discusses global efforts against the phenomenon and policy recommendations. (For more information, please contact: Akanksha Jalan, Indian Institute of Management Bangalore, India: akanksha.jalan10@iimb.ernet.in)

\section{Business Group Affiliation and Risk: A Contrast between Developed and Emerging Economies Vivek Kumar, XLRI Jamshedpur}

Business groups are ubiquitous in developing economies but less visible in developed economies. Related literature has theorized that business groups serve three purposes in developing economies one of which is risk diversification. Earlier studies have used accounting data to test the risk diversification hypothesis of business groups, and found risk of business group affiliated firms to be less than standalone firms only in J apan, South Korea, and Thailand. However accounting data is prone to creative accounting, and may not be veracious. Stock market data does not suffer from such deficiencies. In this study we compare the risk level of business group affiliated firms with standalone firms using stock market data. The study, a replication of Chen, Guo and 
$\operatorname{Tay}(2010)$ who found less risk for business group affiliated firms, found that the risk of business group affiliated firms is more than that of standalone firms. This contrasting result suggests that risk insurance function of business groups may not be true in developing economies. The results were found to persist over time in different institutional environments India has witnessed since economic liberalization. (For more information, please contact: Vivek Kumar, XLRI Jamshedpur, India: fb11004@astra.xIri.ac.in)

\section{Patterns of International Competitiveness of Indian MNEs: Which Capabilities Explain Sustained Internationalization? \\ K. S. Momaya, Indian Institute of Technology Bombay}

Emerging economy multinationals' (EMNEs) strategies for international competitiveness seem to have resulted in contrasting outcomes, particularly in terms of enterprise sustainability. We attempt to explain the phenomenon of 'low international sustainability', taking the case of pharmaceutical industry, and through polarity between type of capabilities. We studied the patterns of competitiveness journey of carefully selected six focal firms, and then by taking a comparative case of two polar firms. Emerging findings indicate that while catch-up with advanced economy MNEs (AMNEs) may be feasible on output capabilities, sustenance demands better balances, consistency and innovation capabilities. (For more information, please contact: K. S. Momaya, Indian Institute of Technology Bombay, India: momaya@iitb.ac.in)

\section{Foreign Direct Investment and BRICS economies \\ Gaurav Agrawal, ABV-I ndian Institute of Information Technology and Management}

This research paper analyzes the impact of foreign direct investment (FDI) on the economic growth of the BRICS nations. Cointegration and causality analysis have been used at the panel level. Findings confirm that foreign direct investment and economic growth are cointegrated at the panel level that indicates that FDI is having long term impact on the economic growth. Causality analysis indicates that there is long run causality running from foreign direct investment to economic growth in these economies. It is thus important for the policymakers in the BRICS nations that they try to remove obstacles for FDI inflows in their nation and improve the respective absorptive capacity in order to reap maximum positive economic growth individually as well as jointly. (For more information, please contact: Gaurav Agrawal, ABV-Indian Institute of Information Technology and Management, India: drgauravagrawal@gmail.com)

Organizing the Unorganized? Towards a Model of the Emerging Market Informal Firms K. Indu Rao, University of Twente

No abstract available. (For more information, please contact: $K$. Indu Rao, University of Twente, Netherlands: i.kaveti@utwente.nl) 


\title{
ABSTRACTS FOR TUESDAY, J UNE 30, 2015
}

\author{
Session: 3.1.1 - Panel \\ Special Session

\section{Learning from External Knowledge Sources in the Global Economy}

Presented On: J une 30, 2015 - 09:00-10:15

Chair: Stephen Tallman, University of Richmond

Panelists:

Stephen Tallman, University of Richmond

Anupama Phene, George Washington University

Farok J. Contractor, Rutgers Business School

Manish K. Srivastava, Michigan Technological University

Sai Yayavaram, Indian Institute of Management Bangalore

External sources of knowledge have become more important to firms as they have dispersed their value-adding operations around the globe and outsourced them to suppliers. The global network firm has access to a rich store of external knowledge - but what do we know about accessing this treasure trove? This panel will address four alternative structures for organizing inter-organizational knowledge transfer and learning that are relevant to firms in the international market for knowledge. These are inter-firm spillovers of knowledge, licensing, exchange within cooperative ventures, and acquisition of a target firm. On the surface, these different routes for accessing knowledge held outside the focal firm seem to be an ad hoc list of sources, some studied in detail and others less so. The concept of the panel will be to highlight the positive and negative aspects of each mode of access and to compare them to each other. The major innovation will be to organize the presentations within a common conceptual framework built around the idea of definable market for information exchange. (For more information, please contact: Stephen Tallman, University of Richmond, USA: stallman@richmond.edu)

\section{Session: 3.1.2 - Competitive}

\section{Track: 8 - Global Strategy, M\&As and Competitiveness}

\section{Competing with Local Firms in Global Settings}

Presented On: June 30, 2015 - 09:00-10:15

Chair: Moritz Putzhammer, WU Vienna

Global Diversification Discount and Its Discontents: A Bit of Self-Selection Makes a World of Difference Sungyong Chang, Columbia University

Bruce Kogut, Columbia University

Jae-Suk Yang, Columbia University

The documented discount on globally diversified firms is often cited, but a correlation is not per se evidence that global diversification destroys firm value. Firms choose to globally diversify based on their firm attributes, some of which may be unobservable. Given these exogenous firm attributes, the decision to diversify globally is endogenous and self-selected. Using the same specifications save for the Heckman selection instrument, our results contradict past research that did not address endogeneity. We posit that the global premium should reflect the value of multinational operating flexibility. We use the 2008-2009 financial crisis as creating exogenous variation to permit a test for the positive change in firm valuation due to global diversification. 
During and after the 2008-2009 financial crisis, the premium associated with global diversification became larger and more significant than before the 2008-2009 financial crisis. The churn of subsidiaries entering and exiting countries increased during the crisis, pointing to the value of an operating flexibility to restructure the geography of the multinational network. In all, the results contradict past findings and provide evidence that operating flexibility is more valued during times of high volatility, thus generating the diversification premium. (For more information, please contact: Sungyong Chang, Columbia University, USA: sc3339@columbia.edu)

\section{The Second-Home Model toward the Dual-Core Paradigm: The Implications of ISE for MNES from Advanced and Emerging Economies}

Peter Ping Li, Copenhagen Business School

The primary purpose of this study is to identify the unique novel perspective of second-home model toward the dual-core paradigm, which has the potential to be the biggest research question for international business (IB) scholars as well as the most unique contribution from IB scholars to the non-IB fields. In particular, the novel perspective of second-home model has the potential to contribute to the research on strategy and entrepreneurship by contextualizing and integrating the separated streams on business model (business model innovation or BMI ), dynamic capability, and strategic entrepreneurship. The final outcome is the development of a novel integrative domain of international strategic entrepreneurship (ISE), where the novel constructs of global divide, cross-divide entry, cross-divide unlearning, and dual-core paradigm will be housed, and where BMl and dynamic capability are framed as two most salient mechanisms for enduring entrepreneurship ( For more information, please contact: Peter Ping Li, Copenhagen Business School, Denmark: ppl.int@cbs.dk)

\section{Do Birds of a Feather Flock Together? Asymmetric Rivalry between Multinational and Local Firms in a Multimarket Setting \\ Rupanwita Dash, Indian Institute of Management Bangalore}

This study seeks to analyze the joint effect of multimarket contact and firm nationality on the likelihood of market exit for pharmaceutical companies. Past research on the relation between multimarket contact and exit has established that multimarket contact leads to mutual forbearance, which reduces the likelihood of exit. I propose to examine the same link between multimarket contact and exit with two extensions: first, explaining role of inter-firm coordination resulting in a U-shaped relationship between multimarket contact and exit; and second, the differences in exit likelihood between MNCs and domestic firms at similar levels of multimarket contact. Using cardiovascular drug sale data for 179 firms in 200 pharmaceutical molecules in India for the period of 2008-2011, this study shows that multimarket contact has a U-shaped relation with exit likelihood of firms, and this likelihood for multinationals varies from the local firms. (For more information, please contact: Rupanwita Dash, Indian Institute of Management Bangalore, India: rupanwita.dash10@iimb.ernet.in)

\section{The Role of Experiential Learning in Overcoming the Liability of Foreignness Yunok Cho, Renmin University of China Jaeyong Song, Seoul National University}

We investigate the role of experiential learning in overcoming the liability of foreignness, building on organizational learning theories in the international business literature. We distinguish between learning from accumulated experience, or learning-by-doing, and learning from a parent firm's prior foreign investment, and further investigate how the concurrence of the two types of learning changes the effect of each learning. We depart from the traditional perspective of the liability of foreignness, by conceptualizing it as the disadvantage of operating in a foreign country in relation to that of operating in a home country. Our empirical test uses a multi-country database of European operations. We find support for our framework in a panel analysis based on the matched sample of foreign and domestic operations owned by the same MNCs. The effect of institutional distance is also addressed. (For more information, please contact: Yunok Cho, Renmin University of China, China: yunok.cho@gmail.com) 


\section{Session: 3.1.3 - Competitive \\ Track: 5 - The Geography of I nternational Business and Global Value Chains}

\section{Offshoring and Governance}

Presented On: June 30, 2015 - 09:00-10:15

Chair: Marcus M. Larsen, Copenhagen Business School

Disentangling the Role of Modularity and Bandwidth in the Entry Mode Choice

Stefano Elia, Politecnico di Milano

Silvia Massini, University of Manchester

Rajneesh Narula, University of Reading

This paper investigates the role of modularity on entry mode choice of companies undertaking offshoring of business services. We distinguish between Functional modularity, reflecting the possibility to separate the business function into smaller modules, and Architectural modularity, reflecting a low interdependence among the modules, and, a hence, a small bandwidth in terms of frequency of interactions among people that is needed to reintegrate the module into the system. We explain how these two dimensions of modularity interfere with the traditional theories explaining entry mode choice, such as Transaction Costs Economics and Resource Based View. We show how the possibility to take advantage of modularity decreases the transaction costs and the risks of knowledge leakages associated to offshoring, thus increasing the probability to opt for less hierarchical entry mode choices. Our empirical analysis, which involves 490 offshoring initiatives of business functions, confirms this relationship, especially in high-tech and knowledge-intensive industries. We also provide evidence that inexperienced firms tend to neglect the role of Architectural modularity when selecting the entry mode choice, thus increasing the risk of a failure of the offshoring initiatives due to the high costs arising when the need for bandwidth is high. (For more information, please contact: Stefano Elia, Politecnico di Milano, Italy: stefano.elia@polimi.it)

\section{A Multi-level Analysis of Project, Firm and Country Characteristics on Offshoring Decisions for Clinical Trials in the Biopharmaceutical Industry \\ Pooja Thakur Wernz, Virginia Tech University \\ Christian Wernz, Virginia Tech University}

This paper identifies factors at the project, firm and country level that influence a firm's offshore make-or-buy decision for R\&D activities. Specifically, the paper studies multi-level factors and their interactions that affected the decisions of biopharmaceutical firms of whether to conduct clinical trials within their own foreign affiliates (captive offshoring) or with foreign third party vendors (offshore outsourcing). Drawing upon institutional theory and RBV, we posit that the type of projects offshored (core versus non-core), the size of the R\&D pipeline, the quality of regulatory institutions specific to the industry and the overall country affect the captive offshoring vs. offshore outsourcing decision. Our empirical research setting is the global biopharmaceutical industry from 1997 to 2005. We test our hypotheses using detailed data from 9,808 clinical trials undertaken by 57 firms across 48 countries. Our results show that core projects tend to be retained within the firm boundary. Firms are also overall more likely to choose offshore outsourcing when they have larger R\&D pipelines. However, this changes when they offshore to countries with weaker institutions. A counterintuitive finding of this study is that overall country level institutions are important for the offshore sourcing decision while industry specific institutions are not relevant. (For more information, please contact: Pooja Thakur Wernz, Virginia Tech University, USA: pthakur@vt.edu) 
Coordination Difficulty and Performance in the Offshoring Context: The Moderating Effect of Governance Mode and Experience

Niccolo Pisani, University of Amsterdam

Kannan Srikanth, Singapore Management University

Joan Enric Ricart, IESE

Western companies have increasingly relocated parts of their value chain to offshore locations in the recent past. Extant research documents that offshoring exposes firms to challenges associated with coordinating geographically dispersed work. In this paper, we argue that vertical integration mitigates the negative effect of coordination difficulty between onsite and offshore locations and the performance of the offshoring venture. However, with experience, firms are able to manage coordination difficulty without recourse to unified governance. We test our theory by analyzing survey data from 709 offshoring implementations executed by 245 multinational companies based in the U.S. and Western Europe. (For more information, please contact: Niccolo Pisani, University of Amsterdam, Netherlands: n.pisani@uva.nl)

\section{Session: 3.1.4 - Competitive}

Track: 6 - Innovation and Knowledge Management

\section{Exploration and Exploitation Across Borders}

Presented On: June 30, 2015 - 09:00-10:15

Chair: Kazuhiro Asakawa, Keio University

Managing Innovation Ambidexterity via Supplier Involvement: The Advantage of Foreignness in the Brazilian Automotive Industry

Denise Dunlap, Northeastern University

J ose-Mauricio G. Geleilate, Florida International University

Ronaldo Parente, Florida International University

Tucker Marion, Northeastern University

The incipient study of how firms manage their explorative and exploitative innovation activities still needs further clarification on the interplay between foreign subsidiaries and local firms. Drawing from a knowledgebased perspective, we empirically investigate how local firms and foreign subsidiaries operating in an emerging economy manage their innovation ambidexterity. Specifically, we examined two types of innovation ambidexterity: the balanced dimension (i.e., the capacity to simultaneously create new and incremental innovations) and the combined dimension (i.e., the capacity to leverage new and incremental innovations). Our findings suggest that firms performed better when they achieved the balanced dimension rather than the combined dimension of innovation ambidexterity. Moreover, firms achieve better performance from their combined dimension of ambidexterity when they also possess higher levels of supplier involvement. Yet, for both the combined and balanced dimensions of innovation performance, being a foreign subsidiary yielded a positive advantage over local firms in reaping performance benefits, indicating that these firms are better able to realize the benefits of both local and multi-national knowledge to improve innovation ambidexterity. (For more information, please contact: Jose-Mauricio G. Geleilate, Florida International University, USA: jgeleila@fiu.edu) 
Solving Environmental Problems: Coordination in Collaborative Search across Borders

Christoph Grimpe, Copenhagen Business School

Anders Oerding Olsen, Copenhagen Business School

Wolfgang Sofka, Copenhagen Business School

Recent innovation and strategy research emphasizes the importance of firm's search for external knowledge to improve innovation performance. We focus on such search strategies within the domain of sustainable innovation in which problems are inherently complex and the relevant knowledge is widely dispersed. As a result, firms frequently collaborate with international partners. We shed new light on collaborative search strategies led by firms in general and for solving environmental problems in particular. Both topics are largely absent in the extant open innovation literature. Using data from the European Seventh Framework Programme for research and technological development, our results indicate that the problem solving potential of a search strategy increases with the diversity of existing knowledge of the partners in a consortium and with the experience of the partners involved. Moreover, we identify a substantial negative effect from involving partners in a search consortium who collaborate for the first time. Our findings have implications for both the literature on firms' search and the creation of sustainable innovation. (For more information, please contact: Christoph Grimpe, Copenhagen Business School, Denmark: cg.ino@cbs.dk)

\section{Search Scope and Innovation Performance: The Moderating Role of Technological Spillovers, Product Market} Rivalry and Geographic Proximity

J ie Wu, University of Macau

Ajai Gaur, Rutgers University

Debmalya Mukherjee, University of Akron

Shuaihe Zhuo, University of Macau

This study examines the boundary conditions for the relationship between knowledge search scope and firm innovation performance. We argue that firms are embedded in the external environment, which is pluralistic in nature. The interactions that firms have with other actors in different spaces affect the extent of knowledge spill over, which in turn may affect the relationship between search scope and firm innovation performance. We identify three distinct spaces in which firms operate and interact with other actors - technological space, product market space, and geographic space. We assume a baseline curvilinear relationship between search scope and innovation performance, consistent with extant literature. Further, we propose that knowledge spillover in any of the three spaces will diminish the positive relationship between search scope and firm innovation performance. Our findings, based on a sample of 715 firms during 1980 and 2001 suggest that knowledge spillover in the technology space and product market space has a negative moderating effect on search scope and firm innovation performance relationship; however spillover in the geographic space has no significant effect. (For more information, please contact: Ajai Gaur, Rutgers University, USA: ajai@business.rutgers.edu)

In Harsh and Slack Times: How Does Prior Performance Affect the Intensity, The Structure and the Direction of Knowledge Seeking Boundary Spanning?

Felipe Monteiro, INSEAD

Thomas Klueter, IESE

Despite our understanding of the importance of boundary spanning for subsequent performance, we know little about how prior performance influences the global technology sourcing activities of multinational corporations (MNCs). To shed light on this question, we draw upon the behavioral theory of the firm, and examine how crossing organizational boundaries may be sensitive to how managers evaluate their own organizational performance. We develop several propositions linking whether MNCs find themselves in "harsh times"-i.e. performing below their aspiration levels_or in "slack times"-i.e. performing above their aspiration levels-to the intensity, the structure and the direction of knowledge seeking boundary spanning. In so doing, the paper not only provides novel insights on how performance affects a MNC's boundary spanning behavior, but also reveals important boundary conditions that influence how a MNC manages its broader set of R\&D activities. (For more information, please contact: Felipe Monteiro, INSEAD, France: felipe.monteiro@insead.edu) 


\section{Session: 3.1.5 - Panel}

Track: 9 - Emerging Economies and Emerging Economy MNEs

\section{A Continent Whose Time has Come: How to Explode the Amount of I B Research in and on Africa}

Presented On: June 30, 2015 - 09:00-10:15

Chair: Michael J Mol, Copenhagen Business School

Panelists:

Michael J Mol, Copenhagen Business School

Amon Chizema, University of Birmingham

John Manuel Luiz, University of Cape Town

Although IB research has started to embrace emerging markets and now looks at ever more parts of the world, Africa is still not looming large on the radar screen, despite it becoming more significant in recent years. Africa is a complex and interesting continent for IB researchers. The purpose of this panel is twofold. First, we want to speed up the Africa research agenda within IB. Second, we want to bring together a community of interested IB scholars and hope to increase the size of that community. The panel tackles three related questions: 1) How much IB research is there on Africa and what have we learned from it; 2) why is there not more research on Africa; 3) what can we do to increase the quantity and quality of research on Africa? The panel is set up to include significant audience participation rather than long monologues from the panelists. (For more

information, please contact: Michael/ Mol, Copenhagen Business School, Denmark: mmo.smg@cbs.dk)

\section{Session: 3.1.6 - Panel}

Track: 15 - Teaching I B (Special Track)

\section{X-Culture: Optimizing the Use of X-Culture in International Business Courses}

Presented On: J une 30, 2015 - 09:00-10:15

Chair: Vasyl Taras, University of North Carolina at Greensboro

Panelists:

Karen Lynden, Rowan-Cabarrus Community College

Vasyl Taras, University of North Carolina at Greensboro

Soma Arora, Institute of Management Technology

Cyntia Calixto, Fundação Getúlio Vargas

Susan Forquer Gupta, Monmouth University

Suchita J ha, Symbiosis Institute of International Business

Raghu Kurthakoti, Arcadia University

Daniel Rottig, Florida Gulf Coast University

Fabiana Sciarelli, Unitelma Sapienza University of Rome

Bindu Vyas, King's College

X-Culture (www.X-Culture.org), launched in 2010, is gaining popularity: over 100 IB professors from 40 countries take part in this international collaboration project with their 4,000 students every semester. The students from different countries work in GVTs and complete a business project, learning in the process the challenges and best practices of global cross-cultural collaboration. While the concept of X-Culture is very 
simple, some instructors have difficulties finding optimal ways to integrate the project in their course, devising a grading systems that, and helping students to fully utilize the opportunities offered by the project. The purpose of this session is to bring together academics with X-Culture experience and those who may only be considering adding this IB experiential learning project to their course so that they could share insights, ideas, concerns, and best practices and collectively develop ways to optimize the use of experiential learning projects, including $\mathrm{X}$-Culture, in their IB courses. (For more information, please contact: Karen Lynden, Rowan-Cabarrus Community College, USA: karen.lynden@rccc.edu)

\section{Session: 3.1.7 - Competitive \\ Track: 12 - The Institutional and Political Environment of I B}

\section{CSR and Ethics in IB}

Presented On: June 30, 2015 - 09:00-10:15

Chair: Ivan Montiel, Loyola Marymount University

Rise of Chinese Banks and the Melding of Institutional Logics and Ethics in International Banking W Travis Selmier II, Indiana University

As Chinese banks expand overseas through acquisition and branch establishment, scholars and policy-makers wonder how such expansion will affect the ethics of international banking. Three required types of banking capital- financial, expertise and reputation- and archetypal financial intermediation models lead to some isomorphism. Banking ethics have been defined partly through this isomorphism, partly through hypernorms, and partly through a legacy of Anglo-American institutional logics. But important institutional and ethical differences exist, and these arise through development of national banking systems and local culture. These manifest as local forms of banking institutional logics. Chinese international bank expansion carries with it three important differences in institutional logics: Chinese banks' tilt toward relationship banking, the pervasive impact of guanxi in financial contracting, and the fact that internationally-expanding Chinese banks are largely stateowned. Examining the intersection between incumbent and emergent institutional logics helps to predict how Chinese banking may affect international banking ethics and vice versa. (For more information, please contact: W Travis Selmier II, Indiana University, USA: wselmier@indiana.edu)

\section{American MNEs: In Search of Legitimacy When You're WEIRD \\ Matthew Mitchell, Drake University \\ Craig VanSandt, University of Northern I owa \\ Mukesh Sud, Fairfield University}

Presuming that American Multinational Enterprises (AMNEs) prefer to be viewed as legitimate, socially responsible firms in their host countries, we seek to provide answers to the question of how they can best determine ethical standards when faced with multiple, frequently conflicting operating environments? After exploring many of the reasons that identifying and understanding hosts' moral matrices is extremely confounding, the authors review historic and existing efforts to bridge them, and suggest specific steps that AMNEs can employ to better accommodate their ethics to the vastly different cultures in which they operate. (For more information, please contact: Matthew Mitchell, Drake University, USA: matthew.mitchell@drake. edu) 
Gender Diversity in Central Banks

Amir Shoham, Temple University

Patricia Charlety, ESSEC Business School and THEMA

Davide Romelli, ESSEC Business School and THEMA

Estefania Santacreu-Vasut, ESSEC Business School and THEMA

We provide the first systematic analysis on the determinants of presence of female in Central Bank boards. In particular, we analyze the impact of the overall presence of women in the economy and of a cultural expression of gender inequality based on linguistic female/male distinctions on female involvement in Central Bank top management. We code the Central Bank Directory 2008 which contains information for a total number of 168 countries and find evidence that women are less represented in Central Bank boards and top management in countries with high gender inequality, as captured by language, even after controlling for economic and institutional factors. (For more information, please contact: Amir Shoham, Temple University, USA:

amir.shoham@temple.edu)

\section{Political Competition and Corporate Bribery: Evidence from South Korea \\ Yujin J eong, American University \\ J ordan Siegel, Harvard Business School}

Bribery is an important phenomenon because it is costly to economic development, because it detracts from public confidence in the rule of law, and because it may distort who ends up being the winners and losers in economic competition. We study the relationship between political competition and bribery by firms as the channel through which firms may win and lose in economic competition. Using a novel dataset with a comprehensive coverage of high-level corporate bribery in South Korea, we test whether and how the degree of political competition affects firms' bribery behavior and decisions on the types of money that they pay to political parties. Results show that corporate bribery is reduced while charity contributions go up in economic size and frequency as political competition is increased. We show that this interesting combination of results is highly robust and consistent across measures and specifications. (For more information, please contact: Yujin Jeong, American University, USA: yjeong@american.edu)

\section{Session: 3.1.10 - I nteractive}

Track: 9 - Emerging Economies and Emerging Economy MNEs

\section{Entry Mode Strategy}

Presented On: June 30, 2015 - 09:00-10:15

Chair: Satyanarayana Rentala, Pondicherry University

\section{Market Entry Strategies and Performance of Indian Firms in Germany: The Moderating Effect of International Experience}

Dirk Holtbruegge, Friedrich-Alexander University of Erlangen-Nuremberg

Ritam Garg, Friedrich-Alexander University of Erlangen-Nuremberg

This study examines the relationship between market entry strategies of Indian firms and their post entry performance in Germany. Based on the learning theory of internationalization we develop hypotheses on the impact of different market entry modes on performance, and on the moderating effect of international experience on this relationship. The hypotheses are tested against a dataset of 159 Indian firms in Germany, using secondary data. Multivariate regression analyses show strong interaction effects of international experience, but only weak direct effects of different market entry modes on performance. Our study contributes to the market entry literature in general and provides new insights to the analysis of market entry strategies of 
emerging market firms in particular. (For more information, please contact: Ritam Garg, Friedrich-Alexander University of Erlangen-Nuremberg, Germany: ritam.garg@fau.de)

Testing the Internalization Theory in Emerging Markets: The Role of Firm-Specific Assets and Asset Dispersion in the Multinationality-Performance Relationship

Sumit Kumar Kundu, Florida International University

Ahmet Kirca, Michigan State University

Whitney Douglas Fernandez, San Diego State University

Testing the predictions of internalization theory with a large sample of MNCs from India, this study confirms that MNCs from emerging markets do possess traditional firm-specific assets and the successful internationalization of these MNCs essentially depends on the possession and exploitation of these productive assets in international markets. In addition, this study reveals that internalization theory requires further specification with a focus on the critical roles of industry context and asset dispersion to explain the success or failure of MNCs from emerging markets in international settings. As such, the present study provides a better understanding of firm internationalization, the role of firm-specific assets, and their financial impact in the context of MNCs from emerging markets. (For more information, please contact: Sumit Kumar Kundu, Florida International University, USA: kundus@fiu.edu)

Business Model Internationalization in the Automotive Industry - A Process Model of Market Entry into Emerging Markets

Christian Landau, EBS Business School

Amit Karna, Indian Institute of Management Ahmedabad

Miriam Sailer, EBS Business School

Emerging markets offer a wide range of opportunities, especially in terms of high growth potential. However, business models that enable firms to achieve competitive advantage in their home markets are often challenged by the characteristics of emerging markets. We argue that firms internationalizing into emerging markets have to reduce product adaptations and focus on business model adaptation. Based on an in-depth case study of a German luxury automobile manufacturer's internationalization to India, we develop a phase model of the process of internationalization from a business model adaptation perspective. We find that firms adapt their business models in four phases: international extension, local emergence, local expansion, and local consolidation. During this business model adaptation process the firm adapts the value creation and delivery component of its business model in the context of its activity system, whilst making only slight adjustments to its value proposition and value capturing. (For more information, please contact: Amit Karna, Indian Institute of Management Ahmedabad, India: karna@iimahd.ernet.in)

Balancing Headquarters Commitment and Subsidiary Employee Involvement: An Examination of Japanese MNC Country Managers in Asean

Katsuhiko Shimizu, Keio University

From the perspective of country managers of multinational corporations (MNCs), simultaneously dealing with both headquarters and local subsidiary is difficult, particularly in emerging markets where competitive and institutional environments are very different from those of developed home markets. This issue is highly critical to J apanese MNCs that experienced success in US and Europe, and thus need to unlearn their earlier success formula and develop a new one in emerging markets. In this paper, we examine how J apanese MNC country manages deal with both headquarters and local employees in three ASEAN countries, Singapore, Thailand, and Indonesia. Understanding the struggle and success of J apanese country managers will provide important insights with managers of MNCs in terms of how to refine and implement their global strategy in emerging markets. (For more information, please contact: Katsuhiko Shimizu, Keio University, Japan:

kshimizu22@gmail.com) 
Institutional Signatures or Firm-specific Imprints?: Joint Venture Profiles and the Performance Panorama in Five Emerging Market Groups

Hemant Merchant, University of South Florida St. Petersburg

This study investigates the relative significance of two sets of variables-institutional variables and firm-specific variables-vis-à-vis their influence on the expected performance of American firms who announce their participation in international joint ventures. Using Dunning's OLI paradigm as its theoretical anchor, this chapter discusses the performance role of institutional as well as firm-specific factors and puts forth two propositions. Recognizing the tremendous diversity in the institutional architecture across emerging markets, this study tests its propositions across five distinct emerging market groups: i) Asia, ii) BRICS, iii) Europe, iv) Latin America, and v) Rest of the world. Results indicate that the expected performance of American firms is largely homogeneous across these five groups. However, the institutional profiles of these groups vary considerably more than those of partner firms. Thus, this study underscores the theoretical as well as empirical imperative to model the role of institutions in studies of firm performance. (For more information, please contact: Hemant Merchant, University of South Florida St. Petersburg, USA: hmerchant@usfsp.edu)

\section{Antecedents to Different Motives of Foreign Expansion of Emerging Economy Firms Faisal Ahsan, Indian I nstitute of Management Lucknow \\ Ashutosh Sinha, Indian Institute of Management Lucknow}

Emerging economy firms are increasingly pursuing foreign expansion to seek newer markets and to acquire strategic assets and key resources. However, there is limited research on the factors that shape up the different motives of foreign expansion. Controlling for the changes in the institutional environment and using a multitheoretic approach, we study firm level and industry level factors that influence the foreign expansion motives of emerging economy firms. Using a sample of 293 I ndian manufacturing firms, from the pharmaceutical, chemical and automotive industries, we find that, firm's endowment of intangible assets, ownership structure, resource slack and exposure to foreign competition influence the motive of foreign expansion. (For more information, please contact: Faisal Ahsan, Indian Institute of Management Lucknow, India: fpm12005@iiml.ac.in)

\section{Subsidy, Learning, Innovation and Export: How Do Emerging Economy Firms Become Exporters? Yong J u Shim, FGV-EAESP Paulo Roberto Arvate, FGV-EAESP}

This study aims to look for new theoretical frameworks, in order to understand the process of becoming exporter through the combination of political economics and RBV approach's ideas. We argue that in the context of emerging economy, the governmental status towards their own firms is the most crucial factor, as well as the firms' own resources gained through learning ability. Empirical theory test has been done through robust regression based on the firm level data set, acquired from a survey done with 140 manufacturing firms located in four major emerging economies (BRICS). (For more information, please contact: Yong Ju Shim, FGV-EAESP, Brazil: morangoum@naver.com)

\section{Location Decisions of Chinese Multinationals: The Role of Overseas Communities \\ Bas Karreman, Erasmus University Rotterdam \\ Martijn Burger, Erasmus University Rotterdam \\ Frank van Oort, Utrecht University}

Overseas Chinese communities are an important determinant in the location choice of greenfield investments made by mainland Chinese multinational enterprises across European regions. This is shown by empirical 
analysis of an exhaustive set of projects across NUTS- 1 regions in 27 European countries for the period 20032010. When controlling for endogeneity bias and the embeddedness of existing Chinese economic activity, our results suggest that the significance of overseas communities is based on increased access to strategic information. Our findings are particularly robust in the context of knowledge intensive sectors and high value added functions. (For more information, please contact: Bas Karreman, Erasmus University Rotterdam, Netherlands: karreman@ese.eur.nl)

\section{Session: 3.1.11 - I nteractive}

\section{Track: 3 - International Human Resource Management and Organizational Behavior}

\section{Global HRM Practical Challenges}

Presented On: June 30, 2015 - 09:00-10:15

Chair: Anna Katharina Hildisch, University of Goettingen

You've Come a Long Way, Baby? Women in the Maritime Industry: Identifying Attitudinal and Structural Impediments

J oan P. Mileski, Texas A\&M University at Galveston

Wyndylyn von Zharen, Texas A\&M University

Sustainable careers for women in the maritime industry may look very different from maritime careers of the past. We propose the "WARS" model incorporating factors of wealth, appreciation, risk, and sacrifice, drawn from the job satisfaction research be used to address the need of maritime women. First, wealth/compensation must be competitive. Second, women must see appreciation for their unique perspectives and skills. Third, the risk that women encounter must be addressed through providing mentoring, sponsorship, and networking opportunities. Finally, sacrifice of time to the career away from family demands must be acknowledged with good practices toward gender-related work issues. (For more information, please contact: Joan P. Mileski, Texas A\&M University at Galveston, USA: mileskij@tamug.edu)

\section{Cultural Diversity as a Competitive Tool}

P.T. Navendra, University of Dallas

Sri Beldona, University of Dallas

A 2011 race relations survey found that contrary to government claims, racism ran deep among the ethnic groups in Malaysia. Diversity training is not viewed as a trust-building competitive tool. In this paper, we argue that diversity training, shared values, and cultural collectivism influence intra-organization trust within the IT Focused MNEs operating in Malaysia. From an economic perspective, shared values and diversity awareness induced trust can arguably result in the positive spillover of knowledge-sharing. This paper highlights both the challenges facing Malaysian MNEs and the latent opportunities for improved collaboration, innovation, and firm performance through diversity and trust. (For more information, please contact: Sri Beldona, University of Dallas, USA: sbeldona@udallas.edu)

\section{Does Size Matter? The Effect of Institutional Size on Global Virtual Teams \\ Grishma Shah, Manhattan College \\ A.F.M Jalal Ahamed, Binus Business School \\ Pawel Bryla, University of Lodz}

With the convergence of globalization and technology, we find more and more students and employees immersing themselves in virtual environments. They are no longer bound by physical space or time and can collaborate with multifaceted and highly functional technologies in real-time. The corporate world is witnessing 
a race for low cost, high value talent globally and is replete with employees working on Global Virtual Teams (GVTs). GVTs are relatively new and while there is a growing body of research, the incessantly evolving nature of technologies, demographics, institutions and globalization demand an ongoing examination of these dynamics. In this study, we address this demand by investing the effects of institutional size on GVT performance. We analyses data from thousands of students working on GVTs in hybrid (partially online and partially in traditional classrooms) courses to understand whether the size of their physical class plays a role in GVT performance. Our initial findings suggest that class size does play a negative role on performance as measured by peer evaluations and student grades. Time invested on the team task plays a moderating role on peer evaluations but not on student grades. Implications for both educational institutions and firms are discussed. (For more information, please contact: Grishma Shah, Manhattan College, USA: grishma.shah@manhattan.edu)

\section{A Yin-Yang Harmony Perspective to Labor-Management Conflicts in China: A 5C Model for Conflict Resolution Tachia Chin, Guangdong University of Foreign Studies Tony Fang, Stockholm University}

Despite increasing labor-management conflicts in the field of human resource management (HRM) in China's manufacturing, Western theories still can't well explain how Chinese culture influences the way Chinese companies deal with employee disputes due to the use of the inappropriate paradigm. To fill this gap, this paper employing a Yin Yang harmony perspective proposes a 5C model delineating a unique "harmonizing process" for solving labor-management conflicts in the Chinese context. We used a ground theory approach to investigate how the world's largest footwear manufacturer in China handle a severe labor strike that just happened in April, 2014 to examine the proposed 5C model. This study contributes to the literature by suggesting an emic, context-specific concern- concern for harmony in China, which is beyond the Western dual-concern model regarding the choice of coping with labor conflicts. More specifically, the novel, circled $5 \mathrm{C}$ framework (conflict, clash, communication, comprise and consensus) characterizes the dynamic, contingent and art-oriented nature embedded in the resolving process of labor-capital conflicts in Chinese HRM. (For more information, please contact: Tachia Chin, Guangdong University of Foreign Studies, China: tachia1231@yahoo.com.sg)

\section{Managerial Assignments of Credit and Blame: A Three Country Study C Lakshman, Tongji University}

Despite the importance of credit- and blame-attributions, the literature has not examined managerial assignments of credit and blame respectively, to subordinate behaviors. Arguing that these types of managerial attributions are likely to have a strong impact on what constitutes desirable leadership behaviors, we examine them in three different countries, viz., USA, France, and India. We contribute to the literature by examining the influence of credit- and blame-attributions on subordinate performance and satisfaction, unaddressed in the literature to date. We also contribute by demonstrating the importance of these attributions for acceptance of leadership in three different cultures. Our results are supportive of the hypotheses developed and suggest the important moderating role of subordinate performance for leadership acceptance. We discuss the findings in the context of the literature, highlight contributions, and identify limitations and directions for future research. (For more information, please contact: C Lakshman, Tongji University, China: clakshman2007@gmail.com)

\section{Foreign Employee's Responses to the 2011 Triple Disaster in Japan \\ Ralf Bebenroth, Kobe University \\ Nir Kshetri, University of North Carolina at Greensboro}

The purpose of this work is to examine how the Great East J apan Earthquake impacted foreign employees at subsidiaries in J apan. We divide foreign employees in three types, classic expatriates, locally hired foreigners and short term employees which are known in the literature as frequent fliers. We found that foreign employees differ according to their type in adjusting in case of an emergency. Expatriates exhibited a higher 
tendency to leave the country than locally hired foreigners. Frequent fliers hardly arrived to the Japanese subsidiary anymore after the triple disaster. Surprisingly, subsidiaries faced more hardship because of the lack of frequent fliers than to a lack of locally hired foreigners. Results also depend on the respondents' nationality. Japanese respondents expressed more concern that it was difficult at the triple disaster to retain expatriates in their J apanese jobs. German respondents were more concerned about retaining locally hired and frequent fliers at their subsidiaries. Our surprising results of more hardship when frequent fliers left than when local hired foreigner left was given by a higher importance of J apanese respondents. Furthermore, German respondents see still nowadays difficulties to get local hired foreigners back to their subsidiaries when they left the country. Implications are discussed. (For more information, please contact: Ralf Bebenroth, Kobe University, Japan: rbeben@rieb.kobe-u.ac.jp)

\section{Session: 3.1.12 - I nteractive \\ Track: 10 - Theories of the MNE and of FDI}

\section{Determinants and Consequences of Internationalization Strategies}

Presented On: J une 30, 2015 - 09:00-10:15

Chair: Pallavi Shukla, Rutgers University

Strategic Fit in Emerging Economy Firms' Internationalization: A Configurational Approach

Yi Li, Australian National University

Lin Cui, Australian National University

Di Fan, Deakin University

Despite the "strategic fit" concept is intuitively appealing, researchers pay relatively little explicit attention to strategic fit in recent strategic management (SM) and international business (IB) studies. To contribute the further development of this important theoretical paradigm, this study seeks to develop a theoretical framework of emerging economy (EE) firms' strategic fit that is configurational, multidimensional, and within TMT's structure. To test this framework, we follow a configurational perspective of theory building, and a fuzzy-set qualitative compariative analysis (fSQCA) method in particular. Empirically, we choose the context of Chinese outward foreign direct investment (FDI). Our findings indicate that multiple equifinal configurations of environment, FDI strategy, and TMT's structure factors will help EE firms to achieve strategic fit that deliver high internationalization performance. (For more information, please contact: Di Fan, Deakin University, Australia: david.fan@deakin.edu.au)

\section{Performance Feedback and International Expansion \\ K. Skylar Powell, Western Washington University}

This study argues that we can better understand the internationalization process if we clearly specify problems that 'trigger' a firm's search for, and evaluation of, foreign market opportunities. From the perspective of performance feedback theory, a failure to meet organizational goals is likely to trigger strategic action and change. Goals are frequently framed in terms of aspirational performance goals, and shortfalls in actual performance vis-à-vis aspirational performance can trigger search and learning behavior directed at resolving problems. It follows that there should be a positive relationship between shortfalls in aspirational performance and internationalization in many contexts. Consistent with expectation, shortfalls in aspirational performance are positively associated with decisions to enter new foreign markets in this empirical context of prestigious New York law firms from 1984 through 2008. (For more information, please contact: K. Skylar Powell, Western Washington University, USA: skylar.powell@wwu.edu) 


\section{Reconstructing Hennart's Transaction Costs Theory of Equity J oint Ventures}

Shih-Fen Chen, I vey Business School

This paper reconstructs Hennart's transaction costs theory of equity joint ventures by clarifying three major misconceptions in his original analysis. Published in this journal in 1988, Hennart's theory has garnered over 2,000 Google Scholar citations and the 2013 Academy of International Business Annual Meeting devoted one plenary session to celebrating the 25th anniversary of this theory. It is unusual that three major misconceptions in such an influential paper have remained unnoticed over a long period of 26 years. After clarifying these three misconceptions, we rebuild Hennart's theory to illustrate how equity joint ventures work to link a multi-step value chain that cannot be easily structured in the market or under the hierarchy. Our reconstructed model allows scholars to better analyze, and executives to better manage, equity joint ventures as a governance device of inter-firm cooperation. (For more information, please contact: Shih-Fen Chen, Ivey Business School, Canada: sfchen@ivey.uwo.ca)

\section{Do Resource Seeking Motivations Impact the Performance of Acquisitions? \\ Sathyajit Gubbi, University of Groningen \\ B. Elango, Illinois State University}

The use of acquisitions to obtain desired or missing resources and capabilities is a well-accepted concept in the IB literature. With focus on the resource-based view of the firm, we identify two motivations for asset-seeking acquisitive behavior: resource deepening and resource extension. Using a sample of 1004 acquisitions conducted by Indian firms during the period 2000-2010, we find support for the notion that the underlying motivation for the acquisition is a significant factor impacting performance. This study extends existing knowledge on emerging market firm acquisitions in several ways. First, it differentiates acquisitions using specific motivations of acquiring firms and shows that performance outcomes vary by nature of resource sought by the firm in the transaction. Second, while we find that the results for resource extension acquisitions are not favorable to firms, this study offers important insights using knowledge and learning theories as to how a firm could address this disappointing outcome in a favorable manner. (For more information, please contact: $B$. Elango, Illinois State University, USA: elango@ilstu.edu)

\section{The Impact of Multinationality on Performance: A Comment Keith J Kelley, University of Michigan-Flint}

Recent research has made use of meta-analytic techniques to conclude that some of the spurious findings in multinationality and performance (MP) research is partially attributable to a number of moderating influences. While future research will undoubtedly adopt improved methodologies, a recent pair of articles by Hennart (2007; 2011) questions whether or not MP research adds any value beyond such traditional theories as transaction cost economics and internalization (TCl). In this article we seek to comment on Hennart's (2011) arguments surrounding the value of future MP research in consideration of internalization theory, various dimensions of multinationality, and in so doing provide insight into solving some methodological challenges surrounding optimal fit of a firm's foreign 'footprint' (i.e., multinationality). (For more information, please contact: KeithJ Kelley, University of Michigan-Flint, USA: keithkel@umflint.edu)

\section{Export Intermediary Firms: The Study of Performance Determinants via Structural Equation Modeling Pornlapas Suwannarat, Mahasarakham University}

This study has primarily focused on the competence of export intermediary firms in Thailand in reducing transaction costs, which relates to their performance. Structural equation modeling (SEM) technique has been adopted to test the relationship of each variable in the model of the study and to investigate the direct influence, indirect influence, and total influence of the independent variables on the dependent variables. Based on three theories, transaction cost, agency and resource-based theory, the results indicate that intangible 
resource of negotiation skills has positive effect on the competence to reduce clients' negotiation costs and the competence to reduce clients' monitoring and enforcement costs. Specialisation and trustworthiness has been found the significant impact on the competence to reduce clients' monitoring and enforcement costs and the competence to reduce clients' search costs. The findings reveal that three valuable resources of export knowledge, negotiation skills, and specialization and trustworthiness have both direct and indirect effect on export intermediary performance. Further, the result of the study indicate that model of the study has good fit to the empirical data. (For more information, please contact: Pornlapas Suwannarat, Mahasarakham University, Thailand: pornlapas.s@mbs.msu.ac.th)

\section{The Internationalization of Distribution Channels in Semiconductor Industry: The Uppsala Business Network Model \\ J oseph Shyh-Yeu Tzou, National Taiwan University}

This study argues that firm's network positions play important roles in its internationalization, and intends to provide a network explanation of the revised Uppsala model for the purported relationship between firm's network positions and its internationalization in the context of semiconductor industry. This study adopts the quantitative methodology to explore the research questions. Drawing on thirty years of observations for each of the five years from 1980 to 2010 for distributor firms in the semiconductor industry, this study utilizes panel data regression to examine the effects of a firm's network positions on its internationalization. The sample of this study is the 113 franchised semiconductor distributor firms in Taiwan of the WSTS's (World Semiconductor Trade Statics) 63 member companies. WSTS's member companies represent more than $75 \%$ of the world semiconductor market. Worldwide top 20 semiconductor suppliers are WSTS members. This virtually includes all major semiconductor manufacturers from 4 major regions, namely America, EMEA(Europe, Middle East, and Africa), Japan and Asia Pacific. Results of this study support the positive relationship between firm's centrality of its network positions and its internationalization, the positive relationship between firm's structural holes of its network positions and its internationalization, and the positive relationship between firm's brokerage of its network positions and its internationalization. The moderator effects of firm's joining association and going initial public offering (IPO) in the structural holes-internationalization relationship are supported by this study, while the moderator effects of firm's joining association and going IPO in the centrality-internationalization relationship are not supported by this study. (For more information, please contact: Joseph Shyh-Yeu Tzou, National Taiwan University, Taiwan: joesytzou@gmail.com)

\section{Food Retail FDI: Learning Through History Brenda Sternquist, Michigan State University \\ Blake Runnalls, Michigan State University}

Most theories of internationalization assume that the company has an export option; this export option is likely a company's first exposure to international markets. Retailers do not have this option. Food retailers represent a type of soft service, where production and consumption of the service cannot be separated. Retailers sell or provide services to the ultimate customer; they must therefore have a physical presence in the country. What makes a country an attractive target for a retailer? We are developing a database of food retailers' international activities. This database includes retailers from throughout the world from 1991 to 2007. Three levels of data are included: company financial data, company dates of international expansion and country statistics. We discuss a portion of the findings for four firms (468 observations) in this preliminary paper. (For more information, please contact: Brenda Sternquist, Michigan State University, USA: sternqui@msu.edu) 


\section{Session: 3.2.1 - Special Session}

Investing in Development - Reforming International Governance and MNEs: International Investment Agreements and Taxation (UNCTAD Special Session)

Presented On: J une 30, 2015 - 10:45-12:00

Chairs: Hafiz Mirza, UNCTAD and Axèle Giroud, UNCTAD

Panelists:

Peter Buckley, University of Leeds

Sarianna Lundan, University of Bremen

Premila Nazareth, UNCTAD

Felipe Monteiro, INSEAD

Ram Mudambi, Temple University

(For more information, please contact: Hafiz Mirza, UNCTAD, Switzerland: hafiz.mirza@unctad.org)

\section{Session: 3.2.2 - Panel \\ Special Session}

\section{Think Global, Act Local: Best Practices for Managing and Developing AI B Chapters}

Presented On: June 30, 2015 - 10:45-12:00

Chairs: Daniel Rottig, Florida Gulf Coast University and Elizabeth Rose, University of Otago

Panelists:

Daniel Rottig, Florida Gulf Coast University

Elizabeth Rose, University of Otago

Kazuhiro Asakawa, Keio University

T.S. Chan, Lingnan University

Abel Kinoti, Riara University

Stephan Manning, University of Massachusetts Boston

William Newburry, Florida International University

Lukasz Puslecki, Poznan University of Economics

S. Raghunath, Indian Institute of Management Bangalore

Piotr Trapczynski, Poznan University of Economics

The Academy of International Business (AlB) currently operates 18 chapters in order to facilitate networking and the exchange of knowledge at the local level. The purpose of this panel is to bring together former and current AlB chapter chairs to discuss the best practices for managing and developing chapters. Former and current chapter chairs from the 6 continents on which AlB is operating chapters as well as the AlB vice president in charge of chapters will attend the panel and provide the interested audience with an overview of AlB chapters, share valuable information about AlB's chapter strategy and how to establish a new chapter including related AlB policies, discuss best practices for managing and growing a young chapter, for institutionalizing and developing an established chapter, and for operating and developing chapters in emerging markets. Panelists will further share knowledge and experiences across chapters, discuss best practices for cooperating with other organizations and developing cross-disciplinary meeting formats, discuss opportunities and challenges when managing chapters, and provide advice to current and future chairs and organizers of already established and potentially new chapters. (For more information, please contact: Daniel Rottig, Florida Gulf Coast University, USA: drottig@fgcu.edu) 


\section{Session: 3.2.3 - Competitive}

\section{Track: 3 - International Human Resource Management and Organizational Behavior}

\section{Diversity and Creative Friction}

Presented On: June 30, 2015 - 10:45-12:00

Chair: Helene Tenzer, Tuebingen University

Lesbian, Gay, Bisexual and Trans (LGBT) Expatriates- An Intersectionality Perspective

Varina Paisley, University of New South Wales

Mark Tayar, Macquarie University

This paper addresses a significant gap in the International Human Resource Management (IHRM) literature on lesbian, gay, bisexual and trans (LGBT) expatriates. Using the theoretical perspective of intersectionality, we explain the factors influencing the choices made by LGBT employees regarding expatriation and how changes in cultural context influences their multiple identities. It is estimated that LGBT individuals make up to $10 \%$ of the population, with some estimates much higher, and yet this group is often overlooked. This article gives a voice to LGBT expatriates and questions the traditional expatriate management model. In particular, it addresses the role of cultural norms and social roles, the perception and negative attitudes towards non-traditional expatriates and discrimination and silencing in organisations. The paper concludes with suggestions for pre-assignment, onassignment, and post-assignment strategies to overcome these barriers. (For more information, please contact: Varina Paisley, University of New South Wales, Australia: v.paisley@unsw.edu.au)

The Impact of Language Barriers on Interaction Processes, Knowledge Exchange and Team Cognition in Multinational Teams

Helene Tenzer, Tuebingen University

Markus Pudelko, Tuebingen University

Mary Zellmer-Bruhn, University of Minnesota

Mary Maloney, University of St. Thomas

Based on qualitative case studies of 15 MNTs in three major German automotive corporations, this study investigates how language barriers influence the formation of team cognition in multinational teams (MNTs). Our findings uncovered divergent influences of language on task-related technical exchange compared social interaction with emotional content. We show how these processes affect the exchange of task- and teamrelated knowledge, which in turn form the building blocks for task mental models, team mental models and transactive memory systems in MNTs. Our study advances research on team cognition by introducing language barriers as a previously neglected antecedent, by connecting the concept to emotionally laden interaction and by extending its investigation to multinational settings. It contributes to MNT research by encouraging more interdisciplinary theorizing on team processes. (For more information, please contact: Helene Tenzer, Tuebingen University, Germany: helene.tenzer@uni-tuebingen.de)

Creativity and Social Alienation: The Costs of Being Creative

Guangxi Zhang, Zhejiang University of Technology

Are creative employees likely to feel social alienation? Although creativity is a widely praised merit, the costs an individual may pay for being creative is generally less examined. The present study hypothesizes that because of internal control, divergent thinking, and change orientation, creative employees are more likely to have conflicts with the environment and hence feel socially alienated. Moreover, the extent to which creative employees perceive social alienation varies with individual and contextual differences, exemplified by individuals' networking ability and the perceived organizational value for creativity. A two-wave survey among 311 
employees from 59 organizations supported the hypotheses. Implications of the findings are discussed for creativity and alienation literature as well as management practice. (For more information, please contact: Guangxi Zhang, Zhejiang University of Technology, China: gxzhang@zjut.edu.cn)

\author{
Transformational Leadership Behaviors in Different Contexts: A Comparison of Russia and Finland \\ Alexei Koveshnikov, Aalto University \\ Mats Ehrnrooth, Hanken School of Economics
}

In this paper we contribute to the literature on transformational leadership in cross-cultural settings by examining the influences of core transformational leadership and supportive leadership behaviors - the two behavioral dimensions of transformational leadership - on followers' organizational identification in two culturally distinct countries of Russia and Finland. We also test how these relationships are mediated by followers' role ambiguity. Empirically, we use Linear Mixed Modeling to analyze a sample of white-collar employees in four Finland-based multinational corporations (295 employees in 72 teams) and their subsidiaries in Russia (104 employees in 28 teams). We find that whereas in Russia both core transformational and supportive leadership behaviors facilitate followers' organizational identification, in Finland only core transformational leadership behavior does so. Moreover, even though in both contexts role ambiguity appears to mediate the relationship between transformational leadership and followers' organizational identification, in Russia it fully mediates only the relationship between supportive leadership behavior and followers' identification and in Finland it partially mediates only the core transformational leadership behavior - followers' identification relationship. (For more information, please contact: Alexei Koveshnikov, Aalto University, Finland: alexei.koveshnikov@aalto.fi)

\title{
Session: 3.2.4 - Competitive Track: 6 - Innovation and Knowledge Management
}

\section{MNC Structure and I nnovation}

Presented On: J une 30, 2015 - 10:45-12:00

Chair: C. Annique Un, Northeastern University

Does Strategic Planning determine Innovation in Organizations?

Safal Batra, IMT Ghaziabad

Sunil Sharma, Indian Institute of Management Ahmedabad

Mukund R Dixit, Indian I nstitute of Management Ahmedabad

Neharika Vohra, Indian Institute of Management Ahmedabad

While some researchers argue that strategic planning assists new product development and can be seen as a framework for innovation, others believe that it restricts creativity and innovation. Despite the appreciation in literature that strategic planning is linked to innovation, the nature of this relationship remains ambiguous. In this study, we propose that strategic planning assists innovation when organizations utilize it as a learning mechanism, rather than a to-do activity. Data for this study, measuring the extent of strategic planning, organizational structure, commitment to learning and innovation in organizations, were collected by administering standardized survey questionnaires to entrepreneurs or other senior executives of small and medium businesses. Quantitative analysis (structural equation modeling) of data obtained from 123 SMEs in the manufacturing sector yielded a positive relationship between strategic planning and innovation. This study also established a significant positive moderating role of commitment to learning on the relationship between strategic planning and innovation. Strategic planning, coupled with organizational learning, helps organizations in responding to the contingencies effectively and makes them more capable of assessing the complexities of their external environment, thus enhancing their likelihood of successful innovation. (For more information, please contact: Safal Batra, IMT Ghaziabad, India: safalb@iimahd.ernet.in) 
Exploring the Role of Formalization, Centralization, and Coordination on Cultural Competitiveness and Firm Performance

Artur Baldauf, University of Bern

Simone Schweiger, University of Bern

In this study, we focus on the strategy and structure paradigm by integrating ideas from the behavioral theory of the firm. Viewing strategy from a cultural competitiveness perspective, we conceptualize this construct as multi-dimensional comprising market, learning, entrepreneurial, and innovation orientations. For structure, we refer informal coordination, formalization, and centralization. Investigating multi-business firms, we choose a conceptualization of coordination that focuses on efforts for synergy creation between business units. We draw on the confines of organizational actions put forward by behavioral theorists (i.e., bounded rationality, imperfect environmental matching, and unresolved conflict) when providing a logic as to why structure precedes strategy, ultimately leading to superior performance. Further, we argue that two of our structure determinants, i.e., coordination and formalization exhibit an interaction effect in its relationship to strategy. Performing regression analysis, we test our hypothesis with a sample of 244 multi-business firms located in a German-speaking environment. We find support for our hypotheses, and thereby contribute to the understanding of the strategy and structure discussion by adopting a context-specific research approach. In addition, we extend the cultural competitiveness literature by revealing antecedents of its relationship to performance. (For more information, please contact: Artur Baldauf, University of Bern, Switzerland: baldauf@imu.unibe.ch)

\section{How Valuable Is Information and Communication Technology? A Study of Emerging Economy Enterprises Yadong Luo, University of Miami \\ J uan Bu, University of Miami}

Anchored at the knowledge management perspective, we address how information and communication technology (ICT) improves the productivity of emerging market firms. We present the logic that ICT enhances firm performance because it is an important channel or facilitator of effective knowledge sharing and knowledge integration. We further argue that the conditions characterizing an emerging economy (i.e., a country's economic development) and emerging economy businesses (i.e., internationalization and quality) would affect the extent to which ICT contributes to knowledge management, and thus to firm performance. Our hierarchical linear modeling analysis of 6,236 firms from 27 emerging economies lends support to our arguments and predictions, suggesting that ICT is a critical investment that generates satisfactory returns for emerging economy enterprises, yet this investment-return relationship is further contingent upon the macro- and microlevel conditions facing these enterprises. ICT actually adds more value to productivity when a focal emerging economy is less economically developed, and when a focal firm reaches foreign markets or its quality control and assurance is superior. (For more information, please contact: J uan Bu, University of Miami, USA: j.bu@umiami.edu) 


\title{
Session: 3.2.5 - Competitive
}

\section{Track: 10 - Theories of the MNE and of FDI}

\section{Again, but This Time Better - Leveraging Experience When Going International}

Presented On: June 30, 2015 - 10:45-12:00

Chair: Timothy Devinney, University of Leeds

\author{
The Impact of Prior Subsidiary Relatedness on New Subsidiary Performance \\ Dwarkaprasad Chakravarty, I vey Business School \\ Andreas Schotter, I vey Business School \\ Brian Pinkham, I vey Business School \\ Paul W. Beamish, Western University
}

We examine how prior host country cultural and industry sector experience impacts new subsidiary performance. Using Japanese MNE investment data in 29 countries, we find that the likelihood of superior performance increases when new subsidiaries are established in countries which are culturally similar to prior subsidiaries. In addition, when cultural similarity is complemented by industry sector relatedness, new subsidiaries are three times more likely to succeed. Such complementarity has a stronger effect on joint venture performance relative to wholly owned subsidiary performance. This study emphasizes the subsidiary to subsidiary perspective, contributes to experiential learning and sequential strategy theories, and provides managerial implications for expansion choices. (For more information, please contact: Dwarkaprasad Chakravarty, Ivey Business School, Canada: dchakravarty.phd@ivey.ca)

\section{The Effects of Experience on Choosing an International Entry Mode} Ryan Tang, University of Technology Sydney Timothy Devinney, University of Leeds

This study investigates how managers' international experience and its interaction with firms' global experience may influence managers' choices of entry modes. We find that managers' international experience can both increase the probability of entering a growing market and make managers prefer joint ventures to non-equity means in the market, despite the fact that firms' global experience may negatively influence managers' preference. Also, while the depth of firms' global experience strengthens the probability of entering an international market, the geographic breadth of firms' global experience does not. Our experimental design complements the extant literature in three ways: (a) incorporating managers' and firms' experience for examining variations among decision-making models; (b) investigating interactions of their experience by observing covariate and moderating effects in the decision-making models, (c) providing direct evidence of heterogeneity in decision-makers. We join the prior findings of both managers' and firms' experience together, and contribute literature with new insights into the effects of experience on strategic decision making. (For more information, please contact: Ryan Tang, University of Technology Sydney, Australia: ryan.w.tang@gmail.com)

\section{Re-Born Internationally! Do Past Antecedents Affect Performance in Re-Internationalizing Firms? Salman Siddeeque Ali, Indian Institute of Management Ahmedabad Ajeet Narain Mathur, Indian Institute of Management Ahmedabad}

This paper presents findings from research into a hitherto unexplored phenomenon of re-internationalization by firms, i.e. re-entry to international operations after a complete withdrawal and time-out from all prior international operations. The study was undertaken to examine the nature of continuities and discontinuities, if any, between previous experience and later attempts in terms of contributions to firm performance. An exhaustive survey was administered among top-level managers in Indian business entities that had gone 
through the process of re-internationalization, exhausting almost the entire population of re-internationalized firms in India ( 71 responses from 73 qualified firms). The data analysis using Partial-Least-Squares based Structural-Equation-Modelling technique provided support for the following hypothesized relationships: initial international experiences, organizational commitment to internationalization, and the presence of dynamic capabilities are positively related to re-internationalization performance. However, continuity in networks and key personnel from initial through re-entry phases of internationalization was not found to have a significant effect on re-internationalization performance as hypothesized earlier. This could be primarily due to the relatively smaller sizes of the firms studied as most of the firms were found not to have formed networks or assigned specific individuals for international operations in the initial phase. The findings have implications for strategies of internationalizing and re-internationalizing firms and for policies that support subventions for internationalization. (For more information, please contact: Salman Siddeeque Ali, Indian Institute of Management Ahmedabad, India: salman@iimahd.ernet.in)

\title{
Session: 3.2.6 - Competitive
}

Track: 9 - Emerging Economies and Emerging Economy MNEs

\section{EMNCS and Entry Modes}

Presented On: June 30, 2015 - 10:45-12:00

Chair: Helena Barnard, GIBS, University of Pretoria

\author{
Home Country Effects in Cross-Border Acquisitions: Gaining Confidence in Majority Control Abroad \\ Zhenzhen Xie, Tsinghua University \\ Jiatao Li, Hong Kong University of Science and Technology
}

Building on the concept of "home country effect", we argue that the information signaled by the strategic choices of home country firms previously invested in a host country will influence the decisions of later arrivals by affecting their level of confidence in that strategy. Combining institutional theory with an organizational learning perspective, we show that in the context of cross-border acquisitions (CBAs), the number of majority CBAs previously made by home country firms in a host country increased a follower firm's confidence about imitating this control mode. Due to a higher level of uncertainty and commitment involved in a majority control CBA, the confidence gained from home country acquirers about imitating majority CBAs is stronger than that gained about imitating minority CBAs. The number of majority or minority CBAs made by acquirers from other countries in the same host country did not contribute to the imitation confidence of follower firms. In addition, the confidence gained from compatriots about imitating majority or minority CBAs is weakened when the acquirer is state-owned. The above argument is supported by empirical work on 604 CBAs made by Chinese firms during 1986-2008. (For more information, please contact: Zhenzhen Xie, Tsinghua University, China: xiezhzh3@sem.tsinghua.edu.cn)

\section{Opportunities and Challenges for Multiple-Embeddedness through Mergers and Acquisitions in Emerging Economies \\ Surender Munjal, University of Leeds \\ Vijay Edward Pereira, University of Portsmouth}

The paper seeks to examine opportunities and challenges from multiple-embeddedness of developed countries multinational enterprises (DMNEs) in emerging economies. Utilising a 10 years long panel data spread prior and during the 2008 financial crisis, it finds that opportunities of embeddedness increased but challenges have decreased over time. (For more information, please contact: Surender Munjal, University of Leeds, United Kingdom: smu@lubs.leeds.ac.uk) 
The Impact of Institutional Distance, Government Support and Firms' Acquisition Capability on the Equity Ownership Acquired by Emerging Multinationals in Cross-Border Acquisitions

Claudia Frias Pinto, EAESP-FGV

Christian Falaster, Universidade Nove de J ulho

Renata Canela, Universidade Nove de J ulho

Maria Tereza Fleury, Fundacao Getulio Vargas

Afonso Fleury, Universidade de Sao Paulo

In this study we analyze the impact of institutional distance, government support and acquisition capability on the equity ownership acquired by emerging market multinationals (EMNCs) in cross-border acquisitions (CBAs). Extant research has already delved into why EMNCs acquire foreign firms abroad, but we have an incomplete understanding of the impact of the institutional differences between countries and of the role of government support on the EMNCs' CBAs. Based on secondary data of 278 CBAs deals by Brazilian multinationals, over a seven years period (2006-12), we examine how the choice between partial and full ownership may be explained by the institutional distance, government support - operationalized in terms of (a) financing, (b) stock participation, and (c) political ties - and EMNCs' acquisition capability. We find that the likelihood of engaging in a full acquisition is higher when the institutional distance between acquirer and target countries is high, and when the EMNCs benefit from government support both as a shareholder and via political ties. However, the likelihood of a partial acquisition increases when the EMNCs have an acquisition capability. This study contributes to the understanding of EMNCs' acquisitions and adds to the literature on the impact of government intervention on EMNCs foreign strategies. (For more information, please contact: Claudia Frias Pinto, EAESPFGV, Brazil: claudia. frias.pinto@gmail.com)

\section{Legitimate and I/legitimate Constraints: Managing in Limited Mandate Subsidiaries Helena Barnard, GIBS, University of Pretoria Deepa Sita, GI BS, University of Pretoria}

MNCs are increasingly organised as a differentiated network within which developing country subsidiaries typically have only a limited mandate. Since MNCs in those countries are often desirable employers, the limited mandate introduces a tension for the often skilled individuals who manage those subsidiaries. Interviewing both current and former managers of three foreign MNCs in South Africa, we found that managers resolve this tension by distinguishing between legitimate and illegitimate headquarter directives. Understanding their distinction criteria helps advance theory about how MNC mandates function and can help MNCs to develop mandates that do not alienate managers in limited mandate subsidiaries. (For more information, please contact: Helena Barnard, GIBS, University of Pretoria, South Africa: barnardh@gibs.co.za)

\section{Session: 3.2.7 - Competitive}

\section{Track: 6 - Innovation and Knowledge Management}

\section{Innovation and the Drivers of MNCs Performance}

Presented On: J une 30, 2015 - 10:45-12:00

Chair: Deepak Somaya, University of Illinois at Urbana-Champaign

\section{Is There a Multinationality-Performance Effect? Exploring the Multinationality-Performance Relationship Heather Berry, George Washington University \\ Aseem Kaul, University of Minnesota}

We examine the effect of multinationality on firm performance using detailed longitudinal data for a comprehensive sample of US manufacturing MNCs from 1989 to 2007, while accounting for the endogeneity of multinationality. We show that while firm performance has a positive effect on multinationality, multinationality 
has an insignificant effect on performance once we account for its endogeneity. Looking beyond an aggregate measure of multinationality, we show that firms do benefit from investments in foreign R\&D activities and product transfers between subsidiaries. Overall, these findings offer fresh empirical insight into the relationship between multinationality and performance, providing a substantially more rigorous test of the relationship while opening the way for further exploration of the effect of foreign knowledge seeking and subsidiary networks on firm performance. (For more information, please contact: Heather Berry, George Washington University, USA: berryh@gwu.edu)

\section{Of Marshallian Scissors \& Blades: Demand Shocks, Import Exposure, and Innovation in Indian Influenza-Vaccine Markets \\ Matej Drev, Georgia Institute of Technology \\ Chirantan Chatterjee, Indian Institute of Management Bangalore \\ Arzi Adbi, INSEAD}

This paper examines the relationship between exogenous shocks in the demand for pharmaceuticals and the structure of I ndia's vaccine markets. Using a novel dataset of detailed purchasing information for vaccines in the country between 2007 and 2013, and exploiting the occurrence of the 2009-10 global H1N1 pandemic as an exogenous shock to demand for influenza vaccines, we document a significant effect of the demand shock on inventive activity in the influenza vaccine market, leading to a sizable market structure shift in India toward declining import exposure and market share gains by domestic vaccine producers. The declining imports of vaccines produced by multinational pharmaceutical firms occur as a result of a proliferation in new product introductions by domestic firms in influenza vaccine markets. We also document the effects of targeted policies by health authorities aimed at spurring development and introduction of new vaccines in India. Our results contribute to an ongoing debate on the relationship between market size and innovation and offer novel policy implications for innovation and competition in a globalized world. (For more information, please contact: Matej Drev, Georgia Institute of Technology, USA: matej.drev@pubpolicy.gatech.edu)

\section{How Does Technological Relatedness between Cross-Border M\&A Partners Affect Innovation Performance? A Moderating Role of Organizational Integration Youngwoo Lee, Korea University Moonsik Shin, Korea University}

This study examines the effects of both pre-merger knowledge relatedness dimensions between merging firms and post-merger organizational integration on the subsequent innovation performance in the cross-border M\&A context. Although external knowledge acquisition has been a primary motive for cross-border M\&As in the last decades, its differential effect of knowledge relatedness on performance in contrast to domestic M\&A has been overlooked in the M\&A literature. By analyzing 130 cross-border M\&As in high-technology industries, we find that technological complementarity and diversity have contrasting contributions to different types of innovation; complementarity facilitates exploitive innovation while diversity increases exploratory innovation. Furthermore, our research investigates whether the degree of integration after the merger either propels or restrains the knowledge absorption process by the acquirer depending on the relatedness between existing knowledge and newly acquired knowledge. Our result suggests that the high degree of integration is beneficial to expedite knowledge exploitation due to relatively high absorptive capacity, while it has no effect on knowledge exploration. (For more information, please contact: Youngwoo Lee, Korea University, Korea, South: lee3992@korea.ac.kr) 
This paper explores the role of organizational architecture mechanisms in capability evolution. The existing literature describes the role of organizational traits in capability creation, but a detailed account of how changes in the firm's architecture enable the development of capabilities has not yet been explored. Drawing on two indepth case studies developed in the context of business service offshoring, this paper contributes to the literature on capability evolution in three ways. First, it theorizes that narrow scope roles and performance metrics are fundamental channels used to guide capability emergence in the initial stage of offshoring, in both in-house and outsourced centers. Second, it suggests that incipient capability emergence creates a push for increases in functional integration with other firms' units, regardless of whether the firm adopts in-house or outsourced offshoring methodologies. Third, it theorizes that advanced stages offshoring show a larger dispersion of organizational architecture tools in either in-house or outsourced offshoring settings, as the tools are selected to tie the leveraging of already developed capabilities to support the firm at large. (For more information, please contact: Carlos Adrian Rodriguez, INCAE, Nicaragua: carlos.rodriguez@incae.edu)

\title{
Session: 3.2.8 - Competitive
}

\section{Track: 4 - International Marketing, Cross-Cultural Marketing, Social Communities}

\section{Insights into Buyer-Supplier Relationships}

\author{
Presented On: J une 30, 2015 - 10:45-12:00 \\ Chair: Susan Mudambi, Temple University \\ Exploring Coopetition - When Partners Start to Compete \\ Emilene Leite, Uppsala University \\ Susanne Aberg, Uppsala University \\ Cecilia Pahlberg, Uppsala University
}

The paper explores the concept of coopetition by addressing a situation when business partners start to compete. Specifically, it intends to understand why firms move from cooperation to competition. To achieve this, case studies based on the development of an IT-solution for urban mobility in Brazil are undertaken. The cases show local and foreign MNEs interactions during and after the project. The findings suggest that the higher the level of interdependence, the higher likelihood of developing a cooperative strategy. In contrast, the lower the level of interdependency, the higher is the degree of competition; meaning that partners will choose a competitive strategy instead. (For more information, please contact: Emilene Leite, Uppsala University, Sweden: emilene.leite@fek.uu.se)

\section{The Effect of Economic Crises on Long-Term Orientation in Buyer-Supplier Relationships Brenda Sternquist, Michigan State University Lindsey M Cohan, Perishables}

Although recessionary impacts have been researched extensively in other sectors, there is a lack of research specifically investigating retail buyer-supplier relationships, and the effect of recession on them. More specifically, long and short term business orientations, in light of economic recession, and particularly from a retail perspective, have seen little attention. We seek to fill this literature void. What is more, the purpose of this paper is to further comprehend how severe economic recessions effect perception of deception, opportunism, relationship termination, and dependence. First, a theoretical background is provided, followed by a review of retail buyer-supplier relationships, long-term orientation and recessions. Next, a framework and series of propositions are given to clarify the pertinent relationships. Finally, we provide managerial implications and 
directions for future research. (For more information, please contact: Brenda Sternquist, Michigan State

University, USA: sternqui@msu.edu)

\section{Curtailing Ex-Post Opportunism from a Symbiotic Relationship in High-Tech Industry Pei-Li Yu, National Cheng Kung University}

Constraining partners' ex-post opportunism is the central managerial focus in interorganizational exchange relationships. By incorporating insights from the perspective of organizational learning theory to complement transaction cost economics, we propose a mediated moderation model integrating the link between exploratory and exploitative innovations and ex-post opportunism and submit that this relationship depends on interfirm adaptation, such as a symbiosis mechanism, as well as on a contingent role of organizational cultural differences. The findings indicate that firms can effectively discourage ex-post opportunism when an exploratory innovation interacts with a lower level of organizational cultural differences and via the mediating effect of interfirm adaptation while exploitative innovation is not.

(For more information, please contact: Pei-Li Yu, National Cheng Kung University, Taiwan: r4895113@mail.ncku.edu.tw)

\section{Export Performance, Marketing Mix Adaptation and Contingency Theory: An Empirical Study of Austrian SMEs Manfred Fuchs, University of Graz Mariella Koestner, University of Graz}

This study investigates the relationships between organizational factors (export market experience, international commitment), external environment (competitive intensity), export marketing strategy, and export success. Using data from 220 export ventures, this study confirms that export market specific experience and international commitment are significant drivers of export success. In addition, the results indicate that the degree of product adaptation is positively related to profitability and overall success. Price and distribution adaptation to local conditions have a direct impact on sales growth. Finally, we found evidence that international commitment exerts a positive effect on the adaptation of marketing strategies to country-specific requirements (For more information, please contact: Manfred Fuchs, University of Graz, Austria:

manfred.fuchs@uni-graz.at)

\section{Session: 3.2.10 - I nteractive}

\section{Track: 10 - Theories of the MNE and of FDI}

\section{International Business in Emerging Markets}

Presented On: June 30, 2015 - 10:45-12:00

Chair: Zliang Deng, Renmin University of China

\section{Early Internationalization and Contemporary Foreign Expansion in China J iangling Yi, Nanjing University \\ Christopher Marquis, Harvard Business School}

This paper was to develop an understanding of the imprinting effect of early internationalization exposure over the past century of Chinese history on the contemporary foreign expansion of Chinese cities and firms that reside in them. The results demonstrate that early internationalization in China has a great positive imprinting effect on modern foreign investment of Chinese cities. Besides, the non-founding sensitive periods such as the Cultural Revolution period has the negative interaction moderating on the imprinting effect mentioned above. That is to say, the Cultural Revolution breaks up the international imprint. At the same time, the policy focusing on inland policy also has the interaction effect on the international exposure. Finally,the results clearly show 
that the open zone policy served as a significant positive intermediary between the early internationalization and the current international expansion of cities in China. (For more information, please contact: Jiangling Yi, Nanjing University, China: yijiangling@smail.nju.edu.cn)

\section{Strategy for International Expansion and Overcoming Liability of Foreignness: Analysis of Multinationals of Emerging Economy \\ Anish Purkayastha, Indian Institute of Management Ahmedabad}

We have theorized international expansion strategy of multinationals from emerging economies (EMNEs) to overcome liability of foreignness (LOF) by integrating prior works of interrelated research efforts in international business and international management along with their theoretical underpinnings \& empirical coverage. We have first integrated extant literature on emerging economies to identify prevalent definition, typologies, differences with multinationals from developed economies \& challenges of EMNEs. We have also organized LOF literatures into origin, characteristics, antecedents \& outcomes of LOF. Subsequently, we have conceptualized expansion strategy of EMNEs that leads to internationalization through overcoming LOF. We have created a conceptual model and multiple propositions articulating adopted strategy of EMNEs. Finally, we have discussed the theoretical and practical implications of this research effort. (For more information, please contact: Anish Purkayastha, Indian Institute of Management Ahmedabad, India: anishp@iimahd.ernet.in)

\section{Inter-organizational Imitation in the Choice of FDI-based Market Entry Modes: Evidence from Indian Multinationals \\ K.V. Mukundhan, Indian Institute of Management Kozhikode \\ Nandakumar Mankavil Kovil Veettil, University of Salford}

The rapid internationalization of emerging market firms (EMFs) has challenged the sufficiency of conventional IB theories to explain the emerging patterns of international activity. The dominant perspectives in entry mode research - transaction costs view and Dunning's OLI paradigm - have come under criticism from researchers who recommend integrating efficiency considerations with behavioural and strategic considerations. This study offers a complementary perspective to entry mode choices by drawing upon inter-organizational imitation literature and anecdotal evidence from internationalization experiences of Indian firms. Specifically, we study the FDI-based entry mode decisions of Indian firms to explain how a firm's likelihood of choosing a particular entry mode (JV or WOS) relates to its organizational form, investment motivations, international focus, industry patterns of internationalization and the level of economic development in host countries. We tested the hypotheses on a sample of 2244 international entries made by Indian firms between 2007 and 2013. The results indicate that entry mode choices are influenced by mimetic pressures operating at the industry and business group level. The mimetic pressures were significantly higher when Indian firms entered developed markets and when firms internationalized to seek access to tangible and intangible assets. We conclude this paper by discussing the limitations of this study and suggest some directions along which future research can be carried out. (For more information, please contact: K.V. Mukundhan, Indian Institute of Management Kozhikode, India: kidambim@gmail.com)

\section{What Drives Africa's FDI Inflows \& Outflows? A Macro-Theoretic Perspective \\ Everlyne Misati, Florida International University \\ Sumit Kumar Kundu, Florida International University \\ Fred O. Walumbwa, Florida International University}

Africa's inward and outward Foreign Direct Investment (FDI) flows are experiencing modest and steady growth despite the continent's myriad challenges. The outlook remains promising, however, the factors determining these FDI flows are not well understood. This study offers some important contributions to knowledge about Africa's FDI flows in several important ways. First, the study contributes to international business (IB) literature by attempting to review and evaluate the major macro FDI theories to help identify factors that determine 
Africa's FDI flows. A better understanding of these factors will inform IB scholars' future research, help business practitioners to formulate and execute investment strategies, and help governments to formulate and execute policies for attracting inward FDI and promoting outward FDI. Second, the study integrates ideas from institutional theory and signaling theory with mainstream FDI theories to improve understanding of the factors determining FDI flows, and in doing so, extends and broadens the use of these theories in a meaningful new way. (For more information, please contact: Everlyne Misati, Florida International University, USA: emisa001@fiu.edu)

\section{Zooming in On the Cross-Border M\&As of Emerging Market MNEs through the Internationalization Process Model \\ Manish Popli, Indian Institute of Management Indore}

The rapid arrival of emerging market multinational enterprises (EM MNEs) has sparked a debate for reassessment of the established theoretical paradigms in international business (IB) literature. This paper intends to contribute in this direction by finding out the commonalities and idiosyncrasies in the internationalization pattern of EM MNEs. Specifically, this article zooms in on the majority stake cross-border M\&As of Indian firms and conceptualize that firms could be sensitive to the risk involved in higher equity entry modes of internationalization. In this paper, we develop testable propositions to advance the argument that the risk taken by the EM MNEs is a function of the motive(s) of acquisition, switching cost and the cumulative cultural experience reserve (CER) of the acquirer firm. The results of this study should inform the debate towards the usability of existing internationalization process models in the EM context. (For more information, please contact: Manish Popli, Indian Institute of Management Indore, India: manishp@iimidr.ac.in)

\section{The Locational Strategies of MNEs Committed to Reducing Greenhouse Gas Emissions Jerry Patchell, Hong Kong University of Science and Technology}

This paper proposes a framework for analysing GHG based locational strategies. The framework uses the GHG Protocol's characterization of a corporation's lifecycle emissions responsibilities to establish four basic lifecycle stage regions where MNEs may choose to locate activities to reduce greenhouse gas emissions. The framework then borrows from with Iammarino and McCann's (2013) analysis of MNE location decisions, supplemented with industrial ecology's perspectives to elaborate on the different characteristics that may be found in any of the lifecycle stage regions. Evidence that leading firms have chosen different regions for these activities is (will be) provided. (For more information, please contact: Jerry Patchell, Hong Kong University of Science and Technology, Hong Kong, SAR-PRC: sopatch@ust.hk)

\section{Session: 3.2.11 - I nteractive}

\section{Track: 12 - The I nstitutional and Political Environment of I B}

\section{CSR in IB}

Presented On: June 30, 2015 - 10:45-12:00

Chair: Maria-Alejandra Gonzalez-Perez, Universidad EAFIT

Institutional Sophistication and Stakeholder Management in Emerging Markets Meng Zhao, Renmin University of China Seung Ho Park, China Europe International Business School

There has been a transformation of institutional voids into sophistication in China along with the top-down maturation of the formal governance system and bottom-up growth of the grassroots legitimation. Institutional sophistication brought in new roles and expectations for stakeholders toward multinational companies. It has 
incurred distrustful stakeholder environment, causing stakeholder-initiated public crises and threats to sustainable operation of multinational companies. Heightened consumer awareness and media reporting create adverse effects of social exchanges in the distrustful environment. Following the social exchange theory and stakeholder management theory, the study conceptualizes MNCs' stakeholder strategies in such distrustful environment with two specific approaches of dialogue and philanthropy. Dialogue facilitates developing the perception of justice for stakeholders, leading to a trust-based firm-stakeholder relationship and a lower chance of experiencing public crises. But, signaling goodwill through philanthropy works against building stakeholders' trust and reducing potential public crises over MNCs' misbehaviors. The empirical testing utilizes qualitative and qualitative analyses of 58 multinational companies in consumer goods that encountered 74 public crises over 2000-2013. (For more information, please contact: Meng Zhao, Renmin University of China, China: mengzhao521@yahoo.com)

Follow the Herd: How Organizational Level Legitimacy Can Give a Helping Hand to Bridge Formal Institutional Voids - The Case of Employee Stock Ownership Plans in MNES Jana Oehmichen, Georg-August University of Goettingen Michael Wolff, Georg-August University of Goettingen Ulrike Zschoche, Georg-August University of Goettingen

We investigate the influence of country level institutions on foreign subsidiary employee participation in employee stock ownership plans (ESOPs) - an important vehicle for MNEs to reduce employee turn-over and increase employee commitment. Based on the theory of the institutional void, we argue that weak country level institutions deter MNEs from successfully introducing ESOPs due to reduced employee participation in the program. However, we expect that MNEs can overcome these effects of the institutional void by enhancing the program's legitimacy through organizational level effects of group behavior and hierarchy. We conduct an empirical analysis based on a MNE's voluntary participation in stock plans of about 188,000 employees in 31 different countries. Our empirical results show that weak formal host-country institutions (e.g. weak governance effectiveness) reduce foreign subsidiary employee participation in ESOPs but also that this negative effect can be reduced, when (1) the employee observes high participation in its near organizational environment (group behavior) and (2) the employee observes participation of its superior (hierarchy). (For more information, please contact: Jana Oehmichen, Georg-August University of Goettingen, Germany: jana.oehmichen@wiwi.unigoettingen.de)

\author{
Three Pillars of CSR: A Firm-Level Analysis of Korean MNEs in China \\ Soonkyoo Choe, Yonsei University \\ Jihoon Kang, Yonsei University \\ Younghoon An, Yonsei University
}

While corporate social responsibility (CSR) has been concerned in the context of single determinant, this research analyzes the different levels of CSR activities by integrating institutional, resource-based and internationalization theory. This research divides the CSR determinants into local demand, necessity and firm capability. Using a large-scale empirical survey conducted on Korean MNCs in China, we test hypotheses on antecedent of CSR activities and the result shows that external factors such as GDP and brand awareness as well as firm capabilities including ownership and firm size positively affect the level of CSR activity. In terms of CSR motivation, we found that corporate social performance is not only driven by single pressure from host country, but also necessity and capability of firms. (For more information, please contact: Soonkyoo Choe, Yonsei University, Korea, South: skychoe@yonsei.ac.kr) 


\section{CSR Practices in China: A Comparative Study of Indigenous and Multinational Companies \\ Xiangxiang Hong, Nottingham University Business School China \\ Michal Lemanski, Nottingham University Business School China}

The confluence of social inequalities and natural disasters mark the contemporary landscape for corporate social responsibility (CSR) in emerging markets like China. Multinational corporations (MNCs) attracted to China as the largest emerging market demonstrate increasingly active approach responding to the calls upon them to respond to social issues in areas where they operate. This paper aims to explore how MNCs approach CSR in China. The research design comprises two stages using two qualitative research methods. First a content analysis is used to analyze 100 companies: 50 best local (Chinese), and 50 best foreign (MNC) social performers according to the 2013 Fortune China CSR Ranking. Next, in-depth interviews were conducted with CSR practitioners within a sub-sample of 10 of those companies. The results show that Chinese and foreign companies are similar in addressing the four categories of CSR themes: economic, legal, ethical and discretionary. Economic and discretionary CSR issues are their primary concern, showing that many major companies have passed the stage where legal compliance is the overriding priority and are in a better shape to conduct CSR beyond bringing their business practices in line with the laws, rules and regulations. We discuss findings and outline directions for future studies, and managerial relevance for both the Chinese and foreign companies and the Chinese government is also discussed. (For more information, please contact: Michal Lemanski, Nottingham University Business School China, Poland: michal.lemanski@nottingham.edu.cn)

\section{Selective Engagement in Corporate Social Responsibility: An Institutional Perspective \\ J ie Zou, Peking University \\ Changqi Wu, Peking University}

This paper attempts to understand why and how firms behave differently in the realm of CSR. A firm can be responsible in some dimensions of CSR while acting irresponsibly in some other dimensions. We propose a multilevel framework to understand selective engagement in CSR. At the organizational level, we focus on the logic of legitimacy seeking and maintaining, and examine how firms employ a decoupling strategy to manage legitimacy while keeping discretion over firms' internal operations. At institutional level, we investigate how variation in regional institutional development and the complexity of institutional pressures shape firms' selective engagement in CSR. We also examine the cross-level interaction effects to see how institutional factor moderates the effect of organizational factor on firms' selective engagement. Exploring a unique survey database of more than 1,000 industrial firms in China, we find that private firms are more likely to engage in corporate philanthropy and SOEs will engage more in employee welfare; while weak market institution encourages corporate philanthropy, strong market institutions stimulates employee welfare; and the effect of institution is more pronounced on SOEs. This study contributes to institutional theory and CSR research in transitional economies, and has important implications for CSR practitioners. (For more information, please contact: Jie Zou, Peking University, China: zoujie@pku.edu.cn)

\section{Institutional Change and MNE CSR Learning \\ J ing'an Tang, Sacred Heart University}

Corporate social responsibility (CSR) has become an important component on the strategic agenda of Multinational Enterprises (MNEs), yet there is only a limited understanding of how CSR has evolved over time to such strategic importance. This study explores theories of organizational learning, internationalization and institutional change and develops a process-based model to explain how MNEs learn to care about CSR. The research demonstrates how the CSR learning is internalized into corporate dominant logic during the business internationalization process. It is argued that such learning goes through a bipartite process in which the micro organizational learning mechanism builds a vehicle for CSR learning to be carried through, while the macro institutional paradigm shift provides thrust that propels the learning vehicle to run on the CSR course. (For more information, please contact: Jing'an Tang, Sacred Heart University, USA: tangj3@sacredheart.edu) 
The Influence of MNES on Indigenous Firms' Involvement of Voluntary CSR Activities in an Emerging Market -

The Chinese Context

Ying Liu, Florida International University

Mary Ann Von Glinow, Florida International University

In this study, we address how the presence of foreign MNEs influences regional institutions and how the joint force of these two factors influences indigenous firms' involvement of CSR-related activities at a regional level within a country. We set an emerging market, China, as the research context. The findings largely support our arguments that the presence of foreign MNEs positively influences indigenous firms' involvement of CSR-related activities directly and indirectly via the dynamic regional CSR-related institutions. The contributions, limitations and implications are discussed. (For more information, please contact: Ying Liu, Florida International University, USA: lilianeliuying@gmail.com)

\section{Session: 3.2.12 - I nteractive \\ Track: 11 - Corporate Governance in I B}

\section{Transparency and Accountability: Diverse Contexts, Diverse Research Methods}

Presented On: June 30, 2015 - 10:45-12:00

Chair: Deepak K Datta, University of Texas at Arlington

Improving Transparency in Governance: The Case of Sustainability Reporting

Rama Kumari J ayanti, Cleveland State University

By providing sustainability related information on the internet, modern global corporations harness the power of sustainability as a core element or ethos of transparency driven effective governance. In an attempt to examine the disclosure intent of transparency driven governance strategy, a content analysis of the top global 100 sustainable companies was performed. The analysis reveals that sustainable governance is organized around environmental, social, and economic dimensions that constitute the triple bottom line conceptualization of the global reporting initiative. Findings evidence wide variation in the aspects used as well as the type of information reported. Further, assigning equal weights to all aspects skew the data in favor of companies that demonstrate average performance on a number of dimensions to the exclusion of companies that provide exemplary performance on a few dimensions. The paper concludes with practical implications of transparency driven governance policies with specific reference to sustainability disclosures and future research directions. (For more information, please contact: Rama Kumari Jayanti, Cleveland State University, USA: r.jayanti@csuohio.edu)

\section{International Accountability Standards Across National Business Systems: A Neo-Institutional Perspective Steve Michael Loren, University of Massachusetts Boston \\ Suhaib Riaz, University of Massachusetts Boston}

We explore Corporate Social Responsibility (CSR) as a mechanism of Global Corporate Governance in the age of globalization. Previous research has looked at how differences in National Business Systems (NBS) impact national level differences in CSR but has neglected how these mechanisms can account for the gap between the differential national commitment to, and achievement of, the objectives related to CSR. We theorize about the potential to discover NBS characteristics that can explain the extent of the 'gap' between the commitment to, and achievement of, objectives related to CSR and how this can inform future research and policy making. (For more information, please contact: Steve Michael Loren, University of Massachusetts Boston, USA: sloren63@msn.com) 
The global financial crisis has demonstrated the weakness of corporate governance systems to properly manage risk, produce sustainable growth, and properly align shareholder interest with managerial activities. Financial institutions have been particularly impacted by poor corporate governance practices and throughout Eastern Europe central banks and national governments have had to step into to recapitalize failing financial institutions. This paper examines the relationship between corporate governance practices of banks and their financial performance. The sample is drawn from 28 publicly listed financial institutions based in Russia, Ukraine and Kazakhstan. Results indicate that cross-listed financial institutions and those financial institutions with substantial non-emerging market ownership from emerging markets have better corporate governance practices. (For more information, please contact: Alexander Settles, Rutgers University, USA: asettles@business.rutgers.edu)

\section{Earnings Quality and Corporate Social Responsibility: Evidence from China \\ J ie Zhang, China Huarong Asset Management Co., Ltd. \\ Chang Song, Renmin University of China \\ Xiyou Liu, Audit Research Institute of China's National Audit Office \\ Jiawen Yang, George Washington University}

In this study, we examine whether firms project a socially-friendly image to disguise earnings management. Using a large sample of China's publicly listed firms, we find that corporate social responsibility (CSR) is relevant to earnings management decisions. Specifically, the results indicate that firms with increased CSR activities exhibit lower performance-adjusted discretionary accruals, consistent with greater earnings management. Further, we find that performance-adjusted discretionary accruals continue to exhibit a significant negative association with CSR among state-owned firms, but not among non-state-owned firms. The results above are robust to various specifications. (For more information, please contact: Jie Zhang, China Huarong Asset Management Co., Ltd., China: jacqueline35@sina.com)

\section{Religion and Corporate Governance \\ Jaehyeon Kim, University of Hawaii at Manoa \\ Shirley Daniel, University of Hawaii at Manoa}

This study examines the association between religion, particularly Protestantism, and corporate governance practices of 37 countries. We find that the proportion of Protestants is positively related to corporate governance practices after controlling for cultural, economic, legal, and institutional factors. The finding of this study contributes to the accounting, finance, and management literature by showing that social norms, especially religion, have a strong effect on corporate governance practices. (For more information, please contact: Jaehyeon Kim, University of Hawaii at Manoa, USA: jaehyeon@hawaii.edu)

\section{Efficiency and CSR - The Role Corporate Governance \\ Barbara Brenner, Danube University Krems \\ Shalini Rogbeer, European Business School}

We attempt to move beyond the 'Friedman vs. Freeman' debate by exploring the relation between efficient governance and sustainable technology (RDI). We hypothesize that though it is costly to invest in sustainable technologies a firm's ability to capitalize on sustainability, and so profit from CSR, depends upon the firm's governance capability. We show that efficient governance can constrain agency conflicts between senior management and the shareholders by putting a focus on sustainable technologies. Our results suggest that effective corporate governance is key for building and investing in sustainable technologies that has a positive 
effect on firm performance. Our study extends the burgeoning theoretical and empirical literature and provides novel insights into the contingent relationship between sustainability and governance. (For more information, please contact: Barbara Brenner, Danube University Krems, Austria: barbara.brenner@donau-uni.ac.at)

\section{Developing a Model for Introducing a Social Credits Trading Scheme in India: International business considerations}

Raji Ajwani, Symbiosis Centre for Management

The landmark Section 135 of the Indian Companies Act 2013 makes it mandatory for companies having roughly USD $\$ 800,000$ or more in profits over the last three years to spend 2 percent of their average net profit on CSR activities. This mandate is expected to result in about USD three billion of CSR spend annually (Forbes, 2013). The underlying premise is that companies need to contribute in the process of finding creative solutions for the socio-economic and environmental issues prevailing in the country, either individually or by working in clusters, since the challenges in these areas are much too complex for the government to handle alone (Confederation of India Industries, 2013). Today, India is the first country in the world where CSR expenditure has been made mandatory. Most companies are still trying to decipher the way to approach this issue. Some companies believe that 'pay and comply' option in order to fulfill a mandate may be simpler than devising impact making CSR projects. This may result in lack of value creation for the involved stakeholders and failure of the concept. (For more information, please contact: Raji Ajwani, Symbiosis Centre for Management, India:

raji.ajwani@gmail.com)

\section{Session: 3.2.13 - Interactive \\ Track: 6 - Innovation and Knowledge Management}

\section{Innovation in the Multinational Context}

Presented On: J une 30, 2015 - 10:45-12:00

Chair: Michael J Mol, Copenhagen Business School

Entrepreneurship and Innovation in New Venture Teams: Outlining the Role of Culture Mourad Dakhli, Georgia State University

Wade Danis, University of Victoria

Qing Li, Shanghai University

Entrepreneurship is considered an important driver of economic development and prosperity. In this paper, we review advances in entrepreneurship and innovation literature and extend research in this area by proposing that innovation and entrepreneurial undertaking are also functions of the underlying processes that take place within new venture teams, and offer a process model of entrepreneurial innovation across different cultural settings. In our model, trust and organizational commitment are proposed as critical factors that determine the nature and degree of cooperative and conflictual exchange processes that underlie innovation. By looking at the interpersonal processes that facilitate innovation across cultures, our model contributes to an area of entrepreneurship research that warrants further investigation. Namely, a consideration of the multilevel nature of innovation processes that drive entrepreneurship, and the delimitation of the role of culture. Our model integrates prior research on conditions affecting innovation with insights from in-depth interviews. The model is based on grounded theory methodology and supports the "equifinality" premise of innovation. In other words, innovation occurs through different paths depending on the cultural context. (For more information, please contact: Mourad Dakhli, Georgia State University, USA: mdakhli@gsu.edu) 
Do Institutions Matter in Knowledge Creation? Quantifying the Impact of Institutional Affiliation on R\&D

Professionals

Lynn Pyun, Baruch College, CUNY

J ordan Siegel, Harvard Business School

Whereas the prior research has clearly illustrated the importance of firm-specific resources, little attention has been paid how institutions across borders impact the knowledge creation activities of the individuals with the highest potential. This study is an attempt to fill this vacuum by tracing elite engineers' knowledge creation activities over time and across institutional boundaries in U.S. and South Korea. Combining multiple databases in an unprecedented fashion, we find that institutions do matter in how elite engineers produce meaningful knowledge, which will in turn, become valuable resource to MNEs. (For more information, please contact: Lynn Pyun, Baruch College, CUNY, USA: Iynn.pyun@baruch.cuny.edu)

\title{
Innovation, Globalization and the Size of Indian Firms: Unpacking Heterogeneity Patterns Sumit Majumdar, University of Texas at Dallas \\ Abhijit Mandal, City University, London
}

This article examines the impact of $R \& D$ on I ndian manufacturing firms' exports. Data are stratified by size into four quartiles, for a large firm-level panel, for the years 1999-00 to 2005-06. We examine heterogeneity patterns using quantile regression analysis. For the smallest firms, the exports impact for firms undertaking R\&D is small and rises with greater exports but the impact of $R \& D$ is negative for the heaviest exporters. For smallto-medium firms, at all export levels, the pay-offs to exports of R\&D are an increase in levels of exports for firms that engage in $R \& D$ activities. Medium-to-large firms enjoy an initial positive benefit from doing $R \& D$. For the largest firms, there are small initial impacts of $R \& D$ on exports but the $R \& D$ and exports relationship turns negative at higher export levels. These details highlight varied patterns of heterogeneity in firms' strategies and their outcomes. We explore the globalization strategy and public policy implications of the findings. (For more information, please contact: Sumit Majumdar, University of Texas at Dallas, USA: majumdar@utdallas.edu)

\author{
Exploring the Link between Open Innovation and Internationalization in High-Tech SMES \\ Matevz Raskovic, University of Ljubljana \\ Miha Cerne, University of Ljubljana \\ Desislava Dikova, Vienna University of Economics and Business \\ Andreja J aklic, University of Ljubljana
}

Knowledge and innovation are important sources of competitive advantage for all types of firms, but particularly for SMEs. Despite growing empirical work on the general relationship between innovation and internationalization and vice-versa, less is known about the specifics of high-tech SMEs. The purpose of this paper is to examine the relationship between open innovation, internationalization and innovation commercialization abroad among high-tech SMEs, as well as the moderating role of proactive market orientation. By testing proactive market orientation as a double moderator in the relationships between open innovation and internationalization, as well as internationalization and commercialization of innovation abroad, we add to the literature on the role of strategic orientations in the upstream internationalization process of hightech SMEs, as well as provide an empirical contribution to the scant literature on open innovation among SMEs. Our results show internationalization to be an important mediator between open innovation and innovation commercialization abroad among high-tech SMEs. More importantly, the positive relationships between open innovation and internationalization, as well as between internationalization and innovation commercialization abroad are moderated by proactive market orientation with stronger impact on the relationship related to innovation commercialization. (For more information, please contact: Matevz Raskovic, University of Ljubljana, Slovenia: matevz.raskovic@ef.uni-lj.si) 


\section{Internationalization Experience and the Evolution of Dynamic Capability in the Indian IT Firms}

Partha Mukerjee, University of New South Wales

Pradeep Kanta Ray, University of New South Wales

Sangeeta Ray, University of Sydney

Zahid Riaz, Lahore School of Economics

This study examines what capabilities enable emerging market firms (EMFs) to catch-up in knowledge intensive industries in global markets. Whereas firms from the OECD are well endowed in resources, latecomer firms from developing countries are generally resource poor and their establishment, development and international expansion have taken place within an institutional environment that is different from those found in Western economies. Hence, despite not being endowed with resources, how EMFs learn to transform comparative advantages into firm-specific capabilities deserves attention. The study explores how Indian IT firms "moved up the value ladder," moving out of the "low road" where the barriers to entry are low and competition is based mainly on price and squeezing wages, to the "high road" where competition is based on differentiation. The research entailed deductive econometric studies on 703 companies using panel data method, which tested the hypothesis which factors contribute to globalization of EMFs. The findings of our panel data analysis reveal that globalisation of EMFs is an evolutionary process and in each phases of evolution, the EMFs linkages with MNCS enable them to leverage resources to learn and globalise rapidly. Through linkages with MNCs, EMFs gain access to markets, technology, and reputation. The findings of this study present a novel and contemporary insight on how EMFs evolve to develop higher level capability, which enables them to leapfrog in a fast changing technology space. (For more information, please contact: Pradeep Kanta Ray, University of New South Wales, Australia: pray@unsw.edu.au)

\section{Session: 3.3.1 - Special Session}

\section{JIBS Decade Award}

Presented On: June 30, 2015 - 13:00-14:15

Chair: J ohn Cantwell, Rutgers University

The 2015 JIBS Decade Award, sponsored by Palgrave Macmillan, honors the most influential paper published in the 2005 volume of the J ournal of International Business Studies.

A Retrospective on their article by the winners of this year's JIBS Decade Award - Reconsidering the issues raised in their 2005 JIBS paper on "Probing theoretically into Central and Eastern Europe: transactions, resources, and institutions"

Klaus Meyer, CEIBS

Mike Peng, University of Texas at Dallas

\section{Discussants and Commentators:}

Mark Peterson, Florida Atlantic University

Tatiana Kostova, University of South Carolina

G. Tomas M. Hult, Michigan State University

(For more information, please contact: John Cantwell, Rutgers University, USA: cantwell@business.rutgers.edu) 


\section{Session: 3.3.2 - Competitive}

\section{Track: 9 - Emerging Economies and Emerging Economy MNEs}

\section{Business Groups}

Presented On: June 30, 2015 - 13:00-14:15

Chair: Ilya Cuypers, Singapore Management University

The Relationship between FDI Decisions and Business-Group Insider Control: Evidence from Taiwanese GroupAffiliated Firms Investing in Chinese Market

Wen-Ting Lin, National Chung Cheng University

Ming-Chang Huang, Providence University

Ya-Ping Chiu, Chung Yuan Christian University

Hsiang-Lin Cheng, National Chung Cheng University

Based on resource-dependence theory, we develop three hypotheses regarding the negative relationship between the equity stake in group-affiliated firms held by business-group headquarters and (1) the slack resources of group-affiliated firms, (2) the products of group-affiliated firms that are similar to those of the business-group headquarters, and (3) directors' ties with managers of group-affiliated firms. I use GLS fixedeffects models to test the hypotheses, and use longitudinal data on China-oriented direct investments undertaken by 194 business groups, including 377 group-affiliated firms from Taiwan over the period of eight years from 2004 through 2011. The results support all of the hypotheses both cross-sectionally and longitudinally. This study points to a new avenue for research in the international-business and business-group literature regarding the ownership relationship between group-affiliated firms and business-group headquarters. (For more information, please contact: Wen-Ting Lin, National Chung Cheng University, Taiwan: wentinglin@ccu.edu.tw)

Does Business Group Affiliation Help Translate Research Inputs into Export Expansion? Empirical Evidence from the Indian Pharmaceutical Sector

Tatiana S. Manolova, Bentley University

Saptarshi Purkayastha, Indian Institute of Management Calcutta

Linda F. Edelman, Bentley University

We combine perspectives from theories of internationalization and diversification in order to explore the moderating role of business group characteristics on the link between research expenditure and export intensity for business-group affiliated companies in the context of the pharmaceutical sector in India. Our three hypotheses are tested on a sample of 219 Indian pharmaceutical firms affiliated with business groups, over a five-year period (2005-2010) in a panel of 1096 firm-year observations. Results indicate that, contrary to our contention, research expenditure is negatively associated with export intensity, implying that firms in the Indian pharmaceutical sector may face a trade-off between investing in innovation and international expansion. As expected, business group characteristics significantly impact the strength of the relationship between innovation and internationalization. The degree of business group differentiation enhances the negative effect of research expenditure on export intensity, whereas the degree of business group internationalization alleviates this negative effect. Theoretical and practitioner implications are discussed. (For more information, please contact: Tatiana S. Manolova, Bentley University, USA: tmanolova@bentley.edu) 
Partnering with Business Group Firms: The Effect of Group Size on Value Creation and Value Distribution

Ilya Cuypers, Singapore Management University

Youtha Cuypers, University of Hong Kong

Jung Yeon Lee, California State University, Fullerton

We examine international joint ventures (IJVs) between members of business groups and foreign partners. Business group firms are potentially attractive IJ V partners due to their connections and access to resources. However, drawing on Resource Dependence Theory, we suggest that local firms belonging to larger business groups have less incentive to contribute to the IJV, resulting in lower value creation. In addition, we suggest that the larger the business group a local partner belongs to, the more bargaining power they have over the foreign partner, allowing them to capture more of the value that is created in the IJ V. Using a sample of foreign-Korean IJ Vs established between 1989 and 2007, we find evidence of (1) a negative relationship between the size of the business group of the Korean partner and the value that is created in the IJVs, and (2) a positive relationship between group size and how much value the Korean partner captures. (For more information, please contact: Ilya Cuypers, Singapore Management University, Singapore: ilyacuypers@smu.edu.sg)

\section{Centralization of Intra-Group Equity Ties and Performance of Business Group Affiliates Ishtiaq Mahmood, National University of Singapore Hongjin Zhu, McMaster University \\ Akbar Zaheer, University of Minnesota}

Although prior research has suggested that business groups create value to member firms, less attention has been paid to the specific mechanisms through which business groups enhance performance of affiliated firms. In this study, we focus on the performance effects of the centralization of equity ties within a group, or the degree of variation in centrality among affiliates and argue that the effect of equity tie centralization on affiliate performance is contingent on environmental turbulence and organizational diversity. Using longitudinal data of business group affiliates in Taiwan, our analysis shows that while equity tie centralization enhances affiliate performance by facilitating resource mobilization within group in a stable environment, it depresses affiliate performance in a turbulent environment due to loss of local autonomy and adaptability. Moreover, the performance effects of equity tie centralization strengthen when the business group diversifies into unrelated industries. (For more information, please contact: Ishtiaq Mahmood, National University of Singapore, Singapore: bizipm@nus.edu.sg)

\section{Session: 3.3.3 - Competitive}

\section{Track: 8 - Global Strategy, M\&As and Competitiveness}

\section{Managing Alliances and Networks in the Global Context}

Presented On: June 30, 2015 - 13:00-14:15

Chair: Snehal Suyash Awate, Indian School of Business
A Yin-Yang Perspective of Relational (Guanxi) Gatekeeping in Business Network Management in China Hongzhi Gao, Victoria University of Wellington
Monica Ren, Macquarie University
Qing Miao, Jilin University

China is an extremely complex and 'controlled' market for foreign businesses. This paper investigates how Western companies engage with local (guanxi) market insiders through relational gatekeepers. In-depth interviews are conducted with 40 Chinese and Western business managers based in China, Australia and New Zealand. Guided by the Yin-Yang balancing logic, we find six key gatekeeping constructs in both cross-cultural 
and cross-network business relationships that connect local guanxi insiders with foreign outsiders. These key constructs are: (1) local guanxi network power and foreign organizational power as gatekeeping antecedents; (2) gatekeeper resources and gatekeeping capability as two key gatekeeping process constructs; (3) access to (powerful) guanxi networks, and long-term inter-organizational relationships as two gatekeeping outcomes. The Yin-Yang balancing logic adds an important new dimension to the understandings of guanxi and intercultural dynamics in Western-Chinese business relationships. (For more information, please contact: Monica Ren, Macquarie University, Australia: monica_ren@yahoo.com)

\section{Think Globally, Act Cooperatively: Exploring Internationalization and Innovation Strategies at the MNE-INV Interface}

Shameen Prashantham, Nottingham University Business School China

Anoop Madhok, York University

Little is known about how new venture internationalization is influenced by large multinational enterprises (MNEs) and, conversely, how MNE innovation is influenced by international new ventures (INVs). We analyse the MNE-INV interaction from the perspective of both actors and examine the MNE-INV interface, specifically the conditions under which MNEs constitute an internationalization pathway for INVs, and the process through which this occurs. Based on three in-depth longitudinal cases in the Bangalore software industry, we identify three distinct pathways: passive, active and co-creation. These three pathways exhibit increasing levels of technological innovation on the part of the new venture and partnering innovation by the MNE. Crucially, the particular pathway adopted results from the match or co-alignment between these two innovation levels, which on their part reflect the respective strategies of the two actors and the "effortful" nature of MNE-INV engagement. The local milieu - as well as the MNE's wider network - provides enabling conditions for differing levels of these two types of innovation. (For more information, please contact: Shameen Prashantham, Nottingham University Business School China, China: shameen.prashantham@nottingham.edu.cn)

\section{Institutional and Cultural Dynamics in Strategic Decision-Making to Innovate Hong-J en C. Chiu, National Taiwan University}

How do entrepreneurs manage ambidexterity in exploring new offshore businesses, while exploiting these opportunities with institutional and cultural barriers? These constraints are embedded into the decision-making to innovate. We intend to examine the extent to which psychic distance and interdependency moderates the relations between absorptive capacity and strategic variables (i.e., regulatory focus, export orientation, and organizational cultural intelligence). Our findings showed that both regulatory focus and export orientation did affect the exporting venture's absorptive capacity and innovativeness differentially. Nevertheless, expected negative moderating effects of psychic distance and interdependency only occurred in the relationship between export orientation and absorptive capacity. (For more information, please contact: Hong-Jen C. Chiu, National Taiwan University, Taiwan: hongjen@ntu.edu.tw)

\section{Institution and Location Strategies of Multinationals Corporations from Emerging Economies: Evidence from China's Cross-border Merger and Acquisitions}

Peter Buckley, University of Leeds

Pei Yu, Wuhan University of Technology

Qing Liu, University of International Business and Economics Beijing

Surender Munjal, University of Leeds

Pan Tao, Shandong University of Finance and Economics

Using Heckman's two-stage model this study investigates the institutional influence in location strategies of Chinese cross-border mergers and acquisitions (M\&A) during the period 1985-2012 and across 146 economies. Results suggest that Chinese MNCs are "shortsighted" and present dual character when confronting host 
institutions. On the one hand, they demonstrate "risk aversion" by avoiding countries with high corruption level; while on the other hand, they show "risk preference", through conducting larger M\&A volume in country with higher political and economic risk, which may damage a firm's long term profit. Besides, China's institutional change towards "Go global" pushes Chinese MNCs to locate increasingly in countries with abundant strategic assets. (For more information, please contact: Surender Munjal, University of Leeds, United Kingdom: smu@lubs.leeds.ac.uk)

\section{Session: 3.3.4 - Competitive}

\section{Track: 12 - The I nstitutional and Political Environment of I B}

\section{Country-Level I nstitutions and Their I mpact}

Presented On: June 30, 2015 - 13:00-14:15

Chair: Bo Bernhard Nielsen, University of Sydney

Configuring an Innovative Society: A Dual Perspective of Cultural and Institutional Varieties

Di Fan, Deakin University

Yi Li, Australian National University

Liang Chen, University of Leeds

Why are some societies more innovative than others? Reconciling both the culture and institutional theoretical perspectives, the study generates nine causal conditions, namely, uncertainty avoidance, masculinity, individualism and power distance as the culture indicators, and union density, skill development, market capitalization to credit, prevalence of cluster, and state dominance as the institutional indicators. Applying the configurational approach, we conducted fuzzy-set qualitative comparative analysis (fsQCA) for testing the nine causal conditions and identifying configurations leading to national innovative performance of Organisation for Economic Co-operation and Development (OECD) member countries. This study contributes to the international business (IB) field by confirming the equal importance of both cultural and institutional mechanisms contributing to national innovation, and identifying equifinal configurations of cultural and institutional varieties leading to an innovative society. Finally, if cultural and institutional varieties are configurational, a society can adjust or develop their cultural and/or institutional conditions to maintain or create their innovation leadership. (For more information, please contact: Di Fan, Deakin University, Australia: david.fan@deakin.edu.au)

\section{Institutions and the Strategic Choices of Multinational Enterprises}

J in Uk Kim, University of Illinois at Urbana Champaign

The nexus between institutions and the strategic choices of multinational enterprises (MNEs) is a burgeoning field of academic inquiry. The purpose of the current paper is to provide a comprehensive review and offer concrete future directions for this research stream. Our assessment is that while significant progress has been made through the integration of new institutionalism and neo-institutional theory into strategy and allied disciplines, existing research has become stalled and over-reliant on a select set of ideas from new institutionalism and neo-institutional theory thus limiting the analyses to a narrow and thin view of institutions. We propose that the time is ripe to widen and deepen the view of institutions within MNE research, and we offer specific suggestions towards this end. (For more information, please contact: Jin Uk Kim, University of Illinois at Urbana Champaign, USA: jkim198@illinois.edu) 
Host-Country Networks and Institutional Awareness

Angelika Lindstrand, Stockholm School of Economics/Stanford University

Kent Eriksson, Stanford University

We develop the concept 'Institutional Awareness'; the internationalizing firm's understanding that host country institutions can create impediments to international operations, and study factors that influence institutional awareness. Our main focus lies on investigating how business networks in the foreign host country influences this awareness. In addition we study the influence of host country societal organizations such as banks, industry organizations and authorities, and the firms' commitment, to business relationships. A survey of 255 internationalizing firms, show that the firm's network in the host country has an effect on institutional awareness. The findings also demonstrate that dependency on societal organizations and commitments, to business relationships influence institutional awareness. In conclusion we show that Institutional Awareness affect firm performance. The theoretical contribution of this paper provides an increased understanding of the role of institutions in international business. (For more information, please contact: Angelika Lindstrand, Stockholm School of Economics/Stanford University, Sweden: alindstr@stanford.edu)

\section{Session: 3.3.5 - Panel Special Session}

\section{New Perspectives on Foreign Cultures as Sources of New Knowledge for I ndividuals and Firm}

Presented On: June 30, 2015 - 13:00-14:15

Chair: Arie Lewin, Duke University

Panelists:

Arie Lewin, Duke University

Shige Makino, Chinese University of Hong Kong

Rosalie Tung, Simon Fraser University

There is a long tradition in international business (IB) research to view culture as a challenge. When individuals and firms function in a foreign culture, they may encounter many culture-related problems, ranging from differences in values and business practices. The literature on cultural distance exemplifies this orientation, suggesting that individuals and firms operating in more distant cultures may encounter more difficulties. Another stream of IB research takes a different perspective and views the exposure to a foreign culture as a source of learning and knowledge acquisition. Firms from emerging economies such as China and India enter developed economies to learn about management knowhow and new technologies. Firms from developed economies may learn about low cost operation when they compete with local firms in emerging markets. At the individual level, exposure to foreign cultures, including working in a multicultural team, can spur creativity. This showcase panel explores new perspectives on how the exposure to diverse cultures facilitates learning and knowledge acquisition of individuals and firms. Four leading scholars, two macro and two micro, will share their insights on this theme. (For more information, please contact: Shige Makino, Chinese University of Hong Kong, Hong Kong, SAR-PRC: makino@baf.cuhk.edu.hk) 


\section{Session: 3.3.6 - Competitive}

\section{Track: 11 - Corporate Governance in I B}

\section{New Perspectives on Ownership Heterogeneity}

Presented On: June 30, 2015 - 13:00-14:15

Chair: Rakesh Sambharya, Rutgers University

Ownership, Time Horizon and Corporate Social Responsibility around the World J ongmoo J ay Choi, Temple University

Jimi Kim, Temple University

Drawing from agency and stakeholder theories, we examine the effects of corporate ownership on CSR using comprehensive dataset for 44 countries around the globe for the period 2010-2012. We find that concentrated ownership measured by the shares of the largest shareholders decreases CSR. However, the effects vary significantly across the types of the largest controlling owners. CSR decreases when the largest owner is an institutional investor but increases in the case of public owners. Importantly, time horizon matters at the level of the firm, controlling owner and home country. The CSR index is higher if the firm is from a country with longterm orientation culture, if the controlling owner is long-term oriented, and if a firm is concerned with future value-creating investments such as R\&D. (For more information, please contact: Jongmoo Jay Choi, Temple University, USA: jjchoi@temple.edu)

\section{Do Expatriates, as MNE Parent-Foreign Subsidiary Governance Mechanisms, Really Impact Foreign Subsidiary Performance?}

Bassam E. Farah, American University of Beirut

Rida Elias, American University of Beirut

Paul W. Beamish, Western University

We investigate whether expatriates, as MNE parent-foreign subsidiary governance mechanisms, really impact foreign subsidiary performance, and if so, how and when. We draw on agency theory and corporate governance (CG) literatures to develop a theoretical model that describes the relationship between expatriates (as MNE parent-foreign subsidiary governance mechanisms) and foreign subsidiary performance, and the moderating effect of parent-subsidiary ownership (as an MNE parent-foreign subsidiary governance mechanism) on this relationship. To test this model, we designed a multi-country study that used survival analysis and multinomial logistic regression techniques to perform various analyses on large longitudinal and cross-sectional datasets for the years 1990-2008. Based on a dataset including 84,369 observations, 1,540 MNE parents, and 12,101 foreign subsidiaries in multiple countries, the results show that the number of expatriates and an MNE parent's ownership in a foreign subsidiary, both similarly impact its survival in a positive logarithmic manner; and both similarly do not significantly impact its profitability. The results also show ownership moderates the relationship between the number of expatriates and subsidiary survival. (For more information, please contact: Bassam $E$. Farah, American University of Beirut, Lebanon: bassam.farah@aub.edu.lb)

\section{Promoter Ownership and Performance in Publicly Listed Firms in India: Does Group Affiliation Matter?}

Ansgar Richter, University of Liverpool

Indrani Chakraborty, Institute of Development Studies Kolkata

Many of the largest Indian firms are characterized by promoter ownership, a hybrid form of ownership and governance in which the companies' founders or their heirs hold controlling stakes, while inviting external minority shareholders to contribute capital, and outside managers to participate in the day-to-day administration of the companies concerned. We analyze a sample of 360 publicly quoted firms with promoter ownership in India during the 2006-2013 period. We find that in group-affiliated firms, the level of promoter ownership is 
positively associated with capital market performance, whereas in stand-alone firms there is a U-shaped relationship between promoter ownership and capital market performance. There are only minor performance differences between group-affiliated and stand-alone firms, once other performance determinants are controlled for. Our findings cast doubt on the idea that group affiliation in promoter-owned firms allows promoters to extract value for themselves at the expense of outside shareholders. (For more information, please contact: Ansgar Richter, University of Liverpool, United Kingdom: a.richter@liverpool.ac.uk)

\section{Session: 3.3.7 - Panel}

Track: 13 - I nternational Economics, Finance and Accounting

\section{Three Decades of SAARC: Delayed Promises or Dashed Hope?}

Presented On: J une 30, 2015 - 13:00-14:15

Chair: Mohammad Niamat Elahee, Quinnipiac University

Panelists:

Mohammad Niamat Elahee, Quinnipiac University

Sumit Kumar Kundu, Florida International University

Basu Sharma, University of New Brunswick-Fredericton

Home to over one-fifths of humanity, the nine countries of South Asia, despite their millennia old cultural and historic ties, are currently suffering from lack of cross-border investment, meager intra-regional trade, and limited people-to-people interaction. Once considered the richest corner of the world for which Columbus sailed his ships, South Asian countries suffer from myriad economic and political problems some of which could be mitigated by promoting regional trade. Partly with this end in view, the South Asian Association for Regional Cooperation (SAARC) was created in 1985 amidst much fanfare. Despite 30 years of annual summits, countless ministerial meetings, and drafting of numerous policy papers, the progress in bolstering intra-regional trade in South Asia remains trivial. Notwithstanding this lack of intra-regional trade, the SAARC countries have witnessed impressive growth in their international trade. The panel will discuss the challenges and opportunities for developing SAARC into a close-knit regional economic and trading bloc as a means for both promoting economic development and reducing military tension. The discussion will specifically focus on India, Pakistan, Nepal and Bangladesh- the four largest SAARC countries bound by the waterways originating from the Himalayan Mountains. (For more information, please contact: Mohammad Niamat Elahee, Quinnipiac University, USA: mohammad.elahee@quinnipiac.edu)

\section{Session: 3.3.8 - Competitive}

Track: 2 - Entrepreneurship, Micro-Multinationals, and I nternational New Ventures

\section{Emerging Markets, Transnationality, and I nward-Outward Internationalization}

Presented On: June 30, 2015 - 13:00-14:15

Chair: Arpita J oardar, Clark University

To Benefit or Not To Benefit? Examining the Duality of Foreignness and Entrepreneurial Orientation on Performance

Arpita J oardar, Clark University

Sibin Wu, University of Texas Pan American

This paper provides an in-depth examination of foreignness at the individual level by studying it in the context of entrepreneurs. Based on data collected from 470 domestic and foreign entrepreneurs operating in the U.S., it 
was concluded that foreignness has a curvilinear relationship with entrepreneur performance. There was also evidence of moderating effects of entrepreneurial orientation on the relationship between foreignness and performance. Implications of the findings are discussed. (For more information, please contact: Arpita Joardar, Clark University, USA: ajoardar@clarku.edu)

\author{
Social Ties, Venture Creation, and Home Country Entry by Transnational Entrepreneurs \\ Sarika Pruthi, San J ose State University \\ Mike Wright, Imperial College London
}

The transnational linkages of ethnic minority entrepreneurs are extensive and rapidly being recognized as a key element of economic development, yet the networks facilitating their emergence are little understood. Based on 15 case studies of transnational entrepreneurs (TEs) of Indian origin in the UK, we explore the nature and role of their social ties for entry into the home country. Our findings show that the role of different types of network relationships for home country entry is contingent on a) whether TEs enter the home country based on prior work experience with a former employer and b) whether the business idea for the transnational venture (TNV) is based on prior work experience in the host country. The implications of these findings for theory, policy and practice are discussed. (For more information, please contact: Sarika Pruthi, San Jose State University, USA: sarika.pruthi@sjsu.edu)

\title{
Explaining the Degree and Scope of Internationalization of Russian New Ventures Irina Mihailova, Aalto University Igor Laine, Lappeenranta University of Technology Galina Shirokova, Saint Petersburg University
}

The topics related to international expansion of new ventures (NV) from emerging markets (EM) only recently came into the focus of IE research. Therefore there is a need to get a better understanding of the applicability of the theoretical premises from developed market literature explaining the features of their internationalization in an EM context. In this study we aim to contextualize the existing conceptual assumptions with regards to determinants of scope and degree of NV internationalization using empirical evidence from Russian NV. We develop theoretical framework integrating entrepreneur-, firm-, industry- and institution- specific factors associated with scope and degree of NV internationalization. Also, we attempt to overcome the challenges of doing research in EM context and adopt a mixed method approach by first conducting a quantitative survey and then expanding further on the survey findings through the implementation of qualitative study. The empirical findings reveal that institutional factors have the strongest relationship with the degree and scope of internationalization in Russian context while entrepreneur- and firm-related factors are not associated with degree and scope of internationalization in an expected manner. Based on these findings, we develop four puzzles and extend theoretical development about the nature of NV internationalization from EM. We also make an empirical contribution by enhancing knowledge about Russian NVs. (For more information, please contact: Irina Mihailova, Aalto University, Finland: irina.mihailova@aalto.fi) 


\section{Session: 3.3.9 - Competitive}

\section{Track: 14 - Research Methodology in I B (Special Track)}

\section{Research Methods in IB}

Presented On: J une 30, 2015 - 13:00-14:15

Chair: David Reeb, National University of Singapore

MNC Structure, Complexity, and Performance: Insights from NK Methodology

Sokol Celo, Suffolk University

I. Kim Wang, Suffolk University

James F. Nebus, Suffolk University

We introduce NK-simulation models to international business research and more specifically show how this methodology provides insights into the effects of MNC structure and internal complexity on performance. The interdependence of decisions made in different MNE-units is theorized as an underlying mechanism by which structure and complexity affect performance. The performance of three organizational structures, regional, matrix, and network, discussed in the IB literature is compared at the various levels of complexity. The results of our simulations show that the relationship between internal complexity and firm performance is an inverted $U$ for all three organizational structures. Furthermore, at high levels of complexity the network structure has the best performance, followed by the matrix, with the regional last. However, at low levels of complexity the rank order of structure performance is reversed. In addition to these conclusions, this paper contributes to the international business research by demonstrating how the methodology's power can help scholars answer fundamental questions regarding other IB phenomena. (For more information, please contact: Sokol Celo, Suffolk University, USA: scelo@suffolk.edu)

The Efficacy of Formative versus Reflective Measures of Corporate Reputation: An Information Processing Approach

James Agarwal, University of Calgary

Oleksiy Osiyevskyy, Northeastern University

In this paper, we study the factorial structure of the multidimensional construct 'corporate reputation' by examining two competing measurement conceptualizations: second-order reflective versus second-order formative models. Acknowledging their ontological and epistemological differences, we advocate an 'information processing approach' in attempting to resolve a long-standing debate. Specifically, we use 'Need for Cognition' (NC) as a potential moderator to theoretically argue for the efficacy of both measurement conceptualizations of corporate reputation depending on the level of the moderator. Consistent with dual process theories (Petty and Wegener, 1999; Chen and Chaiken, 1999; Kahneman and Frederick, 2002), we argue that individuals with low and high NC tend to activate a formative conceptualization of corporate reputation whereas those with moderate NC tend to activate a reflective conceptualization. Four hypotheses are empirically tested using structural equations modeling (SEM) in two samples collected in the U.S. and I ndia. A key finding of this study is the conceptual argument for and empirical validation of both conceptualizations of the construct 'corporate reputation'. The role of culture/country is also modeled in the study. (For more information, please contact: Oleksiy Osiyevskyy, Northeastern University, USA: o. osiyevskyy@neu.edu)

(Mis)using Interaction terms in International Business

David Reeb, National University of Singapore

No abstract available. (For more information, please contact: David Reeb, National University of Singapore, Singapore: bizdmr@nus.edu.sg) 


\section{Session: 3.3.10 - Interactive}

\section{Track: 12 - The I nstitutional and Political Environment of I B}

\section{Government and Politics}

Presented On: J une 30, 2015 - 13:00-14:15

Chair: Alice de J onge, Monash University

Minority State Ownership and Investment Risk Management in Mega-Projects Around the World Paul Martin Vaaler, University of Minnesota

Barclay Edward J ames, Universidad San Francisco de Quito School of Business

Management research largely assumes that state ownership in firms raises risk for private co-investors, but we theorize that minority state ownership may decrease investment risk in countries where state policy stability is low. Non-controlling but still substantial equity credibly signals to private investors that states will maintain initial investment project terms, yet limit state interference in project management under the same initial terms. Analyses of 1376 investment projects announced in 95 countries from 1990-2012 support this proposition with greatest risk-decreasing effects when the state holds from $21-30 \%$ of investment project equity. Private investors use state equity partnership as a risk-reducing strategy in response to low policy stability. We highlight where these 'minority rules' hold and state ownership signals credible assurance to private investors. (For more information, please contact: Paul Martin Vaaler, University of Minnesota, USA: vaal0001@umn.edu)

Governments and Businesses in the Context of International Competitiveness: An Analysis of the Role of Government Policies on Diversification of Exports of Brazilian Companies of Capital Goods

Diego Bonaldo Coelho, ESPM

Moacir Miranda Oliveira, University of São Paulo

Felipe Borini, ESPM

The paper investigates the role of the Brazilian government in the internationalization of national companies, in order to examine whether their support instruments can be considered from the perspective of business (policytakers), a specific and relevant resource in the insertion process of international business, characteristic of its origin. We chose to study the diversification of markets for exports of Brazilian industry of capital goods. The methodology is a survey of 104 companies in the sector, where the chosen analysis technique was the linear regression. The results showed that the government has some participation in the diversification of destinations, even in unexpected situations: large companies and potentially experienced ones. (For more information, please contact: Diego Bonaldo Coelho, ESPM, Brazil: diego.coelho@espm.br)

\section{SOE Strategic Behavior When Entering Competitive Host Markets \\ Birgitte Grogaard, University of Calgary \\ Asmund Rygh, BI Norwegian Business School \\ Gabriel R.G. Benito, BI Norwegian Business School}

There are growing concerns about the increase of state-owned enterprises' (SOE) cross-border activities. Do SOEs differ from privately-owned enterprises (POE) in terms of their strategic decisions when internationalizing? Focusing on the oil and gas industry in Canada, a country that represents an open and competitive business environment, we specifically consider the impact of state-ownership on strategic decisions such as whether to acquire stand-alone assets versus complete firms, joint ventures versus wholly owned subsidiaries, exploration versus producing assets, and the size of the investment as reflected in the price of the acquisition. Based on internalization theory and agency theory we develop hypotheses on potential differences in SOE and POE behavior. Our empirical analysis, considering transactions of assets or firms in the Canadian oil and gas industry 
between 2005 and 2013, do not suggest significant differences between SOEs and POEs, with one key exception: There is some evidence that SOEs tend to make larger acquisitions, even when controlling for variables such as investor size, public listing, and whether the home country is a developed economy. (For more information, please contact: Birgitte Grogaard, University of Calgary, Canada: bgrogaar@ucalgary.ca)

\author{
Government Specific Advantage (GSA): An Extention of Ownership Advantages \\ Dale Dejong, Florida International University \\ Yue Zhao, Florida International University \\ Lucas Wenger, Florida International University
}

This paper presents a new addition to the concept of FSA and CSA that we call government specific advantage (GSA). We first define what GSA is and then elaborate on the different aspects involved with this new classification. We give examples of how this is different from FSA and CSA and why GSA is important to a better understanding of the complex world of international business. (For more information, please contact: Dale Dejong, Florida International University, USA: ddejo002@fiu.edu)

Strategies and Government Policies that Support Internationalization in Brazil: The National Financing System and the Role Played by BNDES

Diego Bonaldo Coelho, ESPM

Moacir Miranda Oliveira, University of São Paulo

This study examines the Brazilian government's involvement in the national financing system for international business, which plays an outstanding role regarding internationalization. Based on a detailed historical and institutional analysis of the government's role with respect to the funding of international businesses and the internationalization of national companies, one can verify the status of such activity and the important role played by the National Bank for Economic and Social Development (BNDES). The findings further underline the relevance of the Brazilian government when financing international business activities, since it has become currently an intentional strategic option, avoiding the emergence of barriers related to internationalization, which occur due to the inexistence of a proper institutional strategic framework that covers all guidelines and government actions regarding internationalization. (For more information, please contact: Diego Bonaldo Coelho, ESPM, Brazil: diego.coelho@espm.br)

\title{
Militarization as an Institutional Context for Entrepreneurial Innovation Todd Masaru Inouye, University of Hawaii at Manoa
}

Country level militarization is negatively related to new technology ventures' ability to innovate. Using multilevel mixed effects logistic regression modeling, this study finds that country militarization should be considered when predicting firm level outcomes in high technology industries. These findings contribute to the entrepreneurial innovation literature by specifically focusing upon the both the institutional and spatial contexts in which entrepreneurs innovate. New technology ventures should take this into account when considering R\&D financing and product development strategy. National governments should also assess the potential for narrowing of innovation scope when public $R \& D$ initiatives are being formulated. In cases of high country militarization, respective governments are at risk for confining innovation to military related applications. (For more information, please contact: Todd Masaru Inouye, University of Hawaii at Manoa, USA: toddi@hawaii.edu)

\section{Impact of Corruption on Competition in India: Which Partner Gets to Acquire the IJ V? Shailen Kumar Dalbehera, Indian Institute of Management Bangalore}

Corruption matters. It impacts competition among firms in many ways. I look at the same in detail through a case study of the $2 \mathrm{G}$ spectrum auction in India. Delving further into how corruption impacts competition 
between local and foreign firms I designed a quantitative study. I look at whether the foreign or the local parent of an international joint venture (IJV) in India gets to acquire it upon its termination. I find that in highly regulated industries the local firms have an advantage over the foreign firms in line of arguments by public choice theory. (For more information, please contact: Shailen Kumar Dalbehera, Indian Institute of Management Bangalore, India: dshailenk@gmail.com)

\section{Session: 3.3.11 - I nteractive}

\section{Track: 11 - Corporate Governance in IB}

\section{Leveraging Social Responsibility for Competitive Success}

Presented On: J une 30, 2015 - 13:00-14:15

Chair: Thomas Andre, Ecole Polytechnique

Disasters that Never Were: What Helps Prevent CSR Failure in Offshore Outsourcing Relationships? Michael J Mol, Copenhagen Business School

Kamel Mellahi, University of Warwick

Sun Hye Lee, University of Warwick

Organizations face major challenges in managing CSR in offshore outsourcing relationships. We investigate how the impact of CSR failure, i.e. its likelihood and severity, can be minimized. Since this requires an understanding of actions of individuals, stakeholder pressures exerted on buyer and supplier firms, interorganizational relationships, and home and host institutional contexts we present a multilevel theory. Our theory suggests that managerial attention, low buyer firm stakeholder expectations, buyer dominated relationships, cooperative relationships and strict host country institutions all serve to lower the impact of CSR failure. By contrast misalignment between the firms in stakeholder expectations and institutional distance between the countries increase that impact. This latter effect is negatively moderated by managerial attention, a buyer dominated relationship, and a cooperative relationship. Our theory generates important new insights for literatures on CSR and offshore outsourcing as well as providing an exemplar for multilevel theorizing in international management. (For more information, please contact: Michael/ Mol, Copenhagen Business School, Denmark: mmo.smg@cbs.dk)

\section{Gaining Competitive Advantage through Carbon Constrained Strategies: Evidence from Brazilian Energy Sector Monica Cavalcanti Sa de Abreu, Federal University of Ceara \\ Ana Rita Pinheiro de Freitas, Federal University of Ceara Silvia Maria Dias Pedro Rebouças, Federal University of Ceara}

Although existing research evaluates how the adoption of proactive environmental strategies affects corporate performance, there is little understand of the mechanism that links climate change strategy and competitive advantages. It is, therefore, unclear how driven forces relate to climate change risk and stakeholder pressure could lead to a innovative and compensation practices. In this paper, we analyze the relationship between risk, pressure from stakeholders, strategic responses and competitive advantage. We develop and test a structural equation model to assess the effects of carbon constrained strategies on competitive advantage in the energy sector. Results from a survey of 105 Brazilian energy firms strongly support the model. (For more information, please contact: Monica Cavalcanti Sa de Abreu, Federal University of Ceara, Brazil: mabreu.ufc@gmail.com) 
Social Risk and the Management of MNCS - From Risk Society to International Business Management Jacob Taarup-Esbensen, Copenhagen Business School

Multinational companies (MNCs) are increasing being exposed to risk that originate from local communities in the business environment where they operate. The response has been to implement systems for stakeholder engagement by including social issues into their risk management systems. However, these seem to be ineffective in mitigating local community grievances. In this paper I argue that social risk is to be defined in terms of how local stakeholders construct reality through a sensemaking and framing process which constitute at risk by its combined capability to influence organisational value creating activities. By utilizing a crossdisciplinary approach founded in a sociological conceptualization of risk I show that there is an alternative to identification and mitigation of social risk. I propose that MNCs could improve their performance by organizing their efforts using "intelligent" systems, which are based on the capability to identify frames and sensemaking processes. This paper show how social risk management can be conventionalised using distinct theoretical domains taking its outset in a sociological perspective on risk, linking International Business (IB) risk management practices, operationalised through Corporate Social Responsibility (CSR) systems, with communication theory and risk as frames of meaning constructed by community members and corporate decision makers. (For more information, please contact: Jacob Taarup-Esbensen, Copenhagen Business School, Denmark: jta.ikl@cbs.dk)

\author{
New Approaches in Overcoming Liability of Foreignness: The Effects of Strategic Social Responsibility \\ Jialiang Zhang, Renmin University of China \\ Majid Ghorbani, Renmin University of China \\ Weiguang Li, Renmin University of China \\ Zyang Liu, Renmin University of China
}

The rapidly-changing business environment in emerging markets makes the competition between firms from these countries fiercer than ever. While most firms are still searching for new ways to compete, some emerging market MNES (EMNE) have realized that adapting to and complying with socially and environmentally friendly practices is a new strategic approach to gain competitive advantage and even an effective solution to overcome obstacles such as liability of foreignness (LOF). We qualitatively explore whether Strategic CSR (SCSR) can reduce LOF, by analyzing the case of China Minmetals Corporation to show that Chinese multinationals have been able to build a better relationship with local and international stakeholders through adaption and implementation of SCSR. (For more information, please contact: Jialiang Zhang, Renmin University of China, China: ruczj1123@163.com)

\author{
How Do CEOs Perceive Business-Aligned CSR? Co-word Analysis of U.S., Korean and Japanese CEO Letters \\ Youjin Baik, Yonsei University \\ Soonkyoo Choe, Yonsei University \\ Young-Ryeol Park, Yonsei University
}

The purpose of this article is to explore how CEOs at U.S., Korean and J apanese firms perceive business-aligned corporate social responsibility (CSR). By using mixed methods of social network analysis, the article analyzes the CEO letters in 2012 sustainability reports of 60 listed companies in the U.S., Korea and J apan. It is concluded that CEOS of U.S., Korean and Japanese firms perceive business-aligned CSR differently. While CEOs at U.S. firms emphasize the importance of innovation and efficiency, J apanese CEOs focus on technological development. Interestingly, Korean firms as the late-movers in international markets consider competition and competitiveness important issues even in the context of CSR. (For more information, please contact: Youjin Baik, Yonsei University, Korea, South: youjin.baik@gmail.com) 
Session: 3.3.12 - Interactive

Track: 3 - International Human Resource Management and Organizational Behavior

\section{Nurturing Global Talent}

Presented On: June 30, 2015 - 13:00-14:15

Chair: Dhara Shah, Griffith University

Managing Talents in Nordic MNCs: The Impact of Socialization Mechanisms

Violetta Khoreva, Hanken School of Economics

Edyta Kostanek, Hanken School of Economics

Maarten van Zalk, Orebro University

We study what is more decisive in predicting talents' attitudes - talent identification or organizational identification, and investigate whether corporate socialization mechanisms influence these relationships. Our analyses of 439 talents in eleven Nordic MNCs reveals the importance of both talent identification and organizational identification in predicting commitment to building relevant competences and acceptance of increasing performance demands. Our results also show that the maintenance of corporate socialization mechanisms increases the likelihood of talents to accept increasing performance demands, which in turn may have a positive effect on the overall business performance of MNCs. (For more information, please contact: Edyta Kostanek, Hanken School of Economics, Finland: kostanek.edyta@hanken.fi)

\section{An Employability Skill Assessment Framework for Indian Organizations Chithra Rema, Pondicherry University}

The purpose of this paper is to suggest a suitable employability skill assessment model for Indian organizations. It is an exploratory study. The aim of this paper is to focus on an acceptable and practical framework of engineering employability skills framework for Indian engineering graduates that are firmly based on requirement by accrediting bodies and professional bodies and existing research findings in employability skills. The framework is developed by the author after an extensive research in employability skills. The study used Borich needs assessment model to identify the training needs of an organization, Normalized skill weight method to prioritize the skill weightage. The proposed model can be used in organizations for recruitment, selection, training and competency assessment (For more information, please contact: Chithra Rema, Pondicherry University, India: chithrarema@gmail.com)

\section{Corporate Social Initiatives and Employee Retention \\ Christiane Bode, INSEAD \\ Jasjit Singh, INSEAD \\ Michelle Rogan, INSEAD}

Firms are increasingly launching initiatives with explicit societal mandates, often justified through one aspect of talent management: employee retention. Although prior empirical studies have demonstrated a link between social initiatives and intermediate outcomes like motivation and identification with the firm, the ultimate relationship between employee participation in these initiatives and actual retention has not been investigated. Our study fills this gap. While the related theoretical literature has focused on potential treatment effects from employee participation in corporate social initiatives, we also take into account the possibility of sorting effects based on heterogeneity in employee preferences for making a social impact. Using individual-level project participation and employee retention data for about 10,000 employees from a global management consulting firm, we present empirical evidence in support of a retention effect associated with participation in an initiative involving projects with an explicit social impact goal. In addition, we offer arguments for moderating conditions that might weaken this relationship, and present evidence consistent with these suggestions. Our choice of 
research context, stringent matching criteria and additional analysis of survey and interview data are most consistent with the finding being driven by a combination of employee sorting and actual treatment effects from participation. (For more information, please contact: Jasjit Singh, INSEAD, Singapore: jasjit.singh@insead.edu)

The Influence of Talent Management Practices on Competitive Advantages in Emerging Market Firms: Evidence from Russian Companies

Marina Latukha, St. Petersburg State University

This study aims to prove the influence of talent management (TM) practices on competitive advantage (CA) in internationalized Russian firms. As a phenomenon TM is rather new and controversial in the emerging market context and despite the certain amount of research devoted to TM practices, there is little said about how TM influence CA in emerging market firms. This study explores the peculiarities of TM practices in Russian companies and empirically proves a positive relationship between TM practices and CA in the emerging market context. The paper identifies a number of specific TM practices, which influence CA. We argue that succession planning and career development have a greater impact on CA in Russian firms. (For more information, please contact: Marina Latukha, St. Petersburg State University, Russia: marina.latuha@gsom.pu.ru)

\title{
Change in China? Taking Stock of Blue Collars' Work Values Marina Schmitz, University of Goettingen
}

According to generational cohort theory, value change is often a combination of generational replacement and other influence factors, such as economic development. As Chinese society has experienced rapid economic development over the past 30 years, I assume that blue collar workers in China have been influenced by this enormous transformation. To confirm my assumption, I conducted semi-structured interviews with 25 employees (17 blue collar workers, 8 HR managers) of foreign companies in Shanghai and assigned the statements of the blue collar cohort to the respective work values, proving the existence of instrumental, cognitive, social and prestige value categories. My findings revealed a high importance of instrumental values, showing a tendency towards growth needs. Drawing on my findings, I further deduct vital theoretical and especially practical implications. (For more information, please contact: Marina Schmitz, University of Goettingen, Germany: mschmit5@uni-goettingen.de)

\author{
On the Psychological Effects of Talent Status Self-Awareness among High-Potentials in MNCS \\ Jennie Sumelius, Hanken School of Economics \\ Mats Ehrnrooth, Hanken School of Economics \\ Ingmar Bjorkman, Aalto University \\ Kristiina Makela, Aalto University \\ Adam Smale, University of Vaasa \\ Susanna Taimitarha, Hanken School of Economics
}

Due to organizational concerns about the effects of openly communicating employees' talent status, employees may not be aware of having been identified as talent. In this paper, we examine how talent status selfawareness on the part of the individual influences the social exchange relationship between the employer and the talent. We propose that the relationship between psychological contract fulfillment, promised inducements and performance appraisal is modified by talent status self-awareness. Based on analyses of a sample of 313 talents - as identified by their respective multinational corporations (MNCS) - we find that talent status selfawareness makes talent more demanding in terms of psychological contract fulfillment, and performance appraisal less effective as a management tool for those who are aware of being talent. The findings have important implications for talent status communication and talent management, as well as future research on these topics. (For more information, please contact: Jennie Sumelius, Hanken School of Economics, Finland: jennie.sumelius@hanken.fi) 
Training and Development in Talent Management Process: A Case Study in Turkey

Berrin Guner, Rowan University

Nevin Deniz, Marmara University

Busra Ercokses, Yapi Kredi Bankasi

Today's businesses face many challenges that impact their human resource practices; growing trends in globalization and technology, demographic shifts in workforce, and instant access to information. The growth potential of organizations worldwide depends on the ability of companies to have the right people in the right place at the right time. Hence, it is imperative for firms to attract, develop, and retain the skilled workers they need to meet their organizational goals. To this end, this study focuses mainly on the training and development processes of talent management practices in a major bank in Turkey. The aim of this research is to determine how talent management process is practically applied to identify the key variables that will help researchers develop more theoretically sound frameworks in later studies. (For more information, please contact: Berrin Guner, Rowan University, USA: guner@rowan.edu)

\section{Managing Yopatriates: A Longitudinal Study of Generation Y Expatriates in an Indian MNC Liza Howe-Walsh, University of Portsmouth \\ Vijay Edward Pereira, University of Portsmouth \\ Ashish Malik, Newcastle Business School}

This paper provides a longitudinal study of HRM practices for a new category of non-traditional expatriates who we term as "Yopatriates", are young, highly qualified and mobile, Generation Y (Gen Y) knowledge workers. In contrast to traditional expatriates Yopatriates seek short-term international assignments that suit their individual (internal, rather than organisational or external) career orientations of learning and travel. We study this group using a longitudinal, extremely unusual revelatory case study analysis of a large Indian Multinational Corporation (MNC) delivering global IT and business process offshoring (BPO) services. Our study presents the HRM practices adopted to manage expatriates as well as a growing population of Yopatriates. More specifically, this paper identifies distinctive HR practices employed for managing Yopatriates, within India. We further evaluate the extent to which internal HR practices of Yopatriates in Indian BPO firms were characterised, by a desire to emulate or adopt what were regarded as global 'HR best practices'. We extend the literature by developing a distinctive theoretical category of non-traditional expatriates that highlights a need for a different set of HRM practices. Findings suggest that these practices were complementary and at the same time contradictory to 'indigenous' localised practices during the period of research. (For more information, please contact: Liza Howe-Walsh, University of Portsmouth, United Kingdom: liza.howe-walsh@port.ac.uk)

\section{Session: 3.3.13 - Interactive}

\section{Track: 9 - Emerging Economies and Emerging Economy MNEs}

\section{Networks and Business Groups}

Presented On: J une 30, 2015 - 13:00-14:15

Chair: Gaurav Agrawal, ABV-Indian Institute of Information Technology and Management

Do Business Group Affiliated Firms Benefit from Knowledge Sharing When They Go Abroad? The Moderating Role of Diversification

J iyoung Shin, Korea University

Kihyun Kim, Korea University

Youngwoo Lee, Korea University

In this study, we attempt to explain how knowledge sharing within business group influences the level of an affiliated firm's internationalization. By utilizing resource-based view and network perspective, we examined 
whether business group network help an affiliated firm going abroad through knowledge sharing. The knowledge sharing is of particular interest in this paper, as business group is essentially a network that consists of relationships among member firms. Using samples of Korean business group from 2000 to 2012, we found that international knowledge and technological knowledge sharing within business group improve the degree of internationalization of an affiliated firm. We also found that the level of diversification of business group positively moderate the relationship between foreign knowledge and internationalization of an affiliated firm. Based on these findings, we conclude by providing implications for researchers and managers. (For more information, please contact: Jiyoung Shin, Korea University, Korea, South: alfolike@korea.ac.kr)

\section{Role of Networks in Internationalization of Emerging Economy MNE Nivisha Singh, Indian Institute of Management Indore}

In this paper I have tried to find the role of networks in internationalization of emerging economy MNEs. It has been realized that as compared to developed economy not much research is done on emerging economy internationalization, where knowledge is considered a strategic asset for competitive advantage. To achieve this advantage role of network becomes critical. I have focused on few characteristics of networks and have proposed what impact each of characteristics will have on internationalization of MNEs. This paper will have theoretical and practical implications and will provide a path for future research. (For more information, please contact: Nivisha Singh, Indian Institute of Management Indore, India: f12nivishas@iimidr.ac.in)

\section{Friends in the Right Places: Non-Market Connections as Emerging Market Strategy Susan Feinberg, Temple University Laura Gasiorowski, Temple University J un Ito, Temple University}

We examine whether the networks of firms' board directors vary systematically with differences in the institutional environment. We predict that in corrupt, underdeveloped institutional environments, firms' board networks will have a greater proportion of "Non-Market" connections, which we define here as connections to actors and organizations in government, NGOs and universities. In contrast, firms will have relatively more "Market" connections to publicly traded firms in highly developed institutional environments. Using a new dataset with detailed information on the resumes of board directors of over 2000 firms in 24 countries, we find support for our predictions. Our work extends the literature on board selection in that we consider how the institutional environment affects the selection of directors with particular network compositions, rather than just work experience or interlocks. We also extend the literature on political connections by theorizing about and demonstrating that firms value a variety of other Non-Market connections in environments where corruption limits the effective functioning of markets. (For more information, please contact: Susan Feinberg, Temple University, USA: feinberg@temple.edu)

\section{An Empirical Study of the Impact of Business Group and Product Market Characteristics on Performance Outcomes of Emerging Market Firms}

B. Elango, Illinois State University

Chinmay Pattnaik, University of Sydney

Jamie R. Wieland, Illinois State University

This study contributes to the current discussion of the impact of Business groups as a powerful economic force in emerging markets by investigating the impact of specific characteristics of business groups along with product market context on affiliate performance. Study findings, based on a sample of 7710 affiliates from 345 business groups, reinforce the significance of business groups in an emerging market and stress the importance of studying the relevance of specific group characteristics rather than the aggregate form. Our findings show that the payoffs from business group diversification may be varied contingent on product market conditions faced by the affiliate. Therefore, firms affiliated with diversified business groups should not assume that benefits 
from diversification will accrue to them and enhance their ability to compete in their industries. This study contributes to the literature by moving the broader discussion on business groups from a monolithic concept to a focus on specific characteristics and integrates the product market context in determining payoffs. (For more information, please contact: B. Elango, Illinois State University, USA: elango@ilstu.edu)

Profit or Relation Matters for Internationalization? The Underlying Mechanism between Small World Network and Internationalization in an Emerging Economy

Yingyu (Kerri) Chen, National Dong Hwa University

Wen-Chiung Chou, Kainan University

Chin-jung Luan, National Dong Hwa University

A small world network featured as an efficient network for divergent resources has received much theoretical attention in diverse fields. Owing to the efficiency in resource exchanging, we postulated the acceleration in internationalization for business network under the embedded small world network mechanism. However, the significant result is not found. We thus posit the untested underlying mediating role of performance between a small world network and internationalization. A business group characterized as a strategic network has been employed as our research context in this study. We analyzed the small world effects by incorporating Taiwanese business group. Using theoretical lens mainly from network perspective, this study extends prior business group research and focuses more on both effects of the group and the cross-level dimensions. We found that the existence of the underlying mediating role between a small world network and internationalization and the dynamic relationship between a small world network and performances of a group and of a core firm under group-embedded structure. Drawing on social network perspective, our research findings contribute literatures of business group and internationalization. (For more information, please contact: Yingyu (Kerri) Chen, National Dong Hwa University, Taiwan: kc615486@gmail.com)

\section{Session: 3.4.1 - Panel}

\section{Track: 11 - Corporate Governance in IB}

\section{Macro-Institutional Change, Corporate Governance and Investment in Emerging Economies: Implications for International Business Research and Practice}

Presented On: J une 30, 2015 - 14:30-15:45

Chair: Tailan Chi, University of Kansas

Panelists:

Anju Seth, Virginia Tech University

Tailan Chi, University of Kansas

Ruth Aguilera, Northeastern University

Luis Alfonso Dau, Northeastern University

This panel session intends to examine the recent and emerging changes in the macro institutions of major emerging economies or economic regions and discuss their implications for corporate governance. The economies or economic regions to be covered are China, India and Latin America. Three of the panelists will each focus on one of these economies or economic regions and outline recent trends in the macro-institutional environment of the country or region covered, implications for corporate governance as well as corporate responses, and potentially interesting research questions to explore. The fourth panelist will examine the diversity of corporate governance systems across emerging economies. After the four presentations, the panelists will engage the audience in an interactive discussion, particularly with regard to the implications of these recent and emerging changes for research in international business and strategy as well as for managerial practice. (For more information, please contact: Anju Seth, Virginia Tech University, USA: aseth@vt.edu) 


\title{
Session: 3.4.2 - Competitive
}

\section{Track: 12 - The Institutional and Political Environment of I B}

\section{The I mpact of I nternational Politics and I nternational I nstitutions}

Presented On: June 30, 2015 - 14:30-15:45

Chair: Michael A. Sartor, Queen's School of Business

\author{
Who Cares about Diplomacy? Location Choice by Multinational Enterprises \\ Klaus Meyer, CEI BS \\ Jing Li, Simon Fraser University \\ Yuan Ding, CEIBS \\ Hua Zhang, CEIBS
}

Do diplomatic relations between countries matter in location choice of multinational enterprises (MNEs), and if so for which MNEs? We propose that MNEs tend to choose and settle successfully in locations with which their home country has good diplomatic relations because they face lower political risks and entry barriers in those locations. However, MNEs vary in their ability to leverage political resources, and those with stronger political connections thus stand to benefit more from good diplomatic relations. Moreover, we argue that MNEs' ability to benefit from diplomatic relations is more important in host countries with weak rule of law. Empirically, we test our hypotheses on overseas investment location decisions by Chinese MNEs, and find empirical support. We discuss implications of our study to research on political risk and international investment strategies, and to research on corporate political connections. (For more information, please contact: Klaus Meyer, CEIBS, China: kmeyer@ceibs.edu)

\section{Does Arbitration Reduce Diplomatic Pressure in Investment Disputes? \\ Srividya J andhyala, ESSEC Business School \\ Geoffrey Gertz, University of Oxford \\ Lauge N. Skovgaard Poulsen, University College London}

We examine whether the presence of International Investment Agreements (IIAs), negotiated among countries for foreign investor protection, alters how MNEs can resolve disputes with the host government. Firms expanding in international markets face a host of political risks, including those arising from changes in government policy or outright expropriation. When confronted with government actions that lower the value of their investment, investment agreements provide the right to sue host government in investor-state arbitration. The legal option was partly intended to lower the extent of costly home state engagement in investment disputes, but the assumption has not been tested in prior literature. We ask if access to arbitration reduces the diplomatic assistance that home governments might otherwise bring to the dispute settlement. We build a unique dataset involving 440 disputes of US investors with foreign governments in 86 countries during the period 1945-2010 using data from US diplomatic cables made available by Wikileaks. We find that the access to investment arbitration for the investing firm has no effect on the presence or extent of US diplomatic support to resolve the dispute. (For more information, please contact: Srividya Jandhyala, ESSEC Business School, Singapore: srividya.jandhyala@essec.edu)

\section{Effects of Deinstitutionalization: Evidence from the Indian Textile Industry \\ Raveendra Chittoor, Indian School of Business \\ Preet Aulakh, York University \\ Oana Branzei, I vey Business School Western University}

In this paper, we focus on the effects of deinstitutionalization and how institutions at multiple levels interact with one another in influencing firm-level outcomes. Our empirical context is the global textile industry. The 
global trade in textiles was governed by Multi-Fibre Agreement (MFA) since 1974, which provided for bilaterally negotiated agreements on import quotas by developed countries. MFA ceased to exist with effect from January 1 2005. We theorize on how the termination of a global institution such as MFA interacts with local institutions such as affiliation to business groups and policy initiatives at the national level. We test our predictions using a comprehensive panel (2001-2010) sample of both publicly listed and private firms in the Indian textile sector. Results show that firms affiliated to business groups lose global market share post-MFA, but improve their profitability when compared to stand-alone firms. We also examine the effect of a major policy initiative called the Technology Upgradation Fund Scheme (TUFS) introduced by the I ndian government to help Indian textile exporters cope with the abolition of the quota regime. We find that accessing the TUFS enhanced firms' global market share post-MFA, but this accrued at the cost of lower profitability (For more information, please contact: Raveendra Chittoor, Indian School of Business, India: raveendra_chittoor@isb.edu)

\section{Does Institutional Distance Still Matter? Industry Standards and Global Sourcing Location Choices}

Stephan Manning, University of Massachusetts Boston

Marcus M. Larsen, Copenhagen Business School

This paper adds nuance to our understanding of institutional antecedents of foreign investment, in particular in global services sourcing. While prior research has stressed the various risks and effects associated with homehost country differences in national-level institutions, e.g. legal systems, we argue that industry-specific, yet often transnational field institutions, e.g. standards, have become critical factors in driving sourcing location decisions. Using data from the Offshoring Research Network, Kaufmann institutional indicators, and data on 'capability maturity model integration' (CMMl) process standard adoption, we show that sourcing location choices are indeed negatively impacted by institutional differences between home and host country, i.e. 'distance' still matters, but they are positively impacted by CMMI standard adoption in host countries, and standard adoption negatively moderates the importance of distance. Findings promote a more contextual, multilevel understanding of institutional antecedents of foreign firm location choices, which also has important policy implications in particular for emerging economies. (For more information, please contact: Stephan Manning, University of Massachusetts Boston, USA: stephan.manning@umb.edu)

\section{Session: 3.4.3 - Competitive}

Track: 1 - People and Careers in Cross-Cultural Business

\section{Cultural Diversity, Cultural I dentity, and Performance}

Presented On: June 30, 2015 - 14:30-15:45

Chair: Paul N. Gooderham, Norwegian School of Economics

Not All Diversity Is the Same: A Comparative Study of the Effects of Diversity in Global Virtual Teams Vasyl Taras, University of North Carolina at Greensboro

Daniel Baack, University of Denver

Douglas Dow, University of Melbourne

Alfredo J imenez, University of Burgos

Peter Magnusson, University of Alabama

The modern workplace necessitates international on-line collaboration. The resulting global virtual teams provide many advantages, but they also pose a range of challenges due to the inherent diversity of member backgrounds. While past research has explored the role of diversity in teams, the present study expands on this by focusing on virtual teams and by exploring a much broader range of distance/diversity dimensions. Specifically, we test multiple approaches, both typical and unique, to measuring cultural diversity. The breadth 
of our data set is unique, as the data were collected as a part of a large academic experiential learning exercise that involved 8,345 EMBA, MBA, and undergraduate business students originally from 134 countries (studying in 44 countries) working together in 1,189 international teams. The reward structure, member interdependence, communication mode, and timing all mimicked those used in the corporate world. The results revealed that diversity clearly affects team performance. National diversity has a positive impact on team effectiveness by increasing the experiences, networks and resource pools of team members. In contrast, individual diversity hinders communication and lowers cohesion and satisfaction with the project. (For more information, please contact: Daniel Baack, University of Denver, USA: dbaack@du.edu)

\title{
The Integration of Cultural I dentity Negotiation Theory into Cross-Cultural Organizational J ustice Theory: A Qualitative Study J oerg Bueechl, Tuebingen University Markus Pudelko, Tuebingen University
}

Enriching organizational justice literature with insights from cultural identity negotiation theory, our explorative, qualitative study develops an evidence-based model illuminating how culturally diverse subordinates and supervisors can achieve through their cross-cultural interactions shared understandings of fairness. Our analysis is based on a complex research design comprising in total 91 interviews in China and in Germany from Chinese subordinates of German supenvisors, German subordinates of Chinese supervisors, Chinese supervisors of German subordinates and from German supervisors of Chinese subordinates. Findings reveal that both, subordinates and supervisors undergo interrelated cultural negotiation processes which lead to an approximation of previously more distinct fairness perceptions and ultimately to a partially shared understanding of fairness. As part of our model, we present a series of personal and contextual moderators, which affect the cultural identity negotiation in a way that the development of shared fairness perceptions is either facilitated or impaired. Based on our findings we formulate specific propositions, guiding future research and practice. (For more information, please contact: Joerg Bueechl, Tuebingen University, Germany: joerg.bueechl@unituebingen.de)

\author{
Crossing Geographic and Organizational Boundaries: A Study of Executives' Access to Help \\ Stewart R. Miller, University of Texas-San Antonio \\ Marie Louise Mors, Copenhagen Business School \\ Michael McDonald, University of Texas-San Antonio
}

We develop a framework to explain expectations for help from professional contacts who (1) reside in the same/ another country and (2) work for the same/ another organization as the focal executive. The framework also considers how relational duration and trust affect these relationships. We find no evidence that executives expect less help from contacts in another country. However, executives have lower expectations for help from contacts outside their firm. This relationship is partially mediated by trust. We find conditional support that relational duration mitigates expectations for less help from external contacts in that this relationship is mediated by trust. (For more information, please contact: Stewart R. Miller, University of Texas-San Antonio, USA: stewart.miller@utsa.edu) 


\section{Session: 3.4.4 - Competitive}

Track: 2 - Entrepreneurship, Micro-Multinationals, and International New Ventures

\section{From International New Ventures to Micromultinationals: Theoretical and Empirical Considerations}

Presented On: J une 30, 2015 - 14:30-15:45

Chair: Tony Fang, Stockholm University

Essential Knowledge Sources for Micromultinationals: A Phenomenographic Approach

Maria-Cristina Stoian, Brunel University London

Pavlos Dimitratos, University of Glasgow

Micromultinationals (mMNE) are a distinct type of internationalised SMEs. Regardless of their innate resource constraints, they are able to engage in high commitment modes of servicing foreign markets, displaying an international behaviour similar to their larger counterparts. Despite their increased potential to yield economic and social benefits, very limited research has been conducted so far into managerial understanding of the essential knowledge sources which enable these smaller-sized firms to operate as mMNEs. Based on the Knowledge-based-View (KBV) and insights from knowledge building and learning in the international business field, we address this gap in the literature by adopting a phenomenographic approach in the UK context. Our results reveal three qualitative distinct conceptions of critical knowledge sources that allowed mMNEs to occur, alluding to different internationalisation behaviours. (For more information, please contact: Maria-Cristina Stoian, Brunel University London, United Kingdom: cristina.stoian@brunel.ac.uk)

\section{Accelerated Small Firm Multinationalization: Studying Born Micromultinationals via Multiple Theoretical Lenses Heini Vanninen, Lappeenranta University of Technology \\ Olli Kuivalainen, Lappeenranta University of Technology Luciano Ciravegna, King's College London}

The multinationalization of young and small firms is not captured by existing theoretical frameworks, because of the literature gap separating studies of born globals and international new ventures from studies of multinational enterprises. However, firms go beyond accelerated internationalization and engage in accelerated multinationalization. Born micromultinationals invest and operate in multiple countries from or soon after their founding. This paper combines the BG/INV research with MNE research to examine the antecedents, processes and outcomes of accelerated small firm multinationalization. We discuss existing theories and examine them in light of four case studies of Finnish born micromultinationals. We develop propositions to characterize born micromultinationals and define a research agenda to study them further. We argue that to explain born micromultinationals it is necessary to examine their smallness, newness and entrepreneurial nature as suggested by the BG/I NV literature, whilst also examining why and how they invest abroad through the theoretical lenses of MNE theories. (For more information, please contact: Heini Vanninen, Lappeenranta University of Technology, Finland: heini. vanninen@/ut.fi)

\section{How Do International New Ventures Create "Valuable" Social Capital? A Local and Global Networking Activities Perspective}

Karl S.R. Warner, Edinburgh Napier University

Marian V. J ones, University of Glasgow

It is widely acknowledged in the international entrepreneurship (IE) literature that social capital is a vital asset for new venture internationalization. Despite a well published body of literature on network based research, it is still unclear as to how international new ventures (INVS) actually create social capital. This is heightened by the fact that there is emerging consensus that social capital has a "dark side" meaning that this intangible can harm 
new venture internationalization. Based on longitudinal multiple case study research, we ask: How do INVs create valuable social capital? Taking a micro-process view of social capital, this paper uncovers five core networking activities that underpin the creation of social capital. Findings depict that INVs use local referring, global referring, local searching, global searching and global accepting as networking activities to create social capital. This paper therefore contributes to the IE literature uncovering some of the networking skills that entrepreneurs and their new ventures require for early internationalization. (For more information, please contact: Karl S.R. Warner, Edinburgh Napier University, United Kingdom: k.warner@napier.ac.uk)

\section{Networking and a Tandem Approach to Foreign Market Entry Decision- Making \\ Sylvie K. Chetty, University of Otago \\ Mitra Etemaddar, University of Otago}

A traditional view of internationalizing firms is that their foreign market entry (FME) is planned but there is an emerging view effectuation (unplanned approach) that challenges the basic assumptions of how SMEs behave under uncertainty such as FME. We use in-depth qualitative research to examine decision-making during foreign market entry and identify how network relationships influence their foreign market entry as the firm expands. While we found two different decision-making processes for FME these are not polar opposites or linear but interwoven as a nonlinear process because entrepreneurs skillfully combine both approaches depending on the context. (For more information, please contact: Sylvie K. Chetty, University of Otago, New Zealand: sylvie.chetty@otago.ac.nz)

\section{Session: 3.4.5 - Competitive Track: 9 - Emerging Economies and Emerging Economy MNEs}

\section{EMNCS and FDI}

Presented On: June 30, 2015 - 14:30-15:45

Chair: Vikas Kumar, University of Sydney

Licking the Dirt to Rise and Shine: EMNCS Path to Competitive Advantage

Tanvi Kothari, San J ose State University

Masaaki Kotabe, Temple University

One of the emerging phenomena of global competition is the increasing number of business innovations originating from emerging economies and dispersing to developed nations. This phenomenon also known as Reverse Innovation is recently being observed by large MNCs operating in developing nations. Another set of key players and perhaps initiators of such reverse innovations are the Emerging Market Multinational Companies (EMNCS) that have survived and succeeded in the rough institutional environments in their home turfs and are now participating in the global marketplace. However, very little is known about how these EMNCs build their competitive advantage as they grow from their home (emerging) markets to developed nation markets. In order to explore this phenomenon further, we conduct a historical analysis of sixteen companies that originated from key emerging markets viz I ndia and China. Finally, based on our analysis we suggest that EMNCs' path to building competitive advantage in the developed nations is, on one hand, based on the EMNCs' ability to acquire resources and absorb them to build their own advantage. On the other hand, it is also based on EMNCs' ability to find some market niches, i.e., entering into markets untapped by traditional MNCs. (For more information, please contact: Tanvi Kothari, San Jose State University, USA: kothari.t@gmail.com) 
FDI Indirect Spillover in Emerging Economies: The Role of Government

Afonso Fleury, Universidade de Sao Paulo

Taotao Chen, Tsinghua University

Maria Tereza Fleury, Fundacao Getulio Vargas

Xiao Chen, Tsinghua University

Liu Shichang, Tsinghua University

Carlos Eduardo Aveline, Fundacao Getulio Vargas

FDI-generated effects may be direct when it impacts the host country economy as a whole or indirect when spillovers increase local producers' productivity and competitiveness. Spillover effects arising from the establishing multinationals will benefit local producers through different channels or mechanisms. Key factors for the enactment of spillovers pervade literature, albeit an overlooked governmental role. We compare FDI spillovers in China and Brazil at both industry and firm levels, aiming at unveiling the influence of government in the configuration of spillover mechanisms and, consequently, in FDI impacts on local industries. Research comparing countries and based on case studies is likely to reveal the subtleties of FDI spillover while micro-level analysis allows a deeper and clearer investigation of the enabling mechanisms of the spillover process. Comparative historical analysis is the central mode of investigation, leading to a commitment to offering historically grounded explanations to important effects. The findings reveal the importance of governmental role in the achievement of positive FDI spillover effects and subsidize a preliminary causal analysis between government intervention and spillover mechanisms. (For more information, please contact: Afonso Fleury, Universidade de Sao Paulo, Brazil: acfleury@usp.br)

Post Financial Crisis Catch-Up Strategies in Emerging Market MNEs: A Comparative Analysis of Strategic Asset Seeking FDI in the United States

John Anderson, University of Northern I owa

Dylan Sutherland, Durham University

Peter Hertenstein, Cambridge University

Some argue emerging market (E)MNEs are different to their developed market (D)MNE counterparts because in the post-financial crisis period they have pursued accelerated internationalization, predominantly using acquisitions to acquire intangible strategic assets in psychically distant developed markets for the purposes of technological 'catch-up'. To date, however, there has been little systematic comparative analysis of whether asset seeking by EMNEs has intensified in the post-financial crisis period or whether their asset seeking orientation is truly different to that of their DMNE counterparts. To address these questions we compare the investment location choice motivations of Chinese, German and British MNEs in the United States. We find Chinese asset seeking activity has intensified in the post crisis period. Importantly, we also find DMNE FDI is strongly motivated by strategic asset seeking. Building from our location choice study intangible asset growth levels of both EMNEs and DMNEs are analyzed. This shows that Chinese MNEs are not 'catching-up'. Our findings bring into question a number of ideas that are increasingly being accepted as received wisdom, namely: the extent to which Chinese MNEs are truly different; whether new theories are required to explain their behaviors; and whether EMNE outward FDI genuinely facilitates technological 'catch-up' with DMNEs. (For more information, please contact: John Anderson, University of Northern Iowa, USA: john. r.anderson@uni.edu) 


\title{
Session: 3.4.6 - Competitive
}

\section{Track: 8 - Global Strategy, M\&As and Competitiveness}

\section{Dealing with Cross National Institutional Differences}

Presented On: J une 30, 2015 - 14:30-15:45

\author{
Chair: Laura Gasiorowski, Temple University
}

The Effect of Political Connections on International Expansion Strategy: Evidence from French Firms, 2003-2012 J oao Albino Pimentel, HEC Paris

This paper examines how different types of political connections influence a firm's international expansion strategy regarding its tolerance to host-country political risk and preference for host-country political alignment with the home-country. Extant research on political connections emphasizes their impact on firm value, overlooking their influence on firm strategy. The main argument in this paper is that a firm's connections with its home-country's political authorities determine the balance between the firm's and its home-country government's political and economic interests, influencing, in turn, the firm's international expansion strategy. Hypotheses regarding the impact of three types of political connections are tested on a sample of international investments by the largest French firms during the 2003-2012 period. Results suggest that politically-connected firms make distinct international expansion strategies relative to non-politically-connected firms. Such heterogeneity is contingent on the type and level of political connections and is influenced by political changes that alter the value of political connections. (For more information, please contact: Joao Albino Pimentel, HEC Paris, France: joao.albino-pimentel@hec.edu)

\section{(How) Do Economic Governance and Location Choices (Jointly) Affect Performance?}

Michael Leiblein, Ohio State University

Kiran Awate, Ohio State University

This paper evaluates the performance consequences of decisions regarding the organization and location of economic activity. While theory leveraging transaction cost logic suggests how the alignment between exchange attributes and governance choice affect subsequent performance, this work has not fully considered the implications of international location choice. While the international business literature relates firms' geographical location decisions to performance consequences via foreign direct investment, "offsoring", or multinationalization, it does not always fully consider the implications of alternative governance modes. This paper jointly considers the performance consequences of decisions regarding whether to organize an exchange via market or hierarchy in one country or another. The paper first reports how the selection of governance form (e.g., make or buy) and location (e.g., USA, Taiwan, J apan, Germany, or France) independently affect technological performance after accounting for firm- and transaction-specific features using a propensity score matching approach. The paper then demonstrates how these results are sensitive to institutional context using a novel extension of propensity score matching techniques. The paper concludes by suggesting that a firm's technological performance is contingent upon the alignment between firms' governance decisions and its environment. (For more information, please contact: Kiran Awate, Ohio State University, USA: awate.3@buckeyemail.osu.edu) 
Internationalization-Performance Relationship: The Moderating Roles of State and Foreign Ownership

Gabriel R.G. Benito, BI Norwegian Business School

Asmund Rygh, BI Norwegian Business School

Randi Lunnan, BI Norwegian Business School

The literature on the relationship between internationalization and performance (I/P-relationship) has provided mixed results. Arguing that one reason may be a lack of attention to corporate governance, we consider the role of state and foreign ownership as moderators of the I/P-relationship. Our theoretical arguments build on corporate governance theory as well as resource based and organizational learning theory. Our hypotheses are tested using a unique panel data set covering listed Norwegian companies over the period 2000 to 2010. Overall, the results indicate that state ownership has a positive moderation effect. We suggest that state owned enterprises may benefit more from internationalization due to greater access to government resources and because international competition may compensate for corporate governance deficiencies. The results for foreign ownership are mixed, sometimes negative, but most often insignificant. We suggest that one explanation may be that several benefits of outward internationalization are already realized by inward internationalization through foreign ownership. (For more information, please contact: Asmund Rygh, BI Norwegian Business School, Norway: asmund.rygh@bi.no)

\section{Session: 3.4.7 - Competitive}

\section{Track: 15 - Teaching I B (Special Track)}

\section{B Course Content and Teaching Innovations}

Presented On: June 30, 2015 - 14:30-15:45

Chair: Shirley Daniel, University of Hawaii at Manoa

\section{International Business and Accounting Practices: A Course Overview \\ Victoria Krivogorsky, San Diego state University \\ Mark Ballam, San Diego State University}

The forces of globalization have fundamentally changed corporate business environment. Predominantly, the firms forming the national economies remain deeply rooted in the national business practices, which might work as conflicting forces (barriers) eliciting the cost of entering global markets to escalate. These course materials are designed to address differences in business practices, as potential obstructions to close business relationships between German and American counterparts. The course is designed to offer an innovative education experience by presenting a dynamic curriculum with a strong emphasis on gaining real world knowledge through the various exercises in conducting research related to specific barriers to transatlantic business relationships. The course employs several active learning strategies including interactive lectures, active review sessions, jigsaw discussions and engaging learning technologies. A focus of the course is the completion of a joint project with students from XXX University in USA and the XXXX University in Germany. Binational student teams are directed to conduct interviews with representatives of firms or industry associations in order to obtain a managerial perspective on impact of differences in business practices. (For more information, please contact: Victoria Krivogorsky, San Diego state University, USA: vkrivogo@mail.sdsu.edu)

\section{Autoethnographic Case Study Research for the Multicultural Classroom on a Masters and MBA Level Ebru I pek, Simon Fraser University}

It is a fact that our classrooms are getting more diverse, and more of our students either have a bi- or multicultural background or have accumulated significant international experience. This organically increasing cultural diversity in our classrooms does not diminish the need for comprehensive education in cross-cultural management. In fact traditional methods of teaching cross-cultural management, such as country-specific 
briefings, are no longer sufficient, as most students with international experiences nowadays may possess content knowledge yet cultural process knowledge, that is culture-general knowledge, is not as accessible for most. In order to pass on a deeper understanding of cultures and provide first hand insights from IB researchers, autoethnographic research and case studies are ideal. By affording students an unparalleled understanding of not only the behaviours of individuals from different cultures, but also how they think and feel, autoethnography arguably gives students an insider's cultural perspective of the multicultural mindset which is an important possession for todays leaders. (For more information, please contact: Ebru Ipek, Simon Fraser University, Canada: eipek@sfu.ca)

\section{International Internship for Business Education: An Experiment to Observe Creation of Trust Haruo H. Horaguchi, Hosei University Yasushi Kodama, Hosei University Seiki Yukimoto, Kanagawa University}

This paper reports an international internship in Malaysia in August 2014. During the internship period, participants answered to questionnaire survey through a web site. The data identify leadership among them and show how the internship created trust among participants. The factory managers evaluated the presentations and some of them were appreciated highly. This experiment reveals two facts. Trust is created through common purpose with pressure. Evaluation methods to students need to be evolved. The upper limit of the evaluation mimics the value of proposals in pecuniary terms. (For more information, please contact: Haruo $\mathrm{H}$. Horaguchi, Hosei University, Japan: horaguch@hosei.ac.jp)

\section{Session: 3.4.8 - Competitive \\ Track: 3 - International Human Resource Management and Organizational Behavior}

\section{Overcoming Challenges of Transitions and Change}

Presented On: J une 30, 2015 - 14:30-15:45

Chair: Vijay Edward Pereira, University of Portsmouth

What factors shape organisational culture of MNEs' subsidiaries? Subsidiary acculturation and its antecedents Innan Sasaki, University of Turku Katsuhiko Yoshikawa, London School of Economics and Political Science

The aim of this conceptual paper is to advance our understanding of interaction between organisational culture and other layers of cultures through exploring the organisational and contextual antecedents of the interaction of organizational culture of MNE with other cultures. We propose that structure (embeddedness and ownership), policy (MNE's management orientation), image (prestige and soft power), and cultural regulation (cultural strength and tightness) affect the relative strength of the HQ's and local market's cultural influence that subsidiary faces in its acculturation scenarios. Our study contributes to the IB literature by introducing contingency approach to the study of organisational culture, by integrating multiple literatures, and finally, by advancing discussion on the cross-level interaction of cultures, providing a framework on its antecedents. (For more information, please contact: Innan Sasaki, University of Turku, Finland: innsas@utu.fi)

\section{Social Ties, Social Capital and Managing Transnational Ventures in the Home Country Sarika Pruthi, San J ose State University Mike Wright, Imperial College London}

The transnational linkages of ethnic minority entrepreneurs are extensive and rapidly being recognized as a key element of economic development, yet the way transnational entrepreneurs (TEs) leverage their social ties to 
manage their transnational ventures (TNVs) is little understood. Based on 17 in-depth interviews with eight TEs of Indian origin in the UK and the key personnel that they recruit to manage their TNVs, our findings show that TEs substitute or complement international and local social capital to manage their TNV in India contingent on a) whether they stay in the UK or relocate to live in India, and b) whether they leverage social ties in the UK or develop local ties in India to recruit key personnel to connect with India for managing their transnational activities. We discuss the implications of these findings for theory and practice. (For more information, please contact: Sarika Pruthi, San Jose State University, USA: sarika.pruthi@sjsu.edu)

\section{Repatriation Outcomes Affecting Corporate ROI: A Critical Assessment and Agenda for Future Research Anika Breitenmoser, University of Hamburg \\ Benjamin Bader, Leuphana University of Lüneburg}

Over the past three decades, scholarly interest in the topic of repatriation has increased considerably. In this regard, research has addressed various repatriation outcomes which can have an effect on the eventual corporate return on investment (cROI) of international assignments. In order to further enhance the strategic integration of repatriation into the expatriate management process, as well as to pave the way for future research on this important topic, this study reviews the current state of literature on CROI-affecting repatriation outcomes. By examining 51 articles, we classify variables according to their cost and benefit potential for the assigning firm. We reveal how repatriation outcomes can affect $\mathrm{CROI}$ and outline potential shortcomings in existing research, to then deduce an agenda for future research in this area. (For more information, please contact: Anika Breitenmoser, University of Hamburg, Germany: anika.breitenmoser@uni-hamburg.de)

\section{Economic Distance, Local Managers' Competence, and Localization of Foreign Subsidiaries Naoki Ando, Hosei University}

Staffing of a foreign subsidiary changes over time depending on organizational and environmental factors. However, it has been treated as a static construct in previous studies. Shedding light on an evolutionary nature of foreign subsidiary staffing, this study addresses the way in which a change in subsidiary staffing affects subsidiary performance. To this end, it focuses on localization in terms of human resources, which is a change in subsidiary staffing toward reliance on host country nationals. Assuming a nonlinear relationship between localization and subsidiary performance, this study explores moderators that interact with localization. The panel dataset consisting of 8,024 observations of foreign subsidiaries owned by multinational corporations is analyzed to test hypotheses. This study demonstrates that the relationship between localization and subsidiary performance is positively moderated by the competence of local managers. It also finds that the relationship between localization and subsidiary performance is negatively moderated by difference in economic development between the home country and the economically less developed host country. (For more information, please contact: Naoki Ando, Hosei University, Japan: nando@hosei.ac.jp)

\section{Session: 3.4.9 - Special Session}

\section{Special Session of AJ BS Best Papers}

Presented On: June 30, 2015 - 14:30-15:45

Chair: Carol Reade, San J ose State University

The Association of J apanese Business Studies (AJ BS) travels with AlB and meets on the day before the AlB conference. This session includes the best papers presented at this year's AJ BS conference, studying the J apanese business system and its economic, social and cultural environment. 
Profitability and Competitiveness of U.S. and Japanese Defense Contractors: Implications for IB Research

Tanya Andrea Peacock, Army-Baylor University

Kiyohiko Ito, University of Hawaii at Manoa

Kazuhiro Asakawa, Keio University

We study the competitive advantage of firms that conduct business with their home country governments. The advantage exists in terms of government sales as well as the nongovernmental sales sector. By estimating the implicit return on military sales for defense contractors, we found context-specific competitive advantages gained from the home government sales segment. Large U.S. defense contractors earn profits from military sales, while large J apanese defense contractors do not. Both U.S. and J apanese firms appear to leverage competitive advantage by conducting business with their home governments. J apanese firms appear to value the home government relationship for intangible benefits. (For more information, please contact: Tanya Andrea Peacock, Army-Baylor University, USA: peacock4@hawaii.edu)

\section{Subsidiary Initiative for Transfer of Headquarters' Strategic Practice: A Case Study in Japanese ICT Company's Finnish Subsidiary \\ Naoto Nadayama, University of Otago}

This study aims at contribution to further discussions about a transfer of strategic practice from headquarters to foreign subsidiaries in MNEs. Especially, it specifies a type of transfer as "autonomous adoption" in which subsidiaries autonomously adopt headquarters' strategic practice. Through one and half year's participant observation of the transfer in a Japanese company's Finnish subsidiary, this study shows a description of details and some propositions about the autonomous adoption. (For more information, please contact: Naoto Nadayama, University of Otago, New Zealand: naoto.nadayama@otago.ac.nz)

\section{Roles of Japanese MNEs' Subsidiaries for National Innovation Systems in the South East Asian Countries Chie Iguchi, Keio University}

This paper tries to generate a theoretical framework for better understanding factors affecting the shifting technological level of local suppliers within a National Innovation System (NIS), focusing on Multinational Enterprise (MNE) subsidiaries' governance structures in sectors producing for global markets, structures we refer to as 'Global Value Chains' (GVC). Our intention is to bring arguments concerning the roles of MNE subsidiaries and potential inter-organizational linkages within the GVC to the NIS framework by focusing on the case of the Philippines. One of our hopes is that the theoretical frameworks connecting the NIS and GVC developed in this paper will be helpful in reconsidering the roles of MNE subsidiaries and the effectiveness of each actor in a NIS, and the crafting of effective policy tools related to industrial upgrading, economic development, employment creation, and poverty reduction in host developing countries. (For more information, please contact: Chie Iguchi, Keio University, Japan: iguchi@fbc.keio.ac.jp)

\section{Discrimination and Turnover of Self-Initiated Expatriates in Asia \\ Fabian J intae Froese, University of Goettingen \\ Anna Katharina Hildisch, University of Goettingen \\ Soo Min Toh, University of Toronto}

Despite its potential relevance to the everyday life of expatriates, discrimination is not often directly addressed in expatriate research. Building on relative deprivation theory, our research aims at filling this gap by investigating the detrimental effects of discrimination on expatriates' turnover. Data from 449 self-initiated expatriate academics in South East Asia (J apan, Korea, and Singapore) provide empirical evidence that discrimination is associated with higher turnover intention, which in turn increases the likelihood of actual turnover. Moreover, country, organization, and individual level factors moderate this relationship. Implications for theory and practice are provided. (For more information, please contact: Fabian Jintae Froese, University of Goettingen, Germany: fabian.froese@gmail.com) 
Session: 3.4.10 - Interactive

Track: 13 - I nternational Economics, Finance and Accounting

\section{Development, Institutions and Finance}

Presented On: J une 30, 2015 - 14:30-15:45

Chair: Serkan Akguc, King Abdulaziz University

Understanding the International Trade-Corruption Linkage: Panel Data Findings from 145 countries

Deepraj Mukherjee, Kent State University

Debmalya Mukherjee, University of Akron

While many studies have shown that increased levels of trade openness diminish the level of corruption among trading partners, there are studies that have raised doubts against this claim. In this paper, we explore this relationship in a large sample of 145 countries and find that the effect of trade openness on the level of corruption is not significant. The findings however are not indicative of the undesirability of trade-openness and/or globalization. In fact, we find that greater levels of economic development are associated with lower levels of corruption. Nevertheless, it does call for some precaution and monitoring as trade-openness alone may not be the panacea for controlling corruption and other factors, for example, the importance of building of relevant institutions to curb corruption may need to be considered. Additionally, it is important to identify moderator and mediator variables that may change the strength and direction of the aforementioned relationship. We conclude by explaining the implications of our findings for international business. (For more information, please contact: Debmalya Mukherjee, University of Akron, USA: dmukher@uakron.edu)

\section{The Impact of the Global Financial Crisis on Corporate Valuation in Mexico}

Robert Grosse, American University of Sharjah

Diana Bustani, Sintec

One of the most intractable problems of a financial crisis is valuation of assets during the crisis. We know from the most recent crisis that traditional valuation models lost their ability to explain asset prices in many cases. We demonstrate that during crisis periods, macroeconomic, system wide factors tend to drive firm value in Mexico, whereas in normal times firm-specific factors dominate valuation. This paper values traded Mexican firms before and after, as well as during the 2008-9 global financial crisis, explaining Market Cap and Tobin's Q values. Before and after the financial crisis, traditional factors including firm sales, sales growth, profits, and risk measures contributed significantly to explain firm value. During the financial crisis various risk factors, plus US and Mexico GDP growth, were significant in explaining company valuation, while firm-specific factors were not. This implies that both company managers and long-term investors need to hold their course during a (shortlived) crisis; while short-term investors can look for company valuations to depend on the macroeconomy and government policies such as government bond interest rates. (For more information, please contact: Robert Grosse, American University of Sharjah, United Arab Emirates: rgrosse@aus.edu)

Firm Level Internal Corporate Governance and Foreign Exchange Exposure: New Evidence from an Emerging Market

Ekta Sikarwar, Indian Institute of Management Indore

Ganesh Kumar Nidugala, Indian Institute of Management Indore

We examine the impact of firm-level internal corporate governance on exchange rate exposure using a sample of 651 Indian firms over the period 2001 to 2013. By constructing an aggregate firm level internal corporate governance index based on the ownership structure of firms, we uncover that the strong firm level internal governance, in which the agency costs and monitoring problems are lower, is associated with reduced level of 
exchange rate exposure. The effect is also economically significant with an impact of $8.7 \%$ on exchange rate exposure from a one standard deviation change in the firm level internal governance index. Our evidence also reveals that currency derivative usage is associated with greater reduction in exchange rate exposure for firms that have strong internal governance. These results are robust to alternative sub-periods and to the use of different hedging proxies. The generality of these findings can be extended to other emerging markets where ownership structure serves as a major internal governance mechanism in the absence of external legal protection. (For more information, please contact: Ekta Sikarwar, Indian Institute of Management Indore, India: f10ektas@iimidr.ac.in)

\section{Intellectual Property Rights and Innovation: A Panel Analysis \\ Nikolaos Papageorgiadis, University of Liverpool \\ Abhijit Sharma, University of Bradford}

This paper investigates the relationship between intellectual property rights (IPR) and innovation, for a panel of 48 countries for the period 1998-2011, with particular focus on patent systems. Prior empirical studies mainly focus on the strength of patent regulations but do not consider the enforcement of such laws in practice. In our analysis we employ a new longitudinal index that accounts for the enforcement related component of the patent system together with the index of patent regulatory strength developed by Ginarte and Park (1997) and Park (2008a). The use of two indices allows us to account for two crucial elements of a national patent system, the de jure position relating to book law, as reflected in IPR regulations and the de facto position relating to IPR enforcement. This enables us to more effectively assess the strength of IPR systems, as well as understand the potential effect of IPR systems on innovation. Our study contributes to a more effective understanding of the role of IPR systems on innovation by both making use of an appropriate IPR enforcement index, which has been a hitherto neglected aspect in the literature, and by identifying nonlinearities between IPR and innovation. (For more information, please contact: Nikolaos Papageorgiadis, University of Liverpool, United Kingdom: n.papageorgiadis@liverpool.ac.uk)

\section{IFRS Convergence: Evidence of Accounting Quality \\ Erick Rading Outa, Strathmore Business School}

The purpose of this study is to examine the characteristics of accounting amounts arising from convergence and annual revisions to IFRS/IAS between 2006 and 2011 when there was accelerated changes in standards to close the convergence process in addition to IASB's annual improvements projects. The research design follows extant literature and tests the hypothesis that firms with higher accounting quality exhibit less earnings management, high frequency of large losses and higher association between stock prices and earnings book values. Applying 302 firm year observations for companies quoted in East African Security Exchanges, the multivariate results analyzed using $p$ and $t$ values reported quality improvements contrary to the mixed findings in prior research. The current study offers insights on the impact of convergence and annual revisions and provides empirical evidence that convergence projects and revisions has given rise to quality of accounting .It will be useful to Professional accounting institutes and their global partners (IASB, OECD), regulators, boards and governments given that East Africa is a major jurisdiction for IFRS and its economies have been growing rapidly thus making it an attractive investment destination. (For more information, please contact: Erick Rading Outa, Strathmore Business School, Kenya: otxeri001@gsba.uct.ac.za)

The Impact of Country Risk on Financing FDI in Developing Countries Thomas Lindner, WU Vienna Jakob Muellner, WU Vienna Jonas Puck, WU Vienna

International strategy literature does not fully account for the effect of financing cost on internationalization decisions. We take a trade-off theory perspective on international investments and develop hypotheses on the 
effect of country risk and its anticipated change on the cost of financing greenfield FDI. Using a dataset of 7,087 investments from developed into developing markets, we show that the financial consequences of internationalization into risky countries depend on both home and host country risk characteristics, since companies compete for financial capital globally. Furthermore, expectations of future risk development are shown to play a role in determining post entry financing costs. We test our hypotheses using hierarchical linear modelling and controlling for diversification effects and obtain evidence in support of our theoretic argumentation. (For more information, please contact: Thomas Lindner, WU Vienna, Austria: thomas.lindner@wu.ac.at)

\section{Session: 3.4.11 - I nteractive \\ Track: 12 - The I nstitutional and Political Environment of I B}

\section{Domestic Institutions and Varieties of Capitalism}

Presented On: J une 30, 2015 - 14:30-15:45

Chair: Ivan Montiel, Loyola Marymount University

\section{Chinese Distinct Form of Capitalism}

Rui Torres Oliveira, Manchester Business School

Societies develop differently, and successful societal development can be achieved via a number of strategies. Even typically poor societal arrangements, from a Western theories point of view, can produce positive economic output since ends and means are not necessarily in common worldwide. China is seen as a successful economic grown case and the analysis of how the economic agents are organized is relevant, even as a way to understand its future path. Although the purpose of this study is an economic analysis, the political framework and its path-evolution are indistinguishably entangled. In fact, the analysis of capitalism system can not be dissociated from a multi-disciplinary approach where sociology, politics, economics and even management have a word to say. In this perspective paper we explain how Chinese capitalism is organised and how it is tied to its own history and groups of interests. We argue that Chinese institutions are too present where they should not be and not present enough where they should. Furthermore, we contend that Chinese state control enterprises and private firms will be better prepared to circumvent the institutional voids if they cooperate with international firms. (For more information, please contact: Rui Torres Oliveira, Manchester Business School, United Kingdom: rui.oliveira@postgrad.manchester.ac.uk)

The Erosion of 'Japaneseness' in the Japanese Business System: The Fukushima Accident as Catalyst for International Collaboration

Camilla Nellemann, Rikkyo University

Miguel Matos Torres, University of Aveiro

Japanese firms are known to collaborate extensively among each other in corporate groups. On one hand, corporate grouping in J apan is argued to be a competitive advantage as member firms may draw on a pool of shared resources, notably patient capital. On the other hand, it is claimed to breed insular and inflexible management practices. Meanwhile, a number of J apanese firms have entered into foreign alliances motivated by, e.g. access to capital and know-how. In this paper, we analyze why the diversified manufacturer Mitsubishi Heavy I ndustries chose to join forces with Vestas, a world leader in wind energy solutions, in the wake of the Fukushima nuclear accident. We hereby put forward fresh insights on decision making in a Japanese multinational cooperation confronted with the opportunities and constraints of operating in a global economy. Our study supports the hypothesis developed by management scholars in the 1990s predicting a gradual erosion of 'J apaneseness' in the J apanese business system. Additionally, we help fill a gap in the literature on the state of J apanese business groups. (For more information, please contact: Camilla Nellemann, Rikkyo University, Japan: camillanellemann@gmail.com) 
The Influence of Institutional Development on $R \& D$ Investment, Internationalization and Performance of Emerging Market Firms

Sreevas Sahasranamam, Indian Institute of Management Kozhikode

Saptarshi Purkayastha, Indian Institute of Management Calcutta

Somnath Lahiri, Illinois State University

Institutional development in emerging markets (EMs) and the entry of multinational enterprises spur significant changes to the industry and institutional environment encountered by domestic firms. In this paper, we study how institutional development influences the relationship of (a) R\&D investment and (b) degree of internationalization with financial performance of EM firms. Drawing on institutional theory, we find support for the argument that greater competition in domestic market discourages EM firms from increasing their internationalization activities. However, contrary to the argument that institutional reforms by lowering the fear of expropriation provide greater incentives to the firm to make R\&D investments, we find that institutional developments have a negative moderating effect on the relationship between $R \& D$ investment and financial performance. We tested our hypotheses on a sample of Indian firms using a fixed effect panel model for the time period 1991-2006. The study findings offer important implications for research and practice. (For more information, please contact: Somnath Lahiri, Illinois State University, USA: slahiri@ilstu.edu)

\section{Role of Institutional Context in Diversification-Performance Relationship: A Meta-Analysis Monika Schommer, EBS Business School \\ Amit Karna, Indian Institute of Management Ahmedabad Ansgar Richter, University of Liverpool}

Diversification is a key strategic response to (changes in) the institutional environment in which the firms are operating. In this paper, we analyze both the strength of this relationship and the moderating effects of institutional developments such as antitrust regulations, external capital market development, and shareholder protection on the diversification-performance relationship. We look at the institutional differences across countries, and their development over time. Using a sample of 103 studies published during the 1971-2005 investigating data from 14 different countries, we undertake meta-analytic regression analysis (MARA) and Hedges and Olkin meta-analysis (HOMA) to analyze the inverted U-shaped relationship between diversification and performance, and the role that institutional developments have played in it. We find little support for the role of various institutional developments that have long been thought to have affected diversification and its performance consequences. Interestingly, our analysis suggests that the diversification-performance relationship has shifted over time. The effect size first increases, then decreases, indicating an inverted Ushaped relationship between effect size and time. (For more information, please contact: Amit Karna, Indian Institute of Management Ahmedabad, India: karna@iimahd.ernet.in)

\section{Going Abroad? Deciding Factors for Foreign Banks Investing in Africa \\ Kweku Adams, University of Calgary \\ Yaw A. Debrah, Swansea University}

While sub-Saharan African countries have been able to attract some degree of resource-seeking FDI due to their abundant natural resources, financial FDI inflows have proved to be elusive for the region, in spite of the widespread financial sector adjustment programmes which offer attractive incentive packages for financial MNCs. Literature surrounding the determinants of FDI inflows has mainly focused on manufacturing and real production activity. We analyzed the root causes of the weak administrative and institutional framework in Africa's banking industry, using Ghana as a case in point. Focusing on two financial MNCs as case studies, this paper validates the significance of a thorough qualitative investigation in evaluating the explanations as to why most foreign banks do not invest in sub-Saharan Africa, and the few that do, have relatively insignificant operations. The study also reveals that despite the far reaching reforms, there are several structural constraints and deficiencies placed on financial MNCs which affect the size of the business they can conduct and their 
future investment decisions. One of the major issues prior to the financial sector reforms in Africa was disintegration and the restructuring was not designed to create an attractive location for foreign capital; hence the low financial FDI inflows to Ghana in particular and Africa in general. (For more information, please contact: Kweku Adams, University of Calgary, Canada: kweku.adams@haskayne.ucalgary.ca)

\author{
Institutions, e-Governance and Competitiveness: A Study of Asian Countries \\ Hernan Roxas, Deakin University \\ Doren Chadee, Deakin University \\ Alfred Presbitero, Deakin University
}

Information and communications technology (ICT) has been shown to impact positively on the overall quality of governance on a national scale. In this study, we offer a more nuanced explanation on how ICT in governance contributes to the competitiveness of countries. Drawing on institutional theory, we examine the dynamic effects of changes in the formal institutional environment and the quality of e-Governance on the national competitiveness of selected Asian countries using partial-least square approach to path analysis. Results of our cross-lagged panel analysis of data from three time periods suggest that e-Governance quality is a critical conduit that fully mediates the effects of formal institutions on a country's competitiveness. The research and policy implications of the findings are also discussed. (For more information, please contact: Hernan Roxas, Deakin University, Australia: banjo.roxas@deakin.edu.au)

\title{
Session: 3.4.12 - I nteractive
}

\section{Track: 2 - Entrepreneurship, Micro-Multinationals, and I nternational New Ventures}

\section{Towards a Theory and Practice of Micromultinationals}

Presented On: J une 30, 2015 - 14:30-15:45

Chair: Amanda Bullough, University of Delaware

\author{
The Born Global Phenomenon: Flat World Enablers and Spiky World Levelers \\ Marleen McCormick, Butler University \\ Deepak Somaya, University of Illinois at Urbana-Champaign
}

We propose that the born global phenomenon is associated with two distinct mechanisms that are simultaneously at play. First, new firms are able to considerably reduce the costs of learning about foreign markets and develop knowledge about these markets through "flat world" enablers. The increasing interconnections between countries unleashed by technological and institutional forces have made it possible for firms to acquire even distant knowledge more easily, thus reducing the costs and risks of foreign expansion. Second new firms may take advantage of "spiky world" levelers. We define spiky world levelers as the diverse formal and informal institutional arrangements that entrepreneurs may use to gain a competitive advantage for the firm. The world is spiky and firms located in countries with fewer institutional barriers (better infrastructure and less corruption) may leverage those differences to enter international markets. Using a sample of firms located in 36 countries, we find that new ventures are more likely to internationalize than established firms if they take advantage of flat world enablers and spiky world levelers. (For more information, please contact: Marleen McCormick, Butler University, USA: memccorm@butler.edu)

\section{Antecedents and Organizational Learning in International New Ventures Teemu Kalevi J ohannes Tuomisalo, J yäskylä University}

The international new ventures (INVs) start internationalization straight from their inception due to the different external and internal factors. We focus on the latter one and aim to find the antecedents of INV formation 
before the legal foundation and observe the organizational learning in the very early phase of its internationalization. The preliminary results from an article review is presented in this paper and it can be carefully presented that there is space for a more disciplined research that concerns the antecedents of INV foundation and the organizational learning in the early phase of internationalization. (For more information, please contact: Teemu Kalevi Johannes Tuomisalo, Jyväskylä University, Finland: tekajotu@student.jyu. fi)

\section{Revisiting the Entrepreneurship-Subsidiary Initiative Relationship: A Contingency Model of Cognition Ming-Chang Huang, Providence University Hsiang-Lin Cheng, National Chung Cheng University}

This study analyzes the personal effect of subsidiary executives on subsidiary initiative actions. It focuses on the role of subsidiary executive entrepreneurial cognition, including both psychological cognition (Psycho-cognition) and social cognition (Social-cognition). This study tests their joint effects upon subsidiary initiative actions. Empirical evidence was obtained for 185 subsidiaries of Taiwan multinational enterprises (MNEs) operating in China. Data were triangulated by connecting two data sources: a secondary dataset and a survey conducted firsthand. First, the result indicates a positive joint effect of entrepreneurial psycho-cognition and entrepreneurial social cognition (from normative, dependence and attachment signals) upon subsidiary initiative actions. Second, the entrepreneurial cognition bias of social regulatory cognition is found. It weakens the positive impact of entrepreneurial psycho-cognition upon subsidiary initiative actions. Similarly, the social cognition of the credibility signal has a negative and direct impact upon subsidiary initiative actions. (For more information, please contact: Ming-Chang Huang, Providence University, Taiwan: mchuang@pu.edu.tw)

\section{Managerial Ties, Trust and Cooperation in Exporters: The Case of Taiwanese Small Trading Firms Hui Yun Chiu, National Chengchi University Chwo Ming Yu, National Chengchi University}

Based on social capital perspective, this paper investigates the impact of managerial ties (consisting of ties with other firms) and trust on cooperation. By using case study method on three small trading firms from Taiwan, we find that managerial ties particularly related-industry ties have positive effect on forming cooperation with other firms. In addition, we also find that power imbalance moderates the relationship between trust and cooperation. In sum, our findings suggest managerial ties leads to cooperation, however even with level of trust between two parties, to form types of cooperation is determined by power imbalance. (For more information, please contact: Hui Yun Chiu, National Chengchi University, Taiwan: hychiucandy@hotmail.com)

\section{How Do Born Global Firms Overcome Resource Constraints? Niche Marketing Strategies of a Micro-Multinational Brand \\ Sonya H. Wen, Tamkang University \\ Yen Hung Chiu, University of Bath}

How do born global firms overcome resource constraints? To explore such a research inquiry, we compared the internationalization processes and marketing strategies of six competitors in the earphones industry. This multiple case study found that Chord \& Major, a born global firm in Taiwan has leveraged niche-marketing strategies in its internationalization path to overcome its resource constraints. In a contrast to the mainstream business model of OEM adopted by its peer SMEs in emerging economies, Chord \& Major entered the earphones industry by specializing in tonality segments, including classical, jazz, rock, and ballad music lovers. Furthermore, in a contrast to the mainstream global model of B2C adopted by resourceful multinational brands like Beats, Chord \& Major has applied the niche marketing strategies like trade shows to build its B2B network with international partners. Such a micro-multinational brand demonstrates the empirical evidence of strategic fitness between born global firms and niche marketing strategies. (For more information, please contact: Sonya H. Wen, Tamkang University, Taiwan: sonya.wen@gmail.com) 


\section{Session: 3.4.13 - Interactive}

\section{Track: 1 - People and Careers in Cross-Cultural Business}

\section{Cross-Cultural Differences in International Business}

Presented On: J une 30, 2015 - 14:30-15:45

Chair: Charles Edward Stevens, Lehigh University

Peer Behavior, Workplace Happiness and Purchasing Social Responsibility: A Comparative Study between Taiwan and China

Yi-Hui Ho, Chang Jung Christian University

Chieh-Yu Lin, Chang J ung Christian University

The involvement of purchasing function in corporate social responsibility significantly affects purchasing decisions, business reputations and consumer evaluations. While most people tend to consider others' thoughts and opinions to ensure that their decisions will be supported by peers, peer behavior may exert great influence on the attitudes toward purchasing social responsibility for purchasing professionals. Furthermore, people may agree that being happy at work is a positive energy that assists encountering challenges in their lives. Studies on the corporate social responsibility in purchasing have gained much attention; however, very few studies analyze the impact of peer behavior and workplace happiness on purchasing professionals' purchasing social responsibility. Consequently, the study will explore the impact of peer behavior and workplace happiness on purchasing professionals' attitudes to purchasing social responsibility. Purchasing professionals in Taiwan and China are taken as research subjects. Research findings reveal that there are significant associations between peer behavior and workplace happiness and purchasing professionals' attitudes to purchasing social responsibility. The difference in purchasing social responsibility attitude between Taiwanese and Chinese purchasing professionals is significant. (For more information, please contact: Chieh-Yu Lin, Chang Jung Christian University, Taiwan: jylin@mail.cjcu.edu.tw)

Guanxi, Renqing and J ustice Theory in the Great China Area: Conceptual Frameworks and Empirical Evidences Lung-Tan Lu, Fo Guang University

This paper aims to develop two conceptual frameworks, on the basis of Hwang's (1987) model and the J ustice Theory. The model suggested that businessperson may seek or reinforce Guanxi (tie) by three types of social ties, instrumental, mixed, and expressive. Therefore, resource allocators in Chinese society may distribute their powers to others with different social ties. Moreover, this study examines the relationships among the three types of social ties and Renqing giving and returning. Data collected form questionnaires completed by 190 J apanese expatriate and 167 Taiwanese managers in Taiwan. The findings are as follows: (1) businessperson with instrumental Guanxi will apply the equity rule, which excludes Renqing giving and returning; (2) businessperson with mixed Guanxi will apply the equality rule, which will request moderate Renqing giving and returning; (3) businessperson with expressive Guanxi will apply the need rule, which will request strong Renqing giving and returning. Managerial implications and further research directions are discussed. (For more information, please contact: Lung-Tan Lu, Fo Guang University, Taiwan: It/u@mail.fgu.edu.tw)

\section{The Role of Sequences of Interactions on Cross-Cultural Inter-Team and Intra-Team Negotiations}

Cheryl Dowie, Manchester Business School

Claudio De Mattos, Manchester Business School

Oscar De Bruijn, Manchester Business School

Many studies show the lack of cross-cultural competence to be a reason for international business failures. The practice of effective communication will help reduce conflict and potentially increase the number of 
opportunities for international business. Studies in social sciences have not fully scrutinised the dynamics of face-to-face negotiations, in particular the behavioural dynamics that may generate negative effects on such interactions. The Bales IPA model (1950) is adapted in this study to analyse behavioural patterns in group negotiations. This working paper aims at identifying sequences of interaction that determine the relative impact on the negotiation process, discusses the relevant frameworks in order to develop a conceptual model primarily based on the literature and suggests suitable research methods. In due course, this study will enable the researcher to unveil implications and provide recommendations for negotiations across cultures in general and for cross-border negotiations in particular. (For more information, please contact: Cheryl Dowie, Manchester Business School, United Kingdom: cheryl.dowie@postgrad.mbs.ac.uk)

\section{Taking a Hit To Save The World: Employee Participation in Social Initiatives \\ Christiane Bode, INSEAD \\ J asjit Singh, INSEAD}

We examine the extent to which strategically managing employee involvement in corporate social responsibility activities (CSR) of the firm can serve as a tool to make such efforts more viable. Specifically, we argue that employee preferences for hybrid careers provide firms with a lever to share the cost of engaging in CSR activities. Our empirical analysis relies on proprietary data from a global management consulting firm. We find that women, young individuals and those without a business education are more likely to be recruited into the firm by the presence of a CSR initiative. However, these demographic characteristics are only weak predictors of actual participation in the initiative, which involves taking a salary cut. We use survey data to analyze the unexplained heterogeneity in employee willingness to accept this personal financial sacrifice in order to participate. We are able to explain these differences in terms of two normally unobserved variables: the extent to which employees feel that their participation would benefit their own careers versus society at large. While career considerations dominate in explaining recruitment to the firm, societal considerations trump career considerations for actual application and participation in the initiative. (For more information, please contact: Jasjit Singh, INSEAD, Singapore: jasjit.singh@insead.edu)

\section{International Mergers \& Acquisitions: Empirical Evidence for Challenges to Global Talent Management Cordula Barzantny, Toulouse Business School Magali Larquey, Toulouse Business School}

Most companies in various industries are compelled to go through external growth and Mergers and Acquisitions (M\&A) if they want a chance to grow and survive on the World market. The search for critical mass and synergies is often what drives these organizations. However, $70 \%$ of $M \& A$ never attain their initial goal, mainly because of the human factor. It is often believed that synergies are solely dependent on numbers and technology when, in fact, the lack of people synergy is what causes M\&A to fail (Cartwright \& Cooper, 1990). Furthermore, in order to leverage the full potential of a corporation fusion, the human element possesses the particular challenge of talent management along the complete value chain: before an acquisition and/or merger, during the process as well as beyond the finalized merged corporate structure.

The purpose of this study is to discover what key elements are necessary for a successful international M\&A, more particularly when the merging organizations are stemming from different countries and cultural origins and how this relates to international, cross-border and cross-cultural talent management. We conducted an empirical study by observations and interviewing several M\&A experts in various high technology industry settings based in Europe.

(For more information, please contact: Cordula Barzantny, Toulouse Business School, France: c.barzantny@tbseducation. fr) 
Reflecting Embeddedness - The Role of Identification for SIES Organizational Integration

Barbara Agha-Alikhani, University of Passau

Andreas Landes, University of Passau

In our paper we discuss the relevance of self-initiated expatriates' reflection on their embeddedness within organizational contexts. We claim that considering those processes can help organizations to develop strategies in order to attract and retain a skilled international workforce.

By combining embeddedness with organizational identification theory we propose a research agenda in order to gain a broader understanding of self-initiated expatriates' identification processes and their reflection about embeddedness. By doing so, we want to enhance strategies for the human resource management of those individuals, which due to the labor shortages in certain branches is clearly needed.

We build on a qualitative methodology and present a research agenda that we developed based on embeddedness and organizational identification theory. The data collection has yet to follow, however we present our research strategy and already discuss important implications of the study. (For more information, please contact: Barbara Agha-Alikhani, University of Passau, Germany: barbara.agha-alikhani@uni-passau.de)

\section{Session: 3.5.1 - Plenary}

\section{AlB Awards Ceremony and Business Meeting}

Presented On: June 30, 2015 - 16:15-17:45

The session will start with the announcement of the winners for the various conference awards including the Buckley and Casson Dissertation Award, Al B/Temple Best Paper Award, and the Rugman Most Promising Scholar Prize. Then, the business meeting will feature a presentation for the 2016 AlB Meeting in New Orleans and a review of the state of AIB and JIBS.The floor will then be open for questions and comments from the membership. 


\section{NDEX OF PROGRAM CONTRI BUTORS}




\section{NDEX OF PROGRAM CONTRI BUTORS}

\section{A}

Aberg, Susanne; Uppsala University, Sweden (susanne.aberg@fek.uu.se): 3.2.8

Abreu, Monica Cavalcanti Sa de; Federal University of Ceara, Brazil (mabreu.ufc@gmail.com): 3.3.11

Adams, Kweku ; University of Calgary, Canada (kweku.adams@haskayne.ucalgary.ca): 3.4.11

Adbi, Arzi; INSEAD, Singapore (arzi.adbi@insead.edu): 3.2.7

Ado, Abdoulkadre; Laval University, Canada (abdoulkadre.ado.1@ulaval.ca): 1.5.9

Agarwal, James; University of Calgary, Canada (james.agarwal@haskayne.ucalgary.ca): 3.3.9

Aggarwal, Raj; University of Akron, USA (aggarwa@uakron.edu): 2.4.6

Agha-Alikhani, Barbara; University of Passau, Germany (barbara.agha-alikhani@uni-passau.de): 1.3.8, 3.4.13

Agrawal, Gaurav; ABV-Indian Institute of Information Technology and Management, India

(drgauravagrawal@gmail.com): 2.5.13, 3.3.13

Aguilera, Ruth; Northeastern University, USA (r.aguilera@neu.edu): 0.2.4, 1.4.3, 2.1.5, 2.3.5, 3.4.1

Ahamed, A.F.M J alal; Binus Business School, Indonesia (ajalal@binus.edu): 3.1 .11

Aharoni, Yair; Tel Aviv University, Israel (prof.yairah@gmail.com): 1.05

Ahi, Ali; Lappeenranta University of Technology, Iran (mohamadali.ahi@lut.fi): 2.5.4

Ahsan, Faisal; Indian Institute of Management Lucknow, India (fpm12005@iiml.ac.in): 2.5.4, 3.1.10

Aichhorn, Nathalie; WU Vienna, Austria (nathalie.aichhorn@wu.ac.at): 2.3.9

Ajwani, Raji; Symbiosis Centre for Management, India (raji.ajwani@gmail.com): 3.2.12

Akguc, Serkan; King Abdulaziz University, Saudi Arabia (sakguc@kau.edu.sa): 1.3.13, 3.4.10

Akter, Shaheen; TMCD, ODID, University of Oxford, United Kingdom (shaheen.akter@qeh.ox.ac.uk): 2.5.4

Al Radadi, Ahmed; Florida International University, USA (a_radadi@hotmail.com): 1.1.9

Alaganandam, Padmaja ; PricewaterhouseCoopers Pvt. Ltd̄., India (padmaja.alaganandan@in.pwc.com): 1.4.7

Alas, Ruth; Estonian Business School, Estonia (ruth.alas@ebs.ee): 2.4 .4

Albertoni, Filippo; Politecnico di Milano, Italy (filippo.albertoni@polimi.it): 2.4 .12

Albino Pimentel, J oao; HEC Paris, France (joao.albino-pimentel@hec.edu): 1.1.5, 3.4.6

Alford, Bruce; Louisiana Tech University, USA (balford@latech.edu): 1.5.13

Ali, Salman Siddeeque; Indian Institute of Management Ahmedabad, India (salman@iimahd.ernet.in): 3.2 .5

Ali-Yrkkö, Jyrki; Research Institute of the Finnish Economy, Finland (jyrki.ali-yrkko@etla.fi): 2.5.2

Alon, Ilan; Rollins College, USA (ialon@rollins.edu): 1.5.11

Alshumaimeri, Ahmed A.; King Saud University, Saudi Arabia (alshum@ksu.edu.sa): 2.2.4

Altuntas, Gultekin; Istanbul University, Turkey (altuntas@istanbul.edu.tr): 1.4.11

Ambos, Björn; University of St. Gallen, Switzerland (bjoern.ambos@unisg.ch): 1.5.4, 2.2.3

Amponsah, Christian Tabi; Skyline University, United Arab Emirates (chris_tabi@hotmail.com): 2.2.4

An, Younghoon; Yonsei University, Korea, South (danysville@yonsei.ac.kr): 3.2.11

Anand, J aideep; Ohio State University, USA (anand.18@osu.edu): 1.3.2

Anbumozhi, Venkatachalam; Economic Research Institute for ASEAN and East Asia (ERIA), Indonesia

(v.anbumozhi@eria.org): 1.1.2

Anderson, John; University of Northern Iowa, USA (john.r.anderson@uni.edu): 3.4 .5

Ando, Naoki; Hosei University, J apan (nando@hosei.ac.jp): 1.1.12, 3.4.8

Andre, Thomas; Ecole Polytechnique, France (thomas.andre@polytechnique.edu): 1.1.12, 2.4.5, 3.3.11

Andrews, Lynda; Queensland University of Technology, Australia (I.andrews@qut.edu.au): 2.4.13

Angulo-Ruiz, Fernando; MacEwan University, Canada (fernando.anguloruiz@macewan.ca): 1.4.11

Annavarjula, Madan; Bryant University, USA (mannavar@bryant.edu): 2.5.12

Antolin-Lopez, Raquel; University of Almeria, Spain (ral252@ual.es): 2.4.5

Arif, Azam ; Universiti Utara, Malaysia, Malaysia (azam_arif@uum.edu.my): 2.5.11

Arora, Soma ; Institute of Management Technology, India (sarora98@hotmail.com): 2.2.11, 3.1.6

Arvate, Paulo Roberto; FGV-EAESP, Brazil (paulo.arvate@fgv.br): 3.1.10

Asakawa, Kazuhiro; Keio University, Japan (asakawa@kbs.keio.ac.jp): 0.2.4, 0.5.6, 2.3.2, 3.1.4, 3.2.2, 3.4.9

Ascani, Andrea; London School of Economics, United Kingdom (a.ascani@lse.ac.uk): 1.5.3

Ashok, Revathy; I ris Consulting, India (revathy@gmail.com): 1.4.7

Asmussen, Christian; Copenhagen Business School, Denmark (cga.smg@cbs.dk): 2.4 .12 
Aulakh, Preet; York University, Canada (paulakh@schulich.yorku.ca): 0.3.5, 0.4.2, 1.3.1, 3.4.2

Ault, J oshua K; University of Victoria, Canada (jault@uvic.ca): 1.4.11

Autio, Erkko ; Imperial College, United Kingdom (erkko.autio@imperial.ac.uk): 2.1.1

Aveline, Carlos Eduardo; Fundacao Getulio Vargas, Brazil (cbdk8594@hotmail.com): 3.4 .5

Awasthi, Kshitij; Indian Institute of Management Bangalore, India (kshitijawasthi@yahoo.co.in): 2.2.10

Awasthy, Richa; International Management Institute, I ndia (richa@imi.edu): 2.3.10

Awate, Kiran; Ohio State University, USA (awate.3@buckeyemail.osu.edu): 2.1.12, 3.4.6

Awate, Snehal Suyash; Indian School of Business, India (snehal_awate@isb.edu): 2.1.3, 2.4.1, 2.5.2, 3.3.3

Ayyagari, Meghana; George Washington University, USA (ayyagari@gwu.edu): 1.1 .5

B

Baack, Daniel; University of Denver, USA (dbaack@du.edu): 2.2.3, 3.4.3

Bader, Benjamin; Leuphana University of Lüneburg, Germany (benjamin.bader@leuphana.de): 1.1.9, 1.3.12, 3.4.8

Baik, Youjin; Yonsei University, Korea, South (youjin.baik@gmail.com): 3.3.11

Bailey, Anastasia Veronica; Ohio State University, USA (bailey.833@fisher.osu.edu): 2.2.12

Balachandran, Sarath; University of Pennsylvania, USA (sarath.bc87@gmail.com): 1.5 .9

Balasubramanian, Bala N; Indian Institute of Management Ahmedabad, India (bala4391@gmail.com): 2.2.10

Baldauf, Artur; University of Bern, Switzerland (baldauf@imu.unibe.ch): 3.2 .4

Ballam, Mark; San Diego State University, USA (mballam@sdsu.edu): 3.4.7

Banai, Moshe; City University of New York, USA (moshe.banai@baruch.cuny.edu): 2.4 .7

Bandeira-de-Mello, Rodrigo; FGV-EAESP, Brazil (rodrigo.bandeira.demello@fgv.br): 2.1.9

Bapuji, Hari; University of Manitoba, Canada (hari.bapuji@umanitoba.ca): 2.4.5

Barnard, Helena; GI BS, University of Pretoria, South Africa (barnardh@gibs.co.za): 1.1.7, 1.3.5, 2.4.12, 3.2.6

Baronchelli, Gianpaolo; University of Bergamo, Italy (gianpaolo.baronchelli@unibg.it): 2.4.13, 2.5 .4

Bartnik, Roman; University of Duisburg-Essen, Germany (roman.bartnik@uni-due.de): 1.1.11

Barua, Samir K; Indian Institute of Management Ahmedabad, India (skbarua@iimahd.ernet.in): 2.2.10

Barulina, Maria; University of Texas at EI Paso, USA (mbarulina@utep.edu): 2.4 .6

Barzantny, Cordula; Toulouse Business School, France (c.barzantny@tbs-education.fr): 3.4.13

Barzotto, Mariachiara; Ca' Foscari University Venice, Italy (mariachiara.barzotto@unive.it): 2.1.10

Basant, Rakesh; Indian Institute of Management Ahmedabad, India (rakesh@iimahd.ernet.in): 2.3.13

Baskar, S; Amagi Media Labs Pvt. Ltd., India (baskar@amagi.com): 1.5.2

Basuil, Dynah A; University of Auckland, New Zealand (d.basuil@auckland.ac.nz): 1.3.9, 1.5.10

Batas, Spiros; University of Northampton - University of Edinburgh, United Kingdom

(spiros.batas@northampton.ac.uk): 1.1.4

Bathula, Hanoku ; University of Auckland, New Zealand (h.bathula@auckland.ac.nz): 1.1.8, 1.3.10

Batra, Safal; IMT Ghaziabad, India (safalb@iimahd.ernet.in): 3.2.4

Bausch, Andreas ; J ustus Liebig University Giessen, Germany (andreas.bausch@wirtschaft.uni-giessen.de):

$1.4 .11,2.3 .8$

Beamish, Paul W.; Western University, Canada (pbeamish@ivey.uwo.ca): 2.2.1, 2.3.5, 3.05, 3.2.5, 3.3.6

Bebenroth, Ralf; Kobe University, Japan (rbeben@rieb.kobe-u.ac.jp): 2.4.10, 3.1.11

Becker, Bettina; Aston Business School, United Kingdom (b. becker@aston.ac.uk): 1.5.3

Belderbos, Rene; KU Leuven, Belgium (rene. belderbos@kuleuven.be): 0.3.5, 1.1.3

Beldona, Sri ; University of Dallas, USA (sbeldona@udallas.edu): 2.5.11, 3.1.11

Benito, Gabriel R.G. ; BI Norwegian Business School, Norway (gabriel.r.g.benito@bi.no): 0.3.5, 3.3.10, 3.4.6

Berg, David M.; Hamline University, USA (dberg06@hamline.edu): 1.3.7

Berning, Sue Claire; Friedrich-Alexander University of Erlangen-Nuremberg, Germany

(sue.claire.berning@fau.de): 2.5 .12

Berry, Heather; George Washington University, USA (berryh@gwu.edu): 3.2.7

Beveridge, Alim; Nottingham University Business School China, China (alim.beveridge@nottingham.edu.cn):

1.3.12

Bhadra, Dhiman; Indian Institute of Management Ahmedabad, India (dhiman@iimahd.ernet.in): 2.5.3

Bhadra, Santanu; Indian Institute of Management Calcutta, India (santanub13@email.iimcal.ac.in): 2.5.13

Bhaduri, Abhijit; Wipro Ltd., India (abhijit.bhaduri@wipro.com): 2.1.2 
Bhagavatula, Suresh; Indian Institute of Management Bangalore, India (sureshbh@iimb.ernet.in): 1.5.2, 2.1.1 Bhasin, Balbir; University of Arkansas at Fort Smith, USA (balbir.bhasin@uafs.edu): 2.3.12 Bhaskaran, R.; Bosch Ltd., India (baskaran.r@in.bosch.com): 1.3.2

Bhatia, Jaspreet N. ; University of New South Wales, Australia (nitasha90@hotmail.com): 1.1 .4 Bi, Kexin; Harbin University of Science and Technology, China (bikx@hrbust.edu.cn): 2.4 .3 Bianchi, Constanza ; Universidad Adolfo Ibanez, Chile (constanza.bianchi@uai.cl): 2.4.13 Bijmolt, Tammo H.A.; University of Groningen, Netherlands (t.h.a.bijmolt@rug.nl): 2.3.4 Binedell, Nick; GI BS Business School, South Africa (binedelln@gibs.co.za): 0.4.2, 0.7.1, 2.5.1

Bird, Allan; Northeastern University, USA (a.bird@neu.edu): 1.1.6, 1.3.7

Bjorkman, Ingmar; Aalto University , Finland (ingmar.bjorkman@aalto.fi): 3.3.12

Blankenburg Holm, Desiree; Uppsala University, Sweden (desiree.holm@fek.uu.se): 2.1.12

Blomkvist, Katarina ; Uppsala University, Sweden (katarina.blomkvist@fek.uu.se): 2.3.11

Bode, Christiane; INSEAD, Singapore (christiane.bode@insead.edu): 3.3.12, 3.4.13

Boehe, Dirk Michael; University of Adelaide, Australia (dirk.boehe@adelaide.edu.au): 1.5 .5

Boglarsky, Cheryl; Human Synergistics International, USA (cab@humansynergistics.com): 2.5.11

Bordia, Sarbari; Australian National University, Australia (sarbari.bordia@anu.edu.au): 1.5.6

Borini, Felipe ; ESPM, Brazil (fborini@espm.br): 2.2.12, 2.3.13, 3.3.10

Boulanger, Michele; Rollins College, USA (mboulanger@rollins.edu): 1.5.11

Bouncken, Ricarda B; University of Bayreuth, Germany (bouncken@uni-bayreuth.de): 1.3.10

Boussebaa, Mehdi; University of Bath, United Kingdom (m_boussebaa@hotmail.com): 1.5.12

Boyacigiller, Nakiye Avdan ; Sabanci University, Turkey (nakiye@sabanciuniv.edu): 0.4.2, 0.7.1, 2.1.4, 2.2.4, 2.5.1

Bozionelos, Nikos; Audencia Nantes Ecole de Management, France (nbozionelos@audencia.com): 1.4.4

Brahmasrene, Tantatape; Purdue University North Central, USA (tapeb@pnc.edu): 2.3.12

Brandl, Kristin ; University of Reading, United Kingdom (kristin.brandl@henley.ac.uk): 1.3.11, 1.4.5, 1.5.5,

2.2.6, 2.4.11

Brannen, Mary Yoko; University of Victoria, Canada (maryyoko@uvic.ca): 1.3.8, 1.5.6

Branzei, Oana; I vey Business School Western University, Canada (obranzei@ivey.uwo.ca): 3.4 .2

Breitenmoser, Anika; University of Hamburg, Germany (anika.breitenmoser@uni-hamburg.de): 3.4 .8

Brenes, Esteban; I NCAE, Costa Rica (esteban.brenes@incae.edu): 1.5.7

Brenner, Barbara; Danube University Krems, Austria (barbara.brenner@donau-uni.ac.at): 2.3.11, 3.2.12

Brewster, Chris; ISCTE Business School, Portugal (c.j.brewster@henley.ac.uk): 1.3 .8

Brown, Cole; Indian Professional Research Project, India (indiacole@icloud.com): 1.5.13

Bryla, Pawel; University of Lodz, Poland (pbryla@uni.lodz.pl): 3.1.11

Bu, Juan; University of Miami, USA (j.bu@umiami.edu): 2.4.11, 3.2.4

Buckley, Peter; University of Leeds, United Kingdom (p.j.buckley@lubs.leeds.ac.uk): 1.4.8, 2.1.1, 3.2.1, 3.3.3

Bueechl, J oerg; Tuebingen University, Germany (joerg.bueechl@uni-tuebingen.de): 3.4 .3

Bullough, Amanda; University of Delaware, USA (bullough@udel.edu): 2.1.4, 3.4.12

Bunz, Thorsten; J ustus Liebig University Giessen, Germany (thorsten.bunz@wirtschaft.uni-giessen.de): 1.4.11

Burger, Martijn ; Erasmus University Rotterdam, Netherlands (mburger@ese.eur.nl): 1.5.7, 3.1.10

Bustani, Diana; Sintec, Mexico (diana.bustani@sintec.com): 3.4.10

C

Cai, Yingdan; University of Groningen, Netherlands (y.cai@rug.nl): 2.3.7

Caldeira, Carlos Afonso; Insper Institute of Education and Research/Getulio Vargas Foudation, Brazil (cac252@yahoo.com): 2.1 .9

Calixto, Cyntia ; Fundação Getúlio Vargas, Brazil (cycalixto@gmail.com): 2.1.11, 3.1.6

Canela, Renata; Universidade Nove de Julho, Brazil (wausma@waus.com.br): 3.2 .6

Cano-Kollmann, Marcelo F; Temple University, USA (mck@temple.edu): 1.4.5, 2.1.9, 2.2.7, 2.5 .2

Cantwell, John; Rutgers University, USA (cantwell@business.rutgers.edu): 0.1.92, 0.71.1, 1.1.10, 2.1.3, 2.4.12, 3.3.1

Cao, Yangfeng; Copenhagen Business School, Denmark (fc.int@cbs.dk): 2.5.7

Carr, Chris; University of Edinburgh, United Kingdom (chris.carr@ed.ac.uk): 2.4.7

Carvalho, Sergio W.; Dalhousie University, Canada (scarvalho@dal.ca): 1.5.13 
Caskey, D'Arcy; Feng Chia University, Taiwan (dcaskey@mail.fcu.edu.tw): 1.5.10

Castelnuovo, Sara; Clifford Chance Pte Ltd, Singapore (srcastelnuovo@gmail.com): 2.4.13

Castro, Alejandro M. Fernández; CESUGA, Spain (afernandez@cesuga.com): 1.5.7, 2.3.7

Castro, Andres Mauricio; Universidad del Rosario, Colombia (andres.castro@urosario.edu.co): 1.1.8

Castro Martins, Henrique; UFRGS, Brazil (hcm@ufrgs.br): 2.2.10

Casulli, Lucrezia ; University of Strathclyde, United Kingdom (lucrezia.casulli@strath.ac.uk): 1.4.11

Cathro, Virginia; University of Otago, New Zealand (virginia.cathro@otago.ac.nz): 1.3.7, 2.1.2

Caviezel, Valeria; University of Bergamo, Italy (valeria.caviezel@unibg.it): 2.4.13

Celo, Sokol; Suffolk University, USA (scelo@suffolk.edu): 1.1.12, 3.3.9

Cerdin, Jean-Luc; ESSEC Business School, France (cerdin@essec.edu): 1.1.13, 1.3.8

Cerne, Miha; University of Ljubljana, Slovenia (miha.cerne@ef.uni-lj.si): 3.2.13

Chabowski, Brian R.; University of Tulsa, USA (brian-chabowski@utulsa.edu): 2.3.4, 2.4.8

Chacar, Aya; Florida International University, USA (chacara@fiu.edu): 0.2.4, 1.1.12, 1.5.4, 2.3.10

Chadee, Doren; Deakin University, Australia (chadee@deakin.edu.au): 2.2.4, 2.5.11, 3.4.11

Chakrabarti, Abhirup; Queen's School of Business, Canada (abhirup.chakrabarti@queensu.ca): 2.1 .10

Chakrabarti, Amit Baran; Indian Institute of Management Calcutta, India (amitbc11@email.iimcal.ac.in): 2.1.10

Chakrabarty, Subrata; University of Texas - El Paso, USA (chakrabarty@gmail.com): 1.4.11

Chakraborty, Indrani; Institute of Development Studies Kolkata, India (indrani.c61@gmail.com): 3.3.6

Chakravarty, Dwarkaprasad; I vey Business School, Canada (dchakravarty.phd@ivey.ca): 2.4.10, 3.2.5

Chan, T.S.; Lingnan University, Hong Kong, SAR-PRC (chants@In.edu.hk): 0.5.6, 3.2.2

Chandna, Vallari; University of North Texas, USA (vallari.chandna@unt.edu): 1.4 .9

Chandra, Pankaj; IIMB, India (chandra@iimb.ernet.in): 0.4.2

Chandra, Rajshree; Delhi University, India (rajshreechandra@yahoo.in): 1.4.12

Chandrasekaran, Deepa; University of Texas at San Antonio, USA (deepa.chandrasekaran@utsa.edu): 0.3.5, 2.4.8

Chang, Sea-J in; National University of Singapore, Singapore (schang@nus.edu.sg): 0.2.4, 1.1.1

Chang, Sheng-Hsiung; Tamkang University, Taiwan (shchang@mail.tku.edu.tw): 2.4.13

Chang, Sungyong; Columbia University, USA (sc3339@columbia.edu): 3.1.2

Charlety, Patricia ; ESSEC Business School and THEMA, France (charlety@essec.edu): 3.1.7

Chatterjee, Chirantan; Indian Institute of Management Bangalore, India (chirantan.chatterjee@iimb.ernet.in): 1.5.2, 3.2.7

Chaudhuri, Kaushik; Shiv Nadar University, India (kaushik.chaudhuri@snu.edu.in): 2.5.11

Chen, Homin; National Taiwan University, Taiwan (hominchen@ntu.edu.tw): 1.5.10, 2.1.11

Chen, Liang ; University of Leeds, United Kingdom (bn10lc@leeds.ac.uk): 3.3 .4

Chen, Shih-Fen; I vey Business School, Canada (sfchen@ivey.uwo.ca): 3.1.12

Chen, Stephen; University of Newcastle, Australia (stephen.chen@newcastle.edu.au): 1.3.4

Chen, Taotao; Tsinghua University, China (chentt@sem.tsinghua.edu.cn): 3.4 .5

Chen, Wei-Lun; National Dong Hwa University, Taiwan (m9733030@ems.ndhu.edu.tw): 2.1 .5

Chen, Xiao; Tsinghua University, China (marskuku@gmail.com): 3.4 .5

Chen, Xiaoyun; University of Macau, Macau (xychen@umac.mo): 2.3.7

Chen, Yingyu (Kerri); National Dong Hwa University, Taiwan (kc615486@gmail.com): 3.3.13

Chen, Zhou; University of Hawaii at Manoa, USA (zhouchen@hawaii.edu): 1.1.8

Cheng, Angela Yazhi; Nottingham University Business School China, China (angelacheng90@gmail.com): 1.3.12

Cheng, Hsiang-Lin; National Chung Cheng University, Taiwan (hlcheng@ccu.edu.tw): 1.1.10, 3.3.2, 3.4.12

Chetty, Sylvie K.; University of Otago, New Zealand (sylvie.chetty@otago.ac.nz): 1.5.12, 3.4.4

Chhajer, Raina; Indian Institute of Management Udaipur, India (raina.chhajer@iimu.ac.in): 1.1 .6

Chi, Tailan; University of Kansas, USA (chi@ku.edu): 1.05, 3.4.1

Chiang, Yi Fang; Feng Chia University, Taiwan (yfchiang@fcuoa.fcu.edu.tw): 1.5.13

Chiao, Yu-Ching ; National Chung Hsing University, Taiwan (chiaoy@nchu.edu.tw): 2.5.10

Child, J ohn; University of Birmingham, United Kingdom (j.child@bham.ac.uk): 2.2.12

Chin, Tachia; Guangdong University of Foreign Studies, China (tachia1231@yahoo.com.sg): 3.1.11

Chiou, Andy; SUNY Farmingdale, USA (chioua@farmingdale.edu): 1.5.6

Chittoor, Raveendra; Indian School of Business, India (raveendra_chittoor@isb.edu): 0.2.4, 1.3.1, 1.4.10, 3.4.2

Chiu, Chih-Fang; National Taiwan University, Taiwan (juju0802@gmail.com): 2.3.10, 2.5.12 
Chiu, Hong-J en C.; National Taiwan University, Taiwan (hongjen@ntu.edu.tw): 2.5.12, 3.3.3

Chiu, Hui Yun; National Chengchi University, Taiwan (hychiucandy@hotmail.com): 3.4 .12

Chiu, Ya-Ping ; Chung Yuan Christian University, Taiwan (yaping@cycu.edu.tw): 3.3 .2

Chiu, Yen Hung; University of Bath, Taiwan (iiaiiaiia3688@hotmail.com): 3.4.12

Chizema, Amon; University of Birmingham, United Kingdom (A.Chizema@bham.ac.uk): 3.1 .5

Chng, Daniel Han Ming; China Europe International Business School, China (dchng@ceibs.edu): 1.4.10, 2.2.10

Cho, Hyuksoo; Chungnam National University, Korea, South (hyuksoo@kmu.ac.kr): 2.2.12

Cho, Se Ho; Rutgers Business School, USA (shcho1981@gmail.com): 2.3.11, 2.4.12

Cho, Theresa S.; Seoul National University, USA (tcho@snu.ac.kr): 2.1.12

Cho, Youngsam; Korea University, Korea, South (zegal82@korea.ac.kr): 2.4.7

Cho, Yunok; Renmin University of China, China (yunok.cho@gmail.com): 3.1.2

Choe, Soonkyoo; Yonsei University, Korea, South (skychoe@yonsei.ac.kr): 3.2.11, 3.3.11

Choi, J ongmoo J ay ; Temple University, USA (jjchoi@temple.edu): 1.3.13, 1.4.6, 3.3.6

Choi, KyuYeong; Florida State University, USA (kc13r@my.fsu.edu): 1.3.11

Chou, Hsin-Hui; National Cheng Kung University, Taiwan (hhchou@mail.ncku.edu.tw): 2.5.12

Chou, Wen-Chiung; Kainan University, Taiwan (joan44@gmail.com): 3.3.13

Christie, Odette ; University of Dallas, USA (ochristie@udallas.edu): 2.5.11

Christmann, Petra; Rutgers University, USA (christmann@business.rutgers.edu): 0.1.92, 1.5.7

Christodoulides, Paul; Cyprus University of Technology, Cyprus (paul.christodoulides@cut.ac.cy): 2.1.8

Chuang, Cheng-Min; National Taiwan University \& Overseas Chinese University, Taiwan

(cmchuang@ntu.edu.tw): 2.3.10

Chung, Sung Hun; Seoul National University, Korea, South (shchung521@gmail.com): 2.1.12

Ciravegna, Luciano; King's College London, United Kingdom (luciano.ciravegna@kcl.ac.uk): 1.1.7, 1.5.7, 2.5.3, 3.4.4

Clapp-Smith, Rachel ; Purdue University Calumet, USA (rachel.smith@purduecal.edu): 1.3.12

Clefberg, Lisa Sofia; Institute for Cognitive Behavior Therapy \& Department of Clinical Neuroscience Karolinska Institute, Sweden (Iclefberg@gmail.com): 1.3.12

Clegg, L. Jeremy; University of Leeds, United Kingdom (I.j.clegg@lubs.leeds.ac.uk): 1.5.12

Coelho, Diego Bonaldo; ESPM, Brazil (diego.coelho@espm.br): 3.3.10

Cohan, Lindsey M; Perishables, USA: 3.2.8

Concer, Ronald de Oliveira; EAESP-FGV, Brazil (ronald.concer@gmail.com): 1.1.12

Conger, Michael; Miami University, USA (congermj@miamioh.edu): 2.3.8

Contractor, Farok J.; Rutgers Business School, USA (farok@andromeda.rutgers.edu): 1.3.1, 2.3.6, 3.1.1

Coro', Giancarlo; Ca' Foscari University Venice, Italy (corog@unive.it): 2.1.10

Cosset, J ean-Claude; HEC Montréal, Canada (jean-claude.cosset@hec.ca): 2.2.7

Crescenzi, Riccardo; London School of Economics, United Kingdom (r.crescenzi@lse.ac.uk): 1.5.3

Cuervo-Cazurra, Alvaro; Northeastern University, USA (a.cuervocazurra@neu.edu): 2.1.11, 2.4.12

Cui, Lin ; Australian National University, Australia (lin.cui@anu.edu.au): 3.1.12

Cuneo, Andres; ESADE Business School, Spain (andres.cuneo@esade.edu): 2.4 .13

Cuypers, Ilya; Singapore Management University, Singapore (ilyacuypers@smu.edu.sg): 2.2.3, 3.3.2

Cuypers, Youtha; University of Hong Kong, Hong Kong, SAR-PRC (ycuypers@hku.hk): 3.3.2

D

D, Karthik; Indian Institute of Management Ahmedabad, India (dkarthik@iimahd.ernet.in): 2.2.10, 2.3.13

Dadhich, Harsh; Indian Institute of Management Ahmedabad, India (harshd@iimahd.ernet.in): 2.3.4

Dai, Li ; Loyola Marymount University, USA (li.dai@Imu.edu): 1.1.3

Dakhli, Mourad; Georgia State University, USA (mdakhli@gsu.edu): 3.2.13

Dalbehera, Shailen Kumar; Indian Institute of Management Bangalore, India (dshailenk@gmail.com): 3.3.10

D'Amelio, Matilde; Politecnico di Milano, Italy (matilde.damelio@polimi.it): 1.3.5

Dandu, Jagadish; Zayed University, United Arab Emirates (jagadish.dandu@zu.ac.ae): 1.3.13, 2.4 .6

Daniel, Shirley; University of Hawaii at Manoa, USA (sdaniel@hawaii.edu): 1.1.8, 3.2.12, 3.4.7

Danis, Wade; University of Victoria, Canada (wdanis@uvic.ca): 3.2.13

d'Arcy, Anne; WU Vienna, Austria (anne.darcy@wu.ac.at): 2.4 .6

Darder, Fidel León; University of Spain, Spain (leonf@uv.es): 1.4.13 
Darendeli, Izzet ; Temple University, USA (tuc71197@temple.edu): 2.4.11

Darwish, Tamer; University of Gloucestershire, United Kingdom (tamer_kd@hotmail.com): 1.4 .4

Das, Shobha; Qatar University, Qatar (shobha.das@qu.edu.qa): 1.4.12

Dasgupta, Pinaki; International Management Institute, India (pinaki@imi.edu): 1.1.10, 1.5.13

Dash, Rupanwita; Indian Institute of Management Bangalore, India (rupanwita.dash10@iimb.ernet.in): 3.1 .2

Dashdeleg, Altan-Uya ; National Dong Hwa University, Mongolia (altanuya@yahoo.com): 2.5.10

Dasi, Angels; University of Valencia, Spain (angels.dasi@uv.es): 2.3.11

Datta, Deepak K; University of Texas at Arlington, USA (ddatta@uta.edu): 1.3.9, 1.5.10, 3.2.12

Dau, Luis Alfonso; Northeastern University, USA (I.dau@neu.edu): 2.4.3, 3.4.1

De Bruijn, Oscar ; Manchester Business School, United Kingdom (o.de-bruijn@mbs.ac.uk): 3.4.13

de la Garza Carranza, María Teresa ; Instituto Tecnológico de Celaya, Mexico (teresa.garza@itcelaya.edu):

\subsubsection{3}

de la Torre, J osé R.; Florida International University, USA (delatorrejoser@gmail.com): 0.4.2, 1.3.7, 2.05

De Mattos, Claudio; Manchester Business School, United Kingdom (claudio.de-mattos@mbs.ac.uk): 2.5.10, 3.4.13

de Vasconcellos, Silvio Luís; Universidade do Vale do Rio dos Sinos, Brazil (silvio@conexo.com.br): 2.1.11

de Vries, Gaaitzen J.; University of Groningen, Netherlands (g.j.de.vries@rug.nl): 2.1 .7

Deboskey, David; San Diego State University, USA (deboskey@mail.sdsu.edu): 1.3.13

Debrah, Yaw A.; Swansea University, United Kingdom (y.a.debrah@swansea.ac.uk): 3.4.11

Decreton, Benoit; WU Vienna, Austria (benoit.decreton@gmail.com): 2.1.12

Dejong, Dale; Florida International University, USA (ddejo002@fiu.edu): 3.3.10

Delios, Andrew; National University of Singapore, Singapore (andrew@nus.edu.sg): 2.3.10

Demirbag, Mehmet; University of Strathclyde, United Kingdom (mehmet.demirbag@strath.ac.uk): 1.3.1, 2.5.6

Deng, Zliang; Renmin University of China, China (dengziliang@rbs.org.cn): 1.4.9, 1.5.8, 3.2.10

Deniz, Nevin; Marmara University, Turkey (deniznevin@yahoo.com): 3.3.12

Devinney, Timothy; University of Leeds, United Kingdom (t.devinney@leeds.ac.uk): 2.3.5, 3.05, 3.2.5

Dhabolkar, Vinay; Catalign Innovation Consulting, India (vinay@catalign.com): 1.3.2

Dhanaraj, Charles ; IMD, Switzerland (charles.dhanaraj@imd.org): 0.4.2, 1.3.1

Dias, Davina; Monash University, Australia (davina.dias@monash.edu): 1.5.11

Dieleman, Marleen; National University of Singapore, Singapore (marleen@nus.edu.sg): 2.5 .6

Dikova, Desislava ; Vienna University of Economics and Business , Austria (desislava.dikova@wu.ac.at): 2.4.9, 3.2.13

Dimitratos, Pavlos; University of Glasgow, United Kingdom (pavlos.dimitratos@glasgow.ac.uk): 3.4 .4

Dimitrova, Mihaela; Oakland University, USA (dimitrova@oakland.edu): 1.1.13

Ding, Daniel Z; City University of Hong Kong, Hong Kong, SAR-PRC (mkding@cityu.edu.au): 1.1.12

Ding, Yuan; CEIBS, Chile (dyuan@ceibs.edu): 3.4 .2

Dixit, Mukund R; Indian Institute of Management Ahmedabad, India (dixit@iimahd.ernet.in): 3.2.4

Doornich, J une Borge; University of Nordland, Norway (june. borge.doornich@uin.no): 2.5.12

Dow, Douglas; University of Melbourne, Australia (d.dow@mbs.edu): 2.1.6, 2.2.3, 2.3.9, 3.4.3

Dowie, Cheryl; Manchester Business School, United Kingdom (cheryl.dowie@postgrad.mbs.ac.uk): 3.4 .13

Doz, Yves; INSEAD, France (yves.doz@insead.edu): 1.5.1, 2.2.1, 2.3.2

Drev, Matej; Georgia Institute of Technology, USA (matej.drev@pubpolicy.gatech.edu): 3.2.7

Driffield, Nigel; Warwick Business School, United Kingdom (n.l.driffield@aston.ac.uk): 1.5.3

Drogendijk, Rian; University of Groningen, Netherlands (rian.drogendijk@rug.nl): 2.1.12

Du, Helen; KU Leuven, Belgium (helendu16@gmail.com): 1.1.3

Du, J ian; Zhejiang Universit, China (dujian@zju.edu.cn): 1.4 .12

Dunlap, Denise; Northeastern University, USA (d.dunlap@neu.edu): 3.1 .4

Dussauge, Pierre; HEC Paris, France (dussauge@hec.fr): 1.1.5

Dutt, Pushan; INSEAD, Singapore (pushan.dutt@insead.edu): 2.4.4

Duvivier, Florence; Universite libre de Bruxelles, Belgium (florence.duvivier@ulb.ac.be): 2.3.11 
E

Eapen, Alex; Australian National University, Australia (alex.eapen@anu.edu.au): 1.5.3

Edelman, Linda F.; Bentley University, USA (ledelman@bentley.edu): 1.3.10, 3.3.2

Eghbali-Zarch, Majid; Memorial University of Newfoundland, Canada (majidez@mun.ca): 1.5.4, 2.1.6

Egri, Carolyn P.; Simon Fraser University, Canada (egri@sfu.edu): 1.4.13, 2.4.4

Ehrnrooth, Mats; Hanken School of Economics, Finland (mats.ehrnrooth@hanken.fi): 3.2.3, 3.3.12

Eiadat, Yousef; University College Dublin, Ireland (yousef.husein@ucd.ie): 1.5.7, 2.3.7

Elahee, Mohammad Niamat; Quinnipiac University, USA (mohammad.elahee@quinnipiac.edu): 3.3.7

Elango, B.; Illinois State University, USA (elango@ilstu.edu): 3.1.12, 3.3.13

Elbanna, Said ; Qatar University, Qatar (said.elbanna@qu.edu.qa): 2.2.12

Elia, Stefano; Politecnico di Milano, Italy (stefano.elia@polimi.it): 2.4.12, 3.1.3

Elias, Rida; American University of Beirut, Lebanon (rida.elias@aub.edu.lb): 2.1.12, 3.3.6

Elliot, Esi; Suffolk University, USA (eaelliot@suffolk.edu): 1.4.13

Elston, Julie Ann ; Oregon State University, USA (julie.elston@osucascades.edu): 1.5.11

Elter, Frank; Telenor Research, Norway (frank.elter@telenor.com): 2.3.11

Ercokses, Busra ; Yapi Kredi Bankasi, Turkey (ercokses@yapikredi.com.tr): 3.3.12

Eriksson, Kent; Stanford University, USA (kenteri@stanford.edu): 3.3.4

Erkus, Ahmet ; Bahcesehir University, Turkey (ahmet.erkus@eas. bahcesehir.edu.tr): 2.4.7

Estrin, Saul; London School of Economics, United Kingdom (s.estrin@lse.ac.uk): 2.5.6

Etemaddar, Mitra ; University of Otago, New Zealand (mitra.etemaddar@otago.ac.nz): 3.4.4

$\mathbf{F}$

Falaster, Christian; Universidade Nove de Julho, Brazil (christianfalaster@gmail.com): 3.2.6

Fan, Di; Deakin University, Australia (david.fan@deakin.edu.au): 2.4.7, 3.1.12, 3.3.4

Fang, Shih-Chieh; National Cheng Kung University, Taiwan (fangsc@mail.ncku.edu.tw): 2.5.12

Fang, Tony; Stockholm University, Sweden (tony.fang@sbs.su.se): 1.4.13, 2.5.7, 3.1.11, 3.4.4

Farah, Bassam E.; American University of Beirut, Lebanon (bassam.farah@aub.edu.lb): 3.3 .6

Farfan , Dalsy Yolima; Universidad del Rosario, Colombia (dalsy.farfan@urosario.edu.co): 1.1.8

Fariborzi, Hadi; University of Calgary, Canada (hadi.fariborzi@ucalgary.ca): 2.3.13

Feinberg, Susan ; Temple University, USA (feinberg@temple.edu): 2.1.7, 3.3.13

Feldman, Maryann; University of North Carolina, USA (maryann.feldman@unc.edu): 1.4.1

Fernandez, Whitney Douglas; San Diego State University, USA (wfernandez@mail.sdsu.edu): 3.1.10

Fey, Carl F.; Nottingham University Business School China, China (carl.fey@nottingham.edu.cn): 1.3.3, 1.4.10

Filatotchev, Igor; WU Vienna, Austria (igor.filatotchev@wu.ac.at): 2.4.6

Fisch, Jan Hendrik; WU Vienna, Austria (jan.fisch@wu.ac.at): 1.5.5

Fleury, Afonso; Universidade de Sao Paulo, Brazil (acfleury@usp.br): 3.2.6, 3.4.5

Fleury, Maria Tereza; Fundacao Getulio Vargas, Brazil (mtereza.fleury@fgv.br): 3.2.6, 3.4.5

Flores, Miguel; Tecnolo' gico de Monterrey, Mexico (miguelflores@itesm.mx): 2.2 .9

Fodchuk, Katy ; Advanced Partnerships, USA (katy@advancedpartnerships.com): 2.2 .8

Ford, J ohn B.; Old Dominion University, USA (ford@olddominion.edu): 2.4.13

Forlani, Emanuele; University of Pavia, Italy (emanuele.forlani@unipv.it): 2.2.6

Fortanier, Fabienne; OECD, France (fabienne.fortanier@oecd.org): 1.5.8

Fortwengel, J ohann; Free University Berlin, Germany (johann.fortwengel@fu-berlin.de): 1.4.8, 2.1.9

Freeman, Susan; University of Adelaide, Australia (susan.freeman@adelaide.edu.au): 2.4.7

Freitas, Ana Rita Pinheiro de; Federal University of Ceara, Brazil (anarita1802@hotmail.com): 3.3.11

Froese, Fabian J intae; University of Goettingen, Germany (fabian.froese@gmail.com): 2.5.11, 3.4.9

Fu, Kun; Imperial College, United Kingdom (k.fu@imperial.ac.uk): 2.5.4

Fu, Qingfen; Tsinghua University, China (fuqf.11@sem.tsinghua.edu.cn): 1.3.11

Fu, Xiaolan; University of Oxford, United Kingdom (xiaolan.fu@qeh.ox.ac.uk): 1.3 .6

Fuchs, Manfred; University of Graz, Austria (manfred.fuchs@uni-graz.at): 3.2.8

Fuller, Douglas B.; Zhejiang University, China (douglas.b.fuller@gmail.com): 1.5.1

Furrer, Olivier; University of Fribourg, Switzerland (olivier.furrer@unifr.ch): 1.4.13, 2.4.4

Furtmueller, Elfi; Innsbruck University, Austria (elfi.furtmueller@amc.or.at): 2.5.10 
G

G. Geleilate, J ose-Mauricio; Florida International University, USA (jgeleila@fiu.edu): 3.1.4

Gadepalli, Sarada Devi; Indian Institute of Management Calcutta, India (saradad11@iimcal.ac.in): 1.4.10, 1.5.4

Galanaki, Eleanna ; Athens University of Economics, Greece (eleanna@aueb.gr): 1.5.11

Galbreath, Jeremy; Curtin Graduate School of Business, Australia (jeremy.galbreath@gsb.curtin.edu.au): 2.1 .5

Gammelgaard, Jens; Copenhagen Business School, Denmark (jg.int@cbs.dk): 2.5.7

Gao, Hongzhi; Victoria University of Wellington, New Zealand (hongzhi.gao@vuw.ac.nz): 3.3.3

Gao, Qian; Anhui University, China (gaoqian0710@sina.com): 2.2.12

Garg, Ritam; Friedrich-Alexander University of Erlangen-Nuremberg, Germany (ritam.garg@fau.de): 3.1.10

Garrido, I van Lapuente; Universidade do Vale do Rio dos Sinos, Brazil (igarrido@unisinos.br): 2.1.11

Garrone, Paola; Politecnico di Milano, Italy (paola.garrone@polimi.it): 1.3.5

Gasiorowski, Laura; Temple University, USA (laura.I.gasiorowski@gmail.com): 3.3.13, 3.4 .6

Gaur, Ajai; Rutgers University, USA (ajai@business.rutgers.edu): 1.3.1, 2.1.6, 3.1.4

Gaur, Sanjaya ; Auckland University of Technology , New Zealand (sgaur@aut.ac.nz): 1.3.10

Ge, Gloria L.; Griffith University, Australia (gloria.ge@griffith.edu.au): 1.1.12

Genc, Omer Faruk; Abdullah Gul University, Turkey (gencomerfaruk@gmail.com): 1.4.6, 1.5.10

Gentile-Luedecke, Simona; Bremen University, Germany (simona.gentile@uni-bremen.de): 2.2.11

Gertz, Geoffrey; University of Oxford, United Kingdom (geoffrey.gertz@politics.ox.ac.uk): 3.4 .2

Ghorbani, Majid; Renmin University of China, Canada (majidghorbani@rbs.org.cn): 1.4.13, 3.3.11

Ghosh, Anindya; IESE, Spain: 2.2.11

Ghosh, Pulak; Indian Institute of Management Bangalore, India (pulak.ghosh@iimb.ernet.in): 1.4 .2

Ghosh, Soumya Kanti; Chief Economic Advisor, State Bank of India, India: 1.4.2

Gibson, Cristina; University of Western Australia, Australia (cristina.gibson@uwa.edu.au): 1.5 .6

Girma, Sourafel; Nottingham University, United Kingdom (sourafel.girma@nottingham.ac.uk): 2.3.7

Giroud, Axèle; UNCTAD, Switzerland (axele.giroud@unctad.org): 2.3.3, 3.2.1

Goerzen, Anthony; Queen's University, Canada (agoerzen@business.queensu.ca): 0.3.5, 1.1.3, 2.2 .9

Gong, Yuanyuan; Nanjing University, China (yy_gong@nju.edu.cn): 1.3.11

Gong, Yundan ; Aston University, United Kingdom (y.gong1@aston.ac.uk): 2.3.7

Gonzalez-Perez, Maria-Alejandra; Universidad EAFIT, Colombia (mgonza40@eafit.edu.co): 2.3.13, 3.2.11

Goodell, J ohn W. ; University of Akron, USA (johngoo@uakron.edu): 2.4.6

Gooderham, Paul N.; Norwegian School of Economics, Norway (paul.gooderham@nhh.no): 2.3.11, 3.4.3

Görg, Holger ; Kiel Institute for the World Economy; University of Kiel; University of Aarhus, Germany

(goerg@economics.uni-kiel.de): 2.3.7

Gorynia, Marian; Poznan University of Economics, Poland (m.gorynia@ue.poznan.pl): 2.3.12

Graf, Nicola; J ohannes Kepler University Linz, Austria (nicola.graf@jku.at): 2.5.10

Grebinevych, Oksana; EMLYON, France (grebinevych@gmail.com): 2.2.6

Grimpe, Christoph; Copenhagen Business School, Denmark (cg.ino@cbs.dk): 3.1 .4

Grogaard, Birgitte; University of Calgary, Canada (bgrogaar@ucalgary.ca): 3.3 .10

Grosse, Robert; American University of Sharjah, United Arab Emirates (rgrosse@aus.edu): 3.05, 3.4.10

Groutsis, Dimitria; University of Sydney, Australia (dimitria.groutsis@sydney.edu.au): 1.1.13

Gruber, Verena; WU Vienna, Austria (verena.gruber@wu.ac.at): 1.5.13

Gubbi, Sathyajit ; University of Groningen, Netherlands (s.r.gubbi@rug.nl): 2.3.7, 3.1.12

Guner, Berrin; Rowan University, USA (guner@rowan.edu): 3.3.12

Gunnigle, Patrick; University of Limerick, Ireland (patrick.gunnigle@ul.ie): 1.1 .3

Gupta, Anupama; National Institute of Fashion Technology, India (anupamaguptanift@gmail.com): 1.1.10

Gupta, Budhaditya; Harvard University, USA (bgupta@hbs.edu): 1.4 .5

Gupta, Deepak ; Amrita School of Business, India (dgshobs@gmail.com): 2.2.11

Gupta, Susan Forquer ; Monmouth University, USA (sgupta@monmouth.edu): 1.3.7, 3.1.6

Guseva, Natalia; National Research University Higher School of Economics, Russia (profguseva@gmail.com):

1.5.11 
Ha, Yoo J ung; University of York, United Kingdom (yoojung.ha@york.ac.uk): 2.4 .2

Haapanen, Mika; University of J yväskylä, Finland (mika.p.haapanen@jyu.fi): 2.4 .4

Hagen, James M.; Hamline University, USA (jhagen05@hamline.edu): 1.3.7

Halaszovich, Tilo; University of Bremen, Germany (tilo.halaszovich@uni-bremen.de): 1.5 .8

Halliday, Cynthia Saldanha; Florida International University, USA (chall061@fiu.edu): 2.5.11

Hamilton III, Robert D.; Temple University, USA (rdhiii@temple.edu): 1.4.5, 2.4.11

Han, Jian; CEIBS, China (jianhan@ceibs.edu): 1.4.10

Hannigan, T.J .; Temple University, USA (tj.hannigan@temple.edu): 2.1.3, 2.3.11, 2.4.1

Hartel, Charmine; University of Queensland, Australia (c.hartel@business.uq.edu.au): 1.1.6

Hatfield, Donald; Virginia Tech University, USA (hatfield@vt.edu): 2.4 .3

Hayashi, Tadashi; Kyoto Sangyo University, Japan (thayashi@cc.kyoto-su.ac.jp): 1.5.12

Hearn, Bruce Allen; University of Sussex, United Kingdom (b.a.hearn@sussex.ac.uk): 1.1.4, 1.3.5, 1.4.12, 2.3.8

Heinberg, Martin; University of Duisburg-Essen, Germany (martin.heinberg@uni-due.de): 1.5.13, 2.3.4

Hennart, J ean-Francois; Tilburg University; University of Pavia; Singapore Management University, Netherlands

(j.f.hennart@uvt.nl): 2.2.6, 2.3.6

Hernandez, Exequiel ; University of Pennsylvania, USA (exequiel@wharton.upenn.edu): 1.5 .9

Hertenstein, Peter; Cambridge University, United Kingdom (ph436@cam.ac.uk): 3.4 .5

Heyden, Mariano; Newcastle Business School , Australia, Australia (mariano.heyden@newcastle.edu.au): 2.1.5

Hildisch, Anna Katharina ; University of Goettingen, Germany (katharina.hildisch@wiwi.uni-goettingen.de):

2.4.10, 3.1.11, 3.4.9

Hildrum, Jarle ; Telenor Research, Norway (jarle.hildrum@telenor.com): 2.3.11

Hirschmann, J ohannes; Trier University, Germany (j.hirschmann@uni-trier.de): 2.4 .8

Hitt, Michael A.; Texas A\&M University, USA (mhitt@mays.tamu.edu): 2.1.6

Ho, Mia Hsiao-Wen; Yuzn Ze University, Taiwan (mia.hohw@saturn.yzu.edu.tw): 1.3.4, 1.5.9

Ho, Yi-Hui; Chang J ung Christian University, Taiwan (vicky@mail.cjcu.edu.tw): 3.4.13

Hofer, Katharina Maria; J ohannes Kepler University Linz, Austria (katharina.hofer@jku.at): 2.5.10

Hofstetter, J oerg Stefan; University of St. Gallen, Switzerland (joerg.hofstetter@unisg.ch): 2.2.9

Holtbruegge, Dirk; Friedrich-Alexander University of Erlangen-Nuremberg, Germany (dirk.holtbruegge@fau.de):

2.5.12, 3.1.10

Hong, Xiangxiang; Nottingham University Business School China, China (francis.hong@nottingham.edu.cn):

3.2.11

Horaguchi, Haruo H.; Hosei University, Japan (horaguch@hosei.ac.jp): 1.1.11, 1.5.3, 3.4.7

Horsburgh, Stuart; Manchester Metropolitan University, United Kingdom (s.horsburgh@mmu.ac.uk): 2.2.8

Hoskisson, Robert; Rice University, USA (robert. hoskisson@rice.edu): 1.3.9

Hou, Sheng-Tsung; Feng Chia University, Taiwan (samuel.hou@gmail.com): 1.1.10, 1.5.13

Howe-Walsh, Liza; University of Portsmouth, United Kingdom (liza.howe-walsh@port.ac.uk): 3.3.12

Hsieh, Kai-Yu; Waseda University, Japan (khsieh@aoni.waseda.jp): 2.2.6

Hsieh, Linda Hsiu-Yun; University of Birmingham, United Kingdom (h.hsieh@bham.ac.uk): 2.2.12

Hu, Dongmei; Shenzhen University; The George Washington University, China (hudm@szu.edu.cn): 2.1.7

Hu, Tianyou; National University of Singapore, Singapore (ht@u.nus.edu): 2.3.10

Huang, Hsiu Ying; Feng Chia University, Taiwan (huanghy@fcu.edu.tw): 1.5.13

Huang, Ming-Chang; Providence University, Taiwan (mchuang@pu.edu.tw): 1.1.10, 3.3.2, 3.4.12

Huang, Victor Zengyu; Zayed University, United Arab Emirates (victor.zuang@zu.ac.ae): 2.2 .4

Huang, Yanjun; China Foreign Affairs University, China (huangyanjun@ruc.edu.cn): 1.3.13

Hui, Kent; Michigan State University, USA (hui@broad.msu.edu): 1.3.11

Hult, G. Tomas M.; Michigan State University, USA (hult@broad.msu.edu): 1.1.2, 2.4.8, 3.3.1

Hundley, Gregory Stephen; Purdue University, USA (ghundley@purdue.edu): 1.4 .5

Hyun, Eunjung; Hitotsubashi University, Japan (ej.hyun@r.hit-u.ac.jp): 2.2.6 
Iammarino, Simona; London School of Economics, United Kingdom (s.iammarino@lse.ac.uk): 1.4.1, 1.5.3

Ianchovichina, Elena ; World Bank, USA (eiachovichina@worldbank.org): 1.5.7

Iguchi, Chie; Keio University, J apan (iguchi@fbc.keio.ac.jp): 3.4 .9

Ikegami, J usuke JJ ; Waseda University, Japan (jjikegami@waseda.jp): 1.3.11

in der Heiden, Peter Thomas; University of Duisburg-Essen, Germany (peter.inderheiden@uni-due.de): 1.3.10,

1.4.12

Inouye, Todd Masaru; University of Hawaii at Manoa, USA (toddi@hawaii.edu): 3.3 .10

Ipek, Ebru ; Simon Fraser University , Canada (eipek@sfu.ca): 1.5.11, 3.4.7

Isakovic, Adrienne A.; Walden University, USA (adrienne.isakovic@waldenu.edu): 2.2.4

Israel, Duraipandian; XLRI-Xavier School of Management, India (disrael@xlri.ac.in): 1.5.13

Ito, J un; Temple University, USA (jun.ito@temple.edu): 3.3 .13

Ito, Kiyohiko; University of Hawaii at Manoa, USA (k.ito@hawaii.edu): 3.4 .9

Ivanova-Gongne, Maria; Abo Akademi University, Finland (mivanova@abo.fi): 1.4.13

Iyer, Rajesh; Bradley University, USA (riyer@bradley.edu): 1.5.13

J

Jadhav, Aditya Mohan; T. A. Pai Management Institute, India (adityajadhav@tapmi.edu.in): 2.5.13

Jahanbakht, Mohammad; Carnegie Mellon University, Canada (mjahanbakht@cmu.edu): 1.3.5

Jain, Naveen K.; University of Akron, USA (njain@uakron.edu): 2.4.11, 2.5.3

Jain, Neera; Management Development Institute, India (njain@mdi.ac.in): 1.1 .9

Jaklic, Andreja; University of Ljubljana, Slovenia (andreja.jaklic@fdv.uni-lj.si): 3.2.13

J alan, Akanksha ; Indian Institute of Management Bangalore, India (akanksha.jalan10@iimb.ernet.in): 2.5.13

J ames, Barclay Edward; Universidad San Francisco de Quito School of Business, Ecuador

(bjames@usfq.edu.ec): 2.2.11, 3.3.10

James, Gareth M.; University of Southern California, USA (gareth@usc.edu): 2.4 .8

J andhyala, Srividya; ESSEC Business School, Singapore (srividya.jandhyala@essec.edu): 2.5.6, 3.4.2

J anssen, Frank; Université Catholique de Louvain, Belgium (frank.janssen@uclouvain.be): 2.1.8

J asovska, Pavlina; University of South Australia, Australia (pavlina.jasovska@mymail.unisa.edu.au): 1.4.10,

2.1.10

Jaura, Manya; Copenhagen Business School, Denmark (mj.smg@cbs.dk): 1.3.11

Jayanti, Rama Kumari; Cleveland State University, USA (r.jayanti@csuohio.edu): 3.2.12

J ean, Ruey-J er "Bryan"; National Chengchi University, Taiwan (bryan@nccu.edu.tw): 1.3.11, 1.5.8

J ensen, Karina R.; NEOMA Business School, France (karina.jensen@neoma-bs.fr): 1.3.6, 1.4.13, 1.5.11

J ensen, Kent W.; University of Southern Denmark, Denmark (kwj@sam.sdu.dk): 1.4.11

J ensen, Peter D. Oerberg; Copenhagen Business School, Denmark (poe.smg@cbs.dk): 1.3.11

J eong, Hyoju; Korea University, Korea, South (jhjryan@hotmail.com): 2.1.5

J eong, Yujin; American University, USA (yjeong@american.edu): 3.1.7

J ha, Suchita ; Symbiosis Institute of International Business, India (suchita.jha@siib.ac.in): 3.1 .6

J iang, Crystal X; Bryant University, USA (cjiang1@bryant.edu): 2.5.12

J iang, Wanxing; Hong Kong Baptist University, Hong Kong, SAR-PRC (wanxing.jiang@gmail.com): 1.4.13, 2.3.9

J iang, Yanbin; Zhejiang University, China (yanbinjiang@126.com): 2.5.4

J imenez, Alfredo; University of Burgos, Spain (ajimenez@ubu.es): 3.4.3

J oardar, Arpita; Clark University, USA (ajoardar@clarku.edu): 1.1.13, 3.3.8

J oh, Gun-Ho; San Diego State University, USA (gun.joh@sdsu.edu): 1.3.13

J ones, Marian V.; University of Glasgow, United Kingdom (marian.v.jones@glasgow.ac.uk): 3.4 .4

J onge, Alice de; Monash University, Australia (alice.dejonge@monash.edu): 1.4.7, 2.2.4, 2.5.12, 3.3.10

J ose, P. D.; Indian Institute of Management Bangalore, India (jose@iimb.ernet.in): 1.1.2

J oseph, Thomas; Indian Institute of Management Udaipur, India (thomas.joseph@iimu.ac.in): 1.1.6

J oshi, Rakesh Mohan; Indian Institute of Foreign Trade, India (rmjoshi@iift.ac.in): 2.5.10

J yotishi, Amalendu; Amrita University, India (amalendu.jyotishi@gmail.com): 1.3.13 
Kabiraj, Sajal; Dongbei University of Finance \& Economics, China (skabiraj@dufe.edu.cn): 2.2.10

Kähäri, Perttu; Aalto University, Finland (perttu.kahari@aalto.fi): 2.3.1

Kalafatoglu, Tugba ; ESADE Business School , Spain (tugba@tugbakalafatoglu.com): 2.1 .4

Kallapur, Sanjay; I ndian School of Business, Hyderabad, India (sanjay_kallapur@isb.edu): 0.4 .2

Kallas, David; Insper Institute of Education and Research, Brazil (davidk@insper.edu.br): 2.1.9

Kalra, Komal Kiran; University of Victoria, Canada (komal@uvic.ca): 2.4.11

Kang, J ihoon; Yonsei University, Korea, South (lyon0725@hanmail.net): 3.2.11

Kannothra, Chacko George; University of Massachusetts Boston, USA (chackog@gmail.com): 1.3.4, 2.3.12

Kano, Liena; University of Calgary, Canada (liena.kano@haskayne.ucalgary.ca): 2.1.6

Kappen, J effrey; Drake University, USA (jeffrey.kappen@drake.edu): 2.5.11

Kappen, Philip ; CBS, Denmark (philip.kappen@fek.uu.se): 2.3.11

Karamongikar, Sandeep; Infosys Technologies, India (tbd1@tbd.com): 2.2.2

Karia, Manisha; Auckland Institute of Studies, New Zealand (manishak@ais.ac.nz): 1.3.10

Karimi, Mehrnaz; University of Bayreuth, Germany (mehrnaz.karimi@uni-bayreuth.de): 1.3.10

Karna, Amit; Indian Institute of Management Ahmedabad, India (karna@iimahd.ernet.in): 2.5.3, 3.1.10, 3.4.11

Karreman, Bas; Erasmus University Rotterdam, Netherlands (karreman@ese.eur.nl): 3.1.10

Kaul, Aseem; University of Minnesota, USA (akaul@umn.edu): 2.4.9, 3.2.7

Kedia, Ben; Univerity of Memphis, USA (bkedia@memphis.edu): 1.1.8, 2.1.12

Kelley, Keith J; University of Michigan-Flint, USA (keithkel@umflint.edu): 3.1.12

Kelly, Aidan; University College Dublin, I reland (aidan.kelly@ucd.ie): 1.5.7, 2.3.7

Kent, Stephanie J o; UMass Amherst, USA (steph@comm.umass.edu): 2.5.11

Ketencioglu, Elif ; Optus, Australia (elif.ketencioglu@optus.com.au): 2.5.12

Khan, Zaheer; University of Sheffield, United Kingdom (khan.zaheer@gmail.com): 1.4.5

Khoreva, Violetta; Hanken School of Economics, Finland (violetta.khoreva@hanken.fi): 2.4.10, 3.3.12

Kim, Brian; Yonsei University, Korea, South (tskim87@yonsei.ac. kr): 2.2.6

Kim, Daekwan; Florida State University, USA (dkim@business.fsu.edu): 1.3.11

Kim, Heechun; Georgia State University, USA (heechunkim@gsu.edu): 1.3.9

Kim, Heeyon; National University of Singapore, Singapore (heeyon@nus.edu.sg): 2.3 .1

Kim, Hyun Gon; Rutgers Business School, USA (hgkim.econ@gmail.com): 2.3.11, 2.4.13

Kim, Jaehyeon; University of Hawaii at Manoa, USA (jaehyeon@hawaii.edu): 3.2.12

Kim, J imi; Temple University, USA (jimi.kim@temple.edu): 3.3.6

Kim, J in Uk; University of Illinois at Urbana Champaign, USA (jkim198@illinois.edu): 2.1.10, 2.4.2, 3.3.4

Kim, Kihyun; Korea University , Korea, South (rlgus9218@korea.ac.kr): 3.3.13

Kim, Kowoon ; Florida International University, USA (kkim021@fiu.edu): 2.5.11

Kim, Min Jung; University of Minnesota, USA (mj.kim1120@gmail.com): 1.3.10

Kim, Philsoo; Yonsei University, Korea, South (kimseolhyun@yonsei.ac.kr): 1.3.9, 1.5.10

Kim, Youngok; University of New South Wales, Australia (y.kim@unsw.edu.au): 1.1.4

Kinoti, Abel; Riara University, Kenya (akinoti@riarauniversity.ac.ke): 0.5.6, 3.2.2

Kirca, Ahmet; Michigan State University, USA (kirca@broad.msu.edu): 2.3.6, 3.1.10

Kirch, Guilherme; EA-UFRGS, Brazil (gkirch@ea.ufrgs.br): 1.3.13

Klopf, Patricia Reneé; WU Vienna, Austria (pklopf@wu.ac.at): 1.1.5

Klueter, Thomas; IESE, Spain (tmklueter@iese.edu): 3.1.4

Knight, Gary; Willamette University, USA (gknight@willamette.edu): 0.5.6, 2.1.1, 2.5.7

Knill, April; Florida State University, USA (aknill@business.fsu.edu): 1.1.5

Knocke, J an Sebastian; Friedrich-Alexander University of Erlangen-Nuremberg, Germany (jan.knocke@fau.de):

1.1 .13

Koch, Bradley James; Grand Valley State University, USA (kochb@gvsu.edu): 2.5.11

Koch, Pamela Tremain; Grand Valley State University, USA (pltkoch@gmail.com): 2.5.11

Kodama, Yasushi; Hosei University, J apan (yass@stu.hosei.ac.jp): 3.4.7

Koestner, Mariella; University of Graz, Austria (mariella.koestner@uni-graz.at): 3.2 .8

Kogut, Bruce; Columbia University, USA (bruce.kogut@columbia.edu): 3.1.2

Konara, Palitha; University of Huddersfield, United Kingdom (palitha.konara@hud.ac.uk): 1.1.3, 1.4.8, 2.1 .9

Koning, Ruud H.; University of Groningen, Netherlands (r.h.koning@rug.nl): 2.3.4 
Koshy, Abraham ; Indian Institute of Management Ahmedabad, India (akoshy@iimahd.ernet.in): 2.3.4

Kostanek, Edyta; Hanken School of Economics, Finland (kostanek.edyta@hanken.fi): 3.3.12

Kostova, Tatiana; University of South Carolina, USA (kostova@moore.sc.edu): 3.3 .1

Kotabe, Masaaki ; Temple University, USA (mkotabe@temple.edu): 3.4 .5

Kothari, Tanvi ; San J ose State University, USA (kothari.t@gmail.com): 3.4 .5

Koveshnikov, Alexei; Aalto University , Finland (alexei.koveshnikov@aalto.fi): 1.3.8, 3.2.3

Krammer, Sorin; University of Groningen, Netherlands (sorin.krammer@gmail.com): 1.4.8

Krishnamurty, Keshav; University of Massachusetts Boston, USA (keshav.krishnamur001@umb.edu): 1.1.12

Krishnan, Rishikesha; Indian Institute of Management, India (rishi@iimidr.ac.in): 0.4.2, 1.1.1, 1.3.2, 2.1 .1

Krivogorsky, Victoria ; San Diego state University, USA (vkrivogo@mail.sdsu.edu): 1.3.13, 3.4.7

Kshetri, Nir ; University of North Carolina at Greensboro, USA (nbkshetr@uncg.edu): 2.4.10, 3.1.11

Kuanr, Abhisek; XLRI-Xavier School of Management, India (r13002@astra.xlri.ac.in): 1.5.13

Kuivalainen, Olli; Lappeenranta University of Technology, Finland (olli.kuivalainen@lut.fi): 2.2.12, 2.5.4, 3.4.4

Kumar, K; Indian Institute of Management Bangalore, India (kumark@iimb.ernet.in): 1.5.2, 2.1.1

Kumar, Kunal Kamal; T. A. Pai Management Institute, India (kumarkunalkamal@gmail.com): 1.4.13

Kumar, Pamela; HP Enterprise R\&D, India (Pamela.kumar@hp.com): 1.4.7

Kumar, Vikas; University of Sydney, Australia (vikas.kumar@sydney.edu.au): 0.3.5, 1.3.1, 2.4.9, 2.5.3, 3.4.5

Kumar, Vivek; XLRI Jamshedpur, India (fb11004@astra.xlri.ac.in): 2.5.13

Kundu, Sumit Kumar; Florida International University, USA (kundus@fiu.edu): 1.1.7, 2.3.6, 2.5.4, 3.1.10,

3.2.10, 3.3.7

Kunisch, Sven; University of St. Gallen, Switzerland (sven.kunisch@unisg.ch): 1.5.4

Kurthakoti, Raghu; Arcadia University, USA (kurthakotir@arcadia.edu): 3.1.6

Kwantes, Catherine; University of Windsor, Canada (catherine.kwantes@uwindsor.ca): 2.5.11

\section{$\mathbf{L}$}

Ladha, Rani ; Indian Institute of Management Kozhikode, India (rani.ladha@iimk.ac.in): 1.4 .4

Lahiri, Nandini; Temple University, USA (nlahiri@temple.edu): 0.2.4, 1.3.11, 1.5.9

Lahiri, Somnath; Illinois State University, USA (slahiri@ilstu.edu): 2.1.11, 2.3.13, 3.4.11

Laine, Igor; Lappeenranta University of Technology, Finland (igor.laine@lut.fi): 3.3 .8

Lakshman, C; Tongji University , China (clakshman2007@gmail.com): 1.4.4, 3.1.11

Laleman, Francis ; Beyond Borders, Belgium (francis.laleman@dunya.be): 1.5.11

Lamb, Peter; La Trobe Business School, Australia (p.lamb@latrobe.edu.au): 2.5.7

Lan, J unbang; Hong Kong Baptist University, China (13480367@life.hkbu.edu.hk): 2.4.10

Lancheros, Sandra; University of Nottingham, China (sandra.lancheros@nottingham.edu.cn): 1.5.3, 2.3.7

Landau, Christian; EBS Business School, Germany (christian.landau@ebs.edu): 3.1 .10

Landes, Andreas; University of Passau, Germany (andreas.landes@uni-passau.de): 3.4.13

Laplume, André ; Michigan Technological University, USA (aolaplum@mtu.edu): 2.3 .8

Larquey, Magali; Toulouse Business School, France (m.larquey@toulouse-bs.org): 3.4 .13

Larsen, Marcus M.; Copenhagen Business School, Denmark (mml.smg@cbs.dk): 1.3.4, 2.1.3, 2.5.2, 3.1.3, 3.4.2

Latukha, Marina; St. Petersburg State University, Russia (marina.latuha@gsom.pu.ru): 1.1.5, 3.3.12

Lavelle, J onathan; University of Limerick, Ireland (jonathan.lavelle@ul.ie): 1.1.3

Lazarova, Mila; Simon Fraser University, Canada (mbl@sfu.ca): 1.5.11

Lee, Ahreum; Temple University, USA (ahreum.lee@temple.edu): 2.1.3, 2.4.5

Lee, Hyun-J ung; London School of Economics and Political Science, United Kingdom (h.lee@lse.ac.uk): 2.2.8

Lee, J ason Kyewon; Korea University, Korea, South (kyewon.jason.lee@gmail.com): 1.4.10

Lee, J ongmin; University of Reading, United Kingdom (j.m.lee@pgr.reading.ac.uk): 1.3.10, 2.2.8

Lee, Jung Wan; Boston University, USA (jwlee119@bu.edu): 2.3.12

Lee, J ung Yeon; California State University, Fullerton, USA (junlee@exchange.fullerton.edu): 3.3.2

Lee, Sun Hye; University of Warwick, United Kingdom (phd13sl@mail.wbs.ac.uk): 3.3.11

Lee, Yeon; Seoul National University, Korea, South (yeonlee.snu@gmail.com): 2.4 .4

Lee, Youngwoo; Korea University, Korea, South (lee3992@korea.ac.kr): 3.2.7, 3.3.13

Leiblein, Michael; Ohio State University, USA (leiblein.1@fisher.osu.edu): 3.4.6

Leinemann, Alexander; University of St. Gallen, Switzerland (alexander.leinemann@unisg.ch): 1.5.4

Leite, Emilene ; Uppsala University, Sweden (emilene.leite@fek.uu.se): 3.2.8 
Lema, Rasmus; Aalborg University, Denmark (lema@business.aau.dk): 1.1.10

Lemanski, Michal; Nottingham University Business School China, Poland (michal.lemanski@nottingham.edu.cn):

1.4.10, 3.2.11

Lenway, Stefanie; University of St. Thomas, USA (stefanie.lenway@stthomas.edu): 0.3.5

Leonidou, Leonidas C.; University of Cyprus, Cyprus (leonidas@ucy.ac.cy): 2.1.8

Leppaaho, Tanja; Jyvaskyla University School of Business and Economics, Finland (tanja.c.leppaaho@jyu.fi):

1.1 .4

Lewin, Arie; Duke University, USA (ayl3@duke.edu): 1.5.1, 2.2.1, 3.3.5

Li, Huiping ; Ramapo College of New Jersey, USA (hli@ramapo.edu): 1.3.7

Li, Ji; Hong Kong Baptist University, Hong Kong, SAR-PRC (jili@hkbu.edu.hk): 1.4.13, 2.3.9

Li, Jiatao; Hong Kong University of Science and Technology, Hong Kong, SAR-PRC (mnjtli@ust.hk): 0.2.4,

1.4.13, 3.2.6

Li, Jing; Simon Fraser University, Canada (jingli@sfu.ca): 3.4 .2

Li, Jing; Zhejiang University, China (lijing89@zju.edu.cn): 2.5.4

Li, Lei; Nottingham University Business School China, China (lei.li@nottingham.edu.cn): 2.1.11, 2.3.6, 2.5.12

Li, Lydia Qianqian; Shanghai University, China (qianqian.li@shu.edu.cn): 2.1.8

Li, Peter Ping; Copenhagen Business School, Denmark (ppl.int@cbs.dk): 2.5.7, 3.1.2

Li, Qing; Shanghai University, China (qingli_sbs@126.com): 3.2.13

Li, Weiguang; Renmin University of China, China (liweiguang198708@163.com): 3.3.11

Li, Xiaomei; Tianjin University, China (Ixm@tju.edu.cn): 1.4.5

Li, Yi ; Australian National University, Australia (yi.li@anu.edu.au): 3.1.12, 3.3.4

Li, Yongjuan; Chinese Academy of Sciences, China (liyj@psych.ac.cn): 1.4.13

Li, Yuanxu; Fudan University, China (yxli@fudan.edu.cn): 1.5.10

Li, Yuanyuan; Rutgers Business School, USA (yuanyuan.li@rutgers.edu): 2.1.10

Liberman, Leonardo; Universidad de los Andes, Chile (leonardo.liberman@gmail.com): 1.3.12

Lien, Wan-Chien; ChengChi University, Taiwan (shingday@gmail.com): 1.1.10

Liesch, Peter W.; University of Queensland, Australia (p.liesch@business.uq.edu.au): 0.3.5, 1.4.9, 2.2.12, 2.5.7

Liesenkötter, Bernd; University of Muenster, Germany (petertidh@gmail.com): 1.3.10

Lin, Chieh-Yu; Chang Jung Christian University, Taiwan (jylin@mail.cjcu.edu.tw): 3.4.13

Lin, Chih-An; National Taiwan University, Taiwan (elintc@yahoo.com): 2.1.11

Lin, Daomi; Sun Yat-sen University, China (lindm6@mail.sysu.edu.cn): 2.4.11

Lin, Jung Ching; National Taiwan University, Taiwan (dorislin1011@gmail.com): 1.5.10

Lin, Nidthida; University of Newcastle, Australia (nidthida.lin@newcastle.edu.au): 1.3.4

Lin, Wen-Ting; National Chung Cheng University, Taiwan (wentinglin@ccu.edu.tw): 3.3.2

Lin, Ying-Jan; National Taiwan University, Taiwan (d98724007@ntu.edu.tw): 1.5.10

Lindner, Thomas; WU Vienna, Austria (thomas.lindner@wu.ac.at): 2.1.9, 3.4.10

Lindorfer, Robert; WU Vienna, Austria (robert.lindorfer@wu.ac.at): 2.4.6

Lindstrand, Angelika; Stockholm School of Economics/Stanford University, Sweden (alindstr@stanford.edu):

3.3.4

Liou, Rushiun; Texas A\&M University -Central Texas, USA (r.liou@tamuct.edu): 2.4 .9

Liu, Mark; University of Kentucky, USA (mark.liu@uky.edu): 1.3.13

Liu, Qing; University of International Business and Economics Beijing, China (leoneco@gmail.com): 3.3.3

Liu, Tao; Southwest University, China (missliu619@sina.com): 1.4.13, 2.3.9

Liu, Wei; Manchester Business School, United Kingdom (wei.liu-2@postgrad.mbs.ac.uk): 2.5.10

Liu, Xiyou; Audit Research Institute of China's National Audit Office, China (jhqslxy@163.com): 3.2.12

Liu, Ying; Florida International University, USA (lilianeliuying@gmail.com): 3.2.11

Liu, Zyang; Renmin University of China, China (liuziyang0128@126.com): 3.3 .11

Lopez, Luis; INCAE, Costa Rica (luis.lopez@incae.edu): 2.5.3

Lopez, Ricardo; Universidad Autónoma de Occidente, Colombia (ralopez@uao.edu.co): 1.1.8

Loren, Steve Michael; University of Massachusetts Boston, USA (sloren63@msn.com): 3.2.12

Lorenzen, Mark; Copenhagen Business School, Denmark (ml.ino@cbs.dk): 2.4 .1

Love, James; Warwick Business School, United Kingdom (jim.love@wbs.ac.uk): 1.5.3

Lu, Jane; University of Melbourne, Australia (jane.lu@unimelb.edu.au): 0.2.4

Lu, Lung-Tan; Fo Guang University, Taiwan (Itlu@mail.fgu.edu.tw): 3.4.13 
Lu, Qiang (Steven) ; University of Sydney, Australia (steven.lu@sydney.edu.au): 2.1.8

Lu, Vinh N.; Australian National University, Australia (vinh.lu@anu.edu.au): 1.3.7

Luan, Chin-jung; National Dong Hwa University, Taiwan (cjluan@mail.ndhu.edu.tw): 2.1.5, 3.3.13

Luiz, J ohn Manuel; University of Cape Town, South Africa (john.luiz@gsb.uct.ac.za): 2.4.12, 3.1.5

Lundan, Sarianna ; University of Bremen, Germany (s.lundan@uni-bremen.de): 1.5.8, 2.2.11, 2.3.3, 3.2.1

Lunnan, Randi; BI Norwegian Business School, Norway (randi.lunnan@bi.no): 3.4 .6

Luo, Yadong; University of Miami, USA (yluo@bus.miami.edu): 2.4.11, 3.2.4

Lupton, Nathaniel Curtis; Fordham University, USA (nlupton@fordham.edu): 2.4 .5

Lynden, Karen; Rowan-Cabarrus Community College, USA (karen.lynden@rccc.edu): 1.3.7, 3.1.6

M

Ma, J ieqiong; Saint Louis University, USA (jma17@slu.edu): 1.1.12, 1.5.8

Mack, Elizabeth; Michigan State University, USA (lizmack287@gmail.com): 2.2 .9

Madhavan, Ravi; University of Pittsburgh, USA (rmadhavan@katz.pitt.edu): 1.4 .6

Madhavan, Shobhana ; Amrita School of Business , India (shobsdg@gmail.com): 2.2.11

Madhok, Anoop; York University, Canada (amadhok@schulich.yorku.ca): 0.3.5, 2.3.10, 3.3.3

Magelssen, Catherine; London Business School, United Kingdom (cmagelssen@london.edu): 1.3.3, 2.3.1

Magnusson, Peter; University of Alabama, USA (peter.magnusson@fiu.edu): 0.5.6, 1.1.9, 3.4.3

Mahmood, Arif; Hong Kong Baptist University, Hong Kong, SAR-PRC (14485206@life.hkbu.edu.hk): 1.4.13,

2.3.9

Mahmood, Ishtiaq; National University of Singapore, Singapore (bizipm@nus.edu.sg): 3.3 .2

Majocchi, Antonio; University of Pavia, Italy (antonio.majocchi@unipv.it): 2.2.6, 2.4.13

Majumdar, Sumit; University of Texas at Dallas, USA (majumdar@utdallas.edu): 2.1.7, 2.4.6, 3.2.13

Makela, Kristiina; Aalto University, Finland (kristiina.makela@aalto.fi): 3.3.12

Makhija, Anil; Ohio State University, USA (makhija.1@osu.edu): 2.1.12

Makhija, Mona; Ohio State University, USA (makhija.2@osu.edu): 2.1.12

Makino, Shige; Chinese University of Hong Kong, Hong Kong, SAR-PRC (makino@baf.cuhk.edu.hk): 3.3 .5

Maksimov, Vladislav ; University of Miami, USA (vmaksimov@bus.miami.edu): 2.4 .11

Malen, J oel; Hitosubashi University Institute of Innovation Research, Japan (malen@iir.hit-u.ac.jp): 2.1.5

Malhotra, Shavin; Ryerson University, Canada (shavinm@ryerson.ca): 2.1.6

Malik, Ashish ; Newcastle Business School, Australia (ashish.malik@newcastle.edu.au): 1.5.11, 3.3.12

Malik, Omar; Higher Education , USA (ormalik@aol.com): 2.5.12

Malla, Sweta Srivastava; I ndian Institute of Foreign Trade, India (sweta@iift.ac.in): 2.5.13

Mallick, Sushanta; Queen Mary University of London, United Kingdom (s.k.mallick@qmul.ac.uk): 2.1.7

Maloney, Mary; University of St. Thomas, USA (mmmaloney@stthomas.edu): 3.2 .3

Mandal, Abhijit; City University, London, United Kingdom (am4096@gmail.com): 3.2.13

Mankavil Kovil Veettil, Nandakumar; University of Salford, United Kingdom (nmkveettil@gmail.com): 2.2.12, 3.2.10

Manning, Stephan; University of Massachusetts Boston, USA (stephan.manning@umb.edu): 0.5.6, 1.1.11, 2.2.9, 3.2.2, 3.4.2

Manolova, Tatiana S.; Bentley University, USA (tmanolova@bentley.edu): 1.3.10, 3.3.2

Manotas, Eva Cristina; Universidad Nacional, Colombia (ecmanota@unal.edu.co): 2.3.13

Mansion, Stephanie Elisabeth; J ustus Liebig University Giessen, Germany (stephanie.mansion@wirtschaft.unigiessen.de): 2.3.8

Manwani, Harish; Chairman, Hindustan Unilever Ltd and former COO, Unilever, India: 1.2.1

Marinov, Marin Alexandrov; Aalborg University, Denmark (marinov@id.aau.dk): 2.2.5, 2.4.13

Marinova, Svetla Trifonova; Aalborg University, Denmark (svetla@business.aau.dk): 2.2.5, 2.4.13

Marion, Tucker; Northeastern University, USA (t.marion@neu.edu): 3.1.4

Markoczy, Livia; University of Texas at Dallas, USA (livia.markoczy@utdallas.edu): 1.5.12

Marquis, Christopher; Harvard Business School , USA (cmarquis@hbs.edu): 3.2.10

Martinez de I barreta, Carlos ; Universidad Pontificia Comillas, Spain (charlie@upcomillas.es): 1.5.11

Maseland, Robbert; University of Groningen, Netherlands (r.k.j.maseland@rug.nl): 1.5.12

Masino, Serena; University of Oxford, United Kingdom (serena.masino@qeh.ox.ac.uk): 1.3.6

Mason, Elizabeth Louise; University of Leeds, United Kingdom (e.I.mason@lubs.leeds.ac.uk): 1.1.11 
Massini, Silvia; University of Manchester, United Kingdom (silvia.massini@mbs.ac.uk): 3.1.3

Mathur, Ajeet Narain; Indian Institute of Management Ahmedabad, India (anmathur@iimahd.ernet.in): 3.2 .5

Mazumdar-Shaw, Kiran; Biocon Limited, India: 0.7.1

McCormick, Marleen; Butler University, USA (memccorm@butler.edu): 3.4.12

McDonald, Michael; University of Texas-San Antonio, USA (michael.mcdonald@utsa.edu): 3.4 .3

McDougall-Covin, Patricia P. ; Indiana University-Bloomington, USA (mcdougal@indiana.edu): 2.1.4, 3.05

McGuinness, Martina; University of Sheffield, United Kingdom (m.mcguinness@sheffield.ac.uk): 2.5.6

Mehta, Anju; University of Northern I owa, USA (anju.mehta@uni.edu): 2.2.8, 2.4.10

Melgarejo, Mauricio ; INCAE, Nicaragua (mauricio.melgarejo@incae.edu): 2.5 .3

Melkumov, Dmitri; Hanken School of Economics, Finland (dmitri.melkumov@gmail.com): 2.4.10

Mellahi, Kamel; University of Warwick, United Kingdom (kamel.mellahi@wbs.ac.uk): 3.3.11

Menon, Aditya; Citibank, India (adityal.menon@citi.com): 2.2 .2

Menon, Anil; Cisco Systems, USA (animenon@cisco.com): 1.1 .2

Menon, Tanya; Ohio State University, USA (menon.53@osu.edu): 2.5.11

Merchant, Hemant; University of South Florida St. Petersburg, USA (hmerchant@usfsp.edu): 3.1 .10

Meyer, Klaus; CEIBS, China (kmeyer@ceibs.edu): 1.05, 1.5.7, 2.5.6, 3.3.1, 3.4.2

Meyers, Judith ; University of California, USA (judith.meyers0@gmail.com): 1.5.11

Miao, Qing; Jilin University, China (mfhtmfht@163.com): 3.3.3

Michael, Ian; Zayed University, United Arab Emirates (ian.michael@zu.ac.ae): 2.2.4

Migendt, Michael; EBS Business School, Germany (michael@migendt.net): 1.4 .12

Mihailova, I rina; Aalto University , Finland (irina.mihailova@aalto.fi): 1.4.9, 3.3.8

Mileski, J oan P.; Texas A\&M University at Galveston, USA (mileskij@tamug.edu): 2.1.4, 3.1.11

Miller, Stewart R.; University of Texas-San Antonio, USA (stewart.miller@utsa.edu): 0.3.5, 2.1.6, 3.4.3

Mirza, Hafiz; UNCTAD, Switzerland (hafiz.mirza@unctad.org): 2.3.3, 3.2.1

Misati, Everlyne; Florida International University, USA (emisa001@fiu.edu): 3.2.10

Mishra, Mridula Savitri; IIFT, India (mridula@iift.ac.in): 1.1.10

Mitchell, Matthew; Drake University, USA (matthew.mitchell@drake.edu): 3.1.7

Mittal, Rakesh; New York Institute of Technology, USA (rmittal@nyit.edu): 2.4.10

Moeller, Miriam; University of Queensland, Australia (m.moeller@business.uq.edu.au): 1.5.11

Mol, Michael J; Copenhagen Business School, Denmark (mmo.smg@cbs.dk): 3.1.5, 3.2.13, 3.3 .11

Momaya, K. S.; Indian Institute of Technology Bombay, India (momaya@iitb.ac.in): 2.3.12, 2.5.13

Monaghan, Sinead; Rutgers Business School, USA (smonaghan@business.rutgers.edu): 1.1.3, 1.5 .5

Mondal, Arindam; Indian Institute of Management Calcutta, India (arindamm11@iimcal.ac.in): 1.4.10, 1.5.4

Monteiro, Felipe; INSEAD, France (felipe.monteiro@insead.edu): 1.3.3, 2.3.2, 3.1.4, 3.2.1

Monticelli, J efferson Marlon; Universidade do Vale do Rio dos Sinos, Brazil (jeffmarlon@hotmail.com): 2.1.11

Montiel, I van; Loyola Marymount University, USA (ivanmontiel@gmail.com): 1.5.7, 2.4.5, 3.1.7, 3.4.11

Moon, Hwy-Chang ; Seoul National University, Korea, South (cmoon@snu.ac.kr): 2.1.11, 2.4 .4

Moon, J on J ungbien; Korea University, Korea, South (jonjmoon@korea.ac.kr): 1.3.10, 2.1.5

Moore, Fiona; Royal Holloway, University of London, United Kingdom (fiona.moore@rhul.ac.uk): 1.1.13, 2.1.4, 2.3.9

Morgulis-Yakushev, Sergey; Stockholm School of Economics, Sweden (sergey.morgulis-yakushev@hhs.se):

1.3.3

Moris, Francisco A; George Washington University, USA (fmoris@nsf.gov): 2.3 .11

Morris, Shad; Brigham Young University, USA (morris@byu.edu): 1.4.10

Mors, Marie Louise; Copenhagen Business School, Denmark (Im.smg@cbs.dk): 3.4 .3

Mostafa, Romel; I vey Business School, Canada (rmostafa@ivey.uwo.ca): 1.3.5, 2.2.12

Mudambi, Ram; Temple University, USA (rmudambi@temple.edu): 0.7.1, 0.71.1, 1.2.1, 2.1.3, 3.2.1

Mudambi, Susan; Temple University, USA (smudambi@temple.edu): 1.1.2, 2.4.13, 3.2.8

Muehlfeld, Katrin; University of Trier, Germany (muehlfeld@uni-trier.de): 1.1.13

Mueller, Marc; University of St. Gallen, Switzerland (marc.mueller@unisg.ch): 2.2 .9

Muellner, Jakob; WU Vienna, Austria (jakob.muellner@wu.ac.at): 3.4.10

Mukerjee, Partha ; University of New South Wales, Australia (partham29@yahoo.com): 3.2.13

Mukherjee, Debmalya ; University of Akron, USA (dmukher@uakron.edu): 2.4.11, 3.1.4, 3.4.10

Mukherjee, Deepraj; Kent State University, USA (dmukherj@kent.edu): 3.4.10 
Mukherji, Shoma; Management Development Institute, India (shoma126@gmail.com): 1.1 .9

Mukundhan, K.V.; Indian Institute of Management Kozhikode, India (kidambim@gmail.com): 3.2.10

Muncy, J ames A. ; Valdosta State University, USA (muncyj@valdosta.edu): 1.5.13

Muniz-Ferrer, Marta ; Universidad Pontificia Comillas, Spain (mferrer@upcomillas.es): 1.5.11

Munjal, Surender; University of Leeds, United Kingdom (smu@lubs.leeds.ac.uk): 3.2.6, 3.3.3

Muralidharan, Etayankara ; MacEwan University, Canada (muralidharane@macewan.ca): 1.4.11, 2.3.8, 2.4.5

Murray, J anet; University of Missouri-St. Louis, USA (murrayjan@umsl.edu): 1.4.7, 2.3.3

Mutlu, Canan; University of Texas at Dallas, USA (cxm101020@utdallas.edu): 2.2.10

\section{$\mathbf{N}$}

Nachum, Lilac; Baruch College, CUNY, USA (lilac.nachum@baruch.cuny.edu): 1.1.5

Nadayama, Naoto; University of Otago, New Zealand (naoto.nadayama@otago.ac.nz): 3.4 .9

Naidoo, Vik; University of Sydney, Australia (viknaidoo@gmail.com): 2.5.10

Nam, Dae-il; Korea University, Korea, South (namdaeil@korea.ac.kr): 1.4.10

Nanda, Ashis; Indian Institute of Management Ahmedabad, India (ananda@iimahd.ernet.in): 0.4.2

Naoumova, I rina; University of Hartford, USA (naoumova@hartford.edu): 1.3.7, 1.4.13

Narain, Kalpana; Director, Full Spectrum Consulting , India (kalpana.narain@gmail.com): 2.3.10

Narula, Rajneesh; University of Reading, United Kingdom (r.narula@henley.ac.uk): 1.3.10, 3.1.3

Navendra, P.T.; University of Dallas, USA (ptnavendra@udallas.edu): 3.1.11

Nayyar, Rishika; PGDAV College, Delhi University, India (rishikadse@gmail.com): 2.3.10

Nazareth, Premila ; UNCTAD, Switzerland (premila@foreigndirectinvestment.in): 2.3.3, 3.2.1

Nebus, James F.; Suffolk University, USA (jnebus@suffolk.edu): 3.3.9

Nell, Phillip; WU Vienna, Austria (phillip.nell@wu.ac.at): 1.1.5, 2.1.12

Nellemann, Camilla; Rikkyo University, Japan (camillanellemann@gmail.com): 3.4.11

Newburry, William ; Florida International University, USA (newburry@fiu.edu): 0.5.6, 1.4.4, 2.1.4, 2.4.11, 3.2.2

Newenham-Kahindi, Aloysius; University of Saskatchewan, Canada (newenham-kahindi@edwards.usask.ca):

1.4.8

Ng, Eddy; Dalhousie University, Canada (edng@dal.ca): 1.1.13

Ngo, Vi Dung; Vietnam National University, Vietnam (dungnv@hsb.edu.vn): 2.1 .8

Nguyen, Linh Khanh; HHL Leipzig Graduate School of Management, Germany (linh.nguyen@hhl.de): 1.1.6

Nicholson, Rekha; University of the West of England, United Kingdom (rao.rekha@gmail.com): 2.4 .9

Nidugala, Ganesh Kumar; Indian Institute of Management Indore, India (ganesh@iimidr.ac.in): 3.4.10

Nielsen, Anders; Aalborg University, Denmark (ana@plan.aau.dk): 1.1.10

Nielsen, Bo Bernhard; University of Sydney, Australia (b.nielsen@econ.usyd.edu.au): 2.4.12, 2.5.6, 3.3.4

Nielsen, Sabina; Copenhagen Business School, Denmark (sta.int@cbs.dk): 0.2.4, 2.5.6

Noh, Ki Bum; Korea Economic Research Institute (KERI), Korea, South (kibum.noh@keri.org): 2.4 .2

Nummela, Niina ; University of Turku, Finland (niina.nummela@utu.fi): 2.2.12

O

Oehmichen, Jana; Georg-August University of Goettingen, Germany (jana.oehmichen@wiwi.uni-goettingen.de):

3.2.11

Ojha, Abhoy; Indian Institute of Management Bangalore, India (aojha@iimb.ernet.in): 1.3.2

Okimoto, Tyler G; University of Queensland, Australia (t.okimoto@business.uq.edu.au): 1.5.11

Oldroyd, James; Ohio State University, USA (oldroyd@fisher.osu.edu): 1.4.10

Oliveira, Fernando; ESSEC Business School, Singapore (fernando.oliveira@essec.edu): 2.5 .6

Oliveira, Moacir Miranda; University of São Paulo, Brazil (mirandaoliveira@usp.br): 2.2.12, 3.3.10

Olsen, Anders Oerding; Copenhagen Business School, Denmark (aoo.ino@cbs.dk): 2.4.1, 3.1.4

Ordoobody, J oobin; University of Victoria, Canada (joobin@uvic.ca): 1.1.12

Osiyevskyy, Oleksiy; Northeastern University, USA (o.osiyevskyy@neu.edu): 3.3 .9

Outa, Erick Rading; Strathmore Business School, Kenya (otxeri001@gsba.uct.ac.za): 3.4.10

Oxelheim, Lars ; Lund University and Research I nstitute of Industrial Economics, Sweden

(lars.oxelheim@fek.lu.se): 1.3.5 
P., Srikanth; T. A. Pai Management Institute, India (psrikanth@tapmi.edu.in): 2.5.13

Pahlberg, Cecilia; Uppsala University, Sweden (cecilia.pahlberg@fek.uu.se): 3.2 .8

Paisley, Varina; University of New South Wales, Australia (v.paisley@unsw.edu.au): 1.1.8, 3.2.3

Pak, Yong Suhk; Yonsei University, Korea, South (yspak@yonsei.ac.kr): 1.3.12, 2.5.11

Pandey, Mohan; Bristol-Myers Squibb, India (mohan. pandey@bms.com): 1.5.2

Pangarkar, Nitin; National University of Singapore, Singapore (bizpn@nus.edu.sg): 2.5.3

Panibratov, Andrei; St. Petersburg University, Russia (panibratov@mail.ru): 1.1.5, 2.2.5

Pant, Anirvan; I ndian I nstitute of Management Calcutta, India (anirvan@iimcal.ac.in): 2.4 .2

Papageorgiadis, Nikolaos; University of Liverpool, United Kingdom (n. papageorgiadis@liverpool.ac.uk): 3.4.10

Parc, Jimmyn; Sciences Po Paris, France (jimmynparc@gmail.com): 2.4 .4

Parente, Ronaldo; Florida International University, USA (rcparent@fiu.edu): 1.1.7, 3.1.4

Park, Eunkyung; Aalborg University, Denmark (eunkyung@business.aau.dk): 1.3.4, 2.1.3

Park, Seung Ho; China Europe International Business School, China (spark@ceibs.edu): 1.1.1, 2.05, 2.4.3, 3.2.11

Park, Young-Ryeol; Yonsei University, Korea, South (yrpark@yonsei.ac.kr): 1.3.9, 1.5.10, 3.3.11

Patchell, J erry; Hong Kong University of Science and Technology, Hong Kong, SAR-PRC (sopatch@ust.hk): 3.2.10

Pathak, Saurav ; Michigan Technological University, USA (sauravp@mtu.edu): 2.3.8

Pattnaik, Chinmay; University of Sydney , Australia (chinmay.pattnaik@sydney.edu.au): 2.1.8, 2.5.12, 3.3.13

Paul, J ustin; University of Puerto Rico, USA (justin.paul@upr.edu): 2.5.10

Paulus, Philipp; University of Trier, Germany (paulusph@uni-trier.de): 1.1.13

Peacock, Tanya Andrea; Army-Baylor University, USA (peacock4@hawaii.edu): 3.4 .9

Pedersen, Torben; Bocconi University, Italy (torben. pedersen@unibocconi.it): 0.3.5, 0.71.1, 2.2.7, 2.3.11

Peeters, Carine; Vlerick Business School, Belgium (carine. peeters@vlerick.com): 2.2.9, 2.3.11

Pekerti, Andre Anugerah; University of Queensland, Australia (a. pekerti@business.uq.edu.au): 1.1.9, 1.5.11, 2.2.3

Peng, Mike; University of Texas at Dallas, USA (mikepeng@utdallas.edu): 1.4.12, 2.2.10, 3.3.1

Peng, Yu-Shu ; National Dong Hwa University, Taiwan (yspeng@mail.ndhu.edu.tw): 2.5.10

Pennings, Enrico; Erasmus University Rotterdam, Netherlands (pennings@ese.eur.nl): 1.5.7

Pereira, Vijay Edward; University of Portsmouth, United Kingdom (vijay.pereira@port.ac.uk): 1.4.4, 2.4.11, 3.2.6, 3.3.12, 3.4.8

Pergelova, Albena; MacEwan University, Canada (pergelovaa@macewan.ca): 1.4.11

Perri, Alessandra ; Ca' Foscari University Venice, Italy (alessandra.perri@unive.it): 1.3.10, 2.1 .3

Pesakovic, Gordana ; King University, USA (pesakovic@msn.com): 1.3.7

Pesch, Robin; University of Bayreuth, Germany (robin.pesch@uni-bayreuth.de): 1.3.10

Peterson, Mark; Florida Atlantic University, USA (Mpeterso@fau.edu): 3.3.1

Petrovici, Dan Alex; University of Kent, United Kingdom (petrovici@uni.kent.ac.uk): 2.4.13

Phene, Anupama; George Washington University, USA (anuphene@gwu.edu): 1.3.3, 1.4.6, 2.3.1, 3.1.1

Piantoni, Mariella; University of Bergamo, Italy (mariella.piantoni@unibg.it): 2.5.4

Piao, Xuelian; Korea University, Korea, South (snowlotus@hanmail.net): 1.3.10

Pichardo, Caleb; INCAE, Costa Rica (caleb.pichardo@incae.edu): 1.5.7

Piesse, J enifer; King's College London, United Kingdom (jenifer.piesse@kcl.ac.uk): 1.4.12

Pineros, Rafael Alejandro; Universidad del Rosario, Colombia (rafael.pineros@urosario.edu.co): 1.1.8

Pinkham, Brian; I vey Business School, Canada (bpinkham@ivey.uwo.ca): 1.1.12, 3.2 .5

Pinto, Claudia Frias; EAESP-FGV, Brazil (claudia.frias.pinto@gmail.com): 3.2.6

Pisani, Niccolo; University of Amsterdam, Netherlands (n.pisani@uva.nl): 3.1 .3

Piscitello, Lucia; Politecnico di Milano, Italy (lucia. piscitello@polimi.it): 1.3.5, 1.4.1, 2.4.12

Podesta, Maria Paola; EAFIT, Colombia (mpodesta@eafit.edu.co): 1.1.8

Polzin, Friedemann; Sustainable Business Institute, Germany (polzin@instoec.de): 1.4.12

Ponssard, Jean-Pierre; Ecole Polytechnique \& CNRS, France (jean-pierre. ponssard@polytechnique.edu): 1.1.12

Popli, Manish; Indian Institute of Management Indore, India (manishp@iimidr.ac.in): 2.4.9, 3.2.10

Poulsen, Lauge N. Skovgaard; University College London, United Kingdom (I.poulsen@ud.ac.uk): 3.4 .2

Powell, K. Skylar; Western Washington University, USA (skylar.powell@wwu.edu): 3.1.12 
Pradhan, Debasis; XLRI-Xavier School of Management, India (debasis@xlri.ac.in): 1.5.13

Prasad, Durga; T. A. Pai Management Institute, India (durgaprasad@tapmi.edu.in): 2.5 .13

Prasad, Sathya; Intel, India (sathya.prasad@intel.com): 2.1.2

Prashantham, Shameen; Nottingham University Business School China, China

(shameen.prashantham@nottingham.edu.cn): 0.3.5, 1.3.1, 2.1.1, 3.3.3

Presbitero, Alfred; Deakin University, Australia (alfred.presbitero@deakin.edu.au): 2.5.11, 3.4.11

Procher, Vivien; University of Wuppertal, Germany (procher@uni-wuppertal.de): 1.1.13

Pruthi, Sarika; San J ose State University, USA (sarika.pruthi@sjsu.edu): 3.3.8, 3.4.8

Pu, Ming; Harbin University of Science and Technology, China (puming2004@163.com): 2.4.3

Puck, Jonas; WU Vienna, Austria (jonas.puck@wu.ac.at): 1.1.5, 2.1.9, 2.3.5, 3.4.10

Pudelko, Markus; Tuebingen University, Germany (markus.pudelko@uni-tuebingen.de): 3.2.3, 3.4.3

Puranam, Phanish; INSEAD, Singapore (phanish. puranam@insead.edu): 0.2.4, 0.4.2, 1.1.1, 2.3.2

Purkayastha, Anish; Indian Institute of Management Ahmedabad, India (anishp@iimahd.ernet.in): 1.4.11, 2.5.3, 3.2.10

Purkayastha, Saptarshi ; Indian Institute of Management Calcutta, India (saptarshi@iimcal.ac.in): 3.3.2, 3.4.11

Puslecki, Lukasz; Poznan University of Economics, Poland (lukasz.puslecki@ue.poznan.pl): 0.5.6, 2.2.5, 3.2.2

Putzhammer, Moritz; WU Vienna, Austria (moritz.putzhammer@wu.ac.at): 2.1.9, 3.1.2

Pyun, Eugene C.; Seoul National University, Canada (egpyun@snu.ac.kr): 2.1.12

Pyun, Lynn ; Baruch College, CUNY , USA (lynn.pyun@baruch.cuny.edu): 3.2.13

Q

Qiao, Kun; Dalian University of Technology, China (qiaokun@dlut.edu.cn): 2.4.7

Qiu, Jane; University of New South Wales, Australia (janeq@unsw.edu.au): 2.1.12

Qiu, Ranfeng; California State University, San Bernardino, USA (rqiu@csusb.edu): 2.1 .3

$\mathbf{R}$

Raghunath, S.; Indian Institute of Management Bangalore, India (srnathiimb@gmail.com): 0.5.6, 0.7.1, 1.1.7, 2.1.2, 3.2.2

Raghunath, Usha; Wipro Ltd., India (usha.raghunath1@wipro.com): 1.4.7, 2.1.2

Raghuvanshi, Ashutosh; Deputy Chairman and Group CEO, Narayana Health, India: 1.2.1

Rahman, Mohammad Mahfuzur; Shandong University, China (mohammad.rahman@sdu.edu.cn): 1.1.4

Rajani, Namita; Dalhousie University, Canada (namita.rajani@dal.ca): 1.1.13

Ralston, David A.; Florida International University, USA (ralstond@fiu.edu): 1.4.13

Ramachandran, J; I ndian I nstitute of Management Bangalore, India (jram@iimb.ernet.in): 0.2.4, 0.4.2, 2.4.2

Ramamurti, Ravi; Northeastern University, USA (r.ramamurti@neu.edu): 1.2.1, 2.05

Ramarajan, Lakshmi; Harvard University, USA (Iramarajan@hbs.edu): 1.5.6

Ramboarison-Lalao, Lovanirina; ESC Troyes Business School, France (lovanirina.ramboarison@get-mail.fr): 1.3.8

Randøy, Trond; University of Agder, Norway (trond.randoy@uia.no): 1.3.5, 2.1.5

Ranganathan, Ram; University of Texas - Austin, USA (ram.ranganathan@mccombs.utexas.edu): 2.2.11

Ranjan , Kumar Rakesh ; Indian Institute of Management Tiruchirapalli, India (ranjan@iimtrichy.ac.in): 2.4.13

Rao, K. Indu; University of Twente, Netherlands (i.kaveti@utwente. nl): 2.3.13, 2.5.13

Rao Sahib , Padma ; University of Groningen, Netherlands (p.rao.sahib@rug.nl): 2.4 .9

Raskovic, Matevz; University of Ljubljana, Slovenia (matevz.raskovic@ef.uni-lj.si): 3.2.13

Ray, Amlan; Amrita University, India (amlanray1@gmail.com): 1.3.13

Ray, Pradeep Kanta; University of New South Wales, Australia (pray@unsw.edu.au): 2.4.4, 3.2.13

Ray, Sangeeta; University of Sydney, Australia (sangeeta.ray@sydney.edu.au): 2.4.4, 3.2.13

Ray, Sougata; I ndian I nstitute of Management Calcutta, India (sougata@iimcal.ac.in): 0.2.4, 0.4.2, 1.3.1,

1.4.10, 2.1.10

Ray Chaudhuri, Bikramjit; Indian Institute of Management Calcutta, India (bikramjitr12@email.iimcal.ac.in):

2.5.13

Reade, Carol; San J ose State University, USA (carol.reade@sjsu.edu): 3.4 .9

Rebouças, Silvia Maria Dias Pedro; Federal University of Ceara, Brazil (smdpedro@gmail.com): 3.3.11

Reddy, Rama Krishna; University of Memphis, USA (rreddy1@memphis. edu): 2.1 .12

Reeb, David; National University of Singapore, Singapore (bizdmr@nus.edu.sg): 3.3 .9 
Rema, Chithra; Pondicherry University, India (chithrarema@gmail.com): 3.3.12

Ren , Hong ; University of Wisconsin - Milwaukee, USA (renh@uwm.edu): 2.2.8

Ren, Monica; Macquarie University, Australia (monica ren@yahoo.com): 3.3.3

Rentala, Satyanarayana; Pondicherry University, India (rentsatya@gmail.com): 2.3.12, 3.1.10

Reuber, Becky; University of Toronto, Canada (reuber@rotman.utoronto.ca): 2.1.1

Rezaei, Shamak; Roskilde University, Denmark (shre@ruc.dk): 1.4.11

Riaz, Suhaib; University of Massachusetts Boston, USA (suhaib.riaz@umb.edu): 3.2.12

Riaz, Zahid; Lahore School of Economics, Pakistan (zahidriaz@lahoreschool.edu.pk): 2.2.10, 2.4.4, 3.2.13

Ribeiro Cahen, Fernanda ; University Center of FEI, Brazil (fribeiro@fei.edu.br): 2.2.12, 2.3.13

Ricart, J oan Enric; IESE, Spain (jericart@iese.edu): 3.1.3

Richter, Ansgar; University of Liverpool , United Kingdom (a.richter@liverpool.ac.uk): 3.3.6, 3.4.11

Richter, Cristiano; Federal University of Rio Grande do Sul, Brazil (engrichter@unisinos.br): 1.1.11

Richter, Ulf Henning; Nottingham University Business School China, China (ulf.richter@nottingham.edu.cn):

2.3.12

Rodrigues, Suzana; Erasmus University \& FUMEC University, Brazil (srodrigues@rsm.nl): 2.5.6

Rodriguez, Carlos Adrian; INCAE, Nicaragua (carlos.rodriguez@incae.edu): 2.3.1, 2.5.4, 3.2.7

Rogan, Michelle; INSEAD, France (michelle.rogan@insead.edu): 3.3.12

Rogbeer, Shalini; European Business School, Germany (shalini.rogbeer@gmail.com): 2.3.11, 3.2.12

Romelli, Davide ; ESSEC Business School and THEMA, France (davide.romelli@essec.edu): 3.1 .7

Rose, Elizabeth; University of Otago, New Zealand (elizabeth.rose@otago.ac.nz): 0.5.6, 1.4.9, 2.1.2, 2.5.13,

3.2.2

Rossmann, Alexander ; Reutlingen University, Germany (alexander.rossmann@reutlingen-university.de): 2.4.13

Rottig, Daniel; Florida Gulf Coast University, USA (drottig@fgcu.edu): 2.4.9, 3.1.6, 3.2.2

Roxas, Hernan ; Deakin University, Australia (banjo.roxas@deakin.edu.au): 2.5.11, 3.4.11

Rui, Huaichuan; Royal Holloway, University of London, United Kingdom (huaichuan.rui@rhul.ac.uk): 2.1.11,

2.2.7

Runnalls, Blake ; Michigan State University, USA (runnalls@broad.msu.edu): 3.1 .12

Rygh, Asmund ; BI Norwegian Business School, Norway (asmund.rygh@bi.no): 3.3.10, 3.4.6

\section{S}

Saarenketo, Sami; Lappeenranta University of Technology, Finland (sami.saarenketo@lut.fi): 2.2.12

Sackmann, Sonja; University Bw Munich, Germany (sonja.sackmann@unibw.de): 1.3.7

Sahasranamam, Sreevas; Indian Institute of Management Kozhikode, India (mailsreevas@gmail.com): 2.2.12,

2.3.12, 3.4.11

Saify, Alireza; University of Tehran, Iran (alireza.seify@ymail.com): 1.1.12

Sailer, Miriam; EBS Business School, Germany (miriam. sailer@me.com): 3.1.10

Saiyed, Abrar Ali Mohammad Usman; Indian Institute of Management, India (abrarali@iimahd.ernet.in): 2.3.13

Sakakibara, Mariko; University of California, Los Angeles , USA (mariko.sakakibara@anderson.ucla.edu): 1.5.2

Sakawa, Hideaki ; Nagoya City University, Japan (sakawa@econ.nagoya-cu.ac.jp): 1.3.13

Sakikawa, Takashi; Niigata University, Japan (saki@mot.niigata-u.ac.jp): 2.5.11

Saleh, Sabrina; University of South Carolina, USA (sabrina.saleh@grad.moore.sc.edu): 2.2.10

Salimath, Manjula S.; University of North Texas, USA (manjula. salimath@unt.edu): 1.4.9, 2.3.8

Salmon, J essica Rae; Rutgers University, USA (js1587@scarletmail.rutgers.edu): 1.1.10, 2.1.10

Samant, Shantala; Virginia Tech University, USA (ssamant7@vt. edu): 2.4.3, 2.5.3

Samaratunge, Ramanie; Monash University, Australia (ramanie.samaratunge@monash.edu): 1.1.6, 1.5.11

Sambharya, Rakesh; Rutgers University, USA (sambhary@camden.rutgers.edu): 3.3 .6

Samiee, Saeed; University of Tulsa, USA (ssamiee@utulsa.edu): 0.3.5, 2.4.8

Sanchez-Henriquez, Fernando ; Rutgers University, USA (fernandosanchezrutgers@gmail.com): 2.2.11

Sandberg, Jorgen; UQ Business School, Australia (j.sandberg@business.uq.edu.au): 2.5.7

Sanghavi, Nitin; Manchester Business School, United Kingdom (nitin.sanghavi@mbs.ac.uk): 2.5.10

Santacreu-Vasut, Estefania; ESSEC Business School and THEMA, France (santacreuvasut@essec.edu): 3.1.7

Sartor, Michael A.; Queen's School of Business, Canada (michael.sartor@queensu.ca): 2.3.1, 2.5.6, 3.4.2

Sasaki, Innan; University of Turku, Finland (innsas@utu.fi): 3.4 .8

Satoglu, Emine Beyza; Rutgers University, USA (eminebeyza@gmail.com): 2.3 .12 
Saungikar, Abhijeet; 3M India, India (saabhijeet1@mmm.com): 1.3.2

Sawant, Rajeev; Baruch College, CUNY, USA (rajeev.sawant@baruch.cuny.edu): 1.1.5, 2.2.11

Scalera, Vittoria Giada; Politecnico di Milano, Italy (vittoriagiada.scalera@polimi.it): 1.3.10, 2.1.3, 2.4 .3

Schiehll, Eduardo; HEC Montréal, Canada (eduardo.schiehll@hec.ca): 2.2.10

Schinzel, Ursula; United Business Institutes, Luxembourg (ursula_schinzel@yahoo.com): 2.4 .7

Schlegelmilch, Bodo; WU Vienna, Austria (bodo.schlegelmilch@wu.ac.at): 1.5.13

Schmeisser, Bjoern; WU Vienna, Austria (bjoern.schmeisser@wu.ac.at): 1.5.5

Schmitz, Marina; University of Goettingen, Germany (mschmit5@uni-goettingen.de): 3.3.12

Schock, Florian; EBS Business School, Germany (florian.schock@ebs.edu): 1.4.12

Schoett, Thomas; University of Southern Denmark, Denmark (tsc@sam.sdu.dk): 1.4.11

Schommer, Monika; EBS Business School, Germany (monika.schommer@ebs.edu): 3.4 .11

Schotter, Andreas; I vey Business School, Canada (aschotter@ivey.uwo.ca): 1.1.12, 1.4.3, 3.2 .5

Schulte Steinberg, Adrian; University of St. Gallen, Switzerland (adrian.schultesteinberg@unisg.ch): 1.5.4

Schuster, Tassilo; University of Erlangen-Nürnberg, Germany (tassilo.schuster@fau.de): 1.1.9

Schweiger, Simone; University of Bern, Switzerland (schweiger@imu.unibe.ch): 3.2.4

Sciarelli, Fabiana ; Unitelma Sapienza University of Rome, Italy (fabiana.sciarelli@unitelma.it): 3.1.6

Scott, W. Richard; Stanford University, USA (scottwr@stanford.edu): 2.2.1

Sekiguchi, Tomoki; Osaka University, Japan (tomoki@econ.osaka-u.ac.jp): 2.4 .10

Selmier II, W Travis; Indiana University, USA (wselmier@indiana.edu): 1.4.12, 3.1.7

Semercioz, Fatih; Istanbul University, Turkey (fsemerci@istanbul.edu.tr): 1.4.11

Seppälä, Timo; Research Institute of the Finnish Economy, Finland (timo.seppala@etla.fi): 2.5.2

Seth, Anju; Virginia Tech University, USA (aseth@vt.edu): 0.3.5, 3.4.1

Settles, Alexander; Rutgers University, USA (asettles@business.rutgers.edu): 3.2 .12

Shaffer, Margaret ; University of Wisconsin - Milwaukee, USA (shafferm@uwm.edu): 1.1.13, 2.2.8

Shah, Dhara; Griffith University, Australia (d.shah@griffith.edu.au): 1.1.6, 3.3.12

Shah, Grishma; Manhattan College, USA (grishma.shah@manhattan.edu): 1.4.13, 3.1.11

Shan, Juan; Shanghai University, China (shanjuan@shu.edu.cn): 2.1.8

Sharma, Abhijit ; University of Bradford, United Kingdom (a.sharma12@bradford.ac.uk): 3.4.10

Sharma, Basu; University of New Brunswick-Fredericton, Canada (bsharma@unb.ca): 3.3.7

Sharma, Kushal; ESSEC Business School, France (kushal.sharma@essec.edu): 1.1.13

Sharma, Sunil; Indian Institute of Management Ahmedabad, India (sunilsharma@iimahd.ernet.in): 2.5.3, 3.2.4

Shenkar, Oded; Ohio State University, USA (shenkar.1@osu.edu): 1.3.9, 2.5.11

Shi, Mengze ; University of Toronto, Canada (mshi@rotman.utoronto.ca): 2.1.8

Shi, Yongjiang; Cambridge University, China (ys@eng.cam.ac.uk): 1.4.12

Shichang, Liu; Tsinghua University, China (59423361@qq.com): 3.4 .5

Shim, Yong J u; FGV-EAESP, Brazil (morangoum@naver.com): 3.1.10

Shimizu, Katsuhiko; Keio University, J apan (kshimizu22@gmail.com): 3.1.10

Shin, J iyoung; Korea University , Korea, South (alfolike@korea.ac.kr): 2.1.5, 3.3.13

Shin, Mannsoo; Korea University, Korea, South (shinms@korea.ac.kr): 2.4.7

Shin, Moonsik; Korea University, Korea, South (mshin@korea.ac.kr): 3.2.7

Shirodkar, Vikrant; University of Sussex, United Kingdom (v.shirodkar@sussex.ac.uk): 1.4 .8

Shirokova, Galina; Saint Petersburg University , Russia (shirokova@gsom.pu.ru): 1.3.10, 3.3.8

Shoham, Amir; Temple University, USA (amir.shoham@temple.edu): 1.3.9, 3.1.7

Shukla, Pallavi; Rutgers University, USA (pallavi.shukla@rutgers.edu): 2.4.12, 3.1.12

Siegel, J ordan; Harvard Business School, USA (jsiegel@hbs.edu): 3.1.7, 3.2.13

Sikarwar, Ekta ; Indian Institute of Management Indore, India (f10ektas@iimidr.ac.in): 3.4.10

Siltaoja, Marjo; University of Jyväskylä, Finland (marjo.siltaoja@jyu.fi): 2.4 .4

Sinding, Knud; University of Southern Denmark, Denmark (ksi@sam.sdu.dk): 2.4 .4

Singh, Damandeep; CDP (Carbon Disclosure Project), India (damandeep.singh@cdp.net): 1.1 .2

Singh, Deeksha ; Rutgers University, USA (deeksha.singh@rutgers.edu): 1.1.11, 2.5.12

Singh, Gurneeta Vasudeva; University of Minnesota, USA (gurneeta@umn.edu): 0.2.4

Singh, Harbir; University of Pennsylvania, USA (hsingh@wharton.upenn.edu): 2.2.11

Singh, Jasjit; I NSEAD, Singapore (jasjit.singh@insead.edu): 0.2.4, 2.4.4, 3.3.12, 3.4.13

Singh, Nivisha; Indian Institute of Management Indore, India (f12nivishas@iimidr.ac.in): 3.3.13

AIB 2015 Conference Proceedings

Page 272 
Singh, Ruhee; Indian Institute of Foreign Trade, India (ruheesingh18@gmail.com): 2.5.13

Singh, Satwinder; Brunel University London, United Kingdom (satwinder.singh@brunel.ac.uk): 1.4.4

Sinha, Ashutosh ; Indian Institute of Management Lucknow, India (ashutosh@iiml.ac.in): 2.5.4, 3.1.10

Sinkovics, Rudolf R.; University of Manchester, United Kingdom (rudolf.sinkovics@manchester.ac.uk): 1.5.8

Sita, Deepa; GIBS, University of Pretoria, South Africa (dssita10@gmail.com): 3.2 .6

Smale, Adam ; University of Vaasa, Finland (adam.smale@uwasa.fi): 3.3.12

Soares Terra, Paulo Renato; EAESP-FGV, Brazil (paulo.terra@fgv.br): 1.3.13, 2.2.10

Sofka, Wolfgang; Copenhagen Business School, Denmark (ws.smg@cbs.dk): 3.1 .4

Soh, Pek-Hooi; Simon Fraser University, Canada (psa28@sfu.ca): 2.4 .3

Somaya, Deepak; University of Illinois at Urbana-Champaign, USA (dsomaya@illinois.edu): 0.3.5, 3.2.7, 3.4.12

Some, Yirlier Hyacinthe; Universite de Sherbrooke, Canada (yirlier.hyacinthe.some@usherbrooke.ca): 2.2.7

Sonderegger, Petra; independent, India (petras@gmail.com): 2.5.2

Song, Chang; Renmin University of China, China (changsong@sina.com): 3.2.12

Song, J aeyong; Seoul National University, Korea, South (jsong@snu.ac.kr): 0.5.6, 2.3.2, 2.4.2, 3.1.2

Sono, Hui He; James Madison University, USA (sonohh@jmu.edu): 1.3.13

Sozuer, Aytug; Istanbul University, Turkey (aytugsozuer@gmail.com): 1.4.11

Spicer, Andrew; University of South Carolina, USA (aspicer@moore.sc.edu): 1.4 .11

Srikanth, Kannan; Singapore Management University, Singapore (ksrikanth@smu.edu.sg): 3.1 .3

Srinavasan, Suresh; Co-Founder, Formcept, India (suresh.srinivasan@formcept.com): 1.4 .2

Srinivasan, Vasanthi; Indian Institute of Management Bangalore, India (vasanthi@iimb.ernet.in): 1.1 .6

Srivastava, Manish K. ; Michigan Technological University, USA (mksrivas@mtu.edu): 3.1.1

Stallkamp, Maximilian; Ivey Business School, Canada (mstallkamp.phd@ivey.ca): 1.1.12

Stea, Diego; Copenhagen Business School, Denmark (ds.smg@cbs.dk): 2.1.12

Stefanidis, Abraham; St. John's University, USA (stefania@stjohns.edu): 2.4.7

Stephens Balakrishnan, Melodena; University of Wollongong in Dubai, United Arab Emirates

(melodenabalakrishnan@uowdubai.ac.ae): 0.5.6, 2.2.4

Sternquist, Brenda; Michigan State University, USA (sternqui@msu.edu): 2.1.8, 3.1.12, 3.2.8

Stevens, Charles Edward; Lehigh University, USA (ces213@lehigh.edu): 1.4.8, 3.4.13

Stoian, Maria-Cristina ; Brunel University London, United Kingdom (cristina.stoian@brunel.ac.uk): 3.4 .4

Storper, Michael; UCLA, USA (storper@ucla.edu): 1.4.1, 2.4.1

Strange, Roger; University of Sussex, United Kingdom (r.n.strange@sussex.ac.uk): 1.4.12

Su, Yiyi; Tongji University, China (suyiyi@tongji.edu.cn): 1.1.11

Su, Zhan; Laval University, Canada (zhan.su@mng.ulaval.ca): 1.5.9

Subramanyam, K.R.; University of Southern California, USA (krs@marshall.usc.edu): 0.4.2

Sud, Mukesh; Fairfield University, USA (mukesh.sud@gmail.com): 3.1.7

Sudarshan, Priyanka; WI PRO Limited, India: 2.3.3

Suder, Gabriele; University of Melbourne, Australia (gabriele.suder@unimelb.edu.au): 1.4.7, 2.3.3

Sugathan, Praveen; Indian Institute of Management Bangalore, India (praveen.s@iimb.ernet.in): 2.4.13

Sullivan Mort, Gillian; La Trobe University, Australia (g.sullivan-mort@latrobe.edu.au): 2.5.7

Sumelius, Jennie; Hanken School of Economics, Finland (jennie.sumelius@hanken.fi): 3.3.12

Sun, Qing Lu; Yonsei University, Korea, South (miracle_sql@hotmail.com): 1.3 .12

Supprakit, Sasiya ; Deloittte UK, United Kingdom (imshingy@gmail.com): 2.4.7

Suryaprakash, K.; I nfosys, India (suryks@infosys.com): 2.2.2

Sutherland, Dylan; Durham University, United Kingdom (dylan.sutherland@durham.ac.uk): 3.4 .5

Suwannarat, Pornlapas; Mahasarakham University, Thailand (pornlapas.s@mbs.msu.ac.th): 3.1.12

Swoboda, Bernhard; Trier University, Germany (b.swoboda@uni-trier.de): 2.4 .8

Syvrud, Kelsey Lynne; Florida State University, USA (kIs07f@my.fsu.edu): 1.1.5

Szymanski, Michal; University of Victoria, Canada (mikeszym@uvic.ca): 1.4.12

\section{$\mathbf{T}$}

Taarup-Esbensen, Jacob; Copenhagen Business School, Denmark (jta.ikl@cbs.dk): 3.3.11

Taimitarha, Susanna; Hanken School of Economics, Finland (susanna.taimitarha@hanken.fi): 3.3.12

Taleb, Ali; MacEwan University, Canada (taleba@macewan.ca): 1.3.9

Tallman, Stephen; University of Richmond, USA (stallman@richmond.edu): 1.1.12, 1.3.3, 3.1.1 
Tan, Hao; University of Newcastle, Australia (hao.tan@newcastle.edu.au): 1.3.4

Tan, Kun; Minjiang University, China (tankun@nbs.edu.cn): 2.2.11

Tang, J ing'an; Sacred Heart University, USA (tangj3@sacredheart.edu): 1.3.6, 3.2.11

Tang, Ryan; University of Technology Sydney, Australia (ryan.w.tang@gmail.com): 3.2.5

Tang, Yinuo; University of Pittsburgh, USA (yit14@pitt.edu): 1.4 .6

Tanganelli, David; Universitat Internacional de Catalunya, Spain (databer@uic.es): 1.3.12

Tao, Pan; Shandong University of Finance and Economics, China (angelatao0531@163.com): 3.3.3

Taras, Vasyl; University of North Carolina at Greensboro, USA (v_taras@uncg.edu): 1.3.7, 3.1.6, 3.4.3

Taube, Markus; University of Duisburg-Essen, Germany (markus.taube@uni-due.de): 2.3.4

Täube, Florian; Université libre de Bruxelles, Belgium (ftaube@ulb.ac.be): 1.4.12, 1.5.6, 2.5.2

Taussig, Markus; National University of Singapore, Singapore (markus@nus.edu.sg): 0.3.5, 1.4.3

Tavares-Lehmann, Ana Teresa; University of Porto, Portugal (atavares@fep.up.pt): 2.5.2

Tayar, Mark; Macquarie University, Australia (mark.tayar@mq.edu.au): 1.1.8, 3.2.3

Teegen, Hildy; University of South Carolina, USA (teegen@moore.sc.edu): 0.4 .2

Tellis, Gerard J .; University of Southern California, USA (tellis@usc.edu): 2.4 .8

Tenzer, Helene; Tuebingen University, Germany (helene.tenzer@uni-tuebingen.de): 3.2 .3

Thakur Wernz, Pooja; Virginia Tech University, USA (pthakur@vt.edu): 2.4.3, 2.5.3, 3.1.3

Thams, Yannick; Suffolk University, USA (ythams@suffolk.edu): 2.3.10

Thomke, Stefan; Harvard University, USA (sthomke@hbs.edu): 1.4 .5

Tien, Chengli; National Taiwan Normal University, Taiwan (cltien@ntnu.edu.tw): 2.1.5

Timmer, Marcel P.; University of Groningen, Netherlands (m.p.timmer@rug.nl): 2.1.7

Toh, Soo Min; University of Toronto, Canada (soomin.toh@utoronto.ca): 3.4 .9

Torkkeli, Lasse; Lappeenranta University of Technology, Finland (lasse.torkkeli@lut.fi): 1.4.13, 2.2.12

Torres, Miguel Matos; University of Aveiro, Portugal (miguel.torres@ua.pt): 1.5.12, 3.4.11

Torres Oliveira, Rui; Manchester Business School, United Kingdom (rui.oliveira@postgrad.manchester.ac.uk):

1.3.6, 3.4.11

Toulan, Omar Nohad; McGill University, Canada (omar.toulan@mcgill.ca): 3.2.7

Trapczynski, Piotr; Poznan University of Economics, Poland (piotr.trapczynski@ue.poznan.pl): 2.2.5, 2.3.12, 3.2.2

Trevino, Len J.; Loyola University New Orleans, USA (Itrevino@loyno.edu): 1.4.13

Tsai, Chih-Hao; National Sun Yat-sen University, Taiwan (billy.tsai88@gmail.com): 2.5.12

Tsai, Chin-J u; Royal Holloway, University of London, United Kingdom (chin-ju.tsai@rhul.ac.uk): 2.4 .7

Tsui, Tiffany S.W.; Erasmus University Rotterdam, Netherlands (ttsuimail@gmail.com): 2.1.10

Tsukada, Osamu; Kagawa University, Japan (tsukada@gsm.kagawa-u.ac.jp): 1.3.11

Tsukanova, Tatyana V.; Saint Petersburg University, Russia (tatyana_tsukanova@gmail.com): 1.3.10

Tu, Wenjun; University of Nottingham, Ningbo, China (zx06477@nottingham.edu.cn): 2.5.12

Tung, Rosalie; Simon Fraser University , Canada (tung@sfu.ca): 2.05, 2.1.4, 3.3.5

Tuomisalo, Teemu Kalevi J ohannes; J yväskylä University , Finland (tekajotu@student.jyu.fi): 3.4.12

Turkina, Ekaterina; HEC Montréal, Canada (ekaterina.turkina@hec.ca): 2.5.2

Turunen, Helena; HAMK University of Applied Sciences, Finland (helena.turunen@hamk.com): 2.2.12

Tzou, J oseph Shyh-Yeu; National Taiwan University, Taiwan (joesyłzou@gmail.com): 3.1.12

\section{$\mathbf{U}$}

Ueta, Toshimitsu; NUS Business School, Singapore (toshimitsu.ueta@u.nus.edu): 1.5.3

ul Haq, Hammad; Uppsala University, Sweden (hammad.haq@fek.uu.se): 2.1.12

Ullah, Barkat; Rhode Island College, USA (bullah@ric.edu): 2.4 .6

Un, C. Annique; Northeastern University, USA (a.un@neu.edu): 2.2.7, 2.4.12, 3.2.4

Upadhyayula, Rajesh; Indian Institute of Management Kozhikode, India (rajesh@iimk.ac.in): 2.3.13

Urbig, Diemo; University of Wuppertal, Germany (urbig@uni-wuppertal.de): 1.1.13 
Vaaler, Paul Martin; University of Minnesota, USA (vaal0001@umn.edu): 1.4.3, 2.1.5, 2.3.5, 2.4.4, 3.3.10

Vachani, Sushil; IIM Bangalore, India (sushilvachani@gmail.com): 2.5 .1

Vaidyanathan, R. ; Indian Institute of Management Bangalore, India (vaidya@iimb.ernet.in): 2.5.13

Van Assche, Ari; HEC Montréal, Canada (ari.van-assche@hec.ca): 1.1.10, 2.5.2

van de Vijver, Fons; Tilburg University, Netherlands (fons.vandevijver@uvt.nl): 1.5.11

van Ees, Hans ; University of Groningen, Netherlands (h.van.ees@rug.nl): 2.3.7, 2.4.9

Van Essen, Marc; University of South Carolina, USA (marc.vanessen@moore.sc.edu): 2.2.10

van Oort, Frank; Utrecht University, Netherlands (f.g.vanoort@geo.uu.nl): 3.1.10

van Veen, Kees; University of Groningen, Netherlands (k.van.veen@rug.nl): 2.3.7

van Witteloostuijn, Arjen; Tilburg University, Netherlands (a.vanwitteloostuijn@uvt.nl): 1.1.13

van Zalk, Maarten; Orebro University, Sweden (maarten.vanzalk@oru.se): 3.3 .12

Vang, Jan; Aalborg University, Denmark (jv@production.aau.dk): 1.1.10, 1.4.11

Vanninen, Heini; Lappeenranta University of Technology, Finland (heini.vanninen@lut. fi): 3.4.4

VanSandt, Craig; University of Northern Iowa, USA (craig.vansandt@uni.edu): 3.1.7

Varma, Sumati; Sri Aurobindo College (Eve), Delhi University, India (varmasumati@yahoo.co.in): 2.3.10

Varman, Rohit; Indian Institute of Management Calcutta, India (rohit@iimcal.ac.in): 0.4 .2

Varum, Celeste Amorim; University of Aveiro, Portugal (camorim@ua.pt): 1.5.12

Velez-Calle, Andres; Universidad EAFIT/Rutgers University, Colombia (avelezca@eafit.edu.co): 1.5.11, 2.2.11

Veloso, Francisco; Catolica Lisbon School of Business and Economics, Portugal (fveloso@ucp.pt): 1.3.5

Venkataramany, Sivakumar; Ashland University, USA (svenkata@ashland.edu): 2.3.12

Verbeke, Alain; University of Calgary, Canada (alain.verbeke@haskayne.ucalgary.ca): 2.1.6

Vijayaraghavan, Ravi; Vice President and Head Analytics, Flipkart.com, India: 1.4.2

Villarreal Gonzalez, Amado; Tecnolo' gico de Monterrey, Mexico (amado.villarreal@itesm.mx): 2.2 .9

Viswanathan, Siva; University of Maryland, USA (sviswana@rhsmith.umd.edu): 1.4.2, 2.2.2

Vives, Luis; ESADE, Spain (luis.vives@esade.edu): 0.2.4

Vo, Linh-Chi; Normandy Business School, France (Ivo@em-normandie.fr): 1.4 .4

Vogelgesang Lester, Gretchen ; San J ose State University, USA (gretchen.lester@sjsu.edu): 1.3.12

Vohra, Neharika; Indian Institute of Management Ahmedabad, India (neharika@iimahd.ernet.in): 3.2 .4

Vohra, Sanjeev ; Accenture Digital, India (tbd3@tbd.com): 2.2 .2

Volpe, Mario; Ca' Foscari University Venice, Italy (mvolpe@unive.it): 2.1.10

von Flotow, Paschen; Sustainable Business Institute, Germany (flotow@instoec.de): 1.4.12

Von Glinow, Mary Ann; Florida International University, USA (vonglino@fiu.edu): 2.5.11, 3.2 .11

von Zharen, Wyndylyn; Texas A\&M University, USA (dr_vonzharen@msn.com): 3.1.11

Vyas, Bindu; King's College, USA (binduvyas@kings.edu): 3.1.6

\section{W}

Wall, Ronald Sean; Erasmus University Rotterdam, Netherlands (wall@ihs.nl): 2.1.10

Walraven, Merel; Fontys University of Applied Sciences, Netherlands (m.walraven@fontys.nl): 2.3 .4

Walumbwa, Fred O.; Florida International University, USA (owalumbw@fiu.edu): 3.2.10

Wan, Zeying; Saint Mary's University, Canada (zeying.wan@smu.ca): 1.4.9

Wang, Dan; Monash University, Australia (dan.wang@monash.edu): 2.4.7

Wang, I. Kim; Suffolk University, USA (iwang@suffolk.edu): 3.3.9

Wang, Song; Zhejiang University, China (wasofei@zju.edu.cn): 2.4.11

Wang, Taiyuan; IE Business School, Spain (taiyuan.wang@ie.edu): 1.4 .9

Wang, Yinglei; Acadia University, Canada (yinglei.wang@acadiau.ca): 1.4.9

Wang, Yu-Kai (Mike); Soochow University, Taiwan (ywang012@scu.edu.tw): 1.4 .6

Wang, Zeyu; Nankai University/Chinese University of Hong Kong, China (wangze1789@vip.sina.com): 1.4.9

Wang, Zhan; Saint Louis University, USA (zhanwang@slu.edu): 2.4.13

Wang, Zhi ; Manchester Metropolitan University, United Kingdom (zhi.wang@mmu.ac.uk): 1.3.10, 2.2 .8

Wanjiru, Roseline; Northumbria University, United Kingdom (roseline.wanjiru@northumbria.ac.uk): 1.5 .9

Warner, Karl S.R.; Edinburgh Napier University, United Kingdom (k.warner@napier.ac.uk): 3.4 .4

Watanabel, Naoki ; Ritsumeikan University, Japan (n-wata@fc.ritsumei.ac.jp): 1.3.13

Wechtler, Heidi Marie; Macquarie University, Australia (heidiwechtler@gmail.com): 1.3.8 
Weerawardena, J ay; UQ Business School, Australia (j.weerawardena@uq.edu.au): 2.5.7

Wei, Liqun; Hong Kong Baptist University, China (weiliqun@hkbu.edu.hk): 2.4.10

Wei, Tian; Fudan University, China (weitian1983@gmail.com): 1.4.6, 1.5.10

Wei, William; MacEwan University, Canada (weix@macewan.ca): 1.4.11

Wei, Yingqi; University of Leeds, United Kingdom (y.wei@leeds.ac.uk): 2.1 .9

Wei, Zuobao; University of Texas at El Paso, USA (zwei@utep.edu): 2.4.6

Weisang, Guillaume; Clark University, USA (gweisang@clarku.edu): 1.1.13

Welter, Christopher; Xavier University, USA (welterc@xavier.edu): 2.3.8

Wen, Sonya H.; Tamkang University, Taiwan (sonya.wen@gmail.com): 2.3.10, 3.4.12

Wenger, Lucas; Florida International University, USA (Iweng004@fiu.edu): 3.3.10

Wernz, Christian; Virginia Tech University, USA (cwernz@vt.edu): 3.1.3

Westjohn, Stanford A.; University of Toledo, USA (standford.westjohn@utoledo.edu): 1.1.9

Whynacht, Christopher R.; University of Massachusetts Boston, USA (christopher.whyna001@umb.edu): 2.2 .9

Wieland, Jamie R.; Illinois State University, USA (jamie. wieland@ilstu.edu): 3.3.13

Wiersema, Margarethe; University of California, Irvine, USA (mfwierse@uci.edu): 2.3.10

Wijewardena, Nilupama; Monash University, Australia (nilupama.wijewardena@monash.edu): 1.1 .6

Witt, Michael; INSEAD, Singapore (michael.witt@insead.edu): 0.2.4, 1.4.3, 1.5.1

Witte, Caroline ; Erasmus University Rotterdam, Netherlands (witte@ese.eur.nl): 1.5.7

Wolff, Michael; Georg-August University of Goettingen, Germany (michael.wolff@wiwi.uni-goettingen.de):

3.2.11

Wood, Charles; University of Tulsa, USA (charles-wood@utulsa.edu): 1.5.13

Wood, Geoffrey; University of Warwick, United Kingdom (geoffrey.wood@wbs.ac.uk): 1.4.4, 2.5.6

Wood, Nicholas; Velocity Consulting, I ndia (india.nick@icloud.com): 1.5.13

Wright, Mike; Imperial College London, United Kingdom (mike.wright@imperial.ac.uk): 3.3.8, 3.4.8

Wu, Changqi; Peking University, China (topdog@gsm.pku.edu.cn): 0.5.6, 3.2.11

Wu, J ie; University of Macau, Macau (jiewu@umac.mo): 1.5.12, 2.4.3, 3.1.4

Wu, Sibin; University of Texas Pan American, USA (sibinwu@utpa.edu): 3.3 .8

Wu, Terry ; University of Ontario Institute of Technology, Canada (terry.wu@uoit.ca): 2.5 .10

Wu, Xiaobo; Zhejiang Universit, China (xbwu@zju.edu.cn): 1.4.12, 2.5.4

$\mathbf{X}$

Xavier, Wlamir; Eastern New Mexico University and UNISUL, USA (wlamirxavier@gmail.com): 1.1.7, 2.1.9, 2.3.7

Xie, Fujiao; University of Hawaii at Manoa, USA (fujiao@hawaii.edu): 1.1 .8

Xie, Guangbao; Bank of China, Anhui Province Branch, China (snoopy116@126.com): 1.3.12

Xie, Zhenzhen; Tsinghua University, China (xiezhzh3@sem.tsinghua.edu.cn): 3.2 .6

Xu, J ieying; Hong Kong Baptist University, Hong Kong, SAR-PRC (14484919@life.hkbu.edu.hk): 1.4.13, 2.3.9

$\mathrm{Xu}, \mathrm{J}$ in; Central University of Finance and Economics, China (xujin@cufe.edu.cn): 1.3.12

Xu, Kai; Texas A\&M University, China (kaixu@mays.tamu.edu): 2.1.6

Xue, Qiuzhi; Fudan University, China (qzxue@fudan.edu.cn): 2.1.8

Y

Yamada, Ichiro; St. Andrew's University, Japan (i-yamada@andrew.ac.jp): 1.3.11

Yamanoi, J unichi; Waseda University, J apan (junyamanoi@yahoo.co.jp): 1.5.12

Yang, Jae-Suk; Columbia University, USA (mathphy@gmail.com): 3.1.2

Yang, J iawen; George Washington University, USA (jwyang@gwu.edu): 1.3.13, 2.1.7, 3.2.12

Yang, J ie; Saint Louis University, USA (jieyang@slu.edu): 1.5 .8

Yang, Kuen-Shiou; Asia University, Taiwan (kyang@asia.edu.tw): 2.3.10

Yang, Xiaohua; University of San Francisco, USA (xyang14@usfca.edu): 1.5 .8

Yang, Yi; Yonsei University, Korea, South (yangyi0403@gmail.com): 2.5.11

Yayavaram, Sai; Indian Institute of Management Bangalore, India (sai.yayavaram@iimb.ernet.in): 1.5.2, 3.1.1

Ye, Qing; China Europe International Business School, China (qye@ceibs.edu): 2.2.10

Ye, Wendy; Nottingham University Business School China, China (wendy.ye@nottingham.edu.cn): 2.1.11

Yeh, Chih-Chuan; National Taiwan University, Taiwan (robert@ocu.edu.tw): 2.3.10

Yen, Su-Chuan; National Chung Hsing University, Taiwan (dora@yourcarbon.com.tw): 2.5.10

AIB 2015 Conference Proceedings

Page 276 
Yeravdekar, Vidya; Symbiosis International University, India (dr-vidya@symbiosis.ac.in): 2.5.1

Yeung, Bernard; National University of Singapore, Singapore (bizdean@nus.edu.sg): 2.5 .1

Yi, J iangling; Nanjing University, China (yijiangling@smail.nju.edu.cn): 3.2.10

Yie, Xiaoqian; Wuhan University, China (00200653@whu.edu.cn): 2.2.8

Yildiz, H. Emre; Uppsala University, Sweden (emre.yildiz@fek.uu.se): 1.3.3

Yim, Sohyun; Seoul National University, Korea, South (sohyunyim@gmail.com): 2.1.11

Yin, Wenyan; Seoul National University, Korea, South (wenyanyin2012@gmail.com): 2.1.11

Yoshikawa, Katsuhiko; London School of Economics and Political Science, United Kingdom

(k.yoshikawa@lse.ac.uk): 2.2.8, 3.4.8

Young, Susan L; Seton Hall University, USA (susan.young@shu.edu): 2.3.8

Yu, Chwo Ming; National Chengchi University, Taiwan (yu54@nccu.edu.tw): 3.4 .12

Yu, Pei ; Wuhan University of Technology , China (peguyvincent@hotmail.com): 3.3.3

Yu, Pei-Li; National Cheng Kung University, Taiwan (r4895113@mail.ncku.edu.tw): 2.3.10, 3.2.8

Yu, Yang; Hong Kong Baptist University, China (irinayu1120@163.com): 1.4.13, 2.3.9

Yuan, Lin; University of Macau, Macau (linyuan@umac.mo): 2.3.7, 2.4.11, 2.5.3

Yukimoto, Seiki; Kanagawa University, Japan (yukimoto@kanagawa-u.ac.jp): 3.4.7

Yunlu, Dilek Gulistan; Northeastern Illinois University , USA (d-yunlu@neiu.edu): 1.3.12, 2.2.8

\section{Z}

Zaheer, Akbar; University of Minnesota, USA (azaheer@umn.edu): 3.3.2

Zaheer, Sri; University of Minnesota, USA (szaheer@umn.edu): 0.4.2, 0.71.1

Zander, Ivo; Uppsala University, Sweden (ivo.zander@fek.uu.se): 2.1.1, 2.3.11

Zdziarski, Michael; University of Warsaw, Poland (mzdziarski@wz.uw.edu.pl): 2.2 .5

Zellmer-Bruhn, Mary; University of Minnesota, USA (zellm002@umn.edu): 3.2 .3

Zhang, Guangxi; Zhejiang University of Technology, China (gxzhang@zjut.edu.cn): 3.2 .3

Zhang, Hua; CEIBS, China (zhua@ceibs.edu): 3.4 .2

Zhang, Jialiang; Renmin University of China, China (ruczjl123@163.com): 3.3.11

Zhang, J ie; China Huarong Asset Management Co., Ltd., China (jacqueline35@sina.com): 3.2.12

Zhang, Man; Bowling Green State University, USA (mzhang@bgsu.edu): 0.5.6, 2.2.12

Zhang, Megan (Min); I vey Business School, Canada (mzhang.phd@ivey.ca): 1.5.8, 2.2.11

Zhang, Miao; Kingston University, United Kingdom (miao.zhang@kingston.ac.uk): 2.2.7

Zhao, John; Saint Louis University, USA (zhaox@slu.edu): 1.1.7, 1.5.8

Zhao, Meng; Renmin University of China, China (mengzhao521@yahoo.com): 3.2.11

Zhao, Youzhen; Fudan University, China (yzzhao@fudan.edu.cn): 1.5.10

Zhao, Yue; Florida International University, USA (yzhao028@fiu.edu): 2.5.11, 3.3.10

Zheng, Xiaolan; University of Nottingham, Ningbo, China (xiaolan.zheng@nottimgham.edu.cn): 2.5.12

Zheng, Yao; Nottingham University Business School China, China (zx14549@nottingham.edu.cn): 2.1.11

Zheng, Zunxin; Shenzhen University, China (zxzheng@szu.edu.cn): 2.1.7

Zhou, Abby Jingzi ; University of Nottingham, Ningbo, China (jingzi.zhou@nottingham.edu.cn): 1.4.10

Zhou, J ieyu; Hong Kong University of Science and Technology, Hong Kong, SAR-PRC (jyzhouaa@ust.hk): 1.4.13

Zhou, Lianxi; Brock University, Canada (Izhou@brocku.ca): 1.3.12

Zhou, Xiaoyu; ShanghaiTech University, China (zhouxy@shanghaitech.edu.cn): 2.4 .11

Zhu, Cherrie; Monash University, Australia (cherrie.zhu@monash.edu): 1.5.11, 2.4.7

Zhu, Hongjin; McMaster University, Canada (zhuhongjin@mcmaster.ca): 3.3 .2

Zhu, PengCheng; University of San Diego, USA (pzhu@sandiego.edu): 2.1.6

Zhuo, Shuaihe; University of Macau, Macau (svicjo@gmail.com): 2.4.3, 3.1.4

Zink, Trevor; Loyola Marymount University, USA (tzink@lmu.edu): 1.5.7

Zou, J ie; Peking University, China (zoujie@pku.edu.cn): 3.2.11

Zou, Xi; London Business School, United Kingdom (czou@london.edu): 2.4.10

Zschoche, Ulrike; Georg-August University of Goettingen, Germany (ulrike.zschoche@wiwi.uni-goettingen.de):

3.2.11 
Proceedings of the

$57^{\text {th }}$ Annual Meeting

of the

Academy of International Business

"Global Networks: Organizations and People"

Bengaluru, India

June 27-30, 2015

ISSN: 2078-0435

(C) 2015 Academy of International Business

For more information, please contact:

AIB Executive Secretariat

G. Tomas M. Hult, Executive Director, or

Tunga Kiyak, Managing Director

Eppley Center

465 N. Shaw Ln Rm 7

Michigan State University

East Lansing, MI 48824, USA

Phone: +1 (517) 432-1452 • Fax: +1 (517) 432-1009

E-mail: aib@aib.msu.edu •Web: http://aib.msu.edu/ 